\title{
FINITE GROUPS WITH QUASI-DIHEDRAL AND WREATHED SYLOW 2-SUBGROUPS
}

\author{
BY \\ J. L. ALPERIN( $\left.{ }^{(}\right)$, RICHARD BRAUER( $\left.{ }^{2}\right)$ AND DANIEL GORENSTEIN $\left({ }^{3}\right)$
}

\begin{abstract}
The primary purpose of this paper is to give a complete classification of all finite simple groups with quasi-dihedral Sylow 2-subgroups. We shall prove that any such group must be isomorphic to one of the groups $L_{3}(q)$ with $q \equiv-1(\bmod 4)$, $U_{3}(q)$ with $q \equiv 1(\bmod 4)$, or $M_{11}$. We shall also carry out a major portion of the corresponding classification of simple groups with Sylow 2-subgroups isomorphic to the wreath product of $Z_{2^{n}}$ and $Z_{2}, n \geqq 2$.
\end{abstract}

\section{TABle OF CONTENTS}

\section{ChAPTER I. INTRODUCtion}

Chapter II. Groups WITH QUASI-DiHedRal OR WREATHED Sylow 2-SUbGroups

1. Fusion of 2-elements

2. $Q$-groups, $D$-groups, and $Q D$-groups

3. The characteristic power of $Q$-groups, $D$-groups, and $Q D$-groups

4. $Q$-groups with quasi-dihedral or wreathed Sylow 2-subgroups

5. D-groups

6. $p$-constraint and $p$-stability

7. An extension of Glauberman's $Z J$-theorem

8. Miscellaneous results

Chapter III. Character theory of Groups with Quasi-dihedral Sylow 2-subgroups

1. Introduction

2. The principal 2-block

3. Regular $Q D$-groups

4. Proof of Theorem A

5. Subsections associated with a 2-block of full defect

6. Continuation. Blocks of full defect of type $\left({ }^{*}\right)$ and the corresponding decomposition numbers

7. Formulas for the order of $G$

8. $Q D$-groups of small characteristic power

9. Various results concerning $Q D$-groups

Received by the editors August 4, 1969.

AMS Subject Classifications. Primary 2027, 2029.

Key Words and Phrases. Quasi-dihedral, wreathed, $Q$-group, $D$-group, $Q D$-group, regular group.

(1) Supported in part by National Science Foundation grant GP-8425.

(2) Supported in part by Air Force Office of Scientific Research grant AF-AFOSR-1468-68 and National Science Foundation grant GP-6432.

( ${ }^{3}$ ) Supported in part by Air Force Office of Scientific Research grant AF-AFOSR-1468-68 and National Science Foundation grant GP-9314.

Copyright (C) 1970, American Mathematical Society 
Chapter IV. The structure of $O(N)$. Localization

1. Preliminary discussion of Theorem B

2. Stationary nonconstrained primes

3. Proof of Theorem 1.1

4. Structure of $L_{0}$

5. Reduction to local subgroups

6. Properties of the elements of $\mathscr{L}(p)$

7. A subgroup of $C$

8. A second family of local subgroups

9. Constraint for elements of $\mathscr{M}^{*}(p)$

Chapter V. The structure of $O(N)$. Proof of Theorem B*

1. Outline of chapter

2. Elimination of the first set of primes

3. A family of cyclic subgroups

4. Properties of signalizers

5. Construction of a certain signalizer

6. Elimination of the second set of primes

7. The structure of $N$

8. An omnibus lemma for signalizers

9. A subgroup which is not a p-group

10. A transitivity theorem

11. Elimination of the last set of primes

Chapter Vi. The structure of $N$. Proof of Theorem C

1. Outline of chapter and some consequences of Theorem $B$

2. Preliminary results

3. Elimination of the case $O(N)=1$

4. Elimination of the case $A=1, B \neq 1$

5. The case $A=1, B \neq 1$ (continued)

6. Double transitivity of $G$ in the case $\varepsilon=1$

7. The value of $d$ in the case $\varepsilon=1$

\section{Chapter I. Introduction}

The object of this paper is to carry out a complete classification of simple groups with quasi-dihedral Sylow 2-subgroups and a major portion of the classification of simple groups with wreathed Sylow 2-subgroups. Recall that a group of order $2^{n+1}, n \geqq 3$, defined by generators $z, s$ and relations

$$
z^{2}=s^{2^{n}}=1, \quad s^{z}=s^{-1+2^{n-1}},
$$

is called quasi-dihedral, while a group of order $2^{2 n+1}, n \geqq 2$, defined by generators $z, s, t$ and relations

$$
z^{2}=s^{2^{n}}=t^{2^{n}}=1, \quad s^{z}=t, \quad t^{z}=s, \quad s t=t s,
$$

is said to be wreathed.

The only simple groups known with such Sylow 2-subgroups are $L_{3}(q)$ $(=\operatorname{PSL}(3, q)), U_{3}(q)(=P S U(3, q))$ for $q$ odd and $M_{11}$. More specifically,

$$
\begin{array}{lll}
L_{3}(q), & q \equiv-1 & (\bmod 4), \\
U_{3}(q), & q \equiv 1 & (\bmod 4), \\
M_{11} & &
\end{array}
$$


have quasi-dihedral Sylow 2-subgroups, while

$$
\begin{array}{lll}
L_{3}(q), & q \equiv 1 & (\bmod 4), \\
U_{3}(q), & q \equiv-1 & (\bmod 4),
\end{array}
$$

have wreathed Sylow 2-subgroups.

The methods used to study simple groups in this paper are primarily of two kinds: local group-theoretic and character-theoretic. It happens that it is possible to give a unified approach to the group-theoretic material for both the quasidihedral and wreathed cases. On the other hand, the character-theoretic results which are needed require separate analysis for each case. Therefore, we shall deal with both types of Sylow 2-subgroups simultaneously in the group-theoretic arguments. In this paper we shall present the character theory only for the quasidihedral case. Many of the analogous results in the wreathed case have already been established, while work on the remaining ones is in progress. These results in the wreathed case will all appear in a separate paper by one of the authors. We shall therefore proceed to outline and explain the contents of this paper as pertains to groups with quasi-dihedral Sylow 2-subgroups. We shall also make comments concerning the group-theoretic results in the wreathed case which we obtain and concerning the character-theoretic results in that case already established but not included in this paper.

The primary end of this paper is the establishment of the following results:

First MAIN THEOREM. If $G$ is a simple group with quasi-dihedral Sylow 2subgroups and $x$ is an involution of $G$, then $C(x)$ is isomorphic to a quotient of either

or

$$
G L(2, q), \quad q \equiv-1 \quad(\bmod 4)
$$

$$
G U(2, q), \quad q \equiv 1(\bmod 4)
$$

by a central subgroup of odd order $d$.

Since all the involutions in such a simple group are conjugate, the number $q$ depends only on $G$; it is called the characteristic power of $G$. Similarly, the number $d$ depends only on $G$.

SECOND MAIN THeORem. If $G$ is a simple group with quasi-dihedral Sylow 2subgroups, of characteristic power $q$ and $q \equiv \varepsilon(\bmod 4), \varepsilon= \pm 1$, then either

$$
|G|=\frac{1}{d} q^{3}\left(q^{3}+\varepsilon\right)\left(q^{2}-1\right)
$$

or

$$
|G|=7920 .
$$

THIRD MAIN THeOREM. If $G$ is a simple group with quasi-dihedral Sylow 2subgroups and of characteristic power $q$, then one of the following holds:

(i) $q \equiv-1(\bmod 4)$ and $G$ is isomorphic with $L_{3}(q)$ or $M_{11}$;

(ii) $q \equiv 1(\bmod 4)$ and $G$ is isomorphic with $U_{3}(q)$. 
With respect to the wreathed case, we shall discuss later in this chapter how far this paper goes towards proving the analogous main theorems.

We shall now present a brief outline of the contents of the paper, dealing with the chapters in turn. We shall then show how the results achieved in those chapters lead directly to the three main theorems. Finally, we conclude with a list of some nonstandard notation.

Chapter II is devoted to a study of groups whose Sylow 2-subgroups are isomorphic with a section of a quasi-dihedral or wreathed 2-group. This is in preparation for the local group-theoretic analysis to be applied to the proper subgroups of the simple group $G$ under study. Moreover, in this chapter we go only so far as previous classification theorems and extension theory allow us. The subgroups of $G$ fall into four types: there are groups which have normal 2-complements and there are what we shall call $D$-groups, $Q$-groups, and $Q D$-groups. For example, if $H$ is a group of the last sort, then $H$ has quasi-dihedral or wreathed Sylow 2subgroups and has exactly one conjugacy class of involutions and no normal subgroup of index two. It follows that $H / O(H)$ has a normal simple subgroup of odd index. Here, as throughout the paper, $O(H)$ denotes the largest normal subgroup of odd order of the group $H$.

Moreover, in this chapter, we shall derive some preliminary information about $N=$ $C(x)$, where $x$ and $G$ are as in the First Main Theorem. In fact, $N /\langle x\rangle$ is a group with dihedral Sylow 2-subgroups so that the classification theorem of GorensteinWalter [22] may be applied. This yields that $N$ has a series of normal subgroups

$$
N \supseteq L \supset O(N) \supseteq 1
$$

with the following properties:

(i) $O(N)$ is the largest normal subgroup of odd order in $N$;

(ii) $L / O(N)$ is isomorphic to $S L^{ \pm}(2, q)$ with $q \equiv-1(\bmod 4)$ (the group of matrices of determinant +1 or -1$)$ or to $S U^{ \pm}(2, q)$ with $q \equiv 1(\bmod 4)$ (the group of unitary matrices of determinant +1 or -1 );

(iii) $N / L$ is cyclic of odd order.

In particular, $L / O(N)$ has a normal subgroup $S(N) / O(N)$ of order two with $S(N)=\langle O(N), x\rangle$ and it has a normal subgroup $L_{0} / O(N)$ of index two. Moreover, the quotient $L_{0} / S(N)$ is then isomorphic to $L_{2}(q)$. This is all best understood by means of the following diagram:

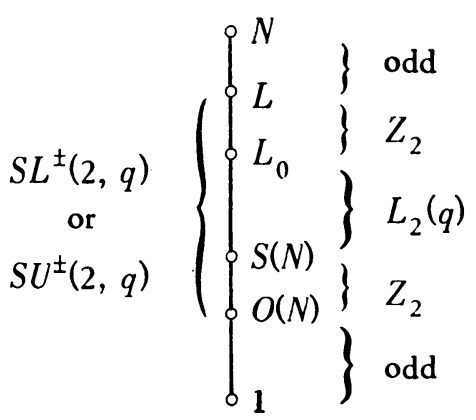


The First Main Theorem simply states that $N=L$ and that $O(N)$ is central in $N$ and cyclic of order dividing $q+\varepsilon$, where $q \equiv \varepsilon(\bmod 4), \varepsilon= \pm 1$.

In any case this result gives us an important invariant of $G$, namely the number $q$ which we shall call the characteristic power of $G$. It is an invariant of $G$ as $G$ does have just one class of involutions.

The same result also is achieved in the case of wreathed Sylow 2-subgroups; in fact, if $G$ is a simple group of such type, $x$ is an involution of $G$ and $N=C(x)$, then $N$ has a series of normal subgroups $N \supseteq L \supset O(N) \supseteq 1$, where $|N: L|$ and $|O(N)|$ are odd and $L / O(N)$ is isomorphic to the subgroup of matrices, whose determinant has order a power of two, of $G L(2, q), q \equiv 1(\bmod 4)$, or $G U(2, q), q \equiv-1(\bmod 4)$. In this case the center $S(N) / O(N)$ of $L / O(N)$ is cyclic of order $2^{n}$, if the Sylow 2subgroups of $G$ have order $2^{2 n+1}$.

The characteristic power can be defined for many subgroups of $G$. For example, if $H$ is a $Q D$-subgroup of $G$ (as described above) and $K / O(H)$ is the normal simple subgroup of odd index in $H / O(H)$, then $K / O(H)$ has a characteristic power, as we may see by applying the above arguments. We call this the characteristic power of $H$; it may also be described by looking directly at the centralizer of involutions in $H$.

The third chapter develops the basic character-theoretic results for the case of groups $G$ with quasi-dihedral Sylow 2-subgroups. As noted above, most of the analogous results have been proved for the wreathed case as well and will appear in a subsequent paper.

As applications of this knowledge we classify all the simple groups $G$ with quasi-dihedral Sylow 2-subgroups of characteristic power three or five; we deal with these two special cases separately since the general group-theoretic arguments break down in these particular circumstances.

Furthermore, in this chapter, there is developed the usual type of group order formulae which express the order of $G$ in terms of the structure of the centralizer of an involution and the degrees of certain characters of $G$.

Another important concept is also introduced; we describe it now. Let $H$ be any $Q D$-subgroup of $G$ (in particular, even $G$ itself). Let $x$ be an involution of $H, T$ a four subgroup of $H, N=C_{H}(x)$ and $C=C_{H}(T)$. If $H$ has characteristic power $q$ and $p$ is the prime divisor of $q$, then we say that $H$ is regular provided that

$$
|H|_{p}\left(|C|_{p}\right)^{2} \geqq\left(|N|_{p}\right)^{3}
$$

The groups $L_{3}(q), q \equiv-1(\bmod 4)$, and $U_{3}(q), q \equiv 1(\bmod 4)$, are regular, while $M_{11}$ is not. Thus if $G$ satisfies the conclusions of the Main Theorems and $q \neq 3$, then $G$ is regular.

This concept can now be used in several ways. First, if $G$ is simple and regular then the degrees of the characters appearing in the group order formulae can be determined; they are polynomials in $q$. In particular, the order of $G$ is then completely determined by the structure of the centralizer of an involution. 
Second, the following basic result is proved:

THEOREM A. If $H$ is a regular $Q D$-subgroup of the $Q D$-group $G$ and $H$ and $G$ have the same characteristic power, then $G$ is regular and $O(H)=H \cap O(G)$.

We shall use this when $G$ is simple so that $O(G)=1$. This yields a fundamental restriction on the nature of the local subgroups of $G$.

The next two chapters are devoted to the bulk of the group-theoretic arguments and achieve a proof of the following:

THEOREM B. Let $G$ be a simple group with quasi-dihedral Sylow 2-subgroups and of characteristic power $q$ greater than five. If every proper simple section of $G$ with quasi-dihedral Sylow 2-subgroups satisfies the conclusions of the Main Theorems and $N$ is the centralizer of an involution of $G$, then

(i) $O(N)=A B$, where $A$ is a cylic subgroup of order dividing $(q+\varepsilon) / 2(q \equiv \varepsilon$ $(\bmod 4), \varepsilon= \pm 1), B$ is an abelian subgroup and $O(N)$ is a Frobenius group with complement $A$ and kernel $B$ provided $A \neq 1$ and $B \neq 1$.

(ii) Any noncentral involution of $N$ inverts $B$ and centralizes some conjugate of $A$.

(iii) The extended centralizer $C^{*}(u)$ lies in $N$ for any nonidentity element $u$ of $O(N)$.

This result also has as a consequence that $L_{0}$, as defined above, is the direct product of $O(N)$ and a subgroup isomorphic with $S L(2, q)$ if $q \neq 9$, in which case there is another possible structure for $L_{0}$ as $S L(2,9)$ has a nontrivial central extension by a group of order three.

For the case of a simple group $G$ with wreathed Sylow 2-subgroups the analogous result is proved provided one also assumes that the obvious analogue of Theorem A holds for $G$. In these two chapters we deal with both types of Sylow 2-subgroups at the same time.

The sixth and final chapter is devoted to the proof of the last basic theorem. We use the above notation.

THEOREM C. Let $G$ be a simple group with quasi-dihedral Sylow 2-subgroups and of characteristic power $q$ greater than five. If, moreover, $G$ fulfills all the conclusions of Theorem B, then

(i) $N=L$;

(ii) $B=1$;

(iii) $G$ is regular;

(iv) If $q \equiv 1(\bmod 4)$, then $d=(q+1,3)$ and $G$ is doubly transitive of degree $q^{3}+1$ such that the subgroup $H$ fixing a letter has a normal subgroup $Q$ of order $q^{3}$, which acts regularly on the set of remaining $q^{3}$ letters, with $H / Q$ cyclic of $\operatorname{order}\left(q^{2}-1\right) / d$.

The proof of this theorem is primarily character-theoretic, but does involve some purely group-theoretic arguments. 
We shall now prove that the three Main Theorems hold provided we assume that all the other results stated above are valid. Let $G$ be a simple group with quasidihedral Sylow 2-subgroups. We may assume without loss of generality that the Main Theorems hold for any simple group with quasi-dihedral Sylow 2-subgroups whose order is less than that of $G$. We let $q$ be the characteristic power of $G$. If $q$ is three or five, then by the results of Chapter III, $G$ is isomorphic with $L_{3}(3), M_{11}$ or $U_{3}(5)$ and so the Main Theorems hold for $G$, as is easily verified.

On the other hand, if $q$ is greater than five, then the hypotheses of Theorem B are fulfilled by $G$ inasmuch as every proper simple $Q D$-section of $G$ has lower order than $G$ and so $G$ satisfies all the conclusions of that result. Thus, the hypotheses of Theorem $\mathrm{C}$ are fulfilled and so $G$ also satisfies the conclusions of that theorem. In the notation of these results, $O(N)$ is a central cyclic subgroup of order $(q+\varepsilon) / 2 d$, for some odd number $d$, and $N / O(N)$ is isomorphic with $S L^{ \pm}(2, q)$ or $S U^{ \pm}(2, q)$, as the case may be. By results of Chapter IV, mentioned just after the statement of Theorem B, it follows that $N$ is the direct product of a group isomorphic with $N / O(N)$ and the subgroup $A$. However, this is precisely the statement of the First Main Theorem.

As for the Second Main Theorem, $G$ is regular by Theorem C, so by the results of Chapter III discussed above, there is a formula for the order of $G$ in terms of the structure of $N$. However, we also know that $N=L$ and $B=1$, so the parameters $e, a, b$ of that formula satisfy $e=1, a=|A|$, and $b=1$ and the Second Main Theorem is valid.

We now verify the Third Main Theorem. If $q \equiv-1(\bmod 4)$, then the hypotheses of a theorem of Brauer [8] hold since $G$ does satisfy the First Main Theorem. Thus, $G$ is isomorphic with $L_{3}(q)$ or $M_{11}$ by the results of that paper. On the other hand, if $q \equiv 1$ (mod 4), part (iv) of Theorem $C$ shows that $G$ fulfills all the hypotheses of a theorem of O'Nan [24*] and it follows from his results that $G$ is isomorphic to $\operatorname{PSU}(3, q)=U_{3}(q)$. In the case $d=1$, this result was previously obtained by Suzuki [28].

In order to prove corresponding results in the wreathed case, it will be necessary to publish the theorems which are the analogues of most of the results of Chapter III as well as to complete the proof of the analogue to Theorem $\mathrm{C}$.

The notation used is standard, with the following exception. If $X$ and $Y$ are subgroups of the group $H$ and $p$ is a prime then

$$
\eta_{H}(X, Y ; p)
$$

is the collection of all elements of $\eta_{H}(X ; p)$ (which is the set of $p$-subgroups of $H$ intersecting $X$ trivially and normalized by $X$ ) which are also permutable with $Y$. The set of maximal elements of $И_{H}(X, Y ; p)$ is denoted by $И_{H}^{*}(X, Y ; p)$.

It will also be convenient to introduce the so-called "bar" convention: If $\bar{H}$ is a homomorphic image of the group $H$ and if $X$ is a subgroup, subset, or element of $H$, then $\bar{X}$ will always denote the image of $X$ in $\bar{H}$. 
Finally, we repeatedly use the fact that a group of odd order is necessarily solvable, without making explicit reference to the theorem of Feit and Thompson [15].

We add a word concerning the numbering system to be used in the paper: Lemma $\mathrm{x}$ of section $\mathrm{y}$ of Chapter $\mathrm{z}$ is designated as Lemma $\mathrm{x}$ throughout the given section $y$, as Lemma $y . x$ in all subsequent sections of Chapter $z$, and as Lemma z.y.x in all subsequent chapters. A similar convention applies for propositions, theorems, and corollaries with the exception of Theorems A, B, and C which are denoted only in this one way.

Chapter II. Groups WITH QUASI-DIHEDRAL OR WREATHED Sylow 2-SUbGroups

1. Fusion of 2-elements. In this section we shall give a complete analysis of the possible types of fusion of 2-elements in a group $G$ with quasi-dihedral or wreathed Sylow 2-subgroups. Apart from the case that $G$ has a normal 2-complement, it will turn out that the fusion pattern in $G$ can be of any one of exactly three distinct types. Corresponding to each of these, we shall introduce the concept of a $Q D$ group, a $Q$-group, and a $D$-group. These three types of groups will play an essential role throughout the paper. In succeeding sections of this chapter we shall derive a very large number of properties of these groups which we shall need for the proof of the Main Theorems.

Let us first indicate by a simple table the four possible types of fusion of 2elements in a group $G$ with quasi-dihedral or wreathed Sylow 2-subgroup $S$. As we shall see, $S$ has exactly one conjugacy class of both maximal noncyclic abelian subgroups and of quaternion subgroups. If $U$ and $V$ denote representatives of these two conjugacy classes respectively, the fusion of 2-elements is completely determined by the structure of $N(U) / C(U)$ and $N(V) / V C(V)$. In the following table, $Z_{2}$ and $\Sigma_{3}$ denote respectively a cyclic group of order two and the symmetric group of degree three.

\begin{tabular}{|c|c|c|c|c|c|}
\hline \multirow[b]{2}{*}{$N(U) / C(U)$} & \multirow[b]{2}{*}{$N(V) / V C(V)$} & \multicolumn{2}{|c|}{$\begin{array}{l}\text { Number of Conjugacy Classes } \\
\text { in } G \text { of Involutions }\end{array}$} & \multicolumn{2}{|c|}{ Sylow 2-Subgroup of $G^{\prime}$} \\
\hline & & Quasi-Dihedral & Wreathed & Quasi-Dihedral & Wreathed \\
\hline$Z_{2}$ & $Z_{2}$ & 2 & 3 & Cyclic & Cyclic \\
\hline$Z_{2}$ & $\Sigma_{3}$ & 2 & 2 & $\begin{array}{l}\text { Generalized } \\
\text { Quaternion }\end{array}$ & $\begin{array}{l}\text { Generalized } \\
\text { Quaternion }\end{array}$ \\
\hline$\Sigma_{3}$ & $Z_{2}$ & 1 & 2 & Dihedral & $\begin{array}{l}\text { Noncyclic } \\
\text { Abelian }\end{array}$ \\
\hline$\Sigma_{3}$ & $\Sigma_{3}$ & 1 & 1 & Quasi-Dihedral & Wreathed \\
\hline
\end{tabular}

In Propositions 1 and 2 below we shall give a more detailed description of the fusion in each of these four cases. We note that in the first case, $G$ will have a 
normal 2-complement. Cases two, three, and four will lead to what we shall call respectively a $Q$-group, a $D$-group, or a $Q D$-group.

Before establishing these results, we shall give three lemmas which list a large number of basic properties of quasi-dihedral and wreathed 2-groups which will be used throughout the paper. However, only a portion of these are needed for the specific problem of analyzing fusion of 2-elements.

LEMMA 1. If $S$ is quasi-dihedral of order $2^{n+1}$, then the following conditions hold:

(i) S has two conjugacy classes of involutions and of elements of order 4.

(ii) $S$ has one conjugacy class of four subgroups and of quaternion subgroups. If $T$ is a four subgroup or a quaternion subgroup of $S$, then $C_{S}(T)=Z(T)$ and $\left|N_{S}(T): T\right|=2$.

(iii) $S$ possesses precisely three maximal subgroups, respectively cyclic, generalized quaternion $\left({ }^{4}\right)$, and dihedral.

(iv) $S^{\prime}=\Phi(S)$ is cyclic of order $2^{n-1}$.

(v) $Z(S)$ has order 2 and $S / Z(S)$ is dihedral.

(vi) $\Omega_{1}(S)$ is dihedral of order $2^{n}$.

(vii) A proper normal subgroup of $S$ is either maximal or is cyclic and contained in $S^{\prime}$.

(viii) If $D$ is a dihedral subgroup of $S$ of order at least 8 , then the maximal cyclic subgroup of $D$ is contained in the maximal cyclic subgroup of $S$.

Proof. These results follow directly from the definition of $S$ given in $§ 1.1$. The details are left to the reader. Compare also Theorem 5.4.3 and Exercise 7.6 of [19].

We list the analogous properties for wreathed 2-groups in a more explicit form. Let then $S$ be a wreathed 2-group, given by generators $s, t, z$ satisfying the relations of the Introduction. We first introduce some additional notation:

$$
\begin{gathered}
u=s t, \quad r=s t^{-1}, \quad x=x_{1}=u^{2^{n-1}}=r^{2^{n-1}}, \quad x_{2}=s^{2^{n-1}}, \quad x_{3}=t^{2^{n-1}}, \quad d=x_{2} z, \\
U=\langle s, t\rangle, \quad T=\left\langle x_{1}, x_{2}, x_{3}\right\rangle, \quad T_{0}=\left\langle x_{1}, z\right\rangle, \quad Y=\langle r, d\rangle .
\end{gathered}
$$

With this notation, we have

LEMMA 2. If $S$ is wreathed of order $2^{2 n+1}$, then the following conditions hold:

(i) $S$ has three conjugacy classes of involutions, represented respectively by $x, x_{2}$, and $z$.

(ii) $U$ is an abelian maximal subgroup of $S$ of type $\left(2^{n}, 2^{n}\right)$ and is the unique abelian subgroup of its order in $S$.

(iii) $T=\Omega_{1}(U)$ is a four group and is normal in $S$.

(iv) $T_{0}$ is a four group and is not conjugate to $T$. Every four subgroup of $S$ except $T$ is conjugate to $T_{0}$.

(v) $Y$ is generalized quaternion of order $2^{n+1}, Y$ is normal in $S$ and is the unique generalized quaternion subgroup of its order in $S$.

$\left.{ }^{4}\right)$ We use the term generalized quaternion to include the quaternion group of order 8 . 
(vi) $S^{\prime}=\langle r\rangle$ is cyclic of order $2^{n}$.

(vii) $Z(S)=\langle u\rangle$ is cyclic of order $2^{n}$ and $S / Z(S)$ is dihedral.

(viii) $\Omega_{1}(S)=T Y$ and $S / \Omega_{1}(S)$ is cyclic.

(ix) $Y$ is the normal closure of $\langle d\rangle$ in $S$.

(x) $(s z)^{2}=u$ and $S=\langle Y, s z\rangle$.

(xi) If $X$ is a proper subgroup of $S$ with $X \supset Y$, then $X=Y Z(X)$ and $X$ contains $T$.

(xii) If $X$ is a nonabelian subgroup of $S$, then $Z(X) \subseteq Z(S)$.

(xiii) If $X$ is a subgroup of $S$ invariant under a cyclic subgroup $A$ of $S$ of order $2^{n}$ with $A$ disjoint from $X$, then either $X$ is cyclic or $S=X A$.

Proof. These properties of $S$ are a straightforward consequence of its definition and are left to the reader to verify.

Our last lemma, whose proof is also left to the reader, will be used in the analysis of 2-fusion which is to follow.

LEMMA 3. If $S$ is a wreathed 2-group of order $2^{2 n+1}$ and $X$ is a subgroup of $S$ such that $C_{S}(X) \subseteq X$ and Aut $(X)$ is not a 2-group, then

(i) Either $X=U$ or $X$ is isomorphic to a central product of a quaternion group with $Z(S)$.

(ii) $\left|N_{S}(X): X\right|=2$.

(iii) $S$ possesses only one conjugacy class of subgroups which are isomorphic to $X$.

There are several alternate methods for analyzing the fusion of 2-elements for arbitrary groups with a given Sylow 2-subgroup $S$; in particular, the focal subgroup theorem (Theorem 7.3.4 of [19]), Grün's theorem (Theorem 7.4.2 of [19]) or Alperin's fusion theorem (the main theorems of [1] or Theorem 7.2.6 of [19]). The case that $S$ is quasi-dihedral is very similar to that in which $S$ is dihedral, the latter case being completely described in Theorem 7.7.3 of [19]. Moreover, the results are given in Exercise 7.7 of [19]. Hence we shall content ourselves in the quasi-dihedral case with a statement of the results. On the other hand, we shall give a detailed analysis in the case that $S$ is wreathed. Because the use of Alperin's fusion theorem is the least familiar of the above-mentioned methods, we shall use it in our proof.

Proposition 1. Let $G$ be a group with a quasi-dihedral Sylow 2-subgroup $S$ and let $T, Q$ be representatives of the conjugacy classes of four subgroups and quaternion subgroups respectively of $S$. Then one of the following four statements holds:

(i) $G$ has no normal subgroups of index $2, G$ has one conjugacy class of involutions and one of elements of order $4,|N(T): C(T)|=6$ and $|N(Q): Q C(Q)|=6$.

(ii) $G$ has a normal subgroup $K$ of index 2 with generalized quaternion Sylow 2subgroups, $K$ has no normal subgroups of index $2, Z(S)$ is weakly closed in $S$ with respect to $G, G$ has two conjugacy classes of involutions and one of elements of order 4 , $|N(T): C(T)|=2$ and $|N(Q): Q C(Q)|=6$.

(iii) $G$ has a normal subgroup $K$ of index 2 with dihedral Sylow 2-subgroups, $K$ has no normal subgroups of index $2, G$ has one conjugacy class of involutions and two of elements of order $4,|N(T): C(T)|=6$ and $|N(Q): Q C(Q)|=2$. 
(iv) $G$ has a normal 2-complement, $G$ has two conjugacy classes of involutions and two of elements of order $4,|N(T): C(T)|=2$, and $|N(Q): Q C(Q)|=2$.

In deriving the corresponding result for groups with wreathed Sylow 2-subgroups, we shall use Alperin's fusion theorem in the following form: If $G$ is a group with Sylow $p$-subgroup $S$ and if $v, w$ are elements of $S$ conjugate in $G$, but not in $S$, then there exists a Sylow 2-subgroup $R$ of $G$ with the following properties:

(a) $S \cap R$ is a tame intersection of Sylow 2-subgroups.

(b) $C_{S}(S \cap R) \subseteq S \cap R$.

(c) $v \in S \cap R$.

(d) $v$ is conjugate in $N(S \cap R)$ to an element $w_{0}$ of $S \cap R$ with $v$ and $w_{0}$ not conjugate in $S$.

Indeed, we recall that by definition $S \cap R$ is a tame intersection if $N_{S}(S \cap R)$ and $N_{R}(S \cap R)$ are each Sylow $p$-subgroups of $N(S \cap R)$. To see that the above conditions actually hold, we apply the second form of the main theorem of [1] together with Theorem 5.2 of [1] and conclude that there exist elements $v_{1}, v_{2}, \ldots, v_{n}$ in $S$ with $v_{1}=v$ and $v_{n}=w$ and Sylow $p$-subgroups $R_{2}, R_{3}, \ldots, R_{n}$ of $G$ such that each $S \cap R_{i}$ is a tame intersection and such that either $v_{i}=v_{i+1}$ or $v_{i}$ and $v_{i+1}$ are in $S \cap R_{i+1}, \quad v_{i}$ and $v_{i+1}$ are conjugate in $N\left(S \cap R_{i+1}\right)$, and $C_{S}\left(S \cap R_{i+1}\right)$ $\subseteq S \cap R_{i+1}$. We now choose $i$ maximal such that $v_{1}, v_{2}, \ldots, v_{i}$ are conjugate in $S$. Since $v$ and $w$ are not conjugate in $S$, we have $i<n$. Let $u$ be an element of $S$ such that $v_{i}^{u}=v$, set $R=R_{i+1}^{u}$ and $w_{0}=v_{i+1}^{u}$. Then one sees at once that $R$ and $w_{0}$ satisfy the required conditions (a)-(d).

We note also that in the above situation, $N(S \cap R) / C(S \cap R)$ is not a 2-group. Indeed, suppose this is the case and set $K=N(S \cap R)$. Since $S \cap K=N_{S}(S \cap R)$ is a Sylow 2-subgroup of $K$, it follows that $K=C(S \cap R)(S \cap K)$. But then if $w_{0}=v^{k}$, $k \in K$, we can write $k=b c$, where $b \in C(S \cap R)$ and $c \in S \cap K$, whence $w_{0}=v^{b c}=v^{c}$ as $b$ centralizes $v$, contrary to the fact that $w_{0}$ is not conjugate to $v$ by an element of $S$.

Proposition 2. Let $G$ be a group with a wreathed Sylow 2-subgroup $S$ of order $2^{2 n+1}$, let $U$ be the unique abelian maximal subgroup of $S$ and let $V$ be a representative of the conjugacy class in $S$ of central products of quaternion groups with $Z(S)$. Then one of the following four statements holds:

(i) $G$ has no normal subgroups of index $2, G$ has one conjugacy class of involutions, $|N(U): C(U)|=6$, and $|N(V): V C(V)|=6$.

(ii) $G$ has a normal subgroup $K$ of index $2^{n}$ with generalized quaternion Sylow 2subgroups, $K$ has no normal subgroups of index 2, every subgroup of $Z(S)$ is weakly closed in $S$ with respect to $G, G$ has two conjugacy classes of involutions, $|N(U): C(U)|=2$ and $|N(V): V C(V)|=6$.

(iii) $G$ has a normal subgroup $K$ with Sylow 2-subgroup $U, K$ has no normal subgroups of index 2, G has two conjugacy classes of involutions, $|N(U): C(U)|=6$, and $|N(V): V C(V)|=2$. 
(iv) $G$ has a normal 2-complement, $G$ has three conjugacy classes of involutions, $|N(U): C(U)|=2$, and $|N(V): V C(V)|=2$.

Proof. Let $R$ be a Sylow 2-subgroup of $G$ such that $S \cap R$ is a tame intersection satisfying conditions (a)-(d) for some element $v$ of $S$. Then, as we have seen, $N(S \cap R) / C(S \cap R)$ is not a 2-group and consequently either $S \cap R=U$ or $S \cap R$ is a conjugate of $V$ by an element of $S$ by Lemma 3. We shall see that the fusion of 2-elements in $G$ is completely determined by the structure of $N(U)$ and $N(V)$.

Suppose $U$ is such a tame intersection. Since the Frattini factor group of $U$ is abelian of type $(2,2)$ and $S \subseteq N(U)$, it follows that $N(U) / C(U)$ is isomorphic to the symmetric group of degree 3 and so has order 6. Moreover, a 3-element of $N(U)-C(U)$ has no fixed points on $U^{\#}$ and consequently $U \subseteq N(U)^{\prime}$. For the same reason the three involutions $x=x_{1}, x_{2}, x_{3}$ of $U$ are conjugate in $G$. On the other hand, if $U$ is not such a tame intersection, then $N(U)=S C(U)$ and $|N(U): C(U)|=2$, since otherwise $|N(U) / C(U)|=6$ and $N(U)$ would possess a second Sylow 2subgroup $S^{*}$ of $G$ such that $S \cap S^{*}=U$. But then $U$ would be the tame intersection of $S$ and $S^{*}$.

Next consider $N(V)$. Any element $h$ of $N(V)-C(V)$ of odd order centralizes $Z(S)$ as $Z(S)$ is a cyclic characteristic subgroup of $V$ and so $h$ acts nontrivially on $V / Z(S)$, which is elementary abelian of type $(2,2)$. Thus $h^{3}$ centralizes $V$. Furthermore, if $V \subseteq S^{*}$ for some Sylow 2-subgroup $S^{*}$ of $G$, then $Z(V)=$ $Z(S) \subseteq Z\left(S^{*}\right)$ by Lemma 2 applied to $S^{*}$ and its nonabelian subgroup $V$. Since $|Z(S)|=\left|Z\left(S^{*}\right)\right|$, we have, in fact, $Z(S)=Z\left(S^{*}\right)$. But now Lemma 3 applied to $S^{*}$ and $V$ yields that $\left|N_{S^{*}}(V): V C_{S^{*}}(V)\right|=2$. Since we can choose $S^{*}$ so that $N_{S^{*}}(V)$ is a Sylow 2-subgroup of $N(V)$, it follows that $|N(V): V C(V)|=2$. Our argument thus shows that $N(V) / V C(V)$ is either of order 2 or is isomorphic to the symmetric group of degree 3 and is of order 6 . As with $U$, the latter case occurs if and only if $V$ is a tame intersection. Continuing this analysis, suppose now that $V$ is a tame intersection. Since $S$ has only one conjugacy class of subgroups isomorphic to $V$ by Lemma 3, we can assume without loss by replacing $V$ by an appropriate conjugate by an element of $S$ that $V=\left\langle r^{2^{n-2}}, d, Z(S)\right\rangle$. Since $N(V)$ in this case contains a 3element which cyclically permutes the involutions of $V / Z(V)$ and since $U \cap V$ has index two in $V$, we see that the involution $c_{1}=d u^{2^{n-2}}$ is conjugate in $N(V)$ to one of the involutions $x_{i}$ of $U$. But $x_{1}$ is conjugate only to itself in $N(V)$, so $i=2$ or 3 . Moreover, by Lemma 2, $c_{1}$ is conjugate to $z$ and $x_{2}$ to $x_{3}$ in $S$. We conclude therefore that $z$ and $x_{2}$ are conjugate in $G$.

With this preliminary information, we can now establish the proposition. Suppose first that both $U$ and $V$ are tame intersections satisfying (a)-(d). Then our argument shows that $|N(U): C(U)|=|N(V): V C(V)|=6$ and that the three conjugacy classes of involutions of $S$, represented by $x, x_{2}$, and $z$ are all fused in $G$. Furthermore, the focal subgroup $F$ of $S$ contains both $U$ and $x_{2}^{-1} z$ and so $F=S$. Hence $G$ has no normal subgroups of index 2. Thus (i) holds in this case. 
Suppose next that $U$, but not $V$, is such a tame intersection. In this case no element of $S-U$ can be conjugate to an element of $U$, whence $U=F$. Thus $G$ has a normal subgroup $K$ with Sylow 2-subgroup $U$ and such that $K$ has no normal subgroup of index 2. Since $x$ and $x_{2}$ are conjugate in $G$, while $x$ and $z$ are not, $G$ has two conjugacy classes of involutions. Moreover, $|N(U): C(U)|=6$ and $|N(V): V C(V)|=2$ in this case, so all parts of (iii) hold.

Now assume that neither $U$ nor $V$ is such a tame intersection, in which case two elements of $S$ conjugate in $G$ are necessarily conjugate in $S$. This implies that $F=S^{\prime}$ and so $G$ possesses a normal subgroup $K$ with Sylow 2-subgroup $S^{\prime}$ and such that $G / K$ is a 2-group. But as $S^{\prime}$ is cyclic, $K$ has a normal 2-complement by Burnside's transfer theorem. Since $O(K)$ char $K$, we have $O(K)$ normal in $G$ and hence $G$ has a normal 2-complement. Furthermore, $G$ has three conjugacy classes of involutions and $|N(U): C(U)|=|N(V): V C(V)|=2$, so (iv) holds in this case.

Finally consider the case that $V$, but not $U$, is such a tame intersection. Then with $V$ generated as above, we see that $V$ contains exactly three cyclic subgroups of order four which do not lie in $Z(S)$; namely, $\left\langle r^{2^{n-2}}\right\rangle,\left\langle r^{2^{n-2}} d\right\rangle$, and $\langle d\rangle$. Hence these must be cyclically permuted by a 3-element $h$ of $N(V)$. Since $\langle r\rangle=S^{\prime} \subseteq F$, it follows that $\langle r, d\rangle \subseteq F$. On the other hand, the only tame intersections containing $Z(S)$ in the present case are the conjugates of $V$ by elements of $S$. But $\langle r, d\rangle$ is normal in $S$ by Lemma 2 and so contains every conjugate of $d$ in $S$. Since the focal subgroup is completely determined by the set of all tame intersections containing $Z(S)$, we conclude that $\langle r, d\rangle=F$. In this case $G$ possesses a normal subgroup $K$ of index $2^{n}$ with Sylow 2-subgroup $F$. Furthermore, the element $h$ above of $N(V)$, being a 3-element, lies in $K$. By the action of $h$ on $\left\langle r^{2^{n-2}}, d\right\rangle$, we see that $d \in K^{\prime}$. Since $K^{\prime}$ is characteristic in $K$ and $K$ is normal in $G$, it follows that the normal closure of $\langle d\rangle$ in $S$ lies in $K^{\prime}$. But this normal closure is $\langle r, d\rangle=F$ by Lemma 2 and so $F \subseteq K^{\prime}$. Thus $F$ is the focal subgroup of $K$ and therefore $K$ has no normal subgroups of index two.

Observe next that $Z(S)=Z(V)$ and hence $Z(S)=Z\left(V^{s}\right)$ for any $s$ in $S$. Thus any subgroup $Z_{0}$ of $Z(S)$, being characteristic in $Z(S)$ as $Z(S)$ is cyclic, is normal in $N\left(V^{s}\right)$ for any $s$ in $S$. Since fusion of 2-elements in the present case is determined by the conjugates of $V$ in $S$, it follows that a generator $u_{0}$ of $Z_{0}$ is not conjugate in $G$ to any element of $S-Z_{0}$. In other words, $Z_{0}$ is weakly closed in $S$ with respect to $G$. For the same reason, $x$ is not conjugate in $G$ to any other involution of $S$ and so $G$ has two conjugacy classes of involutions. Finally $|N(U): C(U)|=2$ and $|N(V): V C(V)|=6$ in the present case, so all parts of (ii) hold and the proposition is completely proved.

2. $Q$-groups, $D$-groups, and $Q D$-groups. There exists a close parallel between the conclusions of corresponding parts of Propositions 1.1 and 1.2. Because of the importance of these groups in the analysis to follow, we shall give them special names. Thus we make the following definitions: 
Definition 1. A group $G$ with quasi-dihedral or wreathed Sylow 2-subgroup $S$ will be called a

$Q D$-group if $G$ satisfies condition (i) of Proposition 1.1 or 1.2;

$Q$-group if $G$ satisfies condition (ii) of Proposition 1.1 or 1.2 ;

$D$-group if $G$ satisfies condition (iii) of Proposition 1.1 or 1.2.

In particular, then, a $Q D$-group $G$ has no normal subgroups of index two and has exactly one conjugacy class of involutions. Furthermore, a $Q$-group $G$ has a normal subgroup $K$ with generalized quaternion Sylow 2-subgroups and with no normal subgroups of index two, $K$ is of index 2 in $G$ if $S$ is quasi-dihedral and of index $2^{n}$ in $G$ if $S$ is wreathed of order $2^{2 n+1}$, and every subgroup of $Z(S)$ is weakly closed in $S$ with respect to $G$. Moreover, a $D$-group $G$ has a normal subgroup $K$ of index two with dihedral Sylow 2-subgroups if $S$ is quasi-dihedral and with abelian Sylow 2-subgroups of type $\left(2^{n}, 2^{n}\right)$ if $S$ is wreathed of order $2^{2 n+1}$.

Note that if $S$ is wreathed of order $2^{2 n+1}$ and $G$ is a $Q$-group, then the index of $K$ in $G$ depends upon the integer $n$. Likewise the order of $Z(S)$ depends on $n$. Hence it will be convenient to have a term for this integer. Thus we make the following definition:

DEFINITION 2. If $S$ is a wreathed 2-group of order $2^{2 n+1}, n \geqq 2$, we say that $S$ is of height $n$. For completeness, we say that $S$ has height 1 if $S$ is quasi-dihedral. Moreover, if $G$ is a group with $S$ as Sylow 2-subgroup, we say that $G$ is of height $n$ if $S$ has height $n$.

Thus if $S$ is quasi-dihedral or wreathed of height $n$, it follows in all cases that $Z(S)$ is cyclic of order $2^{n}$ and that if $G$ is a $Q$-group with Sylow 2 -subgroup $S$, then $G$ possesses a normal subgroup $K$ of index $2^{n}$ in $G$ having generalized quaternion Sylow 2-subgroups and no normal subgroups of index two.

We shall have some occasion in our work to consider groups of the same general form as $Q$-groups or $D$-groups, but with Sylow 2-subgroups which are isomorphic to a proper subgroup of a quasi-dihedral or wreathed 2-group. For this reason we enlarge the definitions of these concepts.

Definition 3. Let $S$ be a quasi-dihedral or wreathed 2-group and let $R$ be a subgroup of $S$ containing the unique generalized quaternion subgroup $Y$ of maximal order in $S$. Then a group $G$ with Sylow 2-subgroup $R$ will be called a $Q$-group provided $G$ contains a normal subgroup $K$ of index a power of 2 with Sylow 2-subgroup $Y$ and no normal subgroups of index 2 and if every subgroup of $Z(R)$ is weakly closed in $R$ with respect to $G$.

Thus if $S$ is quasi-dihedral, either $R=S$ or $R=Y$; and if $S$ is wreathed, then by Lemma 1.2 , either $R=S$ or $R=Y Z(R)$ and $Z(R) \subseteq Z(S)$. In either case, $R \supset Y$ if and only if $R$ contains at least two involutions and hence if and only if $R$ contains a four group. In particular, if $R \supset Y$ and $S$ is wreathed, then $R$ contains the unique normal four subgroup of $S$ by Lemma 1.2.

Definition 4. A group $G$ will be called a $D$-group if either $G$ has quasi-dihedral or wreathed Sylow 2-subgroups and is a $D$-group in accordance with Definition 1 
or if $G$ has dihedral Sylow 2-subgroups and does not possess a normal 2complement.

A complete classification of $Q$-groups and $D$-groups can be given in terms of known classification theorems. The pertinent results which we shall need will be derived in $\$ \S 3,4$, and 5 . In this section we shall establish some basic properties of $Q D$-groups and shall then present some standard examples of $Q D$-groups, $Q$-groups, and $D$-groups.

Our first result shows the significance of $Q$-groups in the study of $Q D$-groups and is fundamental for the entire paper.

Proposition 1. If $G$ is a $Q D$-group and $x$ is an involution of $G$, then $C(x)$ is a $Q$ group with quasi-dihedral or wreathed Sylow 2-subgroups.

Proof. By definition of a $Q D$-group, $G$ has only one conjugacy class of involutions and so $x \in Z(S)$ for some Sylow 2-subgroup $S$ of $G$. Thus $S \subseteq N=C(x)$ and so $N$ has quasi-dihedral or wreathed Sylow 2-subgroup. Moreover, if $V$ is the central product of a quaternion subgroup of $S$ with $Z(S)$, Propositions 1.1 and 1.2 show that $|N(V): V C(V)|=6$. But clearly $\langle x\rangle=\Omega_{1}(Z(V))$ and so $\langle x\rangle$ is normal in $N(V)$, whence $N(V) \subseteq N$. Thus $\left|N_{N}(V): V C_{N}(V)\right|=6$. Hence applying Propositions 1.1 and 1.2 to $N$, we see that $N$ is either a $Q$-group or a $Q D$-group. However, $x$, being central in $N$, is conjugate only to itself in $N$. Since $S$ contains an involution other than $x$, it follows that $N$ has more than one conjugacy class of involutions and we conclude therefore from Propositions 1.1 and 1.2 that $N$ is a $Q$-group.

We can also derive the following general structure theorem for $Q D$-groups:

Proposition 2. If $G$ is a $Q D$-group, then $G / O(G)$ contains a simple normal $Q D$-subgroup of odd index.

Proof. By definition, a Sylow 2-subgroup $S$ of $G$ is quasi-dihedral or wreathed and $G$ has no normal subgroups of index 2 . Hence the same holds for $\bar{G}=G / O(G)$ and so $\bar{G}$ is also a $Q D$-group. Since $O(\bar{G})=1$, the proposition follows by induction on $|G|$ if $O(G) \neq 1$. Hence we can suppose that $O(G)=1$.

Let $L$ be a minimal normal subgroup of $G$, so that $L$ is characteristically simple. If $L$ is solvable, then $L$ is an elementary abelian $p$-group for some prime $p$ and, as $O(G)=1$, we must have $p=2$. Hence $L$ has even order in any case. By Propositions 1.1 and 1.2, $G$ has only one conjugacy class of involutions and so all involutions of $S$ must lie in $L$. In particular, $\Omega_{1}(S) \subseteq L$. By Lemmas 1.1 and $1.2, \Omega_{1}(S)$ is nonabelian and $S / \Omega_{1}(S)$ is cyclic. In particular, $L$ is nonabelian and so $L$ is nonsolvable. Furthermore, $S / S \cap L$ is cyclic and so $\bar{G}=G / L$ has cyclic Sylow 2-subgroups. But then if $|\bar{G}|$ were even, $\bar{G}$ and hence also $G$, would have a normal subgroup of index two by Burnside's transfer theorem, which is not the case. Thus $|\bar{G}|=|G / L|$ is odd.

Finally $L$, being nonsolvable and characteristically simple, is the direct product of isomorphic nonabelian simple groups. Since no nonabelian simple group has 
cyclic or generalized quaternion Sylow 2-subgroups, each simple factor of $L$ contains a four subgroup. Therefore if $L$ had more than one simple factor, $L$ and hence also $S$, would contain an elementary abelian subgroup of order sixteen, which is clearly not the case. Thus $L$ is simple and the proposition is proved.

In this paper we shall require only one other noncharacter-theoretic property of $Q D$-groups. The hypotheses for this result will arise out of our inductive proof of the Main Theorems and will be applied to the proper $Q D$-subgroups of a minimal counterexample to those theorems. Since the Main Theorems have been stated only for groups with quasi-dihedral Sylow 2-subgroups, our result is limited to this case. However, in proving the corresponding Main Theorems for groups with wreathed Sylow 2-subgroups, the following result and its proof will hold without change.

Proposition 3. If $G$ is a $Q D$-group with quasi-dihedral Sylow 2-subgroups whose unique nonsolvable composition factor satisfies the conclusion of the First Main Theorem, then for any involution $x$ of $G$ and any Sylow 2-subgroup $S$ of $C(x)$, we have $[S, O(C(x))] \subseteq O(G)$.

Proof. By Proposition 2 and the solvability of groups of odd order, $G$ possesses a unique nonsolvable composition factor $\bar{L}$ which is a simple normal $Q D$-subgroup of $\bar{G}=\bar{G} / O(G)$. Since $\overline{O(C(x))}=O(C(\bar{x}))$, we need only prove that $\bar{S}$ centralizes $O(C(\bar{x}))$. Since $O(\bar{G})=1$, we can assume without loss to begin with that $O(G)=1$ and then prove that $S$ centralizes $O(N)$, where $N=C(x)$.

Let $L$ be the simple normal $Q D$-subgroup of $G$. By Proposition $2,|G: L|$ is odd and so $S \subset L$, whence $[S, O(N)] \subseteq L \cap O(N)$. Since $L \cap O(N)$ is normal in $O(N)$ and is $S$-invariant, it follows that $S$ normalizes $L \cap O(N)$. Thus it suffices to show that $S$ centralizes $L \cap O(N)$, for then $S$ will stabilize the chain: $O(N) \supseteq L \cap O(N) \supseteq 1$ and, as $S$ and $O(N)$ have coprime orders, this will imply that $S$ centralizes $O(N)$.

Clearly $L \cap O(N)=L \cap O(C(x)) \subseteq O\left(C_{L}(x)\right)$. But by assumption $L$ satisfies the conclusion of the First Main Theorem and so $C_{L}(x)$ is isomorphic to a homomorphic image of the matrix groups $G L(2, q)$ or $G U(2, q)$ for some odd $q$ by a suitable central subgroup of odd order. However, $O(G L(2, q))$ and $O(G U(2, q))$ consist of scalar matrices in $G L(2, q)$ and $G U(2, q)$ respectively. It follows therefore that $O\left(C_{L}(x)\right)$ is contained in the center of $C_{L}(x)$. Since $S \subseteq L \cap N=C_{L}(x), S$ centralizes $O\left(C_{L}(x)\right)$ and so centralizes $L \cap O(N)$, as required.

We turn now to some standard examples of $Q D$-groups, $Q$-groups and $D$-groups.

As is well known (cf. [13] and [14]), the matrix groups $G L(2, q), q$ odd, have quasi-dihedral or wreathed Sylow 2-subgroups of height $n$, where $2^{n}$ is the exact power of 2 dividing $q-1$ (in particular, their Sylow 2-subgroups are quasi-dihedral if $q \equiv-1(\bmod 4)$ and wreathed if $q \equiv 1(\bmod 4))$; while the matrix groups $G U(2, q)$ have quasi-dihedral or wreathed Sylow 2-subgroups of height $n$, where $2^{n}$ is the exact power of 2 dividing $q+1$ (in particular, quasi-dihedral if $q \equiv 1(\bmod 4)$ and wreathed if $q \equiv-1(\bmod 4))$. Moreover, their centers consist of their scalar matrices and so are cyclic of orders $q-1$ and $q+1$ respectively. 
We shall be interested in certain specific subgroups of $G L(2, q)$ and $G U(2, q), q$ odd, which we proceed to describe. The determinant of an element $X$ of $G L(2, q)$ is a nonzero element of $G F(q)$ and so has order dividing $q-1$, while the determinant of an element $X$ of $G U(2, q)$ is a nonzero element of $G F\left(q^{2}\right)$ of order dividing $q+1$. Hence in either case, if the determinant of $X$ has order $2^{m}$, then $0 \leqq m \leqq n$, where $2^{n}$ is correspondingly the highest power of 2 dividing $q-1$ or $q+1$. We define, for $0 \leqq m \leqq n$, the subgroups $S L_{m}(2, q)$ and $S U_{m}(2, q)$ of $G L(2, q)$ and $G U(2, q)$ respectively consisting of those elements whose determinants have orders dividing $2^{m}$.

Thus $S L_{0}(2, q)$ is the special linear group $S L(2, q)$ and $S U_{0}(2, q)$ is the special unitary group $S U(2, q)$, while $S L_{1}(2, q), S U_{1}(2, q)$ are the subgroups $S L^{ \pm}(2, q)$, $S U^{ \pm}(2, q)$ respectively consisting of the elements of determinant \pm 1 . It is clear from the definition that for $1 \leqq m \leqq n, S L_{m}(2, q)$ contain $S L_{m-1}(2, q)$ as a subgroup of index two and that $S U_{m}(2, q)$ contains $S U_{m-1}(2, q)$ as a subgroup of index two.

We now prove

LEMMA 1. The following conditions hold:

(i) $S L_{n}(2, q)$ and $S U_{n}(2, q)$ are Q-groups with quasi-dihedral or wreathed Sylow 2-subgroup $S$ of height $n$.

(ii) The center of $S$ is contained in the center of $G L(2, q)$ or $G U(2, q)$ respectively.

(iii) $S L_{m}(2, q)$ and $S U_{m}(2, q)$ are $Q$-groups for $0 \leqq m \leqq n$.

(iv) $S L_{m}(2, q)$ and $S U_{m}(2, q)$ are characteristic in $G L(2, q)$ and $G U(2, q)$ respectively for $0 \leqq m \leqq n$.

(v) $S L_{m}(2, q)$ and $S U_{m}(2, q)$ are the central product of a cyclic group of order $2^{m+1}$ with $S L_{0}(2, q)$ and $S U_{0}(2, q)$ respectively for $0 \leqq m<n$.

(vi) $S L_{0}(2, q)$ and $S U_{0}(2, q)$ are isomorphic.

Proof. The groups $S L(2, q)$ and $S U(2, q)$ are known to be isomorphic, to have generalized quaternion Sylow 2-subgroups and to have no normal subgroups of index 2 (cf. [14]). In particular, (vi) holds.

We have $|G L(2, q)|=\left(q^{2}-1\right)\left(q^{2}-q\right)=(q-1)|S L(2, q)|$ and $|G U(2, q)|=$ $\left(q^{2}-1\right)\left(q^{2}+q\right)=(q+1)|S U(2, q)|$. Since $2^{n}$ is the highest power of 2 dividing $q-1$ or $q+1$ respectively and since $\left|S L_{n}(2, q): S L(2, q)\right|=\left|S U_{n}(2, q): S U(2, q)\right|=2^{n}$, we see that $S L_{n}(2, q)$ and $S U_{n}(2, q)$ contain a Sylow 2-subgroup $S$ of $G L(2, q)$ and $G U(2, q)$ respectively. By the remarks preceding the lemma, $S$ is either quasidihedral or wreathed of height $n$. We have also noted that the center $Z$ of $G L(2, q)$ or $G U(2, q)$ is cyclic of order $q-1$ or $q+1$ respectively. Hence by definition of the integer $2^{n}, Z \cap S$ is cyclic of order $2^{n}$. Clearly $Z \cap S \subseteq Z(S)$. On the other hand, $Z(S)$ is cyclic of order $2^{n}$ by Lemmas 1.1 and 1.2 , so $Z \cap S=Z(S)$. In particular, $Z(S)$ is in the center of $G L(2, q)$ or $G U(2, q)$, as the case may be, so (ii) holds. Moreover, every subgroup of $Z(S)$ is weakly closed in $S$ with respect to $S L_{n}(2, q)$ or $S U_{n}(2, q)$ respectively and we conclude from the definition that $S L_{n}(2, q)$ and $S U_{n}(2, q)$ are $Q$-groups, so (i) also holds. 
Again with $S$ a Sylow 2-subgroup of $S L_{n}(2, q)$ or $S U_{n}(2, q)$, correspondingly $S \cap S L_{0}(2, q)$ or $S \cap S U_{0}(2, q)$ is the unique maximal generalized quaternion subgroup $Y$ of $S$. Hence if we set correspondingly $R=S \cap S L_{m}(2, q)$ or $R=S \cap S U_{m}(2, q)$ for any $m, 0 \leqq m \leqq n$, then $R \supseteq Y$ and hence by Lemmas 1.1 and 1.2 , we have $Z(R) \subseteq Z(S)$. In particular, every subgroup of $Z(R)$ is weakly closed. Since $S L_{m}(2, q) \supseteq S L_{0}(2, q)$ and $S U_{m}(2, q) \supseteq S U_{0}(2, q)$, we conclude again from the definition that $S L_{m}(2, q)$ and $S U_{m}(2, q)$ are $Q$-groups, $0 \leqq m \leqq n$, proving (iii).

One can verify directly that $S L_{0}(2, q)$ and $S U_{0}(2, q)$ are the derived groups of $G L(2, q)$ and $G U(2, q)$ respectively. Since the corresponding factor groups are isomorphic to subgroups of the multiplicative group of $G F(q)$ or $G F\left(q^{2}\right)$ respectively, they are cyclic and so each of their subgroups is characteristic. Since the derived group of a group is characteristic, we conclude that $S L_{m}(2, q)$ and $S U_{m}(2, q)$ are characteristic in $G L(2, q)$ and $G U(2, q)$ respectively for all $m$, $0 \leqq m \leqq n$, proving (iv).

It thus remains to prove (v), so assume $m<n$. The arguments are the same for both $S L_{m}(2, q)$ and $S U_{m}(2, q)$; for simplicity we treat only the former. Since $S L_{m}(2, q)$ is characteristic in $G L(2, q), R$ is a Sylow 2-subgroup of $S L_{m}(2, q)$. The remarks preceding the lemma imply that $S L_{m}(2, q)$ contains $S L_{0}(2, q)$ as a subgroup of index $2^{m}$; so, in particular, $S L_{m}(2, q)=S L_{0}(2, q) R$. But $R=Y Z(R)$ by Lemmas 1.1 and 1.2 as $m<n$. Since $Y$ is a Sylow 2-subgroup of $S L_{0}(2, q)$, this yields $S L_{m}(2, q)$ $=S L_{0}(2, q) Z(R)$. But as we have shown above, $Z(R) \subseteq Z(S)$ and $Z(S)$ is in the center of $G L(2, q)$. Moreover, $Z(R) \cap S L_{0}(2, q)=Z(R) \cap Y=Z(Y)$ and so is of order two. Since $\left|S L_{m}(2, q): S L_{0}(2, q)\right|=2^{m}$ and $S L_{m}(2, q)=S L_{0}(2, q) Z(R)$, it follows that $Z(R)$ has order $2^{m+1}$. Since $Z(S)$ is cyclic, so also is $Z(R)$ and we conclude that $S L_{m}(2, q)$ is the central product of a cyclic group of order $2^{m+1}$ with $S L_{0}(2, q)$. Thus (v) also holds and the lemma is proved.

It was shown by Schur in [26] that the group $\operatorname{SL}(2, q)$ (which is isomorphic to $S U(2, q)$ ) for $q$ odd and $q>3$ possesses only trivial central extensions except in the case $q=9$, the group $S L(2,9)$ having a unique nontrivial central extension by a group of order three. We shall denote this covering group by $S L^{\wedge}(2,9)$. It can be shown that this extension lifts to each of the groups $S U_{1}(2,9)$ and $S L_{m}(2,9), 1 \leqq m \leqq 3$. However, it turns out that in the course of our analysis, only the first of these groups needs to be considered. We shall therefore limit our discussion to this one group.

LEMma 2. The nontrivial central extension $S L^{\wedge}(2,9)$ of $S L(2,9)$ by a group of order 3 lifts uniquely to the group $S U_{1}(2,9)$ and we denote this extended group by $S U_{1}^{\wedge}(2,9)$. Furthermore, the following conditions hold:

(i) $S U_{1}(2,9)$ is a Q-group with quasi-dihedral Sylow 2-subgroups.

(ii) $S U_{1}^{\wedge}(2,9)$ contains $S L^{\wedge}(2,9)$ as a subgroup of index 2 .

(iii) The center of $S U_{1}^{\wedge}(2,9)$ has order 2.

Proof. Let $K=S L(2,9), L=S U_{1}(2,9)$, and let $P$ be a Sylow 3-subgroup of $K$. We first argue that $N_{L}(P)=P R$, where $R$ is cyclic of order $16, C_{R}(P)=\Omega_{1}(R)=Z(L)$, 
and $R / \Omega_{1}(R)$ acts regularly on $P$. Indeed, as $q \equiv 1(\bmod 4)$, a Sylow 2 -subgroup $S$ of $L$ is quasi-dihedral. Since $|L|=\left(9^{2}-1\right)\left(9^{2}+9\right),|S|=32$. Setting $\bar{L}=L / Z(L), \bar{K}$ is isomorphic to $\operatorname{PSL}(2,9),|\bar{L}: \bar{K}|=2$, and $\bar{S}$ is dihedral of order 16 . Hence by Lemma 9 of [21], $\bar{L}$ is isomorphic to $P G L(2,9)$. But then by Lemma 3.1 (v) of [22], $N_{L}(\bar{P})=\bar{P} \bar{R}$, where $\bar{R}$ is cyclic of order eight and $\bar{P} \bar{R}$ is a Frobenius group. Hence $N_{L}(P)=P R$, where $|R|=16, R \supset Z(L)$, and $\bar{R}=R / Z(L)$ acts regularly on $P$. Since $Z(L) \subseteq Z(R)$ and $\bar{R}$ is cyclic, we also have that $R$ is abelian. Since the Sylow 2subgroups of $L$ are quasi-dihedral and since a quasi-dihedral group does not contain a noncyclic abelian subgroup of order sixteen, $R$ is necessarily cyclic. This proves the assertion. We note also that $N_{R}(\bar{P})=\bar{P} \mho^{1}(\bar{R})$ by Lemma 3.1 (vi) of [22] and hence $N_{K}(P)=P \mho^{1}(R)$.

Now $P$ is elementary abelian of order nine. Hence $P$ is the homomorphic image of an extra-special group $\hat{P}$ of order twenty-seven and exponent three. Furthermore, it can be shown directly that the action of $R$ on $P$ lifts to $\hat{P}$ and that in this action a generator of $R$ inverts $Z(\hat{P})$. We leave the verification to the reader. In particular, $\mho^{1}(R)$ centralizes $Z(\hat{P})$. Moreover, $P$ is disjoint from its conjugates in $L$ by Lemma 3.1 (vi) of [22]. Since $N_{K}(P)=P \mho^{1}(R)$ and $N_{L}(P)=P R$, it follows now from a result of Cartan-Eilenberg (Theorem XII, 10.1 of [12]) that the extension of $P$ to $P$ lifts to the groups $K$ and $L$, the extension of $K$ being central. We denote the corresponding extended groups by $R$ and $\hat{L}$. Since $R$ is a nontrivial central extension of $S L(2,9)$ by a group of order three, $R$ is isomorphic to $S L^{\wedge}(2,9)$ by Schur's results and so we can identify $R$ with $S L^{\wedge}(2,9)$. Finally the results of Cartan-Eilenberg show also that $N_{\hat{L}}(\hat{P})=\hat{P} \hat{R}$, where $\hat{R}$ is cyclic of order sixteen and a generator of $\hat{R}$ inyerts $Z(\hat{P})$. This last condition implies that $Z(\hat{P}) \nsubseteq Z(\hat{L})$ and hence that $|Z(\hat{L})|=2$. Thus $\mathcal{L}$ is an extension of $S U_{1}(2,9)$ by a group of order three, contains $S L^{\wedge}(2,9)$ as a subgroup of index two, and has a center of order two. In addition, $K$ and hence also $R$, has generalized quaternion Sylow 2-subgroups and no normal subgroups of index two. Moreover, the center of a Sylow 2-subgroup of $\hat{L}$ is obviously weakly closed. Hence $\hat{L}$ is also a $Q$-group and so satisfies all the conclusions of the lemma.

Thus to complete the proof, we need only show that any group $\hat{L}^{*}$ which contains $S L^{\wedge}(2,9)$ as a subgroup of index two and which is an extension of $S U_{1}(2,9)$ by a group of order three is isomorphic to $\hat{L}$. Without loss we can assume that $R$ is a subgroup of index two of both $\hat{L}$ and $L^{*}$ and that $L$ is the image of both $\hat{L}$ and $L^{*}$ by $Z(\hat{P})=O(R)=O(Z(R))$. Since $L$ has quasi-dihedral Sylow 2 -subgroups and $K$ is a subgroup of $L$ with generalized quaternion Sylow 2-subgroups, $L$ contains an involution not in $K$ and so $L=K A$, where $|A|=2$ and $K \cap A=1$. Since the Sylow 2-subgroups of $\hat{L}, \hat{L}^{*}$, and $R$ map isomorphically onto those of $L, L$, and $K$ respectively, it follows that $\hat{L}$ contains a subgroup $\hat{A}$ of order two and $\hat{L}^{*}$ a subgroup $\hat{A}^{*}$ of order two, both of which map onto $A$. In particular, $\hat{L}=R \hat{A}, \hat{L}^{*}=\hat{R}^{*} \hat{A}^{*}$ and $R \cap \hat{A}=R \cap \hat{A}^{*}=1$.

We set $\hat{A}=\langle\hat{a}\rangle, \hat{A}^{*}=\left\langle\hat{a}^{*}\right\rangle$ and let $\phi, \phi^{*}$ be the automorphisms of $R$ induced by conjugation by $\hat{a}, \hat{a}^{*}$ respectively. Since the images of $\phi$ and $\phi^{*}$ determine the same 
automorphism of $K$ as $\hat{A}, \hat{A}^{*}$ both map onto $A$, it follows that the image of $\psi=\phi^{-1} \phi^{*}$ acts trivially on $K$ and hence that $[\psi, \hat{K}] \subseteq Z(\hat{P})$. Since $Z(\hat{P}) \subseteq Z(\hat{K})$, this yields $[\psi, R, R]=1$. But $\hat{R}$ is perfect as $K^{\prime}=K$ and $Z(\hat{P}) \subseteq \hat{P}^{\prime} \subseteq R^{\prime}$. Hence $[\hat{R}, \psi]=1$ by the three subgroup lemma and so $\psi$ is the identity on $\hat{K}$. Thus $\hat{a}$ and $\hat{a}^{*}$ induce the same automorphism of $\hat{R}$ and we conclude that $\hat{L}$ and $\hat{L}^{*}$ are isomorphic, as required.

We turn now to some examples of $D$-groups. The following result is well known (cf. [21] and [22]).

Lemma 3. The groups $\operatorname{PSL}(2, q), q$ odd, $\operatorname{PGL}(2, q)$, and the alternating group $A_{7}$ are D-groups with dihedral Sylow 2-subgroups.

There also exist $D$-groups with quasi-dihedral and wreathed Sylow 2-subgroups. To describe the first type, we consider the group $G=P \Gamma L(2, q), q$ odd, which is the quotient of the group $\Gamma L(2, q)$ by its center. Here $\Gamma L(2, q)$ denotes the semidirect product of $G L(2, q)$ with a cyclic group of automorphisms induced by the Galois group of the field $G F(q)$. By Lemma 3.3 of [22], if $q=p^{r}, p$ a prime, then $G=L E$, where $L=P G L(2, q)$ is normal in $G, E$ is cyclic of order $r$, and $L \cap E=1$. Moreover, $L$ contains $K=\operatorname{PSL}(2, q)$ as a subgroup of index two. Thus when $r$ is even, $|E|$ is even and $G$ possesses three distinct subgroups $G_{1}, G_{2}, G_{3}$ each containing $K$ as a subgroup of index two. We can take $G_{1}$ to be $L$ and $G_{2}$ to be of the form $K\left(G_{2} \cap E\right)$. The remaining group $G_{3}$ has been analyzed in Lemma 2.3 of [20] and it has been shown there that it has quasi-dihedral Sylow 2-subgroups. As in [20], we denote this group by $P G L^{*}(2, q)$. It exists whenever $q$ is an odd square. Since $P G L^{*}(2, q)$ contains $K=P S L(2, q)$ as a normal subgroup of index two and since $\operatorname{PSL}(2, q)$ has dihedral Sylow 2-subgroups and no normal subgroup of index two, we have by definition of a $D$-group:

Lemma 4. The groups $P G L^{*}(2, q), q$ an odd square, are D-groups with quasidihedral Sylow 2-subgroups.

To describe $D$-groups with wreathed Sylow 2-subgroups, we consider the direct product $U$ of two cyclic groups, each of order $2^{n}, n \geqq 2$. It is possible to construct the semidirect product of $U$ with the symmetric group of degree 3 (equivalently with $P G L(2,3)$ ) in such a way that an element of order 3 acts regularly on $U$ and a Sylow 2-subgroup of the resulting group is wreathed of height $n$. Moreover, such an extension of $U$ is uniquely determined up to isomorphism. We leave these easily verified facts to the reader. By analogy with $P G L(2,3)$, we denote the resulting group by $P G L_{n}(2,3)$. Clearly $P G L_{n}(2,3)$ contains a normal subgroup $K$ of index 2 such that $U$ is a Sylow 2-subgroup of $K$ and $U$ is contained in the derived group of $K$. We thus have

Lemma 5. The groups $P G L_{n}(2,3), n \geqq 2$, are D-groups with wreathed Sylow 2subgroups of height $n$. 
For completeness, we set $P G L_{1}(2,3)=P G L(2,3)$, so that $P G L_{1}(2,3)$ is a $D$-group with dihedral Sylow 2-subgroups of order eight.

Finally we have the following well-known examples of simple $Q D$-groups together with a few of their basic properties (cf. [13], [14], [24*]).

Proposition 4. The following results hold:

(i) The groups $P S L(3, q)$ and $P S U(3, q), q$ odd, are simple $Q D$-groups of height $n$, where $2^{n}$ is the highest power of 2 dividing $q-1$ and $q+1$, respectively.

(ii) The Mathieu group $M_{11}$ is a simple $Q D$-group with quasi-dihedral Sylow 2subgroups of order 16.

(iii) The centralizer of an involution in $\operatorname{PSL}(3, q)$ or $\operatorname{PSU}(3, q)$ is isomorphic to a homomorphic image of $G L(2, q)$ or $G U(2, q)$ by a central subgroup of order $d$, where $d=(q-1,3)$ or $d=(q+1,3)$, respectively.

(iv) The centralizer of an involution in $M_{11}$ is isomorphic to $G L(2,3)$.

3. The characteristic power of $Q$-groups, $D$-groups, and $Q D$-groups. As we shall see, there is associated in a natural way with each $Q$-group with quasi-dihedral or wreathed Sylow 2-subgroups, with each $D$-group (except for those involving $A_{7}$ ), and with each $Q D$-group an odd prime power which will be called its characteristic power. This integer will play a very important role in our analysis of $Q D$-groups.

To define the characteristic power of a $Q$-group with quasi-dihedral or wreathed Sylow 2-subgroups, we first derive a basic property of arbitrary $Q$-groups.

Proposition 1. If $H$ is a Q-group with Sylow 2-subgroup $S$, then $H=O(H) C_{H}(Z(S))$.

Proof. If $\bar{H}=H / O(H)$, it will suffice to prove that $Z(\bar{S}) \subseteq Z(\bar{H})$. Indeed, assume this is the case. Since $O(H)$ has odd order, $C_{H}(Z(S))$ maps onto $C_{H}(\overline{Z(S)})$. But $\overline{Z(S)}=Z(\bar{S})$, as $S$ maps isomorphically onto $\bar{S}$, and $C_{H}(Z(\bar{S}))=\bar{H}$ by our assumption. Thus $C_{H}(Z(S))$ maps onto $\bar{H}$ and consequently $H=O(H) C_{H}(Z(S))$, as required.

We claim next that $\bar{H}$ is a $Q$-group. Indeed, the Sylow 2-subgroup $\bar{S}$ of $\bar{H}$ is isomorphic to $S$. Furthermore, if $K$ is a normal subgroup of $H$ of index a power of 2 having no normal subgroups of index 2 and with $S \cap K$ generalized quaternion, then $\bar{K}$ has the corresponding properties in $\bar{H}$. Hence we need only show that any subgroup $\bar{Z}$ of $Z(\bar{S})$ is weakly closed in $\bar{S}$ with respect to $\bar{H}$. But if $\bar{Z}^{h} \subseteq \bar{S}$ for some $\bar{h}$ in $\bar{H}$, then $Z^{h} \subseteq O(H) S$, where $h$ is a representative of $\bar{h}$ in $H$. Since $S$ is a Sylow 2-subgroup of $O(H) S$, there thus exists an element $w$ in $O(H)$ such that $Z^{h w} \subseteq S$. But then $Z^{\text {hw }}=Z$ as every subgroup of $Z(S)$ is weakly closed in $S$ with respect to $H$. Since $\bar{w}=1$, the required conclusion $\bar{Z}^{h}=\bar{Z}$ follows. Thus $\bar{H}$ is a $Q$-group, as asserted, and so it will suffice to prove the proposition for $\bar{H}$. Since $O(\bar{H})=1$, we can therefore assume without loss to begin with that $O(H)=1$.

Let $Z_{0}=Z(S) \cap Z(H)$ and suppose, by way of contradiction, that $Z_{0} \subset Z(S)$. Let $Z$ be a subgroup of $Z(S)$ containing $Z_{0}$ as a subgroup of index two. Setting $\bar{H}=H / Z_{0}$, we have that $\bar{Z}$ is a subgroup of $Z(\bar{S})$ of order two and that $\bar{S}$ is a Sylow 
2-subgroup of $\bar{H}$. Suppose $\overline{Z^{h}} \subseteq \bar{S}$ for some $\bar{h}$ in $\bar{H}$. Then $Z^{h} \subseteq S$, where $h$ is a representative of $\bar{h}$ in $H$, whence $Z^{h}=Z$ and so $\overline{Z^{h}}=\bar{Z}$. Thus $\bar{Z}$ is weakly closed in $\bar{S}$ with respect to $\bar{H}$. Since $O(H)=1$ and $Z_{0} \subseteq Z(H)$, we also have that $O(\bar{H})=1$. It follows therefore from a theorem of Glauberman [18] that $\bar{Z} \subseteq Z(\bar{H})$. Thus $Z$ is normal in $H$ and also $H$ stabilizes the chain $Z \supset Z_{0} \supseteq 1$, whence $H / C_{H}(Z)$ is a 2-group. But also $S \subseteq C_{H}(Z)$ as $Z \subseteq Z(S)$. Since $S$ is a Sylow 2-subgroup of $H$, we conclude that $H=C_{H}(Z)$ and hence that $Z \subseteq Z(H)$, contrary to our maximal choice of $Z_{0}$. Therefore $Z(S) \subseteq Z(H)$, as asserted.

We now prove

Proposition 2. If $H$ is a $Q$-group with quasi-dihedral or wreathed Sylow 2subgroups in which $O(H)=1$, then $H$ possesses a normal subgroup $L_{0}$ isomorphic to $S L(2, q)$ for some odd prime power $q$. Moreover, the subgroup $L_{0}$ and the integer $q$ are uniquely determined.

Proof. Let $S$ be a Sylow 2-subgroup of $H$ and let its height be $n$. Since $H$ is a $Q$-group, $H$ contains a normal subgroup $K$ of index $2^{n}$ with no normal subgroups of index two and with $S \cap K$ generalized quaternion. By Lemmas 1.1 and 1.2, $Z(S)$ is cyclic of order $2^{n}$ and $S / Z(S)$ is dihedral. Moreover, since $S \cap K$ is normal in $K$ and since $Z(S \cap K)$ is of order two, we also have that $Z(S) \cap K=Z(S \cap K)$ and so is of order two.

Since $O(H)=1$, it follows from the preceding proposition that $Z(S) \subseteq Z(H)$. We set $\bar{H}=H / Z(S)$. Since $|Z(S) \cap K|=2,|H: K|=2^{n}$ and $|Z(S)|=2^{n}$, we conclude that $|\bar{H}: \bar{K}|=2$. Furthermore, $\bar{S}$ is dihedral by the preceding paragraph. Since $\bar{S}$ is a Sylow 2-subgroup of $\bar{H}$, it follows now from the main structure theorem for groups with dihedral Sylow 2-subgroups [22] that $\bar{H}$ is isomorphic to a subgroup of $P \Gamma L(2, q)$ containing $\operatorname{PSL}(2, q)$ for some odd prime power $q$.

Let $\bar{L}_{0}$ be the normal subgroup of $\bar{H}$ isomorphic to $\operatorname{PSL}(2, q)$. If $q>3, \bar{L}_{0}$ is simple and is the unique minimal normal subgroup of $\bar{H}$, while if $q=3, \bar{H}$ is isomorphic to $P G L(2,3)$ and so $\bar{L}_{0}$ is the unique normal subgroup of index two in $\bar{H}$. In either case it follows that $\bar{L}_{0}$ is the only normal subgroup of its form in $\bar{H}$, so $\bar{L}_{0}$ and $q$ are uniquely determined. Since $\bar{L}_{0}$ has no normal subgroups of index two, we also have that $\bar{L}_{0} \subseteq \bar{K}$.

If $H_{0}$ denotes the inverse image of $\bar{L}_{0}$ in $H$, it follows that $H_{0} \subseteq Z(S) K$ and that $H_{0}$ is a central extension of $Z(S)$ by $\bar{L}_{0}$. If this extension were trivial, $S \cap H_{0}$ would be the direct product of $Z(S)$ and a dihedral group isomorphic to $\bar{S} \cap \bar{L}_{0}$, in which case $S$ would contain an elementary abelian subgroup of order eight. However, a quasi-dihedral or wreathed 2-group contains no subgroup of this form. Thus $H_{0}$ is a nontrivial central extension and therefore by the results of Schur [26], $H_{0}=Z(S) L_{0}$, where $L_{0}$ is isomorphic to $S L(2, q), L_{0}$ is normal in $H_{0}$, and $\left|L_{0} \cap Z(S)\right|=2$. But then $L_{0}$ has no normal subgroups of index two and so $L_{0}=O^{2}\left(H_{0}\right)$. Thus $L_{0}$ is characteristic in $H_{0}$ and, as $H_{0}$ is normal in $H$, we conclude that $L_{0}$ is normal in $H$.

Suppose finally that $L_{1}$ is a normal subgroup of $H$ isomorphic to $S L(2, r)$ for 
some odd $r$. Then $Z\left(L_{1}\right)$ is normal in $H$ and so $Z\left(L_{1}\right) \subseteq Z(S)$. But then $\bar{L}_{1}$ is a normal subgroup of $\bar{H}$ isomorphic to $\operatorname{PSL}(2, r)$. Hence by the uniqueness of $\bar{L}_{0}$ we must have $\bar{L}_{1}=\bar{L}_{0}$. Since $L_{0}$ maps onto $\bar{L}_{0}$ and $H_{0}=Z(S) L_{0}$, it follows that $L_{1} \subseteq H_{0}$ and that also $H_{0}=Z(S) L_{1}$. But then $L_{1}=O^{2}\left(H_{0}\right)$ and so $L_{1}=L_{0}$. We conclude that $L_{0}$, and therefore also $q$, is uniquely determined and the proposition is proved.

REMARK. The proposition is not true without the assumption that $H$ has quasidihedral or wreathed Sylow 2-subgroups. Indeed, if $H$ is the nontrivial central extension of $A_{7}$ by a group of order two, then $H$ is a $Q$-group with generalized quaternion Sylow 2-subgroups of order sixteen and with $O(H)=1$, but $H$ does not contain a normal subgroup isomorphic to $\operatorname{SL}(2, q)$ for any odd $q$. However, as we shall prove in Lemma 2 below, such a group $H$ cannot occur as a subgroup of a $Q D$-group.

Now if $H$ is an arbitrary $Q$-group with quasi-dihedral or wreathed Sylow 2subgroups, we have seen in the proof of Proposition 1 that $\bar{H}=H / O(H)$ is also a $Q$-group with quasi-dihedral or wreathed Sylow 2-subgroups and, in addition, satisfies $O(\bar{H})=1$. Hence by Proposition $2 \bar{H}$ possesses a unique characteristic subgroup $\bar{L}_{0}$ isomorphic to $S L(2, q)$ for some odd prime power $q$.

Definition 1. If $H$ is a $Q$-group with quasi-dihedral or wreathed Sylow 2subgroups and $H / O(H)$ possesses a characteristic subgroup isomorphic to $\operatorname{SL}(2, q)$ for some odd prime power $q$, we call $q$ the characteristic power of $H$. If $q=p^{r}$, $p$ a prime, we call $p$ the characteristic of $H$.

By the definition and the discussion preceding it, we have

LEMMA 1. If $H$ is a $Q$-group of characteristic power $q$, then $H / O(H)$ is also a $Q$ group of characteristic power $q$.

We have already shown in Proposition 2.1 that if $H$ is a $Q D$-group, then $C_{H}(x)$ is a $Q$-group with quasi-dihedral or wreathed Sylow 2-subgroups for any involution $x$ of $H$. Hence $C_{H}(x)$ has a characteristic power $q$ and a characteristic $p$. Moreover, the integers $q$ and $p$ are determined independently of the choice of the involution $x$ as a $Q D$-group has only one class of involutions. Thus we have

-DEFinition 2. If $H$ is a $Q D$-group and $x$ is an involution of $H$, then the characteristic power and the characteristic of $C_{H}(x)$ are called the characteristic power and the characteristic of $\boldsymbol{H}$.

As a direct consequence of these definitions and the preceding results, we have

LEMMA 2. If $H$ is a $Q D$-group of characteristic power $q$, then any $Q$-subgroup $K$ of $H$ has a characteristic power $r$ and $r \leqq q$.

Proof. Let $K$ be a $Q$-subgroup of $H$ and let $R$ be a Sylow 2-subgroup of $K$. By Proposition 1, we have $K=O(K) M$, where $M=C_{K}(Z(R))$. This clearly implies that $M$ is a $Q$-group, that $O(M) \subseteq O(K)$ and that $K / O(K)$ contains a normal subgroup isomorphic to $S L(2, r)$ if and only if $M / O(M)$ contains such a normal subgroup. Hence by definition of the characteristic power of a $Q$-group, it will suffice to show that $M / O(M)$ does possess such a normal subgroup and that $r \leqq q$. 
Now $R$ is a Sylow 2-subgroup of $M$. By definition of a $Q$-group and Lemmas 1.1 and 1.2, $R$ is either quasi-dihedral or is a subgroup of a wreathed 2-group $S, Z(R)$ is cyclic, and $R / Z(R)$ is dihedral. Setting $\bar{M}=M / O(M) Z(R)$, we have that $\bar{R}$ is dihedral and is a Sylow 2-subgroup of $\bar{M}$. Moreover, $O(\bar{M})=1$. Indeed, if $X$ denotes the inverse image of $O(\bar{M})$ in $M$, then $X=Z(R) \times O(X)$ as $Z(R) \subseteq Z(M)$ and $Z(R)$ is a Sylow 2-subgroup of $X$. Hence $X=O(M) Z(R)$ and so $O(\bar{M})=1$. Since $M$ is a $Q$-group, $M$ does not possess a normal 2-complement and so neither does $\bar{M}$. Applying the main structure theorem for groups with dihedral Sylow 2-subgroups to $\bar{M}$, [22], we conclude that either $\bar{M}$ is isomorphic to $A_{7}$ or else $\bar{M}$ possesses a normal subgroup $\bar{L}_{0}$ isomorphic to $\operatorname{PSL}(2, r)$ for some odd prime power $r$.

We argue next that the second case must occur and that $r \leqq q$. Indeed, if $x$ is the involution of $Z(R)$, then $M \subseteq N=C_{H}(x)$ and so we need only show that $N$ does not involve $A_{7}$ or $\operatorname{PSL}(2, r)$ for any $r>q$. Indeed, by definition of the characteristic power of a $Q D$-group, $N$ is a $Q$-group with quasi-dihedral or wreathed Sylow 2subgroup $S$ of characteristic power $q$. Hence $N / O(N)$ contains a normal subgroup isomorphic to $S L(2, q)$. Furthermore, the proof of Proposition 2 shows that $O(N) Z(S)$ is normal in $N$ and that $\bar{N}=N / O(N) Z(S)$ is isomorphic to a subgroup of $P \Gamma L(2, q)$ containing a normal subgroup $\bar{L}$ isomorphic to $P S L(2, q)$. By the structure of $P \Gamma L(2, q)$, as described in Lemma 3.3 of [22], $\bar{N} / \bar{L}$ is abelian. Since $O(N) Z(S)$ is solvable, while $A_{7}$ and $P S L(2, r)$ for $r>3$ are simple, we need only show that $\bar{L}$ does not involve $A_{7}$ or $\operatorname{PSL}(2, r)$ for any $r>q$. However, the first conclusion is a consequence of Lemma 3.1 (viii) of [22], while the second follows at once by a comparison of orders. We conclude that $\bar{M}$ possesses a normal subgroup $\bar{L}_{0}$ isomorphic to $\operatorname{PSL}(2, r)$ for some odd prime power $r$.

Finally using the results of Schur, as in the proof of Proposition 2, it follows that $M / O(M)$ possesses a normal subgroup of the form $L_{0} / O(M)$ with $L_{0} / O(M)$ isomorphic to $S L(2, r)$ and with $L_{0}$ mapping onto $\bar{L}_{0}$ in $\bar{M}$. Thus $M / O(M)$ contains a normal subgroup isomorphic to $S L(2, r)$ and the proof is complete.

Before turning to $D$-groups, we shall derive a result which considerably sharpens the conclusion of Proposition 2. Here the group $\Gamma U(2, q)$ denotes the semidirect product of $G U(2, q)$ with a cyclic group of automorphisms induced by the Galois group of $G F\left(q^{2}\right)$. We note also that in view of Proposition 2 and Lemma 2, the hypotheses of this proposition hold for $H / O(H)$ for any $Q$-group $H$ which has quasi-dihedral or wreathed Sylow 2-subgroups or which is a subgroup of a $Q D$ group.

Proposition 3. If $H$ is a Q-group in which $O(H)=1$ which contains a normal subgroup $L_{0}$ isomorphic to $S L(2, q)$ for some odd prime power $q$, then $H$ is isomorphic to a subgroup of one of the groups $\Gamma L(2, q)$ or $\Gamma U(2, q)$. More precisely, we have

(i) $H=L E$, where $L$ is normal in $H, E$ is cyclic of odd order, $L \cap E=1$, and $L \supseteq L_{0}$.

(ii) $L$ is isomorphic to $S L_{m}(2, q)$ or $S U_{m}(2, q)$ respectively for some $m$. 
(iii) The action of $E$ on $L$ is induced from the Galois group of the field $G F(q)$ or $G F\left(q^{2}\right)$, respectively.

(iv) $E=O\left(C_{H}(S)\right)$ for some Sylow 2-subgroup $S$ of $L$.

Proof. Let $S$ be a Sylow 2-subgroup of $H$. By definition of a $Q$-group and Lemmas 1.1 and $1.2, S$ is either quasi-dihedral or is a subgroup of a wreathed 2-group $S^{*}$ and contains the unique maximal generalized quaternion subgroup $Y^{*}$ of $S^{*}$, $Z(S)$ is cyclic, and $S / Z(S)$ is dihedral. Since $S \cap L_{0}$ is generalized quaternion, $\left|Z\left(S \cap L_{0}\right)\right|=2$ and so $Z\left(S \cap L_{0}\right)=Z\left(L_{0}\right)$. Since $Z\left(L_{0}\right)$ is characteristic in $L_{0}$, it is normal in $H$ and, as $Z\left(L_{0}\right)$ is of order two, $Z\left(L_{0}\right) \subseteq Z(H)$. In particular, $Z\left(L_{0}\right) \subseteq Z(S)$ and hence $Z(S) \cap L_{0}=Z\left(L_{0}\right)$.

By Proposition $1, Z(S) \subseteq Z(H)$. Setting $\bar{H}=H / Z(S)$, it follows that $\bar{S}$, which is a Sylow 2-subgroup of $\bar{H}$, is dihedral and that $\bar{L}_{0}$ is a normal subgroup of $\bar{H}$ isomorphic to $L_{0} / Z\left(L_{0}\right)$ and hence to $\operatorname{PSL}(2, q)$. Hence by the structure theorem for groups with dihedral Sylow 2-subgroups, $\bar{H}$ is isomorphic to a subgroup of $P \Gamma L(2, q)$. We set $\bar{L}=\bar{S} \bar{L}_{0}$. Since $\bar{L}$ has dihedral Sylow 2-subgroups, the proof of Lemma 9 of [21] shows that either $\bar{L}=\bar{L}_{0}$ or that $\left|\bar{L}: \bar{L}_{0}\right|=2$ and $\bar{L}$ is isomorphic to $P G L(2, q)$. Since $\bar{S} \subset \bar{L}$, it follows correspondingly that $Z(S)\left(S \cap L_{0}\right)$ has index one or two in $S$. Furthermore, we know from the structure of $P \Gamma L(2, q)$ that $P \Gamma L(2, q) / P S L(2, q)$ is abelian, so $\bar{L}$ is normal in $\bar{H}$. Moreover, since $\operatorname{SL}(2, q)$ is isomorphic with $S U(2, q)$, so also is $\operatorname{PSL}(2, q)$ with $\operatorname{PSU}(2, q)$ and $\operatorname{P\Gamma L}(2, q)$ with $P \Gamma U(2, q)$ modulo its center. (Since $P \Gamma U(2, q)$ is defined to be the factor group of $\Gamma U(2, q)$ by its subgroup of scalar matrices, it is easily checked that $|Z(P \Gamma U(2, q))|=2$.) Since $|\bar{H}: \bar{L}|$ is odd, we can thus identify $\bar{H}$ with a subgroup of $P \Gamma L(2, q)$ or $P \Gamma U(2, q)$ according as $q \equiv-1(\bmod 4)$ or $q \equiv 1(\bmod 4)$. We conclude, again by the structure of $P \Gamma L(2, q)$, that $\bar{H}=\bar{L} \bar{E}$, where $\bar{L}=P S L(2, q)$ or $P G L(2, q)$ if $q \equiv-1(\bmod 4)$ and $\bar{L}=P S U(2, q)$ or $P G U(2, q)$ if $q \equiv 1(\bmod 4), \bar{E}$ is a cyclic group of automorphisms of $\bar{L}$ induced correspondingly from the Galois group of $G F(q)$ or $G F\left(q^{2}\right)$, and $\bar{L} \cap \bar{E}=1$.

The next step in the proof is to show that $Z(S) L_{0}$ is isomorphic to $S L_{r}(2, q)$ or $S U_{r}(2, q)$ for a suitable $r$. Consider first the case that $S$ is a subgroup of the wreathed 2-group $S^{*}$ and contains the unique maximal generalized quaternion subgroup $Y^{*}$ of $S^{*}$. By definition of a $Q$-group, $H$ possesses a normal subgroup $K$ of index a power of two in $H$ such that $K$ has no normal subgroups of index two and has $Y^{*}$ as a Sylow 2-subgroup. Hence $\bar{K}$ has index a power of two in $\bar{H}$ and $\bar{K}$ has no normal subgroups of index two, so $\bar{K}=O^{2}(\bar{H})$. But $\bar{L}_{0}$ has no normal subgroups of index two, $|\bar{E}|$ is odd, and $\bar{L}_{0} \bar{E}$ is normal in $\bar{H}$ of index at most two, so also $\bar{L}_{0} \bar{E}=O^{2}(\bar{H})$. Thus $\bar{K}=\bar{L}_{0} \bar{E}$. Since $\bar{Y}^{*}$ is a Sylow 2-subgroup of $\bar{K}$ and $\bar{L}_{0}$ is normal in $\bar{K}$ of odd index, $\bar{Y}^{*}$ is a Sylow 2-subgroup of $\bar{L}_{0}$ and $Y^{*} \subseteq Z(S)\left(S \cap L_{0}\right)$, the latter group being the central product of the cyclic group $Z(S)$ and the generalized quaternion group $S \cap L_{0}$. Since $Y^{*}$ is itself generalized quaternion, it follows from the structure of $Z(S)\left(S \cap L_{0}\right)$ that $Y^{*} \subseteq S \cap L_{0}$. But $\bar{Y}^{*}=\bar{S} \cap \bar{L}_{0}$ as $\bar{Y}^{*}$ is a 
Sylow 2-subgroup of $\bar{L}_{0}$ and we conclude that $Y^{*}=S \cap L_{0}$ and hence that $Y^{*}$ is a Sylow 2-subgroup of $L_{0}$.

Therefore if $q \equiv \varepsilon(\bmod 4)$ and $2^{n}$ is the highest power of 2 dividing $q-\varepsilon$, we have $\left|Y^{*}\right|=2^{n+1}$ as $\left|L_{0}\right|=q(q-\varepsilon)(q+\varepsilon)$. It follows therefore from Lemma 1.2 that $S^{*}$ is wreathed of height $n$ and that $Z(S)$ has order $2^{r+1}$ with $0 \leqq r \leqq n-1$. On the other hand, by Lemma $2.1, S L_{r}(2, q)$ or $S U_{r}(2, q)$ is the central product of a cyclic group of order $2^{r+1}$ with $S L(2, q)$ according as $\varepsilon=+1$ or $\varepsilon=-1$ for $0 \leqq r \leqq n-1$. Since $Z(S) L_{0}$ is also such a central product, it follows that $Z(S) L_{0}$ is correspondingly isomorphic to $S L_{r}(2, q)$ or $S U_{r}(2, q)$.

On the other hand, if $S$ is quasi-dihedral, then $|Z(S)|=2$ and so $Z(S)=Z\left(L_{0}\right)$. Hence in this case, $Z(S) L_{0}$ is isomorphic to $S L_{0}(2, q)$ or $S U_{0}(2, q)$ according as $\varepsilon=-1$ or $\varepsilon=+1$. Setting $r=0$, the desired assertion follows in this case as well. We shall identify $Z(S) L_{0}$ with its image $S L_{r}(2, q)$ or $S U_{r}(2, q)$.

We now let $L$ be the inverse image of $\bar{L}$ in $H$. We shall argue next that $L$ is isomorphic to $S L_{m}(2, q)$ or $S U_{m}(2, q)$ for some $m$. If $\bar{L}=\bar{L}_{0}$, then $L=Z(S) L_{0}$ and the desired conclusion holds with $m=r$. Hence we can suppose without loss that $\bar{L} \supset \bar{L}_{0}$, in which case $\left|S: Z(S)\left(S \cap L_{0}\right)\right|=2$. Lemmas 1.1 and 1.2 imply now that $S$ is either quasi-dihedral or is wreathed of height $n$. Correspondingly we have $r=0$ or $r=n-1$ above.

If $S$ is quasi-dihedral, set $L^{*}$ equal to $S L_{1}(2, q)$ or $S U_{1}(2, q)$ according as $\varepsilon=-1$ or $\varepsilon=+1$ and if $S$ is wreathed, set $L^{*}$ equal to $S L_{n}(2, q)$ or $S U_{n}(2, q)$ according as $\varepsilon=+1$ or $\varepsilon=-1$. For simplicity, correspondingly set $m=1$ or $m=n$. Lemma 2.1 implies that $L^{*}$ has quasi-dihedral or wreathed Sylow 2-subgroups of height $m$. Note that because of our identification $L^{*}$ contains $Z(S) L_{0}$ as a subgroup of index two. Moreover, by Lemma $2.1, Z(S) \subseteq Z\left(L^{*}\right)$. Since $|Z(S)|=2^{m}$, it follows that $Z(S)$ is the center of a Sylow 2-subgroup of $L^{*}$. Hence, by Lemmas 1.1 and 1.2, we have that $L^{*} / Z(S)$ has dihedral Sylow 2-subgroups. Since $L^{*} / Z(S)$ contain $Z(S) L_{0} / Z(S)$ as a subgroup of index two, the latter group being isomorphic to $\operatorname{PSL}(2, q)$, it follows from Lemma 9 of [21], as with $\bar{L}$ above, that $L^{*} / Z(S)$ is isomorphic to $P G L(2, q)$. Hence without loss we can identify $L^{*} / Z(S)$ with $\bar{L}$.

We now prove that $L^{*}$ and $L$ are isomorphic. Suppose $L$ and $L^{*}$ have quasidihedral Sylow 2-subgroups. Then $Z(S) L_{0}=L_{0}$ has generalized quaternion Sylow 2-subgroups and so not every involution of $L$ or $L^{*}$ lies in $L_{0}$. Hence $L=L_{0} A$ and $L^{*}=L_{0} A^{*}$, where $A=\langle a\rangle$ and $A^{*}=\left\langle a^{*}\right\rangle$ have order two and $L_{0} \cap A=L_{0} \cap A^{*}=1$. On the other hand, suppose $L$ and $L^{*}$ have wreathed Sylow 2-subgroups. Lemma 1.2 implies that $S$ contains an element $a$ such that $\left\langle a^{2}\right\rangle=Z(S)$ and $S=\left\langle Y^{*}, a\right\rangle$. Since $Z(S) Y^{*}$ is a Sylow 2-subgroup of $Z(S) L_{0}, a \notin Z(S) L_{0}$. Hence if we set $A=\langle a\rangle$, we have $L=Z(S) L_{0} A$ and $Z(S) L_{0} \cap A=Z(S)$. A similar reasoning applied to $L^{*}$ shows that $L^{*}=Z(S) L_{0} A^{*}$, where $A^{*}=\left\langle a^{*}\right\rangle$ is cyclic, $\left\langle a^{* 2}\right\rangle=Z(S)$ and $Z(S) L_{0} \cap A^{*}=Z(S)$. Replacing $a^{*}$ by an appropriate power, we can assume in all cases that $a^{* 2}=a^{2}$.

Since $a^{2}=a^{* 2}$ and $a, a^{*}$ each centralizes $Z(S)$, to prove that $L$ and $L^{*}$ are isomorphic, it will suffice to show that $a$ and $a^{*}$ can be chosen to induce the same 
automorphism of $L_{0}$. For then they will induce the same automorphism of $Z(S) L_{0}$ and the desired isomorphism will follow. Note also that if $q=3$, one can verify directly that $L$ and $L^{*}$ are isomorphic; in fact, each is isomorphic to $G L(2,3)$ in the quasi-dihedral case and to $G U(2,3)$ in the wreathed case. Hence we can assume without loss that $q>3$, in which case $L_{0}$ is perfect.

Since $a^{2}$ and $a^{* 2}$ lie in $Z(S)$ in all cases, while $a$ and $a^{*}$ do not, and taking into account our identification of $L^{*} / Z(S)$ with $\bar{L}$, it follows that $\bar{a}, \bar{a}^{*}$ are each involutions of $\bar{L}-\bar{L}_{0}$. But the Sylow 2-subgroup $\bar{S}$ of $\bar{L}$, being dihedral, has three conjugacy classes of involutions, two of which lie in $\bar{L}_{0}$. Hence $\vec{a}$ and $\bar{a}^{*}$ are conjugate in $\bar{L}$ and so replacing $a^{*}$ by a suitable conjugate in $L^{*}$ (which does not affect $a^{* 2}$ as $a^{* 2} \in Z(S) \subseteq Z\left(L^{*}\right)$ ), we can also assume without loss that $\bar{a}=\bar{a}^{*}$. But then if $\phi$ and $\phi^{*}$ are the automorphisms of $Z(S) L_{0}$ determined by conjugation by $a$ and $a^{*}$ respectively and if $\psi=\phi^{*} \phi^{-1}$, our conditions imply that $\psi$ acts trivially on $L_{0} / Z\left(L_{0}\right)$ inasmuch as $L_{0}$ maps onto $\bar{L}_{0}$. Hence $\left[\psi, L_{0}, L_{0}\right] \subseteq\left[Z\left(L_{0}\right), L_{0}\right]=1$. Since $L_{0}$ is perfect, the three subgroup lemma now yields that $\psi$ is the identity on $L_{0}$, so $a$ and $a^{*}$ induce the same automorphism of $L_{0}$, as required. Thus $L$ and $L^{*}$ are isomorphic in all cases.

We can therefore identify $L$ and $L^{*}$. Thus $L=S L_{m}(2, q)$ or $S U_{m}(2, q)$, as the case may be. Now by Lemma 2.1 the subgroups $S L_{m}(2, q)$ of $G L(2, q)$ and $S U_{m}(2, q)$ of $G U(2, q)$ are normal in $\Gamma L(2, q)$ and $\Gamma U(2, q)$ respectively and so are left invariant by the cyclic group $F^{*}$ of automorphisms of $G L(2, q)$ or $G U(2, q)$ induced from the Galois group of $G F(q)$ or $G F\left(q^{2}\right)$ respectively. Since $\bar{H}=\bar{L} \bar{E}$, where $\bar{E}$ is a cyclic group of automorphisms of $\bar{L}$ induced from the Galois group of $G F(q)$, it follows that there exists a subgroup $E^{*}$ of $F^{*}$ which induces the same group of automorphisms of $L / Z(L)$ (which has been identified with $\bar{L}$ ) as $\bar{E}$ induces on $\bar{L}$. Setting $H^{*}=L E^{*}$, we can therefore regard $\bar{H}$ as the homomorphic image of $H^{*}$ with kernel $Z(L)$ in such a way that $\bar{E}^{*}=\bar{E}$. Moreover, we have $L \cap E^{*}=1$.

Now consider $H$. Since $\bar{E}$ is of odd order, the inverse image of $\bar{E}$ in $H$ is of the form $Z(S) \times E$, where $E$ is isomorphic to $\bar{E}$. Since $\bar{H}=\bar{L} \bar{E}$ and $\bar{L} \cap \bar{E}=1$, it follows that $H=L E$ and that $L \cap E=1$. We shall argue finally that $H$ and $H^{*}$ are isomorphic and that for a suitable choice of $\bar{E}$, we have $E=O\left(C_{H}(S)\right)$. Since $H^{*}$ is a subgroup of one of the group $\Gamma L(2, q)$ or $\Gamma U(2, q)$, all parts of the proposition will be established.

By Lemma 3.3 (i) of [22], $\bar{E}=O\left(C_{F}(\bar{R})\right)$ for some Sylow 2-subgroup of $\bar{L}$. Replacing $\bar{E}$ by a suitable conjugate we can assume that $\bar{R}=\bar{S}$. Thus $E$ normalizes $S$ and stabilizes the chain $S \supset Z(S) \supset 1$. Since $|E|$ is odd, $E$ centralizes $S$ and so $E=O\left(C_{H}(S)\right)$. Similarly $E^{*}=O\left(C_{H}(S)\right)$.

Since $E$ and $E^{*}$ have the same images $\bar{E}$ in $\bar{H}$, we can choose generators $e, e^{*}$ of $E, E^{*}$ respectively such that $\bar{e}=\bar{e}^{*}$. If $\zeta, \zeta^{*}$ denote the corresponding automorphisms of $L$, it follows as with $\psi=\phi^{*} \phi^{-1}$ above that, when $q>3, \zeta^{*} \zeta^{-1}$ is the identity on $L_{0}$ and hence that $e, e^{*}$ determine the same automorphism of $L_{0}$. The same holds when $q=3$ as then $E=E^{*}=1$. But $S \subset L$ and $L=S L_{0}$. Since $E$ and $E^{*}$ centralize $S$, 
we see that $e$ and $e^{*}$ determine the same automorphisms of $L$. Since $E$ and $E^{*}$ are disjoint from $L$, we conclude that $H=L E$ is isomorphic to $H^{*}=L E^{*}$ and the proposition is proved.

To define the characteristic power of a $D$-group, we first prove a result analogous to Proposition 3:

Proposition 4. If $H$ is a D-group in which $O(H)=1$, then $H$ is either isomorphic to $P G L_{n}(2,3)$ for some $n$, to $A_{7}$, or to a subgroup of $P \Gamma L(2, q)$ for some odd $q$. In the latter case, we have

(i) $H=L E$, where $L$ is normal in $H, E$ is cyclic of odd order, and $L \cap E=1$.

(ii) $L$ is isomorphic to either $\operatorname{PSL}(2, q), \operatorname{PGL}(2, q)$, or $P G L^{*}(2, q)$.

(iii) The action of $E$ on $L$ is induced from the Galois group of the field $G F(q)$.

(iv) E centralizes a Sylow 2-subgroup of $L$.

Proof. Let $S$ be a Sylow 2-subgroup of $H$. By definition of a $D$-group, $S$ is either dihedral, quasi-dihedral, or wreathed and $H$ contains a normal subgroup $K$ having no normal subgroups of index two with $|H: K| \leqq 2$ and with $S \cap K$ respectively dihedral, dihedral, or abelian of type $\left(2^{n}, 2^{n}\right)$ for some $n \geqq 2$. Since $O(K)$ is characteristic in $K$, it is normal in $H$ and so $O(K)=1$ as $O(H)=1$.

First consider the case that $S$ is wreathed. Then the Sylow 2-subgroup $U=S \cap K$ of $K$ is abelian of type $\left(2^{n}, 2^{n}\right)$ with $n \geqq 2$. Since $O(K)=1$ and $K$ has no normal subgroups of index two, a theorem of Brauer (Theorem 1 of $\S \mathrm{VI}$ of $[6$, II]) implies that $U$ is normal in $K$ of index three and $C_{K}(U)=U$. Thus a Sylow 3-subgroup $P$ of $K$ acts regularly on $U$. Furthermore, $H=U N_{H}(P)$ by the Frattini argument. Since $\left[N_{U}(P), P\right] \subseteq U \cap P=1$, we have $N_{U}(P) \subseteq C_{U}(P)$ and so $N_{U}(P)=1$ as $P$ acts regularly on $U$. Since $|H: U|=6$, it follows that $\left|N_{H}(P)\right|=6$. If $N_{H}(P)$ were abelian, then $S$ would be normal in $H$. But then $P$ would centralize $S$ by Lemma 1.3, which is not the case. Thus $N_{H}(P)$ is nonabelian and we conclude that $H$ is the semidirect product of $U$ and $N_{H}(P)$, the latter group being isomorphic to $P G L(2,3)$. As remarked in $\S 2$, the action of $N_{H}(P)$ on $U$ is uniquely determined and so $H$ is isomorphic to $P G L_{n}(2,3)$.

We can therefore assume henceforth that $S$ is dihedral or quasi-dihedral, in which case the Sylow 2-subgroup $U=S \cap K$ of $K$ is dihedral. Since $O(K)=1$, the main theorem of [22] implies that $K$ is isomorphic to $A_{7}$ or to a subgroup of $P \Gamma L(2, q)$ containing $\operatorname{PSL}(2, q)$ for some odd $q$. Since $O(H)=1$, the same conclusions hold for $H$ if $S$ is dihedral. As in the proof of Proposition 3 above, it follows with the aid of Lemma 9 of [21] that $K$ (and also $H$ if $S$ is dihedral) is of the form $L_{0} E$, where $L_{0}$ is normal in $K$ (or $\left.H\right), L_{0}$ is isomorphic to $\operatorname{PSL}(2, q)$ or $\operatorname{PGL}(2, q)$, $E$ is cyclic of odd order, $L_{0} \cap E=1$, and the action of $E$ on $L_{0}$ is induced from the Galois group of $G F(q)$. Moreover, $E$ centralizes a Sylow 2-subgroup of $L_{0}$ by Lemma 3.3 (i) of [22]. In particular, all parts of the proposition hold (with $L_{0}=L$ ) if $S$ is dihedral. 
It remains to consider the case that $S$ is quasi-dihedral. Since $K$ has dihedral Sylow 2-subgroups and $|H: K| \leqq 2$, we must have $|H: K|=2$ in this case. Furthermore, $K$ cannot be isomorphic to $A_{7}$, for then $H$ would be isomorphic to either the symmetric group $S_{7}$ or to the direct product of $A_{7}$ and a group of order two, neither of which has quasi-dihedral Sylow 2-subgroups. Thus $K=L_{0} E$, where $L_{0}$ and $E$ are as in the preceding paragraph. In the present case, $L_{0}$ must be isomorphic to $\operatorname{PSL}(2, q)$. Indeed, otherwise, $L_{0}$ would be isomorphic to $P G L(2, q)$ and $K$ would contain a normal subgroup $L_{1}$ isomorphic to $\operatorname{PSL}(2, q)$ and of index two in $L_{0}$. But then $K / L_{1}$ would have a Sylow 2-subgroup of order two and so would contain a normal subgroup of index two. Therefore $K$ would also contain such a normal subgroup, which is not the case.

If $q=3$, then $E=1$; while if $q>3$, then $\operatorname{PSL}(2, q)$ and hence $L_{0}$ is simple. In either case, $L_{0}$ is characteristic in $K$ and so is normal in $H$. Thus $L=L_{0} S$ is a group and $L_{0}$ is of index two in $L$. Moreover, $H=S K=S L_{0} E=L E$. Since $|E|$ is odd and $L_{0} \cap E=1$, we also have $L \cap E=1$.

Since $S$ is quasi-dihedral, no involution of $H$ centralizes $L_{0}$ and hence $C_{H}\left(L_{0}\right)$ has odd order. Since $C_{H}\left(L_{0}\right)$ is normal in $H$ and $O(H)=1$, it follows that $C_{H}\left(L_{0}\right)=1$. Thus $H$ is isomorphic to a subgroup of Aut $L_{0}$. But Aut $L_{0}$ is isomorphic to $P \Gamma L(2, q)$ and so we can identify, not only $K$, but also $H$ with a subgroup of $P \Gamma L(2, q)$. In particular, $L_{0}=P S L(2, q)$ and $E$ is induced from the Galois group of $G F(q)$. Furthermore, $L$ is normal in $H$ as $P \Gamma L(2, q) / P S L(2, q)$ is abelian.

Now $L$ is not equal to $\operatorname{PGL}(2, q)$, for then the Sylow 2-subgroup $S$ of $L$ would be dihedral. This forces $q$ to be a square, since otherwise the cyclic subgroup $F$ of $P \Gamma L(2, q)$ induced from the Galois group of $G F(q)$ would have odd order and consequently $\operatorname{PGL}(2, q)$ would be the unique subgroup of $P \Gamma L(2, q)$ containing $\operatorname{PSL}(2, q)$ as a subgroup of index two. Thus $q=r^{2}$ for some odd prime power $r$. If $L=L_{0} A$, where $A=\langle a\rangle \subseteq F$ and $|A|=2$ then $a$ is induced from an automorphism of $G F(q)$ whose fixed subfield is $G F(r)$. Hence $a$ centralizes those elements of $L_{0}$ which are the images of elements of $\operatorname{SL}(2, q)$ whose matrix entries lie in the subfield $G F(r)$ and so $C_{L_{0}}(a)$ contains a subgroup isomorphic to $\operatorname{PSL}(2, r)$. Since $C_{L_{0}}(a)$ contains a four subgroup and $a \notin L_{0}$, it follows that $L$ contains an elementary abelian subgroup of order eight, contrary to the fact that $L$ has quasi-dihedral Sylow 2-subgroups. Hence $L$ is not of this form. Since we have excluded two of the three possible subgroups of $P \Gamma L(2, q)$ which contain $P S L(2, q)$ as a subgroup of index two, we conclude that $L$ is necessarily equal to $P G L^{*}(2, q)$. Therefore all parts of the proposition hold in this case as well, and the proof is complete.

If $H$ is a $D$-group, then clearly so also is $\bar{H}=H / O(H)$. Since $O(\bar{H})=1$, the proposition applies to $\bar{H}$. In particular, $\bar{H}$ is either isomorphic to $A_{7}$, to $P G L_{n}(2,3)$ for some $n$, or contains a normal subgroup isomorphic to $\operatorname{PSL}(2, q)$ for some odd prime power $q$. Thus we have

Definition 3. If $H$ is a $D$-group and $H / O(H)$ possesses a normal subgroup isomorphic to $\operatorname{PSL}(2, q)$ with $q=p^{n}, p$ an odd prime, we call $q$ the characteristic 
power of $H$ and $p$ the characteristic of $H$. Furthermore, if $H / O(H)$ is isomorphic to $P G L_{n}(2,3)$ for some $n$, we say that $H$ has characteristic power 3 and characteristic 3. On the other hand, if $H / O(H)$ is isomorphic to $A_{7}$, we do not define the characteristic power or the characteristic of $H$. Finally it will be convenient to say that $H$ is of linear type whenever $H / O(H)$ contains a normal subgroup isomorphic to $P S L(2, q)$ or is isomorphic to $P G L_{n}(2,3)$.

We conclude this section with a specialized property of groups whose Sylow 2subgroups are isomorphic to subgroups of a wreathed 2-group.

LEMMA 3. Let $H$ be a group and $R$ a Sylow 2-subgroup of $H$ and assume the following conditions hold:

(a) $R$ is isomorphic to a subgroup of a wreathed 2-group of height $n$.

(b) $H$ contains a normal subgroup $K$ such that $H=K A$, where $A$ is cyclic of order $2^{n}$ and $K \cap A=1$.

Under these conditions, either $H$ has a normal 2-complement or $K$ is a Q-group with generalized quaternion Sylow 2-subgroups.

Proof. Without loss we may assume that $A \subseteq R$ and $R \subseteq S$, where $S$ is a wreathed 2-group of height $n$. Setting $X=R \cap K$, our conditions imply that $X$ is normal in $R, X$ is a Sylow 2-subgroup of $K, R=X A$, and $X \cap A=1$. By Lemma 1.2 (xiii), either $X$ is cyclic or $R=S$. In the first case, $K$ has a normal 2-complement by Burnside's transfer theorem, whence $H$ does as well and the lemma holds. We can therefore assume that $R=S$.

Thus $H$ is a group with wreathed Sylow 2-subgroup $S$ and $H$ possesses a normal subgroup $K$ of index $2^{n}$ with $n \geqq 2$. In particular, $H$ is not a $Q D$-group or a $D$-group and consequently, by Proposition 1.2, either $H$ has a normal 2-complement or $H$ is a $Q$-group. Since the lemma holds in the first case, we can also assume that $H$ is a $Q$-group, whence $H$ contains a normal subgroup $L$ of index $2^{n}$ with generalized quaternion Sylow 2-subgroups, and $L$ has no normal subgroups of index 2 . Hence to complete the proof, we need only show that $K=L$, for then $K$ will be a $Q$-group.

We know that $H$ possesses a unique normal subgroup $O^{2}(H)$ which is minimal subject to the condition that $H / O^{2}(H)$ is a 2-group. Our conditions clearly imply that $L=O^{2}(H)$ and that $K \supseteq O^{2}(H)$. Therefore $K \supseteq L$. Since $|H: K|=|H: L|=2^{n}$, we conclude at once that $K=L$, as required.

4. Q-group with quasi-dihedral or wreathed Sylow 2-subgroups. In this section we shall establish a number of basic properties of $Q$-groups with quasi-dihedral or wreathed Sylow 2-subgroups that we shall need for the paper. It will be convenient to fix the notation once and for all that we shall use throughout the section.

Thus $H$ will denote an arbitrary $Q$-group with quasi-dihedral or wreathed Sylow 2-subgroups and we set:

$S$ = Sylow 2-subgroup of $H$,

$n=$ height of $S$, 
$\langle x\rangle=\Omega_{1}(Z(S))$

$T=$ a four subgroup of $S$ with $T$ normal in $S$ if $S$ is wreathed,

$x_{1}, x_{2}, x_{3}=$ the involutions of $T$ with $x_{1}=x$ and $x_{2}=z$ if $S$ is quasi-dihedral,

$D=N_{S}(T)$,

$y=$ an element of order 4 in $S^{\prime}$,

$d=$ an element of order 4 in $D-C_{S}(T)$,

$N=C_{H}(x)$,

$C=C_{H}(T)$,

$E=O\left(C_{H}(S)\right)$,

$L_{0}=$ the subgroup of $N$ containing $O(H) \cap N$ whose image in $H / O(H)$ is the normal subgroup of $H / O(H)$ isomorphic to $S L(2, q)$,

$L=$ the subgroup of $N$ containing $O(H) \cap N$ whose image in $H / O(H)$ is the normal subgroup of $H / O(H)$ isomorphic to one of the groups $S L_{n}(2, q)$ or $S U_{n}(2, q)$,

$q=$ the characteristic power of $H$,

$\varepsilon= \pm 1$, where $q \equiv \varepsilon(\bmod 4)$,

$\delta= \pm 1$, where $\delta=+1$ if $n=1$ and $\delta=-1$ if $n>1$.

A number of comments are in order. First, by definition of the height of $S$, we have

$S$ is quasi-dihedral if $n=1$ and wreathed if $n>1$.

Lemmas 1.1 and 1.2 have several implications. First, $Z(S)$ is cyclic of order $2^{n}$, so $\Omega_{1}(Z(S))=\langle x\rangle$ for some involution $x$. Thus $x \in T$ and hence we can number this involution of $T$ so that $x=x_{1}$. In addition,

$D$ is dihedral of order 8 if $n=1$ and $D=S$ if $n>1$.

Furthermore, $S^{\prime}$ is cyclic of order at least four and if $n=1$, then $S^{\prime} \cap D$ has order four. Hence in all cases $y$ exists and $y \in D$. Moreover, $x \in S^{\prime}$ and so $y^{2}=x$.

Setting $U=C_{S}(T)$ for the moment, we have $U=T$ if $n=1$ and $U$ is the unique abelian maximal subgroup of $S$ if $n>1$. In the latter case, $S^{\prime} \subseteq U$. Hence

$$
y \in D-C_{S}(T) \text { if and only if } n=1 .
$$

Hence when $n=1, d$ is either $y$ or $y^{-1}$. We normalize the notation by setting $d=y$ if $n=1$. Since $d \in S-U$ when $n>1$, it follows that

$$
d=y \quad \text { if and only if } n=1 \text {. }
$$

In addition, when $n>1$, Lemma 1.2 implies that $S-U$ contains an element $d$ of order four and that $d^{2} \in Z(S)$. Hence in all cases, $d^{2}=x$ and $\left(x_{2} d\right)^{2}=1$. Finally since $D$ normalizes $T$, we also have that $C$ is $D$-invariant.

Observe next that if $n=1, T$ is a Sylow 2-subgroup of $C$, since otherwise $H$ would contain a noncyclic abelian subgroup of order eight, which is not the case when $S$ is quasi-dihedral. On the other hand, if $n>1, D=S$ and so $S$ normalizes $C$. Since $U \subseteq C$ and $T \nsubseteq Z(S)$, it follows that $U$ is a Sylow 2-subgroup of $C$. Thus we have

$C_{S}(T)=C_{D}(T)$ is a Sylow 2-subgroup of $C$,

$C_{S}(T)$ is a noncyclic abelian subgroup of $S$ of maximal order,

$C_{S}(T)=T$ if $n=1$ and $C_{S}(T)$ is the unique abelian maximal subgroup of $S$ if $n>1$. 
We claim next that $C$ has a normal 2-complement. Indeed, $T=\Omega_{1}(U)$ and $U$ is abelian. Hence any element of $C$ of odd order which normalizes $U$ necessarily centralizes it. Since $U$ is a Sylow 2-subgroup of $C$, the assertion follows from Burnside's transfer theorem. Thus we have $C=O(C) C_{S}(T)$.

Since $T \subseteq S$, this clearly implies that $E \subseteq O(C)$.

Since $O(C)$ is characteristic in $C$, it also yields that $O(C)$ is $D$-invariant.

By Lemmas 1.1 and 1.2, $S^{\prime}$ is contained in the unique abelian maximal subgroup of $S$ and $S^{\prime} \cap Z(S)$ has order two. Since $S^{\prime}$ is cyclic and $y$ is an element of order four in $S^{\prime}$, it follows that $\langle y\rangle$ is normal in $S$ and that $C_{S}(y)$ is abelian of index two in $S$. Since $S$ normalizes $C_{H}(y)$, we conclude as with $T$ above that $C_{S}(y)$ is a Sylow 2-subgroup of $C_{H}(y)$. But $C_{S}(y)$ is either cyclic or abelian on two generators and so any element of $C_{H}(y)$ of odd order which normalizes $C_{S}(y)$ necessarily centralizes it. Another application of Burnside's theorem yields that $C_{H}(y)$ possesses a normal 2-complement. Hence

$C_{H}(y)=O\left(C_{H}(y)\right) C_{S}(y)$,

$O\left(C_{H}(y)\right)$ is $S$-invariant, and

$C_{S}(y)$ is abelian of index 2 in $S$.

Since $C_{H}(S) \subseteq C_{H}(y)$, it follows from the structure of $C_{H}(y)$ that also $C_{H}(S)$ has a normal 2-complement. Since $E=O\left(C_{H}(S)\right)$ by definition of $E$, this yields $C_{H}(S)=E \times Z(S)$.

Observe next that $H=O(H) C_{H}(Z(S))$ by Proposition 3.1. Since $x \in Z(S)$ and $N=C_{H}(x)$, this implies $H=O(H) N$.

We turn now to the groups $L_{0}$ and $L$. By Lemma $2.1 \bar{H}=H / O(H)$ is a $Q$-group with quasi-dihedral or wreathed Sylow 2-subgroup $\bar{S}$. Hence by Propositions 2.2 and $2.3, \bar{H}$ possesses normal subgroups $\bar{L}_{0}$ isomorphic to $S L(2, q)$ and $\bar{L}$ isomorphic to $S L_{n}(2, q)$ or $S U_{n}(2, q)$ with $\bar{S} \subseteq \bar{L}$. More precisely,

$\bar{L}$ is isomorphic to $S L_{n}(2, q)$ if $q \equiv-\delta(\bmod 4)$ and

$\bar{L}$ is isomorphic to $S U_{n}(2, q)$ if $q \equiv \delta(\bmod 4)$.

Since $H=O(H) N, \bar{N}=\bar{H}$ and consequently $N$ does contain subgroups $L_{0}, L$ with the given properties and we have

$L_{0}$ and $L$ are normal in $N, S \subset L$.

By Lemmas 1.1 and $1.2, S^{\prime}$ is contained in the unique generalized quaternion subgroup $Y$ of $S$ of maximal order. Moreover, the proofs of Propositions 2.2 and 2.3 show that $\bar{Y}$ is a Sylow 2-subgroup of $\bar{L}_{0}$ and that $Z(\bar{S}) \bar{Y}$ is of index two in $\bar{S}$. Hence

$S \cap L_{0}$ is the unique generalized quaternion subgroup of maximal order in $S$, and

$Z(S)\left(S \cap L_{0}\right)$ is of index 2 in $S$.

Lemmas 1.1 and 1.2 also imply that $S=Y C_{S}(T)$, that $T \nsubseteq Y$ if $n=1$, and that $T \subseteq Y Z(S)$ if $n>1$. Thus we also have

$$
L=L_{0} C_{S}(T), \quad L=L_{0} T \text { if } n=1, \quad \text { and } \quad T \subseteq L_{0} Z(S) \text { if } n>1 .
$$


Moreover, $y \in S^{\prime} \subseteq Y$. In addition, as $d$ inverts a generator of $S^{\prime},\left\langle S^{\prime}, d\right\rangle$ is generalized quaternion, so $\left\langle S^{\prime}, d\right\rangle=Y$ as $Y$ is the unique generalized quaternion subgroup of its order in $S$. Hence we also have

$$
y \in L_{0} \text { and } d \in L_{0} .
$$

By Proposition 2.3 and the fact that $C_{H}(S)$ maps onto $C_{H}(\bar{S})$, we have that $\bar{H}=\bar{L} O\left(C_{H}(\bar{S})\right)=\bar{L} \bar{E}$ and $\bar{L} \cap \bar{E}=1$, whence

$$
H=O(H) L E, \quad N=L E \text { and } E \cap L \subseteq O(H) \cap N .
$$

Since $L$ maps onto $\bar{L}$, which is normal in $\bar{H}$, we have $O(\bar{L})$ normal in $\bar{H}$ and hence $O(\bar{L}) \subseteq O(\bar{H})=1$. Thus $O(L) \subseteq O(N)$. Likewise $O(\bar{N})$ is normal in $\bar{H}$ as $N$ maps onto $\bar{H}$, whence $O(N) \subseteq O(H)$ and therefore $O(N)=O(H) \cap N$. On the other hand, clearly $O(H) \cap N \subseteq O(L)$ as $O(H) \cap N$ is a normal subgroup of $L$ and so $O(N) \subseteq O(L)$. We conclude that

$$
O(N)=O(L)=O(H) \cap N .
$$

Throughout the paper these basic properties of $Q$-groups with quasi-dihedral and wreathed Sylow 2-subgroups will often be used without explicit reference.

Finally $H$ is solvable if and only if $q=3$ inasmuch as $O(H)$ is solvable and $S L(2, q)$, $q$ odd, is solvable if and only if $q=3$. As a result, the case $q=3$ is somewhat exceptional. In our general analysis of $Q D$-groups of characteristic power $q$, we shall treat the cases $q=3$ and $q=5$ by a totally separate argument, primarily involving modular character theory. As a consequence, the properties of $Q$-groups that we shall need will be limited almost entirely to the case $q>5$. For simplicity we shall assume throughout this section that $q>3$. Moreover, in a few specific lemmas we shall make the additional assumption that $q>5$. On the other hand, we note that any property of $Q$-groups of characteristic power three that may be required can easily be verified by direct inspection.

We begin with some specialized lemmas in the case that $O(H)=1$. In this case $H=N$ and, by Proposition 3.1, $Z(S) \subseteq Z(H)$. However, $Z(H)$ is a 2-group as $O(H)=1$, so $Z(S)=Z(H)=Z(L)$. In addition, $Z(S) \cap L_{0}$ has order two. Moreover, $H$ has the structure given in Proposition 3.3 and also $\bar{H}=H / Z(H)=\bar{L} \bar{E}$ has dihedral Sylow 2-subgroups, $\bar{L}$ is isomorphic to $\operatorname{PGL}(2, q)$, and the action of $\bar{E}$ on $\bar{L}$ is induced from the Galois group of $G F(q)$. Thus many of the properties of $H$ can be read off from properties of $\bar{H}$, especially those given in Lemmas 3.1 and 3.3 of [22]. Throughout Lemmas $1-11, \bar{H}$ will denote $H / Z(H)$.

LEMMA 1. If $O(H)=1$, then

(i) $|L|=2^{n} q(q-1)(q+1)$ and $|H|=|L||E|$.

(ii) The Sylow p-subgroups of $L$ are cyclic if $p$ divides $q \pm 1$ and are elementary abelian of order $q$ if $p$ divides $q$.

(iii) $C_{H}\left(L_{0}\right)=Z(H)$. 
(iv) $L_{0} Z(H) / Z(H)$ is simple and is the unique minimal normal subgroup of $H / Z(H)$.

(v) $H^{\prime} \subseteq L_{0}$ and $H / L_{0}$ is abelian.

Proof. Lemmas 3.1 (i), (iii), and (vi) of [22], applied to $\bar{L}$, the image of $L$ in $\bar{H}$ yield (ii) and the first assertion of (i) inasmuch as $Z(H)$ has order $2^{n}$ and $\bar{L}$ is isomorphic to $\operatorname{PGL}(2, q)$. As we have noted at the beginning of the section, $H=L E$ and $L \cap E \subseteq O(H) \cap N$. Since $O(H)=1$, the second assertion of (i) also holds. Furthermore, Lemma 3.3 (i) of [22] implies that $C_{H}\left(\bar{L}_{0}\right)=1$, whence $C_{H}\left(L_{0}\right)=Z(H)$, proving (iii). Since $q>3$ by assumption, $\bar{L}_{0}$ is simple by Lemma 3.1 (ii) of [22], and is the unique minimal normal subgroup of $\bar{H}$ by Lemma 3.3 (i) of [22], proving (iv).

Finally as $Y=S \cap L_{0}$ is the unique generalized quaternion subgroup of $S$ of maximal order, $S / Y$ and hence also $L / L_{0}$, is cyclic by Lemmas 1.1 and 1.2. But also $E$ is cyclic and $E$ centralizes $L / L_{0}$ as $E$ centralizes $S$. Since $H=L E$, it follows that $H / L_{0}$ is abelian. Thus $H^{\prime} \subseteq L_{0}$ and so (v) also holds.

Our next set of lemmas gives more detailed information concerning the subgroup structure of $H$.

LEMMA 2. If $O(H)=1$, then

(i) $O(C)=W E$, where $W=O(C) \cap L$ is normal in $O(C), W$ is cyclic or order $(q+\delta \varepsilon) / 2^{n}, W$ is centralized by $C_{S}(T), W$ is inverted by $d, W$ is a Hall subgroup of $L$, and for any nontrivial subgroup $W_{0}$ of $W, N_{L}\left(W_{0}\right)=W D$.

(ii) $C \cap L_{0}$ contains $W$ and is cyclic of order $q+\delta \varepsilon$.

(iii) For any prime $p$, no p-element of $O(C)-(O(C) \cap L)$ centralizes $O_{p^{\prime}}(W)$.

(iv) $C_{O(C)}\left(O(C)^{\prime}\right)=W$.

(v) If $P$ is a $D$-invariant Sylow p-subgroup of $O(C)$, either $Z(P) \subseteq W$ or $P \subseteq E$.

(vi) If $E_{0}$ is a subgroup of $E$ of order $e_{0}$, then $C_{L_{0}}\left(E_{0}\right)$ is isomorphic to $S L\left(2, q_{0}\right)$ with $q=q_{0}^{e_{0}}$.

Proof. First of all, $\bar{T}=\left\langle\bar{x}_{2}\right\rangle$ is of order two. If $n=1$, then $L=L_{0} T$ and so $\bar{x}_{2} \in \bar{L}-\bar{L}_{0}$. Moreover, in this case, $C_{S}(T)=T, D$ is dihedral of order eight, $\bar{D}$ is a four group, and $\delta=+1$. Lemmas 3.1 (iii) and 3.3 (i) of [22] imply that $C_{H}\left(\bar{x}_{2}\right)$ $=C_{L}\left(\bar{x}_{2}\right) \bar{E}$ with $C_{L}\left(\bar{x}_{2}\right)$ being dihedral of order $2(q+\varepsilon)$ and containing $\bar{D}$. Thus $\bar{W}=O\left(C_{L}\left(\bar{x}_{2}\right)\right)$ is cyclic of order $\frac{1}{2}(q+\varepsilon)$ as $q \equiv \varepsilon(\bmod 4)$ and, in addition, $\bar{W}$ is inverted by any element of $\bar{D}-\bar{T}$. Furthermore, $\bar{W}$ is a Hall subgroup of $\bar{L}$ and $N_{L}\left(\bar{W}_{0}\right)=\bar{W} \bar{D}$ for any nontrivial subgroup $\bar{W}_{0}$ of $\bar{W}$ by Lemma 3.1 (v) of [22]. Since $C_{H}(T)$ maps onto $C_{H}\left(\bar{x}_{2}\right)$, all parts of (i) and also (ii) follow in this case.

Suppose now that $n>1$, in which case $T \subseteq Z(S) L_{0}, D=S, C_{S}(T)$ is the unique abelian maximal subgroup of $S$, and $\delta=-1$. In particular, $\bar{x}_{2} \in \bar{L}_{0}$ and so Lemmas 3.1 (iii) and 3.3 (i) of [22] yield in this case that $C_{H}\left(\bar{x}_{2}\right)=C_{L}\left(\bar{x}_{2}\right) \bar{E}$ with $C_{L}\left(\bar{x}_{2}\right)$ dihedral of order $2(q-\varepsilon)$. Hence by definition of $n, \bar{W}=O\left(C_{L}\left(\bar{x}_{2}\right)\right)$ is cyclic of order $(q-\varepsilon) / 2^{n}$. Again Lemma 3.1 (v) of [22] yields that $\bar{W}$ is a Hall subgroup of $\bar{L}$ and that $N_{L}\left(\bar{W}_{0}\right)=\bar{W} \bar{D}$ for any nontrivial subgroup $\bar{W}_{0}$ of $\bar{W}$. Furthermore, $\bar{S} \subseteq C_{L}\left(\bar{x}_{2}\right)$ 
as $\bar{T}=\left\langle\bar{x}_{2}\right\rangle$ is normal in $\bar{S}$. Since $C_{L}\left(\bar{x}_{2}\right)$ is dihedral, $\bar{W}$ centralizes a cyclic subgroup $\bar{U}$ of index 2 in $\bar{S}$ and is inverted by any element of $\bar{S}-\bar{U}$. Moreover, if $U$ denotes the inverse image of $\bar{U}$ in $S$, then $|S: U|=2$ and $\bar{U}=U / Z(S)$. Since $\bar{U}$ is cyclic, $U$ is abelian and so $U=C_{S}(T)$ is the unique abelian maximal subgroup of $S$. Again all parts of (i) and also (ii) hold.

Now $W$ is abelian and $W$ is normal in $W E$. Hence (iii) will follow if we show that no $p$-element of $E^{\#}$ centralizes $O_{p^{\prime}}(W)$. Since $\bar{W} \bar{E}$ is isomorphic to $W E$, we need only prove that no $p$-element of $\bar{E}^{\#}$ centralizes $O_{p^{\prime}}(\bar{W})$ for any prime $p$. But this is one of the assertions of Lemma 3.3 (ii) of [22].

We now prove (iv). If $E=1$, then $O(C)=W$ is cyclic and $O(C)^{\prime}=1$, so (iv) holds trivially. We can therefore assume that $E \neq 1$, in which case $O(C) \supset W$. If $a$ is a $p$ element of $O(C)-W$, then $\left[O_{p^{\prime}}(W), a\right] \neq 1$, by (ii). Since $\left[O_{p^{\prime}}(W), a, a\right]=$ $\left[O_{p^{\prime}}(W), a\right] \neq 1$, it follows that $a$ does not centralize $\left[O_{p^{\prime}}(W), a\right]$. But $\left[O_{p^{\prime}}(W), a\right]$ $\subseteq O(C)^{\prime}$ and so $a$ does not centralize $O(C)^{\prime}$. This implies that $O(C)^{\prime}$ centralizes no element of $O(C)-W$ as $p$ and $a$ were arbitrary. On the other hand, as $E$ is cyclic and $W$ is normal in $O(C)=W E$, we have $O(C)^{\prime} \subseteq W$. But then $W$, being cyclic, centralizes $O(C)^{\prime}$ and we conclude that $C_{O(C)}\left(O(C)^{\prime}\right)=W$, proving (iv).

Since $D$ normalizes $O(C), D$ normalizes a Sylow $p$-subgroup of $O(C)$ for any prime $p$, so $P$ exists in (v). Now $d$ inverts $W$ by (i) and $d$ centralizes $E$ as $S$ centralizes $E$. Since $O(C)=W E$, it follows that $C_{O(C)}(d)=E$. Furthermore, as $W$ is normal in $O(C), P \cap W$ is a Sylow $p$-subgroup of $W, C_{P}(d) \subseteq E$, and $P \cap W=[P, d]$. But $P=[P, d] C_{P}(d)$ by Theorem 5.3.5 of [19] and so $P=(P \cap W)(P \cap E)$. If $P \cap W=1$, then $P \subseteq E$, while if $P \cap E=1$, then $P \subseteq W$. In either case, (v) holds. Thus in proving (v), we can assume that both $P \cap W \neq 1$ and $P \cap E \neq 1$ and argue that $Z(P) \subseteq W$.

Let $a$ be an element of order $p$ in $P \cap E$. Since $P \cap E$ and $P \cap W$ are cyclic and $P=(P \cap W)(P \cap E)$, it will suffice to show that $a$ does not centralize $P \cap W$. Setting $\bar{K}=C_{L}(\bar{a})$, we therefore need only prove that $\overline{P \cap W}=\bar{P} \cap \bar{W}$ is not contained in $\bar{K}$. Let $|P \cap W|=p^{b}$, so that $p^{b}$ is the highest power of $p$ dividing $q+\delta \varepsilon$. Thus we need only show that $|\bar{K}|_{p}<p^{b}$. Now by Lemma 3.3 (i) of [22], $\bar{K}$ is isomorphic to $\operatorname{PGL}\left(2, q_{0}\right)$, where $q=q_{0}^{p}$ and $\bar{K} \cap \bar{L}_{0}$ is isomorphic to $\operatorname{PSL}\left(2, q_{0}\right)$. Furthermore, we know that $\bar{x}_{2} \in \bar{K} \cap \bar{L}_{0}$ if and only if $n>1$, hence if and only if $\delta=-1$. Since $p$ is odd, we also have $q_{0} \equiv q(\bmod 4)$ and so $q_{0} \equiv \varepsilon(\bmod 4)$. Applying Lemma 3.1 (ii) of [22], it follows that $C_{R}\left(\bar{x}_{2}\right)$ is dihedral of order $2\left(q_{0}+\delta \varepsilon\right)$.

On the other hand, $\bar{a}$ centralizes $\Omega_{1}(\bar{P} \cap \bar{W})$ which lies in $C_{\bar{R}}\left(\bar{x}_{2}\right)$ and so $p$ divides $q_{0}+\delta \varepsilon$. Since $q=q_{0}^{p}$ and $p^{b}$ is the highest power of $p$ dividing $q+\delta \varepsilon=q_{0}^{p}+\delta \varepsilon$, we conclude that $p^{b}$ does not divide $q_{0}+\delta \varepsilon$. However, by Lemma 3.1 (i) of [22], $|\bar{K}|_{p}$ is a divisor of $q_{0}+\delta \varepsilon$. Thus $|\bar{K}|_{p}<p^{b}$ and the proof of (v) is complete.

Finally if $E_{0}$ and $e_{0}$ are as in (vi), Lemma 3.3 (i) of [22] implies that $C_{L_{0}}\left(\bar{E}_{0}\right)$ is isomorphic to $\operatorname{PSL}\left(2, q_{0}\right)$, where $q=q_{0}^{e}$. Since $C_{L_{0}}\left(E_{0}\right)$ maps onto $C_{L_{0}}\left(\bar{E}_{0}\right)$ and $L_{0}$ has generalized quaternion Sylow 2-subgroups, we conclude that $C_{L_{0}}\left(E_{0}\right)$ is isomorphic to $S L\left(2, q_{0}\right)$, proving (vi). 
LEMMA 3. If $O(H)=1$, then

(i) $O\left(C_{H}(d)\right)=V E$, where $V=O\left(C_{H}(d)\right) \cap L$ is normal in $O\left(C_{H}(d)\right), V$ is cyclic of order $(q-\varepsilon) / 2^{m}$, where $2^{m}$ is the highest power of 2 dividing $q-\varepsilon$, and $x_{2}$ inverts $V$.

(ii) For any prime $p$, no p-element of $O(C)-(O(C) \cap L)$ centralizes $O_{p^{\prime}}(V)$.

(iii) $C_{H}(D)=Z(D) \times E$.

Proof. The proof of (i) and (ii) is entirely similar to the proof of (i) and (iii) of the previous lemma. We know that $d \in L_{0}$ and that $d^{2}=x$, so $\bar{d}$ is an involution of $\bar{L}_{0}$. Hence applying Lemmas 3.1 (iii) and 3.3 (i) of [22], as above, we have that $C_{H}(\bar{d})=C_{L}(d) \bar{E}, C_{L}(\bar{d})$ is dihedral of order $2(q-\varepsilon)$, and $O\left(C_{L}(\bar{d})\right)=\bar{V}$ is cyclic of order $(q-\varepsilon) / 2^{m}$, where $2^{m}$ is the highest power of 2 dividing $q-\varepsilon$. Furthermore, as $d$ normalizes $T$ and $\bar{T}=\left\langle\bar{x}_{2}\right\rangle$ is of order two, $\bar{d}$ centralizes $\bar{x}_{2}$, so $\bar{x}_{2} \in C_{L}(\bar{d})$. But $\bar{x}_{2} \neq \bar{d}$ as $d \notin T$. Since $C_{L}(\bar{d})$ is a dihedral group, it follows that $\bar{x}_{2}$ inverts $\bar{V}$. Since $C_{H}(d)$ maps onto $C_{H}(\bar{d})$, all parts of (i) now follow.

Since $V$ is abelian and is normal in $V E$, (ii) will follow if we show than no $p$ element of $E^{\#}$ centralizes $O_{p^{\prime}}(\bar{V})$. But this is one of the conclusions of Lemma 3.3 (ii) of [22].

Finally $C_{H}(S)=Z(S) \times E$, so (iii) holds if $S$ is wreathed. Assume then that $S$ is quasi-dihedral, in which case $D$ is dihedral of order eight and $T$ is a Sylow 2subgroup of $C$. Since $C_{H}(D) \subseteq C$, it follows that $Z(D)=\langle x\rangle$ is a Sylow 2-subgroup of $C_{H}(D)$. Hence by Burnside's transfer theorem, $C_{H}(D)$ is the direct product of $Z(D)$ and $O\left(C_{H}(D)\right)$. On the other hand, $d=y \in D$ in this case and so $O\left(\epsilon_{H}(D)\right)$ $\subseteq O\left(C_{H}(d)\right)=V E$. Since $x_{2}$ inverts $V$ and $D$ centralizes $E$, we conclude that $O\left(C_{H}(D)\right)=E$ and (iii) is proved.

LEMMA 4. If $O(H)=1$, then

(i) E normalizes a Sylow p-subgroup $P$ of $L$, where $p$ is the characteristic of $L$.

(ii) $C_{H}(P)=Z(H) P$ for any such Sylow p-subgroup $P$ of $L$.

Proof. By Lemmas 3.1 (vi) and 3.3 (iii) of [22], $\bar{L}$ possesses an $\bar{E}$-invariant Sylow $p$-subgroup $\bar{P}$ and $C_{H}(\bar{P})=\bar{P}$ for any such Sylow $p$-subgroup $\bar{P}$. Passing to $L$ and $H$, we obtain (i) and (ii) at once.

LEMma 5. If $O(H)=1$ and $K$ is a nonabelian subgroup of $L$ of odd order, then

(i) $K$ is a Frobenius group with kernel and complement of orders dividing $q$ and q-1 respectively.

(ii) If $K$ is $T$-invariant, then there is a unique T-invariant Frobenius complement in $K$ and it is contained in $C$.

Proof. We have $K \subseteq L_{0}$ as $|K|$ is odd and $\left|L: L_{0}\right|$ is a power of two. Hence if $V$ denotes the natural vector space of dimension two over $G F(q)$ on which $L_{0}$ acts, then $K$ is faithfully represented on $V$. If $|K|$ is prime to $q$, the minimal degree of a faithful representation of $K$ over $G F(q)$ is at least three as $|K|$ is odd and $K$ is nonabelian. Hence $p$ divides $|K|$, where $p$ is the characteristic of $L$. If $O_{p}(K)=1$, Theorem B of Hall-Higman (Theorem 11.1.1 of [19]) implies that the minimal 
degree of a faithful representation of $K$ over $G F(q)$ is at least $p$ and hence at least three. Hence $O_{p}(K) \neq 1$ and so $V_{0}=C_{V}\left(O_{p}(K)\right)$ is a one-dimensional subspace of $V$. But $V_{0}$ is $K$-invariant as $O_{p}(K)$ is normal in $K$ and so $K$ is contained in the stabilizer of $V_{0}$ in $L_{0}$. But this stabilizer is the normalizer in $L_{0}$ of a Sylow $p$-subgroup $P$ of $L_{0}$ and has the form $P A$, where $P$ is elementary abelian of order $q, A$ is cyclic of order $q-1$, and $A / Z\left(L_{0}\right)$ acts regularly on $P$. Since $K$ is a nonabelian subgroup of $P A$ of odd order, $K$ necessarily has the structure asserted in (i).

Suppose now that $K$ is $T$-invariant. Since $K T$ is solvable, it possesses a Hall $p^{\prime}$-subgroup $R$ with $T \subseteq R$. Since $K$ is normal in $K T, R \cap K=A_{0}$ is a Hall $p^{\prime}$-subgroup of $K$ and hence is a Frobenius complement in $K$. Moreover, $A_{0}$ is $T$-invariant. If $P_{0}$ is the Frobenius kernel of $K$, then $P_{0}$ is characteristic in $K$ and so $P_{0}$ is also $T$-invariant. Since $P_{0} \subseteq L, C_{P_{0}}(T) \subseteq O(C) \cap L$, which is cyclic of order dividing $q+\delta \varepsilon$, by Lemma 2 . Since $C_{P_{0}}(T)$ is also a $p$-group and $p$ divides $q$, this forces $C_{P_{0}}(T)=1$. But $T=\left\langle x, x_{2}\right\rangle$ and $x$ centralizes $P_{0}$ as $x \in Z(H)$. We conclude that $x_{2}$ inverts $P_{\mathbf{0}}$.

Suppose now that $x_{2}$ inverts an element $a$ of $A_{0}$. Then $x_{2}$ inverts the group $P_{0}\langle a\rangle$, which is therefore abelian, and so $a$ centralizes $P_{0}$. Since $K$ is a Frobenius group, this is possible only if $a=1$. Thus $x_{2}$ inverts no element of $A_{0}^{\#}$ and consequently $x_{2}$ centralizes $A_{0}$. Since $x$ centralizes $A_{0}$, it follows that $A_{0} \subseteq C=C_{H}(T)$. Our argument shows that $A_{0}=C_{K}(T)$. Since any two $T$-invariant Frobenius complements of $K$ are conjugate by an element of $C_{K}(T)=A_{0}$, we conclude that $A_{0}$ is the unique such complement, proving (ii).

Lemma 6. Assume $O(H)=1$ and let $X$ be a nontrivial subgroup of $L$ which satisfies one of the following conditions:

(a) $|X|$ is odd dividing $q-\delta \varepsilon$;

(b) $|X|$ is odd dividing $q+\delta \varepsilon$ and $[X, T]=X$;

(c) $|X|$ is odd dividing $q$ and $\delta \varepsilon=+1$;

(d) $X$ is isomorphic to $S L(2,3)$.

Then

(i) $\left\langle W_{0}, X\right\rangle$ has even order for any nontrivial subgroup $W_{0}$ of $O(C) \cap L$.

(ii) $\langle C \cap L, X\rangle=L$ with the following single exception: $q=5, \delta=-1$, and $X$ satisfies condition (a) or (d), in which case $\langle C \cap L, X\rangle$ contains $S$ and is isomorphic to $S U_{1}(2,3)$.

Proof. Set $K=\left\langle W_{0}, X\right\rangle$ and suppose $K$ has odd order, in which case $K$ and $\bar{K}$ are isomorphic as are $K \cap C$ and $\bar{K} \cap \bar{C}$. Setting $W=O(C) \cap L$, Lemma 3.1 (v) of [22] implies that $\bar{W}$ is a cyclic Hall subgroup of $\bar{L}$ and is disjoint from its conjugates in $\bar{L}$. Since $\bar{W}_{1}=\bar{W} \cap \bar{K}$ contains $\bar{W}_{0}$ and $\bar{W}_{0} \neq 1$, it follows that $\bar{W}_{1}$ is a cyclic Hall subgroup of $\bar{K}$ and that $\bar{W}_{1}$ is disjoint from its conjugates in $\bar{K}$. Furthermore, it is a direct consequence of Lemmas 3.1 (iii) and (v) of [22] that $N_{L}\left(\bar{W}_{1}\right)$ is a dihedral group and that $\bar{W}=O\left(N_{L}\left(\bar{W}_{1}\right)\right)$. Thus $N_{K}\left(\bar{W}_{1}\right)=\bar{W} \cap \bar{K}=\bar{W}_{1}$. But now Frobenius' theorem (Theorems 2.7.7 and 4.5.1 of [19]) implies that either $\bar{K}=\bar{W}_{1}$ or $\bar{K}$ is a 
Frobenius group with complement $\bar{W}_{1}$. Since $K \cap C$ and $\bar{K} \cap \bar{C}$ are isomorphic, the inverse image $W_{1}$ of $\bar{W}_{1}$ in $W$ lies in $K$ and we conclude that either $K=W_{1}$ or that $K$ is a Frobenius group with complement $W_{1}$.

If $K=W_{1}$, then $X \subseteq W_{1}$ as $K=\left\langle W_{0}, X\right\rangle$. However, $T$ centralizes $W_{1}$ and $\left|W_{1}\right|$ divides $q+\delta \varepsilon$ by Lemma 2 . But now we see that $X$ cannot satisfy any of conditions (a), (b), (c), or (d). Thus $K$ must be a Frobenius group with complement $W_{1}$. Hence by the preceding lemma, $|K|$ divides $(q-1) q$ and $\left|W_{1}\right|$ divides $q-1$. Since $\left|W_{1}\right|$ divides $q+\delta \varepsilon$, this forces $\delta \varepsilon=-1$, whence $|K|$ is prime to $q-\delta \varepsilon=q+1$. Thus $X$ cannot satisfy condition (a), (c), or (d) and so $X$ satisfies (b). In particular, $X$ is $T$-invariant and hence so also is $K=\left\langle W_{0}, X\right\rangle$ as $T$ centralizes $W_{0}$. Since $T$ centralizes the complement $W_{1}$ of $K$, it follows that $[K, T]$ is contained in the kernel $P_{1}$ of $K$. Since $X=[X, T]$, this yields $X \subseteq P_{1}$. But $\left|P_{1}\right|$ divides $q$, while $|X|$ divides $q+\delta \varepsilon$ under assumption (b). This contradiction completes the proof of (i).

We turn now to (ii). Set $R=\langle C \cap L, X\rangle$. We shall argue that $R$ does not have a normal 2-complement, so assume the contrary. Then certainly (d) does not hold. Thus $|X|$ is odd and so $\langle W, X\rangle \subseteq O(R)$. But then if $W \neq 1$, (i) is contradicted and so $W=1$.

By Lemma $2,|W|=(q+\delta \varepsilon) / 2^{n}$, whence $q+\delta \varepsilon=2^{n}$. Since $q>3$ by assumption, this forces $n>1$ and hence also $\delta=-1$. Thus $q=2^{n}+\varepsilon$ is either a Mersenne or a Fermat prime or else $q=9$. Setting $U=C_{S}(T)$, we know that $U$ is abelian of type $\left(2^{n}, 2^{n}\right)$ and that $U \subseteq R$. Since $Z(S)$ is cyclic of order $2^{n}$ and $Z(S) \subseteq U, \bar{U}$ is cyclic of order $2^{n}$ and $\bar{x}_{2}=\bar{y}$ is the unique involution of $\bar{U}$. By Lemma 3.1 (iii) of [22], $C_{L}(\bar{y})=\bar{S}$ under the present conditions and so $\bar{y}$ inverts $O(\bar{R})$. Hence $O(\bar{R})$ is abelian and consequently by Lemma 3.1 (iii) and (vi) of [22], $O(\bar{R})$ has order dividing $q$ or $q+\varepsilon$. However, in the latter case, $N_{L}(O(\bar{R}))$ is dihedral by Lemma 3.1 (iii) and (v) of [22] as $O(\bar{R}) \neq 1$, in which case $O(\bar{R}) \bar{U}$ is either cyclic or dihedral, contrary to the fact that $\bar{U}$ is cyclic of order $2^{n}$ with $n>1$ and the involution $\bar{y}$ of $\bar{U}$ inverts $O(\bar{R})$. Hence $O(\bar{R})$ has order dividing $q$. Moreover, if $q=9,|\bar{U}|=8$ and, as $\bar{y}$ inverts $O(\bar{R}), O(\bar{R})$ must be of order nine. Thus $|O(\bar{R})|=q$ in all cases. Since $X \subseteq O(R)$, it follows that condition (c) holds. Hence by Lemma 3.1 (vi) of [22], $N_{L}(O(\bar{R}))$ is a Frobenius group of order $(q-1) q$. Since $\bar{U} \subseteq N_{L}(O(\bar{R}))$, we conclude that $2^{n}$ divides $q-1$. Since $2^{n}=q+\delta \varepsilon$, this is possible only if $\delta \varepsilon=-1$, contrary to the assumptions of (c). Thus $R$ does not have a normal 2-complement, as asserted.

Since $\bar{R}$ has dihedral Sylow 2-subgroups, it is thus a $D$-group. Since $L=L_{0} C_{S}(T)$, $R \nsubseteq L_{0}$ and so $R$ contains a normal subgroup of index two. Hence by Lemma 3.1 (viii) of [22], $\bar{R}$ is isomorphic to $\operatorname{PGL}(2,3), \operatorname{PGL}(2,5)$ or $\operatorname{PGL}\left(2, q_{0}\right)$ for some $q_{0}$ dividing $q$. But, as we have seen in Lemma 2, Lemma 3.1 (iii) of [22] implies that $\bar{C} \cap \bar{L}$ is cyclic of index two in $C_{L}\left(\bar{x}_{2}\right)$ and that $C_{L}\left(\bar{x}_{2}\right)$ has order $2(q+\delta \varepsilon)$. By the same lemma of [22], $C_{\bar{R}}\left(\bar{x}_{2}\right)$ is dihedral of the respective order 4,12 , or $2\left(q_{0}+\varepsilon_{0}\right)$ if $\bar{x}_{2} \in \bar{L}-\bar{L}_{0}$ and the respective order 8,8 , or $2\left(q_{0}-\varepsilon_{0}\right)$ if $\bar{x}_{2} \in \bar{L}_{0}$, where $\varepsilon_{0}= \pm 1$ and $q_{0} \equiv \varepsilon_{0}(\bmod 4)$. Since $C \cap L \subseteq R, \bar{C} \cap \bar{L}$ is a cyclic subgroup of $C_{R}\left(\bar{x}_{2}\right)$ of order $q+\delta \varepsilon$. Since the latter group is dihedral, we conclude that $q+\delta \varepsilon$ must, in fact, divide $\frac{1}{2}\left|C_{\bar{R}}\left(\bar{x}_{2}\right)\right|$. 
Suppose first that $q>7$. Since $q+\delta \varepsilon \geqq q-1$ and $q+\delta \varepsilon$ divides $\frac{1}{2}\left|C_{\bar{R}}\left(\bar{x}_{2}\right)\right|$, the only possibility is that $\bar{R}$ is isomorphic to $\operatorname{PGL}\left(2, q_{0}\right)$ and that $q+\delta \varepsilon=q_{0} \pm \varepsilon_{0}$, whence $q=q_{0}$. Thus $|\bar{R}|=|\bar{L}|$ and, as $\bar{R} \subseteq \bar{L}$, it follows that $\bar{R}=\bar{L}$. Since $Z(S) \subseteq C \cap L \subseteq R$, the desired conclusion $R=L$ holds in this case.

Assume next that $q=7$, but that $R \subset L$, whence $\bar{R} \subset \bar{L}$ and so $\bar{R}$ is isomorphic to $\operatorname{PGL}(2,3)$ or $\operatorname{PGL}(2,5)$. We have $q+\delta \varepsilon=6$ or 8 , as $q=7$. Since $q+\delta \varepsilon$ divides $\frac{1}{2}\left|C_{\bar{R}}\left(\bar{x}_{2}\right)\right|, \bar{R}$ must be isomorphic to $\operatorname{PGL}(2,5)$ and so 5 divides $|\bar{R}|$, contrary to the fact that 5 does not divide $|\bar{L}|=336$.

Suppose finally that $q=5$, but that $R \subset L$, whence $\bar{R} \subset \bar{L}$ and $\bar{R}$ is isomorphic to $P G L(2,3)$. Since $\varepsilon=1$ in this case, our conditions force $\delta=-1,|\bar{C} \cap \bar{L}|=4$, and $\left|C_{R}\left(\bar{x}_{2}\right)\right|=8$. Furthermore, since $X \neq 1$ and $X \subseteq R, X$ is not of odd order dividing $q=5$ or $q+\delta \varepsilon=4$. Thus $X$ satisfies condition (a) or (d). Since $q$ is a Fermat prime and $\bar{x}_{2}=\bar{y}$ in the present case, $C_{L}\left(\bar{x}_{2}\right)=\bar{S}$, as we have seen above, and so $\left|C_{L}\left(\bar{x}_{2}\right)\right|=8$. Since $\left|C_{R}\left(\bar{x}_{2}\right)\right|=8$, it follows that $C_{R}\left(\bar{x}_{2}\right)=\bar{S}$. But $Z(S) \subseteq R$ and so $S \subset R$. We conclude at once now from the structure of $R$ that $R$ is, in fact, isomorphic to $S U_{1}(2,3)$. Thus (ii) holds in this case as well and the lemma is proved.

LEMMA 7. If $O(H)=1$, then

(i) $Z(S)=Z(H)$.

(ii) $C_{H}\left(L_{0}\right)=C_{H}(L)=Z(H)$.

(iii) If $K$ is a subgroup of $H$ containing $L_{0}$, then $O(K)=1$.

(iv) $\left[L_{0}, X\right]$ has even order for any nonidentity subgroup $X$ of $H$ of odd order.

Proof. First, (i) has been noted already at the beginning of the section. In addition, we know that $S \cap L_{0}$ is the unique generalized quaternion subgroup of $L_{0}$ of maximal order and so $C_{S}\left(S \cap L_{0}\right)=Z(S)$ by Lemmas 1.1 and 1.2. Since $Z(S)=Z(H)$ and since $C_{S}\left(L_{0}\right)$ is a Sylow 2-subgroup of $C_{H}\left(L_{0}\right)$, it follows that $Z(H)$ is a Sylow 2-subgroup of $C_{H}\left(L_{0}\right)$. Hence $C_{H}\left(L_{0}\right)$ has a normal 2-complement $R$ by Burnside's transfer theorem. But $C_{H}\left(L_{0}\right)$ is normal in $H$ as $L_{0}$ is normal in $H$. Since $R$ is characteristic in $C_{H}\left(L_{0}\right)$, it follows that $R \subseteq O(H)=1$. Thus $C_{H}\left(L_{0}\right)=Z(H)$. Since $Z(H) \subseteq C_{H}(L) \subseteq C_{H}\left(L_{0}\right)$, we also have that $C_{H}(L)=Z(H)$, so (ii) also holds.

Next if $K$ is as in (iii), then $L_{0}$ is normal in $K$ as $L_{0} \subseteq K$ and $L_{0}$ is normal in $H$. But then $\left[L_{0}, O(K)\right] \subseteq L_{0} \cap O(K)=1$ and so $L_{0}$ centralizes $O(K)$, whence $O(K)=1$ by (i) and (ii), proving (iii).

Finally if $X$ is as in (iv) and if we set $F=\left\langle L_{0}, X\right\rangle$, then $\left[L_{0}, X\right]$ is normal in $F$. Hence if $\left[L_{0}, X\right]$ were of odd order, it would lie in $O(F)$. But $O(F)=1$ by (iii) and so $\left[L_{0}, X\right]=1$. Thus $X$ centralizes $L_{0}$ and so $X=1$, again by (i) and (ii), a contradiction. Thus (iv) also holds.

LEMMA 8. If $O(H)=1$ and $K$ is a $Q$-subgroup of $L$, then the following conditions hold:

(i) If $K$ has no normal subgroups of index two, then $K$ is isomorphic to $S L(2, r)$, where $r=3, r=5$, or $r$ divides $q$.

(ii) If $K$ contains $C \cap L$ and $q>5$, then $K$ has characteristic power $q$. 
Proof. Without loss we may assume that $S \cap K$ is a Sylow 2-subgroup of $K$. Since $\bar{S}=S / Z(S)$ is dihedral, $\overline{S \cap K}$ is either dihedral or cyclic. However, $\bar{K}$ does not have a normal 2-complement, as otherwise $K$ would have one, contrary to the fact that $K$ is a $Q$-group. Hence $\overline{S \cap K}$ is not cyclic, otherwise $\bar{K}$ would have a normal 2-complement by Burnside's transfer theorem. Thus $\overline{S \cap K}$, which is a Sylow 2-subgroup of $\bar{K}$, is dihedral and so $\bar{K}$ is a $D$-group. Now Lemma 3.1 (viii) of [22] implies that $\bar{K}$ is isomorphic to $\operatorname{PSL}(2, r)$ or $P G L(2, r)$, where $r=3, r=5$, or $r$ divides $q$.

Assume now that $K$ has no normal subgroups of index two, in which case neither does $\bar{K}$. Since $P G L(2, r)$ contains a normal subgroup of index two, $\bar{K}$ must be isomorphic to $\operatorname{PSL}(2, r)$. Moreover, $\bar{K} \subseteq \bar{L}_{0}$ as $\bar{L}_{0}$ has index a power of 2 in $\bar{L}$. But now it follows from the results of Schur [26] that $K$ is isomorphic to $S L(2, r)$, proving (i).

Suppose next that $K$ contains $C \cap L$ and $q>5$. By Lemma 2, $C \cap L=U W$, where $U=C_{S}(T)=C_{D}(T)$ is abelian of type $\left(2^{n}, 2^{n}\right)$ and $W=O(C) \cap L$ is of order $(q+\delta \varepsilon) / 2^{n}$. Since $Z(S)$ is cyclic of order $2^{n}$, it follows that $\mid C \cap L=q+\delta \varepsilon$. But $C \cap L \subseteq K$, so $\overline{C \cap L} \subseteq C_{R}(\bar{T})$. On the other hand, $\bar{T}=\left\langle\bar{x}_{2}\right\rangle$ is central in $\bar{D}$ as $T$ is normal in $D$ and $\bar{U} \subset \bar{D}$ as $Z(S) \subset U \subset D$. Since $\bar{U}$ is a Sylow 2-subgroup of $\overline{C \cap L}$, we conclude that

$$
\left|C_{R}\left(\bar{x}_{2}\right)\right| \geqq 2(q+\delta \varepsilon) .
$$

However, by Lemma 3.1 (iii) of [22], we have

where $\varepsilon_{0}= \pm 1$.

$$
\left|C_{K}\left(\bar{x}_{2}\right)\right| \leqq 2\left(r \pm \varepsilon_{0}\right)
$$

Suppose $r$ divides $q$. Since $\delta \varepsilon= \pm 1$ and $q$ is an odd prime power, the above inequalities force $r=q$. In the remaining cases, $r=3$ or 5 . Since $q>5$, there is only a single solution of these two inequalities: namely, $q=7, \delta \varepsilon=-1, r=5$, and $\varepsilon_{0}=1$. However, in this case $|\bar{L}|=|P G L(2,7)|=336$ and so is prime to 5, contrary to the fact that 5 divides $|\bar{K}|$ and $\bar{K} \subseteq \bar{L}$. We conclude that $\bar{K}$, and hence also $K$, is of characteristic power $q$. Thus (ii) also holds.

The next three lemmas give information concerning various signalizers in $H$.

LEMMA 9. If $O(H)=1$, then

(i) If $p$ is the characteristic of $H$, then $U_{L}(D ; p)$ is trivial and any element of $U_{H}(D ; p)$ is contained in $C$.

(ii) Any element of $\eta_{H}(D)$ of odd order containing $O(C) \cap L$ lies in $O(C)$.

(iii) Any element of ${U_{H}}_{H}(C(C) \cap L)$ of odd order lies in $L$.

Proof. If $S$ is quasi-dihedral, $\bar{D}$ is a four group, while if $S$ is wreathed, $\bar{D}=\bar{S}$ is dihedral of order $2^{n+1}$. In either case, $U_{L}(\bar{D} ; p)$ is trivial by Lemma 3.1 (vii) of [22] and this yields at once that $\eta_{L}(D ; p)$ is trivial. Furthermore, if $X \in U_{H}(D ; p)$, then so also does $[X, D]$. But $[X, D] \subseteq L$, as $D \subseteq L$, so $[X, D] \in U_{L}(D ; p)$, which we have just shown is trivial. Hence $[X, D]=1$ and so $D$ centralizes $X$. Thus $X \subseteq C$ and both parts of (i) are established. 
Next set $W=O(C) \cap L$ and let $X$ be an element of $\eta_{H}(D)$ of odd order containing $W$. Then by the same lemma of [22], $\bar{X} \subseteq C_{H}(\bar{t})$ for some involution $\bar{t}$ of $Z(\bar{D})$. If $S$ is quasi-dihedral, both $d$ and $d x_{2}$ invert $W$ and $W \neq 1$ by Lemma 2 . Hence $\bar{d}$ and $\bar{d} \bar{x}_{2}$ do not centralize $\bar{X}$, as $\bar{X} \supseteq \bar{W}$, and so $\bar{t}=\bar{x}_{2}$. Thus $\bar{X} \subseteq O(\bar{C})$ and hence $X \subseteq O(C)$. On the other hand, if $S$ is wreathed, $\bar{T}=\left\langle\bar{x}_{2}\right\rangle$ is in the center of $\bar{D}$ as $T$ is normal in $D$ and $\bar{T}$ has order two. However, $\bar{D}$ is a nonabelian dihedral group, as $n \geqq 2$, and so $|Z(\bar{D})|=2$. But then $Z(\bar{D})=\bar{T}$ and hence $\bar{t}=\bar{x}_{2}$, giving the same conclusion $X \subseteq O(C)$ as in the preceding case. Thus (ii) also holds.

Finally let $X$ be an element of $\eta_{H}(W)$ of odd order. Then $W X \cap L=W(X \cap L)$ is normal in $W X$. But $W$ is a Hall subgroup of $L$ by Lemma 2 . Hence if we set $Y=N_{X}(W)$, it follows from the Frattini argument that $W X=W(X \cap L) N_{W X}(W)$ $=W(X \cap L)(W Y)=W(X \cap L) Y$. Thus to prove (iii), it will suffice to show that $Y \subseteq L$. To establish this, we need only prove that $Y \subseteq O(C)$. Indeed, assume this is the case. We have $[W, Y] \subseteq W \cap X$ as $Y$ normalizes $W$ and $W$ normalizes $X$, which contains $Y$. But $W \cap X=1$ as $X \in \eta_{H}(W)$. Thus $Y$ centralizes $W$. However, by Lemma 2 no element of $O(C)-W$ centralizes $W$. Hence $Y \subseteq W \subseteq L$, as required.

We may also assume $W \neq 1$; otherwise as in Lemma $6, q$ is a Mersenne or Fermat prime or $q=9$, whence $H=L$ by Proposition 3.3, in which case clearly $Y \subseteq L$.

Now $C_{L}(\bar{W})$ is dihedral and $\bar{W}=O\left(C_{L}(\bar{W})\right)$ by Lemmas 3.1 (iii) and (v) of [22] as $\bar{W} \neq 1$. Hence $C_{L}(W)=W U$, where $U=C_{S}(T)$, by Lemma 2. But $Y$ normalizes $W U$ as it normalizes both $L$ and $W$. On the other hand, $U$ is abelian of type $\left(2^{n}, 2^{n}\right), Z(S)$ is cyclic of order $2^{n}$ with $Z(S) \subset U$, and $Y$ centralizes $Z(S)$ as $Z(S)$ $=Z(H)$. It follows at once that $Y$ centralizes $U W / W$ and hence that $U W Y / W$ is the direct product of $U W / W$ and $W Y / W$. Thus $U$ stabilizes the chain $W Y \supseteq W \supseteq 1$ and so $U$ centralizes $W Y$. Since $T \subseteq U$ and $C=C_{H}(T)$ has a normal 2-complement, we conclude that $Y \subseteq O(C)$ and (iii) is proved.

LEMMA 10. If $O(H)=1$ and $\mathrm{U}_{H}(C ; p)$ is nontrivial, $p$ an odd prime, then the following conditions hold:

(i) $\delta \varepsilon=-1$ and $p$ is the characteristic of $L$.

(ii) There are exactly two maximal elements $P$ and $P^{*}$ in $\eta_{H}(C)$, each of which is a Sylow p-subgroup of $L$.

(iii) $C \cap L / Z(H)$ and $C \cap L_{0} / Z\left(L_{0}\right)$ act faithfully and irreducibly on $P$ and $P^{*}$ regarded as vector spaces over $G F(p)$.

(iv) $P$ and $P^{*}$ are permuted under conjugation by $d$.

(v) $L_{0}=\left\langle P, P^{*}\right\rangle$.

Proof. As usual, set $W=O(C) \cap L$ and $U=C_{S}(T)$. By Lemma 2, $W$ centralizes $U, d$ inverts $W, C \cap L=W U$, and either $W \neq 1$ or $n>1$. Applying Lemma 3.1 (iii) and (v) of [22], we conclude as usual that $C_{L}\left(\bar{x}_{2}\right)=\bar{W} \bar{D}=\bar{W} \bar{U}\langle\bar{d}\rangle$ is dihedral and that for any $\bar{z} \neq 1$ in $\bar{W} \bar{U}$, we have $C_{L}(\bar{z}) \subseteq C_{L}\left(\bar{x}_{2}\right)$. We use these facts in proving (i). 
Let $P$ be a nontrivial element of $\bigcup_{H}(C ; p)$. Since $p$ is odd by assumption, $P \subseteq L$ by the preceding lemma. Hence by the preceding paragraph, $C_{\bar{P}}(\bar{z}) \subseteq \bar{W} \bar{D}$ for any $\bar{z} \neq 1$ in $\bar{W} \bar{U}$. Since $\left|C_{\bar{P}}(\bar{z})\right|$ is odd and $\bar{W}$ is normal of index a power of two in $\bar{W} \bar{D}$, we have, in fact, $C_{P}(\bar{z}) \subseteq \bar{W} \subseteq \bar{C} \cap \bar{P}$. But $C \cap P=1$, as $P \in U_{H}(C)$, and so $\bar{C} \cap \bar{P}=1$. Thus $\bar{W} \bar{U}$ acts regularly on $\bar{P}$ and so $\bar{W} \bar{U} \bar{P}$ is a Frobenius group. If $W \neq 1$, then $\bar{W} \bar{P}$ is a Frobenius group and it follows from Lemma 5 that $p$ is the characteristic of $L$ and that $|W|$ divides $q-1$. Since $|W|=(q+\delta \varepsilon) / 2^{n}$ by Lemma 2 , this forces $\delta \varepsilon=-1$. Since $|U|=2^{n},|\bar{W} \bar{U}|=q-1$, whence $|\bar{P}|=q, \bar{P}$ is a Sylow $p$ subgroup of $\bar{L}$, and $\bar{W} \bar{U}$ acts faithfully and irreducibly on $\bar{P}$ regarded as a vector space over $G F(p)$. Since $C \cap L_{0}=W U \cap L_{0}$ is cyclic of order $q-1$ and $L_{0} \cap Z(H)$ has order two, it follows directly from this that $\left(C \cap L_{0}\right) Z(H) / Z(H)$ also acts faithfully and irreducibly on $\bar{P}$. In particular, (i) and (iii) hold.

On the other hand, if $W=1$, we have shown in the course of the proof of part (ii) of Lemma 6 (with $\bar{P}$ in the role of $\bar{X}$ ), that $\delta=-1$, that $q=2^{n}+\varepsilon$ is either a Fermat of Mersenne prime or $q=9$, that $|\bar{P}|=q$, that $\bar{P} \bar{U}$ is a Frobenius group of order $q(q-1)$, and that $\varepsilon=1$. Thus $\delta \varepsilon=-1, p$ is the characteristic of $L, \bar{P}$ is a Sylow $p$ subgroup of $\bar{L}$, and $\bar{U}=\bar{W} \bar{U}$ acts faithfully and irreducibly on $\bar{P}$. In particular, (i) and (iii) hold in this case as well.

It remains to establish (ii), (iv), and (v). We know that $D$ normalizes $C, C$ normalizes $P$, and $C \cap P=1$. Hence if we set $P^{*}=P^{d}$, we have that $C=C^{d}$ normalizes $P^{*}$ and $C \cap P^{*}=1$, so $P^{*} \in U_{H}(C ; p)$. Furthermore, $P^{*} \neq P$, since otherwise $D=\langle U, d\rangle$ would normalize $P$, contrary to Lemma 9 . Thus $\bar{P}$ and $\bar{P}^{*}$ are distinct Sylow $p$-subgroups of $\bar{L}_{0}$ and consequently $\bar{L}_{0}=\left\langle\bar{P}, \bar{P}^{*}\right\rangle$ by Lemma 3.1 (vi) and (viii) of [22]. Thus $L_{0}=\left\langle P, P^{*}\right\rangle$. Since $d^{2} \in U$ and $U$ normalizes $P, d$ permutes $P$ and $P^{*}$ under conjugation and we conclude that (iv) and (v) hold.

Finally let $X$ be an arbitrary element of $U_{H}(C ; p)$. We know already that $X$ is a Sylow $p$-subgroup of $L$. To prove (ii), we must show that either $X=P$ or $X=P^{*}$. In any event, we have $X^{b}=P$ for some $b$ in $L$ and so $U^{b}$ normalizes $P$. But as $N_{L}(\bar{P})=\bar{P} \bar{W} \bar{U}$ by Lemma 3.1 (vi) of [22], we have $N_{L}(P)=P W U$, whence $U^{b} \subseteq P W U$. It follows that $U^{b c} \subseteq W U$ for some $c$ in $P$. Since $X^{b c}=P^{c}=P$, we can assume without loss to begin with that $U^{b} \subseteq W U$. Since $W U=W \times U$ and $U$ is a Sylow 2-subgroup of $W U$, we conclude that $U^{b}=U$. But $T=\Omega_{1}(U)$ and so $b \in N_{H}(T)$. But as $H$ is a $Q$-group, $N_{H}(T)=C_{H}(T) N_{S}(T)=C D=C\langle d\rangle$. Since $d^{2} \in U \subseteq C, b$ lies in one of the two cosets $C$ or $d C$ of $C$ in $C D$. Since $P$ is $C$-invariant, it follows correspondingly that $X=P$ or $X=P^{*}$, thus proving (ii).

LEMMA 11. If $O(H)=1$, then any subgroup of $H$ of odd order permutable with $C$ is contained in $C P$ for some element $P$ of $И_{H}(C ; p), p$ an odd prime.

Proof. Let $X$ be a subgroup of $H$ of odd order permutable with $C$. Again let $U=C_{S}(T)$. Then $U$ is a Sylow 2-subgroup of $X C$ and so $\bar{U}$ is a Sylow 2-subgroup of $\bar{X} \bar{C}$. Since $\bar{U}$ is cyclic, $\bar{X} \bar{C}$ has a normal 2-complement by Burnside's transfer theorem and so $X C$ has one as well. Since $C$ has a normal 2-complement of the 
form $W E$, where $W=O(C) \cap L$, we see that $O(X C)=X W E$. Since $H=L E$, it follows that $O(X C)=(X W \cap L) E$. Setting $Y=X W \cap L$, we see that $Y$ is normal $X C, W \subseteq Y$, and $X C=Y E=Y C$. Furthermore, $Y \cap C=C_{Y}(T) \subseteq O(C) \cap L=W$.

Consider first the case $W=1$, in which case $Y \cap C=1$ and so $x_{2}$ inverts $Y$. But then $Y$ is abelian and hence any Sylow $p$-subgroup $P$ of $Y$ is normal in $X C$. Since $P \cap C=1$, this implies that $P \in U_{H}(C ; p)$, whence $p$ is the characteristic of $L$ by the preceding lemma. Thus the prime $p$ is uniquely determined and consequently $P=Y$. We conclude that $X \subseteq P C$, as required.

Suppose next that $W \neq 1$ and $Y$ is abelian. By Lemma 3.1 (v) of [22], $C_{L}(\bar{W}) \subseteq \bar{W} \bar{D}$ and hence $\bar{Y} \subseteq \bar{W} \bar{D}$. But then $\bar{Y} \subseteq \bar{W}$ and we conclude that $Y=W$. Thus $X C=Y C=C$ and so $X \subseteq C$, in which case the lemma clearly holds.

Assume finally that $Y$ is nonabelian. By Lemma $5, Y$ is a Frobenius group with kernel $P$ of order dividing $q$ and complement of order dividing $q-1$. Since $|W|=(q+\delta \varepsilon) / 2^{n}$, and $W \subseteq Y$, it follows that $\delta \varepsilon=-1$ and that $W$ is a Frobenius complement in $Y$. Hence $Y=W P$ and $P$ is normal in $X C$ as $P$ is characteristic in $Y$. Thus $P$ is $C$-invariant and $X \subseteq Y C=P W C=P C$. Since $Y \cap C \subseteq W$, we also have that $P \cap C=1$, so $P \in \eta_{H}(C ; p)$ and the lemma holds in this case as well.

We turn now to some results which do not involve the assumption $O(H)=1$.

LEMMA 12. If $H=S U_{1}^{\wedge}(2,9)$, then

(i) $|O(H)|=3$ and $O(H)$ is inverted by $x_{2}$.

(ii) $|O(C)|=5=\frac{1}{2}(q+\delta \varepsilon)$.

Proof. Since $S L^{\wedge}(2,9)$ has index 2 in $S U_{1}^{\wedge}(2,9), O(H)$ has order three. Moreover, $x_{2}$ inverts $O(H)$ by Lemma 2.2 and so (i) holds.

Setting $\bar{H}=H / O(H)$, we have that $\bar{H}$ is isomorphic to $S U_{1}(2,9)$ and so $S$ has quasi-dihedral Sylow 2-subgroups. Hence $\delta=1, n=1, \varepsilon=1$, and $(q+\delta \varepsilon) / 2^{n}=5$. Thus by Lemma 2, $O\left(C_{H}(\bar{T})\right)$ has order five. But $C \cap O(H)=1$ by (i) and so $O(C)$ maps isomorphically onto $O\left(C_{H}(\bar{T})\right)$, whence $|O(C)|=5$ and (ii) is proved.

Our next set of lemmas give some general properties of $Q$-groups that will be used considerably in the paper. We make a few preliminary comments. By Proposition 3.1, $H=O(H) C_{H}(Z(S))$ and so $O(H) Z(S)$ is normal in $H$. Since $H / O(H)$ is a $Q$-group of the same characteristic power as $H$ and with isomorphic Sylow 2-subgroups, but with no nontrivial normal subgroups of odd order, the proof of Proposition 3.3, applied to $H / O(H)$, shows that $\bar{H}=H / O(H) Z(S)$ has dihedral Sylow 2-subgroups, that $\bar{L}$ and $\bar{L}_{0}$ are isomorphic to $\operatorname{PGL}(2, q)$ and $\operatorname{PSL}(2, q)$ respectively and, as $q>3$, that $\bar{L}_{0}$ is simple and is the unique minimal normal subgroup of $\bar{H}$. In particular, $S(\bar{H})=1$. Since $O(H) Z(S)$ is solvable, it follows also that $S(H)=O(H) Z(S)$.

LEMMA 13. If $K$ is a subgroup of $H$, then the following results hold:

(i) If $K$ covers $H / O(H)$ and $K$ contains $x$, then $K$ covers $N / O(N)$.

(ii) If $K$ covers $L_{0} S(H) / S(H)$, then $K$ covers $L_{0} O(H) / O(H)$. 
Proof. Setting $\bar{H}=H / O(H)$ we have that $\bar{K}=\bar{H}$ as $K$ covers $H / O(H)$. But also $\bar{N}=\bar{H}$ as $H=O(H) N$, whence also $\bar{x} \in Z(\bar{H})$. Thus $\bar{N}=\bar{K}=C_{R}(\bar{x})$. However, $O(H)$ has odd order and $x \in K$, so $C_{K}(x)=K \cap N$ maps onto $C_{R}(\bar{x})=\bar{N}$. Hence $N=(K \cap N)(O(H) \cap N)$. But $O(H) \cap N=O(N)$, as we have noted at the beginning of the section, so $N=(K \cap N) O(N)$ and $K \cap N$ covers $N / O(N)$, proving (i).

Setting $K_{0}=K \cap L_{0} S(H)$, we have $L_{0} S(H) \subseteq K_{0} S(H)$, as $K$ covers $L_{0} S(H) / S(H)$. Since $S(H)=O(H) Z(S)$, as noted above, it follows therefore, if $\bar{H}=H / O(H)$, that $\bar{L}_{0} Z(\bar{S}) \subseteq \bar{K}_{0} Z(\bar{S})$. Since $H=O(H) C_{H}(Z(S))$, we also have that $Z(\bar{S}) \subseteq Z(\bar{H})$. But $\bar{L}_{0}$ is perfect as $\bar{L}_{0}$ is isomorphic to $\operatorname{SL}(2, q)$ and $q>3$. Hence

$$
\bar{L}_{0}=\left[\bar{L}_{0}, \bar{L}_{0}\right] \subseteq\left[\bar{L}_{0} Z(\bar{S}), \bar{L}_{0} Z(\bar{S})\right] \subseteq\left[\bar{K}_{0} Z(\bar{S}), \bar{K}_{0} Z(\bar{S})\right]=\left[\bar{K}_{0}, \bar{K}_{0}\right] \subseteq \bar{K}_{0} .
$$

Thus $L_{0} O(H) \subseteq K_{0} O(H)$. Since $K_{0} \subseteq K$, we conclude that $K$ covers $L_{0} O(H) / O(H)$, so (ii) also holds.

LEMMA 14. The following conditions hold:

(i) $H=O(H) N$ and $O(N) \subseteq O(H)$.

(ii) $S(H)=O(H) Z(S)$.

(iii) $[H, x] \subseteq O(H)$.

Proof. We have remarked at the beginning of the section that $H=O(H) N$. But then $O(H) O(N)$ is normal in $H$ and of odd order, so $O(H) O(N) \subseteq O(H)$, whence $O(N) \subseteq O(H)$, proving (i). Furthermore, (ii) has just been noted above. Finally, as $N$ centralizes $x$, we have

$$
[H, x]=[O(H) N, x]=[O(H), x] \subseteq O(H),
$$

so (iii) also holds.

Collecting the various observations preceding Lemma 13, we also have:

LEMMA 15. The following results hold:

(i) $H / S(H)$ is a D-group of linear type of characteristic power $q$ with dihedral Sylow 2-subgroups.

(ii) $L_{0} S(H) / S(H)$ is isomorphic to $\operatorname{PSL}(2, q)$ and is the unique minimal normal subgroup of $H / S(H)$.

(iii) $L S(H) / S(H)$ is isomorphic to $P G L(2, q)$.

LEMMA 16. If $C_{H}(O(H))$ is not contained in $S(H)$, then $L_{0}=J O(H)$, where

(i) Either $J$ is isomorphic to $S L(2, q)$ or $q=9$ and $J$ is isomorphic to $S L^{\wedge}(2,9)$.

(ii) $J$ is normal in $L_{0}$.

(iii) $J \cap O(H)=O(J)$. In particular, $L_{0}=J \times O(H)$ if $q \neq 9$.

(iv) $J$ centralizes $O(H)$.

Proof. We know that $\bar{L}_{0}$ is the unique minimal normal subgroup of $\bar{H}=H / S(H)$. Hence if $K=C_{H}(O(H))$ and $K \nsubseteq S(H)$, then $\bar{K}$ is a nontrivial normal subgroup of $\bar{H}$ as $K$ is normal in $H$ and consequently $\bar{K} \supseteq \bar{L}_{0}$. Thus $K$ covers $L_{0} S(H) / S(H)$ and so $K$ covers $L_{0} O(H) / O(H)$ by Lemma 13. Therefore $O(H) K$ contains $L_{0}$. However, 
in the present case $O(H) \subseteq L_{0}$. Indeed, $S \cap K \neq 1$ as $S \cap K$ is a Sylow 2-subgroup of $K$ and $K$ is a normal subgroup of $H$ not contained in $S(H)$. Since $S \cap K$ is normal in $S$, it follows that $Z(S) \cap K \neq 1$. But $Z(S)$ is cyclic and $x$ is the unique involution of $Z(S)$, so $x \in K$ and hence $O(H)$ centralizes $x$. Thus $O(H) \subseteq N$. Since $L_{0}$ contains $O(H) \cap N$ by its definition, the desired assertion $O(H) \subseteq L_{0}$ is verified. Since $L_{0} \subseteq O(H) K$, we conclude therefore that $L_{0}=O(H)\left(K \cap L_{0}\right)$.

Set $R=K \cap L_{0}$. Then $L_{0}=O(H) R$ and so $O(R) \subseteq O\left(L_{0}\right)=O(H)$. Since $R \subseteq K=C_{H}(O(H))$, it follows that $O(R) \subseteq Z(R)$. Thus $R$ is a central extension of $O(R)$ by $R / O(R)$, the latter group being isomorphic to $L_{0} / O(H)$ and hence to $S L(2, q)$. The results of [26] now yield that $R=J O(R)$, where $J$ is isomorphic to $S L(2, q)$ or to $S L^{\wedge}(2,9)$ with $q=9$. Since $J$ is perfect and $O(R) \subseteq Z(R), J=R^{\prime}$. Thus $J$ is characteristic in $R$. But $R=K \cap L_{0}$ is normal in $L_{0}$, so $J$ is normal in $L_{0}$. Furthermore, $L_{0}=R O(H)=J O(R) O(H)=J O(H)$ as $O(R) \subseteq O(H)$. In addition, $J \cap O(H)$ $\subseteq O(J)$. Since $O(J)=1$ if $q \neq 9$, we have $L_{0}=J \times O(H)$ in this case. Finally $J$ centralizes $O(H)$ as $J \subseteq R=C_{H}(O(H))$ and all parts of the lemma are proved.

For our final general property of $Q$-groups, we drop the requirement that $S$ be quasi-dihedral or wreathed.

LEMMA 17. If $H$ is an arbitrary $Q$-group, then no subgroup of $H$ is a D-group or a QD-group.

Proof. Proposition 3.1 still yields that $H=O(H) C_{H}(Z(S))$. Moreover, $S$ is not abelian, since otherwise $S=Z(S)$ and $C_{H}(S)$ would have a normal 2-complement by Burnside's transfer theorem and it would follow that $H$ had a normal 2complement, contrary to the definition of a $Q$-group. Thus $S$ is nonabelian and now Lemmas 1.1 and 1.2 yield that $Z(S)$ is cyclic.

Suppose now that $H$ contains a subgroup $K$ which is either a $D$-group or a $Q D$ group. By Propositions 1.1 and 1.2 and Definition 2.1, $K$ contains a four subgroup $T$ such that $\left|A_{K}(T)\right|$ is divisible by three. Replacing $K$ by a conjugate, if necessary, we can assume without loss that $T \subseteq S$. But $S$ is a subgroup of a quasi-dihedral or wreathed 2-group $S^{*}$ and Lemmas 1.1 and 1.2 imply that $\Omega_{1}\left(Z\left(S^{*}\right)\right)=\Omega_{1}(Z(S)) \subseteq T$.

Finally since $\left|A_{K}(T)\right|$ is divisible by three, $K$ contains a 3-element $a$ which cyclically permutes the involutions of $T$. But then if $\bar{H}=H / O(H), \bar{a}$ cyclically permutes the involutions of $\bar{T}$. However, $Z(\bar{S})$ is central in $\bar{H}$ as $H=O(H) C_{H}(Z(S))$. Since $Z(\bar{S})$ is cyclic, $\bar{a}$ thus fixes the unique involution $\bar{x}$ of $Z(\bar{S})$. But $\langle\bar{x}\rangle=\Omega(Z(\bar{S})) \subseteq \bar{T}$ and so $\bar{x} \in \bar{T}$, contrary to the fact that $\bar{a}$ fixes none of the involutions of $\bar{T}$.

The final lemma of the section gives some properties of the representations of $S L(2, q)$.

LEMMA 18. If $H=S L\left(2, p^{m}\right), p$ an odd prime, is faithfully represented on a vector space $V$ over $G F(p)$, then

(i) $\operatorname{dim} V \geqq 2 m$.

(ii) If $\operatorname{dim} V=2 m$, then $\operatorname{dim} C_{V}(P)=m$ for any Sylow p-subgroup $P$ of $H$. 
Proof. The irreducible representations of $H$ on a vector space $V$ over $G F(p)$ of dimension at most $2 m$ are described by Fong and Wong in (1E) of [16]. Their results prove, in particular, that $\operatorname{dim} V=2 m$, so (i) holds. Moreover, if $\operatorname{dim} V=2 m$, they show that $V$ acts faithfully and irreducibly on $V$ and that if $U=V \otimes F$, where $F$ is an algebraic closure of $G F(p)$, then as an $H$-module, $U$ is the direct sum of $m$ twodimensional submodules $U_{i}, 1 \leqq i \leqq m$, one of which is the natural module for $H$, and the remaining are the distinct algebraic conjugate modules. But then if $P$ is a Sylow $p$-subgroup of $H$, we have $\operatorname{dim} C_{U_{i}}(P)=1,1 \leqq i \leqq m$, whence $\operatorname{dim} C_{U}(P)=m$. This implies that $\operatorname{dim} C_{V}(P)=m$ and so (ii) also holds.

5. D-groups. We now establish those properties of $D$-groups which we shall need for the paper. Throughout the section $H$ will denote an arbitrary $D$-group and $T$ a four subgroup of $H$.

It will be convenient to say that $H$ is a $D$-group with respect to $T$ whenever $\left|A_{H}(T)\right|$ is divisible by three. If $H$ contains no normal subgroups of index two, then $H$ has dihedral Sylow 2-subgroups by definition of a $D$-group. In this case Theorem 7.7.3 of [19] implies that $H$ is a $D$-group with respect to any four subgroup $T$ of $H$.

LEMMA 1. The following results hold:

(i) $H$ is a D-group with respect to $T$ if and only if $T \subseteq H^{\prime}$.

(ii) If $H$ is not a D-group with respect to $T$, then $H / O(H)$ contains a normal subgroup of odd index isomorphic to either $P G L(2, q)$ for some odd $q$ or to $P G L_{n}(2,3)$ for some $n$.

Proof. If $\bar{H}=H / O(H)$, then $N_{H}(\bar{T})$ and $C_{H}(\bar{T})$ are the images of $N_{H}(T)$ and $C_{H}(T)$ respectively. Moreover, $T \subseteq H^{\prime}$ if and only if $\bar{T} \subseteq \bar{H}^{\prime}$. Thus it will suffice to prove the lemma for $\bar{H}$. Since $\bar{H}$ is clearly also a $D$-group and $O(\bar{H})=1$, we can therefore assume without loss that $O(H)=1$.

If $H$ is isomorphic to $A_{7}$, then $T \subseteq H^{\prime}$ and $H$ is a $D$-group with respect to $T$, so the lemma holds in this case. On the other hand, if $H$ is isomorphic to $P G L_{n}(2,3)$, (ii) certainly holds and (i) is immediate from the structure of $H$. Hence we can assume that $H$ is of neither of these forms. But now Proposition 3.4 yields that $H=L E$, where $L$ is isomorphic to $\operatorname{PSL}(2, q), P G L(2, q)$, or $P G L^{*}(2, q)$ for some odd $q, E$ is cyclic of odd order, $L \cap E=1$, and $E$ centralizes a Sylow 2-subgroup of $L$. Thus $T \subset L$ and $L$ contains a normal subgroup $L_{0}$ of index at most two isomorphic to $\operatorname{PSL}(2, q)$. If $T \subset L_{0}$, then $L_{0}$, and hence also $H$, is a $D$-group with respect to $T$. Furthermore, $T \subset L_{0}^{\prime}$ as $L_{0}$ has no normal subgroups of index two. Thus the lemma holds in this case.

Suppose, on the other hand, that $T \nsubseteq L_{0}$, in which case $L$ is isomorphic to $\operatorname{PGL}(2, q)$ or $P G L^{*}(2, q)$. However, in the latter case $L$ has quasi-dihedral Sylow 2-subgroups and so by Lemma 1.1 has only one conjugacy class of four groups. Since $L_{0}$ is normal in $H$ and contains a four group, we have $T \subset L_{0}$, contrary to assumption. Thus $L$ is isomorphic to $\operatorname{PGL}(2, q)$. Furthermore, $L_{0}$ is normal in $H$ and $H=L E=L_{0} T E$, so $\bar{T}$ is normal in $\bar{H}=H / L_{0}=\bar{T} \bar{E}$. Since $\bar{T}$ is of order two, we 
have $\bar{H}=\bar{T} \times \bar{E}$, so $\bar{T} \nsubseteq \bar{H}^{\prime}$ and hence $T \nsubseteq H^{\prime}$. Clearly this implies that $\left|A_{H}(T)\right|$ is not divisible by three and so the lemma holds in this case as well.

LemMA 2. If $H$ is a D-group with respect to $T$ and $p$ is an odd prime, then $T \subseteq O_{p^{\prime}}(H)$ if and only if $O_{p^{\prime}}(H) \nsubseteq O(H)$.

Proof. If $T \subseteq O_{p^{\prime}}(H)$, then obviously $O_{p^{\prime}}(H) \nsubseteq O(H)$. Suppose, on the other hand, that $O_{p^{\prime}}(H) \nsubseteq O(H)$, in which case $O_{p^{\prime}}(H)$ has even order as $O_{p^{\prime}}(H)$ is normal in $H$. But then if $S$ is a Sylow 2-subgroup of $H$ containing $T, S \cap O_{p^{\prime}}(H) \neq 1$. Since $S \cap O_{p^{\prime}}(H)$ is normal in $S$, it follows that $Z(S) \cap O_{p^{\prime}}(H) \neq 1$. But $S$ is either dihedral, quasi-dihedral, or wreathed and so by Lemmas 1.1 and 1.2 and the structure of dihedral 2-groups, $\Omega_{1}(Z(S)) \subseteq T$. Thus $T \cap O_{p^{\prime}}(H) \neq 1$. But $\left|A_{H}(T)\right|$ is divisible by three as $H$ is a $D$-group with respect to $T$ and so $H$ contains a 3-element which cyclically permutes the involutions of $T$. We conclude therefore that $T \subseteq O_{p^{\prime}}(H)$, as required.

A similar result is the following:

LEMMA 3. Let $K$ be a normal subgroup of $H$. Then

(i) If $C_{H}(K) \nsubseteq O(H)$, then $C_{H}(K)$ contains a four group.

(ii) If $K$ is inverted by an involution of $H$, then $C_{H}(K) \nsubseteq O(H)$.

Proof. Set $R=C_{H}(K)$ and $\bar{H}=H / O(H)$. Then $R$ is normal in $H$ as $K$ is normal in $H$, so $\bar{R}$ is normal in $\bar{H}$. If $R \nsubseteq O(H)$, then $R$ and hence also $\bar{R}$, has even order. Since $\bar{H}$ is a $D$-group in which $O(\bar{H})=1$, we can apply Proposition 3.4 to $\bar{H}$ and it is immediate from that result that any nonidentity normal subgroups of $\bar{H}$ contains a four group. Thus, $\bar{R}$ and hence also $R$, contains a four group, proving (i).

Suppose next that $K$ is inverted by an involution of $H$, in which case the extended centralizer $R^{*}=C_{H}^{*}(K)$ of $K$ in $H$ is not contained in $O(H)$. But $R^{*}$ is normal in $H$ and so $\bar{R}^{*}$ contains a four group as in the preceding paragraph. Hence also $R^{*}$ contains a four group. However $\left|R^{*}: R\right|=2$ and so $R$ must have even order. Thus $R \nsubseteq O(H)$ and (ii) also holds.

LEMmA 4. If $K \in \mathrm{U}_{H}(T)$ with $K$ of odd order and $[T, K]=K$, then $K / K \cap O(H)$ is cyclic.

Proof. Setting $\bar{H}=H / O(H)$, we have $\bar{K} \in И_{H}(\bar{T}), \bar{K}$ is of odd order, and $[\bar{T}, \bar{K}]=\bar{K}$ and we need only prove that $\bar{K}$ is cyclic. It will suffice to prove the lemma for $\bar{H}$ and so without loss we can assume that $O(H)=1$ and establish that $K$ is cyclic.

If $H$ is isomorphic to $A_{7}$, the maximal elements of $\eta_{H}(T)$ are of order three, while if $H$ is isomorphic to $P G L_{n}(2,3),{U_{H}}_{H}(T)$ is trivial, as is easily verified. In either case it follows that $K$ must be cyclic. Hence we can suppose that $H$ is not of this form, whence $H=L E$, where $L$ and $E$ are as in Proposition 3.4. Since $L$ is normal in $H$ and $E$ has odd order, $T \subseteq L$ and so $K=[T, K] \subseteq L$. Let $L_{0}$ be the subgroup of index at most two in $L$ isomorphic to $\operatorname{PSL}(2, q)$. Then $K \subseteq L_{0}$. If $L$ is isomorphic to $P G L^{*}(2, q)$, it follows as in the proof of Lemma 1 that $T \subset L_{0}$. Since $L$ is isomorphic 
to $\operatorname{PSL}(2, q), P G L(2, q)$, or $P G L^{*}(2, q)$, we conclude at once that $L_{0} T$ is isomorphic to $\operatorname{PSL}(2, q)$ or $\operatorname{PGL}(2, q)$. But now Lemmas 3.1 (iii) and (vii) of [22] imply that every element of $U_{L_{0}}(T)$ is cyclic. Thus $K$ is cyclic, as required.

Lemma 5. $H$ does not involve a $Q$-group.

Proof. Suppose $H$ involves a $Q$-group $K$. Then by Propositions 1.1 and 1.2 and the definition of a $Q$-group, $K$ contains a quaternion subgroup $Q$ such that $\left|A_{K}(Q)\right|$ is divisible by three. This implies that $Q \subseteq K^{\prime}$ and hence that $H^{\prime}$ involves a quaternion group. But then so does $\bar{H}^{\prime}$, where $\bar{H}=H / O(H)$. On the other hand, Proposition 3.4, applied to $\bar{H}$, yields that a Sylow 2-subgroup of $\bar{H}^{\prime}$ is either dihedral or is abelian of type $\left(2^{n}, 2^{n}\right)$ for some $n$. In either case it follows that $\bar{H}^{\prime}$ does not involve a quaternion group. This contradiction establishes the lemma.

By analogy with Lemma 3.13 we have

LEMMA 6. If $H / O(H)$ is isomorphic to PSL $(2, q)$ with $q>3$ and $q \neq 9$ and if $C_{H}(O(H)) \nsubseteq O(H)$, then $H=J \times O(H)$, where $J$ is isomorphic to $\operatorname{PSL}(2, q)$.

Proof. Set $K=C_{H}(O(H))$, so that $K$ is normal in $H$ and $O(H) K \supset O(H)$. Since $q>3, H / O(H)$ is simple and hence $O(H)$ is a maximal normal subgroup of $H$, thus forcing $O(H) K=H$. This implies that $O(K) \subseteq O(H)$. Since $K$ centralizes $O(H)$, it follows that $O(K) \subseteq Z(K)$. Since $q \neq 9$ and $q>3$, the results of [26] now yield that $K=J \times O(K)$, where $J$ is isomorphic to $\operatorname{PSL}(2, q)$. Since $J$ is simple and $O(K)$ is solvable, $J$ is characteristic in $K$ and so $J$ is normal in $H$. Moreover, $[J, O(H)]$ $\subseteq O(J)=1$. We conclude that $H=K O(H)=J O(K) O(H)=J O(H)=J \times O(H)$ and the lemma is proved.

The next two lemmas list some properties of $D$-groups $H$ of linear type with dihedral or quasi-dihedral Sylow 2-subgroups in which $O(H)=1$. The structure of such a group is given in Proposition 3.4 and we have $H=L E$, where $L$ and $E$ are as in that proposition. In particular, $E$ centralizes a Sylow 2-subgroup $S$ of $H$, which without loss we may assume contains $T$. We also set $D=N_{S}(T)$, so that $D=T$ if $S=T$ and $D$ is dihedral of order eight if $S \supset T$. In addition, we let $x_{i}, 1 \leqq i \leqq 3$, be the involutions of $T$ with $x_{1} \in Z(D)$, we let $L_{0}$ be the normal subgroup of $H$ of index at most two in $L$ isomorphic to $\operatorname{PSL}(2, q)$, and we set $\varepsilon= \pm 1$, where $q \equiv \varepsilon(\bmod 4)$.

LEMMA 7. If $H$ is of linear type with dihedral or quasi-dihedral Sylow 2-subgroup $S$ and with $O(H)=1$, then

(i) If $x_{i} \in L_{0}$, then $C_{L_{0}}\left(x_{i}\right)$ is a dihedral group of order $q-\varepsilon$.

(ii) If $x_{i} \notin L_{0}$, then $C_{L_{0}}\left(x_{i}\right)$ is a dihedral group of order $q+\varepsilon$.

(iii) $C_{H}(T)=T \times E$ and $C_{L}(T)=T$.

(iv) Every element of ${\eta_{H}}_{H}(T)$ is contained in $C_{H}\left(x_{i}\right)$ for some $i, 1 \leqq i \leqq 3$.

(v) If $D \supset T$, then every element of $\eta_{H}(D)$ is contained in $C_{H}\left(x_{1}\right)$.

(vi) If $L$ is isomorphic to $P G L^{*}(2, q)$, then all involutions of $H$ are contained in $L_{0}$.

Proof. First, if $L$ is isomorphic to $P G L^{*}(2, q)$, we have argued already in Lemma 1 that $L_{0}$ contains every four subgroup of $L$. Since any involution of $H$ lies in $L$ and 
is clearly contained in a four subgroup of $L$, we see that (vi) holds. Furthermore, this argument shows that $L_{0} T$ is isomorphic to either $\operatorname{PSL}(2, q)$ or $\operatorname{PGL}(2, q)$. But now (i) and (ii) are a consequence of Lemma 3.1 (iii) of [22], while (iii) follows from Lemmas 3.1 (iii) and 3.3 (i) of [22] and (iv) follows from Lemma 3.1 (vii) of [22].

Finally if $D \supset T$, then $D=\left\langle T, T^{*}\right\rangle$ for some four subgroup $T^{*}$ of $D$. But then, as with $T$, we have that $L_{0} D$ is isomorphic to $\operatorname{PSL}(2, q)$ or $\operatorname{PGL}(2, q)$. Hence if $K \in h_{H}(D)$, then $K \in C_{H}\left(x_{i}\right) \cap C_{H}\left(x^{*}\right)$ by (iv), where $x^{*}$ is an involution of $T^{*}$, so (v) holds if $x_{i}$ or $x^{*}$ is $x_{1}$. Since $T \cap T^{*}=\left\langle x_{1}\right\rangle$, it follows in the contrary case that $\left\langle x_{i}, x^{*}\right\rangle=D$, whence $K \subseteq C_{H}(D)=E$ by (iii) as $K$ is necessarily of odd order. Thus $K \subseteq C_{H}\left(x_{1}\right)$ in this case as well, so (v) holds.

Lemma 8. If $H$ is a D-group with respect to $T$ of linear type with dihedral or quasidihedral Sylow 2-subgroup $S$ in which $O(H)=1$ and $E_{0}$ is a subgroup of $E$ of order $e_{0}$, then

(i) $C_{L_{0}}\left(E_{0}\right)$ is isomorphic to $\operatorname{PSL}\left(2, q_{0}\right)$, where $q=q_{0}^{e}$.

(ii) $C_{L_{0}}\left(E_{0}\right)$ is a D-group with respect to $T$.

Proof. First, (i) is a consequence of Lemma 3.3 (i) of [22]. Furthermore, $T \subseteq H^{\prime}$ by Lemma 1 and it is immediate from the structure of $H$ that $L_{0}=H^{\prime}$. Hence $T \subset C_{L_{0}}\left(E_{0}\right)$. Since $C_{L_{0}}\left(E_{0}\right)$ is isomorphic to $\operatorname{PSL}\left(2, q_{0}\right)$ with $q_{0}$ odd, it has no normal subgroups of index two and so is a $D$-group with respect to any of its four subgroups. Thus (ii) also holds.

The following properties of the groups $A_{7}$ and $P G L_{n}(2,3)$ are easy to check:

LEMMA 9. The following conditions hold:

(i) If $H$ is isomorphic to $A_{7}$, then a maximal element of $\eta_{H}(S)$ has order 3 and centralizes a four subgroup of $S$.

(ii) If $H$ is isomorphic to $P G L_{n}(2,3)$ for some $n$, then $U_{H}(T)$ is trivial.

We conclude this section with a specialized result concerning the representations of $D$-groups.

Lemma 10. Assume $H$ is a D-group satisfying the following conditions:

(a) $H$ is of linear type with dihedral or quasi-dihedral Sylow 2-subgroups and characteristic power $q>5$.

(b) There is an involution $x$ of $H$ with $x \notin H^{\prime}$.

(c) $H$ is faithfully represented on a vector space $V$ over $G F(p)$, $p$ an odd prime.

(d) $\operatorname{dim} V=1+\operatorname{dim} C_{V}(x)$.

Under these conditions, $x$ inverts an element $u$ of $H$ of odd prime order $r$ with $r \neq p$ and such that $C_{V}(x) \cap C_{V}(u)$ is nontrivial.

Proof. It will suffice to prove that $x$ inverts an $r$-element $u \neq 1$ for some odd prime $r \neq p$. Indeed, if this is the case, we can take $u$ to be of order $r$. Setting $x^{\prime}=u^{-1} x u$, we then have that $u \in\left\langle x, x^{\prime}\right\rangle$ as $x$ inverts $u$ and $u$ has odd order. But $x^{\prime}$, being a conjugate of $x$, also centralizes a subspace of $V$ of codimension one. 
Hence $V_{0}=C_{V}\left(\left\langle x, x^{\prime}\right\rangle\right)$ has codimension at most two in $V$ and both $x$ and $u$ centralize $V_{0}$. Thus the lemma holds if $V_{0}$ is nontrivial. However, in the contrary case, $\operatorname{dim} V \leqq 2$. But $H$, being a $D$-group, contains a four subgroup $T_{0}$ such that $\left|A_{H}\left(T_{0}\right)\right|$ is divisible by three. Clearly then $N_{H}\left(T_{0}\right)$ cannot be faithfully represented on a two-dimensional vector space over $G F(p), p$ odd. Thus we need only prove the existence of the required $r$-element $u$.

Setting $\bar{H}=H / O(H)$, we have that $\bar{H}$ is a $D$-group of linear type with dihedral or quasi-dihedral Sylow 2-subgroups of characteristic power $q>5$ and $\bar{x} \notin \bar{H}^{\prime}$. Proposition 3.4 together with Lemma 7 imply that $\bar{H}=\bar{L} \bar{E}$, where $\bar{L}$ is isomorphic to $P G L(2, q), \bar{L}$ is normal in $\bar{H}$, and $\bar{E}$ is cyclic of odd order. It will clearly suffice to show that $\bar{x}$ normalizes, but does not centralize, an $r$-subgroup $\bar{R}$ of $\bar{L}$ for some odd prime $r \neq p$. Indeed, then the inverse image $R$ of $\bar{R}$ in $H$ has odd order and is $x$-invariant, so $x$ normalizes some Sylow $r$-subgroup $R_{0}$ of $R$. Since $\bar{R}_{0}=\bar{R}, x$ does not centralize $R_{0}$ and consequently $x$ will invert some element $u \neq 1$ of $R_{0}$ by Lemma 10.4.1 of [19]. We argue by contradiction and so assume that no such subgroup $\bar{R}$ exists.

Let $\bar{L}_{0}$ be the subgroup of index two in $\bar{L}$ isomorphic to $\operatorname{PSL}(2, q)$ and let $\bar{x}_{0}$ be an involution of $\bar{L}_{0}$ centralizing $\bar{x}$. As usual, Lemma 3.1 (iii) of [22] implies that $C_{L}\left(\bar{x}_{0}\right)$ and $C_{L}\left(\bar{x}_{0}\right)$ are dihedral groups of orders $2(q-\varepsilon)$ and $2(q+\varepsilon)$ respectively, where $q \equiv \varepsilon(\bmod 4)$ and $\varepsilon= \pm 1$. Now a dihedral group (of order greater than four) possesses a unique central involution, which in the present cases will be $\bar{x}_{0}$ and $\bar{x} \bar{x}_{0}$ respectively. It follows at once from this that $\bar{x}$ inverts both $O\left(C_{I}\left(\bar{x}_{0}\right)\right)$ and $O\left(C_{E}\left(\bar{x} \bar{x}_{0}\right)\right)$. Since no odd prime divides both $q-\varepsilon$ and $q+\varepsilon$, our conditions force one of these numbers to be a power of 2 and the other to be divisible only by the primes 2 and $p$; otherwise $\bar{x}$ would invert a Sylow $r$-subgroup $\bar{R} \neq 1$ of $O\left(C_{E}\left(\bar{x}_{0}\right)\right)$ or $O\left(C_{L}\left(\bar{x} \bar{x}_{0}\right)\right)$ for some odd prime $r \neq p$, contrary to our assumption. We conclude therefore that $q$ is either a Mersenne or Fermat prime or $q=9$ and that either $q+1$ or $q-1$ is of the form $2 p^{a}$ for some $a$. Moreover, $a \geqq 1$ as $q>5$ by assumption and consequently the characteristic $t$ of $\bar{L}_{0}$ is distinct from $p$. But then we must also have $\varepsilon=1$, since otherwise Lemma 3.1 (vi) of [22] would imply that $\bar{x}$ inverts a Sylow $t$-subgroup $\bar{P}$ of $\bar{L}_{0}$ and we could take $t=r$ and $\bar{P}=\bar{R}$. Thus $q$ is, in fact, a Fermat prime or 9 and $q+1=2 p^{a}$.

To treat this case, it will suffice to prove the existence of conjugates $z_{1}, z_{2}$ of $x$ in $H$ such that $\left\langle x, z_{1}, z_{2}\right\rangle$ maps onto $\bar{L}$. Indeed, assume this is the case and set $K=\left\langle x, z_{1}, z_{2}\right\rangle$. By assumption (d), $C_{V}\left(z_{i}\right)$ has codimension one in $V, 1 \leqq i \leqq 3$, and hence $V_{0}=C_{V}(x) \cap C_{V}\left(z_{1}\right) \cap C_{V}\left(z_{2}\right)$ has codimension at most three in $V$. Thus $\tilde{V}=V / V_{0}$ has dimension at most three. Furthermore, $K_{0}=C_{K}(\tilde{V})$ stabilizes the chain $V \supseteq V_{0} \supseteq 0$. Since $K$ is faithfully represented on $V$ and $V$ is a vector space over $G F(p)$, it follows that $K_{0}$ is a $p$-group. But $K / O(K)$ is isomorphic to $\bar{K}=\bar{L}$ which has no nontrivial normal $p$-subgroups and so $K_{0} \subseteq O(K)$. Hence if $\widetilde{K}=K / K_{0}$, we conclude that $\tilde{K}$ is faithfully represented on $\tilde{V}$ and that $\tilde{K} / O(\tilde{K})$ is isomorphic to $\operatorname{PGL}(2, q)$.

But now as $\tilde{K}$ is a $D$-group and $V$ is of dimension at most three over $G F(p)$, Lemma 3.5 (iii) of [22] implies that $q=5,7,9$, or $p$. However, in our case $q \neq p$, 
$q \neq 5$, and $q$ is not a Mersenne prime, so we must have $q=9$. But also $q+1=2 p^{a}$ and so $p=5$. Thus $\widetilde{K}$ is isomorphic to a subgroup of $G L(3,5)$. However, this is impossible as $\widetilde{K}$ involves $\operatorname{PGL}(2,9)$ and so has order divisible by nine, while $|G L(3,5)|$ is not divisible by nine.

Thus it remains to prove the existence of the required conjugates $z_{1}, z_{2}$ of $x$. Clearly we need only prove the existence of conjugates $\bar{z}_{1}, \bar{z}_{2}$ of $\bar{x}$ such that $\left\langle\bar{x}, \bar{z}_{1}, \bar{z}_{2}\right\rangle=\bar{L}$. Now $C_{L}\left(\bar{x}_{0}\right)$ and $C_{L}\left(\bar{x} \bar{x}_{0}\right)$ are dihedral groups of order $2(q-1)=2^{m}$ for some integer $m$ and $2(q+1)=4 p^{a}$ respectively and $\bar{x}$ is not in the center of either group. We can therefore determine conjugates $\bar{z}_{1}$ and $\bar{z}_{2}$ of $\bar{x}$ by an element of $C_{L}\left(\bar{x}_{0}\right)$ and $C_{L}\left(\bar{x} \bar{x}_{0}\right)$ respectively such that $\left\langle\bar{x}, \bar{z}_{1}\right\rangle$ and $\left\langle\bar{x}, \bar{z}_{2}\right\rangle$ are dihedral groups of order $2^{m-1}$ and $2 p^{a}$ respectively. Setting $\bar{K}=\left\langle\bar{x}, \bar{z}_{1}, \bar{z}_{2}\right\rangle$, we shall argue now that $\bar{K}=\bar{L}$ and this will suffice to complete the proof.

We note first that $m \geqq 4$ as $q \geqq 9$. In particular, this implies that eight divides $|\bar{K}|$ and that $\bar{x}_{0} \in\left\langle\bar{x}, \bar{z}_{1}\right\rangle$, whence also $\bar{x}_{0} \in \bar{K}$. Furthermore, if $\bar{P}$ is the Sylow $p$ subgroup of $\left\langle\bar{x}, \bar{z}_{2}\right\rangle$, then $|\bar{P}|=p^{a}, \bar{P}$ is inverted by $\bar{x}$, and $\bar{P}$ is centralized by $\bar{x} \bar{x}_{0}$. Consider first the case that $\bar{K}$ has a normal 2-complement. Then $\bar{P} \subseteq O(\bar{K})$ and $\bar{x} \bar{x}_{0}$ is the only involution of the four group $\left\langle\bar{x}, \bar{x}_{0}\right\rangle$ which centralizes $\bar{P}$. However, $O(\bar{K}) \in U_{L}\left(\left\langle\bar{x}, \bar{x}_{0}\right\rangle\right)$ and hence Lemma 3.1 (iii), (v), and (vii) of [22] yields that $O(\bar{K}) \subseteq C_{L}\left(\bar{x} \bar{x}_{0}\right)$ and that $N_{L}(O(\bar{K}))=C_{L}\left(\bar{x} \bar{x}_{0}\right)$. Since $\bar{K} \subseteq \bar{L}$, it follows that $\bar{K} \subseteq C_{L}\left(\bar{x} \bar{x}_{0}\right)$. But eight divides $|\bar{K}|$ and does not divide $\left|C_{L}\left(\bar{x} \bar{x}_{0}\right)\right|$. This contradiction shows that $\bar{K}$ does not have a normal 2 -complement. Thus $\bar{K}$ is necessarily a $D$-group.

Finally $\bar{K}$ has a normal subgroup of index two as $\bar{x} \in \bar{K}$. Moreover, $\left|C_{\bar{K}}\left(\bar{x}_{0}\right)\right| \geqq q-1=2^{m-1} \geqq 8$ as $\left\langle\bar{x}, \bar{z}_{1}\right\rangle \subseteq \bar{K}$. In particular, $\bar{K}$ is not isomorphic to $P G L(2,3)$ or $P G L(2,5)$. Since $q$ is either 9 or a prime, we conclude therefore from Lemma 3.1 (viii) of [22] that $\bar{K}$ is isomorphic to $P G L(2, q)$. Since $\bar{K} \subseteq \bar{L}$, this forces $\bar{K}=\bar{L}$ and the lemma is proved.

6. $p$-constraint and $p$-stability. The concepts of $p$-constraint and $p$-stability will play an important role in the paper. However, we shall require certain variations of the standard notions. We begin with constraint.

Recall that by definition a group $H$ is $p$-constrained, $p$ a prime, provided $C_{H}(P) \subseteq O_{p^{\prime}, p}(H)$, where $P$ is a Sylow $p$-subgroup of $O_{p^{\prime}, p}(H)(\$ 8.1$ of [19]). However, it may or may not be the case that $O_{p^{\prime}}(H) \subseteq O(H)$ or that $O_{p^{\prime}}(H) \subseteq S(H)$. Thus $H$ may very well be $p$-constrained without $C_{H}(P)$ being contained in $O(H)$ or $S(H)$. We make the following definition:

Definition 1. A group $H$ will be said to be $p$-constrained within $O(H)$ or within $S(H), p$ a prime, provided $H$ is $p$-constrained and $O_{p^{\prime}}(H)$ is contained, respectively, in $O(H)$ or $S(H)$.

Our discussion of $p$-constraint depends upon the following general lemma:

LEMMA 1. If the 2-group $S$ acts on the group $K$ of odd order and if $P$ is an $S$ invariant Sylow p-subgroup of $K$, then $S$ centralizes $P$ if and only if it centralizes $P \cap O_{p^{\prime}, p}(K)$. 
Proof. Set $Q=P \cap O_{p^{\prime}, p}(K)$, so that $K$ is an $S$-invariant Sylow $p$-subgroup of $O_{p^{\prime}, p}(K)$. If $S$ centralizes $P$, then obviously $S$ centralizes $Q$. Suppose conversely that $S$ centralizes $Q$. Setting $H=K S$ and $\bar{H}=H / O_{p^{\prime}}(K)$, we have that $\bar{S}$ centralizes $\bar{Q}=O_{p}(\bar{K})$ and it will suffice to show that $\bar{S}$ centralizes $\bar{P}$. Hence without loss we can assume to begin with that $O_{p^{\prime}}(K)=1$.

But then if $C=C_{H}(Q)$, we have $S \subseteq C$. On the other hand, $K$ is $p$-constrained as $K$, being of odd order, is solvable. Since $O_{p^{\prime}}(K)=1$, it follows that $C \cap K=$ $C_{K}(Q) \subseteq Q$. Since $[S, P] \subseteq C \cap K \subseteq Q$ and since $S$ centralizes $Q, S$ stabilizes the chain $P \supseteq Q \supseteq 1$ and so $S$ centralizes $P$, as required.

LEMmA 2. If $H$ is a $Q$-group with quasi-dihedral or wreathed Sylow 2-subgroup $S$ of characteristic power $q \geqq 5$ and $P$ is an $S$-invariant Sylow p-subgroup of $O(H)$ for some prime $p$, then

(i) $H$ is p-constrained within $S(H)$ if and only if $C_{Z(S)}(P)$ is a Sylow 2-subgroup of $C_{H}(P)$.

(ii) $H$ is p-constrained within $O(H)$ if and only if $C_{H}(P)$ is of odd order.

Proof. We shall first reduce to the case that $O_{p^{\prime}}(O(H))=1$. Indeed, setting $\bar{H}=H / O_{p^{\prime}}(O(H))$, we have that $\overline{O(H)}=O(\bar{H}), \overline{S(H)}=S(\bar{H}), \bar{P}$ is an $\bar{S}$-invariant Sylow p-subgroup of $O(\bar{H}), \bar{S}$ is a Sylow 2-subgroup of $\bar{H}$ and is isomorphic to $S$, and $\bar{H}$ is a $Q$-group of the same characteristic power as $H$. Moreover, since $O_{p^{\prime}}(O(H))$ has order relatively prime to 2 and $p, C_{Z(S)}(P)$ maps onto $C_{Z(S)}(\bar{P})$ and a Sylow 2-subgroup of $C_{H}(P)$ maps onto a Sylow 2-subgroup of $C_{H}(\bar{P})$. Thus $C_{Z(S)}(P)$ is a Sylow 2-subgroup of $C_{H}(P)$ if and only if $C_{Z(S)}(\bar{P})$ is a Sylow 2subgroup of $C_{H}(\bar{P})$ and $C_{H}(P)$ is of odd order if and only if $C_{H}(\bar{P})$ is. In addition, it follows at once from the definitions and the fact that $\overline{S(H)}=S(\bar{H})$ and $\overline{O(H)}=O(\bar{H})$ that $H$ is $p$-constrained within $S(H)$ or $O(H)$ if and only if $\bar{H}$ is $p$-constrained within $S(\bar{H})$ or $O(\bar{H})$ respectively. We conclude that the lemma holds for $H$ provided it holds for $\bar{H}$. Since $O_{p^{\prime}}(O(\bar{H}))=1$, we can therefore assume without loss to begin with that $O_{p^{\prime}}(O(H))=1$.

Set $Q=O_{p}(O(H)), S_{0}=C_{S}(Q)$, and $S_{1}=C_{S}(P)$. Then $S$ normalizes $Q$ as $Q$ is normal in $H$ and $S$ normalizes $P$ by hypothesis, so $S$ normalizes both $C_{H}(Q)$ and $C_{H}(P)$ and consequently $S_{0}, S_{1}$ are Sylow 2-subgroups of $C_{H}(Q)$ and $C_{H}(P)$ respectively. Moreover, $S_{1} \subseteq S_{0}$. We now apply the preceding lemma with $O(H)$ in the role of $K$ and $S_{0}$ in the role of $S$. Since $Q=O_{p^{\prime}, p}(O(H)) \cap P$ in the present case, we conclude from the lemma that $S_{0}$ centralizes $P$, whence $S_{0}=S_{1}$. Thus $C_{Z(S)}(P)$ will be a Sylow 2-subgroup of $C_{H}(P)$ if and only if $C_{Z(S)}(Q)$ is a Sylow 2-subgroup of $C_{H}(Q)$ and $C_{H}(P)$ has odd order if and only if $C_{H}(Q)$ does. Therefore it will suffice to prove that $H$ is $p$-constrained within $S(H)$ or $O(H)$ if and only if $C_{Z(S)}(Q)$ is a Sylow 2-subgroup of $C_{H}(Q)$ or $C_{H}(Q)$ has odd order respectively.

Now by Lemma 4.14, we have $S(H)=O(H) Z(S)$ as $q \geqq 5$. Consider first the case that $O_{p^{\prime}}(H) \subseteq S(H)$. Set $X=O_{p^{\prime}}(H)$. Since $O_{p^{\prime}}(O(H))=1$, it follows that 
$X \subseteq Z(S)$ and that $O_{p^{\prime}, p}(H)=O_{p^{\prime}, p}(S(H))$. Since $O(H)$ centralizes $X$, we see that $O_{p^{\prime}, p}(H)=X \times Q$ and hence that $Q$ is a Sylow $p$-subgroup of $O_{p^{\prime}, p}(H)$. Moreover, $S(H)$ is $p$-constrained as $S(H)$ is solvable and so $C_{S(H)}(Q)=X Z(Q) \subseteq O_{p^{\prime}, p}(S(H))$ $=O_{p^{\prime}, p}(H)$. Hence $H$ is $p$-constrained in the present case if and only if $C_{H}(Q) \subseteq S(H)$ and so if and only if it is $p$-constrained within $S(H)$. However, if $C_{H}(Q) \subseteq S(H)$, then $X$ is a Sylow 2-subgroup of $C_{H}(Q)$. Thus $X=C_{Z(S)}(Q)$ is a Sylow 2-subgroup of $C_{H}(Q)$. Suppose, on the other hand, that $C_{H}(Q) \nsubseteq S(H)$. Since $C_{H}(Q)$ is normal in $H$ as $Q$ is normal in $H, C_{H}(Q)$ is therefore nonsolvable. This implies that a Sylow 2-subgroup of $C_{H}(Q)$ is not cyclic, since otherwise $C_{H}(Q)$ would have a normal 2complement by Burnside's transfer theorem and so would be solvable as all groups of odd order are solvable. Since $Z(S)$ is cyclic by Lemmas 1.1 and 1.2 , we conclude that $C_{Z(S)}(Q)$ is not a Sylow 2-subgroup of $C_{H}(Q)$. Therefore (i) holds in this case.

Furthermore, if $H$ is $p$-constrained within $O(H)$ in the present case, then $C_{H}(Q) \subseteq S(H)$ and $O_{p^{\prime}, p}(H) \subseteq O(H)$. The latter condition implies that $X=1$ and so $C_{H}(Q)=Z(Q)$ is of odd order. On the other hand, if $C_{H}(Q)$ is of odd order, then $X=1$ as $X$ centralizes $Q$, so $O_{p^{\prime}, p}(H)=Q \subseteq O(H)$. Moreover, $C_{H}(Q) \subseteq O(H)$ as $C_{H}(Q)$ is normal in $H$. Hence $H$ is $p$-constrained within $O(H)$ and so (ii) also holds.

Finally consider the case that $X=O_{p^{\prime}}(H) \nsubseteq S(H)$. Since $X$ is normal in $H$, this implies as above that a Sylow 2-subgroup of $X$ is noncyclic. Since $X$ centralizes $Q$ and $Z(S)$ is cyclic, it follows that $C_{Z(S)}(Q)$ is not a Sylow 2-subgroup of $C_{H}(Q)$. We shall argue that $H$ is not $p$-constrained within $S(H)$ or $O(H)$ and this will establish (i) and (ii) in the present case.

Let $Q_{1}$ be a Sylow $p$-subgroup of $O_{p^{\prime}, p}(H)$ and let $S_{1}$ be a Sylow 2-subgroup of $X$. Since $X$ is normal in $X Q_{1}$ and $X$ is a $p^{\prime}$-group, it follows by the Frattini argument that $N_{X Q_{1}}\left(S_{1}\right)$ contains a Sylow $p$-subgroup of $X Q_{1}$. But $Q_{1}$ is a Sylow $p$-subgroup of $X Q_{1}$, so by Sylow's theorem a conjugate of $Q_{1}$ by an element of $X$ normalizes $S_{1}$. Hence, replacing $S_{1}$ by a suitable conjugate, we can assume without loss that $Q_{1}$ normalizes $S_{1}$.

It will suffice to prove that $p>3$. Indeed, assume this is the case. Since $S_{1}$ is a subgroup of a quasi-dihedral or wreathed 2-group, no section of $S_{1}$ is elementary abelian of order exceeding four, as is easily checked. Since $p>3$, this implies that $Q_{1}$ centralizes every section of $S_{1}$ that it normalizes and so $Q_{1}$ centralizes $S_{1}$. But as we have shown above, $S_{1}$ is not cyclic. Since $S(H)=O(H) Z(S)$ and $Z(S)$ is cyclic, we conclude that $C_{H}\left(Q_{1}\right) \nsubseteq S(H)$ as $S_{1} \subseteq C_{H}\left(Q_{1}\right)$. However, if $H$ were $p$ constrained within $S(H)$, then by definition $C_{H}\left(Q_{1}\right) \subseteq O_{p^{\prime}, p}(H)$ and $O_{p^{\prime}, p}(H) \subseteq S(H)$, whence $C_{H}\left(Q_{1}\right) \subseteq S(H)$, which is not the case. Thus $H$ is not $p$-constrained within $S(H)$ and so certainly is not $p$-constrained within $O(H)$.

However, as $X \nsubseteq S(H)$, the image $\bar{X}$ of $X$ in $\bar{H}=H / S(H)$ is a nontrivial normal subgroup of $\bar{H}$, so $\bar{X}$ contains the normal subgroup of $\bar{H}$ isomorphic to $\operatorname{PSL}(2, q)$ by Lemma 4.15. But $P S L(2, q)$ has order divisible by three for all odd $q$ and hence $X$ also has order divisible by three. Since $X$ is a $p^{\prime}$-group, we conclude that $p>3$, as required, and the lemma is proved. 
LEMMA 3. Let $H$ be a D-group with respect to the four group $T$ and let $P$ be a $T$ invariant Sylow p-subgroup of $O_{p^{\prime}, p}(O(H))$ and assume that $O_{p^{\prime}}(H) \subseteq O(H)$. Then we have

(i) If $H$ is p-constrained within $O(H)$, then $C_{T}(P)=1$.

(ii) If $H$ is not p-constrained within $O(H)$, then $C_{T}(P)=P$.

Proof. Arguing as in the preceding lemma, we conclude easily that it suffices to consider the case that $O_{p^{\prime}}(O(H))=1$. Since $O_{p^{\prime}}(H) \subseteq O(H)$ by hypothesis, it follows that $O_{p^{\prime}}(H)=1$ and that $O_{p^{\prime}, p}(H)=O_{p^{\prime}, p}(O(H))$, whence $P=O_{p^{\prime}, p}(H)$. Since $O(H)$ is solvable, it is $p$-constrained and so $C_{O(H)}(P)=Z(P) \subseteq O_{p^{\prime}, p}(H)$. It follows therefore from the definition that $H$ is $p$-constrained if and only if $C_{H}(P) \subseteq O(H)$. Since $O_{p^{\prime}, p}(H) \subseteq O(H)$, we see that, in fact, $H$ will be $p$-constrained within $O(H)$ if and only if $C_{H}(P) \subseteq O(H)$.

If $C_{H}(P) \subseteq O(H)$, then obviously $C_{T}(P)=1$, so (i) holds. Suppose, on the other hand, that $C_{H}(P) \nsubseteq O(H)$. If $S$ is a Sylow 2-subgroup of $H$ containing $T$, then $S_{0}=C_{S}(P)$ is a Sylow 2-subgroup of $C_{H}(P)$ as $C_{H}(P)$ is normal in $H$ and so $S_{0} \neq 1$. Since $S_{0}$ is normal in $S$, it follows that $S_{0} \cap Z(S) \neq 1$. However, by definition of a $D$-group, $S$ is either dihedral, quasi-dihedral, or wreathed. One checks in each case that any four subgroup of $S$ necessarily contains $\Omega_{1}(Z(S))$. But $S_{0} \cap \Omega_{1}(Z(S)) \neq 1$ as $S_{0} \cap Z(S) \neq 1$ and so $S_{0} \cap T \neq 1$. Thus $C_{T}(P) \neq 1$. On the other hand, the involutions of $T$ are cyclically permuted by a 3-element of $H$ as $H$ is a $D$-group with respect to $T$. Furthermore, this 3-element leaves $P$ invariant as $P$ is normal in $H$. Since some involution of $T$ centralizes $P$, we conclude that $T$ itself centralizes $P$ and (ii) is proved.

Recall next that a group $H$ in which $O_{p}(H) \neq 1, p$ an odd prime, is said to be $p$ stable provided for any nontrivial $p$-subgroup $P$ of $H$ such that $O_{p^{\prime}}(H) P$ is normal in $H$, we have

$$
A C_{H}(P) / C_{H}(P) \subseteq O_{p}\left(N_{H}(P) / C_{H}(P)\right)
$$

for every $p$-subgroup $A$ of $N_{H}(P)$ for which $[P, A, A]=1$. (Actually this definition differs slightly in form, but not in basic content from that given in $\$ 8.1$ of [19] and removes an error in the former definition. However, it is the present definition which is, in fact, used in the proof of Glauberman's ZJ-theorem (Theorems 8.2.10 and 8.2.11 of [19]).)

We now introduce a slight extension of this concept:

Definition 2. Let $H$ be a group in which $O_{p}(H) \neq 1, p$ an odd prime, and let $R$ be a $p$-subgroup of $H$ such that

(a) $R \cap O_{p^{\prime}, p}(H)$ is a Sylow $p$-subgroup of $O_{p^{\prime}, p}(H)$.

(b) Either $R$ is normal in a Sylow $p$-subgroup of $H$ or $R K / K$ contains $O_{p}(H / K)$ for every normal subgroup $K$ of $H$.

Under these conditions, we say that $H$ is p-stable with respect to $R$ provided for any nontrivial subgroup $P$ of $R$ such that $O_{p^{\prime}}(H) P$ is normal in $H$, we have

$$
A C_{H}(P) / P \subseteq O_{p}\left(N_{H}(P) / C_{H}(P)\right)
$$

for every subgroup $A$ of $R$ for which $[P, A, A]=1$. 
Note that if $R$ is a Sylow p-subgroup of $H$, then automatically $R \cap O_{p^{\prime}, p}(H)$ is a Sylow $p$-subgroup of $O_{p^{\prime}, p}(H)$ and $R K / K$ is a Sylow $p$-subgroup of $O_{p}(H / K)$ for any normal subgroup $K$ of $H$. Moreover, if $A$ is a $p$-subgroup of $N_{H}(P)$, then $A^{x} \subseteq R$ for some $x$ in $N_{H}(P)$, as $R$ is a Sylow $p$-subgroup of $N_{H}(P)$; and $[P, A, A]=1$ if and only if $\left[P, A^{x}, A^{x}\right]=1$. In addition, $A C_{H}(P) / C_{H}(P)$ lies in $O_{p}\left(N_{H}(P) / C_{H}(P)\right)$ if and only if $A^{x} C_{H}(P) / C_{H}(P)$ does. Hence in this case we see that $H$ is $p$-stable with respect to $R$ if and only if it is $p$-stable in the usual sense.

We shall be interested, for certain $Q$-groups and $D$-groups $H$, in the $p$-stability of $H$ with respect to $R$, where $R$ is a maximal element of $\eta_{H}(D ; p)$ and $D$ is an appropriate 2-subgroup of $H$. More precisely, we assume for the balance of the section that one of the following conditions holds for some odd prime $p$ :

(a) $H$ is a $Q$-group with quasi-dihedral Sylow 2-groups of characteristic power $q>5, O_{p^{\prime}}(H) \subseteq S(H)$, and $D$ is a dihedral subgroup of $H$ of order 8;

(b) $H$ is a $Q$-group with wreathed Sylow 2-subgroups of characteristic power $q>5, O_{p^{\prime}}(H) \subseteq S(H)$, and $D$ is a Sylow 2-subgroup of $H$;

(c) $H$ is a $D$-group with dihedral Sylow 2-subgroups of order at least 8 or quasi-dihedral Sylow 2-subgroups, $O_{p^{\prime}}(H) \subseteq O(H)$, and $D$ is a dihedral subgroup of $H$ of order 8;

(d) $H$ is a $D$-group with wreathed Sylow 2-subgroups, $O_{p^{\prime}}(H) \subseteq O(H)$, and $D$ is a Sylow 2-subgroup of $H$.

We also let $S$ be a Sylow 2-subgroup of $H$ containing $D$ and fix this notation.

To analyze $p$-stability in $H$ with respect to a maximal element of $\eta_{H}(D ; p)$ in each of these cases, we need the following lemma:

LEMMA 4. If $R$ is a maximal element of $\eta_{H}(D ; p)$, then

(i) $R \cap O(H)$ is a Sylow p-subgroup of $O(H)$.

(ii) $R \cap O_{p^{\prime}, p}(H)$ is a Sylow p-subgroup of $O_{p^{\prime}, p}(H)$.

(iii) Either $R$ is normal in a Sylow p-subgroup of $H$ or $R K / K \supseteq O_{p}(H / K)$ for every normal subgroup $K$ of $H$.

Proof. If $H$ is a $Q$-group, $O_{p^{\prime}}(H) \subseteq S(H)$ by assumption and so $O_{p^{\prime}, p}(H) \subseteq S(H)$. But $S(H)=O(H) Z(S)$ by Lemma 4.14 as $q>5$. Hence, if (i) holds, $R \cap S(H)$ will be a Sylow $p$-subgroup of $S(H)$ and hence $R \cap O_{p^{\prime}, p}(H)=R \cap O_{p^{\prime}, p}(S(H))$ will be a Sylow $p$-subgroup of $R \cap O_{p^{\prime}, p}(H)$. Similarly if $H$ is a $D$-group, $O_{p^{\prime}}(H) \subseteq O(H)$ by assumption and so $O_{p^{\prime}, p}(H) \subseteq O(H)$. Hence, if (i) holds, $R \cap O_{p^{\prime}, p}(H)$ $=R \cap O_{p^{\prime}, p}(O(H))$ will be a Sylow p-subgroup of $R \cap O_{p^{\prime}, p}(H)$. Thus (ii) will follow from (i), which we proceed now to verify.

Set $\bar{H}=H / S(H)$ or $\bar{H}=H / O(H)$ according to whether $H$ is a $Q$-group or a $D$ group. If $H$ is a $Q$-group, then $S(H)=O(H) Z(S)$ by Lemma 4.14, and $\bar{H}$ is a $D$ group of characteristic power $q$ with dihedral Sylow 2-subgroups by Lemma 4.15. Furthermore, $\bar{D}$ is a four group if $S$ is quasi-dihedral and $\bar{D}=\bar{S}$ is a Sylow 2subgroup of $\bar{H}$ if $S$ is wreathed. On the other hand, if $H$ is a $D$-group, then so is $\bar{H}$ and $\bar{D}$ is either dihedral of order eight or $S$ is wreathed and $\bar{D}=\bar{S}$. Let $\bar{X}$ be a 
maximal element of $И_{H}(\bar{D} ; p)$ containing $\bar{R}$ and let $X$ be the inverse image of $\bar{X}$ in $H$. Since $Z(S)$ is a Sylow 2-subgroup of $X$ and $Z(S)$ is cyclic, $X$ has a normal 2complement. Hence $R \subseteq O(X)$ and, as $O(X)$ is $D$-invariant, $X$ possesses a $D$ invariant Sylow $p$ subgroup $Q$ with $Q \supseteq R$. But then $Q \in h_{H}(D ; p)$ and so $Q=R$ by the maximality of $R$. Since $O(H)$ is normal in $O(X)$, it follows that $R \cap O(H)$ is a Sylow $p$-subgroup of $O(H)$, proving (i). We note also that $\bar{R}=\bar{X}$ as $\bar{X}$ is a $p$-group and so $\bar{R}$ is, in fact, a maximal element of $\eta_{H}(\bar{D} ; p)$.

It remains to prove (iii). Let $K$ be a normal subgroup of $H$ and suppose first that $K \subseteq S(H)$ if $H$ is a $Q$-group and $K \subseteq O(H)$ if $H$ is a $D$-group. If $Y$ denotes the inverse image of $O_{p}(H / K)$ in $H$, then correspondingly $Y \subseteq S(H)$ or $Y \subseteq O(H)$. Since $Y$ is normal in $H$, it follows in either case from the above argument that $R \cap Y$ is a Sylow $p$-subgroup of $Y$, whence $R K / K \supseteq O_{p}(H / K)$. Thus (iii) holds in this case and so we can assume correspondingly that $K \nsubseteq S(H)$ or $K \nsubseteq O(H)$.

Suppose next that $\bar{H}$ is isomorphic to $A_{7}$, in which case $H$ is $D$-group. Since $\bar{K}$ is a nontrivial normal subgroup of $\bar{H}$, we have $\bar{K}=\bar{H}$ and so $H=K O(H)$, whence $O_{p}(H / K)$ is the image of a $p$-subgroup of $O(H)$. Again (iii) follows from (i). Now assume that $\bar{H}$ is isomorphic to $\operatorname{PSL}(2,3)$ or to $P G L_{n}(2,3)$ for some $n \geqq 1$. Since $q>5$ if $H$ is a $Q$-group, $H$ must be a $D$-group in any of these cases and so $\bar{H}=H / O(H)$. Since $R \cap O(H)$ is a Sylow $p$-subgroup of $O(H)$, we see that $R$ is a Sylow $p$-subgroup of $H$ if $p \neq 3$, while $R$ is of index $p$ in a Sylow $p$-subgroup of $H$ if $p=3$. In either case, $R$ is normal in a Sylow $p$-subgroup of $H$ and so (iii) holds in this case as well. We can therefore assume that $\bar{H}$ is of none of these forms.

Hence by Proposition 3.4, $\bar{H}$ is isomorphic to a subgroup of $P \Gamma L(2, q)$ containing $\operatorname{PSL}(2, q)$ for suitable odd $q \geqq 5$ and $\bar{H}=\bar{L} \bar{E}$, where $\bar{L}, \bar{E}$ are as in that proposition. Since $q \geqq 5$ and $\bar{K}$ is nontrivial, $\bar{K}$ contains the unique minimal normal subgroup $\bar{L}_{0}$ of $\bar{H}$ isomorphic to $\operatorname{PSL}(2, q)$. Since $\left|\bar{L}: \bar{L}_{0}\right| \leqq 2$, it follows that $|\bar{H}: \bar{K} \bar{E}| \leqq 2$ and hence that $\bar{K} \bar{E}$ contains a Sylow $p$-subgroup of $\bar{H}$. It will suffice to prove that $\bar{K} \bar{R} / \bar{K}$ contains a Sylow $p$-subgroup of $\bar{K} \bar{E} / \bar{K}$, for then $\bar{K} \bar{R} / \bar{K}$ will contain a Sylow $p$-subgroup of $\bar{H} / \bar{K}$ and consequently will necessarily contain $O_{p}(\bar{H} / \bar{K})$, which will establish (iii) in this final case.

As we have noted above, $\bar{R}$ is a maximal element of $И_{H}(\bar{D} ; p)$. By Lemma 3.3 (i) of [22], $\bar{E}$ centralizes some Sylow 2-subgroup of $\bar{L}$, which without loss we may assume to be $\bar{S}$. Since $\bar{D} \subseteq \bar{S}$ and $\bar{D}$ is dihedral, Lemmas 3.1 (iii) and 3.3 (i) of [22] imply that $\bar{R} \subseteq C_{H}(\bar{x})$ for some central involution $\bar{x}$ of $\bar{D}$ and that $C_{H}(\bar{x})=C_{L}(\bar{x}) \bar{E}$ with $C_{L}(\bar{x})$ being dihedral. This in turn implies that $C_{H}(\bar{x})$ has a normal 2-complement $\bar{V}=O\left(C_{E}(\bar{x})\right) \bar{E}$. Thus $\bar{R} \subseteq \bar{V}$ and $\bar{V}$ is $\bar{D}$-invariant as $\bar{D} \subseteq C_{H}(\bar{x})$. Hence $\bar{D}$ normalizes some Sylow p-subgroup of $\bar{V}$ containing $\bar{R}$. Since $\bar{R}$ is a maximal element of $\bigcup_{H}(\bar{D} ; p)$, we conclude that $\bar{R}$ itself is a Sylow $p$-subgroup of $\bar{V}$. On the other hand, clearly $\bar{V} \subseteq \bar{L}_{0} \bar{E} \subseteq \bar{K} \bar{E}$. Since $\bar{E} \subseteq \bar{V}$ and $\bar{R}$ contains a Sylow $p$-subgroup of $\bar{V}$, it follows therefore that $\bar{K} \bar{R} / \bar{K}$ contains a Sylow $p$-subgroup of $\bar{K} \bar{E} / \bar{K}$, as required.

We now prove 
Proposition 1. The following results hold:

(i) If $H$ is a $Q$-group and $p$ is not the characteristic of $H$ or if $H$ is a D-group, then $H$ is p-stable.

(ii) If $H$ is a $Q$-group of characteristic $p$ or if $H$ is a $D$-group and if $R$ is a maximal element of $U_{H}(D ; p)$, then $H$ is p-stable with respect to $R$.

Proof. Clearly $H$ is $p$-stable or $p$-stable with respect to $R$ if and only if $\bar{H}=H / O_{p}(O(H))$ is $p$-stable or $p$-stable with respect to $\bar{R}$, as follows directly from the definition of $p$-stability and $p$-stability with respect to $R$. Hence without loss we can assume that $O_{p^{\prime}}(O(H))=1$. Since $O_{p^{\prime}}(H) \subseteq S(H)$ by assumption if $H$ is a $Q$ group and since $S(H)$ has a normal 2-complement by Lemma 4.14, it follows that $O_{p^{\prime}}(H)=O_{p^{\prime}}(O(H))$ and hence that $O_{p^{\prime}}(H)=1$. The same result holds if $H$ is a $D$ group since $O_{p^{\prime}}(H)=O_{p^{\prime}}(O(H))$ in this case as $O_{p^{\prime}}(H) \subseteq O(H)$ by assumption. Thus we are reduced to verifying the proposition in the case that the subgroup $P$, appearing in the definition of $p$-stability and $p$-stability with respect to $R$, is normal in $H$.

By the preceding lemma, $R$ satisfies conditions (a) and (b) in the definition of $p$-stability with respect to $R$. For uniformity, in proving (i), we let $R$ be a Sylow $p$-subgroup of $H$ and again the same conditions (a) and (b) hold, as we have noted above. Thus under any of the various hypotheses on $H$, we must show that if $A$ is any $p$-subgroup of $R$ such that $[P, A, A]=1$, then

where $C=C_{H}(P)$.

$$
A C / C \subseteq O_{p}(H / C)
$$

Let $P=P_{1} \supset P_{2} \supset \ldots \supset P_{n+1}=1$ be an $H$-invariant normal series of $P$ such that each $\bar{P}_{i}=P_{i} / P_{i+1}$ is elementary abelian and such that $H$ acts irreducibly on $\bar{P}_{i}$, $1 \leqq i \leqq n$. Let $K_{i}$ be the kernel of the representation of $H$ on $\bar{P}_{i}$, so that $\bar{H}_{i}=H / K_{i}$ acts faithfully and irreducibly on $\bar{P}_{i}$, whence also $O_{p}\left(\bar{H}_{i}\right)=1,1 \leqq i \leqq n$. It will suffice to prove that $A \subseteq K_{i}$ for all $i$, for then $A \subseteq K=\bigcap_{i=1}^{n} K_{i}$. But $K$ stabilizes the chain $P=P_{1} \supset P_{2} \supset \cdots \supset P_{n+1}=1$ and also $K$ contains $C$. Since $K / C$ is a subgroup of Aut $(P)$, P. Hall's theorem (Corollary 5.3.3. of [19]) implies that $K / C$ is a $p$-group. Since $K$ is normal in $H$ and $A \subseteq K$, we obtain the desired conclusion $A C / C \subseteq O_{p}(H / C)$.

We can suppose that the image $\bar{A}_{i}$ of $A$ in $\bar{H}_{i}$ is nontrivial for some $i$. Clearly $\left[\bar{P}_{i}, \bar{A}_{i}, \bar{A}_{i}\right]=1$. Since $O_{p}\left(\bar{H}_{i}\right)=1$, the representation of $\bar{H}_{i}$ on $\bar{P}_{i}$ is therefore not $p$-stable. Hence by Theorem 3.8 .3 of [19], $\bar{H}_{i}$ involves $S L(2, p)$. But $\bar{H}_{i}$, being a homomorphic of $H$, is itself either a $Q$-group or a $D$-group or else has a normal 2-complement. Since $S L(2, p)$ is a $Q$-group, Lemma 5.5 implies that $\bar{H}_{i}$ is not a $D$-group and since $S L(2, p)$ does not have a normal 2-complement, neither does $\bar{H}_{i}$. Hence $\bar{H}_{i}$ must be a $Q$-group, which is possible only if $H$ itself is a $Q$-group and $K_{i} \subseteq O(H)$. In particular, the proposition holds if $H$ is a $D$-group.

Since $K_{i} \subseteq O(H), \bar{H}_{i}$ has the same characteristic power $q$ as $H$ and the image $\bar{D}_{i}$ of $D$ is isomorphic to $D$. The image $\bar{R}_{i}$ of $R$ in $\bar{H}_{i}$ is obviously a Sylow $p$-subgroup of $\bar{H}_{i}$ if $R$ is a Sylow p-subgroup of $H$. Moreover, we claim it is a maximal element of $U_{H_{i}}\left(\bar{D}_{i} ; p\right)$ if $R$ is a maximal element of $U_{H}(D ; p)$. Indeed, if $\bar{X}_{i}$ is a maximal 
\left. element of ${V_{H_{i}}}_{(} \bar{D}_{i} ; p\right)$ containing $\bar{R}_{i}$ and $X$ denotes the inverse image of $\bar{X}_{i}$ in $H$, then $R \subseteq X$ and $X$ has odd order as $X / K_{i}=\bar{X}_{i}$ and both $K_{i}$ and $\bar{X}_{i}$ have odd order. Hence $X$ contains a $D$-invariant Sylow $p$-subgroup $R^{*}$ with $R \subseteq R^{*}$. But then $R^{*} \in U_{H}(D ; p)$ and so $R=R^{*}$ by the maximality of $R$. Thus $R$ is a Sylow $p$ subgroup of $X$ and therefore $X=K_{i} R$ as $\bar{X}_{i}$ is a $p$-group. We conclude that $\bar{R}_{i}=\bar{X}_{i}$ and hence that $\bar{R}_{i}$ is a maximal element of ${U_{H_{i}}}_{(} \bar{D}_{i} ; p)$, as asserted.

Since we shall be concerned in the balance of the proof solely with the action of $\bar{H}_{i}$ on $\bar{P}_{i}$, no confusion will arise if for simplicity we drop the subscript $i$ and the "bars." Thus we assume that our $Q$-group $H$ is faithfully and irreducibly represented on the vector space $P$ over $G F(p)$, so that, in particular, $O_{p}(H)=1$. Moreover, we must derive a contradiction under the appropriate hypotheses on $H$ from the assumption that $[P, A, A]=1$.

Set $X=O_{p^{\prime}}(S(H))$ and suppose first that $A_{0}=C_{A}(X) \subset A$. Then $A_{0}$ is normal in $A X$ and $O_{p}\left(A X / A_{0}\right)=1$. Since $X A_{0}=X \times A_{0}$, Theorem 5.3.4 of [19] implies that $X$ is faithfully represented in $P_{0}=C_{P}\left(A_{0}\right)$. But $A$ leaves $P_{0}$ invariant and, as $O_{p}\left(A X / A_{0}\right)=1$, it follows that $A X / A_{0}$ is faithfully represented on $P_{0}$. However, $\left[P_{0}, A, A\right]=1$ and a Sylow 2-subgroup $X$ is cyclic as $X \subseteq S(H)=O(H) Z(D)$ and $Z(D)$ is cyclic. Thus the Hall-Higman theorem (Theorem 11.1.1 of [19]) is contradicted. Hence $A_{0}=A$ and so $A$ centralizes $X$. On the other hand, since $O_{p}(S(H))=1$ and $S(H)$, is solvable, Theorem 6.3.2 of [19] implies that $C_{S(H)}(X) \subseteq X$. We therefore also conclude that $Y=C_{H}(X) \nsubseteq S(H)$. Since $Y$ is normal in $H$ and $O(Y)$ is characteristic in $Y, O(Y)$ is normal in $H$, whence $O(Y) \subseteq O(H)$. But now $O(Y) \subseteq X$ as $C_{O(H)}(X) \subseteq X$. Thus we also have that $O(Y) \subseteq Z(Y)$.

Since $Y \nsubseteq S(H)$, the image of $Y$ in $H / S(H)$ contains the unique minimal normal subgroup of $H / S(H)$ isomorphic to $P S L(2, q)$ and this implies that $Y$ contains a normal subgroup $Y_{0}$ with $O(Y) \subseteq Y_{0}$ such that $Y_{0} / O(Y)$ is isomorphic to $S L(2, q)$. Application of Lemma 4.16 now yields that $Y_{0}=J O(Y)$, where $J$ is normal in $Y$ and either $J$ is isomorphic to $S L(2, q)$ or to $S L^{\wedge}(2,9)$ with $q=9$.

To treat this case we shall apply the results of $\$ 3.8$ of [19] in a more precise form than above. The proofs of Theorems 3.8.1, 3.8.2, and 3.8.3 of [19] together with Dickson's theorem (Theorem 2.8.4 of [19]), in fact, establish the following result: For any $a$ in $A^{\#}$, there exists a conjugate $b$ of $a$ in $Y$ such that $F=\langle a, b\rangle$ is not a $p$-group and, furthermore, for any such choice of $b, F$ contains a normal subgroup $F_{0}$ such that $F / F_{0}$ is either isomorphic to $\operatorname{SL}\left(2, p^{m}\right)$ for some $m$ or to $\operatorname{SL}(2,5)$ with $p=3$. In particular, if $F / F_{0}$ is not isomorphic to $S L(2,3)$, we have that $a \in F^{\prime}$. However, $F \subseteq Y$ and, as $Y$ is a $Q$-group, $Y / Y_{0}$ is abelian by Lemma 4.1. Thus $a \in Y_{0}$ in this case. Furthermore, $O(Y)$ is abelian as $O(Y) \subseteq Z(Y)$ and $O(Y)$ is normal in $H$ as we have noted above. Since $O_{p^{\prime}}(H)=1$, this implies that $O(Y)$ is a $p^{\prime}$-group. But $Y_{0}=J O(Y)$ with $J$ normal in $Y_{0}$. Since $a$ is a $p$-element, we conclude therefore that $a \in J$ whenever $F / F_{0}$ is not isomorphic to $\operatorname{SL}(2,3)$.

We argue next that the same conclusion holds if $F / F_{0}$ is isomorphic to $\operatorname{SL}(2,3)$. In this case, we necessarily have $p=3$. Since $O(J) \subseteq O(Y)$ and $O(Y)$ is a $p^{\prime}$-group, 
we see that $J$ cannot be isomorphic to $S L^{\wedge}(2,9)$ and so $J$ is isomorphic to $S L(2, q)$. Suppose, by way of contradiction that $a \notin J$, in which case $a \notin J O(Y)$. Setting $\bar{Y}=Y / Z(J) O(Y)$, we have that $\bar{a} \notin \bar{J}$, that $\bar{J}$ is isomorphic to $P S L(2, q)$, and that $\bar{Y}$ is isomorphic to a subgroup $P \Gamma L(2, q)$. Now $\bar{a}$ normalizes some Sylow 3-subgroup $\bar{Q}$ of $\bar{J}$ and $\bar{Q} \neq 1$ as $|\bar{J}|$ is divisible by three. Thus $\bar{a}$ normalizes $\bar{V}=N_{\mathrm{J}}(\bar{Q})$. If 3 divides $q-1$ or $q+1$, Lemmas 3.1 (v) and 3.3 (ii) of [22] imply that $\bar{V}$ is dihedral of order $q-1$ or $q+1$ respectively and that $\bar{a}$ does not centralize $O_{3^{\prime}}(O(\bar{V}))$. But then $a$ does not centralize $O_{3^{\prime}}(O(V))$, where $V$ is the inverse image of $\bar{V}$ in $Y$, whence $[P, a, a] \neq 1$, again by the Hall-Higman theorem. Since $|\bar{J}|=\frac{1}{2} q(q-1)(q+1)$, this forces 3 to divide $q$. In this case, $\bar{V}=\bar{Q} \bar{U}$ is a Frobenius group of order $\frac{1}{2} q(q-1)$ with complement $\bar{U}$ of order $\frac{1}{2}(q-1)$ by Lemma 3.1 (vi) of [22]. Since $\bar{U}$ is a Hall subgroup of $\bar{V}$ and $\bar{V}$ is normal in $\langle\bar{V}, \bar{a}\rangle$, it follows by the Frattini argument that we can write $\bar{a}=\bar{a}_{1} \bar{a}_{0}$, where $\bar{a}_{1}$ normalizes $\bar{U}$ and $\bar{a}_{0} \in \bar{Q}$. Since $\bar{a} \notin \bar{J}$, we have $\bar{a}_{1} \neq 1$ and again by Lemmas 3.1 (v) and 3.3 (ii) of [22], $\bar{a}_{1}$ does not centralize $O_{3^{\prime}}(O(\bar{U}))$. Choose $\bar{u}$ in $O_{3^{\prime}}(O(\bar{U}))$ not centralized by $\bar{a}_{1}$. Then $\left\langle\bar{a}, \bar{a}^{\bar{u}}\right\rangle$ is not a 3-group and is of odd order. Hence for an appropriate representative $u$ of $\bar{u}$ in $Y$, we have found $F^{*}=\left\langle a, a^{u}\right\rangle$ is not a 3-group and that a Sylow 2-subgroup of $F^{*}$ is contained in $Z(J)$. However, this is impossible as the preceding argument shows that $S L(2, r)$ is a homomorphic image of $F^{*}$ for some odd $r$. We conclude that $A \subseteq J$ in all cases.

Now let $a, b, F, F_{0}$ be as above. Since $J$ and $F / F_{0}$ have generalized quaternion Sylow 2-subgroups, $F_{0}$ must have odd order and so $F$ is a $Q$-group. Since $F$ is generated by its $p$-elements, $F$ has no normal subgroups of index two. Hence by Lemma 4.8, applied to $J / O(J)$, we conclude that $F_{0} \subseteq O(J)$ and that $F / F_{0}$ is isomorphic to $S L(2,3), S L(2,5)$ or $S L\left(2, q_{0}\right)$ for some divisor $q_{0}$ of $q$. But we know that $F / F_{0}$ is isomorphic to either $S L\left(2, p^{m}\right)$ for some $m$ or to $S L(2,5)$ with $p=3$. Thus in the first case, if $p^{m}>5$, we must have $p^{m}=q_{0}$ and so $p$ is the characteristic of $H$. Under the hypotheses of the proposition, this implies that $R$ is necessarily a maximal element of $\bigcup_{H}(D ; p)$. Since $A \subseteq R$ and $A \subseteq J$, we see that $U_{H}(D ; p)$ is nontrivial. Moreover, in the present case $J$ is not isomorphic to $S L^{\wedge}(2,9)$, for then it would follow that $p=3$ and that $O(Y)$ would not be a $p^{\prime}$-group, a contradiction. Thus $J$ is isomorphic to $S L(2, q)$. But by assumption $D$ is dihedral of order eight if $S$ is quasi-dihedral and $D=S$ if $S$ is wreathed. In either case Lemma 4.9, applied to the $Q$-group $J D$, yields that $U_{J}(D ; p)$ is trivial. This contradiction shows that both parts of the proposition hold when $p^{m}>5$.

It remains therefore to treat the cases in which $F / F_{0}$ is isomorphic to $\operatorname{SL}(2,3)$ or $S L(2,5)$. In the first case $p=3$ and in the second case $p=3$ or $p=5$. Furthermore, we reach the same contradiction as in the preceding paragraph if $p$ is the characteristic of $H$. Hence we can also assume that the characteristic $t$ of $H$ is distinct from $p$. Since $p$ divides $|J|$, it follows that $p$ divides $q-1$ or $q+1$. We claim that, in fact, $p$ divides $q+1$. Indeed, assume the contrary. Then by Lemma 3.1 (i) and (vi) of [22], $\bar{A}$ normalizes some Sylow $t$-subgroup $\bar{Q}$ of $\bar{J}$ and $\bar{Q} \bar{A}$ is a Frobenius group. But 
then $A$ acts regularly on some Sylow $t$-subgroup $Q$ of $J$. Since $t \neq p$, the HallHigman theorem implies that $[P, A, A] \neq 1$, a contradiction. Thus $p$ divides $q+1$, as asserted.

We first consider the subcase $q>11$. In particular, $J$ is isomorphic to $S L(2, q)$. We identify $J$ with its natural representation by $2 \times 2$ matrices over $G F(q)$. Now $A \subset J$ and hence if $a$ is an element of order $p$ in $A$, then $a$ can be represented by the companion matrix

$$
\left(\begin{array}{rr}
0 & 1 \\
-1 & \alpha
\end{array}\right)
$$

for suitable $\alpha \in G F(q)$. If $u$ is the element of $J$ represented by

$$
\left(\begin{array}{ll}
\lambda^{-1} & 0 \\
0 & \lambda
\end{array}\right)
$$

where $\lambda$ is a primitive element of $G F(q)$, and if we set $b=a^{u}$, then the element $u^{-1} a u a=b a^{-1}$ lies in $F=\langle a, b\rangle$ and a direct computation shows that it is represented by the matrix

$$
\left(\begin{array}{ll}
\lambda^{2} & 0 \\
0 & \lambda^{-2}
\end{array}\right)
$$

Since $\lambda$ has order $q-1$, it follows that $b a^{-1}$ has order $\frac{1}{2}(q-1)$. Moreover, $q \neq 13$ as $p$ divides $q+1$ and $p=3$ or 5 , and so $q \geqq 17$. If $q=17$, then $\left|b a^{-1}\right|=8$; if $q=19$, then $\left|b a^{-1}\right|=9$; and if $q>19$, then $\left|b a^{-1}\right|>10$. But $S L(2,3)$ and $S L(2,5)$ contain no elements of order 8,9 , or exceeding 10 . Since $b a^{-1} \in F$, we see that $F$ is not isomorphic to $S L(2,3)$ or $S L(2,5)$. On the other hand, we know that $F / F_{0}$ is isomorphic to $S L(2,3)$ or $S L(2,5)$ and that $F_{0} \subseteq O(J)$. But $O(J)=1$ as $q>11$, so $F_{0}=1$ and $F$ is isomorphic to $S L(2,3)$ or $S L(2,5)$, a contradiction. Thus $q \leqq 11$.

If $q=11$, then $p=3$, as $p$ divides $q+1$, so $a$ can be represented by the matrix

$$
\left(\begin{array}{rr}
0 & 1 \\
-1 & -1
\end{array}\right)
$$

Taking $b=a^{u}$ with $u$ represented by

$$
\left(\begin{array}{rr}
1 & 0 \\
-1 & 1
\end{array}\right)
$$

we compute that $b a$ is represented by

$$
\left(\begin{array}{rr}
-1 & -2 \\
0 & -1
\end{array}\right)
$$

which shows that $b a$ has order 22 and yields the same contradiction as in the preceding paragraph.

By assumption, $q>5$. Furthermore, $q \neq 7$ as $p$ divides $q+1$ and $p=3$ or 5 . Thus we are left with the single case $q=9$. In this case, $p=5$ and $F / F_{0}$ must be isomorphic to $S L(2,5)$. Setting $\bar{J}=J / Z(J)$, we have that $\bar{J}$ is isomorphic to $\operatorname{PSL}(2,9)$ and $\bar{F}$ to 
$\operatorname{PSL}(2,5)$. In particular, $\bar{F}$ contains no elements of order four. We shall argue, to the contrary, that for a suitable choice of $b, \bar{F}$ contains an element of order four, which will dispose of the case $q=9$ and complete the proof of the proposition.

As is well known, $\operatorname{PSL}(2,9)$ is isomorphic to $A_{6}$. We shall use the latter representation for the elements of $\bar{J}$. Since $\bar{a}$ is of order five, we can assume without loss that $\bar{a}$ is represented by the cycle (12345). Now $A_{6}$ has only one conjugacy class of cyclic subgroups of order five. Moreover, for a suitable choice of $b=a^{u}$ with $u$ in $J$, we can make $\langle\bar{b}\rangle$ any preassigned conjugate of the group $\langle\bar{a}\rangle$. Hence we can choose $u$ so that $\langle\bar{b}\rangle$ contains an element represented by the cycle (23465). For such a choice of $u, \bar{F}=\langle\bar{a}, \bar{b}\rangle$ contains an element represented by $(12345)(23465)=(1365)(24)$ and so $\bar{F}$ contains an element of order four, giving the desired contradiction.

REMARK. We note that the groups $S L_{2}(2,5)$ and $S U_{1}(2,5)$ are not 3-stable, for they are isomorphic to subgroups of $G L(2,9)$ and $G L(2,81)$ respectively. However, in the natural representation of these latter groups, every nontrivial 3-element has a quadratic minimal polynomial. Thus the assumption $q>5$ in Proposition 1 is essential in the case $p=3$.

7. An extension of Glauberman's $Z J$-theorem. An important tool in the paper will be Glauberman's so-called ZJ-theorem (Theorem 8.2.11 of [19]) which for the sake of clarity we restate now.

THEOREM 1. If $G$ is a group with $O_{p}(G) \neq 1, p$ an odd prime, which is $p$-constrained and p-stable and if $P$ is a Sylow p-subgroup of $G$, then

$$
G=O_{p^{\prime}}(G) N(Z(J(P))) .
$$

In some situations we shall need a slight extension of this theorem, which is implicitly contained in its proof, namely:

THEOREM 2. If $G$ is a group with $O_{p}(G) \neq 1, p$ an odd prime, which is p-constrained and is p-stable with respect to the p-subgroup $P$ of $G$, then

$$
G=O_{p^{\prime}}(G) N(Z(J(P)))
$$

Proof. To establish this result, one has only to examine the proofs of Theorems 8.2.10 and 8.2.11 of [19] to see that they carry through under these slightly more general circumstances. Indeed, following the notation of those theorems, but with $P$ as in the present theorem, we outline the argument. First of all, $B \subseteq P$ as $P$ contains a Sylow $p$-subgroup of $O_{p^{\prime}, p}(G)$ by definition of $p$-stability with respect to $P$. Now one reduces by induction in the same way as in [19] to the case that $O_{p^{\prime}}(G)=1$ and that $B$ has the structure required for application of Glauberman's replacement theorem (Theorem 8.2.7 of [19]), which we note does not involve conditions on $p$-stability. Furthermore, because $G$ is $p$-stable with respect to $P$, we again obtain the conclusion $A C / C \subseteq O_{p}(G / C)$, where $C=C_{G}(B)$, which in turn implies that $A L / L \subseteq O_{p}(G / L)$, where $L$ is the largest normal subgroup of $G$ leaving $Z(J(P)) \cap B$ invariant. Let $K$ be the inverse image of $O_{p}(G / L)$ in $G$. Again by the 
$p$-stability of $G$ with respect to $P$, either $P L / L \supseteq O_{p}(G / L)$ or $P$ is normal in a Sylow $p$-subgroup of $G$. Since $K / L$ is a $p$-group and $K$ is normal in $G$, it follows in the first case that $K=L(P \cap K)$ and in the second case that $K \subseteq L N(P)$. Since both $P \cap K$ and $N(P)$ normalize $Z(J(P)) \cap B$ as $Z(J(P))$ is characteristic in $P$ and $B$ is normal in $G$, we conclude in either case that $K$ normalizes $Z(J(P)) \cap B$, whence $K=L$ by our choice of $L$. Thus $O_{p}(G / L)=1$ and so $A \subseteq L$. But now the balance of the proof of Theorem 8.2.10 of [19] applies without change.

Since $P$ contains a Sylow $p$-subgroup of $O_{p^{\prime}, p}(G)$, the proof of Theorem 8.1.3 of [19] shows that $Z(J(P)) \subseteq O_{p^{\prime}, p}(G)$. But now the proof of Theorem 8.2.11 of [19] carries over without change and so Theorem 2 is valid.

Combining Theorems 1 and 2 with the results of the preceding section, we have the following two basic propositions which we shall prove simultaneously:

Proposition 1. Let $H$ be a $Q$-group of characteristic power $q>5$ with quasidihedral or wreathed Sylow 2-subgroup $S$ such that $O_{p}(H) \neq 1$ and $H$ is p-constrained within $S(H)$ for some odd prime $p$. If $p$ is not the characteristic of $H$, let $R$ be a Sylow p-subgroup of $H$ and if $p$ is the characteristic of $H$, let $R$ be a maximal element of $U_{H}(D ; p)$, where $D$ is a dihedral subgroup of $S$ of order 8 if $S$ is quasi-dihedral and $D=S$ if $S$ is wreathed. Under these conditions, we have

$$
H=O_{p^{\prime}}(H) N_{H}(Z(J(R)))
$$

Proposition 2. Let $H$ be a D-group with Sylow 2-subgroup $S$ which is either dihedral of order at least 8, quasi-dihedral, or wreathed such that $O_{p}(H) \neq 1$ and $H$ is p-constrained within $O(H)$ for some odd prime $p$; and let $R$ be either a Sylow $p$ subgroup of $H$ or a maximal element of $h_{H}(D ; p)$, where $D$ is a dihedral subgroup of $S$ of order 8 if $S$ is dihedral or quasi-dihedral and $D=S$ if $S$ is wreathed. Under these conditions, we have

$$
H=O_{p^{\prime}}(H) N_{H}(Z(J(R))) .
$$

Proof. If $H$ is a $Q$-group, then $H$ is $p$-constrained and $O_{p^{\prime}}(H) \subseteq S(H)$ by definition of $p$-constraint within $S(H)$, while if $H$ is a $D$-group, then $H$ is $p$-constrained and $O_{p^{\prime}}(H) \subseteq O(H)$ by definition of $p$-constraint within $O(H)$. Thus we see that $H$ satisfies one of conditions (a), (b), (c), or (d) of the preceding section and so Propositions 6.1 and 6.2 can be applied to $H$ to yield that $H$ is $p$-stable when $R$ is defined to be a Sylow $p$-subgroup of $H$ and that $H$ is $p$-stable with respect to $R$ when $R$ is defined to be a maximal element of $\eta_{H}(D ; p)$. Correspondingly we see that the hypotheses of Theorem 1 or Theorem 2 are satisfied and now both propositions follow from these two theorems.

8. Miscellaneous results. Our arguments will frequently involve the action of an involution or of a four group on a group of odd order. We collect here some basic well-known properties concerning this situation. 
LEMMA 1. If $K$ is a group of odd order acted on by an involution $\alpha$, then

(i) $K=F I=I F$, where $F=C_{K}(\alpha)$ and $I$ is the subset of $K$ whose elements are inverted by $\alpha$.

(ii) If $K$ is of prime power order and $K \supset F$, then $C_{I}(F) \neq 1$.

Proof. First, (i) is proved in Lemma 10.4.1 (i) of [19]. As for (ii), we have $F \times\langle\alpha\rangle$ acting on $K$, which is a $p$-group for some odd prime $p$. Since $K \supset F, \alpha$ acts nontrivially on $K$. Hence by Theorem 5.3.4 of [19], $\alpha$ acts nontrivially on $C=C_{K}(F)$, whence $C \supset F \cap C$. But $C$ is $\alpha$-invariant as $F$ is, and consequently $C=(F \cap C)(I \cap C)$ by (i), applied to $C$. Thus $C \cap I \neq 1$ and so $C_{I}(F) \neq 1$, proving (ii).

The following basic result is proved in Lemma 4 of [21] (cf. also Lemma 10.5.1 of [19]).

LEMMA 2. If $K$ is a group of odd order acted on by a four group $T$ with involutions $\alpha_{1}, \alpha_{2}, \alpha_{3}$, then

$$
K=C_{K}\left(\alpha_{1}\right) C_{K}\left(\alpha_{2}\right) C_{K}\left(\alpha_{3}\right)
$$

If $K$ is a group of odd order acted on by a four group $T$ with involutions $\alpha_{1}, \alpha_{2}, \alpha_{3}$, set $K_{i}=C_{K}\left(\alpha_{i}\right), 1 \leqq i \leqq 3$, and $K_{0}=C_{K}(T)$. Clearly $K_{0} \subseteq K_{i}$ for each $i, 1 \leqq i \leqq 3$. Furthermore, since $\alpha_{i}$ centralizes $K_{i}$, both involutions of $T-\left\langle\alpha_{i}\right\rangle$ induce the same automorphism of $K_{i}$. Moreover, if $j \neq i$, then $C_{K_{i}}\left(\alpha_{j}\right)=C_{K_{i}}\left(\left\langle\alpha_{i}, \alpha_{j}\right\rangle\right)=C_{K_{i}}(T)$, as $\alpha_{i}$ centralizes $K_{i}$. Since $K_{0} \subseteq K_{i}$, it follows that $C_{K_{i}}\left(\alpha_{j}\right)=K_{0}$ for all $i, j, 1 \leqq i$, $j \leqq 3, i \neq j$. Hence if $K_{i}^{\prime}$ denotes the subset of $K_{i}$ inverted by $\alpha_{j}$ for $j \neq i$, we conclude from Lemma 1 that

$$
K_{i}=K_{0} K_{i}^{\prime}=K_{i}^{\prime} K_{0}, \quad 1 \leqq i \leqq 3 .
$$

But now combining these relations with the conclusion of Lemma 2, we obtain the basic equalities:

$$
K=K_{1} K_{2} K_{3}=K_{0} K_{1}^{\prime} K_{2}^{\prime} K_{3}^{\prime} \text {. }
$$

We shall refer to (8.1) as the $T$-decompositions of $K$. Throughout the paper we shall use this notation for $T$-decompositions whenever we have a group of odd order acted on by a four group $T$.

We also need the following elementary result of a different nature.

LEMMA 3. Let $X$ and $Y$ be permutable subgroups of relatively prime orders of a group $G$. If $N$ is a normal subgroup of $G$, then

(i) $X$ and $Y \cap N$ are permutable;

(ii) $X$ normalizes $Y \cap N$ if $X \cap N=1$.

Proof. Suppose that $x \in X$ and $y \in Y \cap N$, so that there is $x^{\prime} \in X$ and $y^{\prime} \in Y$ with $x y=y^{\prime} x^{\prime}$. Thus, $y^{\prime}=x y x^{\prime-1} \in X N X=X N$, so $y^{\prime} \in N$ as $X N / N$ has order prime to the order of $Y$. Hence, $y^{\prime} \in Y \cap N$ and (i) is proved. Moreover, $x \equiv x^{\prime}$ $(\bmod N)$, so that $x=x^{\prime}$ if $X \cap N=1$. This in turn yields that $x y x^{-1}=y^{\prime}$ and so $Y \cap N$ is normalized by $X$ if $X \cap N=1$, as required. This proves the lemma. 
Our next result is a slight extension of Theorem 3.4.3 of [19].

LEMMA 4. Let $H$ be a Frobenius group with abelian kernel $K$ and cyclic complement $A$. If $H$ is faithfully and irreducibly represented on a vector space $V$ over a field $F$, then $V$ is free as an A-module.

Proof. If $\bar{F}$ is an algebraic closure of $F$ and we set $\bar{V}=\bar{F} \otimes V$, then as an $\bar{F}(H)$ module, $\bar{V}$ is the direct sum of algebraically conjugate irreducible submodules. Since $V$ is a faithful $H$-module, so also is each of these submodules. Furthermore, it will clearly suffice to prove that each of these submodules is free as an $A$-module. Hence without loss we can assume to begin with that $F$ is algebraically closed.

Let $V_{i}, 1 \leqq i \leqq n$, be the Wedderburn components of $V$ with respect to the normal subgroup $K$ of $H$. Since $H=K A$ and $K$ leaves each $V_{i}$ invariant, it follows from Clifford's theorem (Theorem 3.4.1 of [19]) that $A$ induces a transitive permutation of the $V_{i}$. In order to show that $V$ is free as an $A$-module, we need only prove that this transitive permutation representation of $A$ is, in fact, the regular representation of $A$. Clearly the latter result holds if and only if the number of Wedderburn components is exactly the order of $A$.

However, this last conclusion is proved in Theorem 3.4 .3 of [19] under the stronger hypothesis that $K$ is an elementary abelian $q$-group for some prime $q$. But an examination of the argument shows that it holds without change, as do Theorems 3.2.3 and 3.2.4 of [19] which are used in its proof, if $K$ is an arbitrary abelian group, inasmuch as $F$ contains a primitive $|K|$ th root unity under our present assumptions.

We shall next prove a pair of lemmas concerning incidence and covering properties of sections:

Lemma 5. Suppose that $H, H_{1}, H_{2}$ are subgroups of a group $G, K_{i}$ is a normal subgroup of $H_{i}, i=1,2$, and the following conditions are satisfied:

(a) $H_{1} / K_{1}$ and $H_{2} / K_{2}$ are incident sections;

(b) $H$ covers $H_{1} / K_{1}$;

(c) $H_{1} \subseteq H_{2}$.

Under these conditions, $\mathrm{H}$ covers $\mathrm{H}_{2} / \mathrm{K}_{2}$.

Proof. Since $H_{1} / K_{1}$ and $H_{2} / K_{2}$ are incident sections, we have $H_{2}=\left(H_{2} \cap H_{1}\right) K_{2}$ by definition of this concept, so $H_{2}=H_{1} K_{2}$ as $H_{1} \subseteq H_{2}$. But $H$ covers $H_{1} / K_{1}$, so $H_{1} \subseteq H K_{1}$. Thus

$$
H_{2}=H_{1} K_{2} \subseteq H K_{1} K_{2}
$$

However, again because of our incidence assumption, we also have that $K_{1} \cap H_{2} \subseteq K_{2}$, so $K_{1} \subseteq K_{2}$ as $K_{1} \subseteq H_{1} \subseteq H_{2}$. Thus,

$$
H_{2} \subseteq H K_{1} K_{2}=H K_{2},
$$

and so $\mathrm{H}$ covers $\mathrm{H}_{2} / K_{2}$, as asserted. 
LEMMA 6. If $H_{1}$ is a subgroup of the group $H_{2}, K_{i}$ is a normal subgroup of $H_{i}$, $i=1,2, H_{1} / K_{1}$ and $H_{2} / K_{2}$ are isomorphic and $K_{2} \cap H_{1} \subseteq K_{1}$, then $H_{1} / K_{1}$ and $H_{2} / K_{2}$ are incident sections.

Proof. The projection of $K_{2}$ into $H_{1} / K_{1}$ is just $K_{1}$ as $K_{2} \cap H_{1} \subseteq K_{1}$ and the projection of $H_{2}$ is $H_{1}$ as $H_{1} \subseteq H_{2}$. Hence, by the Zassenhaus lemma (p. 58 of [30]), the projection of $H_{1} / K_{1}$ into $H_{2} / K_{2}$ is a section isomorphic with $H_{1} / K_{1}$. But $\left|H_{1} / K_{1}\right|=\left|H_{2} / K_{2}\right|$ as $H_{1} / K_{1}$ and $H_{2} / K_{2}$ are isomorphic by assumption. Hence $H_{2} / K_{2}$ itself is the projection of $H_{1} / K_{1}$ into $H_{2} / K_{2}$ and so the sections $H_{1} / K_{1}$ and $\mathrm{H}_{2} / \mathrm{K}_{2}$ are, in fact, incident.

Lemma 7. Let $H$ be a group of odd order acted on by a 2-group $T$ and let $P$ be $a$ $T$-invariant Sylow p-subgroup of $H$ for some prime $p$. Suppose that for every odd prime $q \neq p,[P, T] \subseteq O_{p}(P Q)$ for some $T$-invariant Sylow q-subgroup $Q$ of $H$ permutable with $P$. Under these conditinos, $[P, T] \subseteq O_{p}(H)$.

Proof. Since $H$ is of odd order, $H T$ is a solvable group and so a $T$-invariant Sylow $q$-subgroup $Q$ of $H$ permutable with $P$ does, in fact, exist for each odd prime $q \neq p$.

To prove the lemma, set $\bar{H}=H / O_{p}(H)$ and observe that $O_{q}(\bar{H}) \subseteq \bar{Q}$ for each prime $q$ as $\bar{Q}$ is a Sylow $q$-subgroup of $\bar{H}$. Hence,

$$
\left[[\bar{P}, T], O_{q}(\bar{H})\right] \subseteq O_{p}(\bar{P} \bar{Q}) \cap O_{q}(\bar{H})=1,
$$

and so $[\bar{P}, T]$ centralizes $O_{q}(\bar{H})$. Moreover, this holds for each odd prime $q \neq p$ dividing $|H|$. Since $O_{p}(\bar{H})=1$, we conclude that $[\bar{P}, T]$ centralizes the Fitting subgroup $F(\bar{H})$ of $\bar{H}$. However, $\bar{H}$ is solvable and therefore $[\bar{P}, T] \subseteq F(\bar{H})$ by Theorem 6.1 .3 of [19]. Since $F(\bar{H})$ is a $p^{\prime}$-group, this yields $[\bar{P}, T]=1$ and consequently $[P, T] \subseteq O_{p}(H)$, as asserted.

LEMMA 8. Let $K$ be a group of odd order acted on by a 2-group $S$ and assume that $K=X Y$, where $X$ and $Y$ are $S$-invariant subgroups of $K$ and $X$ is a Hall subgroup of $K$. Then $C_{K}(S)=C_{X}(S) C_{Y}(S)$.

Proof. Let $\pi$ be the set of primes dividing $|X|$, so that $X$ is a Hall $\pi$-subgroup of $K$. Since $K$ is of odd order, $K$ and hence also $Y$ is solvable, and so $Y$ contains a $S$-invariant Hall $\pi^{\prime}$-subgroup $Z$. But $|Y|$ is divisible by $|K| /|X|$ as $K=X Y$. Since $|K| /|X|$ is relatively prime to $|X|$, it follows that $|Z|=|K| /|X|$. Since $X$ and $Z$ are of coprime orders, we conclude that $K=X Z$ and $X \cap Z=1$. Since $Z \subseteq Y$, it will therefore suffice to prove that $C_{K}(S)=C_{X}(S) C_{Z}(S)$. Since obviously $C_{X}(S) C_{Z}(S)$ $\subseteq C_{K}(S)$, we need only demonstrate the reverse inclusion.

Let $c \in C_{K}(S)$ and let $\alpha \in S$. Since $K=X Z$, we have $c=x z$, where $x \in X$ and $z \in Z$, whence also $c^{\alpha}=x^{\alpha} z^{\alpha}$. But $c^{\alpha}=c$ and so $x z=x^{\alpha} z^{\alpha}$. Thus $\left(x^{\alpha}\right)^{-1} x=z^{\alpha} z^{-1}$ $\in X \cap Z=1$, so $x=x^{\alpha}$ and $z=z^{\alpha}$. Since $\alpha$ is arbitrary in $S$, it follows that $x \in C_{X}(S)$ and $z \in C_{Z}(S)$. Since $c$ is arbitrary in $C_{K}(S)$, we conclude that $C_{K}(S) \subseteq C_{X}(S) C_{Z}(S)$, and the lemma is proved. 
Chapter III. Character THeORY OF GROUPS WITH QUASI-DiHEDRAL Sylow 2-SUBGROUPS

1. Introduction. In Chapter III, we consider finite groups $G$ with quasidihedral Sylow 2-subgroups $S=\langle s, z\rangle$,

$$
s^{2^{n}}=1, \quad z^{2}=1, \quad s^{z}=s^{-1+2^{n-1}}
$$

of order $2^{n+1}, n \geqq 3$. In particular, we are interested in the groups $G$ which do not have a normal subgroup of index 2. In the terminology introduced in Definition 2.2.1, these are the $Q D$-groups. Since we wish to include the groups which are centralizers of involutions along with $Q D$-groups, we also consider $Q$-groups $G$. We recall that among the groups $G$ with Sylow 2-subgroup $S$, the $Q D$-groups and $Q$-groups together can be characterized by the fact that the elements of order 4 lie in a single conjugate class. In a $Q D$-group, there is only one class of involutions while in a $Q$-group, there are two such classes $\left({ }^{5}\right)$. Similar ideas can be used for the study of the two other types of groups $G$ with Sylow 2-subgroup $S$, but this will not become necessary for us.

The structure of the principal 2-block $B_{0}(2, G)$ of $Q D$-groups and $Q$-groups has already been discussed in [6, III, §8]. Since these results are basic for us, we summarize them in $\$ 2$ without proofs. Actually, it will become necessary for us later to generalize these results for the case of certain other 2-blocks of full defect. However, the proofs there are more difficult. We have postponed this discussion and give it in $\S \S 5,6$, and 7 . Since these later results include those stated in $\$ 2$, our present paper is, in fact, independent of [6, III, $\S 8]$. $\$ 2$ also contains a few supplementary results.

In $\S 3$, the notion of a regular $Q D$-group is introduced. We then proceed in $\$ 4$ to prove Theorem A as stated in Chapter I. We have already mentioned the contents of $\S \$ 5,6$, and 7. In $\S 8$, we discuss $Q D$-groups of small characteristic power $q$. We determine explicitly all simple $Q D$-groups with $q=3$ and $q=5$. This allows us to assume $q \geqq 7$ in all the following chapters. This is a necessary assumption since a number of the local group-theoretic arguments in Chapters IV and V break down for these low values of $q$. We remark that Theorem A together with the classification of simple $Q D$-groups of characteristic power $q=3$ and $q=5$ are the only results of Chapter III which are needed in Chapters IV and V.

In $\S 8$, we also prove some partial results for the cases $q=7$ and $q=9$. These are needed in Chapter VI.

One of the major aims of the whole paper is to show that a $Q D$-group of characteristic power $q \neq 3$ is necessarily regular. With this in mind, we give in $\S 9$ sufficient

$\left({ }^{5}\right)$ Some of the elementary remarks are also given in [6, III, §8]. With the notation used there, we could define the characteristic power $q$ of $G$ as the absolute value of $m$, cf. Proposition 2.7 below. However, it would then not be clear that $q$ is a prime power.

Of course, only results of Chapter II concerned with groups with quasi-dihedral Sylow 2-subgroups are needed in the present chapter. 
conditions for the regularity of $Q D$-groups. We also derive some other results concerning the structure of $Q D$-groups which are needed in Chapter VI.

In general, we follow the notation introduced in $\$ \$ 1$ and 4 of Chapter II. Thus $x=s^{2^{n-1}}$, so that $x$ is the central involution of the Sylow 2-subgroup $S$ of our group $G$. We have $T=\langle x, z\rangle, N=C(x), C=C(T), E=O(C(S))$, and $y=s^{2^{n-2}}$. We know that $C=T \times O(C)$ and we set $C_{0}=O(C)$. We also set $S_{0}=\langle s\rangle$. If $G$ is a $Q D$ group, Proposition 2.1.1 implies that there exists a 3-element $t$ in $N(T)$ which cyclically permutes the three involutions of $T$. Replacing $t$ by its inverse, if necessary, we may assume that $t$ maps

$$
t: x \rightarrow x z \rightarrow z \rightarrow x
$$

under conjugation. We note also that $N(T)=\langle C, y, t\rangle$ and that $N(T) / C(T)$ is isomorphic with a symmetric group on three letters. Thus

$$
t y \equiv y t^{-1} \quad(\bmod C) .
$$

In addition, $L_{0}, L$ denote respectively the normal subgroups of $N$ containing $O(N)$ such that $L_{0} / O(N)$ is isomorphic to $S L(2, q)$ and $L / O(N)$ is isomorphic to $S L^{ \pm}(2, q)$ or $S U^{ \pm}(2, q)$ according as the characteristic power $q$ of $G$ and of $N$ is congruent to -1 or +1 modulo 4 . In particular, $N=L E$ and $N / L$ is cyclic.

2. The principal 2-block. As stated in $\S 1, G$ will always be a finite group with quasi-dihedral Sylow 2-subgroup $S$. We state without proof some of the results of $[6, \mathrm{III}, \S 8]\left({ }^{6}\right)$, adapting the notation to that used in the preceding chapters.

Proposition 1. If $G$ is a $Q D$-group or a $Q$-group and if the Sylow 2-subgroup $S$ of $G$ has order $2^{n+1}$, the principal 2-block $B_{0}=B_{0}(2, G)$ consists of $4+2^{n-1}$ irreducible characters. Exactly four of these characters have odd degrees.

A great deal of information is available concerning the values of these characters. One of the characters of odd degree is the principal character $\chi_{0}=1$. The remaining three characters of odd degrees will be denoted by $\chi_{1}, \chi_{2}, \chi_{3}$ and the $2^{n-1}$ characters of even degrees by $\chi_{4}, \chi^{(j)}$ with

$$
j= \pm 1, \pm 3, \ldots, \pm\left(2^{n-2}-1\right) ; \quad 2,4, \ldots, 2^{n-1}-2 .
$$

Let $s$ be a fixed element of order $2^{n}$ in $S$ and let $\zeta$ be a fixed primitive $2^{n}$ th root of unity. If this notation is chosen in a suitable manner, we have the following results.

Proposition 2. Let $w$ be an element of $G$ whose order is divisible by 4 . If the 2factor of $w$ is conjugate to $s^{h}$, then

$$
\begin{aligned}
& \chi_{1}(w)=\delta_{1}, \quad \chi_{2}(w)=\delta_{2}(-1)^{h+1}, \quad \chi_{3}(w)=\delta_{3}(-1)^{h+1}, \\
& \chi_{4}(w)=0, \quad \chi^{(j)}(w)=\delta_{1}\left(\zeta^{j h}+(-\zeta)^{-j h}\right) .
\end{aligned}
$$

Here, $\delta_{1}, \delta_{2}, \delta_{3}$ are signs, exactly two of which are -1 .

${ }^{(6)}$ We have corrected some misprints in $[6, \operatorname{III}(8 \mathrm{~A})]$ where the range of $j$ was given erroneously and where the formula for $\chi_{4}\left(J_{\rho}\right)$ and the congruence for $\phi_{1}^{\prime}(1)$ have to be changed. In (8D), the second congruence should read $x_{2} \equiv-\delta_{2} m$. 
The group $N=C(x)$ is a $Q$-group and the preceding results apply to $N$ instead of $G$. We shall denote by $\psi$ the character of $N$ in the principal block $B_{0}(2, N)$ which plays the role $\chi_{1}$ here $\left({ }^{7}\right)$.

Proposition 3. Let $w$ be an element of $G$ which is conjugate to $x r$, where $r$ is a 2-regular element of $N$. Then

$$
\begin{aligned}
& \chi_{1}(w)=\delta_{1} \varepsilon \psi(r), \quad \chi_{2}(w)=-\delta_{2} \varepsilon \psi(r), \quad \chi_{3}(w)=-\delta_{3}, \\
& \chi_{4}(w)=\delta_{2}(\varepsilon \psi(r)-1), \quad \chi^{(j)}(w)=(-1)^{j} \delta_{1}(\varepsilon \psi(r)+1) .
\end{aligned}
$$

Here, $\varepsilon$ is a sign such that

$$
\psi(1)=\psi(x) \equiv \varepsilon+2^{n-1} \quad\left(\bmod 2^{n}\right) .
$$

We shall see below (Proposition 7) that $\psi(1)=q$ is the characteristic power of $G$ (Definition 2.3.2). It will then be clear that $\varepsilon$ has the same significance as in Chapter II.

In the case of a $Q D$-group, every element $u$ of even order is either of the type treated in Proposition 2 or Proposition 3. In the case of $Q$-groups, one can give analogous formulas for the remaining elements of even order, but these will not be needed.

Proposition 4. For elements $r \in G$ of odd order,

$$
1+\delta_{1} \chi_{1}(r)=\delta_{1} \chi^{(j)}(r)=-\delta_{2} \chi_{2}(r)-\delta_{3} \chi_{3}(r),
$$

all $\chi^{(j)}(r)$ being equal, and

$$
1+\delta_{2} \chi_{2}(r)=\delta_{2} \chi_{4}(r) .
$$

In particular, this holds for $r=1$ and if we set

$$
f_{i}=\chi_{i}(1), \quad f=\chi^{(j)}(1),
$$

then (as $\left.\delta_{1} \delta_{2} \delta_{3}=1\right)$,

$$
1+\delta_{1} f_{1}+\delta_{2} f_{2}+\delta_{3} f_{3}=0, \quad \delta_{1}+f_{1}=f, \quad \delta_{2}+f_{2}=f_{4} .
$$

Proposition 5. If $G$ is a $Q$-group, then $\delta_{3}=-1, \delta_{1}=-\delta_{2}$. For $r \in G$ of odd order, $\chi_{1}(r)=\chi_{2}(r), \chi_{3}(r)=1$ and, in particular, $f_{1}=f_{2}, f_{3}=1$.

The next proposition will be formulated only for the case of $Q D$-groups. We anticipate here the result already mentioned that $\psi(1)$ coincides with the characteristic power $q$ of $G$ (cf. Proposition 7).

Proposition 6. Let $G$ be a $Q D$-group of characteristic power $q$. Then

$$
g=|G|=\frac{|N|^{3}}{|C|^{2}} \frac{f_{1}\left(f_{1}+\delta_{1}\right)}{\left(f_{1}-\varepsilon \delta_{1} q\right)^{2}} \frac{q+\varepsilon}{q}=\frac{|N|^{3}}{|C|^{2}} \frac{f_{2}\left(f_{2}+\delta_{2}\right)}{\left(f_{2}+\varepsilon \delta_{2} q\right)^{2}} \frac{q-\varepsilon}{q}
$$

( ${ }^{7}$ In the notation of $[6, \mathrm{III}]$, this character was $\tilde{\chi}_{1}$. For 2-regular $\rho \in N$, we have $\psi(\rho)=\delta_{1}^{*} \phi_{1}^{J}(\rho)-\delta_{1}^{*} ; \delta_{1}^{*}=\varepsilon$ in the notation of $[6, \mathrm{III}]$. 
where the $f_{i}, \delta_{i}$ are as in Proposition 4. Also,

$$
f_{1} f_{2}=q^{2} f_{3}
$$

Finally,

$$
f_{1} \equiv \delta_{1}(2-\varepsilon q), \quad f_{2} \equiv-\delta_{2} \varepsilon q, \quad f_{3} \equiv-\delta_{3}+2^{n} \quad\left(\bmod 2^{n+1}\right) .
$$

COROLlary 1 . In the case of a QD-group, the odd degrees $1, f_{1}, f_{2}, f_{3}$ are distinct. The degree $f_{4}$ is divisible by $2^{n-1} \geqq 4$ while $f \equiv 2(\bmod 4)$.

The first statement is immediate from $(6)$ since $q \equiv \varepsilon+2^{n-1}\left(\bmod 2^{n}\right)$. The other statements also follow from (6).

COROLlARY 2. The characters $\chi_{0}, \chi_{1}, \chi_{2}, \chi_{3}, \chi_{4}$ and $\chi^{\left(2^{n-2}\right)}$ take only rational values.

Proof. An algebraic conjugate of a character in the principal block $B_{0}$ always lies in $B_{0}$ again, cf. [6, I, §III, Lemma 2]. In the case of $Q D$-groups, it is now evident that each of $\chi_{1}, \chi_{2}, \chi_{3}, \chi_{4}$ is algebraically conjugate only to itself. In the case of $\chi^{\left(2^{n-2}\right)}$, this is still true as can be seen from Proposition 2. If we also use Proposition 3, we see that Corollary 2 holds for $Q$-groups, too.

We add some supplements. As already mentioned, we wish to show

Proposition 7. If $G$ is a $Q D$-group or a $Q$-group and if $q$ is its characteristic power, then in the notation of Proposition 3, $\psi(1)=q$.

Proof. We determine the four odd degrees in the principal 2-block $B_{0}(2, N)$ of $N=C(x)$. These remain the same if $N$ is replaced by $\bar{N}=N / O(N)$, cf. [6, I, Theorem 1]. According to Proposition 2.3.3, $\bar{N}$ has a normal subgroup $\bar{L}$ such that $\bar{L} /\langle\bar{x}\rangle \simeq P G L(2, q)$ and such that $\bar{N} / \bar{L}$ is cyclic of odd order $e$.

The principal 2-block $B_{0}(2, \bar{L})$ of $\bar{L}$ contains that of $\bar{L} /\langle\bar{x}\rangle \simeq P G L(2, q)$ (if, of course, characters of $\bar{L} /\langle\bar{x}\rangle$ are interpreted as characters of $\bar{L}$ ). As is well known, the group $\operatorname{PGL}(2, q)$ has exactly four irreducible characters of odd degree and these degrees are $1,1, q, q$, cf. [27]. These degrees then occur in $B_{0}(2, \bar{L})$. If for a moment the notation of Proposition 2 is used for the $Q$-group $\bar{L}$, then by Proposition 5 , $f_{3}=1, f_{1}=f_{2}=q$ and $\delta_{1}=-\delta_{2}$. By Proposition $2, \chi_{1}$ and $\chi_{2}$ have opposite values for elements of order $2^{n}$ and consequently, they are not associated in $\bar{N}$. Each of them then has $e$ extensions to irreducible characters of $\bar{N}$. In the terminology introduced in [7, I, $\S 4$, the principal 2-block $B_{0}(2, \bar{N})$ covers the principal 2-block $B_{0}(2, \bar{L})$. It follows that $B_{0}(2, \bar{N})$ contains two irreducible characters of degree $q$ in addition to two characters of degree 1 and this implies the statement.

We use a similar argument to prove a lemma that will be needed in $\$ 4$.

LEMMA 1. Let $G$ be a $Q D$-group with the characteristic power $q$. The principal 2-block $B_{0}(2, N)$ (with $N=C(x)$ ) contains exactly one modular irreducible character $\phi$ different from the principal modular character. If $\psi$ is as in Proposition 3, then for $r \in N$ of odd order, we have formulas

$$
\psi(r)=d_{0}+d_{1} \phi(r),
$$


where $d_{0} \geqq 0$ and $d_{1}>0$ are integers (decomposition numbers) which do not depend on $r$. Here, $\phi(1), d_{0}$ and $d_{1}$ are determined by $q$ alone and do not depend otherwise on $G$.

Proof. If $H$ is a $Q$-group and if we restrict the characters of $B_{0}(2, H)$ to the set of 2-regular elements, Propositions 4 and 5 show that at most two of these restrictions are linearly independent. Hence the number $l$ of modular irreducible characters in $B_{0}(2, H)$ is at most equal to 2 . If we had $l=1$, then $H$ would have a normal 2-complement $[9, \S 29]$. This is not true, since in a $Q$-group all elements of order 4 are conjugate while the Sylow 2-subgroup has two conjugate classes of elements of order 4 . Thus $l=2$. Of course, one of the two modular irreducible characters is the principal modular character of $H$ which we shall denote by $1_{H}$.

In particular, this applies for the $Q$-group $H=N$ in Lemma 1 and this yields the first part of the lemma including the existence of the equation (7). We have $d_{1}>0$, since otherwise $\psi$ would be constant on the set of 2-regular elements of $N$. By Propositions 4 and 5, the same would be true for all other irreducible characters in $B_{0}(2, N)$. This is impossible as we have $l=2$.

By Theorem 1 of $[6, I], \psi$ can be considered as an irreducible character of $\bar{N}=N / O(N)$ and then $\psi \in B_{0}(2, \bar{N})$. Likewise, $\phi$ can be identified with the modular irreducible character different from $1_{N}$ in $B_{0}(2, \bar{N})$. The proof of Proposition 7 shows that $\psi \mid \bar{L}$ is irreducible and, in fact, $\psi \mid \bar{L}$ is one of the two irreducible characters of degree $q$ in $B_{0}(2, \bar{L})$. Let $1_{L}$ and $\phi^{*}$ denote the two modular irreducible characters in the principal 2-block of the $Q$-group $H=\bar{L}$. Clearly, they are not associated in $\bar{N}$ and each of them is only associated to itself in $\bar{N}$. Since $\bar{N} / \bar{L}$ is cyclic of odd order $e$, both $1_{L}$ and $\phi^{*}$ have $e$ extensions each to modular irreducible characters of $\bar{N}$. Since $B_{0}(\bar{N})$ covers $B_{0}(\bar{L})$, it follows easily from [7, I, (4A)] that $\phi$ is one of the extensions of $\phi^{*}$. In particular, $\phi(1)=\phi^{*}(1)$. By (7), $d_{0}$ and $d_{1}$ are the decomposition numbers of the character $\psi \mid \bar{L} \in B_{0}(2, \bar{L})$ of degree $q$. Note that by Proposition 5 , both characters of degree $q$ coincide for 2-regular elements and hence both have the same pair $\left(d_{0}, d_{1}\right)$ of decomposition numbers.

Since $\bar{L} \simeq S L^{ \pm 1}(2, q)$ for $q \equiv-1(\bmod 4)$ and $\bar{L} \simeq S U^{ \pm 1}(2, q)$ for $q \equiv 1(\bmod 4)$, the group $\bar{L}$ is uniquely determined by $q$ and so are then $\phi^{*}(1)=\phi(1)$ and $\left(d_{0}, d_{1}\right)$. This completes the proof of Lemma 1.

We conclude this section with the proof of some inequalities for the degrees in $B_{0}(2, G)$.

Proposition 8. Let $G$ be a $Q D$-group with the characteristic power $q$. Let $f_{i}, \delta_{i}$ have the same significance as above.

(i) If $\delta_{1}=1$, then $f_{2}<q^{2}$ and if we set

$$
f_{2}=q^{2}-h, \quad 0<h<q^{2},
$$

we have

$$
f_{1}=\frac{q^{2}\left(q^{2}-h-1\right)}{h}, \quad f_{3}=\frac{\left(q^{2}-h\right)\left(q^{2}-h-1\right)}{h}
$$


(ii) If $\delta_{2}=1$, then $f_{1}<q^{2}$ and if we set

$$
f_{1}=q^{2}-h, \quad 0<h<q^{2},
$$

we have

$$
f_{2}=\frac{q^{2}\left(q^{2}-h-1\right)}{h}, \quad f_{3}=\frac{\left(q^{2}-h\right)\left(q^{2}-h-1\right)}{h} .
$$

(iii) If $\delta_{3}=1$ and if

$$
f_{\lambda}=\operatorname{Min}\left(f_{1}, f_{2}\right), \quad f_{\mu}=\operatorname{Max}\left(f_{1}, f_{2}\right)
$$

then $q^{2}<f_{\lambda}<2 q^{2}$ and if we set

$$
f_{\lambda}=q^{2}+h, \quad 0<h<q^{2},
$$

we have

$$
f_{\mu}=\frac{q^{2}\left(q^{2}+h-1\right)}{h}, \quad f_{3}=\frac{\left(q^{2}+h\right)\left(q^{2}+h-1\right)}{h} .
$$

In each case, $h$ divides $q^{2}\left(q^{2}-1\right)$.

Proof. (i) If $\delta_{1}=1$, then $\delta_{2}=\delta_{3}=-1$ and, by (3) $1+f_{1}=f_{2}+f_{3}$. On account of (5),

$$
q^{2}+q^{2} f_{1}=q^{2} f_{2}+q^{2} f_{3}=q^{2} f_{2}+f_{1} f_{2}>q^{2}+f_{1} f_{2} .
$$

Hence $f_{1} f_{2}<q^{2} f_{1}$, i.e. $f_{2}<q^{2}$. If we set $f_{2}=q^{2}-h$, we have

$$
q^{2}+q^{2} f_{1}=q^{2}\left(q^{2}-h\right)+f_{1}\left(q^{2}-h\right)
$$

which yields the first formula (9). The second formula then is obtained from (5).

(ii) The case $\delta_{2}=1$ is treated in an analogous manner with $f_{1}$ and $f_{2}$ interchanged.

(iii) If $\delta_{3}=1$, then $\delta_{1}=\delta_{2}=-1$. Here,

$$
\begin{aligned}
1+f_{3} & =f_{1}+f_{2}=f_{\lambda}+f_{\mu}, \\
q^{2}+f_{\lambda} f_{\mu} & =q^{2}+q^{2} f_{3}=q^{2}\left(f_{\lambda}+f_{\mu}\right) \leqq 2 q^{2} f_{\mu} .
\end{aligned}
$$

This implies $f_{\lambda}<2 q^{2}$ and we set $f_{\lambda}=q^{2}+h$ with $-q^{2}<h<q^{2}$. Now

$$
q^{2}+\left(q^{2}+h\right) f_{\mu}=q^{2}\left(q^{2}+h\right)+q^{2} f_{\mu},
$$

whence $h f_{\mu}=q^{2}\left(q^{2}+h-1\right)$. Since $q^{2}+h-1 \geqq 0$, we have $h \geqq 0$. Actually, $h=0$ would lead to $q^{2}\left(q^{2}-1\right)=0$ which is absurd. Thus, $h>0$ and $0<h<q^{2}$. We also obtain the first equation (9) $)_{111}$. The second equation then follows from (5).

In the case (i), the numerator of $f_{1}$ must be divisible by $h$ and this shows that $h$ divides $q^{2}\left(q^{2}-1\right)$. A similar argument applies in the cases (ii) and (iii).

We prove one more corollary of a different nature.

COROLlaRY 3. If $G$ is a $Q D$-group and if $\chi$ is a character in the principal block $B_{0}(2, G)$, then for $\chi \neq \chi_{0}$, the kernel $K$ of $\chi$ is $O(G)$. 
Proof. By [6, I, Theorem 1], we have $K \supseteq O(G)$. If we have inequality, then $|K: O(G)|$ is even and hence $K$ contains all involutions of $G$. Thus $x \in K, s^{2} x \in K$ and hence $s^{2} \in K$. Since $\chi(1) \neq 1$ by Corollary 1, it follows from Proposition 2 that $\chi \neq \chi_{1}, \chi_{2}, \chi_{3}, \chi_{4}$. If $\chi=\chi^{(j)}$ for some $j$, necessarily $f=2$. Proposition 3 shows that $\varepsilon q+1= \pm 2$ and hence $q=3$. It follows from Proposition 4 that $f_{1}=3, \delta_{1}=-1$. Now, (6) leads to a contradiction. Thus, $K=O(G)$.

3. Regular $Q D$-groups. Let $G$ be a $Q D$-group of characteristic power $q$. Then $q$ is a power of a prime $p$, the characteristic of $G$.

Definition 1. The $Q D$-group is regular, if $p$ occurs in $|G|$ with at least the same exponent with which it divides $|N|^{3} /|C|^{2}$.

Here, $N$ and $C$ have the same significance as before.

Proposition 1. If $G$ is a regular $Q D$-group, then with the notation introduced in $\S 1$, we have one of the following two cases:

Case I. $\varepsilon=1, \delta_{1}=1, \delta_{2}=\delta_{3}=-1, f_{1}=q^{3}, f_{2}=q^{2}-q+1, f_{3}=\left(q^{2}-q+1\right) q$, $f_{4}=q^{2}-q, f=q^{3}+1$.

Case II. $\varepsilon=-1, \delta_{3}=1, \delta_{1}=\delta_{2}=-1, f_{1}=q^{3}, f_{2}=q^{2}+q+1, f_{3}=\left(q^{2}+q+1\right) q$, $f_{4}=q^{2}+q, f=q^{3}-1$.

Proof. Let $\nu(h)$ denote the exact exponent with which the prime $p$ divides an integer $h$. Set $\nu\left(f_{i}\right)=\alpha_{i}, \nu\left(f_{i}+\delta_{i}\right)=\beta_{i}(i=1,2), \nu\left(f_{1}-\varepsilon \delta_{1} q\right)=\gamma_{1}, \nu\left(f_{2}+\varepsilon \delta_{2} q\right)=\gamma_{2}$. Then by (2.4), for $i=1,2$,

$$
\nu(g)=3 \nu(|N|)-2 v(|C|)+\alpha_{i}+\beta_{i}-2 \gamma_{i}-\nu(q) .
$$

If $G$ is regular, by definition $\nu(g) \geqq 3 \nu(|N|)-2 \nu(|C|)$ and hence

$$
\alpha_{i}+\beta_{i} \geqq 2 \gamma_{i}+\nu(q) \text {. }
$$

By (2.5), we have $\alpha_{1}+\alpha_{2} \geqq 2 v(q)$. We can therefore choose $i=1$ or 2 such that $\alpha_{i} \geqq v(q)$. This implies $\beta_{i}=0$ and $\gamma_{i} \geqq v(q)$. Hence by (1) $\alpha_{i} \geqq 3 \nu(q)$ and $q^{3}$ divides $f_{i}$. If $i^{\prime}$ is the index 1 or 2 different from $i$, by (2.5) $q \mid f_{3}$ and by (2.3) $\alpha_{i^{\prime}}=0$.

We now distinguish the three cases in Proposition 2.8 .

(i) If $\delta_{1}=1$, we have $f_{2}<q^{2}$ and hence $i=1, i^{\prime}=2$. If we set $f_{1}=q^{3} f^{*}$, by $\left(2.9_{1}\right)$,

$$
q^{2}-h-1=q h f^{*} \text {. }
$$

Then $h \equiv-1(\bmod q)$, say $h=h_{0} q-1$ with integral $h_{0}>0$. Thus,

If $f^{*} \geqq 2$, we find

$$
q-h_{0}=\left(h_{0} q-1\right) f^{*} \text {. }
$$

$$
q-h_{0} \geqq 2\left(h_{0} q-1\right) \geqq 2(q-1)>q,
$$

which is impossible. Thus $f^{*}=1$ and then $q-h_{0}=h_{0} q-1$, which implies $h_{0}=1$, $h=q-1$. Now, the Proposition 2.8(i) yields

$$
f_{1}=q^{3}, \quad f_{2}=q^{2}-q+1, \quad f_{3}=\left(q^{2}-q+1\right) q .
$$


In particular, $f_{1} \equiv \varepsilon^{3} \equiv \varepsilon(\bmod 4)$. Since by $(2.6) f_{1} \equiv \delta_{1}(\bmod 4)$ in the present case, $\varepsilon=1$. The values of $f_{4}$ and $f$ can be obtained from (2.3) and this establishes the statements for Case I.

(ii) If $\delta_{2}=1$, an analogous argument yields

$$
f_{1}=q^{2}-q+1, \quad f_{2}=q^{3}, \quad f_{3}=\left(q^{2}-q+1\right) q .
$$

By (2.6), (as $\left.\delta_{1}=-1\right)$

$$
q^{2}-q+1 \equiv \varepsilon q-2 \quad\left(\bmod 2^{n+1}\right) .
$$

In particular, $2-\varepsilon \equiv-1(\bmod 4)$, whence $\varepsilon=-1$. But then $q^{2} \equiv-3\left(\bmod 2^{n+1}\right)$, which is impossible as $q^{2} \equiv 1(\bmod 8)$. Thus, the case (ii) does not arise.

(iii) If $\delta_{3}=1$, then in $\left(2.8_{111}\right), f_{\lambda}$ is not divisible by $q^{3}$, we have $i=\mu$ and $i^{\prime}=\lambda$. Set here $f_{\mu}=q^{3} f^{*}$. By $\left(2.9_{\mathrm{it1}}\right)$,

$$
q h f^{*}=q^{2}+h-1
$$

and $h \equiv 1(\bmod q)$, say, $h=1+q h_{0}$ with integral $h_{0} \geqq 0$. Actually, as $q$ and $f_{\lambda}$ are odd $h$ in $\left(2.8_{111}\right)$ is even and $h_{0} \geqq 1$. Now,

$$
\left(1+q h_{0}\right) f^{*}=q+h_{0}
$$

Clearly, $f^{*}<2$, i.e. $f^{*}=1$. This yields $h_{0}=1, h=q+1$. Now, Proposition 2.8(iii) gives $f_{\lambda}=q^{2}+q+1, f_{\mu}=q^{3}, f_{3}=\left(q^{2}+q+1\right) q$. Since then

$$
f_{3}=q^{3}+q^{2}+q \equiv 1+2 q \equiv 1+2 \varepsilon \quad(\bmod 8),
$$

the congruence (2.6) with $\delta_{3}=1$ shows that $\varepsilon=-1$. If we had $\lambda=1$, by (2.6)

$$
q^{2}+q+1 \equiv-(2+q) \quad\left(\bmod 2^{n+1}\right),
$$

i.e. $(q+1)^{2} \equiv-2\left(\bmod 2^{n+1}\right)$, which is absurd. Thus, $\lambda=2, \mu=1$. This shows that the values of $f_{1}, f_{2}, f_{3}, \varepsilon, \delta_{1}, \delta_{2}, \delta_{3}$ are as given in the Case II of the proposition. The values of $f_{4}$ and $f$ are again obtained from (2.3).

Proposition 2. If $G$ is a regular $Q D$-group of characteristic power $q$, then

$$
|G|=\frac{|N|^{3}}{|C|^{2}} \frac{q^{2}-\varepsilon q+1}{(q-\varepsilon)^{2}}
$$

In particular, $p$ divides $|G|$ with exactly the same exponent with which it divides $|N|^{3} /|C|^{2}$.

This is immediate from Proposition 1 and (2.4).

By Lemmas 2.4.1(i) and 2.4.2(i), we have

$$
|N / O(N)|=2 q(q-1)(q+1) e, \quad \text { where } e=|N / L|=|E / E \cap O(N)|
$$

and

$$
|C / C \cap O(N)|=2 e(q+\varepsilon) .
$$


We now set $a=|C \cap O(N)|$ and $b=|O(N): C \cap O(N)|$. Then by equations (2) and (3), we have

$$
|N|=2 a b e q(q-1)(q+1) \text { and }|C|=2 e a(q+\varepsilon)
$$

Hence in this notation, Proposition 2 can be expressed as follows:

Proposition 2*. For a regular $Q D$-group of characteristic power $q$, we have

$$
|G|=2 a b^{3} e q^{3}(q+1)(q-1)\left(q^{2}-\varepsilon q+1\right) .
$$

Proposition 3. Let $G$ be a $Q D$-group of characteristic power $q$. Each of the following three conditions is necessary and sufficient for the regularity of $G$.

(i) $q^{3} \mid f_{1}$.

(ii) $f_{2}=q^{2}-\varepsilon q+1$.

(iii) $q \mid f_{3},\left(q, f_{1}\right) \neq 1$.

Proof. The necessity of each of these conditions is clear from Proposition 1. If $q^{3} \mid f_{1}$, the factor

$$
\frac{f_{1}\left(f_{1}+\delta_{1}\right)}{\left(f_{1}-\varepsilon \delta_{1} q\right)^{2}} \frac{q+\varepsilon}{q}
$$

in (2.4) contains $p$ with a nonnegative exponent and then $G$ is regular by definition. The conditions (iii) imply (i) by (2.3) and (2.5).

Finally, if $f_{2}=q^{2}-\varepsilon q+1$, by (2.6)

$$
2-\varepsilon q \equiv-\varepsilon \delta_{2} q(\bmod 8)
$$

whence $\delta_{2}=-1$. If $\delta_{2}=-1$, the second form of $|G|$ in (2.4) shows that $G$ is regular. This completes the proof.

We add a corollary.

COROLlaRY 1. The $Q D$-groups $G$ and $G / O(G)$ are regular at the same time.

This is a consequence of the fact that the principal 2-blocks of $G$ and of $G / O(G)$ can be identified, cf. [6, I, Theorem 1].

4. Proof of Theorem A. The theorem has already been stated in Chapter I.

TheOREM A. Let $G$ be a $Q D$-group and assume that $G$ contains a regular $Q D$ subgroup $H$ of the same characteristic power $q$ as $G$. Then $G$ is regular and $O(H)=O(G) \cap H$.

Proof. We use the same notation for $G$ as before while in the case of $H$, asterisks will be added. Thus, $B_{0}(2, H)$ consists of the characters $\chi_{0}^{*}=1, \chi_{1}^{*}, \chi_{2}^{*}, \ldots$ Since $H$ is regular, the degrees are

$$
\begin{aligned}
& f_{1}^{*}=q^{3}, \quad f_{2}^{*}=q^{2}-\varepsilon q+1, \quad f_{3}^{*}=q\left(q^{2}-\varepsilon q+1\right) \\
& f_{4}^{*}=q^{2}-\varepsilon q, \quad f^{*}=q^{3}+\varepsilon
\end{aligned}
$$


and the corresponding signs are

$$
\begin{array}{ll}
\delta_{1}^{*}=1, \quad \delta_{2}^{*}=\delta_{3}^{*}=-1 & \text { for } \varepsilon=1, \\
\delta_{1}^{*}=\delta_{2}^{*}=-1, \quad \delta_{3}^{*}=1 & \text { for } \varepsilon=-1 .
\end{array}
$$

Since $q \equiv \varepsilon+2^{n-1}\left(\bmod 2^{n}\right)$, the value of $n$ is determined by $q$ and it is the same for $G$ and $H$. In this section, $j$ will always range over the values in (2.1).

Since $\chi_{i} \mid H$ is a character of $H,(i=1,2,3)$, we may set

$$
\chi_{i} \mid H=\sum_{\lambda=0}^{4} a_{i \lambda} \chi_{\lambda}^{*}+\sum_{j} b_{i j} \chi^{(j)^{*}}+\Xi_{i} .
$$

Here, the $a_{i \lambda}$ and $b_{i j}$ are nonnegative integers and $\Xi_{i}$ is a character of $H$ whose irreducible constituents do not belong to the principal 2-block $B_{0}(2, H)$.

We first note that it will suffice to prove that

$$
\chi_{2} \mid H=\chi_{2}^{*} \text {. }
$$

Indeed, then $f_{2}=f_{2}^{*}=q^{2}-\varepsilon q+1$ and Proposition 3.3 shows that $G$ is regular. By [6, I, Theorem 1] $\chi_{2}^{*}$ is trivial on $O(H)$ and so is then $\chi_{2}$. Now Corollary 2.3 implies that $O(H) \subseteq O(G) \cap H$. Since the reverse inclusion is obvious, Theorem A will then be established.

We now come to the proof of $\left(^{*}\right)$. Let $r$ range over the 2-regular elements of $C_{H}(s)$. Substituting $u=s r$ in (3) and applying Proposition 2.2 both to $G$ and to $H$, we obtain

$$
\delta_{i}=\sum_{\lambda=0}^{3} a_{i \lambda} \delta_{\lambda}^{*}+\delta_{1}^{*} \sum b_{i j}\left(\zeta^{j}+(-\zeta)^{-\jmath}\right)+\Xi_{i}(s r)
$$

$(i=1,2,3)$. Here, we set $\delta_{0}^{*}=1$. As always in this section, $j$ ranges over the vaues (2.1).

It follows from the main result of [5, II] that we may set $\Xi_{i}(s r)=\theta_{i}(r)$, where $\theta_{i}$ is a linear combination of 2-modular irreducible characters of $C_{H}(s)$, none of which lies in $B_{0}\left(2, C_{H}(s)\right)$. Since then 1 and $\theta_{i}$ are linearly independent, (4) implies $\theta_{i}=0$. Thus

$$
\delta_{i}=\sum_{v=0}^{3} a_{i v} \delta_{v}^{*}+\delta_{1}^{*} \sum_{j} b_{i j}\left(\zeta^{j}+(-\zeta)^{-\jmath}\right) .
$$

Here, $j$ ranges over the values (2.1). Since $\zeta$ is a primitive $2^{n}$ th root of unity, we have $\zeta^{2^{n-1}}=-1, \zeta^{2^{n-2}}+(-\zeta)^{-2^{n-2}}=0$ and

$$
\begin{aligned}
& \zeta^{j}+(-\zeta)^{-j}=-\left(\zeta^{-j}+(-\zeta)^{-(-j)}\right) \quad(j \text { odd }), \\
& \zeta^{j}+(-\zeta)^{-j}=-\left(\zeta^{2^{n-1}-j}+(-\zeta)^{-\left(2^{n-1}-j\right)}\right) \quad(j \text { even }) .
\end{aligned}
$$

Now $\left(4^{*}\right)$ can be written in the form

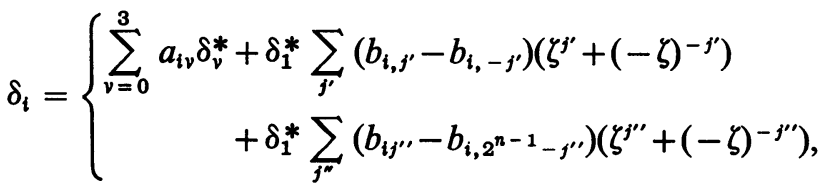


where $j^{\prime}$ ranges over the values $1,3,5, \ldots, 2^{n-2}-1$ and where $j^{\prime \prime}$ ranges over the values $2,4, \ldots, 2^{n-2}-2$.

Since $1, \zeta, \ldots, \zeta^{2^{n-1}-1}$ are linearly independent over the field $\boldsymbol{Q}$ of rational numbers, so are

$$
\zeta^{-2^{n-2}+1}, \zeta^{-2^{n-2}+2}, \ldots, \zeta^{2^{n-2}}
$$

On comparing coefficients of powers of $\zeta$ in our equation, we have

$$
\begin{aligned}
& \delta_{i}=\sum_{v=0}^{3} a_{i v} \delta_{v}^{*} \\
& b_{i, j^{\prime}}=b_{i,-j^{\prime}} \quad \text { for } j^{\prime}=1,3, \ldots, 2^{n-2}-1 \text {, } \\
& b_{i, j^{\prime \prime}}=b_{i, 2^{n-1}-j^{\prime \prime}} \text { for } j^{\prime \prime}=2,4, \ldots, 2^{n-2}-2 \text {. }
\end{aligned}
$$

We next substitute $u=s^{2} r$ in (3), where $r$ now ranges over the 2-regular elements of $C_{H}\left(s^{2}\right)$. An analogous argument yields

$$
\pm \delta_{i}=\left\{\begin{array}{r}
a_{i 0}+a_{i 1} \delta_{1}^{*}-a_{i 2} \delta_{2}^{*}-a_{i 3} \delta_{3}^{*} \\
+\delta_{1}^{*} \sum_{j} b_{i j}\left(\zeta^{2 j}+\zeta^{-2 j}\right)
\end{array}\right.
$$

where we have the + sign on the left for $i=1$ and the - sign for $i=2$ and 3 . The term with $j=2^{n-2}$ in the sum is $-2 b_{i, 2^{n-2}}$. For all other $j$, we have formulas $\zeta^{2 j}= \pm \zeta^{k}, \zeta^{-2 j}= \pm \zeta^{l}$ with $\zeta^{k}$ and $\zeta^{l}$ occurring in (5) and being different from 1. On using this in (8) and comparing the coefficient of 1 , we find

$$
a_{i 0}+a_{i 1} \delta_{1}^{*}-a_{i 2} \delta_{2}^{*}-a_{i 3} \delta_{3}^{*}-2 b_{i, 2^{n-2}} \delta_{1}^{*}=\left\{\begin{aligned}
\delta_{1}, & (i=1) \\
-\delta_{2}, & (i=2) \\
-\delta_{3}, & (i=3)
\end{aligned}\right.
$$

We shall later apply the same method once more using elements $u=x r$ with 2-regular $r \in C_{H}(x)$, but it is better to postpone this.

We now separate the cases $\varepsilon=-1$ and $\varepsilon=1$.

Case $\varepsilon=-1$. If $\delta_{1}=1$, by Proposition 2.8(i), $f_{2}<q^{2}$. Since here $\delta_{2}=-1$, (6) for $i=2$ reads

$$
-1=a_{20}-a_{21}-a_{22}+a_{23},
$$

cf. (2). Hence $a_{21}+a_{22}>0$. By (1), $f_{1}^{*}>q^{2}, f_{2}^{*}>q^{2}$. Neither $\chi_{1}^{*}$ nor $\chi_{2}^{*}$ can occur as a constituent of $\chi_{2} \mid H$ of degree less than $q^{2}$, so we have a contradiction with (3).

Likewise, if $\delta_{2}=1$, we have $f_{1}<q^{2}$. Here, $\delta_{1}=-1$ and (6) for $i=1$ shows that $a_{11}+a_{12}>0$. Again, (3) leads to a contradiction since $f_{1}^{*}>q^{2}, f_{2}^{*}>q^{2}$.

Thus, $\delta_{3}=1$ and then $\delta_{1}=\delta_{2}=-1$. Here, (9) for $i=1$ reads

$$
a_{10}-a_{11}+a_{12}-a_{13}+2 b_{1,2^{n-2}}=-1 \text {, }
$$

cf. (2). Then $a_{11}+a_{13}>0$, i.e. $\chi_{1} \mid H$ contains $\chi_{1}^{*}$ or $\chi_{3}^{*}$ as constituent. By (1) $f_{3}^{*}>f_{1}^{*}=q^{3}$. Since then $f_{1} \geqq q^{3}$, in Proposition 2.8(iii), we must have $\mu=1, \lambda=2$ and

$$
f_{2}=q^{2}+h, \quad q^{2}\left(q^{2}+h-1\right)=h f_{1} \geqq h q^{3} .
$$


It follows that $h q \leqq q^{2}+h-1$. Thus, $h \leqq q+1$ and so

$$
f_{2} \leqq q^{2}+q+1 \text {. }
$$

Again, by (6) with $i=2, a_{21}+a_{22}>0$, i.e. $\chi_{1}^{*}$ or $\chi_{2}^{*}$ occurs as constituent of $\chi_{2} \mid H$. However, by (1) $f_{1}^{*}$ is larger than $f_{2}$ and $\chi_{2} \mid H$ cannot contain $\chi_{1}^{*}$. Since $f_{2}^{*}=q^{2}+q+1$, (10) shows that necessarily $\chi_{2} \mid H=\chi_{2}^{*}$, i.e. that $\left(^{*}\right)$ holds. As remarked, this establishes Theorem $\mathrm{A}$ in the Case $\varepsilon=-1$.

Case $\varepsilon=1$. For $i=1$, the equation (9) reads here

$$
a_{10}+a_{11}+a_{12}+a_{13}-2 b_{1,2^{n-2}}=\delta_{1},
$$

cf. (2). By (6) for $i=1,2,3$,

$$
a_{i 0}+a_{i 1}-a_{i 2}-a_{i 3}=\delta_{i} .
$$

It follows from (11) that if $\delta_{1}=-1$, necessarily $b_{1,2^{n-2}}>0$. Since $f^{*}=q^{3}+1$ by (1), it follows from (3) with $i=1$ that

$$
f_{1} \geqq q^{3}+1 \quad\left(\text { for } \delta_{1}=-1\right) .
$$

In particular, this is true in the case $\delta_{2}=1$. However then Proposition 2.8(ii) yields $f_{1}<q^{2}$, a contradiction. Thus $\delta_{2}=-1$ and hence $\delta_{1}=1$ or $\delta_{3}=1$.

If $\delta_{3}=1$, (13) applies. Now Proposition 2.8(iii) shows that $\lambda=2, \mu=1$ and that

$$
f_{2}=q^{2}+h, \quad h f_{1}=q^{2}\left(q^{2}+h-1\right), \quad 0<h<q^{2} .
$$

It follows from (13) that $h q<q^{2}+h-1$. Then $h<q+1$ and

$$
f_{2}<q^{2}+q+1 \quad\left(\text { for } \delta_{3}=1\right) \text {. }
$$

If $\delta_{1}=1$, we can apply Proposition 2.8(i) and find

$$
f_{2}<q^{2} \quad\left(\text { for } \delta_{1}=1\right) .
$$

Since $\delta_{2}=-1$, the equation (12) shows that $a_{22}+a_{23}=0$. On account of (14), $\chi_{3}^{*}$ of degree $q\left(q^{2}-q+1\right)$ is not a constituent of $\chi_{2} \mid H$. Hence $a_{23}=0$ and $a_{22}>0$. This means that $\xi=\chi_{2} \mid H-\chi_{2}^{*}$ is a character of $H$. By (14),

$$
\xi(1)=f_{2}-f_{2}^{*}<q^{2}+q+1-\left(q^{2}-q+1\right)=2 q .
$$

It follows that $\xi$ cannot contain any irreducible constituent in $B_{0}(2, H)$ except possibly $\chi_{0}^{*}$, cf. (1). In particular, $a_{22}=1$ and then (12) shows that $a_{20}=0$. Thus, $\chi_{0}^{*}$ is not an irreducible constituent of $\xi$ either. In other words, in (3) for $i=2, \Xi_{2}$ coincides with $\xi$, we have

$$
\chi_{2} \mid H=\chi_{2}^{*}+\Xi_{2} .
$$

We shall need some more relations between the coefficients of (3). Substitute $u=x r$ in (15) where $r$ is a 2-regular element of $C_{H}(x)=N \cap H$. As we have $\varepsilon=1$, $\delta_{2}=\delta_{2}^{*}=-1$, Proposition 2.3 yields $\chi_{2}(x r)=\psi(r), \chi_{2}^{*}(x r)=\psi^{*}(r)$, where $\psi^{*}$ plays the 
same role for $H$ as $\psi$ does for $G$, i.e. where $\psi^{*}$ is an irreducible character of degree $q$ in $B_{0}\left(2, C_{H}(x)\right)=B_{0}(2, N \cap H)$.

On the other hand, the main result of $[5$, II $]$ shows that $\Xi_{2}(x r)=\theta_{2}^{\prime}(r)$, where $\theta_{2}^{\prime}$ is a linear combination of 2-modular irreducible characters of $N \cap H$ which do not lie in $B_{0}(2, N \cap H)$. We then have $\psi(r)=\psi^{*}(r)+\theta_{2}^{\prime}(r)$ for all 2-regular $r$ in $N \cap H$.

Apply now Lemma 2.1 to both $G$ and $H$. As before, let $\phi$ be the nonprincipal 2-modular irreducible character in $B_{0}(2, N)$. Let $\phi^{*}$ be the nonprincipal modular irreducible character in $B_{0}(2, N \cap H)$. Since $G$ and $H$ have the same characteristic power $q$, the lemma shows that

$$
\phi(1)=\phi^{*}(1)
$$

and that we have formulas $\psi(r)=d_{0}+d_{1} \phi(r), \psi^{*}(r)=d_{0}+d_{1} \phi^{*}(r)$ with the same integers $d_{0} \geqq 0$ and $d_{1}>0$ in both. These integers do not depend on $r$. Then $\phi(r)=\phi^{*}(r)+\left(1 / d_{1}\right) \theta_{2}^{\prime}(r)$ for all 2-regular $r \in H \cap N$. Since $\phi \mid N \cap H$ is a modular character of $N \cap H$, it follows now that $\left(1 / d_{1}\right) \theta_{2}^{\prime}$ is the sum of the constituents of $\phi \mid N \cap H$ which do not belong to $B_{0}(2, N \cap H)$. Hence $\left(1 / d_{1}\right) \theta_{2}^{\prime}$ is a modular character of $N \cap H$ and since (16) implies that $\left(1 / d_{1}\right) \theta_{2}^{\prime}(1)$ vanishes, we conclude that $\left(1 / d_{1}\right) \theta_{2}^{\prime}$ vanishes. Hence

$$
\psi(r)=\psi^{*}(r)
$$

for all 2-regular $r$ in $N \cap H$.

Apply again Proposition 2.3 to $G$ and $H$. Firstly, we have

$$
\chi_{1}(x r)=\delta_{1} \varepsilon \psi(r)=\delta_{1} \psi^{*}(r) \text {. }
$$

Then, if we substitute $u=x r$ in (3) for $i=1$, we find

$$
\begin{aligned}
\delta_{1} \psi^{*}(r)= & a_{10}+a_{11} \psi^{*}(r)+a_{12} \psi^{*}(r)+a_{13}-a_{14}\left(\psi^{*}(r)-1\right) \\
& +\sum_{j}(-1)^{j} b_{1 j}\left(\psi^{*}(r)+1\right)+\Xi_{1}(x r) .
\end{aligned}
$$

Again $\Xi_{1}(x r)=\theta_{1}^{\prime}(r)$, where $\theta_{1}^{\prime}$ is a linear combination of modular irreducible characters of $N \cap H$ which do not lie in $B_{0}(2, N \cap H)$. Then $1, \phi^{*}, \theta_{1}^{\prime}$ are linearly independent and since $\psi^{*}(r)=d_{0}+d_{1} \phi^{*}(r)$ with $d_{1} \neq 0$, we can compare the coefficients of 1 and of $\psi^{*}$ in the equation. This yields

$$
\begin{aligned}
& a_{10}+a_{13}+a_{14}+\sum_{j}(-1)^{j} b_{1 j}=0, \\
& a_{11}+a_{12}-a_{14}+\sum_{j}(-1)^{j} b_{1 j}=\delta_{1} .
\end{aligned}
$$

We next wish to improve $\left(14_{1}\right)$. Assume then that $\delta_{1}=-1$. Suppose first that $f_{1}<4 q^{3}$. Since $f^{*}=q^{3}+1$, it follows from (3) that

$$
\sum_{j} b_{i j}<4 .
$$


By (11), $b_{1,2^{n-2}}>0$ and (18) shows that the sum of the $b_{i j}$ with odd $j$ is at least 1 . By (7), this sum is even. On account of (20), it has the value 2 . Moreover $b_{1,2^{n-2}}=1$, while all other $b_{1}$, with even $j$ vanish. Now, (11), (12), (18) and (19) read

$$
\begin{aligned}
a_{10}+a_{11}+a_{12}+a_{13} & =1, & a_{10}+a_{11}-a_{12}-a_{13} & =-1, \\
a_{10}+a_{13}+a_{14} & =1, & a_{11}+a_{12}-a_{14} & =0 .
\end{aligned}
$$

Hence $a_{10}=a_{11}=0$ and either

$$
a_{12}=0, \quad a_{13}=1, \quad a_{14}=0 \quad \text { or } \quad a_{12}=1, \quad a_{13}=0, \quad a_{14}=1 .
$$

In the former case, (3) yields

$$
f_{1} \geqq q\left(q^{2}-q+1\right)+3\left(q^{3}+1\right)=4 q^{3}-q^{2}+q+3
$$

and, in the latter case,

$$
f_{1} \geqq\left(q^{2}-q+1\right)+\left(q^{2}-q\right)+3\left(q^{3}+1\right)>3 q^{3}+2 q^{2}-2 q .
$$

Since $q^{3} \geqq 3 q^{2}$, it is now clear that in all cases

$$
f_{1}>3 q^{3}+2 q^{2}-2 q .
$$

This is also true in the case $f_{1} \geqq 4 q^{3}$, which was at first excluded.

Apply now Proposition 2.8(iii). We must have $\lambda=1, \mu=2$ since $f_{1}>2 q^{2}$. Then $f_{2}=q^{2}+h, h f_{1}=q^{2}\left(q^{2}+h-1\right)$ and we find $q^{2}\left(q^{2}+h-1\right)>h\left(3 q^{3}+2 q^{2}-2 q\right)$, whence

$$
q^{4}-q^{2}>h\left(3 q^{3}+q^{2}-2 q\right)=h q(3 q-2)(q+1) .
$$

Thus, $h<q(q-1) /(3 q-2)<\frac{1}{3} q$, and this implies that, for $\delta_{1}=-1$,

$$
f_{2}<q^{2}+\frac{1}{3} q .
$$

On account of $\left(14_{2}\right)$, this is also true for $\delta_{1}=1$.

Now, (15) and (21) imply that

$$
\Xi_{2}(1)=f_{2}-f_{2}^{*}<q^{2}+\frac{1}{3} q-\left(q^{2}-q+1\right)<4 q / 3 .
$$

If $\Xi_{2}=0$, then (15) shows that $\left(^{*}\right)$ holds and we are finished.

The proof of Theorem 1 will be complete if we can show that the assumption $\Xi_{2} \neq 0$ leads to a contradiction. Suppose then that $\Xi_{2} \neq 0$. Set

$$
\Xi_{2}=c_{1} \eta_{1}+c_{2} \eta_{2}+\cdots+c_{m} \eta_{m},
$$

where $\eta_{1}, \eta_{2}, \ldots, \eta_{m}$ are distinct irreducible characters of $H$, where the $c_{i}$ are positive integers and where $m \geqq 1$.

Since we have $\varepsilon=1$ and $\delta_{2}=\delta_{2}^{*}=-1$, it follows from Propositions 2.2 and 2.3 applied to $G$ and $H$, and from (17) that $\chi_{2}(w)=\chi_{2}^{*}(w)$ for all 2-singular elements $w$ of $H$. Thus,

$$
\Xi_{2}(w)=0
$$

for these $w$. 
Assume first that some $\eta_{v}$ in (23) has 2-defect 0 . Then $\eta_{v}$ can be interpreted as a chief indecomposable character of $H$ (for the prime $p=2$ ). It follows that $\eta_{v} \overline{\eta_{v}}$ is a sum of principal indecomposable characters of $H$ amongst which the principal indecomposable character $\Phi_{0}^{*}$ belonging to the modular irreducible character 1 of $H$ occurs (cf. [9, §§23 and 24]). Hence, by (22),

$$
\Phi_{0}^{*}(1) \leqq \eta_{\nu}(1)^{2} \leqq 16 q^{2} / 9 .
$$

Here, $\Phi_{0}^{*}$ may be considered as an ordinary character of $H$. One of its irreducible constituents is $\chi_{0}^{*}$, which occurs with the multiplicity 1 . All irreducible constituents of $\Phi_{0}^{*}$ belong to $B_{0}(2, H)$. Since

$$
16 q^{2} / 9<q^{3}-q^{2}+q<q^{3},
$$

it follows from (1) that $\chi_{1}^{*}, \chi_{3}^{*}$ and the $\chi^{(j) *}$ cannot occur as constituents of $\Phi_{0}^{*}$. For $q \geqq 9$, at most one of $\chi_{2}^{*}, \chi_{4}^{*}$ can occur since

$$
2\left(q^{2}-q\right) \geqq 16 q^{2} / 9 .
$$

Moreover, the multiplicity of the constituent $\chi_{2}^{*}$ or $\chi_{4}^{*}$ must be 1 . Hence $\Phi_{0}^{*}=\chi_{0}^{*}+\chi_{2}^{*}$ or $\Phi_{0}^{*}=\chi_{0}^{*}+\chi_{4}^{*}$. Accordingly, $\Phi_{0}^{*}(1)=q^{2}-q+2$ or $\Phi_{0}^{*}(1)=q^{2}-q+1$. However, this is impossible since $\Phi_{0}^{*}(1)$ is divisible by the order $2^{n+1}$ of a Sylow 2-group of $H$, while $q^{2}-q+2 \equiv 2(\bmod 4)$ and $q^{2}-q+1$ is odd.

If $q<9$, then as $\varepsilon=1$ we have $q=5$. Here $f_{2}^{*}=21, f_{4}^{*}=20$, and $\Phi_{0}^{*}(1) \leqq 44$. It is seen easily that this also leads to a contradiction.

Hence none of the $\eta_{v}$ in (23) has 2-defect 0 . This implies that none of the $\eta_{v}$ vanishes for all elements of $S-\{1\}$. Now (23) and (24) show that $m \geqq 2$. Moreover, if $u$ ranges over all 2-singular elements of $H$, we have

$$
-c_{m} \overline{\eta_{m}(u)}=\sum_{\kappa=1}^{m-1} c_{\kappa} \overline{\eta_{k}(u)}
$$

and hence

$$
-c_{m} \eta_{m}(u) \overline{\eta_{m}(u)}=\sum_{\kappa=1}^{m-1} c_{\kappa} \eta_{m}(u) \overline{\eta_{\kappa}(u)}
$$

If we add over all 2-singular $u \in H$, the left hand side is negative. By the orthogonality relations for group characters, the right hand side can be written in the form

$$
-\sum_{\kappa=1}^{m-1} c_{\kappa} \sum_{r} \eta_{m}(r) \bar{\eta}_{\kappa}(r)
$$

where $r$ ranges over all 2-regular elements of $H$. It follows that for some $\kappa \leqq m-1$, we have $\sum_{r} \eta_{m}(r) \overline{\eta_{k}}(r) \neq 0$. Then, for some irreducible constituent $\chi^{*}$ of $\eta_{m} \overline{\eta_{\kappa}}$, the sum $\sum_{r} \chi^{*}(r)$ does not vanish. If we express $\chi^{*}(r)$ by the modular irreducible characters, it follows from the orthogonality relations for modular characters $[9$, 
$\S 8]$ that $\chi^{*} \in B_{0}(2, H)$. Here $\chi^{*} \neq \chi_{0}^{*}$ since $\kappa \neq m$. Now (1) shows that $\chi^{*}(1) \geqq q^{2}-q$ and hence $q^{2}-q \leqq \eta_{m}(1) \eta_{\kappa}(1)$. By (22) and (23), $\eta_{k}(1)<4 q / 3-\eta_{m}(1)$. Thus,

$$
q^{2}-q<\eta_{m}(1)\left(4 q / 3-\eta_{m}(1)\right) \leqq(2 q / 3)^{2} .
$$

Since this implies $q<9 / 5$, we have a contradiction. Thus, the case $\Xi_{2} \neq 0$ cannot arise and the proof of Theorem $\mathrm{A}$ is complete.

5. Subsections associated with a 2-block of full defect. As before, let $G$ be a $Q D$ group or a $Q$-group. We shall discuss the 2-blocks of full defect $n+1$ applying the general results of [7]. The fixed prime $p$ will always be taken as 2 . The 2-section of a 2-element $u$ of $G$ will be denoted by $\mathscr{S}(u)$. This is the set of all elements $w$ of $G$ whose 2-factor $w_{2}$ is conjugate to $u$.

We first note that

$$
N(S)=S C(S)=S \times E ; E=O(C(S)) .
$$

Here, $E$ is a subgroup of odd order. The 2-blocks of $G$ of full defect are in one-to-one correspondence with the irreducible characters $\theta$ of $E$. We may consider $\theta$ as an irreducible character of $N(S)$, which is trivial on $S$, i.e. for which $\theta(u)=\theta(1)$ for $u \in S$. If $b_{\theta}$ is the block of $N(S)$ containing $\theta$, the corresponding block of $G$ is $b_{\theta}^{G}$. This block will be denoted by $B_{\theta}$. Thus, our correspondence is given by

$$
\theta \rightarrow B_{\theta}=b_{\theta}^{G}
$$

If $\theta=1$ is the principal character of $E$, then $B_{\theta}$ is the principal block $B_{0}(2, G)$ of $G$.

By a subsection $(u, b)$ of a section $\mathscr{S}(u)$, we mean a pair consisting of a 2-element $u$ of $G$ and a 2-block $b$ of $C(u)$. The subsection is associated with the 2-block $B$ of $G$, if $b^{G}=B$. It suffices to take $u$ in a fixed set of representatives for the conjugate classes of 2-elements of $G$. In particular, we shall always take $u \in S$.

Our first aim is to determine the subsections $(u, b)$ associated with a fixed 2block $B_{\theta}$ of full defect. In other words, we wish to characterize the 2-blocks $b$ of $C(u)$ for which

$$
b^{G}=B_{\theta}
$$

for a given irreducible character $\theta$ of $E$. We first show:

Proposition 1. Let $u$ be an element of $S$ of order more than 4. There exists a unique subsection $(u, b)$ of $\mathscr{S}(u)$ associated with $B_{\theta}$. Here, $b$ is the unique block $b_{u}$ of $C(u)$ covered by $b_{\theta}^{S C(u)}$ (in the sense of $[7, \mathrm{I}]$ ). The defect group of $b_{u}$ is the cyclic subgroup $S_{0}=\langle s\rangle$ of $S$ of order $2^{n}$. If $u \in S_{0}$ has order 4 and if $b_{u}$ is defined in the same way, then $\left(u, b_{u}\right)$ is the only subsection of $\mathscr{S}(u)$ of defect more than 2 associated with $B_{\theta}$. The defect group of $b_{u}$ in this case is still $S_{0}$.

Proof. Let $(u, b)$ be a subsection associated with $B_{\theta}$ and let $D_{1}$ be its defect group. Since $\langle u\rangle$ is normal in $C(u)$, we have $u \in D_{1}[5, \mathrm{I},(9 \mathrm{~F})]$. If $u \in S_{0}$ has order at 
least 4 , then $S_{0}$ is a Sylow 2-group of $C(u)$ and we may choose $D_{1} \subseteq S_{0}$. Of course $D_{1}$ is cyclic and in the cases considered in Proposition 1, its order is at least 8.

Set $M=N(\langle u\rangle)$. It is clear that $S C(u)$ is contained in $M$ and that $M / C(u)$ is a 2group. Hence $M=S C(u),|M: C(u)|=2$. Since $C\left(D_{1}\right) \subseteq C(u)$, the block $b^{M}$ of $M$ is defined [5, II, (2E)]. Let $D_{2} \supseteq D_{1}$ be a defect group of $b^{M}$. We have

$$
\left(b^{M}\right)^{G}=b^{G}=B_{\theta} .
$$

This implies that $D_{2}$ is contained in a defect group of $B_{\theta}$, i.e., in a quasi-dihedral group. It follows that the cyclic subgroup $D_{1}$ of order at least 8 is characteristic in $D_{2}$. Then $\langle u\rangle$ is also characteristic in $D_{2}$ and hence

$$
N\left(D_{2}\right) \subseteq N(\langle u\rangle)=M \text {. }
$$

Now, the first main theorem on blocks [5, I, II] shows that $D_{2}$ is a defect group of $\left(b^{M}\right)^{G}=B_{\theta}$. Then $\left|D_{2}\right|=2^{n+1}$ and $D_{2}$ can be chosen as the Sylow 2-subgroup $S$ of $M$. Since $N(S) \subseteq M$, the first main theorem implies

$$
b^{M}=b_{\theta}^{M} .
$$

It follows from [7, I,(4D)] that the block $b$ of $C(u)$ is covered by the block $b_{\theta}^{M}$ of $M$.

Conversely, let $b$ be a block of $C(u)$ covered by $b_{\theta}^{M}$. Since $b_{\theta}^{M}$ has the defect group $S$ and since $C(S) \subseteq C(u), b_{\theta}^{M}$ is regular with regard to $C(u)$ and we conclude that $b_{\theta}^{M}=b^{M}[7, \mathrm{I},(4 \mathrm{D})]$. This implies (3). Moreover, as $b_{\theta}^{M}$ has full defect, it contains an irreducible character $\chi^{*}$ of odd degree. Since $|M: C(u)|=2, \chi^{*} \mid C(u)$ is irreducible. As it lies in $b, b$ is unique and $b$ also has full defect, i.e. the Sylow 2-subgroup $S_{0}$ of $C(u)$ is a defect group of $b$. This completes the proof.

Proposition 2. The subsection $\left(x, b_{x}\right)$ with $b_{x}=b_{\theta}^{N}$ is associated with $B_{\theta}$ and its defect group is $S$. It is the only subsection of $\mathscr{S}(x)$ associated with $B_{\theta}$ whose defect group is not elementary abelian of order 4.

Proof. It is clear that $N(S) \subseteq N$ and then $\left(b_{\theta}^{N}\right)^{G}=b_{\theta}^{G}=B_{\theta}$. The first statement is now obvious.

Conversely, assume that the subsection $(x, b)$ is associated with $B_{\theta}$ and let $D_{1}$ denote its defect group. We may assume that $D_{1}$ belongs to the Sylow 2-subgroup $S$ of $N$. Since $x \in D_{1}$, we see that either $x$ is the unique involution in the center of $D_{1}$ or that $D_{1}$ is elementary abelian of order 4 . If the latter case is excluded, $\langle x\rangle$ is characteristic in $D_{1}$ and hence

$$
N\left(D_{1}\right) \subseteq N(\langle x\rangle)=N .
$$

It then follows from the first main theorem on blocks that $b^{G}=B_{\theta}$ has the defect group $D_{1}$. Thus, $D_{1}=S$, and then $b=b_{\theta}^{N}$ as we wished to show.

We now study the cases not covered by the first two propositions. We use the method developed in $[7, \mathrm{I}, \S 6]$. We summarize some of the results. 
Let $D_{1}$ be a 2-subgroup of $G$ and let $b_{1}$ be a 2-block of $F_{1}=D_{1} C\left(D_{1}\right)$ with the defect group $D_{1}$. We construct a sequence

$$
D_{1}, D_{2}, \ldots, D_{h}
$$

of 2-subgroups of $G$ and of 2-blocks $b_{j}$ of $F_{j}=D_{j} C\left(D_{j}\right)$ with the defect group $D_{j}$ as follows. Suppose that, for some $j \geqq 1$, the group $D_{j}$ and the block $b_{j}$ of $F_{j}$ have already been obtained.

Case 1. There exists a subgroup $H_{j} \supset F_{j}$ of $N\left(D_{j}\right)$ with $\left|H_{j}: F_{j}\right|=2$ such that $b_{j}$ is stable in $H_{j}$.

Choose $D_{j+1}$ as defect group of the (well-defined) block $b_{j}^{H}$, of $H_{j}$ and choose $b_{j+1}$ as a block of $F_{j+1}=D_{j+1} C\left(D_{j+1}\right)$ with

$$
b_{j}^{H_{j}}=b_{j+1}^{H_{j}}
$$

(which is possible).

Case 2. No group $H_{j+1}$ with the required properties exists.

Then we terminate the sequence (4) with $D_{j}$, i.e. we take $h=j$.

Our conditions imply that

$$
D_{j} \subset D_{j+1}, \quad\left|D_{j+1}: D_{j}\right|=2
$$

and that

$$
H_{j}=D_{j+1} C\left(D_{j}\right), \quad D_{j+1} \cap F_{j}=D_{j}, \quad H_{j} / F_{j} \simeq D_{j+1} / D_{j}
$$

for $1 \leqq j<h$. We have the result that $b_{1}^{G}$ has full defect, if and only if $D_{h}$ is a Sylow 2-subgroup $S^{w}$ of $G, w \in G$. If this is so, we have $b_{1}^{G}=B_{\theta}$ if and only if $b_{h}=b_{\theta}^{w}$. We are using here the fact that because of (1), we have

$$
N(S)^{w}=(S C(S))^{w}=D_{h} C\left(D_{h}\right)=F_{h} ;
$$

and that therefore $b_{\theta}^{w}$ is the only block $B^{*}$ of $F_{h}$ with $\left(B^{*}\right)^{G}=B_{\theta}$.

In general, if $D_{1}$ and $D_{h}$ are 2-subgroups of $G$, if $b_{1}$ is a 2-block of $D_{1} C\left(D_{1}\right)$ with the defect group $D_{1}$ and if $b_{h}$ is a 2-block of $D_{h} C\left(D_{h}\right)$ with the defect group $D_{h}$, we say that $b_{1}$ and $b_{h}$ are linked, if there exist groups $D_{j}$ and blocks $b_{j}$ for $1 \leqq j \leqq h$ for which the conditions above are satisfied.

We can now study subsections $(x, b)$ associated with $B_{\theta}$ which are not given by Proposition 2, i.e. which have defect 2. In the terminology of [7], they are minor subsections. In some of the later developments the occurrence of such minor subsections will play an important role.

We first introduce some notation. With $S=\langle s, z\rangle$ as before, set

$$
s_{j}=s^{2^{n-j}}, \quad T_{j}=\left\langle s_{j}, z\right\rangle, \quad(1 \leqq j \leqq n) .
$$

Thus, $s_{j}$ has order $2^{j}, s_{n}=s, s_{1}=x, s_{2}=y$. Also, $\left|T_{j}\right|=2^{j+1}, T_{n}=S$, and $T_{1}=T$ is elementary abelian of order 4 . For $1<j<n, T_{j}$ is dihedral. As is seen without difficulty,

$$
N\left(T_{j}\right)=T_{j+1} C\left(T_{j}\right) \text { for } 1<j<n .
$$


If $G$ is a $Q$-group, this is still true for $j=1$. However, if $G$ is a $Q D$-group, then $t$ as defined in $\S 1$, equation (1) lies in $N(T)$. Also, $y \in N(T)$. Since $N(T) / C$ is isomorphic to a subgroup of the symmetric group on 3 letters, we now see easily that

$$
N\left(T_{1}\right)= \begin{cases}\langle C, y\rangle, & (G \text { a } Q \text {-group }) \\ \langle C, y, t\rangle, & (G \text { a } Q D \text {-group }) .\end{cases}
$$

We next prove two lemmas.

LEMMA 1. If $D_{j} \supseteq T$ is a subgroup of order $2^{j+1}$ of $S(1 \leqq j \leqq n)$, then $D_{j}=T_{j}$.

This is obvious, since $z \in D_{j}$ and since $\left|D_{j} \cap S_{0}\right| \geqq 2^{j}$, whence $s_{j} \in D_{j}$.

LEMma 2. There exists a unique block $b_{C}$ of $C$ which is linked with the block $b_{\theta}$ of $S C(S)$. We have $\left(b_{C}\right)^{y}=b_{C}$.

Proof. Since the Sylow 2-subgroup $T$ of $C$ is normal in $C$, the group $T$ is the unique defect group of any block $b_{1}$ of $C$. Likewise, $S$ is the unique defect group of $b_{\theta}$. If $b_{1}$ and $b_{\theta}$ are linked and if we use the same notation as in the definition of linkage, we have $D_{1}=T, D_{h}=S$ and, necessarily, $\left|D_{j}\right|=2^{j+1}, h=n$. It follows from Lemma 1 that $D_{j}=T_{j}$ for $1 \leqq j \leqq n$.

Conversely, if we choose $D_{j}=T_{j}, F_{j}=D_{j} C\left(D_{j}\right)$ for $1 \leqq j \leqq n$ and $H_{j}=D_{j+1} C\left(D_{j}\right)$ for $j<n$, the relations (6) and (7) hold.

Suppose that $b_{j+1}$ is a block of $F_{j+1}$ with the defect group $D_{j+1}, j<n$. Since $C\left(D_{j+1}\right) \subseteq F_{j+1}$, the block $B^{*}=b_{j+1}^{H_{j}}$ of $H_{j}$ is defined. A suitable defect group contains $D_{j+1}$ and, as $D_{j+1}$ is a Sylow 2-subgroup of $H_{j}$, the group $D_{j+1}$ itself is a defect group of $B^{*}$. Since $C\left(D_{j+1}\right) \subseteq C\left(D_{j}\right) \subseteq F_{j}$, it is now clear that $B^{*}$ is regular with regard to $F_{j}$ and that there exist blocks $b_{j}$ of $F_{j}$ with

$$
b_{j}^{H_{j}}=B^{*}=b_{j+1}^{H_{j}},
$$

cf. [7, I,(4B), (4D)]. Moreover, $B^{*}$ covers $b_{j}$. Since $B^{*}$ has full defect in $H_{j}$, there exists an irreducible character $\chi^{*} \in B^{*}$ of odd degree. Then all irreducible constituents of $\chi^{*} \mid F_{j}$ have odd degree and as some of these constituents lie in $b_{j}$, the block $b_{j}$ has full defect. Clearly, $D_{j}$ is a Sylow 2-subgroup of $F_{j}$ and it is now clear that $D_{j}$ is a defect group of $b_{j}$. Since $B^{*}$ has larger defect than $b_{j}$, it follows from [7, I, (5C)] that $b_{j}$ is stable in $H_{j}$. In particular, $b_{j}$ is the only block of $F_{j}$ covered by $B^{*}$. This implies that $b_{j}$ is uniquely determined by $b_{j+1}$.

If we start with $b_{n}=b_{\theta}$ and apply our argument successively for $j=n-1$, $n-2, \ldots, 1$, we obtain a block $b_{1}=b_{C}$ of $C$ which is linked with $b_{\theta}$. Moreover, we see at the same time that $b_{C}$ is unique. Since $b_{C}$ is stable in $H_{1}$ and since $y \in T_{2}$, we have $b_{C}^{y}=b_{C}$ and the proof is complete.

We now show:

Proposition 3. If $G$ is a $Q$-group, there do not exist minor subsections $(x, b)$ associated with $B_{\theta}$. The same is true in the case of $Q D$-groups, if and only if $b_{C}^{t}=b_{C}$ 
for the block $b_{C}$ defined in Lemma 2. If $b_{C}^{t} \neq b_{C}$, there exists a unique minor subsection $(x, b)$ associated with $B_{\theta}$. Here, $b=\left(b_{C}^{t}\right)^{N}$; the defect group is $T$.

Proof. Suppose that $(x, b)$ is a minor subsection associated with $\boldsymbol{B}_{\boldsymbol{\theta}}$. Proposition 2 shows that the defect group $D_{1}$ is elementary abelian of order 4 . Since $D_{1}$ can be replaced by a conjugate in $N$, we may take $D_{1}=T$. There exist then blocks $b_{1}$ of $F_{1}=C\left(D_{1}\right)=C$ with the defect group $T$ such that

$$
b_{1}^{N}=b .
$$

Since $b$ and $b_{1}$ have the same defect, the stabilizer of $b_{1}$ in $N(T) \cap N$ has odd index over $C,[5, \mathrm{II}(2 \mathrm{~F})]$. In particular, this implies

$$
b_{1}^{y} \neq b_{1} .
$$

Apply now the construction above using the same notation as before. Since $b_{1}^{G}=B_{\theta}$ by (11) and (3), the group $D_{h}$ is a conjugate $S^{w}$ of $S, w \in G$. As $\left|D_{j}\right|=2^{j+1}$, we have $h=n$. We also note that $b_{n}=b_{\theta}^{w}$ and that by (6), (7)

$$
F_{j} \subset H_{j} \subseteq N\left(D_{j}\right), \quad\left|H_{j}: F_{j}\right|=2 .
$$

For $j=1, F_{1}=C$ and $H_{1} / F_{1}$ is a subgroup of order 2 of $N(T) / C$. If $G$ is a $Q$ group, (10) shows that $H_{1}=\langle C, y\rangle$. However, since $b_{1}$ is stable in $H_{1}$, this leads to a contradiction with (12). This case is impossible and we have already proved the first statement of the proposition.

Suppose then that $G$ is a $Q D$-group. Now (10) shows that $N(T)$ has exactly three subgroups $\langle C, y\rangle,\left\langle C, y^{t}\right\rangle,\left\langle C, y^{t^{-1}}\right\rangle$ which contain $C$ as subgroup of index 2 . Since $y \notin H_{1}$ on account of (12), we must have $H_{1}=\left\langle C, y^{t^{\prime}}\right\rangle$, where $t^{\prime}=t^{ \pm 1}$. Since $t^{\prime}$ and $y$ normalize $T$, the group $H_{1}$ has the Sylow 2-subgroup $\left\langle T, y^{t^{\prime}}\right\rangle=\langle T, y\rangle^{t^{\prime}}=T_{2}^{t^{\prime}}$. As $\left|D_{2}\right|=8=\left|T_{2}^{t^{\prime}}\right|$, we may choose the Sylow 2-group $T_{2}^{t^{\prime}}$ as defect group $D_{2}$ of the block $b_{1}^{H_{1}}$. Suppose that we have already shown for some $j \geqq 2$ that we can take $D_{j}=T_{j}^{t^{\prime}}$. It follows immediately from (13) and (9) that

$$
H_{j}=N\left(T_{j}^{t^{\prime}}\right)=T_{j+1}^{t^{\prime}} C\left(T_{j}^{t^{\prime}}\right)
$$

and that we may choose $D_{j+1}$ as the Sylow 2-subgroup $T_{j+1}^{t^{\prime}}$ of $H_{j}$.

Hence we can assume that $D_{j}=T_{j}^{t^{\prime}}$ for all $j$. In particular, $D_{n}=S^{t^{\prime}}$ and the element $w$ can be taken as $t^{\prime}$.

The blocks $b_{1}$ and $b_{n}=b_{\theta}^{w}=b_{\theta}^{t^{\prime}}$ are linked. If we transform all $D_{j}, F_{j}, H_{j}$ and the blocks $b_{j}$ by $\left(t^{\prime}\right)^{-1}$, we see that the block $b_{1}^{\left(t^{\prime}\right)-1}$ of $C^{\left(t^{\prime}\right)^{-1}}=C$ is linked with $b_{\theta}$. Now, Lemma 2 yields $b_{1}^{\left(t^{\prime}\right)^{-1}}=b_{C}$. Thus, $b_{1}=b_{C}^{t}$ or $b_{1}=b_{C}^{t^{-1}}$. Both choices for $b_{1}$ yield the same $b$ in (11) since $b_{C}^{y}=b_{C}$ and, by equation (2) of $\S 1$,

$$
\left(b_{C}^{t}\right)^{N}=\left(b_{C}^{y t}\right)^{N}=\left(b_{C}^{t^{-1} y}\right)^{N}=\left(\left(b_{C}^{t^{-1}}\right)^{N}\right)^{y}=\left(b_{C}^{t^{-1}}\right)^{N} .
$$

Hence we may take $b_{1}=b_{C}^{t}, b=\left(b_{C}^{t}\right)^{N}$. The stabilizer of $b_{C}$ in $N(T)$ includes $\langle C, y\rangle$. If the stabilizer was not $\langle C, y\rangle$ itself, it would be $N(T)$ and then (12) would be 
false. In other words, if $b_{C}^{t}=b_{C}$, we have a contradiction and no minor subsection $(x, b)$ can exist.

On the other hand, if $b_{C}^{t} \neq b_{C}$, the stabilizer of $b_{C}$ in $N(T)$ is $\langle C, y\rangle$ and the stabilizer of $b_{1}=b_{C}^{t}$ is $\left\langle C, y^{t}\right\rangle$. Then $b_{1}$ has the stabilizer $C$ in $N(T) \cap N$ and, by [7, I,(5C)], $b=b_{1}^{N}$ has the same defect group $T$ as $b_{1}$. Since

$$
b^{G}=b_{1}^{G}=\left(b_{C}^{t}\right)^{G}=\left(b_{C}^{G}\right)^{t}=b_{C}^{G}=B_{\theta},
$$

the subsection $(x, b)$ is associated with $B_{\theta}$, its defect group is $T$. This completes the proof.

A similar argument can be used to discuss if there exist subsections $(y, b)$ of defect 2 associated with $B_{\theta}$. We shall need only the following result:

Proposition 4. If $(y, b)$ is a subsection of defect 2 associated with $B_{\theta}$, the analogous statement is true, if $G$ is replaced by $N$ and $B_{\theta}=b_{\theta}^{G}$ is replaced by $B_{\theta}^{*}=b_{\theta}^{N}$.

Proof. Suppose that the subsection $(y, b)$ is associated with $\boldsymbol{B}_{\theta}$ and that it is not the subsection obtained in Proposition 1 for $y=u$. Then $b$ has defect 2 and since its defect group $D_{1}$ contains $y$, we have $D_{1}=\langle y\rangle$. We use again the construction above with $D_{1}=\langle y\rangle, b_{1}=b$. Again, $D_{h}$ must be a conjugate of $S$ and $h=n$. It is seen easily that $D_{2}, D_{3}, \ldots, D_{n}$ are nonabelian groups with the center $\langle x\rangle$, [7, I,(7A), (7B)]. It follows that the groups $D_{j}, F_{j}, H_{j}$ lie in $N$ and that the whole construction remains the same, if $G$ is replaced by $N$. It is now clear that the subsection $(y, b)$ of $N$ is associated with $B_{\theta}^{*}=b_{\theta}^{N}$. Of course, its defect group remains $\langle y\rangle$.

If $G$ is a $Q D$-group, all conjugate classes of 2-elements of $G$ contain elements $u \in S_{0}$. If $u$ has order 4 , we can choose $u=y$. Hence our propositions give information concerning all 2-singular subsections associated with $B_{\theta}$. In the case of $Q$ groups, there is one other class, that of $z$.

Proposition 5. If $G$ is a $Q$-group, there exists a unique subsection $\left(z, b_{z}\right)$ associated with $B_{\theta}$. Here, $b_{z}=\left(b_{C}\right)^{C(z)}$, where $b_{C}$ is defined in Lemma 2. The defect group is $T$.

Proof. If $G$ is a $Q$-group, the Sylow 2-subgroup of $C(z)$ is $T$. If $(z, b)$ is associated with $B_{\theta}$, the defect group $D_{1}$ of $b$ can be chosen as a subgroup of $T$. Since $z \in D_{1}$ and since $D_{1}=\langle z\rangle$ is impossible, $D_{1}=T$. Then there exists a block $b_{1}$ of $C$ with $b_{1}^{C(z)}=b$. Again, we use the construction above. Since $G$ is a $Q$-group, $N(T)$ $=\langle C, y\rangle$ by (10). Then we must have $H_{1}=\langle C, y\rangle$ and we can choose $D_{2}=T_{2}$. It follows successively that we may take $D_{j}=T_{j}$ for $j=3,4, \ldots, n$. Hence $b_{n}=b_{\theta}$ and Lemma 2 shows that $b_{1}=b_{C}$. Then, $b=b_{C}^{C(z)}$. Conversely, if $b$ is this block, then $(z, b)$ is associated with $B_{\theta}$.

6. Continuation. Blocks of full defect of type $\left(^{*}\right)$ and the corresponding decomposition numbers. In continuing the work in $\$ 5$, we shall now study the decomposition numbers belonging to a 2-block $B_{\theta}$ of full defect. It will suffice for 
our purpose to consider only characters $\theta$ of $N(S)$ which satisfy the following condition:

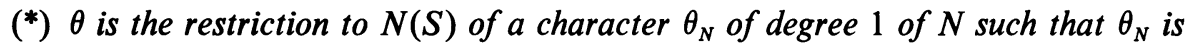
trivial on $S$.

Of course, then $\theta$ is trivial on $S$ as required in $\S 5$. If the condition (*) is satisfied we shall say that $B_{\theta}$ is a block of type $\left(^{*}\right)$. Clearly, the principal 2-block $B_{0}(2, G)$ of $G$ is of type (*).

If $\theta$ satisfies (*) and if $M$ is a subgroup of $N$ we set

$$
\theta_{M}=\theta_{N} \mid M \text {. }
$$

If $B_{0}(2, M)$ is the principal 2-block of $M$ and if we multiply all the irreducible characters in $B_{0}(2, M)$ with $\theta_{M}$, we obtain the characters of a block of $M$ which we denote by $\theta_{M} B_{0}(2, M)$. It is clear that $\theta_{M} B_{0}(2, M)$ has full defect and that it contains the linear character $\theta_{M}$. We prove a lemma.

LEMMA 1. If $\theta$ satisfies the condition (*) and if $M$ is a subgroup of $N$ which contains $N(S)$, then $\theta_{M} B_{0}(2, M)=\left(b_{\theta}\right)^{M}$, where $b_{\theta}$ (as in $\left.\S 5\right)$ is the block of $N(S)=S \times E$ which contains $\theta$.

Proof. Since $\theta_{M} B_{0}(2, M)$ has the defect group $S$ and since $N_{M}(S)=S \times E$, there exists an irreducible character $\theta_{1}$ of $S \times E$ which is trivial on $S$ such that

$$
\theta_{M} B_{0}(2, M)=\left(b_{\theta_{1}}\right)^{M} .
$$

We claim that if $\mathfrak{p}$ is a suitable prime ideal divisor of 2 , then for $r \in E$, we have

$$
\theta_{M}(r) \equiv \theta_{1}(r) \quad(\bmod \mathfrak{p})
$$

Indeed, we can apply [7, II,(2D)] to the character $\theta_{M} \in\left(b_{\theta_{1}}\right)^{M}$. Since here $N_{M}(S)=S C(S)$, the residue system $R$ can be taken as $\{1\}$. The canonical character in $b_{\theta_{1}}$ is $\theta_{1}$. Since $\theta_{M}$ has height 0 , the rational number $A_{i}$ in [7, II,(2D)] can be written with odd numerator and denominator and we obtain (3).

Since $E$ has odd order, the congruence (3) implies that $\theta_{M} \mid E=\theta_{E}$ and $\theta_{1} \mid E$ have an.irreducible constituent in common. Since both are irreducible, they are equal. Since $\theta$ and $\theta_{1}$ are trivial on $S$, then $\theta=\theta_{1}$, and the statement is obvious from (2).

For blocks $B_{\theta}$ of type $\left(^{*}\right)$, we can now supplement Proposition 5.1.

Proposition 1. If $B_{\theta}$ is a block of type (*), no subsection $(y, b)$ of defect 2 is associated with $B_{\theta}$.

Proof. It follows immediately from Proposition 5.4 that it suffices to prove this in the special case where $G=N$. The lemma with $M=N$ shows that then

$$
B_{\theta}=b_{\theta}^{G}=b_{\theta}^{N}=\theta_{N} B_{0}(2, N) .
$$

If the proposition was not true, there would exist a block $b$ of $C(y)$ of defect 2 for which

$$
b^{N}=\theta_{N} B_{0}(2, N)
$$


If $b^{*}$ is the block of $C(y)$ obtained by multiplying the characters in $b$ with $\theta_{c(y)}^{-1}$, it follows that

$$
\left(b^{*}\right)^{N}=B_{0}(2, N) \text {. }
$$

However, since $b^{*}$ also has defect 2 and not full defect in $C(y)$, this is incompatible with [6, I, Theorem 3]. This establishes the proposition.

We can also characterize the block $b_{C}$ in Lemma 5.2 in a more explicit form.

Proposition 2. Let $B_{\theta}$ be a block of type (*). The block $b_{C}$ in Lemma 5.2 is the block of $C$ which contains $\theta_{C}$ as its canonical character. In particular, there exists a minor subsection $(x, b)$ associated with $B_{\theta}$ if and only if $G$ is a $Q D$-group (not a $Q$ group) and if $\theta_{C}^{t} \neq \theta_{C}$ for the element $t$ in $\S 1,(1)$. Then, $b=\left(b_{C}^{t}\right)^{N}$.

Proof. We choose again $F_{j}=T_{j} C\left(T_{j}\right), H_{j}=T_{j+1} C\left(T_{j}\right)$. Let $b_{j}^{*}$ denote the block of $F_{j}$ which contains the character $\theta_{F_{j}}, 1 \leqq j \leqq n$. Then $b_{j}^{*}$ has full defect and the Sylow 2-subgroup $T_{j}$ of $F_{j}$ is a defect group of $b_{j}^{*}$. Since $\theta_{N}$ is trivial on $S, \theta_{F_{j}}$ is trivial on $T_{j}$. It is now clear that $\theta_{F}$, is the canonical character of $b_{j}^{*}$. It is also clear that for $j<n, \theta_{F_{j}}$ and hence $b_{j}^{*}$ are stable in $H_{j}$. Moreover

$$
\theta_{C\left(T_{j}\right)} \mid C\left(T_{j+1}\right)=\theta_{C\left(T_{j+1}\right)} .
$$

It now follows from $[7, \mathrm{I},(6 \mathrm{D})]$ that

$$
\left(b_{j+1}^{*}\right)^{H_{j}}=\left(b_{j}^{*}\right)^{H_{j}} .
$$

In the terminology introduced in $\S 5$, the block $b_{1}^{*}$ of $C$ is linked with the block $b_{n}^{*}$ of $N(S)=S C(S)$. Since $\theta=\theta_{N(S)}$ belongs to $b_{n}^{*}$, we have $b_{n}^{*}=b_{\theta}$. Now Lemma 5.2 shows that $b_{1}^{*}=b_{C}$. Hence $\theta_{F_{1}}=\theta_{C}$ is the canonical character of $b_{C}$. The remaining statements in Proposition 2 are immediate consequences of Proposition 5.3.

We review some developments given in [6]. If $B$ is a $p$-block, we consider columns $R$ of complex numbers whose length is the number of irreducible characters $\chi \in B$. We use these $\chi$ as row indices for the column $R$. Thus, for each $\chi \in B$, we have a coefficient $R_{\chi}$ of $R$. If all $R_{x}$ are rational integers, we speak of an integral column. The (unitary) inner product $\left(R, R^{\prime}\right)$ of two columns is defined as

$$
\left(R, R^{\prime}\right)=\sum_{x} R_{x} \overline{R_{\chi}^{\prime}}
$$

with $\chi$ ranging over the irreducible characters of $B$.

If $w$ is a fixed element of $G$, we can form the column $R$ with $R_{\chi}=\chi(w)$. This column will be denoted by $X(w),(X(w))_{x}=\chi(w)$.

If $u$ is a $p$-element of $G$ and if $\phi$ is a modular irreducible character of $C(u)$, we can form the column $D^{(u)} \phi$ whose entry for $\chi \in B$ is the generalized decomposition number $d^{(u)}(\chi, \phi)$ belonging to $\chi$ and $\phi$. The main result of $[5, \mathrm{II}]$ asserts that $D^{(u)}(\phi)$ is the zero column 0 , except when $\phi$ belongs to a $p$-block $b$ of $C(u)$ with 
$b^{G}=B$, i.e. when the subsection $(u, b)$ is associated with $B$. This implies that if $r$ is a $p$-regular element of $C(u)$, we have

$$
X(u r)=\sum_{b} \sum_{\phi} D^{(u)}(\phi) \phi(r)
$$

where $b$ ranges over all $p$-blocks of $C(u)$ for which $(u, b)$ is associated with $B$. For each $b, \phi$ ranges over the modular irreducible characters of $b$. If $u^{\prime}$ is also a $p$ element of $G$ and $\phi^{\prime}$ a modular irreducible character of $C\left(u^{\prime}\right)$, then

$$
\left(D^{(u)}(\phi), D^{\left(u^{\prime}\right)}\left(\phi^{\prime}\right)\right)=0
$$

if $u$ and $u^{\prime}$ are not conjugate in $G$. On the other hand, if $u=u^{\prime}$, we have

$$
\left(D^{(u)}(\phi), D^{(u)}\left(\phi^{\prime}\right)\right)=c\left(\phi, \phi^{\prime}\right),
$$

where $c\left(\phi, \phi^{\prime}\right)$ is the Cartan invariant of $C(u)$ belonging to the modular irreducible characters $\phi, \phi^{\prime}$ of $C(u)$. In all these considerations, we can replace the set of modular irreducible characters of a block $b$ of $C(u)$ by an arbitrary basic set for $b$ $[6, \mathrm{I}, \S \mathrm{V}]$.

We return now to the particular case of groups $G$ considered in this paper. The following lemma is obvious from Chapter II or from [6, III, §VIII].

Lemma 2. Let $G$ be a $Q D$-group or a $Q$-group. Form the sets

$$
Y_{1}=\left\{ \pm 1, \pm 3, \ldots, \pm\left(2^{n-2}-1\right)\right\}, \quad Y_{2}=\left\{2,4, \ldots, 2^{n-1}-2\right\} .
$$

The elements $s^{\alpha}$ with $\alpha \in Y_{2}$ form a set of representatives for the conjugate classes of $G$ consisting of elements of an order $2^{\mu}$ with $2 \leqq \mu \leqq n-1$. The elements $s^{\beta}$ with $\beta \in Y_{1}$ form a set of representatives for the conjugate classes of $G$ consisting of elements of order $2^{n}$. Set $m=2^{n-1}$. Then two powers $s^{i}$ and $s^{j}$ of $s$ are conjugate in $G$ if and only if $j \equiv i$ or $j \equiv i(m-1)\left(\bmod 2^{n}\right)$.

In the rest of $\S 6, Y_{1}, Y_{2}$ and $m$ will always have the same significance as in Lemma 2. We shall reserve the letters $\alpha, \alpha^{\prime}$ for indices ranging over $Y_{2}$ and the letters $\beta, \beta^{\prime}$. for indices ranging over $Y_{1}$.

Proposition 3. Let $B_{\theta}$ be a fixed block of type (*). For each $u=s^{j}$ with $j \in Y_{1} \cup Y_{2}$, we have a column $R(u)$ such that, for each 2-regular $r \in C(u)$, we have $X(u r)=R(u) \theta_{N}(r)$. Moreover, the columns $R(u), R\left(u^{\prime}\right)$ belonging to different elements $u, u^{\prime}$ of the form $s^{j}$ with $j \in Y_{1} \cup Y_{2}$ are (unitarily) orthogonal, while $(R(u), R(u))=2^{n}$.

Proof. It follows from Propositions 5.1 and 6.1 that there exists a unique subsection $\left(u, b_{u}\right)$ associated with $B_{\theta}$. Here, $b_{u}$ is the block of $C(u)$ covered by $\left(b_{\theta}\right)^{S C(u)}$. Since $S_{0} \subseteq C(u)$, it is clear that $|S C(u): C(u)|=2$. On account of Lemma 1 , we have

$$
\left(b_{\theta}\right)^{S C(u)}=\theta_{S C(u)} B_{0}(2, S C(u)) \text {. }
$$

It is now clear that this block covers the block $\theta_{C(u)} B_{0}(2, C(u))$. Thus,

$$
b_{u}=\theta_{C(u)} B_{0}(2, C(u)) \text {. }
$$


Since $C(u)$ has a cyclic Sylow 2-subgroup $S_{0}$, it has a normal 2-complement. This implies that the only modular irreducible character in $B_{0}(2, C(u))$ is the modular 1-character $[9, \$ 29]$ and then our formula for $b_{u}$ shows that the only modular irreducible character $\phi_{u}$ in $b_{u}$ is $\theta_{C(u)}$ or, rather the restriction of $\theta_{C(u)}$ to the set of 2-regular elements of $C(u)$. Moreover, since $b_{u}$ has defect $n$, the Cartan invariant $c\left(\phi_{u}, \phi_{u}\right)$ is $2^{n}$, e.g. by [5, I,(6C)]. If we set $R(u)=D^{(u)}\left(\phi_{u}\right)$, the proposition is immediate from (4) and (5).

Consider next the case $u=x$. Here, $C(u)=N$. By Proposition 5.2 and Lemma 1, the major subsection $\left(x, b_{x}\right)$ associated with $N$ is given by

$$
b_{x}=b_{\theta}^{N}=\theta_{N} B_{0}(2, N) \text {. }
$$

Set $\bar{N}=N /\langle x\rangle$. The principal 2-block $B_{0}(2, \bar{N})$ can be considered as a subset of $B_{0}(2, N)$. Since $\langle x\rangle$ is a 2-group, both blocks $B_{0}(2, N)$ and $B_{0}(2, \bar{N})$ contain the same modular irreducible characters, [5]. Hence a basic set for $B_{0}(2, \bar{N})$ can serve as a basic set for $B_{0}(2, N)$. The Cartan invariants in the latter case are twice those for $B_{0}(2, \bar{N})$.

The Sylow 2-subgroup $\bar{S}$ of $\bar{N}$ is dihedral of order $2^{n}$. The central involution in $\bar{S}$ is the image $\bar{y}$ of $y$. It is clear then that $\bar{y}$ is not conjugate in $\bar{N}$ to the involution $\bar{z} \in \bar{S}$. On the other hand, since $G$ is a $Q D$-group or a $Q$-group, the elements $y$ and $s z$ of order 4 are conjugate in $G$. Since both elements have the square $x$, they are conjugate in $C(x)=N$. It follows that the images $\bar{y}$ and $\bar{s} \bar{z}$ in $\bar{S}$ are conjugate in $\bar{N}$. It is now clear that in $\bar{N}$, we have the situation discussed in [6, III, §VII]. In the notation employed there, we have Case II. As shown there, $B_{0}(2, \bar{N})$ contains exactly four irreducible characters of odd degrees. Two of these degrees are 1 , the other two have the same value $q_{0} \neq 1$. [We shall see later by the argument used in the proof of Proposition 2.7 that $q_{0}=q$.] Both characters of degree $q_{0}$ have the same restriction $\psi$ to the set of 2-regular elements of $\bar{N}$. We can then choose

$$
\phi_{0}=1, \quad \phi_{1}=1+e^{*} \psi
$$

as basic set for $B_{0}(2, \bar{N})$. Here, $\varepsilon^{*}$ is a sign which can be fixed by the condition

$$
q_{0}=\psi(1) \equiv \varepsilon^{*}+2^{n-1} \quad\left(\bmod 2^{n}\right)
$$

or, equivalently, by

$$
\phi_{1}(1) \equiv 2+2^{n-1} \quad\left(\bmod 2^{n}\right) .
$$

The corresponding Cartan matrix $\left({ }^{8}\right)$ is

$$
\left(\begin{array}{cc}
4 & -2 \\
-2 & 2^{n-2}+1
\end{array}\right)
$$

$\left(^{8}\right)$ Cf. [6, III,(7J)]. In the notation used there, $\varepsilon^{*}$ is $\delta_{1}$. Also, $\varepsilon^{*} \phi_{1}$ is the restriction of the character denoted there by $\chi^{(1)}$ to the set of 2-regular elements. 
As already remarked, then 1 and $\phi_{1}$ (considered as functions defined on $N$ ) form a basic set for $B_{0}(2, N)$. The Cartan invariants are twice those obtained for $B_{0}(2, \bar{N})$. Then (6) shows that

$$
\theta_{N} \phi_{0}=\theta_{N}, \theta_{N} \phi_{1}
$$

form a basic set for $b_{x}$. It is seen easily that the Cartan invariants are the same as for $\phi_{0}, \phi_{1}$. (For instance, we can use [9, (21)].)

Form now the columns $U_{0}=D^{(x)}\left(\theta_{N}\right), U_{1}=D^{(x)}\left(\theta_{N} \phi_{1}\right)$. In applying (4), we have to observe that there may exist a minor subsection $(x, b)$ associated with $B_{\theta}$. We obtain

Proposition 4. There exist two integral columns $U_{0}$ and $U_{1}$ for $B_{\theta}$ which belong to the major subsection $\left(x, b_{x}\right)$ associated with $B_{\theta}$. We have

$$
\left(U_{0}, U_{0}\right)=8, \quad\left(U_{0}, U_{1}\right)=-4, \quad\left(U_{1}, U_{1}\right)=2^{n-1}+2 .
$$

Moreover, these columns are unitarily orthogonal to the columns $R(u)$ of Proposition 3 and to the columns $X\left(r_{0}\right)$ for 2-regular $r_{0} \in G$. For 2-regular $r \in N$, we have

$$
X(x r)=U_{0} \theta_{N}(r)+U_{1} \phi_{1}(r) \theta_{N}(r)+X^{*}(x r) .
$$

Here $X^{*}$ is the contribution to (4) of the minor subsection $(x, b)$ if such a minor subsection exists, and $X^{*}=0$ otherwise.

Our aim is to determine explicitly the columns $R(u)$ of Proposition 3 and $U_{0}, U_{1}$ of Proposition 4. We shall apply a method used already in [6].

Let $\eta$ denote a generalized character of $S_{0}$. We form the column

$$
A(\eta)=2^{-n} \sum_{u} X(u) \overline{\eta(u)}
$$

where $u$ ranges over $S_{0}$. Since $A(\eta)_{\chi}=\left(\chi \mid S_{0}, \eta\right)$ for $\chi \in B_{0}$, it is clear that $A(\eta)$ is an integral column.

LEMmA 3. If $\eta_{1}$ and $\eta_{2}$ are two generalized characters of $S_{0}$ which vanish for 1 and $x$, then

$$
\left(A\left(\eta_{1}\right), A\left(\eta_{2}\right)\right)=\left(\eta_{1}, \eta_{2}+\eta_{2}^{z}\right),
$$

where the inner product on the right is the usual inner product of generalized characters of $S_{0}$.

Proof. We have

$$
2^{2 n}\left(A\left(\eta_{1}\right), A\left(\eta_{2}\right)\right)=\sum_{u, u^{\prime}}\left(X(u), X\left(u^{\prime}\right)\right) \eta_{1}(u) \overline{\eta_{2}\left(u^{\prime}\right)}
$$

where $u, u^{\prime}$ range over $S_{0}$. It suffices to take $u$ and $u^{\prime}$ different from 1 and $x$. It follows from Proposition 3 and (5a) that $\left(X(u), X\left(u^{\prime}\right)\right)$ vanishes if $u^{\prime}$ is not conjugate to $u$ in $G$, i.e. if $u^{\prime} \neq u, u^{z}$. If $u^{\prime}$ is conjugate to $u$, then $\left(X(u), X\left(u^{\prime}\right)\right)=2^{n}$. We obtain

and the lemma becomes evident.

$$
2^{n}\left(A\left(\eta_{1}\right), A\left(\eta_{2}\right)\right)=\sum_{u} \eta_{1}(u)\left(\overline{\eta_{2}}(u)+{\overline{\eta_{2}}}^{z}(u)\right)
$$


If $\zeta$ is a fixed primitive $2^{n}$ th root of unity, we denote by $\lambda$ the linear character of $S_{0}$ for which $\lambda(s)=\zeta$. It follows from the orthogonality relations for group characters and (9) that

$$
X(u)=\sum_{i=0}^{2^{n}-1} A\left(\lambda^{i}\right) \lambda^{i}(u)
$$

for $u \in S_{0}$. Clearly, $\lambda^{i}$ remains unchanged if $i$ is changed $\left(\bmod 2^{n}\right)$. Since $u$ and $u^{m-1}$ (with $m=2^{n-1}$ ) are conjugate in $G$, we have

$$
\begin{aligned}
2^{n} A\left(\lambda^{i}\right) & =\sum_{u} X(u) \lambda^{-i}(u)=\sum_{u} X\left(u^{m-1}\right) \lambda^{-i}(u) \\
& =\sum_{u} X(u) \lambda^{-i}\left(u^{m-1}\right)=2^{n} A\left(\lambda^{i(m-1)}\right) .
\end{aligned}
$$

Thus,

$$
A\left(\lambda^{i}\right)=A\left(\lambda^{i(m-1)}\right)
$$

We now form the columns

$$
V_{\beta}=A\left(\lambda-\lambda^{\beta}\right) \text { for } \beta \in Y_{1}, \quad V_{j}=A\left(1-\lambda^{j}\right) \text { for } j \in Y_{2} \text { and } j=m .
$$

Of course, $V_{1}$ is the zero column 0 .

We note that the generalized characters $\lambda-\lambda^{\beta}, 1-\lambda^{\alpha}, 1-\lambda^{m}$ vanish on $\{1, x\}$. Hence Lemma 3 can be applied. We find

LeMma 4. For $\alpha, \alpha^{\prime} \in Y_{2}$ and $\beta, \beta^{\prime} \in Y_{1}-\{1\}$ we have

$$
\begin{array}{lll}
\left(V_{\alpha}, V_{\alpha^{\prime}}\right)=2+\delta_{\alpha, \alpha^{\prime}}, & \left(V_{\beta}, V_{\beta^{\prime}}\right)=1+\delta_{\beta, \beta^{\prime}}, & \left(V_{\alpha}, V_{\beta}\right)=0, \\
\left(V_{m}, V_{m}\right)=4, & \left(V_{m}, V_{\alpha}\right)=2, & \left(V_{m}, V_{\beta}\right)=0 .
\end{array}
$$

We note next

LEMMA 5. The columns $V_{\alpha}, V_{\beta}, V_{m}, U_{0}, U_{1}$ are rational integral. Each $V_{i}$ is unitarily othogonal to each $U_{j}$. Moreover, if $r \in G$ is 2-regular, each $V_{i}$ and each $U_{j}$ is unitarily orthogonal to $X(r)$. No linear combination of columns $V_{i}$ and $U_{j}$ can contain a single nonzero coefficient. With the exception of $V_{1}=0$, each of the columns $V_{i}, U_{j}$ has both positive and negative coefficients.

Proof. Since as remarked above the columns $A(\eta)$ are rational integral, so are the columns $V_{i}$. Since $x$ is an involution, the generalized decomposition numbers for the section $\mathscr{S}(x)$ are integers in the field of the second roots of unity, i.e. in the rational field. This remains true if basic sets are used. Hence $U_{0}, U_{1}$ are rational integral columns. The definition of the columns $V_{i}$ in conjunction with (9) shows that each $V_{i}$ is a linear combination of columns $X(u)$ with $u \in S_{0}-\{1, x\}$. Now all the orthogonality relationships stated in the lemma are immediate consequences of (5a). Since $X(1)$ has only positive coefficients, no column $T$ orthogonal to $X(1)$ can have a single nonzero entry. If $T$ has real coefficients and is not zero it has both 
positive and negative coefficients. It follows from Proposition 4 and Lemma 4 that $U_{i}$ and $V_{j}$ with $j \neq 1$ do not vanish.

We also need the following lemma:

LEMMA 6. If $h$ is a member of $Y_{1} \cup Y_{2}$, then

$$
X\left(s^{h}\right)=-\sum_{j} V_{j}\left(\zeta^{h j}+(-\zeta)^{-h j}\right)-V_{m} \zeta^{m h},
$$

where $j$ ranges over the elements of $Y_{1} \cup Y_{2}$. Moreover,

$$
\begin{aligned}
X(x) & \equiv-2 \sum_{\alpha} V_{\alpha}+2 \sum V_{\beta}-V_{m}\left(\bmod 2^{n-1}\right) \\
& \equiv U_{0}+2 U_{1} .
\end{aligned}
$$

Proof. If $u \in S_{0}$, then on account of (11), the formula (10) can be written in the form

$$
\begin{aligned}
X(u)= & A(1)+A\left(\lambda^{m}\right) \lambda^{m}(u)+\sum_{\alpha} A\left(\lambda^{\alpha}\right)\left(\lambda^{\alpha}(u)+\lambda^{\alpha(m-1)}(u)\right) \\
& +\sum_{\beta} A\left(\lambda^{\beta}\right)\left(\lambda^{\beta}(u)+\lambda^{\beta(m-1)}(u)\right) .
\end{aligned}
$$

If we introduce the $V_{i}$ from (12), we find

$$
\begin{aligned}
X(u)= & A(1) \sum_{k} \lambda^{k}(u)+A(\lambda) \sum_{l} \lambda^{l}(u)-V_{m} \lambda^{m}(u) \\
& -\sum_{\alpha} V_{\alpha}\left(\lambda^{\alpha}(u)+\lambda^{\alpha(m-1)}(u)-\sum_{\beta} V_{\beta}\left(\lambda^{\beta}(u)+\lambda^{\beta(m-1)}(u)\right),\right.
\end{aligned}
$$

where $k$ ranges over the even indices with $0 \leqq k<2^{n}$ and where $l$ ranges over the odd indices with $0 \leqq l<2^{n}$.

If $u=s^{h}$, then $\lambda(u)=\zeta^{n}$ is a $2^{n}$ th root of unity. If $u \neq 1, x$, we have $\lambda(u) \neq \pm 1$. The sums

$$
\sum_{k} \lambda^{k}(u), \quad \sum_{l} \lambda^{l}(u)
$$

can be computed easily as sums of geometric progressions. Both sums (13) vanish and we obtain the first part of the lemma.

For $u=x, \lambda^{k}(u)=1$ for even $k$ and $\lambda^{l}(u)=-1$ for odd $l$. Hence the sums (13) are equal to $\pm 2^{n-1}$ and we obtain the first congruence for $X(x)$. On the other hand, we can apply Proposition 4 with $r=1$ and find

$$
X(x)=U_{0}+U_{1} \phi_{1}(1)+X^{*}(x) .
$$

Here, $\phi_{1}(1) \equiv 2\left(\bmod 2^{n-1}\right)$. If no minor subsection $(x, b)$ is associated with $B_{0}, X^{*}=0$ and we obtain the last part of the lemma. If a minor subsection $(x, b)$ occurs, then $b$ has defect 2 by Proposition 5.2. Then the degree of a modular irreducible character in $b$ is divisible by $2^{n-1}$. This means that in (4) for $u=x$, the 
degrees $\phi(1)$ are divisible by $2^{n-1}$ for all $\phi$ which do not belong to the major subsection $\left(x, b_{x}\right)$. Since the coefficients of $D^{(u)}(\phi)$ are algebraic integers, we find

$$
X^{*}(x) \equiv 0 \quad\left(\bmod 2^{n-1}\right)
$$

and the last part of the lemma holds in this case too.

We can now state the basic result of this section.

Proposition 5. Let $G$ be a $Q D$-group or a $Q$-group and let $B_{6}$ be a fixed block of type (*). Then $B_{\theta}$ consists of $4+2^{n-1}$ irreducible characters. These can be denoted by $\chi_{0}, \chi_{1}, \chi_{2}, \chi_{3}, \chi_{4}, \chi^{(\alpha)}$ with $\alpha \in Y_{2}, \chi^{(\beta)}$ with $\beta \in Y_{1}$ in such a way that the columns $V_{m}, V_{\alpha}$ with $\alpha \in Y_{2}, V_{\beta}$ with $\beta \in Y_{1}-\{1\}, U_{0}, U_{1}$ are as follows:

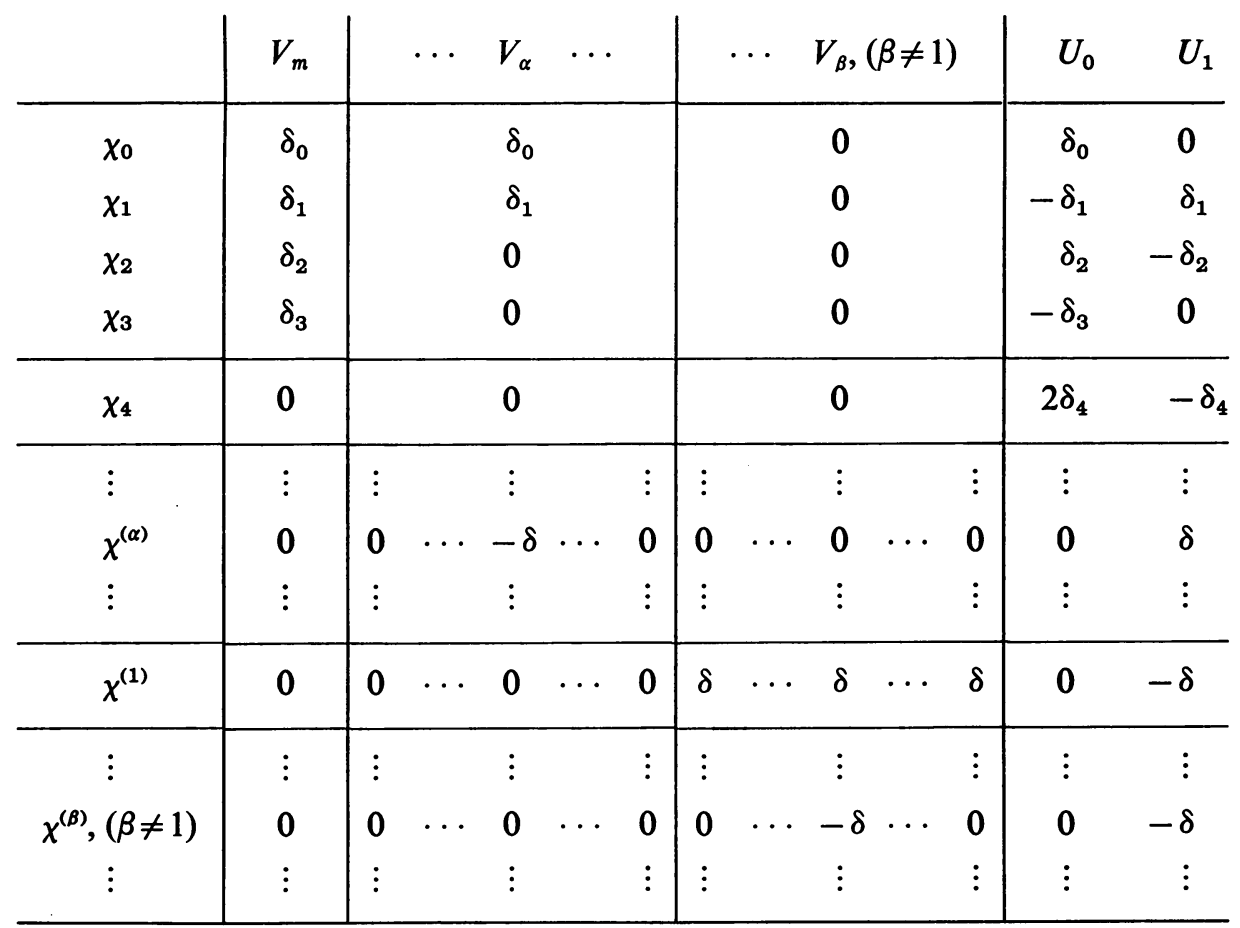

Here, $\delta_{0}, \delta_{1}, \delta_{2}, \delta_{3}, \delta_{4}$ and $\delta$ are signs \pm 1 .

REMARK. The characters $\chi_{i}, \chi^{(\alpha)}, \chi^{(\beta)}$ and the signs $\delta_{i}, \delta$ depend on $\theta$. If necessary, this will be indicated by adding $\theta$ as a superscript.

Proof. Since $\left(V_{m}, V_{m}\right)=4$, the column $V_{m}$ has four nonzero coefficients and these are necessarily all $\pm 1\left({ }^{9}\right)$. Since $V_{m} \equiv U_{0}(\bmod 2)$ by Lemma $6, U_{0}$ has odd coefficients in the rows for which $V_{m}$ has coefficients \pm 1 and even coefficients in the other rows. Since $\left(U_{0}, U_{0}\right)=8$, one of these even coefficients is \pm 2 , the others

$\left({ }^{9}\right)$ The results of Lemmas 4 and 5 as well as the values of the inner products given in Proposition 4 are used constantly throughout the proof. No explicit reference will be made. 
are 0 and the four odd coefficients are \pm 1 . Finally, as $\left(V_{m}, U_{0}\right)=0$ we see that we can choose characters $\chi_{0}, \chi_{1}, \chi_{2}, \chi_{3}, \chi_{4}$ in $B_{\theta}$ and signs $\delta_{0}, \delta_{1}, \delta_{2}, \delta_{3}, \delta_{4}$ such that the columns $V_{m}$ and $U_{0}$ have the form given in the proposition. We shall denote the coefficient of a column $R$ for a character $\chi_{i}$ by $R_{i}$. Likewise, if in the following, a character of $B_{\theta}$ is denoted by $\chi^{(j)}$, the corresponding coefficient of $R$ will be denoted by $R^{(j)}$.

If $\alpha \in Y_{2}$, we have $\left(V_{\alpha}, V_{\alpha}\right)=3$, and hence $V_{\alpha}$ has three nonzero coefficients each of which is \pm 1 . Since $\left(V_{\alpha}, V_{m}\right)=2,\left(V_{\alpha}, U_{0}\right)=0$, we find

$$
\delta_{0}\left(V_{\alpha}\right)_{0}+\delta_{2}\left(V_{\alpha}\right)_{2}+\delta_{4}\left(V_{\alpha}\right)_{4}=\delta_{1}\left(V_{\alpha}\right)_{1}+\delta_{3}\left(V_{\alpha}\right)_{3}-\delta_{4}\left(V_{\alpha}\right)_{4}=1 \text {. }
$$

If here $\left(V_{\alpha}\right)_{4}=\delta_{4}$, necessarily $\delta_{1}\left(V_{\alpha}\right)_{1}=\delta_{3}\left(V_{\alpha}\right)_{3}=1$ and then $\left(V_{\alpha}\right)_{0}=\left(V_{\alpha}\right)_{2}=0$. It follows that $V_{m}-U_{0}-2 V_{\alpha}$ has a single nonzero coefficient, which is impossible. Likewise, if $\left(V_{\alpha}\right)_{4}=-\delta_{4}$, we obtain $\delta_{0}\left(V_{\alpha}\right)_{0}=\delta_{2}\left(V_{\alpha}\right)_{2}=1$ and then $\left(V_{\alpha}\right)_{1}=\left(V_{\alpha}\right)_{3}=0$. Again, this is impossible, since $V_{m}+U_{0}-2 V_{\alpha}$ would have a single nonzero coefficient.

Hence $\left(V_{\alpha}\right)_{4}=0$ for all $\alpha \in Y_{2}$. It follows that

$$
\left(V_{\alpha}\right)_{0}=\delta_{0}, \quad\left(V_{\alpha}\right)_{2}=0 \quad \text { or } \quad\left(V_{\alpha}\right)_{0}=0, \quad\left(V_{\alpha}\right)_{2}=\delta_{2},
$$

and

$$
\left(V_{\alpha}\right)_{1}=\delta_{1}, \quad\left(V_{\alpha}\right)_{3}=0 \quad \text { or } \quad\left(V_{\alpha}\right)_{1}=0, \quad\left(V_{\alpha}\right)_{3}=\delta_{3} .
$$

Moreover, there must exist a character $\chi$ in $B_{\theta}$ different from $\chi_{0}, \chi_{1}, \ldots, \chi_{4}$ for which $V_{\alpha}$ has a coefficient \pm 1 . Let $\chi^{(\alpha)}$ designate this character and set $\left(V_{\alpha}\right)^{(\alpha)}=-\delta^{(\alpha)}= \pm 1$.

Since we can interchange $\chi_{0}$ and $\chi_{2}$ and also $\chi_{1}$ and $\chi_{3}$, we can assume that for $\alpha=2$, we have the first alternative in both cases above. If we set $\delta=\delta^{(2)}$, then $V_{2}$ has the form stated in the proposition.

Suppose that for some $\alpha \in Y_{2}$, we do not have $\left(V_{\alpha}\right)_{0}=\delta_{0},\left(V_{\alpha}\right)_{1}=\delta_{1}$. Since $Y_{2}$ for $n=3$ consists of 2 only, necessarily $n>3$ and $\left|Y_{2}\right|=2^{n-2}-1 \geqq 3$. The above discussion applies to $V_{\alpha}$. Since $\left(V_{2}, V_{\alpha}\right)=2$, we see easily that we have one of the cases:

Case (a). $\left(V_{\alpha}\right)_{0}=\delta_{0},\left(V_{\alpha}\right)_{3}=\delta_{3},\left(V_{\alpha}\right)^{(2)}=-\delta$,

Case (b). $\left(V_{\alpha}\right)_{1}=\delta_{1},\left(V_{\alpha}\right)_{2}=\delta_{2},\left(V_{\alpha}\right)^{(2)}=-\delta$,

while all other coefficients of $V_{\alpha}$ vanish. Choose now $\alpha^{\prime} \in Y_{2}$ different from 2 and $\alpha$. If we do not have $\left(V_{\alpha^{\prime}}\right)_{0}=\delta_{0},\left(V_{\alpha^{\prime}}\right)_{1}=\delta_{1}$, then for $V_{\alpha^{\prime}}$ we must have Case (a) or Case (b) and then we could not have $\left(V_{\alpha}, V_{\alpha^{\prime}}\right)=2$. On the other hand, if $\left(V_{\alpha^{\prime}}\right)_{0}=\delta_{0}$, $\left(V_{\alpha^{\prime}}\right)_{1}=\delta_{1}$ then $\left(V_{\alpha}, V_{\alpha^{\prime}}\right)=2$ implies that $\left(V_{\alpha^{\prime}}\right)^{(2)}=-\delta$. It then follows that $V_{\alpha^{\prime}}=V_{2}$. This is impossible, since $\left(V_{2}, V_{2}-V_{\alpha^{\prime}}\right)=1$.

Thus, for all $\alpha \in Y_{2}$, we have $\left(V_{\alpha}\right)_{0}=\delta_{0},\left(V_{\alpha}\right)_{1}=\delta_{1}$. Since $\left(V_{\alpha}, V_{\alpha^{\prime}}\right)=2$ for $\alpha, \alpha^{\prime} \in Y_{2}$ with $\alpha \neq \alpha^{\prime}$, the characters $\chi^{(\alpha)}$ with $\alpha \in Y_{2}$ are necessarily distinct. If a column $R$ is orthogonal to all $V_{\alpha}$, we have

$$
\delta_{0} R_{0}+\delta_{1} R_{1}-\delta^{(\alpha)} R^{(\alpha)}=0 .
$$

This implies $\delta^{(2)} R^{(2)}=\delta^{(\alpha)} R^{(\alpha)}$. If we take here $R=X(1)$, we see that all $\delta^{(\alpha)}$ are equal. If we set $\delta=\delta^{(\alpha)}$, the columns $V_{\alpha}$ have the form stated in the proposition. 
If a column $R$ is orthogonal to $V_{m}$, we have

$$
\delta_{0} R_{0}+\delta_{1} R_{1}+\delta_{2} R_{2}+\delta_{3} R_{3}=0 .
$$

If $R$ is orthogonal to $V_{m}$ and $U_{0}$, then

$$
\delta_{0} R_{0}+\delta_{2} R_{2}+\delta_{4} R_{4}=0 .
$$

In particular, (14), (15), (16) hold for $R=V_{\beta}$ with $\beta \in Y_{1}-\{1\}$. Since $\left(V_{\beta}, V_{\beta}\right)=2$, each $V_{\beta}$ has two nonzero coefficients, one of which is +1 and the other -1 . It follows first that $R_{4}=0$, since for $R_{4} \neq 0$, the second nonzero coefficient would be $R_{0}$ or $R_{2}$ and then (15) could not hold. Thus $R_{4}=0$ and $\delta_{0} R_{0}=-\delta_{2} R_{2}$. If $R_{0} \neq 0$, then $R_{1}=0, R^{(\alpha)}=0$ and (14) would not hold. Hence $R_{0}=R_{2}=0$. Again, if $R_{1} \neq 0$, then $R_{3} \neq 0$ and hence $R^{(\alpha)}=0$. Hence (14) could not hold. Thus, $R_{1}=0$ and this now implies $R_{3}=0, R^{(\alpha)}=0$. We now see that the nonzero entries of $V_{\beta}$ occur for characters of $B_{\theta}$ different from $\chi_{0}, \chi_{1}, \ldots, \chi_{4}, \chi^{(\alpha)}$.

Suppose first that $n>3$. Then $3 \in Y_{1}$. Since $\left(V_{-1}, V_{3}\right)=1$, there exists a character $\chi^{(1)} \in B_{\theta}$ such that $\left(V_{-1}\right)^{(1)}=\left(V_{3}\right)^{(1)}=\delta^{*}$, where $\delta^{*}$ is \pm 1 . We also must have two characters in $B_{\theta}$ to be denoted by $\chi^{(-1)}$ and $\chi^{(3)}$ such that $\left(V_{-1}\right)^{(-1)}=-\delta^{*}$, $V_{3}^{(3)}=-\delta^{*}$. Consider now any $\beta \neq 1,-1,3$ in $Y_{1}$. Since $\left(V_{\beta}, V_{-1}\right)=\left(V_{\beta}, V_{3}\right)=1$, it follows easily that $\left(V_{\beta}\right)^{(1)}=\delta^{*}$. Moreover, there exists a character $\chi^{(\beta)} \in B_{\theta}$ for which $\left(V_{\beta}\right)^{(\beta)}=-\delta^{*}$. It is also clear that the $2^{n-2}=\left|Y_{1}\right|$ characters $\chi^{(\beta)}$ are distinct. Let $V_{\beta}^{*}$ denote the columns given in the proposition for $V_{\beta}$. It is now clear that $V_{\beta}=\delta \delta^{*} V_{\beta}^{*}$ (i.e. we shall have to show $\delta=\delta^{*}$ eventually).

For $n=3, Y_{1}=\{ \pm 1\}$. It is here clear that we can choose $\chi^{(1)}, \chi^{(-1)}$ such that $V_{-1}$ has the value given in the proposition. We may assume $\delta=\delta^{*}$ in this case.

In order to find $U_{1}$, we apply the congruence in Lemma 6 . Using the values obtained for the columns $V_{\alpha}, V_{\beta}, V_{m}, U_{0}$, we obtain easily that

$$
\begin{aligned}
& \left(U_{1}\right)_{0} \equiv 0, \quad\left(U_{1}\right)_{1} \equiv \delta_{1}, \quad\left(U_{1}\right)_{2} \equiv-\delta_{2}, \quad\left(U_{1}\right)_{3} \equiv 0 \quad\left(\bmod 2^{n-2}\right) . \\
& \left(U_{1}\right)_{4} \equiv-\delta_{4}, \quad\left(U_{1}\right)^{(\alpha)} \equiv \delta, \quad\left(U_{1}\right)^{(\beta)} \equiv-\delta^{*} \quad
\end{aligned}
$$

If there exist $\chi \in B_{\theta}$ different from $\chi_{0}, \ldots, \chi_{4}, \chi^{(\alpha)}, \chi^{(\beta)}$, then $\left(U_{1}\right)_{\chi} \equiv 0\left(\bmod 2^{n-2}\right)$. It follows that $U_{1}$ has exactly

$$
3+\left|Y_{2}\right|+\left|Y_{1}\right|=3+\left(2^{n-2}-1\right)+2^{n-2}=2^{n-1}+2
$$

odd coefficients. Since $\left(U_{1}, U_{1}\right)=2^{n-1}+2$, the odd coefficients of $U_{1}$ are \pm 1 and the even coefficients are 0 . If $n \geqq 4$, it is now clear that our congruences for the coefficients of $U_{1}$ are actually equalities.

We claim that this is still true when $n=3$, where $Y_{1}=\{ \pm 1\}, Y_{2}=\{2\}$. Our argument shows that $\left(U_{1}\right)_{0}=0,\left(U_{1}\right)_{3}=0$ and that if characters $\chi \in B_{\theta}$ different from $\chi_{0}, \chi_{1}, \ldots, \chi_{4}, \chi^{(2)}, \chi^{( \pm 1)}$ exist, then $\left(U_{1}\right)_{\chi}=0$. Since $\left(U_{1}, U_{0}+V_{m}\right)=-4$, we have

$$
2 \delta_{2}\left(U_{1}\right)_{2}+2 \delta_{4}\left(U_{1}\right)_{4}=-4
$$

and as $\left(U_{1}\right)_{2}= \pm 1,\left(U_{1}\right)_{4}= \pm 1$, this implies that $\left(U_{1}\right)_{2}=-\delta_{2},\left(U_{1}\right)_{4}=-\delta_{4}$. It now 
follows from $\left(U_{1}, V_{m}\right)=0$ that $\left(U_{1}\right)_{1}=\delta_{1}$. Since $\left(U_{1}, V_{2}\right)=0$, we find $\left(U_{1}\right)^{(2)}=\delta$. Finally, as $\left(U_{1}, V_{-1}\right)=0$, we have

$$
U_{1}^{(1)}=U_{1}^{(-1)}= \pm 1 .
$$

If we have $U_{1}^{(1)}=\delta^{*}$, we interchange $\chi^{(1)}$ and $\chi^{(-1)}$ which is permitted for $n=3$. Then $\delta^{*}$ is replaced by $-\delta^{*}$ while $U_{1}^{(1)}$ remains the same. Hence we have $U_{1}^{(1)}=U_{1}^{(-1)}=-\delta^{*}$ after the interchanging. This shows that our result concerning $U_{1}$ still holds for $n=3$.

Consider a column $R$ which is orthogonal to all $V_{\alpha}, V_{m}, V_{\beta}, U_{0}, U_{1}$. Then (14), (15), (16) hold, all $R^{(\alpha)}$ are equal, all $R^{(\beta)}$ are equal and

$$
\delta_{1} R_{1}-\delta_{2} R_{2}-\delta_{4} R_{4}+\left(2^{n-2}-1\right) \delta R^{(\alpha)}-2^{n-2} \delta^{*} R^{(\beta)}=0 .
$$

Add here (16) and use (14). We obtain

$$
\delta R^{(\alpha)}=\delta^{*} R^{(\beta)} \quad\left(\alpha \in Y_{2}, \beta \in Y_{1}\right) .
$$

If we take here $R=X(1)$, we find $\delta=\delta^{*}$. It now follows that the columns $V_{\beta}$ and $U_{1}$ have the form stated in Proposition 5. The proof of the proposition will be complete when we can show that $B_{\theta}$ does not contain any character $\chi$ different from $\chi_{0}, \ldots, \chi_{4}, \chi^{(\alpha)}, \chi^{(\beta)}$. If such $\chi$ existed, we would have

$$
\left(U_{0}\right)_{x}=\left(U_{1}\right)_{\chi}=0 .
$$

However, this is impossible on account of Theorem [7, II,(4C)] since the decomposition number $d(\chi, \phi)$ would vanish for each modular irreducible character $\phi \in b_{x}$ and since $\left(x, b_{x}\right)$ is a major subsection associated with $\boldsymbol{B}_{\theta}$.

We now give some consequences of Proposition 5 and the lemmas used in the proof of the proposition. We have already noted that if a column $R$ is orthogonal to the columns $V_{\alpha}$ with $\alpha \in Y_{2}, V_{\beta}$ with $\beta \in Y_{1}, V_{m}, U_{0}, U_{1}$ the equations (14), (15), (16) and (17) hold and that $\delta=\delta^{*}$. If $r$ is a 2-regular element of $G$, we can take $R=X(r)$. This yields

COROLlaRY 1. If $r$ is a 2-regular element of $G$, we have

$$
\begin{aligned}
& \delta_{0} \chi_{0}(r)+\delta_{1} \chi_{1}(r)+\delta_{2} \chi_{2}(r)+\delta_{3} \chi_{3}(r)=0, \\
& \delta_{0} \chi_{0}(r)+\delta_{1} \chi_{1}(r)=\delta \chi^{(\alpha)}(r)=\delta \chi^{(\beta)}(r),
\end{aligned}
$$

for $\alpha \in Y_{1}, \beta \in Y_{2}$ and

$$
\delta_{0} \chi_{0}(r)+\delta_{2} \chi_{2}(r)+\delta_{4} \chi_{4}(r)=0 .
$$

In particular, we may take $r=1$.

COROLlaRY 2. All $\chi^{(\alpha)}$ and $\chi^{(\beta)}$ have the same degree $f$. If $\chi_{i}$ has degree $f_{i}$ for $0 \leqq i \leqq 4$, we have

$$
\begin{gathered}
\delta_{0} f_{0}+\delta_{1} f_{1}+\delta_{2} f_{2}+\delta_{3} f_{3}=0 \\
\delta_{0} f_{0}+\delta_{1} f_{1}-\delta f=0, \quad \delta_{0} f_{0}+\delta_{2} f_{2}+\delta_{4} f_{4}=0
\end{gathered}
$$


If $u=s^{h}$ has order at least 4 , we can compute $X(u)$ from Lemma 6 and Proposition 5. If $r \in C(u)$ is 2-regular, by Proposition 3, we have $X(u r)=X(u) \theta_{N}(r)$. In this manner the following result is obtained without difficulty.

COROLlARY 3. Let $u=s^{h}$ be an element of $S_{0}$ of order at least 4 and let $r$ be a 2-regular element of $C(u)$. If $\zeta$ is a fixed $2^{n}$ th root of unity, then

$$
\begin{gathered}
\delta_{0} \chi_{0}(u r)=\delta_{1} \chi_{1}(u r)=\theta_{N}(r), \quad \delta_{2} \chi_{2}(u r)=\delta_{3} \chi_{3}(u r)=(-1)^{h+1} \theta_{N}(r), \\
\chi_{4}(u r)=0, \quad \chi^{(j)}(u r)=\delta\left(\zeta^{j h}+(-\zeta)^{-j h}\right)
\end{gathered}
$$

for $j \in Y_{1} \cup Y_{2}$.

We have already used [7, II,(4C)] in the proof of Proposition 5. Actually, this theorem shows that the exact power of 2 dividing the degree $\chi(1)$ of a character $\chi \in B_{\theta}$ is the same as the exact power of 2 dividing $\left(U_{0}\right)_{x}+\left(U_{1}\right)_{x} \phi_{1}(1)$. If we substitute the values obtained in Proposition 5 and use (8), we find

Corollary 4. The degrees $f_{0}, f_{1}, f_{2}, f_{3}$ are odd. We have $f_{4} \equiv 2^{n-1}\left(\bmod 2^{n}\right)$ and $f \equiv 2(\bmod 4)$.

In other words, $\chi_{0}, \chi_{1}, \chi_{2}, \chi_{3}$ have "height" $0, \chi_{4}$ has the maximal possible height $n-1$ and the $\chi^{(\alpha)}$ and $\chi^{(\beta)}$ have height 1 .

It will be convenient to separate now the case that $B_{\theta}$ has a minor subsection $(x, b)$ and the case that no minor subsection exists.

COROllary 5. Let $B_{\theta}$ denote a fixed block of type $\left(^{*}\right)$ such that no minor subsection $(x, b)$ is associated with $B_{\theta}$. Let $\psi$ denote either one of the two irreducible characters of odd degree different from 1 in the principal block $B_{0}(2, N /\langle x\rangle)$ and define the sign $\varepsilon^{*}= \pm 1$ by the condition $\psi(1) \equiv \varepsilon^{*}(\bmod 4)$. If $r$ is a 2-regular element of $N$, we have

$$
\begin{aligned}
\chi_{0}(x r) & =\delta_{0} \theta_{N}(r), \quad \chi_{1}(x r)=\delta_{1} \varepsilon^{*} \psi(r) \theta_{N}(r), \\
\chi_{2}(x r) & =-\delta_{2} \varepsilon^{*} \psi(r) \theta_{N}(r), \quad \chi_{3}(x r)=-\delta_{3} \theta_{N}(r), \\
\chi_{4}(x r) & =-\delta_{4}\left(\varepsilon^{*} \psi(r)-1\right) \theta_{N}(r), \\
\chi^{(j)}(x r) & =\delta(-1)^{j}\left(\varepsilon^{*} \psi(r)+1\right) \theta_{N}(r)
\end{aligned}
$$

for $j \in Y_{1} \cup Y_{2}$.

This is immediate from Propositions 4 and 5 and (7). Note that here $X^{*}=0$.

COROLlaRY 6. Let $G$ be a $Q D$-group. Let $B_{\theta}$ be a block of type (*) without minor subsection. Then the degrees $f_{i}$ satisfy the following congruences $\left(\bmod 2^{n+1}\right)$ : $\delta_{0} f_{0} \equiv 1, \delta_{1} f_{1} \equiv 2-\varepsilon^{*} \psi(1), \delta_{2} f_{2} \equiv-\varepsilon^{*} \psi(1), \delta_{3} f_{3} \equiv-1+2^{n}$. In particular, $f_{0}, f_{1}, f_{2}, f_{3}$ are distinct.

Proof. If $\chi$ is a character of $G$, we have $\sum \chi(u) \equiv 0\left(\bmod 2^{n+1}\right)$, where $u$ ranges over $S$. Since $G$ is a $Q D$-group, every $u \neq 1$ is conjugate to an element $s^{\alpha}, s^{\beta}$, or $x$ and then $\chi(u)$ can be obtained from Corollaries 3 and 5 . If we substitute these 
values, we obtain the congruences stated in Corollary 6 , since $\varepsilon^{*} \psi(1) \equiv 1+2^{n-1}$ $\left(\bmod 2^{n}\right)$. It is now seen easily that $f_{0}, f_{1}, f_{2}, f_{3}$ are distinct.

Assume next that a minor subsection $(x, b)$ is associated with $B_{\theta}$. Then $b$ is a block of $N$ of defect 2 . Since $b$ corresponds to a block $\bar{b}$ of $N /\langle x\rangle$ of defect $1, b$ contains a single modular irreducible character $\phi^{\#}$ and the Cartan invariant $c\left(\phi_{b}, \phi_{b}\right)$ has the value 4 . Since $b$ has defect $2, \phi^{\#}(1) \equiv 2^{n-1}\left(\bmod 2^{n}\right)$. If we set $R=D^{(x)}\left(\phi^{\#}\right)$, the column $R$ is orthogonal to the columns $X(r)$ for 2-regular $r \in G$ and the columns $V_{\alpha}, V_{\beta}, V_{m}$. It also follows from (5b) that $R$ is orthogonal to $U_{0}, U_{1}$. Finally, $(R, R)=4$. Hence $R$ has four nonzero coefficients. These have the values \pm 1 and both signs occur. As in the proof of Corollary 1 , we see that $R^{(\alpha)}=R^{(\beta)}$ for all $\alpha \in Y_{2}, \beta \in Y_{1}$. Also,

$$
\delta_{0} R_{0}+\delta_{1} R_{1}=\delta R^{(\alpha)}=-\delta_{2} R_{2}-\delta_{3} R_{3}, \quad \delta_{0} R_{0}+\delta_{2} R_{2}+\delta_{4} R_{4}=0 .
$$

If $R^{(\alpha)} \neq 0$, we would have at least five nonzero coefficients, which is impossible. Hence $R^{(\alpha)}=R^{(\beta)}=0$. Since an even number of $R_{0}, R_{1}, R_{2}, R_{3}$ are different from 0 , they are all different from 0 and $R_{4}=0$. If we set $\delta_{0} R_{0}=\delta^{\#}$, then $\delta^{\#}= \pm 1$ and

$$
R_{0}=\delta_{0} \delta^{\#}, \quad R_{1}=-\delta_{1} \delta^{\#}, \quad R_{2}=-\delta_{2} \delta^{\#}, \quad R_{3}=\delta_{3} \delta^{\#} .
$$

If $r$ is a 2-regular element of $G$, the relation $(R, X(r))=0$ now yields

$$
\delta_{0} \chi_{0}(r)-\delta_{1} \chi_{1}(r)-\delta_{2} \chi_{2}(r)+\delta_{3} \chi_{3}(r)=0 .
$$

Then, by Corollary 1 ,

$$
\delta_{0} \chi_{0}(r)+\delta_{3} \chi_{3}(r)=0, \quad \delta_{1} \chi_{1}(r)+\delta_{2} \chi_{2}(r)=0 .
$$

If we first take $r=1$, we find $\delta_{0}=-\delta_{3}, \delta_{1}=-\delta_{2}$. Then, for arbitrary 2-regular elements $r \in G$

$$
\chi_{0}(r)=\chi_{3}(r), \quad \chi_{1}(r)=\chi_{2}(r)
$$

In particular, for $r=1$, we have $f_{0}=f_{3}, f_{1}=f_{2}$. The same results hold in the case that $G$ is a $Q$-group. Here, we have an additional subsection $\left(z, b_{z}\right)$ associated and $b_{z}$ has defect 2, cf. Proposition 5.5. Since $b_{z}$ corresponds to a block of defect 1 of $C(z) /\langle z\rangle$ and we have unique modular irreducible character in $b_{z}$, its Cartan invariant is 4 . There is then a column $R$ belonging to this subsection and we have the same consequences as in the other case. Thus

Proposition 6. Let $B_{\theta}$ be a block of type (*). Assume that either there exists a minor subsection $(x, b)$ associated with $B_{\theta}$ or that $G$ is a $Q$-group. Then in Proposition 5 , we have $\delta_{0}=-\delta_{3}, \delta_{1}=-\delta_{2}$ and, for 2-regular $r \in G, \chi_{0}(r)=\chi_{3}(r), \chi_{1}(r)=\chi_{2}(r)$. In particular, for $r=1, f_{0}=f_{3}, f_{1}=f_{2}$.

Our results show again that in the case of a $Q$-group $G$, no minor subsection $(x, b)$ is associated with $B_{\theta}$. Moreover, in the case of a $Q D$-group, we can have at most one such minor subsection. Indeed, if this was not so, we would have two columns belonging to different subsections which are linearly dependent. This is impossible by (5b), cf. also Proposition 5.3. 
Proposition 4 now yields

COROLlaRY 7. Suppose that $B_{\theta}$ is a block of type $\left({ }^{*}\right)$ such that a minor subsection $(x, b)$ is associated with $B_{\theta}$. Then there exists a unique modular irreducible character $\phi^{\#}$ in $b$. There exists a sign $\delta^{\#}= \pm 1$ with the following property: If $r$ is a 2-regular element of $N$, we have

$$
\begin{array}{ll}
\chi_{0}(x r)=\delta_{0}\left(\theta_{N}(r)+\delta^{\#} \phi^{\#}(r)\right), & \chi_{1}(x r)=\delta_{1}\left(\varepsilon^{*} \psi(r) \theta_{N}(r)-\delta^{\#} \phi^{\#}(r)\right), \\
\chi_{2}(x r)=-\delta_{2}\left(\varepsilon^{*} \psi(r) \theta_{N}(r)+\delta^{\#} \phi(r)\right), & \chi_{3}(x r)=-\delta_{3}\left(\theta_{N}(r)-\delta^{\#} \phi^{\#}(r)\right) .
\end{array}
$$

For $\chi_{4}(x r)$ and $\chi^{(j)}(x r)$ the same formulas hold as in Corollary 5. We have $\phi^{\#}(1) \equiv 2^{n-1}$ $\left(\bmod 2^{n}\right)$,

Our method can also be applied to elements in the 2-section $\mathscr{S}(z)$.

COROLlary 8. Let $G$ be a $Q$-group and let $B_{\theta}$ be a block of type $\left(^{*}\right)$. Let $\left(z, b_{z}\right)$ denote the subsection of Proposition 5.5 associated with $B_{\theta}$. Then $b_{z}$ contains a unique modular irreducible character $\phi^{(z)}$. There exists a sign $\gamma= \pm 1$ with the following property: If $r$ is a 2-regular element of $C(z)$, we have

$$
\begin{array}{ll}
\chi_{0}(z r)=\delta_{0} \gamma \phi^{(z)}(r), & \chi_{1}(z r)=-\delta_{1} \gamma \phi^{(z)}(r), \\
\chi_{2}(z r)=\delta_{1} \gamma \phi^{(z)}(r), & \chi_{3}(z r)=-\delta_{0} \gamma \phi^{(z)}(r),
\end{array}
$$

while $\chi_{4}(z r)$ and all $\chi^{(j)}(z r)$ vanish.

Our results apply when $B_{\theta}=B_{0}(2, G)$ is the principal block. Here, $\theta_{N}$ can be taken as the principal character of $N$ and hence $B_{\theta}$ is of type $\left({ }^{*}\right)$. By [6, I, Theorem 3], no minor subsection $(x, b)$ is associated with $B_{\theta}$. Of course, the principal character of $G$ is one of the characters of $G$. Corollaries 3 and 5 show that this must be the character $\chi_{0}$ in the notation of Proposition $5 ; f_{0}=1, \delta_{0}=1$. It now follows from Corollary 2 that $\delta=\delta_{1}, \delta_{1}+f_{1}=f, \delta_{4}=-\delta_{2}, \delta_{2}+f_{2}=f_{4}$. Proposition 5 and the corollaries yield now Propositions 2.1, 2.2, 2.3, 2.4, 2.5 with the following exceptions:

(i) In the case of a $Q D$-group and of a block $B_{\theta}$ without minor subsection, we still have to show that exactly two of the signs $\delta_{1}, \delta_{2}, \delta_{3}$ are -1 .

(ii) We still have to show $\varepsilon=\varepsilon^{*}, \psi(1)=q$.

(iii) In the case of a $Q$-group, we have to show that we cannot have $f_{0}=f_{1}=f_{2}=f_{3}$ in the case of the principal block $B_{0}(2, G)$.

(i) will be treated in $\$ 7$, where we also prove the Proposition 2.6. The other two statements can be handled easily. We first note that the proof of Proposition 2.7 can now be applied and we find $\psi(1)=q$. Since $\psi(1) \equiv \varepsilon^{*}(\bmod 4)$, we have $\varepsilon \equiv \varepsilon^{*}$ $(\bmod 4)$. Thus $\varepsilon=\varepsilon^{*}$. We state this as a corollary.

COROllaRY 9. Let $G$ have the characteristic power $q$. In Corollary 5, we have $\psi(1)=q, \varepsilon=\varepsilon^{*}$. 
Suppose next that $B_{\theta}$ is a block of type $\left(^{*}\right)$ and that we have $f_{0}=f_{1}=f_{2}$. Then Corollary 2 implies that $f=f_{4}=2 f_{0}$. However, this is impossible since $f \neq f_{4}$ by Corollary 4. This takes care of (iii).

7. Formulas for the order of $G$. We shall assume in this section that $G$ is a $Q D$-group of characteristic power $q$. We shall apply the general results of [6, II, III]. The notation will be the same as in $\S 6$.

If $u$ is an element of $S_{0}$ of order $2^{n}$, then $u$ is not conjugate to $u^{-1}$ in $G$. If $x^{\prime}$ and $x^{\prime \prime}$ are two conjugates of $x$ in $G$, the 2-factor of $x^{\prime} x^{\prime \prime}$ cannot be $u$. If $B$ is any 2-block, $[6$, II, Proposition 4] shows that

$$
\sum_{x} \frac{\chi(x)^{2} \chi(u)}{\chi(1)}=0
$$

where $\chi$ ranges over all characters of $B$. In particular, take $B$ as a block $B_{\theta}$ of type $\left(^{*}\right)$. It follows from $\S 6$, equations (12) and (9) that $V_{m}$ with $m=2^{n-1}$ is a linear combination of columns $X(u)$ such that $u \in S_{0}$ has order $2^{n}$. Thus (1) remains valid if $\chi(u)$ is replaced by $\left(V_{m}\right)_{x}$. Using the coefficients of $V_{m}$ given in Proposition 6.5 , we find

$$
\delta_{0} \frac{\chi_{0}(x)^{2}}{f_{0}}+\delta_{1} \frac{\chi_{1}(x)^{2}}{f_{1}}+\delta_{2} \frac{\chi_{2}(x)^{2}}{f_{2}}+\delta_{3} \frac{\chi_{3}(x)^{2}}{f_{3}}=0 .
$$

Corollary 6.2 yields

$$
\delta_{0} f_{0}+\delta_{1} f_{1}+\delta_{2} f_{2}+\delta_{3} f_{3}=0 .
$$

Suppose first that $B_{\theta}$ does not have a minor subsection $(x, b)$. The values of $\chi_{i}(x)$ can be obtained from Corollary 6.5. Then, $\psi(1)=q$, cf. Corollary 6.9. Since $\theta_{N}(1)=1$, we find

$$
\delta_{0} \frac{1}{f_{0}}+\delta_{1} \frac{q^{2}}{f_{1}}+\delta_{2} \frac{q^{2}}{f_{2}}+\delta_{3} \frac{1}{f_{3}}=0 .
$$

This can be written in the form

$$
\delta_{0} \delta_{3}\left(\delta_{0} f_{0}+\delta_{3} f_{3}\right) /\left(f_{0} f_{3}\right)=-q^{2} \delta_{1} \delta_{2}\left(\delta_{1} f_{1}+\delta_{2} f_{2}\right) /\left(f_{1} f_{2}\right) .
$$

By (3) and Corollary 6.6

$$
-\left(\delta_{1} f_{1}+\delta_{2} f_{2}\right)=\delta_{0} f_{0}+\delta_{3} f_{3} \equiv 2^{n} \quad\left(\bmod 2^{n}\right) .
$$

Since then $\delta_{0} f_{0}+\delta_{3} f_{3} \neq 0$, cancellation of this factor yields

$$
\delta_{0} \delta_{3} /\left(f_{0} f_{3}\right)=q^{2} \delta_{1} \delta_{2} /\left(f_{1} f_{2}\right) \text {. }
$$

Hence we have $\delta_{0} \delta_{1} \delta_{2} \delta_{3}=1, f_{1} f_{2}=q^{2} f_{0} f_{3}$. As shown by (3), not all $\delta_{i}$ have the same sign. Thus, exactly two of them are -1 and we have

Proposition 1. If $B_{\theta}$ is a block of type $\left(^{*}\right)$ without minor subsection $(x, b)$, exactly two of the signs $\delta_{0}, \delta_{1}, \delta_{2}, \delta_{3}$ are -1 and we have

$$
f_{1} f_{2}=q^{2} f_{0} f_{3} .
$$


Assume next that $B_{\theta}$ has a minor subsection $(x, b)$. We now have to use Corollary 6.7. Set $M=\delta^{\#} \phi^{\#}(1)$. Since $\varepsilon=\varepsilon^{*}, \psi(1)=q$ by Corollary 6.9 and since $\delta_{0}=-\delta_{3}$, $\delta_{1}=-\delta_{2}, f_{0}=f_{3}, f_{1}=f_{2}$ by Proposition 6.6, (2) now reads

$$
\delta_{0} \frac{(1+M)^{2}-(1-M)^{2}}{f_{0}}+\delta_{1} \frac{(\varepsilon q-M)^{2}-(\varepsilon q+M)^{2}}{f_{1}}=0 .
$$

Thus $\delta_{0} f_{1} 4 M-\delta_{1} f_{0} 4 \varepsilon q M=0$.

Since $M \neq 0$, we have

Proposition 2. If $B_{\theta}$ is a block of type $\left(^{*}\right)$ with a minor subsection, we have $f_{1}=f_{2}=q f_{0}=q f_{3}$ and $\delta_{1}=\varepsilon \delta_{0}, \delta_{2}=-\varepsilon \delta_{0}, \delta_{3}=-\delta_{0}$.

We next apply [6, III,(2A)]. The subgroup $\tilde{G}$ occurring in this theorem will be taken as $N, p=2, \pi=y_{1}=y_{2}=x$. Then $\tilde{G}$ is itself a $Q$-group of the characteristic power $q$. We set $\widetilde{B}_{\theta}=\left(b_{\theta}\right)^{N}$. Then $\widetilde{B}_{\theta}$ is a block of $N$ of type $\left(^{*}\right)$. We use analogous notation for $\widetilde{B}_{\theta}$ as for $B_{\theta}$ with tilde signs added. Thus, $\widetilde{B}_{\theta}$ consists of characters $\tilde{\chi}_{0}, \ldots, \tilde{\chi}_{4}, \tilde{\chi}^{(\alpha)}, \tilde{\chi}^{(\beta)}$, we have signs $\tilde{\delta}_{0}, \tilde{\delta}_{1}$, etc. The results of $\S 6$ hold for $\widetilde{B}_{\theta}$.

LEMMA 1. We may assume that $\tilde{\chi}_{1}=\psi \theta_{N}$. We have

$$
\begin{gathered}
\tilde{f}_{0}=\tilde{f}_{3}=1, \quad \tilde{f}_{1}=\tilde{f}_{2}=q, \quad \tilde{f}_{4}=q-\varepsilon, \quad \tilde{f}^{\prime}=q+\varepsilon, \\
\tilde{\chi}_{0}(x)=1, \quad \tilde{\chi}_{1}(x)=q, \quad \tilde{\chi}_{2}(x)=q, \quad \tilde{\chi}_{3}(x)=1, \\
\tilde{\chi}_{4}(x)=-(q-\varepsilon), \quad \tilde{\chi}^{(j)}(x)=(-1)^{j}(q+\varepsilon) \quad\left(\text { for } j \in Y_{1} \cup Y_{2}\right), \\
\tilde{\chi}_{0}(z)=1, \quad \tilde{\chi}_{1}(z)=-\varepsilon, \quad \tilde{\chi}_{2}(z)=\varepsilon, \quad \tilde{\chi}_{3}(z)=-1, \quad \tilde{\chi}_{4}(z)=0, \quad \tilde{\chi}(z)=0, \\
\tilde{\delta}_{0}=1, \quad \tilde{\delta}_{1}=\varepsilon, \quad \tilde{\delta}_{2}=-\varepsilon, \quad \tilde{\delta}_{3}=-1, \quad \tilde{\delta}=\varepsilon, \quad \tilde{\delta}_{4}=\varepsilon .
\end{gathered}
$$

Proof. We first take $\theta_{N}$ as the principal character $\theta_{0}$ of $N$. Then $\widetilde{B}_{\theta_{0}}$ is the principal block $B_{0}(2, N)$. The results of $\S 6$ show that the character $\left({ }^{10}\right) \tilde{\chi}_{0}^{\left(\theta_{0}\right)}$ is the principal character $\theta_{0}$ of $N, \tilde{\delta}_{0}^{\left(\theta_{0}\right)}=1$ and that $\tilde{\chi}_{1}^{\left(\theta_{0}\right)}$ and $\tilde{\chi}_{2}^{\left(\theta_{0}\right)}$ of degree $q$ are the two irreducible characters of $B_{0}(2, N)$ of odd degree different from 1 . Either one can be taken for $\psi$ in Corollary 6.5 (in the case of an arbitrary $\theta$ of type $\left({ }^{*}\right)$ ). We shall choose $\psi=\tilde{\chi}_{1}^{\left(\theta_{0}\right)}$. Since

$$
\tilde{f}^{\left(\theta_{0}\right)}=\tilde{\delta}^{\left(\theta_{0}\right)} \tilde{\delta}_{0}^{\left(\theta_{0}\right)} \tilde{f}_{0}^{\left(\theta_{0}\right)}+\tilde{\delta}^{\left(\theta_{0}\right)} \tilde{\delta}_{1}^{\left(\theta_{0}\right)} \tilde{f}_{1}^{\left(\theta_{0}\right)}=\tilde{\delta}^{\left(\theta_{0}\right)}\left(1+\tilde{\delta}_{1}^{\left(\theta_{0}\right)} q\right)
$$

is a degree of an irreducible representation (Corollary 6.2) and since this degree is not divisible by 4 (Corollary 6.4), we must have $\tilde{\delta}_{1}^{\left(\theta_{0}\right)}=\varepsilon, \tilde{\delta}^{\left(\theta_{0}\right)}=\varepsilon$. Now Corollary 6.3 yields

$$
\psi(u)=\varepsilon \quad\left(\text { for } u \in S_{0}-\{1, x\}\right) .
$$

For an arbitrary $\theta$ of type $\left(^{*}\right)$, by Lemma 6.1 ,

$$
\tilde{B}_{\theta}=\left(b_{\theta}\right)^{N}=\theta_{N} B_{0}(2, N) \text {. }
$$

(10) As was mentioned, the characters $\chi_{i}$ of $B_{\theta}$ depend on $\theta$. In order to emphasize this point, we write $\left(\chi_{i}\right)^{(\theta)}$ for $\chi_{i}$ whenever necessary. 
In particular, $\theta_{N}$ and $\theta_{N} \psi$ belong to $\widetilde{B}_{\theta}$. Corollaries 6.3 and 6.5 in conjunction with (5) show that $\tilde{\chi}_{0}^{(\theta)}=\theta_{N}, \tilde{\chi}_{1}^{\theta}=\theta_{N} \psi$, and that $\tilde{\delta}_{0}^{(\theta)}=1, \delta_{1}^{(\theta)}=\varepsilon^{*}$. By Corollary 6.9, $\tilde{\delta}_{1}^{(\theta)}=\varepsilon$. It is also clear that $\tilde{f}_{0}^{(\theta)}=1, \tilde{f}_{1}^{(\theta)}=q$. Proposition 6.6 shows that

$$
f_{2}^{(\theta)}=q, \quad f_{3}^{(\theta)}=1, \quad \delta_{2}^{(\theta)}=-\varepsilon, \quad \delta_{3}^{(\theta)}=-1 .
$$

Corollaries 6.2 and 6.4 yield $\tilde{\delta}^{(\theta)}=\varepsilon, \tilde{f}^{(\theta)}=q+\varepsilon, \tilde{\delta}_{4}^{(\theta)}=\varepsilon, f_{4}^{(\theta)}=q-\varepsilon$.

The values of the characters for the element $x$ are obtained from Corollary 6.5. We have

$$
\tilde{\chi}_{0}^{(\theta)}(z)=\theta_{N}(z)=1 .
$$

Now Corollary 6.8 shows that $\gamma=\delta_{0}^{(\theta)}=1, \phi^{(z)}(1)=1$ and it also gives the values of the other characters for the element $z$.

It is clear that $\psi$ remains the same, if $G$ is replaced by $N$.

For each character $\tilde{\chi} \in \widetilde{B}$, we have to form the expression

$$
h(\tilde{\chi})=\tilde{\chi}(x)|N|^{-1}+\tilde{\chi}(z)|C|^{-1} .
$$

Let $R$ be a column $X(u)$ for the block $B_{\theta}, u \in S_{0}-\{1, x\}$, and let $\tilde{R}$ denote the corresponding column for $\widetilde{B}_{\theta}$. Then $[6, \mathrm{III},(2 \mathrm{~A}),(2 \mathrm{~B})]$ states that

$$
|G| \sum_{x}(R)_{x} \frac{\chi(x)^{2}}{\chi(1)}=|N|^{3} \sum_{\tilde{x}}(\tilde{R})_{z} \frac{h(\tilde{\chi})^{2}}{\tilde{\chi}(1)}
$$

where on the left $\chi$ ranges over the characters of $B_{\theta}$ and where on the right, $\tilde{\chi}$ ranges over the characters of $\widetilde{B}_{\theta}$. The same result holds when $R$ is a column $U_{i}$ $(i=0,1)$ and $\tilde{R}$ the corresponding column $\tilde{U}_{i}$ for $\widetilde{B}_{\theta}$. More generally, (7) holds when $R$ is a linear combination of the columns $X(u), U_{0}, U_{1}$ and $\widetilde{R}$ the corresponding linear combination of the columns $\tilde{X}(u), \tilde{U}_{0}, \tilde{U}_{1}$.

We first take $R=V_{2}$. The equations $\S 6,(12)$ and (9) show that $R$ is indeed a linear combination of columns $X(u)$. Then $\tilde{R}$ is to be the corresponding columns $\tilde{V}_{2}$ for $\widetilde{B}_{\theta}$. The coefficients of $V_{2}$ are given in Proposition 6.5, and we have analogous formulas for $\tilde{V}_{2}$. We obtain

$$
\begin{aligned}
& |G|\left(\delta_{0} \chi_{0}(x)^{2} f_{0}^{-1}+\delta_{1} \chi_{1}(x)^{2} f_{1}^{-1}-\delta \chi^{(2)}(x)^{2} f^{-1}\right) \\
& =|N|^{3}\left(\delta_{0} h\left(\tilde{\chi}_{0}\right)^{2} \tilde{f}_{0}^{-1}+\delta_{1} h\left(\tilde{\chi}_{1}\right)^{2} \tilde{f}_{1}^{-1}-\delta h\left(\tilde{\chi}^{(2)}\right) \tilde{f}^{-1}\right) .
\end{aligned}
$$

Here, by (6) and Lemma 1,

$$
h\left(\tilde{\chi}_{0}\right)=|N|^{-1}+|C|^{-1}, \quad h\left(\tilde{\chi}_{1}\right)=|N|^{-1} q-|C|^{-1} \varepsilon, \quad h\left(\tilde{\chi}^{(2)}\right)=|N|^{-1}(q+\varepsilon),
$$

and the right hand side of (8) becomes

$$
\begin{gathered}
|N|^{3}\left(\left(|N|^{-1}+|C|^{-1}\right)^{2}+\varepsilon q^{-1}\left(|N|^{-1} q-|C|^{-1} \varepsilon\right)^{2}-\varepsilon(q+\varepsilon)^{-1}|N|^{-2}(q+\varepsilon)^{2}\right) \\
=|N|^{3}|C|^{-2}\left(1+\varepsilon q^{-1}\right)>0 .
\end{gathered}
$$

Consider first the case that $\boldsymbol{B}_{\theta}$ does not have a minor subsection. On account of Corollary 6.5 , the left hand side of $(8)$ is

$$
|G|\left(\frac{\delta_{0}}{f_{0}}+\frac{\delta_{1} q^{2}}{f_{1}}-\delta \frac{(q \varepsilon+1)^{2}}{f}\right)
$$


As shown by Corollary 6.2, we have

$$
\delta_{0} f_{0}+\delta_{1} f_{1}-\delta f=0
$$

and our expression becomes

$$
|G| \frac{\delta_{0} \delta_{1}\left(\delta_{1} f_{1}-\varepsilon \delta_{0} f_{0} q\right)^{2}}{f_{0} f_{1}\left(\delta_{0} f_{0}+\delta_{1} f\right)}
$$

Our equation shows that the numerator does not vanish. We now have

Proposition 3. Suppose that $B_{\theta}$ is a block of type $\left(^{*}\right)$ without minor subsection. Set

$$
Q_{1}=\delta_{0} \delta_{1} \frac{\left(\delta_{1} f_{1}-\varepsilon \delta_{0} f_{0} q\right)^{2}}{f_{0} f_{1}\left(\delta_{0} f_{0}+\delta_{1} f_{1}\right)}
$$

Then $Q_{1} \neq 0$ and we have

$$
|G|=\frac{|N|^{3}}{|C|^{2}} \frac{1}{Q_{1}} \frac{q+\varepsilon}{q} .
$$

Consider now the case that $B_{\theta}$ has a minor subsection $(x, b)$. The right hand side of (8) remains the same. If as before $M=\delta^{\#} \phi^{\#}(1)$, we now have (by Corollary 6.7) $\chi_{0}(x)=\delta_{0}(1+M), \chi_{1}(x)=\delta_{1}(\varepsilon q-M), \chi^{(2)}(x)=\delta(\varepsilon q+1)$, and the left hand side of $(8)$ reads

$$
|G|\left(\delta_{0} \frac{(1+M)^{2}}{f_{0}}+\delta_{1} \frac{(\varepsilon q-M)^{2}}{f_{1}}-\delta \frac{(\varepsilon q+1)^{2}}{f}\right) .
$$

Apply here Proposition 2 and (9). The expression can be written in the form $|G| \delta_{0} M^{2}(q+\varepsilon) /\left(q f_{0}\right)$. Here $M= \pm \phi^{\#}(1)$, where $\phi^{\#}$ is the unique modular irreducible character in the block $b$. Since $b$ has defect 2 , then $\phi^{\#}(1)$ is the common degree of the four ordinary irreducible characters in $b$. Since the left hand side of (8) has the sign $\delta_{0}$ and the right hand side is positive, we have $\delta_{0}=1$. We obtain

Proposition 4. Let $B_{\theta}$ be a block of type $\left(^{*}\right)$ which has a minor subsection. Then $\delta_{0}=1$. The block $b$ of $N$ of defect 2 consists of four ordinary irreducible characters all of which have the same degree $f^{\#}$. Then

$$
|G|=\frac{|N|^{3}}{|C|^{2}} \frac{f_{0}}{\left(f^{\#}\right)^{2}}
$$

We can also use (7) in the case that $R=V_{m}+U_{0}$ and $\tilde{R}=\tilde{V}_{m}+\tilde{U}_{0}$. A similar computation yields the following result (which can also be deduced from Propositions 1 and 3).

Proposition 5. Let $B_{\theta}$ be a block of type $\left(^{*}\right)$ which does not have a minor subsection $(x, b)$. Set

$$
Q_{2}=\delta_{0} \delta_{2} \frac{\left(\delta_{2} f_{2}+\varepsilon \delta_{0} f_{0} q\right)^{2}}{f_{0} f_{2}\left(\delta_{0} f_{0}+\delta_{2} f_{2}\right)}
$$


Then $Q_{2} \neq 0$ and

$$
|G|=\frac{|N|^{3}}{|C|^{2}} \frac{1}{Q_{2}} \frac{q-\varepsilon}{q} .
$$

Proof. As shown by Proposition 6.5, (7) reads here

$$
\begin{aligned}
2|G|\left(\delta_{0} \chi_{0}(x)^{2} f_{0}^{-1}+\delta_{2} \chi_{2}(x)^{2} f_{2}^{-1}+\delta_{4} \chi_{4}(x)^{2} f_{4}^{-1}\right) & \\
& =2|N|^{3}\left(\tilde{\delta}_{0} h\left(\tilde{\chi}_{0}\right)^{2} \tilde{f}_{0}^{-1}+\tilde{\delta}_{2} h\left(\tilde{\chi}_{2}\right)^{2} \tilde{f}_{2}^{-1}+\tilde{\delta}_{4} h\left(\tilde{\chi}_{4}\right)^{2} \tilde{f}_{4}^{-1}\right) .
\end{aligned}
$$

Lemma 1 and (6) show that the right hand side is

$$
\begin{gathered}
2|N|^{3}\left(\left(|N|^{-1}+|C|^{-1}\right)^{2}-\varepsilon\left(|N|^{-1} q+|C|^{-1} \varepsilon\right)^{2} q^{-1}+\varepsilon|N|^{-2}(q-\varepsilon)^{2}(q-\varepsilon)^{-1}\right) \\
=2|N|^{3}|C|^{-2}\left(1-\varepsilon q^{-1}\right) .
\end{gathered}
$$

On the other hand, the results of $\S 6$ yield

$$
\begin{gathered}
\delta_{0} f_{0}+\delta_{2} f_{2}+\delta_{4} f_{4}=0, \\
\chi_{0}(x)=\delta_{0}, \quad \chi_{2}(x)=-\delta_{2} \varepsilon q, \quad \chi_{4}(x)=-\delta_{4}(\varepsilon q-1) .
\end{gathered}
$$

The left hand side of our equation then becomes

$$
\begin{aligned}
& 2|G|\left(\delta_{0} f_{0}^{-1}+\delta_{2} q^{2} f_{2}^{-1}-(\varepsilon q-1)^{2}\left(\delta_{0} f_{0}+\delta_{2} f_{2}\right)^{-1}\right) \\
& \quad=2|G| \delta_{0} \delta_{2}\left(\delta_{2} f_{2}+\varepsilon \delta_{0} f_{0} q\right)^{2} f_{0}^{-1} f_{2}^{-1}\left(\delta_{0} f_{0}+\delta_{2} f_{2}\right)^{-1}=2|G| Q_{2}
\end{aligned}
$$

and we obtain the statement.

If $B_{\theta}$ is the principal block $B_{0}(2, G)$, as we have seen at the end of $\S 6, \delta_{0}=1$, $f_{0}=1, \delta=\delta_{1}, \delta_{4}=-\delta_{2}$. Propositions $1,3,5$ and Corollary 6.6 apply. This yields Proposition 2.6 and we see that exactly two of the signs $\delta_{1}, \delta_{2}, \delta_{3}$ are -1 . Now all the statements given in $\$ 2$ without proof have been derived.

We investigate further blocks $B_{\theta}$ of type $\left(^{*}\right)$ without minor subsection. Our aim is a proof of Proposition 6 below.

LEMMA 2. If $B_{\theta}$ is a block of type $\left(^{*}\right)$ without minor subsection $(x, b)$, we have

$$
\delta_{1} f_{0}+\delta_{0} f_{1}>0, \quad \delta_{2} f_{0}+\delta_{0} f_{2}>0 .
$$

The numbers $Q_{1}$ and $Q_{2}$ are positive and they are the same for all choices of $B_{\theta}$ satisfying the hypotheses of the lemma. We have

$$
\begin{gathered}
Q_{1}(q-\varepsilon)=Q_{2}(q+\varepsilon), \\
Q_{1}=\frac{\left(\delta_{1} f_{1}-\varepsilon \delta_{0} f_{0} q\right)^{2}}{\delta_{0} \delta_{1} f_{0} f_{1}\left(\delta_{0} f_{0}+\delta_{1} f_{1}\right)}=-\frac{\left(\delta_{2} f_{2}-\varepsilon \delta_{3} f_{3} q\right)^{2}}{\delta_{2} \delta_{3} f_{2} f_{3}\left(\delta_{2} f_{2}+\delta_{3} f_{3}\right)} \\
Q_{2}=\frac{\left(\delta_{2} f_{2}+\varepsilon \delta_{0} f_{0} q\right)^{2}}{\delta_{0} \delta_{2} f_{0} f_{2}\left(\delta_{0} f_{0}+\delta_{2} f_{2}\right)}=-\frac{\left(\delta_{1} f_{1}+\varepsilon \delta_{3} f_{3} q\right)^{2}}{\delta_{1} \delta_{3} f_{1} f_{3}\left(\delta_{1} f_{1}+\delta_{3} f_{3}\right)}
\end{gathered}
$$

Proof. It follows from (11) and (13) that the expressions $Q_{1}$ and $Q_{2}$ do not depend on the particular choice of $B_{\theta}$. Moreover, $Q_{1}$ and $Q_{2}$ are positive and satisfy (15). The first inequality (14) follows from (10) and the second inequality (14) follows from (12). 
The first part of (16) occurs in (10). On account of (4),

$$
\begin{aligned}
\left(\delta_{1} f_{1}-\varepsilon \delta_{0} f_{0} q\right)^{2} & =f_{2}^{-2} f_{2}^{2}\left(\delta_{1} f_{1}-\varepsilon \delta_{0} f_{0} q\right)^{2}=f_{2}^{-2}\left(\delta_{1} f_{0} f_{3} q^{2}-\varepsilon \delta_{0} f_{0} f_{2} q\right)^{2} \\
& =f_{2}^{-2} f_{0}^{2} q^{2}\left(\delta_{1} f_{3} q-\varepsilon \delta_{0} f_{2}\right)^{2}=f_{0} f_{1} f_{2}^{-1} f_{3}^{-1}\left(\delta_{2} f_{2}-\varepsilon \delta_{0} \delta_{1} \delta_{2} f_{3} q\right)^{2} .
\end{aligned}
$$

If we substitute this in (10) and use (3) and $\delta_{0} \delta_{1} \delta_{2} \delta_{3}=1$, we obtain the last part of (16). The proof of (17) is analogous.

LEMMA 3. We have

$$
2 / 5<Q_{i} \leqq 32 / 19 \quad(i=1,2) .
$$

Proof. We shall prove (18) here for $q \geqq 7$. For $q=3$ and $q=5$, (18) will be obtained in $\$ 8$ by a direct argument. On account of Lemma 2, we may choose $B_{\theta}$ as the principal block $B_{0}(2, G)$. Then $f_{0}=1, \delta_{0}=1$.

Suppose first that $f_{3}>100$. It follows from (4) that we can choose $j=1$ or 2 such that $f_{j}>10 q$. By (16), (17)

$$
Q_{j}=\frac{\left(f_{j}-q\right)^{2}}{f_{j}\left(f_{j} \pm 1\right)} \text { or } \quad Q_{j}=\frac{\left(f_{j}+q\right)^{2}}{f_{j}\left(f_{j} \pm 1\right)}
$$

In the former case, replace $f_{j}$ by a variable $w \geqq 10 q$. We then have an increasing function of $w$ and hence

$$
Q_{f}>\frac{81 q^{2}}{10 q(10 q+1)}=\frac{81}{10} \frac{q}{10 q+1}
$$

This holds in the latter case, too, since here $Q_{j}>1$.

Similarly, we see that in the latter case, $Q_{j}$ is less than the value obtained when $f_{j}$ is replaced by $10 q$,

$$
Q_{j}<\frac{121 q^{2}}{10 q(10 q+1)}=\frac{121}{10} \frac{q}{10 q-1}
$$

This holds in the former case, too, since here $Q_{j}<1$. Now (15) shows that for both values $i=1$ and 2

$$
\frac{81}{10} \frac{q}{10 q+1} \frac{q-1}{q+1}<Q_{i}<\frac{121}{10} \frac{q}{10 q-1} \frac{q+1}{q-1}
$$

Since $q \geqq 7$, then

$$
\frac{81}{10} \frac{7}{71} \frac{3}{4}<Q_{i}<\frac{121}{10} \frac{7}{69} \frac{4}{3}
$$

This shows that (18) holds in this case.

We show next that, for $q \geqq 7$, the case $f_{3}<100$ is impossible. Suppose we have $f_{3}<100$.

Case 1. $\delta_{3}=1$.

By Proposition 2.8(iii), $q^{2}<f_{3}<100$. Since we assume $q \geqq 7$, then $q=7$ or $q=9$. In both cases, $n=4$. By Corollary $6.6, f_{3} \equiv 15(\bmod 32)$. We have then only the possibilities

$$
f_{3}=15,47,79 \text {. }
$$


The equations ( 3 ) and (4) read here

$$
1+f_{3}=f_{1}+f_{2}, \quad f_{1} f_{2}=q^{2} f_{3} .
$$

We easily obtain a contradiction for each of the three values of $f_{3}$ since $f_{1}$ or $f_{2}$ is divisible by the largest prime factor of $f_{3}$.

Case 2. $\delta_{3}=-1$.

Here, by Corollary $6.6, f_{3} \equiv 1+2^{n}\left(\bmod 2^{n+1}\right)$. Since $f_{3}<100$, then $n \leqq 6$ and we have only the following possibilities:

$$
\begin{aligned}
& n=3 \text { and } f_{3}=9,25,41,57,73,89 \\
& n=4 \text { and } f_{3}=17,49,81 ; \\
& n=5 \text { and } f_{3}=33,97 \\
& n=6 \text { and } f_{3}=65 \\
& \text { If } \delta_{i}=1, \delta_{j}=-1 \text { with }\{i, j\}=\{1,2), \text { here }
\end{aligned}
$$

$$
1+f_{i}=f_{j}+f_{3}, \quad f_{i} f_{j}=q^{2} f_{3} .
$$

If $d$ is the greatest common divisor of $f_{1}$ and $f_{2}, d$ divides $f_{3}-1$. Since $d$ then is coprime to $f_{3}$, the second equation (20) shows that $d^{2}$ divides $q^{2}$, i.e. $d$ divides $q$. Now $q$ is a power of a prime $p$, say $q=p^{l}$ and we have $d=p^{\lambda}$ with $0 \leqq \lambda \leqq l$.

Suppose first that $\lambda=0$. By (20), $q^{2}$ divides either $f_{i}$ or $f_{j}$. In the latter case, $f_{i}$ divides $f_{3}$. This is impossible since the first equation (20) shows that $f_{i}>f_{3}$.

Hence $q^{2}$ divides $f_{i}$, say $f_{i}=q^{2} f_{i}^{\prime}$. Then $f_{3}=f_{i}^{\prime} f_{j}$. Since $f_{3} \neq f_{j}$ by Corollary 6.6, we have $f_{i}^{\prime} \geqq 3$ and then

$$
3 q^{2}<1+f_{i}^{\prime} q^{2}=1+f_{i}=f_{j}+f_{3}=f_{3}\left(f_{i}^{\prime-1}+1\right) \leqq 4 f_{3} / 3 .
$$

Thus, $q^{2}<400 / 9$. As we assumed $q \geqq 7$, this is false.

We have now proved that $\lambda \geqq 1$ and that $p^{\lambda}$ divides $f_{3}-1$. The second equation (20) shows that either $f_{i}$ is divisible by $p^{2 l-\lambda}$ and $f_{j}$ divisible by $p^{\lambda}$ or that $f_{i}$ is divisible by $p^{\lambda}$ and $f_{j}$ divisible by $p^{2 l-\lambda}$. We consider two cases:

Case $\alpha . f_{i}=p^{2 l-\lambda} \alpha, f_{j}=p^{\lambda} \beta$.

Case $\beta$. $f_{i}=p^{\lambda} \beta, f_{j}=p^{2 l-\lambda} \alpha$ with integral $\alpha, \beta$.

In both cases

$$
\alpha \beta=f_{3} .
$$

Since $\lambda \leqq l$ and $f_{j}<f_{i}<f_{j}+f_{3}$, we see from (20) that in both cases

$$
\beta>1 \text {. }
$$

Suppose first that $\lambda \geqq 2$. Inspection of the values possible for $f_{3}$ shows that the only value for which $f_{3}-1$ is divisible by a power $p^{\lambda}$ of an odd prime $p$ with an exponent $\lambda \geqq 2$ is the value $f_{3}=73$. We then have $n=3, p=3, \lambda=2$. By (21) and (22) $\alpha=1, \beta=73$. Here, (20) yields

Case $\alpha .1+3^{2 l-2}=3^{2} \cdot 73+73$.

Case $\beta .1+9 \cdot 73=3^{2 l-2}+73$. 
The case $\beta$ is clearly impossible. In the case $\alpha$, we find $3^{2 l-2}=729, l=4, q=81$. However, for $q=81$, we have $n=5$, a contradiction.

Thus $\lambda=1$. On account of (20) and (21),

Case $\alpha .1+p^{2 l-1} \alpha=p \beta+\alpha \beta$.

Case $\beta$. $1+p \beta=p^{2 l-1} \alpha+\alpha \beta$.

If $\alpha=1$, then

$$
f_{3}=\beta=\frac{p^{2 l-1}+1}{p+1} \text { or } f_{3}=\beta=\frac{p^{2 l-1}-1}{p-1} \text {. }
$$

None of the values possible for $f_{3}$ has this form except $f_{3}=57$. For $f_{3}=57$, we must have $n=3, d=p=7$. If $\alpha=1$, we necessarily have Case $\beta$ and $p^{2 l-1}=343, l=2$, $q=49$. However, then $n=5$, a contradiction.

Thus, in addition to (22), we have $\alpha>1$. Since the greatest common divisor $d^{\prime}$ of $\alpha$ and $\beta$ divides $f_{i}$ and $f_{j}$ and hence $d=p$, we find $d^{\prime}=1$. Now (21) shows that $f_{3}$ cannot be a power of a prime. This leaves us only with the cases $f_{3}=57,33,65$. For $f_{3}=33$ and $f_{3}=65$, no odd prime $p$ divides $f_{3}-1$. For $f_{3}=57$ we have $\alpha=19$, $\beta=3$ or $\alpha=3, \beta=19$. Here $p=7$. In the former case, we find $p \beta=21 \equiv \pm 1(\bmod 19)$, which is not true. In the latter case, we have $7^{2 l-1} \cdot 3=p \beta \pm(\alpha \beta-1)=133 \pm 56$. Again, this is impossible.

We have now shown that, for $q \geqq 7$, we cannot have $f_{3}<100$ and the lemma is established (for $q \geqq 7$ ).

Proposition 6. If $B_{\theta}$ is a block of type $\left(^{*}\right)$ without minor subsection, then $\chi_{0}$ has degree $f_{0}=1$.

Proof. Suppose that $f_{0} \neq 1$. Set

$$
f_{0} / f_{1}=\mu / q, \quad f_{0} / f_{2}=\nu / q, \quad f_{3} / f_{1}=\mu^{\prime} / q, \quad f_{3} / f_{2}=\nu^{\prime} / q .
$$

By (4),

$$
\mu \nu^{\prime}=\nu \mu^{\prime}=1 .
$$

We can now write (16) and (17) in the form

$$
\begin{aligned}
& Q_{1}=\frac{\left(1-\varepsilon \delta_{0} \delta_{1} \mu\right)^{2}}{\delta_{0} f_{0}\left(1+\delta_{0} \delta_{1} \mu q^{-1}\right)}=\frac{\left(1-\varepsilon \delta_{2} \delta_{3} \nu^{\prime}\right)^{2}}{\left(-\delta_{3}\right) f_{3}\left(1+\delta_{2} \delta_{3} \nu^{\prime} q^{-1}\right)} \\
& Q_{2}=\frac{\left(1+\varepsilon \delta_{0} \delta_{2} \nu\right)^{2}}{\delta_{0} f_{0}\left(1+\delta_{0} \delta_{2} \nu q^{-1}\right)}=\frac{\left(1+\varepsilon \delta_{1} \delta_{3} \mu^{\prime}\right)^{2}}{\left(-\delta_{3}\right) f_{3}\left(1+\delta_{1} \delta_{3} \mu^{\prime} q^{-1}\right)}
\end{aligned}
$$

We show next that $\mu, \nu, \mu^{\prime}, \nu^{\prime}$ are all larger than $\frac{2}{3}$. Suppose that this is false and that $\xi \leqq \frac{2}{3}$, where $\xi$ is one of $\mu, \nu, \mu^{\prime}, \nu^{\prime}$. By (25), we have a relation

$$
(1 \pm \xi)^{2} /\left(1 \pm \xi q^{-1}\right)= \pm f_{i} Q_{\alpha}
$$

with $i=0$ or 3 and $\alpha=1$ or 2 . Since the expression on the left is positive, the + sign applies on the right. If $i=3$, this means that $\delta_{3}=-1$. It follows from Corollary 6.6 that $f_{i} \geqq 9$ for $i=0$ and $i=3$ (since $f_{0}$ was assumed to be different from 1 ). Using 
Lemma 3, we have $(1 \pm \xi)^{2}>9 \cdot(2 / 5)\left(1-\xi q^{-1}\right)$. Since $0<\xi \leqq \frac{2}{3}$, the left side is at most equal to $25 / 9$. On the other hand, the right hand side is at least $(18 / 5)(1-2 / 9)$ $=14 / 5$. This is a contradiction. Thus $\mu, \nu, \mu^{\prime}, \nu^{\prime}>\frac{2}{3}$. Then, by (24) $\mu, \nu, \mu^{\prime}, \nu^{\prime}<3 / 2$ and, by (23),

$$
f_{0}<f_{1}, \quad f_{0}<f_{2}, \quad f_{3}<f_{1}, \quad f_{3}<f_{2} .
$$

If $\delta_{0}=-1$, then (14) shows that $\delta_{1}>0$ and that $f_{0}>f_{1}$, a contradiction. Thus $\delta_{0}=1$.

If $\delta_{3}=1$, by (3) and Proposition $1, f_{0}+f_{3}=f_{1}+f_{2}$. Again this is not consistent with (26). It follows that $\delta_{0}=1, \delta_{3}=-1$ and that one of $\delta_{1}, \delta_{2}$ is 1 and the other -1 , say $\delta_{i}=1$ with $i=1$ or 2 . Set $\xi=\mu$ when $i=1$ and $\xi=\nu$ when $i=2$. It follows from (25) that

$$
(1 \pm \xi)^{2}=f_{0} Q_{i}\left(1+\xi q^{-1}\right)>f_{0} Q_{i} .
$$

The left side here is less than 25/4. By Corollary $6.6, f_{0} \equiv \delta_{0}=1(\bmod 16)$. Since $f_{0} \neq 1$, then $f_{0} \geqq 17$. Since $Q_{i}>2 / 5$ by Lemma 3 , again, we have a contradiction and the proof is complete.

If $\chi$ is a character of degree 1 of $G$, we shall write $\chi B_{0}(2, G)$ for the set of characters obtained by multiplying the characters in $B_{0}(2, G)$ by $\chi$. Clearly, this is a 2-block of $G$.

COROllary 1. If $B_{\theta}$ is a 2-block of type (*) without minor subsection, then $B_{\theta}=\chi_{0}^{\theta} B_{0}(2, G)$.

This is now clear since as was shown, $\chi_{0}^{\theta}$ is a linear character. Hence we have a 2-block on the right side and $\chi_{0}^{\theta}$ is a member.

We can also show the converse of Proposition 6.

Proposition 7. If $B$ is a 2-block of $G$ which contains a character $\chi$ of degree 1, then $B$ is a block $B_{\theta}$ of type $\left(^{*}\right)$ without minor subsection and $\chi=\chi_{0}^{\theta}$.

Proof. It is clear that $B$ has full defect $n+1$ and then $B$ has the form $B_{\theta}=b_{\theta}^{G}$, where $\theta$ is an irreducible character of $N(S)=S \times E$ which is trivial on $S$. Since $\chi \in b_{\theta}^{G}$, an argument similar to that used in the proof of Lemma 6.1 shows that, for 2-regular $r \in E$,

$$
\chi(r) \equiv \theta(r) \quad(\bmod \mathfrak{p})
$$

where $\mathfrak{p}$ is a suitable prime ideal divisor of 2 . Again, this implies that $\theta \mid E$ and $\chi \mid E$ have an irreducible constituent in common. Since $\theta \mid E$ is irreducible, and since $\chi$ is of degree 1 , this means that $\theta|E=\chi| E$. As a linear character of $G, \chi$ is trivial on $G^{\prime}$. Since $G$ is a $Q D$-group, we have $S \subseteq G^{\prime}$. It is now clear that $\chi \mid N$ is an extension of $\theta$ to $N$ which is trivial on $S$. Thus $\theta$ is of type $\left({ }^{*}\right)$, we may take $\theta_{N}=\chi \mid N$.

We now know that the results of $\S 6$ and the preceding results of $\S 7$ apply to $B=B_{\theta}$. Suppose that $B_{\theta}$ has a minor subsection $(x, b)$. The character $\chi \in B_{\theta}$ can only be one of the four characters $\chi_{0}^{\theta}, \chi_{1}^{\theta}, \chi_{2}^{\theta}, \chi_{3}^{\theta}$ of odd degree in $B_{\theta}$. Proposition 2 
rules out $\chi_{1}^{\theta}, \chi_{2}^{\theta}$. On the other hand, Corollary 6.7 shows that $\chi_{0}^{\theta}(x) \neq 1, \chi_{3}^{\theta}(x) \neq 1$. Since $\chi(x)=1$, this case is impossible.

Hence $B_{\theta}$ has no minor subsection. By Proposition $6, \chi_{0}^{\theta}$ has degree 1. Since Corollary 6.6 shows that no other character in $B_{\theta}$ has degree 1 , the proof is complete.

We shall give another application of Lemma 3.

Proposition 8. Let $G$ be a $Q D$-group. Let $t$ be the 3-element of $G$ in $\$ 1$, equation (1). Then $G=\langle N, t\rangle$.

Proof (for $q \geqq 7$ ). Set $G_{0}=\langle N, t\rangle$. It is clear that $G_{0}$ is a $Q D$-group. Moreover, $N_{G_{0}}(x)=N$ and hence $q, N$ and $C$ are the same for $G_{0}$ as for $G$. We shall use the same notation for $G_{0}$ as for $G$ with superscripts 0 added. Applying Proposition 3 to the principal 2-blocks both of $G$ and $G_{0}$, we have

$$
\left|G: G_{0}\right|=Q_{1}^{(0)} / Q_{1}
$$

Now Lemma 3 yields

$$
\left|G: G_{0}\right| \leqq 5 \cdot 32 /(2 \cdot 19)=160 / 38 .
$$

Hence $\left|G: G_{0}\right|<5$. Since $\left|G: G_{0}\right|$ is necessarily odd, then either $G_{0}=G$ or else $\left|G: G_{0}\right|=3$.

Suppose we have the latter case. Then $Q_{1}^{(0)}=3 Q_{1}$. If $q \geqq 7$, by (19) applied to $Q_{1}$ and $Q_{1}^{(0)}$,

$$
\frac{121}{10} \frac{7}{69} \frac{4}{3}>3 \frac{81}{10} \frac{7}{71} \frac{3}{4}
$$

Since this is false, we have $G=G_{0}=\langle N, t\rangle$ for $q \geqq 7$. As to the cases $q=3$ and $q=5$, the proof of the proposition will be given in $\$ 9$ by means of the following:

Remark. Suppose that for some fixed $q$, there are not two values which $Q_{1}$ can take such that one of these values is three times the other value. Then Proposition 8 holds.

Actually one can give an alternate proof of Proposition 8 by using Bender's classification of groups which contain a strongly embedded subgroup; cf. [2], [3]. Indeed, since $G_{0}$ is a $Q D$-group, it has only one conjugacy class of involutions; and since $N \subseteq G_{0}$, it follows that $G_{0}$ contains the centralizer in $G$ of each of its involutions as well as the normalizer of a Sylow 2-subgroup of $G$. Hence if $G_{0} \subset G$, $G_{0}$ would be a strongly embedded subgroup of $G$. However, this contradicts Bender's theorem and therefore $G_{0}=G$.

Corollary 2. If $G$ is a $Q D$-group and if $O(N)^{t}=O(N)$, then $O(N) \subseteq O(G)$.

Proof. Indeed, if $O(N)^{t}=O(N)$, then the normalizer in $G$ of $O(N)$ includes $\langle N, t\rangle$. By Proposition 8, we have that $O(N)$ is normal in $G$ and this implies $O(N) \subseteq O(G)$. 
8. $Q D$-groups of small characteristic power $q$. As before, $G$ will be a $Q D$-group of characteristic power $q$. We shall work with the principal 2-block $B_{0}(2, G)$ and the notation will be that of $\S 2$. Thus, $\chi_{i}, \chi^{(j)}$ will be the characters of $B_{0}(2, G)$, etc.

For each fixed $q$, Proposition 2.8 allows us to discuss the values possible for the degrees. We have to separate the cases $\delta_{1}=1, \delta_{2}=1$, and $\delta_{3}=1$. In each case, we have a positive integer $h<q^{2}$ such that $h$ divides $q^{2}\left(q^{2}-1\right)$. Then $f_{1}, f_{2}, f_{3}$ are determined by $h$. The congruences (6) of Proposition 2.6 impose further restrictions. If we know $f_{1}, f_{2}, f_{3}$, the degrees $f_{4}$ and $f$ are obtained from Proposition 2.4. Then Proposition 2.6, (4) gives a formula for the order $g=|G|$. As in $\S 3$, we set $A=C \cap O(N),|A|=a$ and $|O(N): A|=b$. Then $|O(N)|=a b$.

In the first part of this section, the discussion will be carried out for $q=3,5,7$, and 9.

In general, if we set $q=p^{m}$, where $p$ is a prime, $e$ is an odd divisor of $m$. In our cases, we must have $e=1$ by Proposition 2.3.3. Then the results of Chapter 2, $\$ 4$ yield

$$
|N|=2 a b q(q-1)(q+1), \quad|C|=4\left|C_{0}\right|=2 a(q+\varepsilon) .
$$

Cf. equation (4) of $\S 3$.

Proposition 1. If $G$ is a $Q D$-group of characteristic power $q=3$, we have one of the following two cases

(I) $f_{1}=11, f_{2}=45, f_{3}=55, f_{4}=44, f=10$,

$$
|G|=a b^{3} \cdot 2^{4} \cdot 3^{2} \cdot 5 \cdot 11=7920 a b^{3}
$$

(II) $f_{1}=27, f_{2}=13, f_{3}=39, f_{4}=12, f=26$,

$$
|G|=a b^{3} 2^{4} \cdot 3^{3} \cdot 13 \text {. }
$$

In both cases, $\delta_{3}=1$. In the case (II), $G$ is regular.

Proof. If $q=3$, we have $n=3, \varepsilon=-1$. Here $0<h<9, h \mid 72$.

If $\delta_{1}=1$, then $f_{2}=9-h$ and $f_{2} \equiv-3(\bmod 16)$ (Propositions 2.4 and 2.6$)$. This is impossible.

If $\delta_{2}=1$, then $f_{1}=9-h$ and $f_{1} \equiv-5(\bmod 16)$. Again, this is impossible.

If $\delta_{3}=1$, either $f_{1}=9+h$ or $f_{2}=9+h$. Since $f_{1} \equiv-5, f_{2} \equiv-3(\bmod 16)$, in the former case $h=2$ and we find the results given in (I). In the latter case, $h=4$ and we have Case II.

CoROllary 1. If $q=3$ and if $Q_{1}, Q_{2}$ are as in Lemma 7.2 we have $Q_{1}=32 / 55$, $Q_{2}=64 / 55$ in the Case I and $Q_{1}=32 / 39, Q_{2}=64 / 39$. In both cases, Lemma 7.3 holds. Also, Proposition 7.8 holds for $q=3$.

As to the last statement, it is immediate that the remark stated in $\$ 7$ in connection with Proposition 7.8 applies. 
Proposition 2. If G is a $Q D$-group of characteristic power 5 , we have one of the following three cases:

(I) $f_{1}=125, f_{2}=21, f_{3}=105, f_{4}=20, f=126$,

$$
|G|=a b^{3} \cdot 2^{4} \cdot 3^{2} \cdot 5^{3} \cdot 7 \text {. }
$$

$G$ is regular and $\delta_{1}=1$.

(II) $f_{1}=19, f_{2}=75, f_{3}=57, f_{4}=76, f=18$,

$$
|G|=a b^{3} \cdot 2^{4} \cdot 3^{2} \cdot 5^{2} \cdot 19
$$

Here, $\delta_{2}=1$.

(III) $f_{1}=35, f_{2}=85, f_{3}=119, f_{4}=84, f=34$,

$$
|G|=a b^{3} \cdot 2^{4} \cdot 3^{2} \cdot 5 \cdot 7 \cdot 17,
$$

$\delta_{3}=1$.

Proof. Here $n=3, \varepsilon=1,1 \leqq h<25, h \mid 25 \cdot 24$.

If $\delta_{1}=1$, then $f_{2}=25-h$ and $f_{2} \equiv 5(\bmod 16)$. Then $f_{2}=5, h=20$ or $f_{2}=21$, $h=4$. In the former case, we find $f_{1}=5$, while by Proposition $2.6, f_{1} \equiv-3(\bmod 16)$. Hence this case is impossible. The latter case leads to Case (I).

If $\delta_{2}=1$, then $f_{1}=25-h$ and $f_{1} \equiv 3(\bmod 16)$. Since $h=22$ is excluded, we must have $f_{1}=19, h=6$. This yields Case II.

If $\delta_{3}=1$, then $f_{1} \equiv 3, f_{2} \equiv 5(\bmod 16)$. We have either $f_{1}=25+h$ or $f_{2}=25+h$. In the former case $f_{1}=35, h=10$ and this leads to Case III. In the latter case, $f_{2}=37, h=12$. However, then $f_{1}=75 \not \equiv 3(\bmod 16)$ and this is impossible.

Corollary 2. For $q=5$, we have

Case I. $Q_{1}=32 / 35, Q_{2}=64 / 105$.

Case II. $Q_{1}=32 / 19, Q_{2}=64 / 57$.

Case III. $Q_{1}=160 / 119, Q_{2}=320 / 357$.

Again, Lemma 7.3 and Proposition 7.8 hold.

Proposition 3. If G is a QD-group of characteristic power 7, we have one of the following two cases:

(I) $f_{1}=55, f_{2}=441, f_{3}=495, f_{4}=440, f=54$

$$
|G|=a b^{3} \cdot 2^{5} \cdot 3^{3} \cdot 5 \cdot 7^{2} \cdot 11 \text {. }
$$

(II) $f_{1}=343, f_{2}=57, f_{3}=399, f_{4}=56, f=342$

$$
|G|=a b^{3} \cdot 2^{5} \cdot 3^{2} \cdot 7^{3} \cdot 19
$$

and $G$ is regular.

Proof. For $q=7$, we have $n=4, \varepsilon=-1,1<h<49, h \mid 49 \cdot 48$.

If $\delta_{1}=1$, then $f_{2}=49-h$ and $f_{2} \equiv-7(\bmod 32)$. Then $f_{2}=25, h=24$. This implies $f_{1}=49$. However, we should have $f_{1} \equiv 9(\bmod 32)$, a contradiction. 
If $\delta_{2}=1$, we have $f_{1}=49-h$ and $f_{1} \equiv-9(\bmod 32)$. Then $f_{1}=23, h=26$. This is impossible since $h$ should divide $49 \cdot 48$.

Thus, $\delta_{3}=1$ and then

$$
f_{1} \equiv-9, f_{2} \equiv-7 \quad(\bmod 32) .
$$

Either $f_{1}=49+h$ or $f_{2}=49+h$. In the former case, necessarily $f_{1}=55, h=6$ and this leads to Case (I). In the latter case, $f_{2}=57, h=8$ and this leads to Case (II).

PROPOSITION 4. If $G$ is a $Q D$-group of characteristic power $q=9$, we have one of the cases:

(I) $f_{1}=729, f_{2}=73, f_{3}=657, f_{4}=72, f=730$,

$$
|G|=a b^{3} \cdot 2^{5} \cdot 3^{6} \cdot 5 \cdot 73
$$

and $G$ is regular.

(II) $f_{1}=71, f_{2}=567, f_{3}=497, f_{4}=568, f=70$,

$$
|G|=a b^{3} \cdot 2^{5} \cdot 3^{4} \cdot 5 \cdot 7 \cdot 71 .
$$

(III) $f_{1}=135, f_{2}=201, f_{3}=335, f_{4}=200, f=134$,

$$
|G|=a b^{3} \cdot 2^{5} \cdot 3^{3} \cdot 5^{3} \cdot 67 .
$$

Proof. For $q=9$, we have $n=4, \varepsilon=1,0<h<81$ and $h \mid 81 \cdot 80$.

If $\delta_{1}=1$, then $f_{2}=81-h$ and $f_{1} \equiv-7, f_{2} \equiv 9(\bmod 32)$. Thus, $f_{2}=9, h=72$ or $f_{2}=41, h=40$ or $f_{2}=73, h=8$. In the first of these cases, we find $f_{1}=9$ which is impossible. Likewise, the second case is impossible since we would find $f_{1}=81$ $\not \equiv-7(\bmod 32)$. Hence $h=8$ and this leads to Case (I).

If $\delta_{2}=1$, then $f_{1}=81-h$ and $f_{1} \equiv 7, f_{2} \equiv-9(\bmod 32)$. Then $f_{1}=7,39$, or 71 , but the condition $h \mid 81.80$ is satisfied only in the last of these cases. Here, $h=10$ and we are led to Case II.

If $\delta_{3}=1$, we have $f_{1} \equiv 7, f_{2} \equiv 9(\bmod 32)$. Either $f_{1}=81+h$ or $f_{2}=81+h$. In the former case $f_{1}=135, h=54$, since for $f_{1}=103, h$ would be 22 and would not divide $81 \cdot 80$. For $h=54$, we are led to Case III. In the latter case, $f_{2}=105, h=24$, since for $f_{2}=137, h$ would not divide $81 \cdot 80$. For $h=24$, we find $f_{1}=351 \not \equiv 7(\bmod 32)$, so that this case does not occur either.

In the rest of the section, we shall determine the corefree $Q D$-groups of characteristic powers $q=3$ and 5. For the cases $q=7$ and 9 this will be done in Chapter VI by the methods used for arbitrary $q$, as particular cases of the Third Main Theorem. In the case $q=3$, we could have referred right from the beginning to a result of W. J. Wong [30].

We start with a number of lemmas which hold for corefree $Q D$-groups $G$ of an arbitrary characteristic power $q$.

We recall that the characters $\chi_{1}, \chi_{2}, \chi_{3}, \chi_{4}$ of the principal block $B_{0}(2, G)$ take only rational values (Corollary 2.2). Moreover, if $G$ is corefree, i.e. if $O(G)=1$, then $\chi_{1}, \chi_{2}, \chi_{3}, \chi_{4}$ are faithful (Corollary 2.3). Then a theorem of Schur [25] applies. We obtain 
LEMMA 1. Let $G$ be a corefree $Q D$-group. Let $p$ be a prime number and let $\alpha(p)$ denote the exact exponent with which $p$ divides $|G|$. Then

for $1 \leqq i \leqq 4$.

$$
\alpha(p) \leqq\left[f_{i} /(p-1)\right]+\left[f_{i} / p(p-1)\right]+\left[f_{i} / p^{2}(p-1)\right]+\cdots
$$

We can also use a similar principle with $G$ replaced by $N$. We show

Lemma 2. Suppose that $O(N) \neq 1$. Let $i$ be one of the indices 1 or 2. There exists a faithful character $\eta_{1}$ of $N$ such that $\eta_{1}(r)$ is rational for $r \in N$ of odd order and that $f^{*}=\eta_{1}(1)$ satisfies the conditions

$$
f^{*} \equiv 0 \quad\left(\bmod 2^{f-1}\right), \quad 2 f^{*} \leqq f_{i}-q .
$$

If $p$ is an odd prime and if $p$ divides $|N|$ with the exact exponent $\alpha^{*}(p)$, then

$$
\alpha^{*}(p) \leqq\left[f^{*} /(p-1)\right]+\left[f^{*} / p(p-1)\right]+\left[f^{*} / p^{2}(p-1)\right]+\cdots .
$$

Proof. Let $\xi$ denote the sum of the irreducible constituents of $\chi_{i} \mid N$ which lie in the principal block $B_{0}(2, N)$ and let $\eta$ denote the sum of the remaining irreducible constituents of $\chi_{i} \mid N$. Thus,

$$
\chi_{i} \mid N=\xi+\eta .
$$

Here, $\eta \neq 0$, since $O(N) \neq 1$ and $\xi$ is trivial on $O(N)[6, \mathrm{I}$, Theorem 1].

Let $u \in S_{0}-\{1\}$ and let $r$ be a 2-regular element of $C(u)$. Then $\chi_{i}(u r)$ and $\xi(u r)$ each are linear combinations of the values $\phi(r)$ with $\phi$ ranging over the modular irreducible characters of the principal block $B_{0}(2, C(u))$, while $\eta(u r)$ is a linear combination of the values $\phi(r)$ with $\phi$ ranging over the modular irreducible characters of $C(u)$ not belonging to $B_{0}(2, C(u))$, cf. [5, II] and [6, I, Theorem 3]. It then follows from the linear independence of the modular irreducible characters that

$$
\eta(u r)=0 .
$$

In particular, $\eta(u)=0$ for all $u \neq 1$ in $S_{0}$ and this implies

$$
\eta(1) \equiv 0 \quad\left(\bmod 2^{n}\right)
$$

If $\omega$ is an irreducible character of $N$, then as $x$ belongs to the center of $N$, either $\omega(x)=\omega(1)$ or $\omega(x)=-\omega(1)$. In the former case, $x$ belongs to the kernel of $\omega$. Let $\xi_{0}$ denote the sum of the irreducible constituents of $\xi$ whose kernel contains $x$. Let $\eta_{0}$ denote the sum of the irreducible constituents of $\eta$ whose kernel contains $x$. Set

$$
\xi=\xi_{0}+\xi_{1}, \quad \eta=\eta_{0}+\eta_{1} .
$$

If $r \in N$ is 2-regular, by (3) with $u=x$,

Hence

$$
0=\eta(x r)=\eta_{0}(x r)+\eta_{1}(x r)=\eta_{0}(r)-\eta_{1}(r) .
$$

$$
\eta_{0}(r)=\eta_{1}(r)
$$


In particular, $\eta_{0}$ and $\eta_{1}$ have the same degree $f^{*} \neq 0$. By $(4), \eta_{1}(1) \equiv 0\left(\bmod 2^{n-1}\right)$. Now (2) and (5) yield

Since $\eta(x)=0$,

$$
f_{i}=\chi_{i}(1)=\xi_{0}(1)+\xi_{1}(1)+2 \eta_{1}(1) .
$$

Hence

$$
\chi_{i}(x)=\xi_{0}(x)+\xi_{1}(x)=\xi_{0}(1)-\xi_{1}(1) \text {. }
$$

$$
\begin{aligned}
& f_{i}+\chi_{i}(x)=2 \xi_{0}(1)+2 \eta_{1}(1) \geqq 2 \eta_{1}(1), \\
& f_{i}-\chi_{i}(x)=2 \xi_{1}(1)+2 \eta_{1}(1) \geqq 2 \eta_{1}(1) .
\end{aligned}
$$

Since $\chi_{i}(x)= \pm q$ for $i=1,2$ (Proposition 2.3), $f^{*}=\eta_{1}(1)$ satisfies the conditions stated in the lemma.

Let $K$ be the kernel of $\eta_{1}$. Since $\eta_{1}(x)=-\eta_{1}(1), x \notin K$. This implies $S_{0} \cap K=\{1\}$. If $K$ has even order, $K$ must contain the element $z$. However, then $K$ would also contain $x z$ which is conjugate to $z$ in $N$. This is impossible, since $x \notin K$. Thus $K$ has odd order and then $K \subseteq O(N)$. If $r \in K$, by [6, I, Theorem 1], $\xi(r)=\xi(1)$, since all irreducible constituents of $\xi$ belong to $B_{0}(2, N)$. On the other hand, by (6), $\eta_{0}(r)=\eta_{1}(r)=\eta_{1}(1)$. Hence

$$
\chi_{i}(r)=\xi(1)+\eta(1)=f_{i}
$$

This means that $r$ belongs to the kernel of $\chi_{i}$. Now Corollary 2.3 shows that $r=1$. Thus, $K=\{1\}$, i.e. $\eta_{1}$ is a faithful character of $N$.

Let $\tau$ be an element of the Galois group of the field of characters of $N$ over the field of rational numbers. As $\chi_{i}$ takes only rational values,

$$
\xi+\eta=\chi_{i}=\chi_{i}^{\tau}=\xi^{\tau}+\eta^{\tau} .
$$

Since $\tau$ permutes the characters of $B_{0}(2, N)$ [6, I, Lemma 2], $\eta^{\tau}=\eta$. It follows that $\eta^{\tau}$ takes only rational values. Now (6) shows that $\eta_{1}(r)$ is rational for 2-regular $r \in N$.

The last part of the lemma is obtained by applying Schur's Theorem [25] to the restriction of $\eta_{1}$ to a Sylow $p$-subgroup of $N$.

LEMMA 3. Let $p$ be a prime which divides $a=|A|=|C \cap O(N)|$ but which does not divide $e(q+\varepsilon)$. Then, for $1 \leqq i \leqq 4,4(p-1) \leqq f_{i}$.

Proof. Let $r$ be a $p$-element of $A$. Then $T \subseteq C(r)$ and hence

$$
\chi_{i}(r)+\chi_{i}(x r)+\chi_{i}(z r)+\chi_{i}(x z r) \equiv 0 \quad(\bmod 4) .
$$

By Proposition 2.3 and [6, I, Theorem 1], $\chi_{i}(x r)=\chi_{i}(x)$ as $r \in O(N)$. If $t$ is as in equation (1) of $\S 1, t$ normalizes $T$ and then $C=C(T)$ and we have

$$
r^{t} \in A^{t} \subseteq C^{t}=C
$$

Since $A=C \cap O(N)$ is normal in $C$ and $|C: A|=2(q+\varepsilon) e$ (cf. equation (4) of $\S 3$ ), it follows from the hypothesis that $r^{t} \in A \subseteq O(N)$. By Proposition 2.3,

$$
\chi_{i}(z r)=\chi_{i}\left((z r)^{t}\right)=\chi_{i}\left(x r^{t}\right)=\chi_{i}(x)
$$


Similarly, $r^{t^{-1}} \in A \subseteq O(N)$ and $\chi_{i}(x z r)=\chi_{i}(x)$. Now (7) yields $\chi_{i}(r) \equiv \chi_{i}(x)(\bmod 4)$. For $r=1$, then $f_{i} \equiv \chi_{i}(x)(\bmod 4)$. Thus, for any $p$-element $r$ of $A, \chi_{i}(r) \equiv f_{i}(\bmod 4)$. Since $\chi_{i}(r) \equiv f_{i}(\bmod p)$, we can set

$$
\chi_{i}(r)=f_{i}-4 p \chi^{*}(r)
$$

with rational integral $\chi^{*}(r)$. As $\chi_{i}$ is faithful, $\chi^{*}(r)>0$ for $r \neq 1$. If we add (8) for all $r$ in a subgroup of $A$ of order $p$, the orthogonality relation for group characters show that

$$
4 p \sum \chi^{*}(r) \leqq p f_{i}
$$

Since the left side here is at least $4 p(p-1)$, we obtain $4(p-1) \leqq f_{i}$ as was stated.

In the following lemma, we consider the case of a prime $p$ which divides $|G|$ with the exact exponent 1 . If $P_{p}$ is a Sylow $p$-subgroup of $G$, we can set

$$
C\left(P_{p}\right)=P_{p} \times V_{p},
$$

where $V_{p}$ is a subgroup of an order $v_{p}$ not divisible by $p$. Moreover, we set

$$
\left|N\left(P_{p}\right): C\left(P_{p}\right)\right|=m_{p}
$$

Then $m_{p}$ divides $p-1$. It follows from Sylow's theorem that

$$
|G| / p \equiv m_{p} v_{p} \quad(\bmod p)
$$

The principal $p$-block $B_{0}(p, G)$ consists of $m_{p}$ "nonexceptional" characters and $(p-1) / m_{p}$ "exceptional" characters, cf. [4]. We show

Lemma 4. Let $p$ be a prime such that the Sylow p-subgroup $P_{p}$ of $G$ has order $p$. Assume further that $p$ does not divide $|N|$ and that $m_{p}=\left|N\left(P_{p}\right): C\left(P_{p}\right)\right|$ is even. Then $v_{p}=\left|C\left(P_{p}\right)\right| / p$ is odd and if $p \mid f_{i}$ for $i=1$ or 2 , then $p q \leqq f_{i}$.

Proof. If $v_{p}$ was even, $G$ would contain elements of order $2 p$ and then $|N|$ would necessarily be divisible by $p$ which was excluded. Hence $v_{p}$ is odd. Since $m_{p}$ is even, there exist 2-elements $u \in N\left(P_{p}\right)$ such that $u$ has order 2 over $C\left(P_{p}\right)$. Then $u^{2}=1$ and $u$ is an involution. After replacing $P_{p}$ by a conjugate, we may assume that $u=x$. The group $M=P_{p}\langle x\rangle$ has order $2 p$ and it consists of $P_{p}$ and $p$ involutions. There is a linear character $\lambda_{1}$ of $M$ which is trivial on $P_{p}$ and takes the value -1 for the $p$ involutions of $M$. Let $\lambda_{0}$ denote the principal character of $M$. Then, for $j=0$ and 1 ,

$$
0 \leqq\left(\chi_{i} \mid M, \lambda_{j}\right)=\chi_{i}(1)+\sum_{r \in P_{p}-\{1\}} \chi_{i}(r)+p \chi_{i}(x)(-1)^{j} .
$$

If $p \mid f_{i}, \chi_{i}(r)=0$ for all $r \in P_{p}-\{1\}$ and taking $j=0$ and 1 , we obtain

$$
p\left|\chi_{i}(x)\right| \leqq f_{i}
$$

Since $\left|\chi_{i}(x)\right|=q$ for $i=1,2$ by Proposition 2.3 , this establishes the lemma. 
We can now determine the corefree $Q D$-groups of characteristic powers 3 and 5 .

Proposition 5. If $G$ is a corefree $Q D$-group of characteristic power 3 , then $G$ is isomorphic to either the Mathieu group $M_{11}$ of order 7920 or to $\operatorname{PSL}(3,3)$ of order 5616.

Proof. If $q=3$, we have $\varepsilon=-1, n=3$. By (1), $|C|=2 e a(q+\varepsilon)=4 a,\left|C_{0}\right|=a$. Thus, $C_{0}=C(z) \cap O(N)=A$.

Suppose first that $b=1$. Then $O(N)=A=C_{0}$. Since $C_{0}$ is normalized by $t, O(N)$ is normalized by $t$. As $G$ is corefree, Corollary 7.2 implies that $O(N)=\{1\}$, i.e. we have $a=1$. It is now clear that $N \simeq G L(2,3)$. Then the statement is immediate from the main result of [30], cf. also [8].

It remains to deal with the case $b \neq 1$. We distinguish the Cases I and II of Proposition 1.

Case I. We apply Lemma 2 using $\chi_{1}$. Since $2 f^{*} \leqq 11-3$ and $f^{*} \equiv 0(\bmod 4)$, we have $f^{*}=4$ and we see that $|N|$ divides $16 \cdot 3^{2} \cdot 5$. Since $e=1$, (1) shows that $a b$ divides 15. Application of Lemma 1 now yields that $|G|$ divides $2^{4} \cdot 3^{6} \cdot 5^{2} \cdot 11$. Hence $a b^{3} \mid 3^{4} \cdot 5$. Since $b \neq 1$, necessarily $b=3$ and $a=1$ or $a=5$. Since $|G|$ is divisible by 11 only with the exponent 1 , the Sylow 11 -subgroup $P_{11}$ has order 11 . Now Lemma 4 shows that $m_{11}$ is odd. Since $\chi_{1}$ vanishes for elements of $G$ of an order divisible by 11 , it follows easily that $\chi_{1} \mid V_{11}=11 \lambda$, where $\lambda$ is a linear character of $V_{11}$. Since $\chi_{1}$ is rational valued and since $V_{11}$ has odd order, $\lambda$ must be the principal character. As $\chi_{1}$ is faithful, it follows that $v_{11}=1$.

Then $B_{0}(11)$ is the only 11 -block of positive defect. Hence $\chi_{0}, \chi_{2}, \chi^{(1)}, \chi^{(-1)}$, $\chi^{(2)}$ are in $B_{0}(11)$ and are nonexceptional. Hence $m_{11} \geqq 5$. As $m_{11}$ divides 10 , necessarily $m_{11}=5$. Now $(11)$ yields $a b^{3} 5 \equiv 5(\bmod 11), a b^{3} \equiv 1(\bmod 11)$. However this does not hold for the values of $a, b$ which can occur and this case is impossible.

Case II. We work here with $\chi_{2}$ of degree 13. The procedure is quite similar to that used in Case I. Again, $f^{*}=4$ in Lemma 2 and we find $a b \mid 15$, while Lemma 1 yields $a b^{3} \mid 3^{5} \cdot 5^{3}$. If $P_{13}$ is the Sylow subgroup of $G$ of order 13 , then $P_{13}$ is selfcentralizing, $v_{13}=1$. Lemma 4 shows that $m_{13}$ is odd and as $\chi_{0}, \chi_{1}$ and $\chi_{4}$ belong to $B_{0}(13, G)$, necessarily $m_{13}=3$. Then the degree equation for $B_{0}(13, G)[4$, Theorem 11] reads

$$
1+27=12+16
$$

the four exceptional characters in $B_{0}(13, G)$ have degree 16 . Now it follows from [11, Lemmas 2 and 3] that 3 divides $|G|$ with the exact exponent 3 . This means that $(a b, 3)=1$. As $b \neq 1$, we find $b=5, a=1$. However, on account of (11) for $p=13$, $a b^{3} \cdot 3 \equiv 3(\bmod 13)$ and this leads to a contradiction. This completes the proof of Proposition 5.

Proposition 6. If $G$ is a corefree $Q D$-group of characteristic power 5 , then $G$ is isomorphic to $\operatorname{PSU}(3,5)$ or to $\operatorname{PGU}(3,5)$. 
Proof. We separate the three cases of Proposition 2. The first case is by far the most difficult one.

Case I. Assume $O(N) \neq 1$. We work here with $\chi_{2}$ of degree 21. In applying Lemma 2 , we have $f^{*} \equiv 0(\bmod 4), 2 f^{*} \leqq 21-5$. Hence $f^{*}=4$ or $f^{*}=8$. This shows that $|N|$ divides $2^{4} \cdot 3^{5} \cdot 5^{2} \cdot 7$. Hence $a b$ divides $3^{4} \cdot 5 \cdot 7$. We then see from Lemma 1 that $a b^{3}$ divides $3^{12} \cdot 5^{3} \cdot 7^{2}$. In particular, $(7, b)=1$. On the other hand, Lemma 3 with $f_{i}=21, p=7$ implies that $(a, 7)=1$. Thus,

$$
a b\left|3^{4} \cdot 5, \quad a b^{3}\right| 3^{12} \cdot 5^{3} .
$$

Thus, 7 divides $|G|$ only with the exponent 1 and $|N|$ is not divisible by 7 . Now Lemma 4 with $f_{2}=21, p=7$ shows that $m_{7}$ is odd.

We claim that $\chi_{0}, \chi_{1}, \chi_{4}$ belong to $B_{0}(7, G)$. This is clear for $\chi_{0}$. If $r$ is an element of $G$ of an order divisible by 7 , then as $|N|$ is not divisible by $7, r$ has odd order. Since the degree $f=126$ of $\chi^{(1)}$ is divisible by $7, \chi^{(1)}(r)=0$. Now Proposition 2.4 shows that $\chi_{1}(r)=-1$ for all 7-singular elements $r$ of $G$. It follows from the main result of $[5, \mathrm{II}]$ that indeed $\chi_{1} \in B_{0}(7, G)$. Likewise, since $f_{2} \equiv 0(\bmod 7)$, we have $1+\chi_{4}(r)=0$ for the same $r$ and this implies that $\chi_{4} \in B_{0}(7, G)$. We have now found three nonexceptional characters in $B_{0}(7, G)$ and this implies that $m_{7} \geqq 3$. Since $m_{7}$ is odd and divides 6 , we have $m_{7}=3$ and $\chi_{0}, \chi_{1}, \chi_{4}$ are the only nonexceptional characters in $B_{0}(7, G)$. Hence the degree equation for $B_{0}(7, G)$ [4, Theorem 11] reads

$$
1+144=125+20
$$

i.e. the two exceptional characters have degree 144.

Consider now the restriction of $\chi_{4}$ to the centralizer $C\left(P_{7}\right)=P_{7} \times V_{7}$ of a Sylow 7-subgroup $P_{7}$ of $G$. We may set

$$
\chi_{4} \mid P_{7} \times V_{7}=\sum_{i=0}^{6} \lambda_{i} \gamma_{i}
$$

where $\lambda_{0}=1, \lambda_{1}, \ldots, \lambda_{6}$ are the seven linear characters of $P_{7}$ and where the $\gamma_{i}$ are characters of $V_{7}$. Since $\chi_{4}$ is a nonexceptional character in $B_{0}(7, G)$ of degree $20 \equiv-1(\bmod 7)$, it takes the value -1 for each element of $P_{7} \times V_{7}$ whose order is divisible by 7 . If $u$ is a generator of $P_{7}$, then

$$
\lambda_{0}(u)\left(1+\gamma_{0}\right)+\lambda_{1}(u) \gamma_{1}+\cdots+\lambda_{6}(u) \gamma_{6}=0
$$

for all elements of $V_{7}$. Since $\lambda_{0}(u)=1, \lambda_{1}(u), \ldots, \lambda_{6}(u)$ are the seven 7 th roots of unity, this implies

$$
1+\gamma_{0}=\gamma_{1}=\cdots=\gamma_{6}
$$

and we find

$$
\chi_{4} \mid V_{7}=\sum_{i=0}^{6} \gamma_{i}=7 \gamma_{0}+6
$$


In particular, $f_{4}=7 \gamma_{0}(1)+6$, i.e. $\gamma_{0}(1)=2$. Moreover, since $\chi_{4}$ is rational valued and faithful on $V_{7}$, so is $\gamma_{0}$. Now Schur's Theorem implies that the odd number $v_{7}$ divides 3. On account of (11), we find that we have one of the following two cases

$$
a b^{3} \equiv 1 \quad \text { or } a b^{3} \equiv 3 \quad(\bmod 7)
$$

according as to whether $v_{7}=1$ or $v_{7}=3$.

Since $\left(v_{7}, 5\right)=1$, no elements of order 35 exist in $G$. Now the method of blockseparation [11, Lemmas 2 and 3] can be applied to $B_{0}(7)$. It follows that $|G|$ contains the prime 5 with the exact exponent 3 . Thus, $(a b, 5)=1$ and

$$
a b\left|3^{4}, \quad a b^{3}\right| 3^{12} .
$$

Let $P_{5}^{*}$ denote a Sylow 5-subgroup of $N$. Then $\left|P_{5}^{*}\right|=5$ and $P_{5}^{*}$ normalizes $O(N)$. Assume first that $P_{5}^{*}$ does not centralize $O(N)$. Then $O(N) \neq 1$ and if $F$ is the Frattini subgroup of $O(N), P_{5}^{*}$ does not centralize $O(N) / F$. Since an elementary abelian group of an order dividing $3^{3}$ does not have an automorphism of order 5, we have $|O(N): F| \geqq 3^{4}$ and since $|O(N)|=a b \leqq 3^{4}, O(N)$ is necessarily elementary abelian of order $3^{4}$. Moreover, since $P_{5}^{*}$ does not centralize $O(N)$, it follows easily that $P_{5}^{*}$ acts without fixed points on $O(N)-\{1\}$. This implies that $P_{5}^{*}$ acts without (nontrivial) fixed points on the character group of $O(N)$. Hence if $\mu$ is a linear character of $O(N)$ which is different from the principal character $\mu_{0}$ of $O(N)$, then the number of conjugates of $\mu$ in $N$ is divisible by 5 .

Let $\rho$ denote an irreducible constituent of the character $\eta_{1}$ of $N$. By Clifford's theorem,

$$
\rho \mid O(N)=c \sum_{j=1}^{k} \mu_{j}
$$

where $c$ is a positive integer and where $\mu_{1}, \mu_{2}, \ldots, \mu_{k}$ is a full system of irreducible characters of $O(N)$ conjugate in $N$. Since $O(N)$ is abelian, $\mu_{j}(1)=1$ and

$$
c k=\rho(1) \leqq \eta_{1}(1)=f^{*} \leqq 8 .
$$

If $\mu_{1}$ is not the principal character $\mu_{0}$, then as we have shown, $k$ is divisible by 5 and hence $k=5, c=1$. It is then clear that $\rho \mid O(N)$ cannot be real valued. Since $\eta_{1}$ was rational valued, the conjugate complex character $\bar{\rho}$ of $N$ would also be a constituent of $\eta_{1}$. However, this is impossible since $\rho(1)=\bar{\rho}(1)=5$ and $\eta_{1}(1) \leqq 8$.

Thus, for any choice of the irreducible constituent $\rho$ of $\eta_{1}$, the character $\mu_{1}$ is the principal character $\mu_{0}$ of $O(N)$. Then $k=1$ and $\rho$ is trivial on $O(N)$. However, this is also impossible, since then $\eta_{1}$ would be trivial on $O(N)$, while $\eta_{1}$ was a faithful character of $N$ and $O(N) \neq 1$.

It now follows that $P_{5}^{*}$ centralizes $O(N)$. If $K$ is the normal closure of $P_{5}^{*}$ in $N$, $K$ also centralizes $O(N)$. Here, $K$ belongs to the normal subgroup $L_{0}$ of index 2 in $N$ and we have

$$
K O(N) / O(N) \triangleleft L_{0} / O(N) \simeq S L(2,5)
$$


The only normal subgroups of $S L(2,5)$ have orders 1,2 , or 120 . Since $K O(N) / O(N)$ contains $P_{5}^{*} O(N) / O(N)$ of order 5 , we must have $K O(N)=L_{0}$. It follows that $K$ contains the Sylow 2-subgroup $\left\langle s^{2}, s z\right\rangle$ of $L_{0}$. Hence $\left\langle s^{2}, s z\right\rangle$ centralizes $O(N)$.

Let $r \neq 1$ be an element of $O(N)$. Since $\left\langle s^{2}, s z\right\rangle$ has order 8, it follows that

$$
\sum_{u} \chi_{4}(r u) \equiv 0 \quad(\bmod 8)
$$

where $u$ ranges over the elements of the quaternion group $\left\langle s^{2}, s z\right\rangle$. On account of Proposition 2.2, $\chi_{4}(r u)=0$ when $u$ has order 4. Using Proposition 2.3 we now find

$$
\chi_{4}(r)-4 \equiv 0 \quad(\bmod 8)
$$

Since $\chi_{4}(r) \equiv 20(\bmod 3)$ and $-20 \leqq \chi_{4}(r)<f_{4}=20$, we obtain $\chi_{4}(r)=-4$. If we add $\chi_{4}(r)$ over all $r$ in $O(N)$, the sum is nonnegative and we find

$$
0 \leqq 20+(|O(N)|-1)(-4) \text {. }
$$

Thus $|O(N)| \leqq 6$ i.e. $a b=|O(N)|=1$ or 3 . Since the case $a=1, b=3$ is excluded by (12), we have $b=1$ and $a=1$ or $a=3$.

The case $a=1, b=1 .|G|=2^{4} \cdot 3^{2} \cdot 5^{3} \cdot 7$.

Here $G$ has the order of $P S U(3,5)$. The results of Harada [24], [24'] apply and shows that $G \simeq P S U(3,5)\left({ }^{11}\right)$.

The case $a=3, b=1 .|G|=2^{4} \cdot 3^{3} \cdot 5^{3} \cdot 7$.

We have here $O(N)=A$, i.e. $T$ centralizes $O(N)$. Then the normal closure $T^{N}$ of $T$ in $N$ centralizes $O(N)$. Since $z \in T$ and $s^{2} z$ are conjugate in $N$ (even in $S$ ), $s^{2} \in T^{N}$ and this implies $y=s^{2^{n-2}} \in T^{N}$. Since $y$ and $s z$ are conjugate in $N$, we see that $S=\langle s, z\rangle \in T^{N}$ and hence $S$ centralizes $O(N)$. Thus, the normal subgroup $C_{N}(O(N))$ of $N$ contains both the cyclic group $O(N)$ and $S$. Since $e=1$, we have $N=L$ and so $N /(\langle x\rangle O(N))$ is isomorphic to $P G L(2,5)$ by Lemma 2.4.15. As $P G L(2,5)$ has no nontrivial normal subgroup of odd index, $C_{N}(O(N))=N$, i.e.

$$
A=O(N) \subseteq Z(N) \text {. }
$$

By the results of Schur [26], it follows that

$$
N=N_{1} \times A,
$$

where $N_{1}$ is a normal subgroup of $N$ of index 3. Furthermore, $C_{0}=\left(C_{0} \cap N_{1}\right) \times A$ and if we set $W=C_{0} \cap N_{1}$, we have that $W$ is a Sylow 3-subgroup of $N_{1}$ of order 3 and that $W$ is inverted by $y$.

Let $u_{1}$ be a generator of $A$ and let $u_{2}$ be a generator of $W$. Then

$$
u_{1}^{y}=u_{1}, \quad u_{2}^{y}=u_{2}^{-1} .
$$

( $\left.{ }^{11}\right)$ Using methods given below in Chapter VI, $\$ 6$ we could also show that $G$ satisfies the hypothesis of O'Nan's paper $\left[24^{*}\right]$ and deduce the statement from his results. 
The 3-element $t$ of $G$ also normalizes $C_{0}$. However, as $G$ is assumed to be corefree, $t$ does not normalize $A=O(N)$ by Corollary 7.2. Since $3^{3}$ is the exact power of 3 dividing $|G|$, it follows that $\left\langle C_{0}, t\right\rangle$ is a Sylow 3-subgroup $P_{3}$ of $G$, that $\left|P_{3}\right|=27$, and that $P_{3}$ is nonabelian. The center $Z\left(P_{3}\right)$ is a subgroup of order 3 of $C_{0}$, say $Z\left(P_{3}\right)=\langle w\rangle$. On account of equation (2) of $\S 1$, we find

$$
w^{y t}=w^{t-1} y=w^{y} .
$$

Hence $w^{y} \in Z\left(P_{3}\right)$ and thus $w^{y}=w$ or $w^{y}=w^{-1}$. In the former case, (15) would show that $Z\left(P_{3}\right)=A$ and then $t$ would normalize $A=O(N)$, which was not true. Thus, $y$ inverts $Z\left(P_{3}\right)$ and then (15) implies that $Z\left(P_{3}\right)=\left\langle u_{2}\right\rangle=W$. We then have formulas $u_{1}^{t}=u_{1}^{\alpha} u_{2}^{\beta}, u_{2}^{t}=u_{2}$, with integral $\alpha, \beta$ and we must have $u_{2}^{\beta} \neq 1$. As we can replace $u_{1}$ by $u_{1}^{-1}$, we can assume $u_{2}^{\beta}=u_{2}$. Since $t^{3} \in C_{0}$ centralizes $u_{1}$, we find $\alpha^{3} \equiv 1(\bmod 3)$. Hence $\alpha \equiv 1(\bmod 3)$ and then

$$
u_{1}^{t}=u_{1} u_{2}, \quad u_{2}^{t}=u_{2} .
$$

If as before $E=O(C(S))$, then as $S$ centralizes $O(N)$, we have $O(N) \subseteq E$. On the other hand, $E \subseteq C=T \times C_{0}$ and hence $E \subseteq C_{0}$. Since $y$ does not centralize $C_{0}$, actually $E \neq C_{0}$ and as $\left|C_{0}\right|=9$, we have $E=O(N)$. Thus, $E$ has three linear characters $1, \theta, \bar{\theta}$ and, consequently, $G$ has exactly three 2-blocks of full defect $B_{0}(2, G)$, $B_{\theta}, B_{\bar{\theta}}$. Clearly, $B_{\bar{\theta}}$ consists of the conjugate complex characters of the characters of $B_{\theta}$. It follows from (14) that $\theta$ can be extended to a character $\theta_{N}$ of $N$ which is trivial on $N_{1}$ and, in particular, on $S$. Thus, $B_{\theta}$ is of type $\left(^{*}\right)$ as defined in $\S 6$. Set $\theta_{C}=\theta_{N} \mid C$. Since $u_{2} \in W \subseteq N_{1}, \theta_{C}\left(u_{2}\right)=1$. On the other hand, $\theta_{C}\left(u_{1}\right)$ is a primitive third root of unity $\omega$. It follows from (16) that

$$
\theta_{C}\left(t^{-1} u_{1} t\right)=\omega=\theta_{C}\left(u_{1}\right), \quad \theta_{C}\left(t^{-1} u_{2} t\right)=1=\theta_{C}\left(u_{2}\right) .
$$

Thus, $\theta_{C}^{t}=\theta_{C}$ and now, Proposition 6.2 shows that $B_{\theta}$ does not contain a minor subsection $(x, b)$. It then follows from Proposition 7.6 that $B_{\theta}$ contains exactly one character of degree 1 . In fact, $G$ possesses exactly three characters of degree 1 . This means that $\left|G: G^{\prime}\right|=3$.

It is clear that $G^{\prime}$ again has the Sylow 2-subgroup $S$. Also, as $G$ is corefree, so is $G^{\prime}$. In particular, $G^{\prime}$ does not possess a normal 2-complement. If $G^{\prime}$ was not a $Q D$-group, it would necessarily possess a unique normal subgroup $H$ of index 2 . Then $H$ would be normal in $G$. The group $G / H$ of order 6 with the normal subgroup $G^{\prime} / H$ of order 2 would be abelian and then $G$ would have a normal subgroup of index 2 , a contradiction.

Hence $G^{\prime}$ is again a $Q D$-group. Since $N^{*}=C_{G^{\prime}}(x)$ is a nonsolvable subgroup of $N$, the characteristic power of $G^{\prime}$ is necessarily 5 . Now Proposition 2 and the preceding discussion show that necessarily $G^{\prime} \simeq P S U(3,5)$.

Note that we can choose $t \in G^{\prime}$ and that $\left|N^{*}\right|=240$. On account of (16), $u_{2} \in G^{\prime}$. Since $\left|N^{*}\right|$ is not divisible by 9 , then $u_{1} \notin G^{\prime}$ and hence

$$
G=\left\langle G^{\prime}, u_{1}\right\rangle \text {. }
$$


By Proposition 7.8 applied to $G^{\prime}, G^{\prime}=\left\langle N^{*}, t\right\rangle$ and hence

$$
G=\left\langle G^{\prime}, u_{1}\right\rangle=\left\langle N^{*}, t, u_{1}\right\rangle \text {. }
$$

Since $u_{1} \in O(N) \subseteq Z(N), u_{1}$ centralizes $N^{*}$ while by (16) $u_{1} t=t u_{1} u_{2}$. It is clear that the action of $u_{1}$ on $G^{\prime}$ is unique and as $u_{1}^{3}=1$, the group $G$ is unique apart from isomorphism. Since $P G U(3,5)$ is a corefree $Q D$-group of characteristic power 5 and the order $2^{4} \cdot 3^{3} \cdot 5^{3} \cdot 7$, we have $G \simeq P G U(3,5)$. This concludes the discussion of the Case I.

Case II. We apply Lemma 2 with $\chi_{i}=\chi_{1}, f_{1}=19$. Then $2 f^{*} \leqq 14, f^{*} \equiv 0(\bmod 4)$. If $a b \neq 1$, then $f^{*}=4$ and this implies that $|N|$ divides $16 \cdot 3^{2} \cdot 5$. Then $a b \mid 3$. In any case, the Sylow 19-subgroup $P_{19}$ of $G$ has order 19 and since we have a faithful irreducible character $\chi_{1}$ of degree $19, P_{19}$ is self-centralizing, i.e. $v_{19}=1$. Lemma 4 shows that $m_{19}$ is odd. Since $\chi_{0}, \chi_{2}, \chi^{(1)}, \chi^{(-1)}, \chi^{(2)}$ belongs to $B_{0}(19, G)$, we have $m_{19} \geqq 5$ and as $m_{19} \mid 18$, then $m_{19}=9$. Now (11) yields $9 a b^{3} \equiv 9(\bmod 19)$ i.e. $a b^{3} \equiv 1$ (mod 19). For $a b \mid 3$, this is only possible when $a=b=1$.

If $a=b=1$, we have $g=2^{4} \cdot 3^{2} \cdot 5^{2} \cdot 19$. Since $m_{19}=9$, we have $\left|N\left(P_{19}\right): C\left(P_{19}\right)\right|=9$. Hence there exist elements $w \in G$ of order 9 and the Sylow 3-subgroup $P_{3}=\langle w\rangle$ is cyclic. We can choose here $w$ such that $w^{3}=u \in C_{0}$. Let $R \supseteq T$ be a Sylow 2subgroup of $C(u)$. If $|R| \geqq 8, R$ contains a conjugate $y^{\prime}$ of $y$. However $C(y) \subseteq N$ and the structure of $N$ shows that $C(y)$ is a 2-group. So is $C\left(y^{\prime}\right)$. But then we cannot have $u \in C\left(y^{\prime}\right)$, a contradiction.

Hence $R=T$. This implies that if an element $r \in G$ has the 3-factor $u$, its 2-factor is either 1 or an involution. In either case, Propositions 2.3 and 2.4 show that

$$
1+\delta_{1} \chi_{1}(r)+\delta_{2} \chi_{2}(r)+\delta_{3} \chi_{3}(r)=0 .
$$

Since $f_{2}=75$ and $f_{3}=57, \chi_{2}$ and $\chi_{3}$ belong to 3-blocks of defect 1 . These must have the defect group $\langle u\rangle$ and then (17) in conjunction with the main result of $[5$, II] shows that necessarily $\chi_{2}$ and $\chi_{3}$ lie in the same 3-block $B(3)$ of defect 1 . Since these characters have degrees 75 and 57 respectively, the third character in $B(3)$ has necessarily degree $75+57=132$. However, this is impossible as $|G|$ is not divisible by 11 . Hence the Case II cannot occur.

Case III. On account of Lemma 1 with $f_{i}=f_{1}=35, \alpha(17) \leqq 2$ and this implies that $(b, 17)=1$. Lemma 3 shows that $(a, 17)=1$ and hence $\alpha(17)=1$. On account of Lemma $4, m_{17}$ is odd and as $m_{17} \mid 16$, we have $m_{17}=1$. Then $B_{0}(17, G)$ consists of 17 linear characters of $G$. Since these form a multiplicative group, $G$ possesses a normal subgroup $G_{1}$ of index 17. Clearly, $N \subseteq G_{1}$ and $t \in G_{1}$ and hence $G_{1}$ is a $Q D$-group of characteristic power $q=5$. It is also clear that $G_{1}$ is corefree. In applying Proposition 2 to $G_{1}$ we must necessarily have Case I. This implies that $5^{3}$ divides $\left|G_{1}\right|$. Also, $O\left(N \cap G_{1}\right)$ has order 1 or 3 by our discussion of Case I. Since $N=N \cap G_{1}$, we have $|O(N)|=1$ or 3 i.e. $a b \mid 3$. However, then Proposition 2 shows that $5^{2}$ does not divide $|G|$. This is a contradiction and the proof of Proposition 6 is complete.

In the case $q=7$, only the following partial result will be needed in Chapter VI. 
Proposition 7. If $G$ is a simple $Q D$-group of characteristic power $q=7$ and if $G$ is not regular, then $a b \equiv 0(\bmod 5)$.

Proof. If $G$ is not regular, we have Case I in Proposition 3. If $a b \not \equiv 0(\bmod 5)$, then $|N|$ is not divisible by 5 and $|G|$ contains 5 only with the exponent 1 . Now the results of [4] and [11] apply. Since $f_{1}=55$ is divisible by 5 and since no elements of order 10 exist, it follows that all characters $\chi^{(j)}$ lie in the principal 5-block $B_{0}(5, G)$. For $q=7$, we have $n=4$ and there are seven characters $\chi^{(j)}$, cf. $\S 2,(1)$. Since $B_{0}(5, G)$ contains at most five characters, we have a contradiction.

9. Various results concerning $Q D$-groups. We shall now draw some conclusions from the results of $\$ \$ 6$ and 7 . They will be fully effective only in conjunction with the results of Chapters IV and V.

Proposition 1. If $G$ is a $Q D$-group, then

$$
\left|G: G^{\prime}\right|=\left|E: E \cap\left[C_{0}, t\right] N^{\prime}\right| \text {. }
$$

Here, $E, C_{0}, t$ are defined in $\S 1$. The prime indicates the commutator-subgroup.

Proof. Since $\left|G: G^{\prime}\right|$ is the number of characters of degree 1 of $G$, it follows from Propositions 7.6 and 7.7 that $\left|G: G^{\prime}\right|$ is equal to the number $k$ of blocks $B_{\theta}$ of $G$ of type $\left(^{*}\right)$ without a minor subsection $(x, b)$. Now Proposition 6.2 shows that $k$ is equal to the number of characters $\theta$ of degree 1 of $E$ such that

(i) $\theta$ can be extended to a character $\theta_{N}$ of $N$ which is trivial on $S$.

(ii) If we set $\theta_{C}=\theta_{N} \mid C$, then $\theta_{C}^{t}=\theta_{C}$.

Since $\theta_{N}$ is trivial on $T$ and has degree 1 , (ii) is equivalent with the condition that $\theta_{N}$ is trivial on $C^{*}=\left[C_{0}, t\right] \subseteq C_{0}$. Since $\theta_{N}$ is trivial on $N^{\prime}$, then $\theta_{N}$ is trivial on $C^{*} N^{\prime}$ and, consequently, $\theta$ is trivial on $E \cap C^{*} N^{\prime}$.

Conversely, assume that $\theta$ is an irreducible character of $E$ which is trivial on $E \cap C^{*} N^{\prime}$. Since $E^{\prime} \subseteq N^{\prime}$, then $\theta$ can be considered as an irreducible character of $E / E^{\prime}$ and hence $\theta(1)=1$. Since $\theta$ is trivial on $E \cap N^{\prime} \subseteq E \cap C^{*} N^{\prime}, \theta$ can be extended to a character of $E N^{\prime}$ which is trivial on $N^{\prime}$. It is now clear that $\theta$ can be extended to a character $\xi$ of $N$. We now set

$$
\theta_{N}=\xi \eta
$$

where $\eta$ is a linear character of $N$. We shall show that $\eta$ can be chosen such that $\theta_{N}$ satisfies the conditions (i) and (ii).

The condition (ii) requires that $\xi \eta$ is trivial on $C^{*}$. Since $\xi$ extends $\theta$, we know that $\xi$ is trivial on $E \cap C^{*} N^{\prime}$ and as $\xi$ is a linear character of $N, \xi$ is trivial on $N^{\prime}$ and then on

$$
\left(E \cap C^{*} N^{\prime}\right) N^{\prime}=E N^{\prime} \cap C^{*} N^{\prime} .
$$

It is then clear that there exist extensions $\eta$ of $\xi^{-1} \mid\left(C^{*} N^{\prime}\right)$ to $N$ such that $\eta$ is trivial on $E N^{\prime}$. If $\eta$ in (2) is chosen in this manner, we have

$$
\theta_{N} \mid C^{*}=\left(\xi \mid C^{*}\right)\left(\eta \mid C^{*}\right)=\left(\xi \mid C^{*}\right)\left(\xi^{-1} \mid C^{*}\right)=1_{C^{*}}
$$


Hence $\theta_{N}$ is trivial on $C^{*}$ and the condition (ii) is satisfied. Moreover, since $\xi$ extends $\theta$, we have

Thus $\theta_{N}$ extends $\theta$.

$$
\theta_{N} \mid E=(\xi \mid E)(\eta \mid E)=\theta \cdot 1_{E}=\theta
$$

We note that $N^{\prime}$ has the Sylow 2-subgroup $\left\langle s^{2}, s z\right\rangle$. In particular, the linear character $\theta_{N}$ of $N$ is trivial on $\left\langle s^{2}, s z\right\rangle$. If $\theta_{N}$ is not trivial on $S=\langle s, z\rangle$, we must have $\theta_{N}(z)=-1$. Clearly, $N$ has a normal subgroup $N_{0}$ of index 2. Hence there exists a linear character $\mu$ of $N$ with the kernel $N_{0}$. Then $\mu(z)=-1$. Since $N^{\prime}, E$, $C^{*}$ all belong to $N_{0}$, we may replace $\eta$ by $\eta \mu$ above and then $\theta_{N}(z)$ will have the value 1 , i.e. $\theta_{N}$ is trivial on $S$ and the condition $\left({ }^{*}\right)$ above is also satisfied.

We have now shown that $\left|G: G^{\prime}\right|$ is equal to the number of linear characters of $E$ which are trivial on $E \cap C^{*} N^{\prime}$. As $C^{*}=\left[C_{0}, t\right]$, this sets $(1)$ in evidence.

As an application, we prove

Proposition 2. Let $G$ be a $Q D$-group. If $M$ is a $t$-invariant subgroup with $C_{0} \supseteq M \supseteq$ $C_{0} \cap L$, then $\left|C_{0}: M\right|$ divides $\left|G: G^{\prime}\right|$.

Here $L$ is defined as in $\S 1$. As we have noted there, $N=L E$ and $N / L$ is cyclic.

Proof. If $M=C_{0}$, the statement is trivial. If $M \neq C_{0}$, we must have $L \neq N$. Since $E \subseteq C_{0}$, we have $E L=C_{0} L=N$ and this implies that

$$
N / L \simeq E /(E \cap L) \simeq C_{0} /\left(C_{0} \cap L\right) \text {. }
$$

These groups are cyclic of order $e$, by definition of $e$. Since $M$ contains $C_{0} \cap L$, it is normal in $C_{0}$. The element $y$ normalizes $C_{0}$ and $C_{0} \cap L$ and it is clear now that $y$ normalizes $M$. As by assumption $t$ normalizes $M$, we have

$$
M \triangleleft\left\langle C_{0}, y, t, z\right\rangle=N(T) .
$$

By (3) and the cyclicity of $N / L$, there exists an element $w$ in $E$ such that $C_{0}$ $=\left(C_{0} \cap L\right)\langle w\rangle$. In particular, $C_{0}=M\langle w\rangle$. As $N(T)$ normalizes $C_{0}$, the action of $N(T)$ on $C_{0} / M$ produces a homomorphism $\omega$ of $N(T)$ into the automorphism group of the cyclic group $C_{0} / M$. It is clear that the kernel $K_{\omega}$ of $\omega$ contains $C_{0}$. Since $y$ and $z$ centralize $w$, we have $y, z \in K_{\omega}$. Finally, as

$$
t y \equiv y t^{-1}, t^{3} \equiv 1 \quad(\bmod C),
$$

(cf. $\S 1,(1)$ and (2)), we have $\omega(t)=\omega\left(t^{-1}\right)$ and $\omega(t)^{3}=1$. Hence $\omega(t)=1$, i.e. $t \in K_{\omega}$. It follows that $\left[C_{0}, t\right] \subseteq M$. Since $N^{\prime} \subseteq L$, then $\left[C_{0}, t\right] N^{\prime} \subseteq M L$.

On account of Proposition 1, we have

$$
\left|G: G^{\prime}\right|=|E: E \cap M L| \cdot\left|E \cap M L: E \cap\left[C_{0}, t\right] N^{\prime}\right| .
$$

Proposition 2 will be proved, if we can show that

$$
|E: E \cap M L|=\left|C_{0}: M\right| \text {. }
$$

Now, as $C_{0} \cap L \subseteq M \subseteq C_{0}$, we have

$$
C_{0} \cap M L=M\left(C_{0} \cap L\right)=M \text {. }
$$


Since $N=E L=E M L$, we find

$$
|N: M L|=|E: E \cap M L|
$$

Likewise, since $N=C_{0} M L$

$$
|N: M L|=\left|C_{0}: C_{0} \cap M L\right|=\left|C_{0}: M\right|
$$

cf. (4). On comparing (5) and (6), we obtain $\left(^{*}\right)$ and the proof is complete.

CoRollary 1. If $G$ is a $Q D$-group and if $C_{0} \cap L$ is t-invariant, then e divides $\left|G: G^{\prime}\right|$.

Indeed, we can apply Proposition 2 with $M=C_{0} \cap L$. We have to observe that

$$
\left|C_{0}: C_{0} \cap L\right|=\left|C_{0} L: L\right|=|N: L|=e .
$$

COROLlaRY 2. If $G$ is a $Q D$-group with $G=G^{\prime}$, there does not exist a t-invariant subgroup $M$ with $C_{0} \supset M \supseteq C_{0} \cap L$.

This is immediate from Proposition 2.

COROLlaRY 3. If $G$ is a $Q D$-group with $G=G^{\prime}$, then $e=|N: L|$ divides $a(q+\varepsilon) / 2$ (where $a$ as always is defined as $a=|C \cap O(N)|$ ). In fact, $a(q+\varepsilon) / 2=c e$ with $c=\left|\left(C_{0} \cap L\right) \cap\left(C_{0} \cap L\right)^{t}\right|$.

Proof. Set $C_{1}=C_{0} \cap L$. Then $C_{1} \triangleleft C_{0}$. Since $t$ normalizes $C_{0}$ and $t^{3} \in C_{0}$, $t$ permutes the subgroups $C_{1}, C_{1}^{t}, C_{1}^{t^{-1}}$ by conjugation. Set $c=\left|C_{1} \cap C_{1}^{t}\right|$. Conjugation shows that

$$
c=\left|C_{1} \cap C_{1}^{t}\right|=\left|C_{1}^{t} \cap C_{1}^{t-1}\right|=\left|C_{1}^{t^{-1}} \cap C_{1}\right| .
$$

Now $C_{0} / C_{1} \simeq N / L$ is cyclic of order $e$. It follows that the groups $C_{1} C_{1}^{t}$ and $C_{1} C_{1}^{t-1}$ are equal, since both lie between $C_{0}$ and $C_{1}$ and both have the same order $\left|C_{1}\right|^{2} / c$. It follows that

$$
C_{1} C_{1}^{t} C_{1}^{t-1}=C_{1} C_{1}^{t}
$$

In particular, $C_{1} C_{1}^{t}$ is $t$-invariant. Corollary 2 shows that $C_{1} C_{1}^{t}=C_{0}$. It follows that

$$
\left|C_{0}\right|=\left|C_{1}\right|^{2} / c
$$

Since $\left|C_{0}: C_{1}\right|=e$, then $\left|C_{0}\right|=c e^{2}$. Since $\left|C_{0}\right|=a e(q+\varepsilon) / 2$, we have $c e=a(q+\varepsilon) / 2$ as we wished to show.

REMARK. Since $C_{0}^{\prime} \subseteq C_{1}$ and hence $C_{0}^{\prime} \subseteq C_{1} \cap C_{1}^{t}$, it follows that $\left|C_{0}: C_{0}^{\prime}\right|$ is divisible by $e^{2}$.

The following two propositions are concerned with sufficient conditions for regularity of a $Q D$-group $G$. It will be convenient to formulate a condition for $N$. We consider 2-blocks $b$ of $N$ with the defect group $T$. Since $b$ corresponds to a block $\bar{b}$ of $N /\langle x\rangle$ of defect $1, \bar{b}$ and then $b$ contains a single modular irreducible 
character $\phi$. Since the corresponding Cartan invariant $c(\phi, \phi)$ then is 4 (e.g. by $[5, I,(6 \mathrm{C})]), b$ consists of four ordinary irreducible characters and each of them coincides with $\phi$ for elements $r \in N$ of odd order. In particular, the four irreducible characters in $b$ have degree $\phi(1)=f(b)$. We shall say now that $N$ satisfies condition (\#) provided that

For each 2-block $b$ of $N$ with the defect group $T$, the degree $f(b)$ of the four characters in $N$ is coprime to the characteristic power $q$ of $N$.

We shall now prove:

Proposition 3. Let $G$ be a $Q D$-group for which $G=G^{\prime}$ and $e>1$. If the centralizer $N$ of an involution satisfies the condition (\#), then $G$ is regular.

Proof. Since $N$ has a normal subgroup $L$ for which $N / L$ is cyclic of order $e \neq 1$, we can choose a linear character $\theta_{N}$ of $N$ with the kernel $L$. If $w$ is chosen as before then $w \in E$ and $\theta_{N}(w) \neq 1$. If $\theta=\theta_{N} \mid E, \theta$ is a linear character of $E$ different from the principal character of $E$, and $\theta$ satisfies the condition $\left({ }^{*}\right)$ of $\S 6$. Then the 2-block $B_{\theta}$ of $G$ is different from the principal block $B_{\theta}(2, G)$. On account of Proposition 7.6, $B_{\theta}$ has a minor subsection $(x, b)$. Here, $b$ is a 2-block of $N$ with the defect group $T$. According to the hypothesis (\#) on $N$, we have $(f(b), q)=1$. In Proposition 7.4 we have $f^{\#}=f(b)$ and then

$$
|G|=\frac{|N|^{3}}{|C|^{2}} \frac{f_{0}^{(\theta)}}{f(b)^{2}}
$$

Now the definition of regularity in $\S 3$ shows that $G$ is regular.

RemarK. A similar proof shows that Proposition 3 remains valid, if the two assumptions $G=G^{\prime}$ and $e>1$ are replaced by the assumption $e>\left|G: G^{\prime}\right|$.

Proposition 4. Assume that $G$ is a $Q D$-group for which the centralizer $N$ of an involution satisfies the condition (\#). Suppose that there exist irreducible characters $\omega$ of $C=T \times C_{0}$, trivial on $T$, which have the stabilizer $C$ in $N(T)$. Then $G$ is regular.

Proof. Let $\omega$ belong to the 2-block $b$ of $C$. Then $\omega$ is the canonical character of $b$ and $b$ also has the stabilizer $C$ in $N(T)$. It follows that $B=b^{G}$ is a 2-block of $G$ with the defect group $T$.

It follows from the general results on 2-blocks of defect $2[6$, IV] that in our present case, $B$ consists of four characters all of the same degree $f(B)$ and that we have a formula

$$
|G|=\frac{|N|^{3}}{|C|^{2}} \frac{\omega(1)^{2}}{f\left(b_{1}\right) f\left(b_{2}\right) f\left(b_{3}\right)} f(B),
$$

where each $b_{i}(i=1,2,3)$ is a 2-block of $N$ with the defect group $T$ and where $f\left(b_{i}\right)$ is the degree of the characters in $b_{i}$. By the condition $(\#),\left(f\left(b_{i}\right), q\right)=1$. Again, the definition of regularity shows that $G$ is regular.

We shall show ultimately that every $Q D$-group of characteristic power $q>3$ is regular. 
Chapter IV. Structure of $O(N)$. Localization

1. Preliminary discussion of Theorem B. This chapter and the next are devoted to the proof of Theorem B and the study of the 2-regular core $O(N)$ of the centralizer $N=C(x)$ of an involution $x$ in a simple group $G$ with quasi-dihedral or wreathed Sylow 2-subgroups. Theorem B has been stated explicitly only in the case that $G$ has quasi-dihedral Sylow 2-subgroups and only if the proper simple $Q D$-sections of $G$ satisfy the conclusions of the Main Theorems. We wish to reformulate the theorem now in a way that will include the wreathed case as well as the quasi-dihedral.

In order to accomplish this, we first list the two consequences of our assumption on the proper simple $Q D$-sections of $G$ that we actually use in the proof of Theorem B. In the quasi-dihedral case these two results are a direct consequence of Proposition 2.3 of Chapter II and Theorem A of Chapter III.

I. If $H$ is a proper $Q D$-subgroup of $G, N_{1}$ is the centralizer of an involution of $H$, and $T$ is a four-subgroup of $H$ containing that involution, then [T,O(N)] $\subseteq O(H)$.

II. If $H$ is a proper $Q D$-subgroup of $G$ of the same characteristic power $q$ as $G$ and if $q>3$, then $O(H)=1$.

Furthermore, in Chapter III we have already established the Main Theorems when $G$ has quasi-dihedral Sylow 2-subgroups and characteristic power $q=3$ or 5. In particular, it is immediate from this that $G$ satisfies the conclusions of Theorem B for these values of $q$. Thus in the quasi-dihedral case it will suffice to establish Theorem B when $q>5$. This is an important point, for as we have noted in $\$ 4$ of Chapter II, $Q$-groups of characteristic power $q=3$ or 5 are somewhat exceptional and as a consequence many of the arguments to be given in the general proof of Theorem B are not valid in the special cases $q=3$ or 5 . Corresponding difficulties occur when $G$ has wreathed Sylow 2-subgroups for the same values of $q$ and so these two cases must also be treated independently in that problem.

We therefore make the following additional assumption:

III. The characteristic power $q$ of $G$ exceeds five.

We shall refer to assumptions I, II, and III together as the working hypotheses and we shall restrict ourselves to a simple group $G$ with quasi-dihedral or wreathed Sylow 2-subgroups which satisfies the working hypotheses. We emphasize that in the quasi-dihedral case the working hypotheses are themselves a consequence of results of Chapters II and III. Moreover, as remarked in the Introduction they will be proved in the wreathed case in a subsequent paper by one of the authors.

Finally, we remark that assumption II is of critical importance to us. Because of it, we are able to show that certain local subgroups which we must construct in the course of our arguments are not $Q D$-groups. As a consequence, the subsequent analysis of these local subgroups requires only a knowledge of properties of $Q$-groups, $D$-groups, and normal 2-complements. 
We can now state Theorem $B$ in a form which covers both the quasi-dihedral and wreathed cases. As in $\S 4$ of Chapter II, we define $\delta, \varepsilon$, and $n$ by the conditions $q \equiv \varepsilon(\bmod 4), \delta=+1$ or -1 according as $G$ has quasi-dihedral or wreathed Sylow 2 -subgroups, and $2^{n}$ is the highest power of 2 dividing $q+\delta \varepsilon$.

THEOREM B. Let $G$ be a simple group with quasi-dihedral or wreathed Sylow 2-subgroups of characteristic power $q$ which satisfies the working hypotheses. If $N$ is the centralizer of an involution in $G$, then the following conditions hold:

(i) $O(N)=A B$, where $A$ is cyclic of order dividing $(q+\delta \varepsilon) / 2^{n}, B$ is abelian, and if $A \neq 1, B \neq 1$, then $O(N)$ is a Frobenius group with kernel $B$ and complement $A$.

(ii) Any noncentral involution of $N$ inverts $B$ and centralizes a conjugate of $A$.

(iii) If $X$ is a noncyclic abelian 2-subgroup of $N$ of maximal order, then $X$ centralizes a conjugate of $A, X$ normalizes $B$, and $X / C_{X}(B)$ is cyclic of order $2^{n}$ and acts regularly on $B$.

(iv) The extended centralizer $C^{*}(u)$ lies in $N$ for any nonidentity element $u$ of $O(N)$.

The goal of the present chapter will be to reduce the proof of Theorem B to the study of certain local subgroups. In particular, we shall construct and examine certain families of local subgroups.

As a first step we wish to restate Theorem B in a more detailed way. We shall then easily see that the version just stated is a consequence of the new version. We begin by introducing some notation and terminology which we shall fix once and for all. We let $G$ be a simple group with quasi-dihedral or wreathed Sylow 2-subgroups of characteristic power $q>5$ and define $\varepsilon, \delta$, and $n$ as above. We fix an involution $x$ of $G$ and we let $N=C(x)=C_{G}(x)$. Thus, $N$ contains a Sylow 2subgroup of $G$ by Propositions 2.2.1 and 2.2.2. We choose notation for elements and subgroups of $N$, in accordance with the opening remarks of $\S 4$ of Chapter II. This includes the following:

$$
x, T, x_{1}, x_{2}, x_{3}, y, d, C, D, E, L_{0}, L .
$$

Here $x=x_{1}, x_{2}, x_{3}$ are the three involutions of the four-subgroup $T, D$ is either dihedral of order eight or is a Sylow 2-subgroup of $N, S$ is a Sylow 2-subgroup of $N$ containing $D, y$ and $d$ are certain elements of $S$, which actually lie in $D, C=C_{N}(T)$ $=C(T), D=N_{S}(T), E=O\left(C_{N}(S)\right)=O(C(S)), L_{0} \supset O(N)$ with $L_{0} / O(N)$ isomorphic to $S L(2, q)$, and $L=L_{0} S$. Furthermore, $C_{D}(T)=T$ if $S$ is quasi-dihedral, while $C_{D}(T)$ is the unique abelian subgroup of index two in $D$ if $D=S$ is wreathed. We also set $M=N(C)$.

Actually in some arguments in the quasi-dihedral case, it is more convenient to fix only $T$ and $D$, but not $S$, and instead to allow $S$ to be an arbitrary Sylow 2-subgroup of $N$ containing $D$. We shall therefore allow this degree of freedom in the choice of $S$. Since $O\left(C_{N}(S)\right)=O\left(C_{N}(D)\right)$ by Lemma 2.4.3 and since $L_{0}$ and $L$ are normal in $N$, this will not affect the definition of $E$ or of $L$. 
We next introduce two concepts which will be very important for us.

Definition 1. If $p$ is a prime divisor of $|O(N)|$, then $p$ is said to be stationary provided there is a Sylow p-subgroup $R$ of $O(N)$ such that $R$ is also a Sylow $p$-subgroup of $O(N(R))$.

Note that this definition is independent of the choice of $R$, by Sylow's theorem, and so is really a property of the prime $p$.

Definition 2. If $p$ is a prime divisor of $|O(N)|$, then $p$ is said to be centralized (inverted) if there is a Sylow p-subgroup $R$ of $O(N)$ normalized by $T$ and centralized by $T$ (inverted by the elements $x_{2}, x_{3}$ of $T-\langle x\rangle$ ).

Again, this does not depend on $R$. Indeed, $O(N)$ is of odd order, so any two $T$ invariant Sylow $p$-subgroups of $O(N)$ are conjugate by an element of $C_{O(N)}(T)$. Futhermore, if $R$ is as in the definition, then $R$ is certainly centralized by $x$, so $p$ is centralized if $x_{2}$ centralizes $R$ and $p$ is inverted if $x_{2}$ inverts $R$.

We now let $A$ be a $D$-invariant Hall subgroup of $O(N)$ for the set of centralized and stationary primes. Such Hall subgroups exist since $O(N)$ is solvable, while $D$ is a 2-group and $O(N)$ is of odd order. Similarly, we let $B$ be a $D$-invariant Hall subgroup of $O(N)$ for the set of inverted and stationary primes. We fix $A$ and $B$. Note that $B$ is, of course, abelian as it is inverted by the involution $x_{2}$.

We can now state the more detailed form of Theorem $B$.

THEOREM B. If $G$ is a simple group with quasi-dihedral or wreathed Sylow 2subgroups which satisfies the working hypotheses, then $G$ has the following properties:

(i) $O(N)=A B, A$ is cyclic of order dividing $(q+\delta \varepsilon) / 2^{n}, B$ is abelian and if $A \neq 1, B \neq 1$, then $O(N)$ is a Frobenius group with kernel $B$ and complement $A$.

(ii) If $U=C_{D}(T)$, then $U$ centralizes $A, U$ normalizes $B$, and $U / C_{U}(B)$ is cyclic of order $2^{n}$ and acts regularly on $B$.

(iii) The extended centralizer $C^{*}(u)$ lies in $N$ for any nonidentity element $u$ of $O(N)$.

(iv) There is a normal subgroup $J$ of $L_{0}$ such that $L_{0}=J O(N)$, $J$ centralizes $O(N)$, and either $J$ is isomorphic to $S L(2, q)$ and $J \cap O(N)=1$ or $q=9, J$ is isomorphic to $S L^{\wedge}(2,9),|J \cap O(N)|=3$, and $G$ has quasi-dihedral Sylow 2-subgroups.

Here $S L^{\wedge}(2,9)$ is the group described in $\$ 2$ of Chapter II which has a central subgroup of order three with quotient isomorphic to $\operatorname{SL}(2,9)$ and is directly indecomposable.

This result has as a consequence the earlier version of Theorem B. Indeed, parts (i) and (iv) of that version follow directly from parts (i) and (iii) here. Furthermore, $x_{2}$ inverts $B$ and centralizes $A$ by definition of $B$ and $A$. If $S$ is quasi-dihedral, every noncentral involution of $S$ is conjugate to $x_{2}$ in $S$ by Lemma 2.1.1 and so any noncentral involution $t$ of $N$ is conjugate to $x_{2}$ in $N$. Since $B$ is characteristic in $O(N)=A B$, we see that $B$ is normal in $N$. It follows therefore that $t$ inverts $B$ and centralizes a conjugate of $A$. Likewise, by the same lemma, any noncyclic abelian 2-subgroup $X$ of $N$ of maximal order is conjugate in $N$ to $T$ and contains $x$. Thus 
$X$ centralizes a conjugate of $A$ and $X / C_{X}(B)=X /\langle x\rangle$ inverts $B$. This shows that parts (ii) and (iii) of the first version also hold in this case.

Suppose, on the other hand, that $S$ is wreathed of height $n$, in which case $S=D$. By the remarks at the beginning of $\S 4$ of Chapter II, $Y=D \cap L_{0}=D \cap J$ is generalized quaternion of order $2^{n+1}$ and hence $Y$ is the unique generalized quaternion subgroup of $D$ of maximal order. But now Lemma 2.1.2 yields that $\Omega_{1}(D)=T Y$. Since $x_{2}$ and $x_{3}$ are conjugate in $D$ and since every involution of $D$ lies in $\Omega_{1}(D)$, we conclude that any noncentral involution $t$ of $N$ is conjugate in $N$ to an involution of the form $x_{2} v$ for some element $v$ of $Y$. But $J$ centralizes $O(N)$ by (iv) and $Y \subseteq J$, so $v$ centralizes $O(N)$. Therefore, $t$ inverts $B$ and centralizes a conjugate of $A$, so part (ii) of the first version holds in this case as well.

Finally any noncyclic abelian 2-subgroup of $N$ of maximal order is conjugate in $N$ to $U$, the unique such abelian subgroup of $D$. Hence part (iii) of the earlier version follows from part (ii) of this version (together with the fact that $B$ is normal in $N$ ). Thus the earlier form of Theorem B does indeed follow from the present one.

It will be understood that it is the second expanded form of Theorem B that we shall establish in Chapters IV and V.

The working hypotheses together with the results of Chapter II have a great number of consequences for the structure of the subgroups of $G$. The most important and widely used of these will now be listed. They can be viewed as an expanded form of our "working hypotheses" for the proof of Theorem B.

Proposition 1. The following conditions are satisfied by $G$ and its subgroups:

(i) If $H$ is a local $Q D$-subgroup of $G$, then $H$ has characteristic power 3 or 5 or a proper divisor of $q$.

(ii) If $H$ is a proper $Q D$-subgroup of $G, N_{1}$ is the centralizer of an involution of $H$, and $T_{1}$ is a four-subgroup of $H$ containing that involution, then $\left[T_{1}, O\left(N_{1}\right)\right] \subseteq O(H)$.

(iii) If $H$ is a subgroup of $G$ with $O(H) \neq 1$ which covers $L_{0} / O(N)$, then $H$ is a $Q$-group of characteristic power $q$ and if $x \in H$, then $H=O(H)(H \cap N)$.

(iv) If $H$ is a subgroup of $G$ containing $T$ with $O(H) \neq 1$ and if $H$ covers $C \cap L / C \cap O(N)$, then $H$ is not a $Q D$-group.

(v) If $H$ is a subgroup of $G$ containing $T$ such that $\left|A_{H}(T)\right|$ is divisible by 3 and either $D \subseteq H$ or $G$ has quasi-dihedral Sylow 2-subgroups, then $H$ is a $Q D$-group or a D-group with respect to $T$.

(vi) If $H$ is a proper subgroup of $G$ containing $T$ and either $D \subseteq H$ or $G$ has quasidihedral Sylow 2-subgroups, then precisely one of the following holds: $H$ is a $Q D$ group, $H$ is a $Q$-group, $H$ is a $D$-group, or $H$ has a normal 2-complement.

Although it is possible to determine the general structure of all proper subgroups of $G$ when $H$ has wreathed Sylow 2-subgroups, the results given in parts (v) and (vi) will suffice for the paper. 
Proof. (i) Let $r$ be the characteristic power of $H$. Without loss we can assume that $x \in H$, in which case $H \cap N=C_{H}(x)$ is a $Q$-group of characteristic power $r$ by definition of the characteristic power of $H$. Setting $\bar{N}=N / O(N)$, we have that $\overline{H \cap N}$ is also a $Q$-group of characteristic power $r$. As such, it contains a $Q$-subgroup $\bar{K}$ of the same characteristic $r$, but with no normal subgroups of index two. Since $\bar{N}=\bar{L} \bar{E}$, where $\bar{L}, \bar{E}$ have the properties listed in Proposition 2.3.3, it follows that $\bar{K} \cap \bar{L}$ is also a $Q$-group of characteristic power $r$ with no normal subgroups of index two. Since $\bar{L}_{0}$ is normal in $\bar{L}$ of index a power of 2 , we see that $\bar{K} \cap \bar{L}$ $\subseteq \bar{L}_{0}$. But now Lemma 2.4.8 yields that either $r=3, r=5$, or $r$ divides $q$. However, since $O(H) \neq 1$, it follows from the working hypotheses on $G$ that $r \neq q$.

(ii) This is simply a restatement of assumption I of the working hypotheses on $G$.

(iii) By assumption, $O(H) \neq 1$ and $L_{0}=O(N) H_{0}$, where $H_{0}=H \cap L_{0}$. Hence $H_{0}$ contains a Sylow 2-subgroup $S_{0}$ of $L_{0}$ and $\bar{H}_{0}=\bar{L}_{0}=L_{0} / O(N)$, so $\bar{H}_{0}$ is isomorphic to $S L(2, q)$. But then if $x_{0}$ is the unique involution of $S_{0}, O(N)\left\langle x_{0}\right\rangle$ is normal in $H_{0}$ and it follows by the Frattini argument that $H_{0}=O\left(H_{0}\right) C_{H_{0}}\left(x_{0}\right)$, so $C_{H_{0}}\left(x_{0}\right)$ also covers $L_{0} / O(N)$. We conclude at once that $C_{H_{0}}\left(x_{0}\right)$ is a $Q$-group of characteristic power $q$.

Now $H$ is not a $D$-group by Lemma 2.5 .5 , as it contains a $Q$-group. Hence, if $H$ were not a $Q$-group, it would necessarily be a $Q D$-group and so $C_{H}\left(x_{0}\right)$ would be a $Q$-group by Proposition 2.2.1. But $C_{H_{0}}\left(x_{0}\right) \subseteq C_{H}\left(x_{0}\right)$ and $C_{H_{0}}\left(x_{0}\right)$ is a $Q$-group of characteristic power $q$, so the characteristic power of $C_{H}\left(x_{0}\right)$ is at least $q$, whence by Lemma 2.3.2, it is exactly $q$. However, this contradicts our working hypotheses on $G$ since $O(H) \neq 1$. Thus $H$ is a $Q$-group and, as $H_{0} \subseteq H$, its characteristic power is $q$.

Finally assume $x \in H$, whence $x \in H_{0}$, and so we can take $x=x_{0}$. Since $S_{0}$ is generalized quaternion, it follows at once from Lemmas 2.1.1 and 2.1.2 that $x \in Z(S)$ for any Sylow 2-subgroup $S$ of $H$ containing $S_{0}$. But $H=O(H) C_{H}(Z(S))$ by Proposition 2.3 .1 as $H$ is a $Q$-group, so $H=O(H) C_{H}(x)=O(H)(H \cap N)$ as $x \in Z(S)$. Thus all parts of (iii) hold.

(iv) Suppose, by way of contradiction, that $H$ is a $Q D$-group. Since $x \in T \subseteq H$, Proposition 2.2.1 implies that $H \cap N$ is a $Q$-group. As in the proof of (i), it follows that $H_{0}=H \cap L_{0}$ is also a $Q$-group. Thus $\bar{H}_{0}$ is a $Q$-subgroup of $\bar{L}_{0}=L_{0} / O(N)$ and so $\bar{H}_{0}$ contains a subgroup isomorphic to $S L(2, r)$, where $r=3, r=5$, or $r$ divides $q$ by Lemma 2.4.8. Since $S L(2, r)$ contains a subgroup isomorphic to $S L(2,3)$ for all odd $r$, we see that $H_{0}$ contains a subgroup $X$ such that $\bar{X}$ is isomorphic to $S L(2,3)$.

Furthermore, $C \cap H$ covers $C \cap L / C \cap O(N)$ by assumption, so

$$
C \cap L=(C \cap O(N))(C \cap H \cap L)
$$

whence $\overline{C \cap L}=\overline{C \cap H \cap L}$. We conclude therefore that $\overline{H \cap L}$ contains $\langle\bar{X}, \overline{C \cap L}\rangle$. On the other hand, $q>5$ by the basic hypotheses of the chapter and 
therefore $\langle\bar{X}, \overline{C \cap L}\rangle=\bar{L}$ by Lemma 2.4.6. Thus $\overline{H \cap L}=\bar{L}$ and so $\overline{H \cap L} \supseteq \bar{L}_{0}$, which implies that $H$ covers $L_{0} / O(N)$. Since $O(H) \neq 1, H$ must therefore be a $Q$ group by (iii) and so cannot be a $Q D$-group. Thus (iv) also holds.

We prove (v) and (vi) simultaneously. If $G$ has wreathed Sylow 2-subgroups, then $D$ is a Sylow 2-subgroup of $G$ by definition of $D$ and $D \subseteq H$ by assumption, so $H$ has wreathed Sylow 2-subgroups in this case. On the other hand, if $G$ has quasi-dihedral Sylow 2-subgroups, then the Sylow 2-subgroups of $H$ are either dihedral or quasi-dihedral as these are the only types of subgroups of a quasidihedral group which contain a four group.

Let $S_{1}$ be a Sylow 2-subgroup of $H$. If $S_{1}$ is dihedral, then by Definition 2.2.4, either $H$ has a normal 2-complement or is a $D$-group; while if $S_{1}$ is either quasidihedral or wreathed, Propositions 2.1.1 and 2.1.2 together with Definition 2.2.1 imply that either $H$ has a normal 2-complement, $H$ is a $Q$-group, $H$ is a $D$-group, or $H$ is a $Q D$-group. Thus (vi) holds.

Suppose finally that $H$ contains a four-subgroup $T_{1}$ such that $\left|A_{H}\left(T_{1}\right)\right|$ is divisible by three. Clearly then, $H$ does not have a normal 2-complement. Hence by (vi), either (v) holds or $H$ is a $Q$-group. Suppose, by way of contradiction, that $H$ is a $Q$-group. Since $\left|A_{H}\left(T_{1}\right)\right|$ is divisible by three, $H$ contains a 3-element $a$ which normalizes but does not centralize $T_{1}$. Then $K=\left\langle T_{1}, a\right\rangle$ is a subgroup of $H$ which does not have a normal 2-complement. Moreover, $T_{1}$ is a Sylow 2-subgroup of $K$ as $T_{1}$ is normal in $K$ of index a power of three. Hence $K$ is a $D$-group by Definition 2.2.4 as $T_{1}$ is a dihedral group of order four. However, this is impossible as no subgroup of a $Q$-group is a $D$-group by Lemma 2.4 .17 . Hence (v) holds and the proposition is proved.

In $\$ 4$ of this chapter we shall show that condition (iv) of Theorem $B$ is a consequence of conditions (i) and (iii). Actually a more general result on the structure of $L_{0}$ is proved which will be used in the next chapter. Then in $\$ 5$ of the chapter we shall show that Theorem $B$ itself is a consequence of the following result:

THEOREM B*. If $p$ is a prime divisor of $|O(N)|$, then $p$ is stationary and is either centralized or inverted.

In order to state yet another equivalent form of our results, we introduce one more bit of terminology.

Definition 3. If $p$ is a prime divisor of $|O(N)|$, then $p$ is ordinary if $p$ is stationary and is centralized or inverted. Otherwise, we say that $p$ is exceptional.

Thus, Theorem $\mathrm{B}^{*}$ states that all the prime divisors of $|O(N)|$ are ordinary; there are no exceptional ones. In order to state a key result on exceptional primes we need one more concept.

Definition 4. If $p$ is a prime divisor of $|O(N)|$, then $\mathscr{L}(p)$ is the collection all $p$-local subgroups of $G$ which cover $L_{0} / O(N)$ and contain $D$ as well as a Sylow $p$-subgroup of $O(N)$. 
In view of Proposition 1 , every element of $\mathscr{L}(p)$ is a $Q$-group of characteristic power $q$.

The important property in the definition of $\mathscr{L}(p)$ is the covering statement. The others are included for technical reasons. Henceforth, if we speak of ordinary, exceptional, centralized, or inverted primes $p$, we shall of course mean that $p$ is a prime divisor of $|O(N)|$ even though we do not state that assumption explicitly.

The following important result which is to be derived in $\$ 3$ gives a criterion for a prime $p$ to be exceptional in terms of the structure of the elements of $\mathscr{L}(p)$. This theorem will constitute our final reduction of Theorem $B$.

THEOREM 1. The prime $p$ is exceptional if and only if there is an element $H$ in $\mathscr{L}(p)$ which is p-constrained within $S(H)$.

This can be restated as follows: The prime $p$ is ordinary if and only if every element $H$ of $\mathscr{L}(p)$ is not $p$-constrained within $S(H)$.

Thus the effect of Theorem 1 is to shift the focus of attention, in deriving Theorems $\mathrm{B}$ and $\mathrm{B}^{*}$, upon properties of the $p$-local subgroups of $G$ for the various primes $p$ dividing $|O(N)|$. The next chapter will be devoted to a proof of Theorem $\mathrm{B}^{*}$; all the results of the present chapter (apart from the derivation of Theorem B from Theorem $\mathrm{B}^{*}$ ) can be regarded as preparatory to that endeavor. Completing the outline of this chapter, in $\$ 2$ we give a sufficient condition for a prime $p$ to be, in fact, ordinary, which is needed for the arguments of $\$ 3,4$, and 5. In $\$ 6$ we develop a number of properties of the family $\mathscr{L}(p)$. Then in the final three sections we introduce and investigate a second important family $\mathscr{M}(p)$ of $p$-local subgroups of $G$, each of whose members is a $D$-group. A large part of the proof of Theorem B* involves a study of the interrelation of the elements of $\mathscr{L}(p)$ and $\mathscr{M}(p)$.

We conclude this section with some useful, basic properties of the elements of $\mathscr{L}(p)$; these can be regarded as an addition to our "working hypotheses" of Proposition 1.

LEMMA 1. If $H \in \mathscr{L}(p)$ for a prime $p$, then

(i) $H$ is a $Q$-group of characteristic power $q$.

(ii) $H=O(H)(H \cap N)$.

(iii) $H$ covers $L / O(N)$.

(iv) $H$ contains a Sylow 2-subgroup of $L$ and of $G$.

(v) $O(H) \cap N \subseteq O(N)$ and $O(N) \cap H \subseteq O(H)$.

(vi) $O(H)$ contains a D-invariant Sylow p-subgroup of $O(N)$.

Proof. (i) As noted above, this is a consequence of Proposition 1.

(ii) Again, by Proposition 1, we only need to show that $x \in H$. However, $x \in D \subseteq H$, by assumption.

(iii) By the remarks of $\S 4$ of Chapter II, we know that $L=L_{0} C_{D}(T)$. But $C_{D}(T)$ $\subseteq D \subseteq H$ and $H$ covers $L_{0} / O(N)$, so (iii) is proved.

(iv) Since $x \in H$, it follows from (iii) that $H$ contains a Sylow 2-subgroup of $L$. But a Sylow 2-subgroup of $L$ is also one of $G$, proving (iv). 
(v) Clearly $O(H) \cap N \subseteq O(H \cap N)$. But $(H \cap N) O(N) / O(N)$ has no nontrivial normal subgroups of odd order by Lemma 2.4.7 as it contains $L_{0} / O(N)$. Hence, $O(H \cap N) \subseteq O(N)$ and so $O(H) \cap N \subseteq O(N)$. Similarly, $O(N) \cap H \subseteq O(N \cap H)$ and $O(N \cap H) \subseteq O(H)$ as $H / O(H)$ has no normal subgroups of odd order and $N$ covers $H / O(H)$ by (ii).

(vi) Let $R$ be a Sylow $p$-subgroup of $O(N)$ contained in $H$. Hence $R \subseteq O(H)$ by (iv). Let $R^{*}$ be a $D$-invariant Sylow $p$-subgroup of $O(H)$. Since $R$ and $R^{*}$ are $x$-invariant, there is $u \in C_{O(H)}(x)$ such that $R^{u} \subseteq R^{*}$. But then $u \in N$ and so $R^{u} \subseteq O(N)$ as $R \subseteq O(N)$ and $O(N)$ is normal in $N$. This implies that $R^{u}$ is a Sylow p-subgroup of $O(N)$ and hence that $R^{u}=O(N) \cap R^{*}$. Thus $R^{u}$ is also $D$-invariant. Finally, $R^{u} \subseteq N \cap O(H) \subseteq O(H)$ by (iv). This establishes (vi) and completes the proof of the lemma.

2. Stationary nonconstrained primes. We begin by introducing one more concept.

Definition 1. If $p$ is a prime divisor of $|O(N)|$, then $p$ is constrained if $N$ is $p$-constrained within $S(N)$ and $p$ is nonconstrained in the contrary case.

The following remark is very useful:

LEMMA 1. If $p$ is a prime divisor of $|O(N)|$ and $R$ is a Sylow p-subgroup of $O(N)$, then the following conditions hold:

(i) $p$ is constrained if and only if $C_{N}(R) \subseteq S(N)$.

(ii) $p$ is nonconstrained if and only if $C_{N}(R)$ covers $L_{0} / O(N)$.

Proof. Let $S$ be a Sylow 2-subgroup of $N$ containing $D$. Since our conditions on $R$ hold if and only if they hold for any conjugate of $R$ by an element of $N$ and since $S$ leaves some Sylow $p$-subgroup of $O(N)$ invariant, we can assume that $R$ is $S$-invariant. Then by Lemma 2.6.2, $p$ is constrained if and only if $C_{Z(S)}(R)$ is a Sylow 2-subgroup of $C_{N}(R)$. Suppose $p$ is constrained. Since $C_{Z(S)}(R)$ is cyclic, we conclude from Burnside's transfer theorem that $C_{N}(R)$ has a normal 2-complement and so is solvable. But $O(N) C_{N}(R)$ is normal in $N$ as $C_{N}(R)$ is normal in $N_{N}(R)$ and $N=O(N) N_{N}(R)$ by the Frattini argument. Thus $O(N) C_{N}(R)$ is a solvable normal subgroup of $N$ an we conclude that $C_{N}(R) \subseteq S(N)$. On the other hand, if $p$ is not constrained, $C_{Z(S)}(R)$ is not a Sylow 2-subgroup of $C_{N}(R)$. But $S(N)$ $=O(N) Z(S)$ by Lemma 2.4.14 and so $Z(S)$ is a Sylow 2-subgroup of $S(N)$. We conclude at once that $C_{N}(R) \nsubseteq S(N)$. Thus (i) holds.

If $C_{N}(R)$ covers $L_{0} / O(N)$, then $C_{N}(R)$ is not solvable as $L_{0} / O(N)$ is not solvable. Hence $C_{N}(R) \nsubseteq S(N)$ and so $p$ is nonconstrained by (i). Conversely, if $p$ is nonconstrained, then $C_{N}(R) \nsubseteq S(N)$ by (i). But $N_{N}(R)$ covers $N / O(N)$ as $N=O(N) N_{N}(R)$ and so $N_{N}(R)$ also covers $N / S(N)$. Thus $S(N) C_{N}(R)$ is a normal subgroup of $N$ containing $S(N)$ properly. However, $L_{0} S(N) / S(N)$ is the unique minimal normal subgroup of $N / S(N)$ by Lemma 2.4.15 and therefore $C_{N}(R)$ covers $L_{0} S(N) / S(N)$. But then $C_{N}(R)$ covers $L_{0} / O(N)$ by Lemma 2.4.13. Hence (ii) also holds. 
The main result of this section states that certain primes are indeed ordinary, in the sense of Definition 1.3.

Proposition 1. The prime $p$ is ordinary if and only if it is stationary and nonconstrained.

Before proving this, we require a preliminary lemma.

LEMMA 2. If $p$ is a stationary and nonconstrained prime and $R_{0}$ is a nonidentity T-invariant p-subgroup of $O(N)$, then there is a Sylow p-subgroup of $O\left(N\left(R_{0}\right)\right)$ centralized by $x$.

In particular, any Sylow $p$-subgroup of $O\left(N\left(R_{0}\right)\right)$ normalized by $x$ is centralized by $x$ as $\left\langle x, O\left(N\left(R_{0}\right)\right)\right\rangle$ is a solvable group with Sylow 2-subgroup $\langle x\rangle$.

Proof (of Lemma 2). Let $R$ be a $T$-invariant Sylow p-subgroup of $O(N)$ containing $R_{0}$; such a subgroup exists since $O(N)$ has odd order and $T$ is a 2-group, while $R_{0}$ is $T$-invariant by assumption. First, suppose that $R_{0}=R$. In this case $R_{0}$ is a Sylow $p$-subgroup of $O\left(N\left(R_{0}\right)\right)$ since $p$ is stationary and $R_{0}=R$. Moreover, $x$ centralizes $R_{0}$ as $x \in Z(N)$, so the lemma holds in this case.

We proceed by induction on $\left|R: R_{0}\right|$. We may assume that $R \supset R_{0}$, so $R_{1}$ $=N_{R}\left(R_{0}\right) \supset R_{0}$. We also set $H_{0}=N\left(R_{0}\right)$ and $H_{1}=N\left(R_{1}\right)$.

By induction, $x$ centralizes each Sylow $p$-subgroup of $O\left(H_{1}\right)$ that it normalizes. We claim that $x$ even centralizes each $p$-subgroup of $H_{1}$ that it normalizes. First, we note that $H_{1}$ is a $Q$-group of characteristic power $q$ and $H_{1}=O\left(H_{1}\right) C_{H_{1}}(x)$. Indeed, $H_{1}=N\left(R_{1}\right) \supseteq C\left(R_{1}\right) \supseteq C(R)$, so $H_{1}$ covers $L_{0} / O(N)$ as $C(R)$ does by Lemma 1. Also $x \in H_{1}$, so $H_{1}$ is as described by Proposition 1.1. Next, suppose that $R^{*}$ is a p-subgroup of $H_{1}$ normalized by $x$. It follows that

$$
\left[R^{*}, x\right] \subseteq\left[H_{1}, x\right]=\left[O(H) C_{H_{1}}(x), x\right] \subseteq O\left(H_{1}\right) .
$$

Thus $\left[R^{*}, x\right]$ is a $p$-subgroup of $O\left(H_{1}\right)$ normalized by $x$. Since $O\left(H_{1}\right)$ is of odd order, there is a Sylow p-subgroup of $O\left(H_{1}\right)$ normalized by $x$ and containing $\left[R^{*}, x\right]$. This Sylow subgroup is centralized by $x$, so $x$ centralizes $\left[R^{*}, x\right]$. Since $\langle x\rangle$ and $R^{*}$ have coprime orders, it follows that $x$ centralizes $R^{*}$, as claimed.

Suppose now that the lemma fails for $R_{0}$. Since $R_{1} \subseteq H_{0}, O\left(H_{0}\right) R_{1}$ is a group and is of odd order. Since $x$ normalizes both $O\left(H_{0}\right)$ and $R_{1}$, there is a Sylow $p$-subgroup $R_{2}$ of $O\left(H_{0}\right) R_{1}$ normalized by $x$ and containing $R_{1}$. But $O\left(H_{0}\right)$ is a normal subgroup of $O\left(H_{0}\right) R_{1}$, so $R_{2} \cap O\left(H_{0}\right)$ is an $x$-invariant Sylow p-subgroup of $O\left(H_{0}\right)$. Since the lemma is false for $R_{0}$, no $x$-invariant Sylow $p$-subgroup of $O\left(H_{0}\right)$ is centralized by $x$. Thus $x$ does not centralize $R_{2} \cap O\left(H_{0}\right)$ and so $x$ does not centralize $R_{2}$. It follows therefore from Lemma 2.8.1 that there is a nonidentity element $u$ of $R_{2}$ inverted by $x$ and centralizing $C_{R_{2}}(x)$. But $R_{1} \subseteq C_{R_{2}}(x)$ as $R_{1} \subseteq R_{2}$ and so $u \in H_{1}$. Thus $\langle u\rangle$ is a $p$-subgroup of $H_{1}$ normalized, but not centralized, by $x$. This is a contradiction and the lemma is proved.

Proof (of Proposition 1). We begin with the easier half; we assume that $p$ is ordinary and verify that $p$ has the necessary properties. Thus by definition $p$ is 
stationary and is either inverted or centralized; and we must prove that $p$ is nonconstrained.

Hence if $R$ is a $T$-invariant Sylow $p$-subgroup of $O(N)$, it will suffice by Lemma 1 to show that $C_{N}(R) \nsubseteq S(N)$. However, if $p$ is centralized, then $T \subseteq C_{N}(R)$ and $T \nsubseteq S(N)$ as $Z(D)$ is a Sylow 2-subgroup of $S(N)$ by Lemma 2.4.14 and $Z(D)$ is cyclic as $D$ is either dihedral of order eight or is a wreathed Sylow 2-subgroup of $G$. Suppose, on the other hand, that $p$ is inverted. Let $K$ be the subgroup of $N$ of elements which either invert or centralize $R$. Then $K$ is normal in $N_{N}(R)$ and $T \subset K$. Since $N=O(N) N_{N}(R)=S(N) N_{N}(R)$ by the Frattini argument, it follows that $S(N) K$ is a normal subgroup of $N$ containing $S(N)$ properly. Moreover, $x \in K$ as $x \in Z(N)$. But now we conclude from Lemmas 2.4.13 and 2.4.15, as in Lemma 1, that $K$ covers $L_{0} / O(N)$. Thus $L_{0} \subseteq O(N) K$. Since $L_{0}$ is nonsolvable and $O(N)$ is a solvable normal subgroup of $O(N) K$, we have that $K$ is nonsolvable. But $\left|K: C_{N}(R)\right|$ $=2$ and so $C_{N}(R)$ is also nonsolvable. Therefore $C_{N}(R) \nsubseteq S(N)$, as desired.

Now assume $p$ is stationary and nonconstrained. To prove that $p$ is ordinary, we must show that $p$ is either inverted or centralized. Since $C$ is $D$-invariant and $C$ has a normal 2-complement, $C$ possesses a $D$-invariant Sylow $p$-subgroup $P$. The group $O(N) O(C)$ is of odd order, is normalized by $T$, and contains $P$ as $C$ has a normal 2-complement. Hence there is a $T$-invariant Sylow $p$-subgroup $R^{*}$ of $O(N) O(C)$ containing $P$. It follows that $R=O(N) \cap R^{*}$ is a $T$-invariant Sylow $p$-subgroup of $O(N)$ normalized by $P$ and $R^{*}=R P$. Moreover, $C_{R}\left(x_{2}\right)=P \cap R$; indeed, $P$ is a Sylow $p$-subgroup of $C$ and $x$ is central in $N$. Thus $p$ is inverted if $P \cap R=1$ and $p$ is centralized if $P \cap R=R$. Hence we may assume that

$$
1 \subset P \cap R \subset R
$$

and derive a contradiction. To do this, we set $H=N(P)$ and $K=N(P \cap R)$, so $H$ and $K$ are $p$-local subgroups of $G$. These groups and their properties provide the desired contradiction.

First, $K$ is a $Q$-group of characteristic power $q$ and $K=O(K)(K \cap N)$. Indeed, $C(R)$ covers $L_{0} / O(N)$ as $p$ is nonconstrained, so $C(P \cap R)$ also covers $L_{0} / O(N)$. Thus $K$ is as described, by Proposition 1.1, as $x \in K$.

Second, $H$ is a solvable $D$-group. Indeed, $H=N(P)$ covers $M / C$ by the Frattini argument, so $A_{H}(T)=$ Aut $(T)$. Moreover, $D \subset H$ as $P$ is $D$-invariant. Hence $H$ is either a $Q D$-group or a $D$-group with respect to $T$, again by Proposition 1.1. Suppose that $H$ is a $Q D$-group or a nonsolvable $D$-group with respect to $T$. We shall derive a contradiction. It follows from Propositions 2.2.2 and 2.3.4 and Lemma 2.5.1 that $\bar{H}=H / O(H)$ possesses a normal subgroup $\bar{H}_{1}=H_{1} / O(H)$, which is simple and contains $\bar{T}$. Therefore $\bar{H}_{1}$ is the normal closure of $\bar{T}$ in $\bar{H}_{1}$. Since $C(P)$ contains $T$ and $C(P)$ is normal in $H$, we have $\overline{C(P)} \supseteq \bar{H}_{1}$, so $C(P)$ is also a $Q D$ group or a nonsolvable $D$-group. However, $C(P) \subseteq C(P \cap R) \subseteq K$. But $K$ contains no $Q D$-subgroups or $D$-subgroups by Lemma 2.4 .17 , since $K$ is a $Q$-group. This contradiction forces $H$ to have the desired structure. In particular, since $H$ contains 
a Sylow 2-subgroup of $M$, it follows from Proposition 2.3.4 that $\bar{H}$ is isomorphic to $P G L_{n}(2,3)$ for some $n \geqq 1$.

Since $P \cap R \subset R$ and $R$ is normal in $P R$, it follows that $P_{1}=N_{R}(P) \supset P \cap R$. Hence $P_{1}$ is a $T$-invariant $p$-subgroup of $H$ and so $\bar{P}_{1}$ is a $\bar{T}$-invariant $p$-subgroup of $\bar{H}$. But then $\bar{P}_{1}=1$ by Lemma 2.5 .9 , forcing $P_{1} \subseteq O(H)$; therefore if $P^{*}$ is a $T$-invariant Sylow $p$-subgroup of $O(H)$, then $P \subset P^{*}$ and so $T$ does not centralize $P^{*}$. But $N_{H}\left(P^{*}\right)$ is also a $D$-group with respect to $T$ by the Frattini argument and so all involutions of $T$ are conjugate in $N_{H}\left(P^{*}\right)$. Hence no involution of $T$ centralizes $P^{*}$. In particular, $x$ does not, so by Lemma 2.8 .1 , there is a nonidentity element $u$ of $P^{*}$ inverted by $x$ and centralizing $C_{P^{*}}(x)$. Then $u \in K$ as $u$ centralizes $P \cap R$ and $P \cap R \subseteq C_{P^{*}}(x)$. But $x$ centralizes $K / O(K)$, so $u \in O(K)$ and $\langle u\rangle$ is an $x$-invariant $p$-subgroup of $O(K)$ not centralized by $x$. This contradicts Lemma 2 and the proposition is proved.

We conclude the section by giving a property of ordinary primes.

LEMMA 3. If $p$ is an ordinary prime, $R$ is a T-invariant Sylow p-subgroup of $O(N)$, and $u$ is a nonidentity element of $R$, then

(i) $C(u)$ is $T$-invariant.

(ii) Every T-invariant p-subgroup of $C(u)$ lies in $N$.

Proof. (i) Since $p$ is ordinary, $R$ is inverted or centralized by each element of $T$. Thus $T$ normalizes $\langle u\rangle$ and so normalizes $C(\langle u\rangle)=C(u)$.

(ii) We set $H=N(\langle u\rangle)$ and thus $T \subseteq H$. Furthermore, $C(R)$ covers $L_{0} / N$ as $p$ is nonconstrained by the proposition, so $H$ is a $Q$-group of characteristic power $q$ and $H=O(H) C_{H}(x)$ by Proposition 1.1. Moreover, by Lemma 2, $x$ centralizes every $p$-subgroup of $O(H)$ that it normalizes. Since $x$ centralizes $H / O(H)$, it follows, just as in the proof of Lemma 2, that $x$ centralizers every $p$-subgroup of $H$ that it normalizes. In particular, any $T$-invariant $p$-subgroup of $H$ certainly lies in $N$.

3. Proof of Theorem 1.1. The task to be accomplished in this section is a proof of Theorem 1.1. We must show that a prime $p$ is exceptional if and only if $\mathscr{L}(p)$ has a certain property. We begin as follows:

LEMMA 1. If $p$ is an ordinary prime and $H \in \mathscr{L}(p)$, then

(i) The element $x$ centralizes each p-subgroup of $H$ that it normalizes.

(ii) $H$ is not p-constrained within $S(H)$.

Proof. (i) Since $H \in \mathscr{L}(p), x$ centralizes $H / O(H)$. Thus we need only establish that $x$ centralizes each $p$-subgroup of $O(H)$ that it normalizes. In particular, it suffices to show that $x$ centralizes some Sylow $p$-subgroup of $O(H)$.

Since $H \in \mathscr{L}(p)$, there is a $D$-invariant Sylow $p$-subgroup $R$ of $O(N)$ contained in $O(H)$ by Lemma 1.1. Let $R^{*}$ be a $D$-invariant Sylow $p$-subgroup of $O(H)$ which contains $R$. We shall prove that $R^{*}=R$ and so demonstrate (i).

Suppose that $x$ centralizes $R^{*}$. Thus $R^{*} \subseteq N$, so $R^{*} \subseteq O(N)$ by Lemma 1.1 as 
$R^{*} \subseteq N \cap O(H)$. But $R \subseteq R^{*}$ and $R$ is a Sylow $p$-subgroup of $O(N)$, so $R=R^{*}$ in this case.

Next, assume that $x$ does not centralize $R^{*}$; we shall derive a contradiction. By Lemma 2.8.1, there is $u \in R^{*}, u \neq 1$, such that $u$ is inverted by $x$ and $u$ centralizes $C_{R^{*}}(x)$. Hence $u \in C(R)$ as $R \subseteq R^{*} \cap N=C_{R^{*}}(x)$. But $N(R) \in \mathscr{L}(p)$ as $N(R)$ contains both $D$ and $R$ and covers $L_{0} / O(N)$ by the Frattini argument applied to the normal subgroup $O(N)$ of $N$. Hence $x$ centralizes $N(R) / O(N(R))$, so $u \in O(N(R))$ as $u \in N(R)$ and $x$ inverts $u$. But $p$ is stationary, being ordinary, so $R$ is a Sylow $p$-subgroup of $O(N(R))$. However, $x$ does not centralize $\langle u\rangle$ and does centralize $R$. This is a contradiction and (i) is proved.

(ii) The subgroup $O(N) \cap H$ is a normal subgroup of $L_{0} \cap H$ and contains $R$. Hence by the Frattini argument there is a Sylow 2-subgroup $S_{0}$ of $L_{0} \cap H$ which normalizes $R$. However, $H$ covers $L_{0} / O(N)$ as $H \in \mathscr{L}(p)$, so $S_{0}$ is a Sylow 2-subgroup of $L_{0}$. Since $p$ is ordinary, it is nonconstrained by Proposition 2.1; hence $C_{L_{0}}(R)$ covers $L_{0} / O(N)$ by Lemma 2.1 and so $C_{L_{0}}(R)$ has odd index in $N_{L_{0}}(R)$. Thus $S_{0} \subseteq C_{L_{0}}(R)$. But $S_{0} \subseteq H$, so $S_{0} \subseteq C_{H}(R)$. Since $R$ is a Sylow $p$-subgroup of $O(H)$ and $S_{0}$ is generalized quaternion, it follows from Lemma 2.6.2 that $H$ is not $p$-constrained 'within $S(H)$ and the lemma is proved.

We remark that actually this lemma is superfluous, for the only part of Theorem 1.1 that we require for the proof of Theorem B is the existence of elements $H$ of $\mathscr{L}(p)$ that are $p$-constrained in $S(H)$ when $p$ is exceptional. However, we think the fuller picture is clearer.

LEMMA 2. If $p$ is an exceptional prime and $R$ is a D-invariant Sylow p-subgroup of $O(N)$, then $H=N(R)$ is an element of $\mathscr{L}(p)$ and $H$ is p-constrained within $S(H)$.

This result together with the previous lemma will clearly provide a proof of Theorem 1.1.

Proof. The subgroup $H$ contains both $D$ and $R$ and it covers $N / O(N)$ by the Frattini argument. Thus $H \in \mathscr{L}(p)$ by Definition 1.4 and also $H$ contains a Sylow 2-subgroup of $N$ and hence of $G$. We now prove that $H$ is constrained as stated.

First, suppose that $p$ is constrained; that is, $C_{N}(R) \subseteq S(N)$ by Lemma 2.1 and consequently $C_{D}(R) \subseteq Z(D)$ as $S(N)=O(N) Z(D)$ by Lemma 2.4.14. Let $R^{*}$ be a $D$-invariant Sylow $p$-subgroup of $O(H)$ containing $R$. Hence $C_{D}\left(R^{*}\right) \subseteq Z(D)$. Let $S$ be a Sylow 2-subgroup of $H$ containing $D$ and normalizing $R^{*}$. Thus $S \cap C_{H}\left(R^{*}\right)$ is a Sylow 2-subgroup of $C_{H}\left(R^{*}\right)$ as $S$ is a Sylow 2-subgroup of $N_{H}\left(R^{*}\right)$. But $H$ is a $Q$-group and $S$ is a Sylow 2-subgroup of $G$, so that it now suffices to prove that $C_{S}\left(R^{*}\right)=S \cap C_{H}\left(R^{*}\right) \subseteq Z(S)$ by Lemma 2.6.2. However, $C_{S}\left(R^{*}\right)$ is a normal subgroup of $S$, which is either quasi-dihedral or wreathed, and $C_{S}\left(R^{*}\right)$ intersects $D$ in a subgroup of $Z(D)$. In the latter case, the desired assertion is clear as then $D=S$, while in the former case, it follows from Lemma 2.1.1 as then $D$ is dihedral of order eight. 
Next, assume that $p$ is not constrained. Since $p$ is exceptional, it follows from Proposition 2.1 that $p$ is not stationary. Thus, if $R^{*}$ is as above, then $R^{*} \supset R$. Hence the proof will obviously be concluded once we establish the following result:

LemMA 3. If $H \in \mathscr{L}(p), R$ is a D-invariant Sylow p-subgroup of $O(N)$ contained in $O(H)$ and $R^{*}$ is a D-invariant Sylow p-subgroup of $O(H)$ properly containing $R$, then $H$ is p-constrained within $O(H)$.

Proof. First, $x$ does not centralize $R^{*}$. For if it did, then $R^{*} \subseteq N \cap O(H)$, so $R^{*} \subseteq O(N)$ by Lemma 1.1 and $R=R^{*}$ as $R$ is a Sylow p-subgroup of $O(N)$. This contradicts our assumption that $R^{*} \supset R$.

Let $S$ be a Sylow 2-subgroup of $H$ containing $D$ and normalizing $R^{*}$. Such a subgroup exists by Sylow's theorem applied to $N_{H}\left(R^{*}\right)$. Moreover, since $H \in \mathscr{L}(p)$, $S$ is a Sylow 2-subgroup of $G$ by Lemma 1.1. However, the subgroup $C_{S}\left(R^{*}\right)$ is a normal subgroup of $S$ not containing $x$. But $\langle x\rangle=\Omega_{1}(Z(S))$ by Lemmas 2.1.1 and 2.1.2, so $C_{S}\left(R^{*}\right) \cap Z(S)=1$, which is possible only if $C_{S}\left(R^{*}\right)=1$. Hence $\left|C_{H}\left(R^{*}\right)\right|$ is odd and so $H$ is $p$-constrained within $O(H)$ by Lemma 2.6.2. The lemma is proved, as is the previous one, and so is Theorem 1.1.

4. Structure of $L_{0}$. The proposition to be established here is to be used to prove the third statement of Theorem B, on the assumption that Theorem $\mathrm{B}^{*}$ is valid. We shall prove the result in much greater generality in order to make certain uses of it in the next chapter.

Proposition 1. Let $\pi$ be the set of primes which divide $|O(N)|$ and which are neither centralized nor inverted. Set $\bar{N}=N / O_{\pi}(O(N))$. Then the following conditions hold:

(i) Any $\bar{T}$-invariant Sylow subgroup of $O(\bar{N})$ is centralized or inverted by $\bar{x}_{2}$.

(ii) $L_{0}$ has a normal subgroup $J$ containing $O_{\pi}(O(N))$ such that $L_{0}=J O(N)$ and either

(a) $\bar{J}$ is isomorphic to $S L(2, q)$ and $\bar{J} \cap O(\bar{N})=1$ or

(b) $\bar{J}$ is isomorphic to $S L^{\wedge}(2,9), q=9$, and $|\bar{J} \cap O(\bar{N})|=3$.

(iii) $\bar{J}$ centralizers $O(\bar{N})$.

Proof. We first establish statement (i). Let $X$ be a $T$-invariant Hall $\pi$-subgroup of $O(N)$. By the definition of $\pi$, we need only show that every $\bar{T}$-invariant Sylow subgroup of $\bar{X}$ is centralized by $\bar{T}$. We set $\bar{Y}=O_{\pi^{\prime}}(O(\bar{N}))$. Since $O(\bar{N})$ is solvable, and $O_{\pi}(O(\bar{N}))=1$, Theorem 6.3.2 of [19] implies that $C_{O(\bar{N})}(\bar{Y}) \subseteq \bar{Y}$ and $C_{\bar{X}}(\bar{Y})=1$. Thus it suffices to show that $[\bar{X}, \bar{T}]$ centralizes $\bar{Y}$, as then $[\bar{X}, \bar{T}]=1$ and $\bar{T}$ centralizes $\bar{X}$.

Let $r$ be a prime divisor of $|\bar{Y}|$. There exists a Sylow $r$-subgroup $\bar{R}$ of $\bar{Y}$ that is normalized by $\bar{T}$ and $\bar{X}$. Indeed, $\bar{Y} \bar{X} \bar{T}$ is a solvable group having $\bar{Y}$ and $\bar{X} \bar{T}$ as Hall subgroups, so there is a Sylow $r$-subgroup $\bar{R}$ of $\bar{Y}$ permutable with $\bar{X} \bar{T}$. But 
as $\bar{Y}$ is a $\pi^{\prime}$-group, we see that $\bar{R}=\bar{Y} \cap \bar{R} \bar{X} \bar{T}$. Since $\bar{Y}$ is normal in $O(\bar{N}) \bar{T}$, $\bar{Y} \cap \bar{R} \bar{X} \bar{T}$ is normal in $\bar{R} \bar{X} \bar{T}$ and we conclude that $\bar{R}$ is normalized by $\bar{X} \bar{T}$.

It suffices to prove that $[\bar{X}, \bar{T}]$ centralizes $\bar{R}$, as $r$ was an arbitrary prime divisor of $|\bar{Y}|$. However, each element of $\bar{T}$ either inverts or centralizes $\bar{R}$, by the definition of $\pi$ and the fact that $r \in \pi^{\prime}$. Thus, $\bar{T} C(\bar{R}) / C(\bar{R})$ has order at most two and is normal in $N(\bar{R}) / C(\bar{R})$, so $\bar{T} C(\bar{R}) / C(\bar{R})$ is central in $N(\bar{R}) / C(\bar{R})$ and hence $[\bar{T}, \bar{X}] \subseteq C(\bar{R})$, as was required to be demonstrated. Part (i) is proved.

We now turn our attention to (ii) and (iii). These are simply statements about $\bar{N}$. Since we shall work entirely in that group, we drop the "bars" and for the rest of this proof-only-we use $N, L_{0}, T$, and so forth, for $\bar{N}, \bar{L}_{0}, \bar{T}$.

Let $S$ be a Sylow 2-subgroup of $N$ containing $D$ and let $y$ be the element of $S^{\prime}$ of order four defined in $\S 4$ of Chapter II, so that $y \in L_{0}$ and $y \notin Z(S)$ by the remarks at the beginning of that section. We need only prove that $y$ centralizes $O(N)$, for then $C_{N}(O(N)) \nsubseteq S(N)$ as $S(N)=O(N) Z(S)$ by Lemma 2.4.14. Thus the hypotheses of Lemma 2.4.16 will be satisfied and both (ii) and (iii) will follow from that lemma.

Let $p$ be a prime divisor of $|O(N)|$ and let $R$ be an $S$-invariant Sylow $p$-subgroup of $O(N)$. Also set $K=N_{N}(R)$ and $K_{0}=C_{N}(R)$. It will suffice to show that $K_{0}$ is a $Q$-group. Indeed, assume that this is the case. We have $S \subseteq K$ as $R$ is $S$-invariant and consequently $S \cap K_{0}$ is a Sylow 2-subgroup of $K_{0}$ as $K_{0}$ is normal in $K$. But then $S \cap K_{0}$ clearly contains a quaternion subgroup. However, Lemmas 2.1.1 and 2.1.2 imply that any quaternion subgroup of $S$ contains $y$. Thus $y \in S \cap K_{0}$ and so $y$ centralizes $R$. Since $p$ is arbitrary, it will then follow that $y$ centralizes $O(N)$, as required.

By the Frattini argument $N=O(N) K$, so $K$ is a $Q$-group of characteristic power $q$ and $O(K) \subseteq O(N)$. Furthermore, we have $S \subseteq K, S(K)=O(K) Z(S)$ by Lemma 2.4.14, and $K_{0} T=K_{0}\left\langle x_{2}\right\rangle$ as $x \in Z(N)$. On the other hand, $x_{2}$ either inverts or centralizes $R$, by (i), and so $K_{0}\left\langle x_{2}\right\rangle=K_{0} T$ is normal in $K$. Since $Z(S)$ is cyclic, $K_{0} T \nsubseteq S(K)$. But $L_{0} S(K) / S(K)$ is the unique minimal normal subgroup of $K / S(K)$ and is isomorphic to $\operatorname{PSL}(2, q)$ by Lemma 2.4.15, as $q>5$. Since $K_{0} T S(K) / S(K)$ is a nontrivial normal subgroup of $K / S(K)$, it follows that $L_{0} \subseteq K_{0} T S(K)$. Since $L_{0}$ is nonsolvable and $K_{0} S(K)$ is normal in $K_{0} T S(K)$, this implies that $K_{0}$ is also nonsolvable. Since $K_{0}$ is a subgroup of the $Q$-group $K$ and is invariant under the Sylow 2-subgroup $S$ of $N$, Lemma 2.4.17, together with Proposition 1.1 now yield that $K_{0}$ is a $Q$-group and the proposition is proved.

5. Reduction to local subgroups. This section is devoted to a proof that Theorem $\mathrm{B}^{*}$ implies Theorem B. Hence, we assume throughout that Theorem $\mathrm{B}^{*}$ holds and verify the statements (i), (ii), (iii), and (iv) of Theorem $B$.

We begin with the last assertion. Since every prime divisor of $|O(N)|$ is inverted or centralized, by Theorem $\mathrm{B}^{*}$, the set $\pi$ of prime divisors of $|O(N)|$ which is neither centralized nor inverted is empty and $O_{\pi}(O(N))=1$. Proposition 4.1 applies and gives us the existence of a normal subgroup $J$ of $L_{0}$ such that $L_{0}=J O(N)$, 
$J$ centralizes $O(N)$, and either $J$ is isomorphic to $S L(2, q)$ and $J \cap O(N)=1$ or $J$ is isomorphic to $S L^{\wedge}(2,9), q=9$, and $|J \cap O(N)|=3$. Thus to complete the proof of (iv), it remains to establish that $G$ has quasi-dihedral Sylow 2-subgroups in the case that $J$ is isomorphic to $S L^{\wedge}(2,9)$.

Suppose then that $J$ is isomorphic to $S L^{\wedge}(2,9)$ and assume by way of contradiction that $G$ has wreathed Sylow 2-subgroups, in which case $D$ is wreathed. Since $q=9$, we have $\varepsilon=1$ and $n=3$, whence also $\delta=-1$. Setting $\bar{N}=N / O(N)$, we have $\bar{N}=\bar{L}$ by Proposition 2.3.3 as $q=9$ and hence $\overline{O(C)} \subseteq \bar{L}$. But $(q+\delta \varepsilon) / 2^{n}=1$ in this case and consequently $\overline{O(C)}=1$ by Lemma 2.4.2. Thus $O(C) \subseteq O(N)$.

We claim next that $O(J) \subseteq O(C)$. Indeed, since $J$ is perfect and $O(N)$ is solvable, $J$ is the terminal member of the derived series of $L_{0}$ and so $J$ is characteristic in $L_{0}$. Hence $J$ is $S$-invariant and therefore so also is $J \cap O(N)$. But $J \cap O(N)$ contains $O(J)$ and so $J \cap O(N)=O(J)$ and is of order three. Furthermore, $D \cap J$ is a Sylow 2-subgroup of $J$ and so is generalized quaternion of order sixteen. Since $J$ is isomorphic to $S L^{\wedge}(2,9), O(J) \subseteq Z(J)$ and hence $D \cap J$ centralizes $O(J)$. In addition, we know that $Z(D)$ is cyclic of order eight and, as $|O(J)|=3, \mho^{1}(Z(D))$ necessarily centralizes $O(J)$. But $T \subseteq \mho^{1}(Z(D))(D \cap J)$ by Lemma 2.1 .2 and, as the latter group centralizes $O(J)$, we conclude that $T$ centralizes $O(J)$. Thus $O(J)$ $\subseteq O(C)$, as asserted.

Finally set $R=O_{3}(O(C))$. Since $O(J)$ is normal in $O(N)$ and $O(C) \subseteq O(N)$, we have that $O(J) \subseteq R$ and hence that $R \neq 1$. It follows therefore that $H=N(R)$ is a local subgroup of $G$. Since $J$ centralizes $O(N)$ and $O(N)$ contains $R$, we see that $J \subseteq H$. But clearly $J$ covers $L_{0} / O(N)$, so $H$ must be a $Q$-group of characteristic power $q$ by Proposition 1.1. On the other hand, $R$ is characteristic in $O(C)$ and hence in $C$, while $C$ is normal in $M$, so $R$ is normal in $M$ and $M \subseteq H$. Since $M / C$ is isomorphic to Aut $(T)$, we see that $\left|A_{H}(T)\right|$ is divisible by three. Moreover, since $C$ is $D$-invariant, so also is $R$ and hence $D \subset H$. But now Proposition 1.1 forces $H$ to be a $D$-group or $Q D$-group. This contradiction completes the proof of part (iv) of Theorem B.

The argument we have just given did not use anything about ordinary or exceptional primes, but was solely a consequence of the factorization $L_{0}=J O(N)$ with $J$ isomorphic to $S L^{\wedge}(2,9)$ and normal in $L_{0}$. We record this result here for later use.

Proposition 1. If $L_{0}=J O(N)$, where $J$ is normal in $L_{0}, J$ is isomorphic to $S L^{\wedge}(2,9)$, $q=9$, and $J$ centralizes $O(N)$, then $G$ has quasi-dihedral Sylow 2-subgroups.

We continue our analysis of Theorem B. Let us look a little more at the structure of $N$. First, we have noted above that $J$ is the terminal member of the derived series of $L_{0}$ and so $J$ is characteristic in $L_{0}$ and is canonically determined. Since Theorem $\mathrm{B}^{*}$ holds, certainly $O(N)=A B$, where $A$ and $B$ are as defined in $\S 1$. Let $W=O(C) \cap J$. It follows, since $C$ has a normal 2-complement and $C \cap O(N)$ $=A$, that $O(C) \cap L=W A$. Since $N / L$ is cyclic, we also have that $O(C) / O(C) \cap L$ is cyclic and that $O(C)^{\prime} \subseteq W A$. 
Moreover, $O(C) \cap L$ is the direct product of $W$ and $A$. Indeed, if $J$ is isomorphic to $S L(2, q)$, in which case $J \cap O(N)=1$, this is clear as $W \subseteq J$ and $A \subseteq O(N)$. Suppose, on the other hand, that $q=9$ and $J$ is isomorphic to $S L^{\wedge}(2,9)$, in which case $|J \cap O(N)|=3$. By what we have shown above, the Sylow 2-subgroups of $N$ are quasi-dihedral in this case. Since $x_{2}$ normalizes $J$ as $J$ is characteristic in $L_{0}$, it follows that $\left\langle J, x_{2}\right\rangle$ has quasi-dihedral Sylow 2-subgroups, so that by Lemma 2.2.2, $\left\langle J, x_{2}\right\rangle$ is isomorphic to $S U_{1}(2,9)$ and $x_{2}$ inverts $O(J)$ which has order three. But $J \cap O(N)=O(J)$ and so $J \cap O(N)$ is contained in $B$. Thus, $W \cap A=1$ in this case as well.

Finally, since $M / C$ is isomorphic with Aut $(T)$, it follows that there is a 3-element $a$ in $M$ and not in $C$ such that $x_{1}^{a}=x_{2}, x_{2}^{a}=x_{3}$, and $x_{3}^{a}=x_{1}$ and such that $a$ is inverted by the involution $x_{2} d$ which lies in $D-T$. This last condition holds since the normalizer of a Sylow 3-subgroup of $M$ covers $M / C$ and intersects $C$ in a subgroup of $O(C)$. We set $N_{i}=C\left(x_{i}\right), 1 \leqq i \leqq 3$, and let $J_{i}, W_{i}, A_{i}, B_{i}$, and so on, be the obvious subgroups of $N_{i}$. Here, of course, we have $N=N_{1}$.

The group $W$ is cyclic of order $(q+\delta \varepsilon) / 2^{n}$. In fact, if $J$ is isomorphic to $\operatorname{SL}(2, q)$, this follows from Lemma 2.4.2, while if $J$ is isomorphic to $S L^{\wedge}(2,9)$, this is still true by Lemma 2.4 .12 as $G$ has quasi-dihedral Sylow 2-subgroups and $\left\langle J, x_{2}\right\rangle$ is isomorphic to $S U_{1}(2,9)$ in this case.

We shall now prove assertions (i) and (ii) of Theorem B assuming (iii) holds. It will then remain to prove (iii); this we save for last. Under these hypotheses and with all the above notation, we first demonstrate the

LEMMA 1. (i) $W A$ is normal in $M$;

(ii) $C_{A}(a)=1$;

(iii) $A$ is cyclic of order dividing $|W|$.

Proof. (i) Since $O(C) / O(C) \cap L$ is cyclic, as mentioned above, it follows that $O(C)^{\prime} \subseteq O(C) \cap L$. If $\bar{N}=N / O(N)$, then $O(\bar{C})=\overline{O(C)}$ and $C_{O(\bar{C})}\left(O(\bar{C})^{\prime}\right)=O(\bar{C}) \cap \bar{L}$ $=\bar{W}$ by Lemma 2.4.2. Hence

$$
X=C_{O(C)}\left(O(C)^{\prime}\right) \subseteq O(C) \cap L=W A .
$$

However, $W \subseteq X$ as $O(C)^{\prime} \subseteq O(C) \cap L=W A=W \times A$ and $W$ is cyclic. Now $x_{2}$ centralizes both $A$ and $N / L$. By the remarks at the beginning of $\S 4$ of Chapter II, $d \in L_{0}$ and so $d \in J$. But then also $d$ centralizes both $A$ and $N / L$. Hence, $x_{2} d$ centralizes $O(C) / O(C) \cap L$ and $O(C) \cap L / W$. But $x_{2} d$ is an involution and $O(C)$ is of odd order, so $x_{2} d$ centralizes $O(C) / W$. Thus, $x_{2} d$ centralizes $O(C) / X$. Since $X$ is characteristic in $O(C)$, by its definition, $X$ is normal in $M$. Since $x_{2} d$ inverts $a$, we have that $a$ centralizes $O(C) / X$. However, as $M=\left\langle C, x_{2} d, a\right\rangle$, this implies that every normal subgroup of $C$ contained in $O(C)$ and containing $X$ is normal in $M$. In particular, $X \subseteq W A \subseteq O(C)$ and $W A=O(C) \cap L$ is normal in $C$, so $W A$ is normal in $M$, as desired. 
(ii) If $u \in C_{A}(a)$, then $a \in C(u)$. However, if $u \neq 1, C(u) \subseteq N$, since we are assuming, for now, that part (iii) of Theorem B is valid. But $a \notin N$ as $x_{1}$ and $x_{2}$ are not conjugate in $N$ and $x_{1}^{a}=x_{2}$. Hence $u=1$ and (ii) is proved.

(iii) By (i), we have that $A^{a} \subseteq W A$. Since $W A$ is the direct product of $W$ and $A$, and $W$ is cyclic, it will follow that $A$ is cyclic and is of order dividing $|W|$ provided we can show that $A^{a} \cap A=1$. However, suppose that $A^{a} \cap A \neq 1$. Hence, there exist nonidentity elements $u$ and $v$ of $A$ such that $u^{a}=v$. But $x_{2} d$ centralizes $A$ and inverts $a$, so $u^{a-1}=v$. Thus $u^{a^{2}}=u$, whence $u^{a}=u$ as $a$ is a 3-element and again $a \in C(u) \subseteq N$, giving the same contradiction as in (ii), and (iii) is proved.

Since $W$ is of order $(q+\delta \varepsilon) / 2^{n}$, in order to establish (i) of Theorem B, assuming Theorem $\mathrm{B}^{*}$ and part (iii) of Theorem $\mathrm{B}$, we need only prove that $A B$ is a Frobenius group with kernel $B$ and complement $A$, when $A \neq 1$ and $B \neq 1$. However, if $A \neq 1$ and $B \neq 1$ and this is not true, then there is an element $u$ of prime order $p$ in $A$ such that $C_{B}(u) \neq 1$. Since $A$ and $W$ are cyclic and $|A|$ divides $|W|$ by the preceding lemma, we have that $A W$ possesses a characteristic subgroup $R$ of type $(p, p)$ and that $R=\langle u\rangle(R \cap W)$. But $B$ centralizes $W$, so $C_{B}(R) \neq 1$ as $C_{B}(u) \neq 1$. But $W A$ is normal in $M$ by the preceding lemma and $R$ is characteristic in $W A$, so $H=N(R)$ contains $M$ as well as $C_{B}(R)$. Thus $a \in H$, so $C_{B}(R)^{a} \subseteq C(R) \subseteq C(u)$. Therefore $C_{B}(R)^{a} \subseteq N$ by part (ii) of Theorem B. But $C_{B}(R)^{a}$ is inverted by $x$ since the fact that $x_{3}$ inverts $B$ implies that $x=x_{1}=x_{3}^{a}$ inverts $B^{a}$. This contradiction completes the proof.

We next demonstrate (ii) of Theorem B. Setting $U=C_{D}(T)$, we have that $U$ normalizes both $A$ and $B$ as $A=C_{O(N)}(T)$ and $B=[O(N), T]$. If $G$ has quasi-dihedral Sylow 2-subgroups, then $U=T$, so $U$ centralizes $A$ and $U / C_{U}(B)=T /\langle x\rangle$ has order 2 and inverts $B$. Since $n=1$ in this case, all parts of (ii) hold. Hence we can suppose that $G$ has wreathed Sylow 2-subgroups, in which case $D$ is a Sylow 2-subgroup of $N$ and $U$ is the unique abelian subgroup of index two in $D$. Moreover, $J$ is isomorphic to $S L(2, q)$ by part (iv) of Theorem $\mathrm{B}$.

We known that $D \cap J$ is the unique generalized quaternion subgroup of $D$ of order $2^{n+1}$. It follows therefore from Lemma 2.1.2 that $U \cap J=D^{\prime}$ and that $U=D^{\prime} \times V$, where $V$ is cyclic of order $2^{n}$ and $\Omega_{1}(V)=\left\langle x_{2}\right\rangle$. (In fact, we can take $V=\langle s\rangle$ in the notation of that lemma.) Since $x_{2}$ inverts $B, V$ must act regularly on $B$. On the other hand, $D^{\prime}$ centralizes $B$ as $D^{\prime} \subseteq J$ and $J$ centralizes $O(N)$. We conclude that $D^{\prime}=C_{U}(B)$ and that $U / C_{U}(B)$ is cyclic of order $2^{n}$ and acts regularly on $B$.

Thus to complete the proof of (ii), we need only show that $U$ centralizes $A$. Suppose false, in which case $A_{1}=[U, A] \neq 1$. By Lemma 2.4.2, applied to $J D$, we have that $U$ centralizes $W$ and consequently $[U, W A]=[U, A]=A_{1}$. On the other hand, $U$ is a Sylow 2-subgroup of $C$ and $C$ is normal in $M$, so by the Frattini argument there is a 3-element of $M-C$, which without loss we may assume to be $a$, which normalizes $U$. But $a$ also leaves $W A$ invariant as $W A$ is normal in $M$ by Lemma 1. Therefore $a$ also leaves $A_{1}=[U, W A]$ invariant. Moreover, $D$ leaves $A_{1}$ 
invariant as it normalizes both $U$ and $A$. Hence, if $p$ is a prime divisor of $\left|A_{1}\right|$ and we set $H=N\left(O_{p}\left(A_{1}\right)\right)$, then $H$ is a local subgroup of $G$ containing $a, D$, and $J$. Since $a \in H,\left|N_{H}(T)\right|$ is divisible by three, so $H$ is either a $D$-group or a $Q D$-group by Proposition 1.1. On the other hand, since $J \subseteq H$, the same proposition forces $H$ to be a $Q$-group of characteristic power $q$, a contradiction. Thus $U$ centralizes $A$ and part (ii) of Theorem $\mathrm{B}$ is completely proved.

We are now left with demonstrating part (iii) of Theorem B. We shall accomplish this with a sequence of lemmas. Let $u$ be a nonidentity element of $O(N)$. We wish to prove that $H=N(\langle u\rangle) \subseteq N$; hence we may assume that $u$ has prime order $r$. Moreover, since $O(N)$ is solvable and $A$ and $B$ have relatively prime orders, we may assume that $u \in A$ or $u \in B$. In particular, $\langle u\rangle$ is $T$-invariant, so $T \subseteq H$.

LEMMA 2. The subgroup $H$ contains $J$, is a $Q$-group of characteristic power $q$ and $H=O(H)(H \cap N)$.

Proof. We know that $H$ is a local subgroup of $G$ containing $x$. Furthermore, $J \subseteq H$ as $J$ centralizes $O(N)$ by part (iii) of Theorem B, which we have already proved. Since $J$ covers $L_{0} / O(N)$, the lemma now follows from Proposition 1.1.

In the case that $J \cap O(N) \neq 1$, we have

Lemma 3. If $J$ is isomorphic to $S L^{\wedge}(2,9)$, then $A=1$.

Proof. Indeed, in this case, $q=9,|A|$ divides 5 , and $J \cap O(N) \subseteq B$ as it is inverted by $x_{2}$. But $A(J \cap O(N))$ is a Frobenius group if $A \neq 1$, which is impossible as $|J \cap O(N)|=3$. Hence, $A=1$.

Suppose now that $H \nsubseteq N$. Lemma 2 implies that $O(H) \nsubseteq N$, so there is a $T$ invariant Sylow $p$-subgroup $R$ of $O(H)$ for some prime $p$ dividing $|O(H)|$, such that $R \nsubseteq N$. Hence with the usual $T$-decomposition notation of $\S 8$ of Chapter II, we have $R_{i}^{\prime} \neq 1$ for $i=2$ or 3 . We first investigate the structure of $R$ and $H$.

LEMMA 4. The following conditions hold:

(i) $R$ is abelian and inverted by $x$;

(ii) $R_{1}=1$ and $R_{i}=R_{i}^{\prime}, 2 \leqq i \leqq 3$;

(iii) $R_{i}$ is an abelian subgroup of $J_{i} B_{i}, 2 \leqq i \leqq 3$.

Proof. It will suffice to show that $R$ is inverted by $x$. Indeed, if that is so, then $R$ is abelian and $R_{1}=1$. Thus, $R$ is the direct product of $R_{2}^{\prime}$ and $R_{3}^{\prime}$ and $R_{i}^{\prime}=R_{i}$, $2 \leqq i \leqq 3$. Moreover, $R_{i} \subseteq J_{i} O\left(N_{i}\right)$ as $x$ centralizes $N_{i} / J_{i} O\left(N_{i}\right)$, this quotient being isomorphic to $N / L_{0}, 2 \leqq i \leqq 3$. Hence $R_{i} \subseteq J_{i} B_{i}$ as $x$ centralizes $A_{i}$ and $J_{i} O\left(N_{i}\right) / J_{i} B_{i}$ is isomorphic to $A_{i}, 2 \leqq i \leqq 3$, so all parts of the lemma will hold.

Hence, we assume that $R$ is not inverted by $x$ and derive a contradiction. It follows that $R_{1} \neq 1$. Moreover, $R_{1} \subset R$ as $R_{i}^{\prime} \neq 1$ for some $i, 2 \leqq i \leqq 3$. Hence by Lemma 2.8.1, there is a nontrivial $T$-invariant $p$-subgroup $R^{*}$ of $R$ centralizing $R_{1}$ such that $\left[R^{*}, x\right]=R^{*}$. 
We claim next that $R_{1} \subseteq O(N)$. Indeed, $J \subseteq H$ by Lemma 2 and $R_{1} \subseteq N \cap O(H)$ by definition of $R$ and $H$, so [J, $R_{1}$ ] is a subgroup of $N$ of odd order. Setting $\bar{N}=N / O(N)$, it follows that $\left[\bar{L}_{0}, \bar{R}_{1}\right]$ has odd order as $\bar{J}=\bar{L}_{0}$. Since $\left|\bar{R}_{1}\right|$ is odd, Lemma 2.4.7 now yields that $\bar{R}_{1}=1$, whence $R_{1} \subseteq O(N)$, as asserted.

Thus $R_{1}$ is a subgroup of a $T$-invariant Sylow $p$-subgroup of $O(N)$. Since we are assuming Theorem $\mathrm{B}^{*}$, it follows therefore from Lemma 2.3 for any $v \neq 1$ in $R_{1}$ that every $T$-invariant $p$-subgroup of $C(v)$ lies in $N$. But $R^{*} \subseteq C(v)$ as $R^{*}$ centralizes $R_{1}$ and $R^{*}$ is $T$-invariant by construction. Since $\left[R^{*}, x\right]=R^{*}$, we conclude that $R^{*}=1$, which is not the case. Hence $R_{1}=1$ and the proof is complete.

Before going on, we require a subsidiary result:

LEMMA 5. Any subgroup of $L_{0}$ which covers $L_{0} / O(N)$ contains $J$.

Proof. Let $X$ be a subgroup of $L_{0}$ covering $L_{0} / O(N)$. Since $L_{0} / O(N)$ is perfect, it follows that each member of the derived series of $X$ covers $L_{0} / O(N)$. In particular, the terminal member $X_{0}$ does. However, as we have noted at the beginning of this section, $J$ is the terminal member of the derived series of $L_{0}$, whence $X_{0} \subseteq J$. However, $X_{0}$ covers $L_{0} / O(N)$, so this implies that $X_{0}$ covers $J / J \cap O(N)$. Thus, $X_{0}=J$ and certainly $J \subseteq X$.

LEMMA 6. The following conditions hold:

(i) $J$ normalizes $R$.

(ii) $R_{2}$ and $R_{3}$ are conjugate in $H$.

Proof. We first observe that (ii) will follow from (i). In fact, $d \in J$ and $x_{2}^{d}=x_{3}$. Hence, if $J$ normalizes $R$, so does $d$. Thus,

and (ii) holds.

$$
R_{3}=C_{R}\left(x_{3}\right)=C_{R}\left(x_{2}\right)^{d}=R_{2}^{d}
$$

It remains to establish (i). We set $H_{0}=N_{H}(R)$ and must demonstrate that $J \subseteq H_{0}$. In any case, $J \subseteq H$, by Lemma 2 . It now suffices to show that

$$
H \cap N=\left(H_{0} \cap N\right)(O(H) \cap N) .
$$

Indeed, suppose this factorization holds. Since $[J, O(H) \cap N]$ has odd order, it follows from Lemma 2.4.7 exactly as in the proof of Lemma 4 above that $O(H) \cap N \subseteq O(N)$. Since $J \subseteq H \cap N$, the given factorization thus implies that $\left(H_{0} \cap N\right) O(N) \supseteq J$. Hence, $H_{0} \cap N$ is a subgroup of $N$ covering $L_{0} / O(N)$, so $H_{0} \cap N \supseteq J$ by the previous lemma.

We now derive the required factorization. It suffices to show that $H \cap N$ $\subseteq\left(H_{0} \cap N\right) O(H)$. But $H \cap N$ covers $H / O(H)$ by Lemma 2 and $H_{0} \cap N \subseteq H \cap N$, so it is enough to prove that $H_{0} \cap N$ covers $H / O(H)$. However, $H_{0}$ covers $H / O(H)$ by the Frattini argument. Moreover, $O(H) x$ is central in $H / O(H)$ and $x \in H_{0}$ inasmuch as $x \in H$ and $x$ normalizes $R$. Thus, $\left(O(H) \cap H_{0}\right) x$ is central in $H_{0} / O(H) \cap H_{0}$, so $H_{0}=\left(H_{0} \cap N\right)\left(O(H) \cap H_{0}\right)$ by the Frattini argument and we are done. 
LEMMA 7. Either $R_{i} \cap B_{i} \neq 1,2 \leqq i \leqq 3$, or the following conditions hold:

(i) $q=p^{m}=\left|R_{2}\right|=\left|R_{3}\right|$ for a suitable positive integer $m$;

(ii) $u \in B$.

Proof. Assume for the sake of definiteness that $R_{2} \cap B_{2}=1$. We shall prove that (i) and (ii) hold. Since $R_{2} \subseteq J_{2} B_{2}$ by Lemma 4, it follows that $R_{2}$ is isomorphic to a p-subgroup $P_{2}$ of $J_{2}$.

Suppose that $p$ is not the characteristic of $J_{2}$. It follows from Lemma 2.4.1 that $P_{2}$ is cyclic, so $R_{2}$ is cyclic as well. But $R_{2}$ and $R_{3}$ are conjugate in $H$ by Lemma 6 , so $R_{3}$ is cyclic. Since $x$ inverts $R$, by Lemma 4, and $J$ normalizes $R$, by Lemma 6 , we have that either $J$ or $J / O(J)$ is faithfully represented on the Frattini quotient group of $R$, which is elementary abelian of order $p^{2}$. Hence, $J$ or $J / O(J)$ is isomorphic with a subgroup of $G L(2, p)$. Since $J$ is perfect, $J$ or $J / O(J)$ is isomorphic with a subgroup of $S L(2, p)$. Moreover, $J$ is not solvable and is not of characteristic $p$, so Lemma 2.4 .8 now forces $q=5$, contrary to our assumption $q>5$.

Hence, $p$ is the characteristic of $J_{2}$. Since $J_{2}$ has characteristic power $q$, we have now established that $q=p^{m}$ for a suitable integer $m$. Since $P_{2}$ is a $p$-subgroup of $J_{2}$ and $J_{2}$ has characteristic power $q$, it follows from Lemma 2.4.1 that $\left|P_{2}\right| \leqq q$ and $P_{2}$ is elementary abelian. Thus, $R_{2}$ is elementary abelian of order at most $q=p^{m}$, so $R$ is elementary of order at most $p^{2 m}$, since $R=R_{2} R_{3}$ by Lemma 4 while $\left|R_{2}\right|$ $=\left|R_{3}\right|$ by Lemma 6 . Hence, in order to establish (i), it suffices to show that $|R|$ $\geqq p^{2 m}$. But $J$ or $J / O(J)$ is faithfully represented on $R$, so $|R| \geqq p^{2 m}$ by Lemma 2.4.18.

Suppose now that (ii) is false. In this case $u \in A$, by our choice of $u$, and $T$ centralizes $u$. We shall show that $J_{2} \subseteq H$, which will contradict the factorization $H=O(H) C_{H}(x)$ obtained in Lemma 2.

First, $u \in N_{2}$ since $x_{2} \in T$ and $T$ centralizes $u$. Moreover, $R_{2}$ centralizes $u$. Indeed, Aut $(\langle u\rangle)$ is abelian and $R_{2}=\left[R_{2}, x\right]$ as $R_{2}=R_{2}^{\prime}$ by Lemma 4 , and so $R_{2} \subseteq H^{\prime}$ $\subseteq C(u)$. We set $\bar{N}_{2}=N_{2} / O\left(N_{2}\right)$; it follows from above that $\bar{R}_{2}=\bar{P}_{2}$ and $\bar{R}_{2}$ is a Sylow $p$-subgroup of $\bar{J}_{2}$. Furthermore, $u$ has prime order $r$ by choice. Since $R_{1}=1$ by Lemma 4 and $\langle u\rangle$ is normal in $H$, it follows that $r \neq p$. On the other hand, since $\bar{R}_{2}$ is a Sylow $p$-subgroup of $\bar{J}_{2}$, it follows from Lemma 2.4 .4 that $C_{\bar{N}_{2}}\left(\bar{R}_{2}\right)=$ $Z\left(\bar{N}_{2}\right) \bar{R}_{2}$. Since $r \neq p$ and $Z\left(\bar{N}_{2}\right)$ is a 2-group, $C_{\bar{N}_{2}}\left(\bar{R}_{2}\right)$ is thus an $r^{\prime}$-group. Since $\bar{u} \in C_{\bar{N}_{2}}\left(\bar{R}_{2}\right)$, we conclude that $\bar{u}=1$. Thus $u \in O\left(N_{2}\right)$, so $J_{2}$ centralizes $u$ and $J_{2} \subseteq H$, as desired.

We know that $R_{2}^{\prime} \neq 1$ or $R_{3}^{\prime} \neq 1$ and so $R_{2} \neq 1$ or $R_{3} \neq 1$ by Lemma 4 . But $R_{2}$ and $R_{3}$ are conjugate in $H$ by Lemma 6 , so $R_{2} \neq 1$ and $R_{3} \neq 1$. We now set $K=C\left(R_{2}\right)$ and shall study this group in the next two lemmas. The final contradiction will come from the interrelations of $H$ and $K$.

Lemma 8. $K$ is T-invariant and either $K$ is a Q-group and $K=O(K)\left(K \cap N_{2}\right)$ or $K$ has a normal 2-complement.

Proof. We treat the quasi-dihedral and wreathed cases separately. The latter, being easier, is considered first. In this case, $D$ is wreathed of height $n$ and $D \cap J$ 
is the unique generalized quaternion subgroup of $D$ of order $2^{n+1}$. Moreover, by Lemma 2.1.2, $C_{D \cap J}\left(x_{2}\right)=D^{\prime}$ and so is cyclic of order $2^{n}$. Now $J$, and hence $D \cap J$, normalizes $R$ by Lemma 6 and $T$ normalizes $R$ by construction, so $T D^{\prime}$ normalizes $R_{2}=C_{R}\left(x_{2}\right)$. Thus $K=C\left(R_{2}\right)$ is $T D^{\prime}$-invariant. In addition, since $x$ inverts $R_{2}$ and $x$ is the unique involution of $D^{\prime}$, we have $K \cap D^{\prime}=1$. We see then that all the conditions of Lemma 2.3.3 are satisfied with $K D^{\prime}, K$, and $D^{\prime}$ in the roles of $H, K$, and $A$, respectively. We conclude that either $K D^{\prime}$, and hence also $K$, has a normal 2-complement or that $K$ is a $Q$-group with generalized quaternion Sylow 2-subgroups. Finally, in the latter case, we note that $x_{2} \in K$ as $x_{2}$ centralizes $R_{2}$ and so $\left\langle x_{2}\right\rangle$ is the center of some Sylow 2-subgroup of $K$. Hence $K=O(K) C_{K}\left(x_{2}\right)=$ $O(K)\left(K \cap N_{2}\right)$ by Proposition 2.3.1. Thus the lemma holds when $D$ is wreathed.

Suppose next that $G$ has quasi-dihedral Sylow 2-subgroups. Again we have that $x_{2} \in K$ and that $x$ inverts $R_{2}$, so $K$ is $T$-invariant and $K$ is of index two in $K T$. Thus a Sylow 2-subgroup of $K$ is not a Sylow 2-subgroup of $G$ and so is not quasidihedral, which implies that $K$ is not a $Q D$-group. Hence by Proposition $1.1 K$ is either a $Q$-group, a $D$-group, or has a normal 2-complement. We assert that $K$ is not a $D$-group; we assume that it is and derive a contradiction.

First, $R_{2} \cap B_{2}=1$. Indeed, if $R_{2} \cap B_{2} \neq 1$, then $N\left(R_{2} \cap B_{2}\right)$ contains $J_{2}$ as well as $K$. This implies that $N\left(R_{2} \cap B_{2}\right)$ covers $J_{2} O\left(N_{2}\right) / O\left(N_{2}\right)$ and so is a $Q$-group of characteristic power $q$ by Proposition 1.1. As such it cannot contain the $D$-group $K$ by Lemma 2.4.17. Thus $R_{2} \cap B_{2}=1$ and now the preceding lemma implies that $q=p^{m}$ for some integer $m$.

On the other hand, $K$ is of index two in $K T$ and $K$ is a $D$-group, so $K T$ is also a $D$-group by Proposition 2.1.1. Furthermore, a Sylow 2-subgroup of $K T$ is either dihedral of order at least eight or quasi-dihedral, by the structure of $D$-groups enunciated in Proposition 2.3.4. We choose a Sylow 2-subgroup $S^{*}$ of $K T$ which contains $T$. It follows that $\left|Z\left(S^{*}\right)\right|=2$ and $Z\left(S^{*}\right) \subset T$, no matter which structure $S^{*}$ has. But $K \cap S^{*}$ is normal in $S^{*}$ and is not trivial, so $Z\left(S^{*}\right) \subseteq K$. Since $x_{2}$ is the unique involution of $T$ which centralizes $R_{2}$, we conclude that $\left\langle x_{2}\right\rangle=Z\left(S^{*}\right)$. In particular, $S^{*} \subseteq N_{2}$. If we set $\bar{N}_{2}=N_{2} / O\left(N_{2}\right)$, we have that $\bar{R}_{2}$ is an $\bar{S}^{*}$-invariant $p$-subgroup of $\bar{J}_{2}$. Moreover, $\bar{R}_{2} \neq 1$ as $R_{2} \subseteq J_{2} B_{2}$ and $R_{2} \cap B_{2}=1$. However, this is impossible by Lemma 2.4 .9 as $\bar{J}_{2}$ has characteristic $p$ and $\bar{S}^{*}$ contains a dihedral subgroup of order eight.

Thus, $K$ is a $Q$-group or has a normal 2-complement. It remains to demonstrate in the former case that $K=O(K)\left(K \cap N_{2}\right)$. However, $K \cap S^{*}$ is of index two in $S^{*}$ and $K \cap S^{*}$ is a Sylow 2-subgroup of $K$. Hence, if $K$ is a $Q$-group, $K \cap S^{*}$ is necessarily generalized quaternion and now the desired conclusion follows exactly as in the wreathed case.

LEMMA 9. The following containments hold:

(i) $\left\langle B_{2}, u\right\rangle \subseteq K$;

(ii) $u \in O(K)$ if $u \in B$. 
Proof. We have proved in Lemma 4 that $R_{2} \subseteq J_{2} B_{2}$. Since $B$ centralizes $J$ and $B_{2}$ is abelian, it follows that $B_{2} \subseteq C\left(R_{2}\right)=K$. Moreover, $R_{2}=R_{2}^{\prime}$, so $R_{2}$ is inverted by $x$. Since $R_{2} \subseteq H$, we have that $R_{2}$ normalizes $\langle u\rangle$. But $x$ inverts or centralizes $u$, so $R_{2}$ must centralize $u$. Hence, $u \in K$ and (i) is valid.

Assume next that $u \in B$; we shall establish (ii). If $K$ has a normal 2-complement, then this is automatic. If not, then Lemma 8 yields that $K$ is a $Q$-group and $K=O(K)\left(K \cap N_{2}\right)$, whence $\left[x_{2}, K\right] \subseteq O(K)$. But $u \in B$, so $x_{2}$ inverts $u$ and again $u \in O(K)$.

We are now ready to derive the final contradictions and thus establish assertion (iii) of Theorem B. Suppose first that $u \in B$, so $u \in O(K)$ by the previous lemma. Moreover, $B_{2} \subseteq K$ by the same result. Since $\langle u\rangle, B_{2}$, and $O(K)$ are $T$-invariant and $u$ has prime order $r$, it follows that $O(K) B_{2}$ possesses a $T$-invariant Sylow $r$-subgroup $Q$ which contains $u$. But $x=x_{1}$ centralizes $u$ and $x_{2}$ inverts $u$, as $u \in B$, so $Q_{1}^{\prime} \neq 1$ in the $T$-decomposition of $Q$. On the other hand, $B_{2}$ is inverted by $x$ and centralized by $x_{2}$ and $r$ divides $\left|B_{2}\right|$ as $\left|B_{2}\right|=|B|$ and $u \in B$ has order $r$. Thus, $Q_{2}^{\prime} \neq 1$ as well and so $Q \supset Q_{1}$.

By Lemma 2.8.1, there is a $T$-invariant subgroup $Q^{*}$ of $Q$ centralizing $Q_{1}$ such that $\left[Q^{*}, x\right] \neq 1$. But $u \in Q_{1}$ as $u \in Q$ and so $Q^{*}$ is a $T$-invariant $r$-subgroup of $C(u)$ not centralized by $x$. Since $r$ is an ordinary prime, by Theorem B*, which we are assuming, this contradicts Lemma 2.3.

In particular, we may now assume that if $v$ is any nonidentity element of $B$, then $N(\langle v\rangle) \subseteq N$. We shall use this in dealing with the case that $u \in A$.

Finally, assume that $u \in A$, which is the only remaining possibility, by our choice of $u$. Hence, by Lemma $7, R_{2} \cap B_{2} \neq 1$. If $v \in R_{2} \cap B_{2}$ and $v \neq 1$, then $v^{a^{-1}}$ is a nonidentity element of $B=B_{1}$. Thus $N\left(\left\langle v^{a-1}\right\rangle\right) \subseteq N$, so $N(\langle v\rangle) \subseteq N_{2}$. We shall contradict this fact.

Since $R$ is abelian, we have that $R \subseteq K$. However, $x_{2}$ inverts $R_{3}=R_{3}^{\prime}$, so $R_{3} \subseteq O(K)$ by Lemma 8. In particular, $x_{2}$ does not centralize a $T$-invariant Sylow $p$-subgroup $R^{*}$ of $O(K)$; that is, $R^{*} \supset R^{*} \cap N_{2}$. Thus, by Lemma $2 . \dot{8} .1, C_{R^{*}}\left(R^{*} \cap N_{2}\right) \nsubseteq$ $R^{*} \cap N_{2}$, so $C\left(R^{*} \cap N_{2}\right) \nsubseteq N_{2}$. But $R_{2} \subseteq R^{*} \cap N_{2}$ as $R_{2}$ is normal in $K$. Hence, choosing $v$ as above, we have $v \in R_{2} \subseteq R^{*} \cap N_{2}$ and therefore $C(v) \supseteq C\left(R^{*} \cap N_{2}\right)$. Thus $C(v) \nsubseteq N_{2}$, which yields the desired contradiction $N(\langle v\rangle) \nsubseteq N_{2}$. This completes the proof.

6. Properties of the elements of $\mathscr{L}(p)$. There are two key results on the elements of the important collection $\mathscr{L}(p)$ of $p$-local subgroups of $G$ which we shall establish in this section. They are as follows:

Proposition 1. If $p$ is an exceptional prime and $H \in \mathscr{L}(p)$, then $H$ is p-constrained within $S(H)$.

This is an extension of part of Theorem 1.1, which states that there is at least one element of $\mathscr{L}(p)$ satisfying this proposition. The next result is a "pushing-up" lemma. 
Proposition 2. If $H \in \mathscr{L}(p)$ for a prime $p, R_{1}$ is a nonidentity $D$-invariant p-subgroup of $O(H)$ and $N\left(R_{1}\right)$ covers $H / O(H)$ and contains a Sylow p-subgroup of $O(H)$, then $N\left(R_{1}\right) \in \mathscr{L}(p)$.

This will be used in the following form:

CoRollary 1. If $p$ is an exceptional prime, $H \in \mathscr{L}(p), R^{*}$ is a Sylow p-subgroup of $H$ with $R^{*} \cap O(H)$ being D-invariant and if $O_{p^{\prime}}(H) Z\left(J\left(R^{*}\right)\right)$ is normal in $H$, then $N\left(Z\left(J\left(R^{*}\right)\right)\right) \in \mathscr{L}(p)$.

Proof (of Proposition 1). Let $H$ be an element of $\mathscr{L}(p)$. As usual, it follows from Lemma 1.1 that $H$ contains a $D$-invariant Sylow $p$-subgroup $R$ of $O(N)$ and that $R \subseteq O(H)$. Let $R^{*}$ be a $D$-invariant Sylow $p$-subgroup of $O(H)$ containing $R$.

First, suppose that $R^{*} \supset R$. The hypotheses of Lemma 3.3 apply and we deduce that $H$ is $p$-constrained within $O(H)$ and consequently $p$-constrained within $S(H)$. Hence we may assume that $R^{*}=R$.

Let $S$ be a Sylow 2-subgroup of $H$ normalizing $R$. Since $H$ covers $L / O(N)$ by Lemma 1.1, $S$ is a Sylow 2-subgroup of $G$ and consequently is a Sylow 2-subgroup of $N(R)$. However, $N(R)$ is p-constrained within $S(N(R))$ by Lemma 3.2. Thus $C_{S}(R) \subseteq Z(S)$ by Lemma 2.6.2. But $C_{S}(R)$ is also a Sylow 2-subgroup of $C_{H}(R)$, so $H$ is $p$-constrained within $S(H)$, again by Lemma 2.6.2. This proves the proposition.

Proof (of Proposition 2). First, we note that $N\left(R_{1}\right)$ contains $D$ inasmuch as $R_{1}$ is $D$-invariant by assumption.

Next, we show that $N\left(R_{1}\right)$ contains a Sylow $p$-subgroup of $O(N)$. Since $N_{O(H)}\left(R_{1}\right)$ is $D$-invariant and contains a Sylow $p$-subgroup of $O(H)$, by hypothesis, it follows that there is a $D$-invariant Sylow $p$-subgroup $R^{*}$ of $O(H)$ containing $R_{1}$ as a normal subgroup. However, any two $D$-invariant Sylow $p$-subgroups of $O(H)$ are conjugate by an element of $C_{O(H)}(D)$ and there is a $D$-invariant Sylow $p$-subgroup of $O(N)$ contained in $O(H)$, by Lemma 1.1. It follows that $C_{R^{*}}(x)$ is a Sylow $p$-subgroup of $O(N)$ (and it is $D$-invariant since $R^{*}$ is and $x \in Z(D)$ ). But $N\left(R_{1}\right) \supseteq R^{*}$, so our assertion is valid.

Hence, it remains to establish the covering property to show that $N\left(R_{1}\right) \in \mathscr{L}(p)$. Let $K=N_{H}\left(R_{1}\right)$, so $K \subseteq N\left(R_{1}\right)$; it suffices to show that $K$ covers $L_{0} / O(N)$.

We set $H_{0}=\left(L_{0} \cap H\right) O(H)$ and claim that $H_{0} / O(H)$ and $L_{0} / O(N)$ are incident sections. Indeed, $O(N) \cap H_{0} \subseteq O(H), O(H) \cap N \subseteq O(N), H$ covers $L_{0} / O(N)$, and $N$ covers $H / O(H)$, so this is clear. In particular, $L_{0} / O(N)$ and $H_{0} / O(H)$ are isomorphic.

The subgroup $K$ covers $H / O(H)$ by the Frattini argument. Let $K_{0}=H_{0} \cap K$, so $H_{0} / O(H)$ and $K_{0} / O(H) \cap K$ are incident sections. Moreover, $O(H) \cap K=O(K)$ since $K$ covers $H / O(H)$. Furthermore, $x \in K$ and $K / O(K)$ is a $Q$-group with the image of $x$ central. Thus, $K \cap N$ covers $K / O(K)$. In particular, $K_{0} \cap N / O(K) \cap N$ is incident with $K_{0} / O(K)$. Thus, $L_{0} / O(N)$ is isomorphic to $K_{0} \cap N / O(K) \cap N$. 
However,

$$
K_{0} \cap N \subseteq H_{0} \cap N=O(H)\left(L_{0} \cap H\right) \cap N \subseteq L_{0},
$$

as $O(H) \cap N \subseteq O(N)$. Finally,

$$
O(N) \cap\left(K_{0} \cap N\right) \subseteq O(N) \cap H \cap K \subseteq O(H) \cap K \cap N=O(K) \cap N .
$$

Thus, Lemma 2.8.6 applies to the sections $L_{0} / O(N)$ and $K_{0} \cap N / O(K) \cap N$ and they are incident. In particular, $K_{0}$ covers $L_{0} / O(H)$ and $K_{0} \subseteq K$. This proves the proposition.

Proof (of Corollary 1). We set $Z=Z\left(J\left(R^{*}\right)\right.$ ) and we shall verify that $Z$ satisfies the conditions for $R_{1}$ in Proposition 2; this will suffice for the proof.

First, $Z \subseteq O(H)$. Indeed, $O_{p^{\prime}, p}(H) \subseteq S(H)$ since $H$ is $p$-constrained within $S(H)$ by Proposition 1. Thus, $O_{p^{\prime}}(H) Z \subseteq S(H)$ as $Z$ is a $p$-group and $O_{p^{\prime}}(H) Z$ is normal in $H$ by assumption. However, $H$ is a $Q$-group, since $H \in \mathscr{L}(p)$, so $S(H)$ has $O(H)$ as a normal 2-complement by Lemma 2.4.14. Consequently $Z \subseteq O(H)$, as asserted.

It follows that $Z$ is $D$-invariant. In fact, $Z=O_{p^{\prime}}(H) Z \cap R^{*}$. But $Z \subseteq O(H)$, so

$$
Z=O_{p^{\prime}}(H) Z \cap\left(R^{*} \cap O(H)\right) \text {. }
$$

However, $O_{p^{\prime}}(H) Z$, being normal in $H$, is $D$-invariant and $R^{*} \cap O(H)$ is, by assumption. Thus $Z$ is also $D$-invariant.

Moreover, $Z$ is normal in $R^{*}$, by definition of $Z$; it follows that $N(Z)$ covers $H / O_{p^{\prime}}(H) Z$, so $N(Z)$ covers $H / S(H)$, as we have seen that $O_{p^{\prime}}(H) Z \subseteq S(H)$ : But $S(H)=O(H) Z(D)$ by Lemma 2.4.14 as $H$ is a $Q$-group and $H=O(H)(H \cap N)$. Since $Z$ is $Z(D)$-invariant, it follows that $N(Z)$ covers $H / O(H)$ and the corollary is proved.

7. A subgroup of $C$. In this section and the two that follow, we let $p$ be $a$ fixed prime divisor of $|O(N)|$ such that $p$ divides $|C \cap L|$ and $p$ is not stationary in the case that $N$ is not p-constrained within $S(N)$; our purpose here is to construct a very important nonidentity $p$-subgroup $Q$ of $C$. Its normalizer $N(Q)$ will then be a $p$-local subgroup of $G$ of a very special sort. This will lead to the study of another family of $p$-local subgroups of $G$; the existence of $Q$ will show that the family is nonempty. These local subgroups will then turn out to be $D$-groups which "mesh" with $N$ in a nontrivial way.

We now fix a $D$-invariant Sylow $p$-subgroup $P$ of $C$. Since $D P O(N)$ is solvable, there exist $D P$-invariant Sylow $p$-subgroups of $O(N)$; we let $R$ be such a subgroup and fix it. Hence, by Lemma $2.1, N$ is $p$-constrained within $S(N)$ if and only if $C_{N}(R) \subseteq S(N)$; moreover, if $C_{N}(R) \nsubseteq S(N)$, then $C_{N}(R)$ covers $L_{0} / O(N)$, again by Lemma 2.1.

The main result of this section is as follows:

Proposition 1. There is a nonidentity characteristic subgroup $Q$ of $P$ and $a$ $Q$-subgroup $N^{*}$ of $G$ with the following properties: 
(i) $N^{*}$ is p-constrained within $S\left(N^{*}\right)$, covers $L / O(N)$, and contains $D Q$.

(ii) $C^{*}=N_{C \cap N^{*}}(Q)$ covers $C \cap L / C \cap S(L)$.

(iii) There is a $D Q$-invariant Sylow p-subgroup $R^{*}$ of $O_{p^{\prime}, p}\left(N^{*}\right)$, such that $C_{C}\left(Q C_{R^{*}}(Q)\right) \subseteq C^{*}$.

The exceedingly complicated form of the result is due to the fact that the proof divides into a number of separate cases. Let us, in advance, examine one of the easier possibilities, in order to gain insight into the nature of the proposition.

Thus, suppose that $N$ itself is $p$-constrained in $S(N)$. We set $N^{*}=N$ and also assume that $Q$ is some nonidentity characteristic subgroup of $P$. Let us see what the conditions amount to under these special hypotheses. First, (i) is clear as $N$ certainly covers $L / O(N)$ and contains both the subgroups $D$ and $Q$. Hence, only the second and third conditions need to be met.

However, even that requirement is simplified in this case. Indeed, $C^{*}=N_{C}(Q)$ as $C \cap N^{*}=C \cap N=C$. Moreover, $N$ is $p$-constrained within $S(N)$, so $O_{p^{\prime}, p}(N)$ $\subseteq S(N)$. Since $S(N)$ has a normal 2-complement by Lemma 2.4.14, so does $D Q O_{p^{\prime}, p}(N)$ and hence there does exist a $D Q$-invariant Sylow $p$-subgroup $R^{*}$ of $O_{p^{\prime}, p}(N)$. For any such Sylow subgroup, we have

$$
C_{C}\left(Q C_{R^{*}}(Q)\right) \subseteq C_{C}(Q) \subseteq N_{C}(Q)=C^{*}
$$

Hence the only other condition $Q$ needs to satisfy (besides being a nonidentity characteristic subgroup of $P$ ) is that $N_{C}(Q)$ covers $C \cap L / C \cap S(L)$.

Let us conclude this introductory explanation by exhibiting the appropriate subgroup $Q$ under one further assumption. We suppose that $p$ does not divide $|C: C \cap O(N)|$. This is indeed the case except for a finite number of primes for a given value of the characteristic power $q$ of $G$. With all these hypotheses, we assert that $Q=P$ will do. Indeed, $P \subseteq C \cap O(N)$ as $C \cap O(N)$ is normal in $C$. Since $P$ is a Sylow $p$-subgroup of $C \cap O(N), N_{C}(Q)$ covers $C / C \cap O(N)$ by the Frattini argument, so certainly covers $C \cap L / C \cap S(L)$. Moreover, $P \neq 1$ as $p$ divides $|C \cap L|$ by assumption.

The proof of the proposition is divided into a sequence of lemmas. The first .of these embodies the arguments we have just given. With the above notation, it is as follows:

LEMMA 1. If $N$ is p-constrained within $S(N)$ and $Q$ is a nonidentity characteristic subgroup of $P$ such that $N(Q)$ covers $C \cap L / C \cap S(L)$, then $N^{*}=N$ and $Q$ satisfies the proposition.

We shall begin by considering this case: namely, that $N$ is $p$-constrained within $S(N)$ and we shall construct the desired subgroup $Q$. In fact, we shall show that we may take $Q$ to be $Z(J(P))$ or $\Omega_{m}(P)^{\prime}$ for a suitable positive integer $m$.

We set $K=N_{C \cap L}(P \cap L) P$, so $K$ is a group as $P$ normalizes $P \cap L$ and $C \cap L$. Let $U=O_{p^{\prime}}(K) O_{p}(K)$. 
LEMMA 2. With the above notation, we have

(i) $K$ is a subgroup of $C$.

(ii) $K$ covers $C \cap L / C \cap S(L)$.

(iii) $O_{p}(K)=P \cap L$.

Proof. (i) This is clear as $P$ is a subgroup of $C$.

(ii) In fact, we shall show that $N_{C \cap L}(P \cap L)$ covers $C \cap L / C \cap S(L)$. Since $C \cap L$ is a normal subgroup of $C$, it follows that $P \cap L$ is a Sylow $p$-subgroup of $C \cap L$. Furthermore, $C \cap L / C \cap S(L)$ is abelian by Lemma 2.4.2, so $K_{1}=$ $(C \cap S(L))(P \cap L)$ is a normal subgroup of $C \cap L$. Thus, by the Frattini argument,

$$
\begin{aligned}
C \cap L & =N_{C \cap L}(P \cap L) K_{1} \\
& =N_{C \cap L}(P \cap L)(P \cap L)(C \cap S(L)) \\
& =N_{C \cap L}(P \cap L)(C \cap S(L)),
\end{aligned}
$$

and our assertion is valid.

(iii) Certainly $P \cap L \subseteq O_{p}(K)$ as $P \cap L$ is normal in $N_{C \cap L}(P \cap L)$ and in $P$. Suppose that the inclusion is proper and set $\bar{N}=N / O(N)$. Thus, $\overline{P \cap L} \subset O_{p}(\bar{K})$, so there is a $p$-element $\bar{u}$ of $O_{p}(\bar{K})$ not contained in $\bar{L}$. However, by (ii), it follows that $\bar{W}=\overline{O(C) \cap L}=O(\bar{C}) \cap \bar{L}$ is contained in $\bar{K}$. Hence $\bar{W}$ is normal in $\bar{K}$ as $\bar{K} \subseteq \bar{C}$ and $\bar{W}=\overline{O(C) \cap L}$ is normal in $\bar{C}$. Moreover, $\bar{u}$ centralizes $O_{p^{\prime}}(\bar{W})$ as $\bar{u} \in O_{p}(\bar{K})$ and this contradicts Lemma 2.4.2, applied to $\bar{N}$.

LEMMA 3. If $U=O_{p^{\prime}, p}(K)$, then $Q=Z(J(P))$ satisfies the proposition.

Proof. Indeed, we need only demonstrate that $Q$ is normal in $K$ and then $N(Q)$ covers $C \cap L / C \cap S(L)$ by the preceding lemma. However, $C$ is the product of $C_{D}(T)$ and $O(C), K$ contains $C_{D}(T)$ as $D$ normalizes $P \cap L$, and $K \subseteq C$ by Lemma 2. Since $O(C)$ is a normal 2-complement in $C$, it follows that $K=O(K) C_{D}(T)$. Since $O(K)$ is normal in $K$ and of odd order, Glauberman's theorem now implies that $O_{p^{\prime}}(K) Q$ is a normal subgroup of $K$. But this yields that $Q \subseteq O_{p^{\prime}, p}(K)$ which is, by assumption, the direct product of $O_{p}(K)$ and $O_{p}(K)$. Hence, $Q$ centralizes $O_{p^{\prime}}(K)$ and so $Q$ is normal in $K$.

Therefore, we shall assume for the remaining considerations in the constrained case that

$$
U=O_{p^{\prime}}(K) O_{p}(K) \subset O_{p^{\prime}, p}(K) .
$$

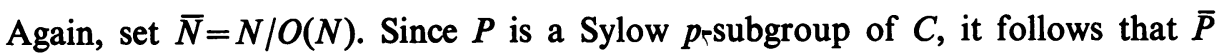
is a Sylow $p$-subgroup of $\bar{C}$. Thus $\bar{P} / \overline{P \cap L}$ is a Sylow $p$-subgroup of $\bar{N} / \bar{L}$, so it is cyclic by Proposition 2.3.3. However, $P \cap L \supseteq P \cap O(N)$, so $P / P \cap L$ is also cyclic. As $O_{p}(K)$ is not a Sylow $p$-subgroup of $O_{p^{\prime}, p}(K)$, neither is $P \cap L$ by Lemma 2. It follows that if $v \in P$ and $v^{p} \in P \cap L$, then $v \in O_{p^{\prime}, p}(K)$. We choose $v \in P$ of minimal order such that $v^{p} \in P \cap L, v \notin P$. Let $v$ have order $p^{m}$.

LEMMA 4. If $\Omega_{m}(P)$ is nonabelian, then $Q=\Omega_{m}(P)^{\prime}$ satisfies the proposition. 
Proof. Because of our assumption on $\Omega_{m}(P), Q$ is nontrivial. As in the preceding lemma, it suffices to prove that $Q$ is normal in $K$. We set $P_{1}=\langle P \cap L, v\rangle$. Our choice of $v$, and the discussion that went with it, shows that $P_{1} / P \cap L$ is of order $p$. Moreover, as $P / P \cap L$ is cyclic, $P \cap L=O_{p}(K)$ and $P_{1} \subseteq O_{p^{\prime}, p}(K)$, it follows that $O_{p^{\prime}}(K) P_{1}$ is a normal subgroup of $K$. Thus, by the Frattini argument, we have $K=O_{p^{\prime}}(K) N_{K}\left(P_{1}\right)$.

We claim that the minimal choice of $v$ forces the containment $\Omega_{m}(P) \subseteq P_{1}$. Indeed, suppose $u \in P, u \notin P_{1}$, and $u^{p^{m}}=1$. Since $P / P \cap L$ is cyclic, this implies that there is a positive integer $r$ such that $P_{1}=\left\langle P \cap L, u^{p^{r}}\right\rangle$. However, $u^{p^{r}}$ has order less than $p^{m}$ and this contradicts our choice of $v$. Hence, we have $\Omega_{m}(P) \subseteq P_{1}$, so $\Omega_{m}(P)=\Omega_{m}\left(P_{1}\right)$.

Thus, the above factorization of $K$ yields now that $K=O_{p^{\prime}}(K) N_{K}\left(\Omega_{m}(P)\right)$, so $K=O_{p^{\prime}}(K) N_{K}(Q)$ as $Q$ is a characteristic subgroup of $\Omega_{m}(P)$. Hence we need only show that $Q$ centralizes $O_{p^{\prime}}(K)$. To do this, we require only that $Q \subseteq P \cap L$ as $O_{p}(K)=P \cap L$. However, since $\Omega_{m}(P)=\Omega_{m}\left(P_{1}\right)$,

$$
Q=\Omega_{m}\left(P_{1}\right)^{\prime} \subseteq P_{1}^{\prime} \subseteq P \cap L
$$

as $P_{1} / P \cap L$ has order $p$ and the result is proved.

To conclude the constrained case, we shall establish that, in fact, $\Omega_{m}(P)$ is not abelian. This will be accomplished once we verify the following complicated, independent property of solvable groups.

LEMMA 5. Assume that $Y$ is a normal subgroup of odd order of the solvable group $X$ and that the following conditions hold:

(a) The quotient $X / Y$ is a Frobenius group of order $2 p r$, where $p$ and $r$ are distinct odd primes, with Frobenius kernel of order $r$ and cyclic complement of order $2 p$.

(b) If $R$ is a Sylow p-subgroup of $Y$, then $C_{X}(R) \subseteq Y$.

(c) There is an involution $t$ and a p-element $u$ of $C_{X}(t)$ such that the image of $t u$ in $X / Y$ generates a cyclic complement of order $2 p$.

Under these conditions, there is a $\langle t u\rangle$-invariant Sylow p-subgroup $R$ of $Y$ such that $u$ does not centralize $\Omega_{1}\left(C_{R}(t)\right)$.

The proof is by induction on $|X|$; the situation finally reached will also motivate the result itself.

Proof. As we have just stated, we shall proceed by induction on $|X|$; we may assume the result holds for all groups of order less than $|X|$ inasmuch as the case $|X|=1$ is vacuous.

Suppose that $X_{0}$ is a subgroup of $X$ containing both $t u$ and a Sylow $p$-subgroup of $Y$ and covering $X / Y$. We set $Y_{0}=Y \cap X_{0}$ and we claim that $X_{0}$ fulfills all the hypotheses of the lemma. Indeed, $Y_{0}$ is a normal subgroup of odd order of $X_{0}$. Moreover, $X_{0} / Y_{0}$ is isomorphic with $X / Y$ since $X_{0}$ covers $X / Y$; hence condition (a) is satisfied by $X_{0}$. Furthermore, if $R_{0}$ is a Sylow $p$-subgroup of $Y$ contained in $X_{0}$, then $C_{X_{0}}\left(R_{0}\right) \subseteq X_{0} \cap Y=Y_{0}$, so (b) holds. Finally, (c) is valid for $X_{0}$ as $\langle t u\rangle$ 
$\subseteq X_{0}$ and the isomorphism between $X / Y$ and $X_{0} / Y_{0}$ shows that $Y_{0} t u$ is a generator for a cyclic complement of the Frobenius group $X_{0} / Y_{0}$.

Thus if $X_{0} \subset X$, we may apply our induction assumption to $X_{0}$. Hence, there is a $\langle t u\rangle$-invariant Sylow $p$-subgroup $R_{0}$ of $Y_{0}$ such that $u$ does not centralize $\Omega_{1}\left(C_{R_{0}}(t)\right)$. However, $R_{0}$ is a Sylow $p$-subgroup of $Y$, by assumption, and so the result holds for $X$. Consequently, we shall assume that if $X_{0}$ is any such subgroup, then $X_{0}=X$.

Since $\langle Y, t, u\rangle$ is a solvable group with Sylow 2-subgroup $\langle t\rangle$, there is a $\langle t u\rangle$ invariant Sylow $p$-subgroup $R$ of $Y$. We now set $X_{0}=N_{X}(R)$ and we claim that $X_{0}=X$. Indeed, $X_{0}$ contains $t, u$ and the Sylow $p$-subgroup $R$ of $Y$. Moreover, $X_{0}$ covers $X / Y$ by the Frattini argument. Thus, $X_{0}=X$ as claimed and so $R$ is normal in $X$.

Next, we assert that if $\pi=\{2, p, r\}$, then $X$ is a $\pi$-group. Since $X$ is solvable, there is a Hall $\pi$-subgroup $X_{1}$ of $X$ containing $t$ and $u$. Moreover, $X_{1}$ certainly contains $R$ and covers $X / Y$. Hence $X_{1}=X$, again by the above discussion, and so $X$ is a $\pi$-group.

Thus if $K / Y$ is the Frobenius kernel of $X / Y$, then $K / R$ is an $r$-group. Indeed, $K / Y$ is of order $r$. Furthermore, $Y$ is a $\{r, p\}$-group since $Y$ is a $\pi$-group of odd order. As $R$ is a normal Sylow $p$-subgroup of $Y$, it follows that $Y / R$ is an $r$-group. Hence, $K / R$ is also an $r$-group.

This now implies that $K / R$ is cyclic. To see this, we let $V$ be the subgroup of $K$ containing $R$ such that $V / R$ is the Frattini subgroup of $K / R$. Since $K / Y$ is of order $r$, it follows that $Y \supseteq V$. Furthermore, $K / R$ is cyclic if $Y=V$. However, $K / V$ is a completely reducible $X$-module since $X / K$ and $K / V$ have coprime orders. Hence if $Y \supset V$, then there are normal subgroups $K_{1}$ and $K_{2}$ of $X$ such that $K_{2} \subset K$, $K=K_{1} K_{2}, K_{1} \cap K_{2}=V$, and $K_{2} / V$ is incident with $K / Y$. Thus, if $X_{2}=\left\langle K_{2}, t u\right\rangle$, then $X_{2} \subset X$. However, $R \subseteq X_{2},\langle t u\rangle \subseteq X_{2}$, and $X_{2}$ covers $X / Y$. This is a contradiction, so we must have that $Y=V$ and so $K / R$ is indeed a cyclic $r$-group.

We now claim that $R$ contains a characteristic subgroup $R^{*}$ of exponent $p$ such that $C_{X}\left(R^{*}\right) \subseteq Y$. Indeed, $R$ contains a characteristic subgroup $R_{1}$ of class at most two such that no $p^{\prime}$-automorphism of $R$ centralizes $R_{1}$, by Theorem 5.3.11 of [19]. Since $C_{X}(R) \subseteq Y$, it follows that $C_{K}\left(R_{1}\right) \subseteq Y$. But $X / Y$ is a Frobenius group, so $C_{X}\left(R_{1}\right) \subseteq Y$. We now set $R^{*}=\Omega_{1}\left(R_{1}\right)$, so that no $p^{\prime}$-automorphism of $R_{1}$ centralizes $R^{*}$, by Theorem 5.3.10 of [19]. Thus, by an argument similar to the one just given, we have that $C_{X}\left(R^{*}\right) \subseteq Y$. Moreover, since $R_{1}$ has class at most two and $p$ is odd, it follows that $R^{*}$ has exponent $p$ and our assertion is valid.

Hence, in order to complete the proof, it suffices to show that there is a chief factor $H / H_{0}$ of $X$ with the following properties:

(1) $H \subseteq R^{*}$;

(2) $t$ does not centralize $H / H_{0}$;

(3) $u$ does not centralize the fixed points of $t$ on $H / H_{0}$.

Indeed, suppose that $H / H_{0}$ is such a factor. Thus, $C_{H}(t)$ covers the fixed points of $t$ on $H / H_{0}$ as $H$ is of odd order. But $u$ normalizes $C_{H}(t)$ as $t$ and $u$ commute, 
so $u$ does not centralize $C_{H}(t) / C_{H_{0}}(t)$ by (3). Thus, $u$ does not centralize $C_{H}(t)$. However,

$$
C_{H}(t) \subseteq C_{R^{*}}(t) \subseteq \Omega_{1}\left(C_{R}(t)\right)
$$

as $R^{*}$ is of exponent $p$ and we obtain the desired conclusion of the lemma.

However, let $R^{*}=H_{1} \supset H_{2} \supset \cdots \supset H_{k}=1$ be part of a chief series of $X$; set $F_{i}=H_{i} / H_{i+1}, 1 \leqq i \leqq k-1$, and set

$$
Z=\bigcap_{i=0}^{k-1} C_{X}\left(F_{i}\right)
$$

Since $R^{*}$ is a $p$-group, it follows that $Z / C_{X}\left(R^{*}\right)$ is a $p$-group. But $C_{X}\left(R^{*}\right) \subseteq Y$, $Z$ is normal in $X$, and $O_{p}(X / Y)=1$, so $Z \subseteq Y$. Moreover, $C_{X}\left(F_{i}\right) \supseteq R$ as $R$ is a $p$-group and $F_{i}$ is a chief factor of $X$. But $K / R$ is a cyclic $r$-group and $K / Y$ is the Frobenius kernel of $X / Y$, so $X / R$ is a Frobenius group with kernel $K / R$. Hence if $C_{X}\left(F_{i}\right) \nsubseteq Y$, then $C_{X}\left(F_{i}\right) \supseteq K$. Thus, there must exist an index $j$ with $1 \leqq j \leqq k-1$ such that $C_{X}\left(F_{j}\right) \subseteq Y$ since $Z \subseteq Y$.

We claim that $F=F_{j}$ is the desired chief factor. Indeed, $X / C_{X}(F)=\bar{X}$ is clearly a Frobenius group with kernel $\bar{K}$ a cyclic $r$-group and with cyclic complement of order $2 p$. Hence, by Lemma $2.8 .4, F$ is a free module for any complement of $\bar{X}$ and, in particular, for the complement $\langle\bar{t} \bar{u}\rangle$. We conclude from this that $u$ does not centralize all the fixed points of $t$ on $F$. This proves the lemma.

At last we can finish the considerations of the constrained case by proving

LEMma 6. The subgroup $\Omega_{m}(P)$ is nonabelian.

Proof. We assume that $\Omega_{m}(P)$ is abelian and derive a contradiction. We begin by setting $\bar{N}=N / O(N)$ and showing that we may assume that $\bar{v} \in \bar{E}$. We were careful to choose $P$ as a $D$-invariant Sylow $p$-subgroup of $C$; consequently, $D$ normalizes $\Omega_{m}(P)$. Thus, $D$ normalizes the section $\Omega_{m}(P) / \Omega_{m}(P) \cap L$, which is incident with $P_{1} / P \cap L$. But $D$ centralizes $N / L$, so $D$ centralizes these sections. Thus, there is $v_{1} \in \Omega_{m}(P) \cap C(D), v_{1} \notin P \cap L$. Since $\Omega_{m}(P)$ is abelian by assumption, this implies that $v_{1}^{p m}=1$, so that $v_{1}$ has order $p^{m}$ precisely by the minimality of $m$. Hence, we may replace $v$ by $v_{1}$; thus, we may assume $v \in C(D)$. However, $C_{\bar{N}}(\bar{D})$ is the direct product of $Z(\bar{D})$ and $\bar{E}$ by Lemma 2.4.3, so that $\bar{v} \in \bar{E}$ as we claimed. In particular, this implies that $v^{p} \in O(N)$ as $\bar{N}$ is the semidirect product of $\bar{L}$ and $\bar{E}$.

Again by the same lemma, there is a cyclic subgroup $\bar{V}$ of odd order in $\bar{L}$ inverted by $\bar{x}_{2}$ such that $\bar{v}$ does not centralize $O_{p^{\prime}}(\bar{V})$. Hence there is an odd prime divisor $r$ of $O_{p^{\prime}}(\bar{V})$ such that $\bar{v}$ normalizes a subgroup $\bar{K}$ of order $r$ of $O_{p^{\prime}}(\bar{V})$ but centralizes no nonidentity element of $\bar{K}$. Moreover, $\bar{x}_{2}$ inverts $\bar{K}$. Since $\bar{v}$ has prime order $p$, it follows that $\left\langle\bar{K}, \bar{x}_{2}, \bar{v}\right\rangle$ is a Frobenius group with Frobenius kernel cyclic of order this prime $r$ and complement $\left\langle\bar{x}_{2}, \bar{v}\right\rangle$ cyclic of order $2 p$.

Thus, if $\bar{K}=K / O(N)$ for a subgroup $K$ of $N$ and if we set $X=\left\langle K, x_{2}, v\right\rangle$, $Y=O(N), t=x_{2}$, and $u=v$, then all the hypotheses of Lemma 5 are satisfied. Hence there is a $\left\langle x_{2} v\right\rangle$-invariant Sylow $p$-subgroup $R$ of $Y=O(N)$ such that $v$ does not 
centralize $\Omega_{1}\left(C_{R}\left(x_{2}\right)\right)$. Hence there is an element $z$ of order $p$ in $C_{R}\left(x_{2}\right)$ such that $z$ and $v$ do not commute, but do generate a $p$-subgroup. Thus, $z$ and $v$, in fact, generate a nonabelian $p$-subgroup of $C$ as each lies in $C$. Let $P^{*}$ be a Sylow $p$ subgroup of $C$ containing $\langle z, v\rangle$. Since $z$ has order $p$ and $v$ has order $p^{m}$, it follows that $\langle z, v\rangle \subseteq \Omega_{m}\left(P^{*}\right)$ and so $\Omega_{m}\left(P^{*}\right)$ is nonabelian. Hence, $\Omega_{m}(P)$ is also nonabelian, which is the contradiction we have been seeking.

This establishes the lemma and concludes the proof of the proposition in the constrained case.

Thus, we assume for the remainder of the section that $N$ is not $p$-constrained within $S(N)$. We let $R$ be as at the beginning of the section, so $C_{N}(R)$ covers $L_{0} / O(N)$, as we remarked much earlier. To verify the proposition in this case, we shall set $Q=Z(P)$. Moreover, we shall take $N^{*}=N(Q \cap O(N))$ if $P \cap O(N) \neq 1$ and $N^{*}=N(R)$ if $P \cap O(N)=1$. Our first result, however, tells us about $Z(P)$.

\section{LEMMA 7. The subgroup $Z(P)$ is contained in $L$.}

Proof. We shall assume that $Z(P) \nsubseteq L$ and derive a contradiction. We begin by observing that $P \cap L \subseteq O(N)$. Indeed, set $\bar{N}=N / O(N)$, so $\bar{P}$ is a $\bar{D}$-invariant Sylow $p$-subgroup of $O(\bar{C})$. Hence, by Lemma 2.4.2, $Z(\bar{P}) \subseteq \bar{L}$ or $\bar{P} \cap \bar{L}=1$. Thus, we must assume that $\bar{P} \cap \bar{L}=1$, whence $P \cap L \subseteq O(N)$ as we claimed. Since $P \cap L \neq 1$ by our choice of the prime $p$, this implies that $P \cap O(N) \neq 1$.

We now distinguish two cases: $P$ abelian; $P$ not abelian. We shall deal with each of these separately, beginning with the easier of the two.

Thus, suppose that $P$ is not abelian. Since $N / L$ is cyclic, by Proposition 2.3.3, it follows that $P^{\prime} \subseteq O(N)$. Hence $P^{\prime} \subseteq P \cap O(N) \subseteq R$ as $R$ is $D P$-invariant. Consequently, $C\left(P^{\prime}\right)$ covers $L_{0} / O(N)$, so $N\left(P^{\prime}\right)$ does also. Hence, $N\left(P^{\prime}\right)$ is a $Q$-group of characteristic power $q$ by Proposition 1.1 as $P^{\prime} \neq 1$. However, $P^{\prime}$ is a characteristic subgroup of $P$, so $N\left(P^{\prime}\right)$ covers $M / C$ by the Frattini argument. Thus, $N_{M}\left(P^{\prime}\right)$ is a $D$-subgroup of the $Q$-group $N\left(P^{\prime}\right)$, contrary to Lemma 2.4.17.

We now assume that $P$ is abelian. We claim that it suffices to show that $N(P)$ contains a $Q$-subgroup. Indeed, if that is the case, then $N(P)$ is a $Q$-group or a $Q D$-group by Proposition 1.1 inasmuch as $N(P)$ contains $D$. But $N(P)$ covers $M / C$, by the Frattini argument; therefore, $N(P)$ must be a $Q D$-group, again by Lemma 2.4.17. Now $C(P) \supseteq T$, so $C(P)$ is a normal subgroup of even order of the $Q D$-group $N(P)$. It follows therefore from Proposition 2.2 .2 that $C(P)$ is also a $Q D$-group. However, $C(P) \subseteq C(P \cap O(N))$, so the latter group is also a $Q D$-group. But $C(R)$ covers $L_{0} / O(N)$; consequently, $C(P \cap O(N))$ covers $L_{0} / O(N)$. Since $P \cap O(N) \neq 1$, it follows from Proposition 1.1 that $C(P \cap O(N))$ must be a $Q$ group of characteristic power $q$, contrary to the fact that it is a $Q D$-group. Thus, we shall conclude the proof by establishing that $N(P)$ contains a $Q$-subgroup.

We set $X_{0}=C_{L_{0}}(R)$ and let $Y_{0}=O(N) \cap X_{0}$. We shall now examine the structure of $X_{0}$. First, $X_{0}$ covers $L_{0} / O(N)$ by hypothesis; therefore $X_{0} / Y_{0}$ is isomorphic to $L_{0} / O(N)$, which is isomorphic to $S L(2, q)$. Furthermore, since $Y_{0}=C_{O(N)}(R)$ and 
$R$ is a Sylow $p$-subgroup of $O(N)$, it follows that $Y_{0}$ is the direct product of $Z(R)$ and a $p^{\prime}$-group $K$. Since $S L(2, q)$ has no nontrivial normal subgroup of odd order, we have that $O\left(X_{0}\right)=Y_{0}$; also $K$ is a characteristic subgroup of $Y_{0}$.

The group $Z(R)$ is in the center of $X_{0}$. Hence $X_{0} / K$ is the extension of the central p-subgroup $Y_{0} / K$ by the group $X_{0} / Y_{0}$, which is isomorphic to $\operatorname{SL}(2, q)$. However, $N \supset L$ as $P \nsubseteq L$, so $q>9$, as $N / L$ is isomorphic with a subgroup of odd order of the group of automorphisms of the Galois field with $q$ elements. Hence by Lemma 2.4.16, $X_{0} / K$ is the direct product of $Y_{0} / K$ and a group $J_{0} / K$, the latter being isomorphic to $S L(2, q)$. Thus, it follows that $J_{0} / K=O^{p}\left(X_{0} / K\right)$ and so $J_{0} / K$ is a characteristic subgroup of $X_{0}$. Moreover, $J_{0} / K$ is clearly incident with $L_{0} / O(N)$.

We now conclude the proof. The subgroups $L_{0}$ and $R$ are normalized by $P$, so $X_{0}$ is $P$-invariant. Consequently, $J_{0}$ and $K$ are also normalized by $P$. Thus $C_{J_{0} / K}(P)$ is isomorphic to $C_{L_{0}}(\bar{P})$, where $\bar{L}_{0}, \bar{P}$ are the images of $L_{0}, P$ respectively in $\bar{N}=N / O(N)$. Moreover, $K$ is a $p^{\prime}$-group; therefore, $C_{J_{0}}(P)$ covers $C_{J_{0} / K}(P)$. Hence it suffices to show that $C_{L_{0}}(\bar{P})$ is isomorphic to $S L\left(2, q_{0}\right)$ for some prime power $q_{0}$. Indeed, this will imply directly that $C_{J_{0}}(P)$ is a $Q$-group. Since $C_{J_{0}}(P)$ $\subseteq N(P)$, this will establish the desired assertion concerning $N(P)$.

However, we are assuming that $Z(P) \nsubseteq L$, so that $Z(\bar{P}) \nsubseteq \bar{L}$. Hence by Lemma 2.4.2, $\bar{P} \subseteq \bar{E}$. Thus, by the same lemma, $C_{L_{0}}(\bar{P})$ is isomorphic to $S L\left(2, q_{0}\right)$, where $q=q_{0}^{m}$ and $m=|\bar{P}|$. The lemma is now proved completely.

The next result will be used to show that the subgroup we take for $N^{*}$ has the desired properties.

LEMMA 8. If $R_{0}$ is a nonidentity DP-invariant subgroup of $R$, then $N\left(R_{0}\right)$ is a $Q$-group which covers $L_{0} / O(N)$, contains $D P$, and is p-constrained within $O\left(N\left(R_{0}\right)\right)$.

Proof. Since $C(R)$ covers $L_{0} / O(N)$, certainly $N\left(R_{0}\right)$ does as well. Moreover, $D P$ is contained in $N\left(R_{0}\right)$ as $R_{0}$ is $D P$-invariant. Hence by Proposition $1.1, N\left(R_{0}\right)$ is a $Q$-group and $N\left(R_{0}\right)=O\left(N\left(R_{0}\right)\right)\left(N\left(R_{0}\right) \cap N\right)$. Thus, all is verified except the last statement: we need only establish that $N\left(R_{0}\right)$ is p-constrained within $O\left(N\left(R_{0}\right)\right)$. It will suffice to show that $x$ normalizes but does not centralize a $p$-subgroup of $N\left(R_{0}\right)$. Indeed, if this is the case, then $x$ will normalize but not centralize a $p$ subgroup of $O\left(N\left(R_{0}\right)\right)$ as the image of $x$ is central in $N\left(R_{0}\right) / O\left(N\left(R_{0}\right)\right)$. But then if $S$ is a Sylow 2-subgroup of $N\left(R_{0}\right)$ containing $D$ and $R^{*}$ is an $S$-invariant Sylow $p$-subgroup of $O\left(N\left(R_{0}\right)\right.$ ), it will follow that $x$ does not centralize $R^{*}$. However, $S$ is quasi-dihedral or wreathed, so $Z(S)$ is cyclic and $\langle x\rangle=\Omega_{1}(Z(S))$. Thus $C_{Z(S)}\left(R^{*}\right)$ $=1$. Since $C_{S}\left(R^{*}\right)$ is normal in $S$, it follows that $C_{S}\left(R^{*}\right)=1$. But $C_{S}\left(R^{*}\right)$ is a Sylow 2-subgroup of $C_{N\left(R_{0}\right)}\left(R^{*}\right)$ and consequently the latter group is of odd order. Now Lemma 2.6.2 implies that $N\left(R_{0}\right)$ is $p$-constrained within $O\left(N\left(R_{0}\right)\right)$, as asserted.

However, $N$ is not $p$-constrained within $S(N)$; our assumptions thus yield that $p$ is not stationary. Let $R_{1}$ be a $\langle x\rangle$-invariant Sylow $p$-subgroup of $O(N(R))$. As usual, $R_{1}$ is not centralized by $x$ and $R=C_{R_{1}}(x)$. Hence by Lemma 2.8 .1 , there is a nonidentity element $u$ of $R_{1}$ which is inverted by $x$ and which centralizes $R$. Thus 
$u \in N\left(R_{0}\right)$ and $x$ normalizes but does not centralize the $p$-subgroup $\langle u\rangle$. This proves the lemma.

We now deal with the first of the two possibilities:

Lemma 9. If $P \cap O(N) \neq 1$, then $Q=Z(P)$ and $N^{*}=N(Q \cap O(N))$ satisfy the conditions of the proposition.

Proof. Since $P \cap O(N)$ is a nonidentity normal subgroup of $P$, it follows that $Q \cap O(N) \neq 1$. Thus, $Q \cap O(N)$ is a nonidentity $D P$-invariant subgroup of $P$; since $R$ is $P$-invariant, we also have that $P \cap O(N) \subseteq R$. The previous lemma now applies with $R_{0}=Q \cap O(N)$ and implies that $N^{*}$ satisfies condition (i) of the proposition.

We claim that in this case we have $C^{*}=N_{C}(Q)$. Indeed, we need only show that $N_{C}(Q) \subseteq N^{*}$ by definition of $C^{*}$. Hence, it suffices to verify that $N_{C}(Q)$ normalizes $Q \cap O(N)$. However, $N_{C}(Q)$ certainly normalizes $Q$ and $C \cap O(N)$ is a normal subgroup of $C$. Thus, $N_{C}(Q)$ normalizes $C \cap O(N) \cap Q=Q \cap O(N)$, as desired.

By Lemma 8, $N^{*}$ is $p$-constrained in $O\left(N^{*}\right)$; thus $O_{p^{\prime}, p}\left(N^{*}\right) \subseteq O\left(N^{*}\right)$ and so $D Q O_{p^{\prime}, p}\left(N^{*}\right)$ has a normal 2-complement. Therefore there is a $D Q$-invariant Sylow $p$-subgroup $R^{*}$ of $O_{p^{\prime}, p}\left(N^{*}\right)$. It follows that

$$
C_{C}\left(Q C_{R^{*}}(Q)\right) \subseteq C_{C}(Q) \subseteq N_{C}(Q)=C^{*},
$$

which establishes part (iii) of the proposition. It now remains to prove that $N_{C}(Q)$ covers $C \cap L / C \cap S(L)$. Since $L=L_{0} D$ with $L_{0}$ normal in $L$ and since $D \subseteq N_{C}(Q)$, it will suffice to show that $N_{C}(Q)$ covers $C \cap L_{0} / C \cap S\left(L_{0}\right)$.

To this end, we set $X=C_{L_{0}}(P \cap O(N))$. Since $P \cap O(N) \subseteq R$, we have $X \supseteq C_{L_{0}}(R)$, which in turn covers $L_{0} / O(N)$. In particular, $X / S\left(L_{0}\right) \cap X$ is isomorphic with $L_{0} / S\left(L_{0}\right)$. This implies that $S(X)=S\left(L_{0}\right) \cap X$.

The subgroups $S(X)$ and $S\left(L_{0}\right)$ are each the direct product of $\langle x\rangle$ and a group of odd order as $X \subseteq L_{0} \subseteq N$. Hence, $C_{L_{0} / S\left(L_{0}\right)}(T)$ is isomorphic to $C \cap L_{0} / C \cap S\left(L_{0}\right)$ and $C_{X / S(X)}(T)$ is isomorphic to $C \cap X / C \cap S(X)$.

We now set $H_{1}=C \cap L_{0}, K_{1}=C \cap S\left(L_{0}\right), H_{2}=C \cap X, K_{2}=C \cap S(X)$, and $H=C^{*}=N_{C}(Q)$. We assert that we need only demonstrate that $H$ covers $H_{2} / K_{2}$. Indeed, we already know that $K_{2} \cap H_{1} \subseteq K_{1}$, that $H_{2} \subseteq H_{1}$, and that $H_{1} / K_{1}$ and $H_{2} / K_{2}$ are isomorphic. Hence, by Lemma 2.8.6, $H_{1} / K_{1}$ and $H_{2} / K_{2}$ are incident. Thus, by Lemma 2.8 .5 , if $H$ covers $H_{2} / K_{2}$, then it covers $H_{1} / K_{1}$. Since $H=C^{*}$, it will therefore follow that $C^{*}$ covers $C \cap L_{0} / C \cap S\left(L_{0}\right)$, which is what we have to prove. Hence our assertion is valid.

We note that $P \cap H_{2}$ is a Sylow $p$-subgroup of $H_{2}$. In fact, $P$ normalizes $L_{0}$ and $P \cap O(N)$. Thus, $P$ normalizes $X$ and so $P$ normalizes $C \cap X$. The subgroup $P(C \cap X)$ has Sylow $p$-subgroup $P$ as $P \subseteq P(C \cap X) \subseteq C$ and has $C \cap X$ as a normal subgroup; consequently, $P \cap H_{2}=P \cap(C \cap X)$ is a Sylow $p$-subgroup of $\mathrm{H}_{2}$ $=C \cap X$. 
Furthermore, $C \cap S\left(L_{0}\right)$ is normal in $C$, so $P \cap S\left(L_{0}\right)=P \cap O(N)$ is a Sylow $p$-subgroup of $C \cap S\left(L_{0}\right)$. Thus, $Z(P \cap O(N))$ is a Sylow $p$-subgroup of $C_{C \cap S\left(L_{0}\right)}(P \cap O(N))$, which is just $C \cap S\left(L_{0}\right) \cap X=K_{2}$ inasmuch as $S\left(L_{0}\right) \cap X$ $=S(X)$ and $K_{2}=C \cap S(X)$.

The subgroup $H_{2} / K_{2}$ is isomorphic to $C_{L_{0} / S\left(L_{0}\right)}(T)$, which is cyclic, by Lemma 2.4.2. But $Q=Z(P) \subseteq C \cap X=H_{2}$, so this implies that $K_{2} Q$ is a normal subgroup of $H_{2}$. However, $Z(P \cap O(N))$ is a Sylow $p$-subgroup of $K_{2}$ and is clearly normalized by $Q$, so $Q^{*}=Z(P \cap O(N)) Q$ is a Sylow $p$-subgroup of $K_{2} Q$. The Frattini argument now yields

$$
H_{2}=N_{H_{2}}\left(Q^{*}\right) K_{2} \text {. }
$$

However, $K_{2} Q / K_{2}$ and $Z(P \cap O(N))$ are central factors of $H_{2}$, inasmuch as $H_{2} / K_{2}$ is cyclic and $Z(P \cap O(N))$ is central in $X \subseteq C(P \cap O(N))$. Hence, since the automorphism group of a stability chain of a $p$-group is also a $p$-group and since $Z(P \cap O(N)) Q$ is normal in the Sylow p-subgroup $P \cap H_{2}$ of $H_{2}$, it follows that

$$
N_{\mathrm{H}_{2}}\left(Q^{*}\right)=\left(P \cap \mathrm{H}_{2}\right) C_{\mathrm{H}_{2}}\left(Q^{*}\right) \subseteq C(Q) .
$$

Thus $N(Q)$ covers $H_{2} / K_{2}$. Since $H_{2} \subseteq C$, it follows that $C^{*}=N_{C}(Q)$ covers $H_{2} / K_{2}$ and the lemma is proved.

The next result is the final one of the section. It completes the nonconstrained case of Proposition 1 and so gives the last step of the proof of that proposition. We note that this last case is the only one in which $C^{*} \neq N_{C}(Q)$.

LEMMA 10. If $P \cap O(N)=1$, then $Q=Z(P)$ and $N^{*}=N(R)$ satisfy the conditions of the proposition. Furthermore, $R \subseteq C_{R^{*}}(Q)$.

Proof. As in the preceding lemma, we have that $N^{*}$ fulfills condition (i) of the proposition as a direct consequence of Lemma 8.

We now claim that $Q$ centralizes $R$. To see this, we set $\bar{N}=N / O(N)$. Lemma 7 implies that $\bar{Q} \subseteq \bar{L}$. Hence, $\bar{Q}$ is a subgroup of $\overline{O(C) \cap L}=O(\bar{C}) \cap \bar{L}$, which is inverted by $\bar{d}$ by Lemma 2.4.2. Since $P \cap O(N)=1, \bar{Q}$ is isomorphic to $Q$ and so $Q$ is inverted by $d$. However, $C(R)$ covers $L_{0} / O(N)$, so $A_{L_{0}}(R)$ is of odd order. Thus, as $d \in L_{0}$ and $d$ normalizes $R$, we have that $d$ centralizes $R$. Hence, $Q=[Q, d]$ also centralizes $R$. Thus, $Q \subseteq N^{*}$.

As in the previous lemma, there is a $D Q$-invariant Sylow $p$-subgroup $R^{*}$ of $O_{p^{\prime}, p}\left(N^{*}\right)$. Moreover, $R \subseteq R^{*}$ as $R$ is obviously normal in $N^{*}$. Hence, $C_{R^{*}}(Q) \supseteq R$ and so

$$
\begin{aligned}
C_{C}\left(Q C_{R^{*}}(Q)\right) & \subseteq C_{C}(R) \cap N_{C}(Q) \\
& \subseteq N^{*} \cap N_{C}(Q) \\
& \subseteq C^{*}
\end{aligned}
$$

This proves part (iii) of the proposition and also establishes the final statement of the lemma. 
We now set $X=C_{L_{0}}(R)$, so $X$ covers $L_{0} / O(N)$ and, as before, $S(X)=S(N) \cap X$. Moreover, just as in the previous result, using Lemmas 2.8.5 and 2.8.6, it suffices to prove that $C^{*}$ covers $C \cap X / C \cap S(X)$. However, $C \cap X \subseteq N^{*}$, so it is enough to show that $N_{C}(Q)$ covers $C \cap X / C \cap S(X)$. Again this quotient is cyclic and $Q \subseteq L_{0}$ by Lemma 7, so $Q \subseteq X$. Thus $(C \cap S(X)) Q$ is normal in $C \cap X$. Moreover, $P \cap O(N)=1$ by assumption, so $x_{2}$ inverts $R$. Hence $C \cap S(X)$ is a $p^{\prime}$-group as $Z(R)$ is a Sylow $p$-subgroup of $S(X)$. Thus, $Q$ is a Sylow $p$-subgroup of $(C \cap S(X)) Q$ and the Frattini argument yields that

$$
C \cap X=N_{C \cap x}(Q)(C \cap S(X)),
$$

as desired. This proves the lemma and completes the proof of the proposition.

8. A second family of local subgroups. We shall define and examine another set of local subgroups of $G$. The preceding section will imply that this collection is nonempty. We preserve all the hypotheses of the preceding section. We choose $Q, N^{*}$, and $R^{*}$ in accordance with Proposition 7.1. Moreover, we set $C^{*}=$ $N_{C \cap N^{*}}(Q)$, as before, and we now let $Q^{*}=Q C_{R^{*}}(Q)$. Hence, $Q^{*}$ is $D$-invariant and $C_{C}\left(Q^{*}\right) \subseteq C^{*}$. To get started, we list some properties of $N(Q)$ :

LEMMA 1. If $H=N(Q)$, then

(i) $H$ contains $D Q^{*}$;

(ii) $A_{H}(T)=$ Aut $(T)$;

(iii) $C^{*} \cap H$ covers $C^{*} \cap L / C^{*} \cap S(L)$;

(iv) $C^{*} \cap O_{p^{\prime}}(H) \subseteq S(L)$.

Proof. The group $Q$ is $D$-invariant, as it is characteristic in $P$, so $D \subseteq H$. Moreover, $Q^{*}$ normalizes $Q$, by its definition. This establishes (i). Furthermore, again as $Q$ is characteristic in $P, N(Q)$ covers $M / C$, so (ii) is valid. Statement (iii) is immediate from Proposition 7.1 as $C^{*} \subseteq N_{C}(Q) \subseteq H$. Finally the last assertion is a direct consequence of the next result:

LEMMA 2. If $K$ is any subgroup of $G$ containing $Q^{*}$, then

$$
C^{*} \cap O_{p^{\prime}}(K) \subseteq S(L) .
$$

Proof. We set $X=C^{*} \cap O_{p^{\prime}}(K)$ and we shall first prove that $X \subseteq S\left(N^{*}\right)$. Let $\bar{N}^{*}=N^{*} / O_{p^{\prime}}\left(N^{*}\right)$. Since $X \subseteq C^{*} \subseteq N^{*}$ and $N^{*}$ is $p$-constrained in $S\left(N^{*}\right)$, it is sufficient to establish that $\bar{X}$ centralizes $\bar{R}^{*}$ inasmuch as $\bar{R}^{*}=O_{p}\left(\bar{N}^{*}\right)$ by definition of $R^{*}$. However, $O_{p^{\prime}}\left(N^{*}\right)$ is certainly a $p^{\prime}$-group, so $\overline{C_{R^{*}}(Q)}$ is the centralizer of $\bar{Q}$ in $\bar{R}^{*}$. Thus, by Theorem 5.3.4 of [19], we need only prove that $\bar{X}$ centralizes both $\bar{Q}$ and $C_{R^{*}}(\bar{Q})$ to conclude that $\bar{X}$ centralizes $\bar{R}^{*}$. But $\left[X, Q^{*}\right] \subseteq O_{p^{\prime}}(K)$, so $[\bar{X}, \bar{Q}]$ and $\left[\bar{X}, \overline{C_{R^{*}}(Q)}\right]$ are $p^{\prime}$-groups, since $Q^{*}=Q C_{R^{*}}(Q)$. Moreover, $X \subseteq C^{*}$ $\subseteq N_{C}(Q)$, so $[\bar{X}, \bar{Q}]$ is a $p$-group and $\bar{X}$ centralizes $\bar{Q}$, which is one of the conclusions we want. Finally, we have

$$
\left[\bar{X}, \overline{C_{R^{*}}(Q)}\right] \subseteq\left[\bar{X}, \bar{R}^{*}\right] \subseteq \bar{R}^{*}
$$

as $\bar{R}^{*}$ is normal in $\bar{N}^{*}$ and so $\bar{X}^{*}$ also centralizes $\overline{C_{R^{*}}(Q)}=C_{\bar{R}^{*}}(\bar{Q})$. 
We claim next that $N \cap S\left(N^{*}\right) \subseteq S(L)$. Indeed, we have $N \cap S\left(N^{*}\right) \subseteq S\left(N \cap N^{*}\right)$. But $N^{*}=O\left(N^{*}\right)\left(N \cap N^{*}\right)$ by Lemma 2.4 .14 as $N^{*}$ is a $Q$-group containing $D$. Since $N^{*}$ covers $L_{0} / O(N)$, it follows that $N \cap N^{*}$ covers $L_{0} / O(N)$, so $S\left(N \cap N^{*}\right)$ $\subseteq S(N)$. Since $S(N)=S(L)$, we conclude that $N \cap S\left(N^{*}\right) \subseteq S(L)$, as claimed.

Finally $X \subseteq S\left(N^{*}\right)$ by the first paragraph of the proof and $X \subseteq C^{*} \subseteq C \subseteq N$, so $X \subseteq N \cap S\left(N^{*}\right)$. Thus, $X \subseteq S(L)$ and the lemma is proved.

We are now ready to define the new collection of $p$-local subgroups.

Definition 1 . The family $\mathscr{M}(p)$ is the collection of all $p$-local subgroups $H$ of $G$ satisfying the following conditions:

(a) $D Q^{*} \subseteq H$;

(b) $A_{H}(T)=\operatorname{Aut}(T)$;

(c) $C^{*} \cap H$ covers $C^{*} \cap L / C^{*} \cap S(L)$;

(d) $C^{*} \cap O_{p^{\prime}}(H) \subseteq S(L)$.

In particular, it is immediate from Lemma 1 that $N(Q) \in \mathscr{M}(p)$. Our next result lists some properties of the elements of $\mathscr{M}(p)$. We shall then state and prove the main result of this section.

Lemma 3. If $H$ is an element of $\mathscr{M}(p)$, then

(i) $H$ is a $D$-group with respect to $T$.

(ii) $O_{p^{\prime}}(H)$ is of odd order.

(iii) $C^{*} \cap O_{p^{\prime}}(H) \subseteq O(L)$.

Notice that the last assertion is an improvement of the statement of Lemma 2 for the elements of $\mathscr{M}(p)$.

Proof. To prove (i), it will suffice to show that $C \cap H$ covers $C \cap L / C \cap O(N)$. Indeed, assume this is the case. Since $H$ contains $D$ by part (a) of Definition 1, Proposition 1.1 implies that $H$ is not a $Q D$-group. But $A_{H}(T)=$ Aut $(T)$ by part (b) of Definition 1, so $H$ must be a $D$-group with respect to $T$, again by Proposition 1.1 , and our assertion is valid.

But $C^{*}$ covers $C \cap L / C \cap S(L)$, by Proposition 7.1, and

$$
C^{*} \cap L \subseteq\left(C^{*} \cap S(L)\right)\left(C^{*} \cap H\right)
$$

by (c) of Definition 1 . Hence

$$
\begin{aligned}
C \cap L & =(C \cap S(L))\left(C^{*} \cap L\right) \\
& \subseteq(C \cap S(L))\left(C^{*} \cap S(L)\right)\left(C^{*} \cap H\right) \\
& \subseteq(C \cap S(L))(C \cap H),
\end{aligned}
$$

as $C^{*} \subseteq C$, so $C \cap H$ covers $C \cap L / C \cap S(L)$. Furthermore, as $D \subseteq H, C_{D}(T)$ $\subseteq C \cap H$. Since $S(L)=S(N)=O(N) Z(D)$ by Lemma 2.4.14 and $Z(D) \subseteq C_{D}(T)$, we conclude that $C \cap H$ covers $C \cap L / C \cap O(N)$, as required. This proves (i).

Next, suppose that $O_{p^{\prime}}(H)$ is of even order; it follows that $T \cap O_{p^{\prime}}(H) \neq 1$. Indeed, since $H$ is a $D$-group, its Sylow 2-subgroups are either dihedral, quasidihedral or wreathed. Now $D \subseteq H$, so if $S$ is a Sylow 2-subgroup of $H$ containing 
$D$, it is immediate that any nonidentity normal subgroup of $S$ has a nontrivial intersection with $T$. In particular, $T \cap O_{p^{\prime}}(H) \neq 1$ as $S \cap O_{p^{\prime}}(H)$ is normal in $S$ and is a Sylow 2-subgroup of $O_{p^{\prime}}(H)$.

However, $H$ is a $D$-group with respect to $T$, so all the involutions of $T$ are conjugate in $H$. Thus, $T \subseteq O_{p^{\prime}}(H)$. But $T \subseteq C^{*}$, by Proposition 7.1, so $T \subseteq C^{*} \cap O_{p^{\prime}}(H)$ and hence $T \subseteq S(L)$ by statement (d) of Definition 1. This is a contradiction as $Z(D)$ is a Sylow 2-subgroup of $S(L)$ by Lemma 2.4 .14 and $Z(D)$ is cyclic. Thus (ii) is proved.

The last statement of the lemma now follows directly. In fact, $C^{*} \cap O_{p^{\prime}}(H)$ $\subseteq S(L)$ by statement (d) of Definition 1 and $C^{*} \cap O_{p^{\prime}}(H)$ is of odd order, by part (ii) of this lemma. But $O(N)=O(L)$ is a normal 2-complement in $S(L)$, again by Lemma 2.4.14 and we conclude that $C^{*} \cap O_{p^{\prime}}(H) \subseteq O(L)$. The lemma is thus completely proved.

The main result of this section is as follows:

Proposition 1. If $H$ is an element of $\mathscr{M}(p), R_{1}$ is a nonidentity $D Q^{*}$-invariant p-subgroup of $H$ and $O_{p^{\prime}}(H) R_{1}$ is normal in $H$, then $N\left(R_{1}\right) \in \mathscr{M}(p)$.

This proposition, which is analogous to Proposition 6.2 for the elements of $\mathscr{L}(p)$, will be used in the next chapter to construct elements of $\mathscr{M}(p)$ containing Sylow $p$-subgroups of $G$. Before giving the proof we shall state and prove an important consequence, which is likewise analogous to Corollary 6.1.

Corollary 1. If $H$ is an element of $\mathscr{M}(p), P_{1}$ is a Sylow p-subgroup of $H$ and $O_{p^{\prime}}(H) Z\left(J\left(P_{1}\right)\right)$ is normal in $H$, then there is a conjugate of $Z\left(J\left(P_{1}\right)\right)$ in $H$ whose normalizer in $G$ is an element of $\mathscr{M}(p)$.

Proof (of the corollary). By the proposition, it suffices to exhibit a $D Q$ *-invariant conjugate in $H$ of $Z=Z\left(J\left(P_{1}\right)\right)$. However, $Q^{*} \subseteq H$ as $H \in \mathscr{M}(p)$, so there is $h \in H$ such that $Q^{*} \subseteq P_{1}^{h}$, by Sylow's theorem. Thus, $Q^{*}$ normalizes $Z^{h}$, since $Z$ is a normal subgroup of $P_{1}$. Moreover, $D$ normalizes $Q^{*}$ and $O_{p^{\prime}}(H) Z^{h}=O_{p^{\prime}}(H) Z$ is normal in $H$, so $D$ normalizes $K=O_{p^{\prime}}(H) Z^{h} Q^{*}$. However, $Z^{h} Q^{*}$ is a Sylow $p$-subgroup of $K, Q^{*}$ is $D$-invariant, and $K$ has odd order as $O_{p^{\prime}}(H)$ is of odd order by (ii) of Lemma 3. Hence, there is a $D$-invariant Sylow $p$-subgroup of $K$ containing $Q^{*}$. We may assume that it is $\left(Z^{h} Q^{*}\right)^{k}$ for some $k$ in $K$, Thus,

$$
Z^{h k}=O_{p^{\prime}}(H) Z \cap\left(Z^{h} Q^{*}\right)^{k}
$$

is both $D$-invariant and $Q^{*}$-invariant and the corollary is demonstrated.

Proof (of Proposition 1). First, $D$ and $Q^{*}$ both normalize $R_{1}$, by assumption, so $D Q^{*} \subseteq H_{1}=N\left(R_{1}\right)$. This establishes (a) of the definition of $\mathscr{M}(p)$ for $H_{1}$. Moreover, $O_{p^{\prime}}(H)$ is of odd order, by (ii) of Lemma 3, so $A_{H}(T)$ and $A_{H}(\bar{T})$ are isomorphic, where $\bar{H}=H / O_{p^{\prime}}(H)$. Similarly, since $H_{1}$ covers $H / O_{p^{\prime}}(H)$, by the Frattini argument, it follows that $A_{H_{1} \cap H}(T)$ and $A_{H}(\bar{T})$ are isomorphic. Thus, $A_{H_{1}}(T)=$ Aut $(T)$ and so $H_{1}$ satisfies (b) of Definition 1. Statement (d) is also clear. 
In fact, $Q^{*} \subseteq H_{1}$, so $C^{*} \cap O_{p^{\prime}}\left(H_{1}\right) \subseteq S(L)$ by Lemma 2 . It therefore remains to demonstrate the covering property given by (c).

However, if we set $K=H \cap H_{1}=N_{H}\left(R_{1}\right)$, then it suffices to show that $C^{*} \cap K$ covers $C^{*} \cap L / C^{*} \cap S(L)$ since $C^{*} \cap K \subseteq C^{*} \cap H_{1}$. This is a question concerning the structure of $H$, rather than of $H_{1}$, and we shall now deal with it.

First of all, since $H \in \mathscr{M}(p), C^{*} \cap H$ covers $C^{*} \cap L / C^{*} \cap S(L)$; that is,

$$
C^{*} \cap L \subseteq\left(C^{*} \cap S(L)\right)\left(C^{*} \cap H\right)
$$

Hence, it is sufficent to prove that

Indeed, then

$$
C^{*} \cap H \subseteq\left(C^{*} \cap O_{p^{\prime}}(H)\right)\left(C^{*} \cap K\right) \text {. }
$$

$$
\begin{aligned}
C^{*} \cap L & \subseteq\left(C^{*} \cap S(L)\right)\left(C^{*} \cap O_{p^{\prime}}(H)\right)\left(C^{*} \cap K\right) \\
& =\left(C^{*} \cap S(L)\right)\left(C^{*} \cap K\right),
\end{aligned}
$$

as $C^{*} \cap O_{p^{\prime}}(H) \subseteq S(L)$, since $H \in \mathscr{M}(p)$. However, this last statement is just the assertion that $C^{*} \cap K$ covers $C^{*} \cap L / C^{*} \cap S(L)$.

The analysis of the preceding section and the proof of Proposition 7.1 showed that there are two possibilities: namely, either

(1) $C^{*}=N_{C}(Q)$; or

(2) $Q=Z(P), N^{*}=N(R)$, and, by Lemma 7.10, $Q$ centralizes $R$.

The first case occurred if $p$ was constrained or if $P \cap O(N) \neq 1$ and $p$ was nonconstrained. The second case was for $p$ nonconstrained, but inverted; that is, $P \cap O(N)=1$. We shall conclude the proof of this proposition by establishing the desired factorization of $C^{*} \cap H$ in each of these cases.

First, we shall deal with case (1), so $C^{*}=N_{C}(Q)$. Suppose that $u \in C^{*} \cap H$. It follows that $R_{1}$ and $R_{1}^{u}$ are Sylow $p$-subgroups of $O_{p^{\prime}}(H) R_{1}$. Moreover, $Q$ normalizes $R_{1}$ as $Q \subseteq Q^{*}$ and $R_{1}$ is $Q^{*}$-invariant, by assumption. Therefore, $Q^{u}$ normalizes $R_{1}^{u}$. However, $u \in C^{*}=N_{C}(Q)$, so $Q=Q^{u}$. Hence $R_{1} Q$ and $R_{1}^{u} Q$ are Sylow $p$-subgroups of $O_{p^{\prime}}(H) R_{1} Q$. Similarly $T$ normalizes $R_{1}$ and $Q$ and $u \in C$, so $R_{1} Q T$ and $R_{1}^{u} Q T$ are Hall $\{2, p\}$-subgroups of $O_{p^{\prime}}(H) R_{1} Q T$ as $O_{p^{\prime}}(H)$ is of odd order by Lemma 3. Hence, $O_{p^{\prime}}(H) R_{1} Q T$ is solvable and there is $z \in O_{p^{\prime}}(H)$ such that

$$
\left(R_{1}^{u} Q T\right)^{z}=R_{1} Q T \text {. }
$$

However, $[Q T, z] \subseteq O_{p^{\prime}}(H)$ as $z \in O_{p^{\prime}}(H)$. In addition,

$$
[Q T, z] \subseteq\left\langle(Q T)^{z}, Q T\right\rangle \subseteq\left\langle\left(R_{1}^{u} Q T\right)^{z}, Q T\right\rangle=R_{1} Q T .
$$

Since $O_{p^{\prime}}(H) \cap R_{1} Q T=1$, it follows, in fact, that $z$ centralizes $Q T$. Thus $z$ centralizes both $Q$ and $T$, so certainly $z \in C^{*}$. Moreover,

$$
R_{1}^{u z}=\left(R_{1}^{u} Q T\right)^{z} \cap O_{p^{\prime}}(H) R_{1} \quad \text { and } \quad R_{1}=R_{1} Q T \cap O_{p^{\prime}}(H) R_{1} .
$$

Thus, $R_{1}^{u z}=R_{1}$ and so $u z \in N_{H}\left(R_{1}\right)=K$. Since $u$ and $z$ are elements of $C^{*}$, so is $u z$. Hence, we have

$$
u=(u z) z^{-1} \in\left(C^{*} \cap K\right)\left(C^{*} \cap O_{p^{\prime}}(H)\right)
$$


The two factors are permutable as $C^{*} \cap O_{p^{\prime}}(H)$ is a normal subgroup of $C^{*} \cap H$. Since $u$ is an arbitrary element of $C^{*} \cap H$, the desired factorization of $C^{*} \cap H$ follows in case (1) described above.

We now turn to case (2); the argument here is entirely similar. In fact, we have simply to replace $Q$ by $Q R$ throughout. To make this change, three points have to be checked. First, that $Q R \subseteq Q^{*}$. Indeed, $Q$ centralizes $R$ in case (2) and $R \subseteq R^{*}$ as $N^{*}=N(R)$ in case (2), so

$$
Q^{*}=Q C_{R^{*}}(Q) \supseteq Q C_{R}(Q)=Q R .
$$

Second, $C^{*}$ normalizes $Q R$. Indeed, in this case,

$$
C^{*}=N_{C \cap N^{*}}(Q)=N_{C}(Q) \cap N(R) \text {. }
$$

Third, $C(Q R T) \subseteq C^{*}$. Indeed,

$$
\begin{aligned}
C(Q R T) & =C(Q) \cap C(T) \cap C(R) \\
& \subseteq N_{C}(Q) \cap N(R)=C^{*} .
\end{aligned}
$$

The argument is now as follows. Again if $u \in C^{*} \cap H$, then $R_{1}$ and $R_{1}^{u}$ are Sylow $p$-subgroups of $O_{p^{\prime}}(H) R_{1}$. Also $Q R$ normalizes $R_{1}$, as $Q^{*}$ does by assumption and $Q R \subseteq Q^{*}$. Since $C^{*}$ normalizes $Q R$, this yields that $Q R=(Q R)^{u}$ normalizes $R_{1}$. Proceeding as above, since $R$ is $T$-invariant, we obtain $z \in O_{p^{\prime}}(H)$ such that

$$
\left(R_{1}^{u} Q R T\right)^{z}=R_{1} Q R T
$$

and also that $z \in C(Q R T)$. Thus, $z \in C^{*}$ and so $z \in C^{*} \cap O_{p^{\prime}}(H)$. Again,

$$
R_{1}^{u}=R_{1}^{u} Q R T \cap O_{p^{\prime}}(H) R_{1}, \quad R_{1}=R_{1} Q R T \cap O_{p^{\prime}}(H) R_{1},
$$

so $R_{1}^{u z}=R_{1}$ and hence $u z \in K$. The rest of the proof is the same and again we obtain the desired factorization of $C^{*} \cap H$. This completes the proof of the proposition.

9. Constraint for elements of $\mathscr{M}^{*}(p)$. We again preserve our assumptions on the prime $p$ and prove a constraint result for element of $\mathscr{M}(p)$. However, it turns out to be easier to work in a larger collection of $p$-local subgroups of $G$ and, moreover, there is one application to come in which we use this larger family instead of $\mathscr{M}(p)$.

The relevant family of subgroups is defined as follows:

Definition 1. We let $\mathscr{M}^{*}(p)$ be the collection of all $p$-local subgroups $K$ of $G$ satisfying the two conditions:

(a) $H$ contains $D$ and is a $D$-group with respect to $T$.

(b) $O_{p^{\prime}}(H)$ is of odd order.

The following remark gives part of the motivation for considering this collection:

LEMMA 1. Each element of $\mathscr{M}(p)$ is in $\mathscr{M}^{*}(p)$.

Proof. If $K$ is an element of $\mathscr{M}(p)$, then $D \subseteq K$ by Definition 8.1, which defines $\mathscr{M}(p)$. Moreover, Lemma 8.3 shows that $K$ is a $D$-group with respect to $T$ and that $O_{p^{\prime}}(H) \subseteq O(H)$. This proves the result. 
The main result of this section is as follows:

Proposition 1. If $K$ is an element of $\mathscr{M}^{*}(p)$, then $K$ is p-constrained within $O(K)$.

The preceding lemma and this proposition have an immediate consequence:

Corollary 1. If $K$ is an element of $\mathscr{M}(p)$, then $K$ is p-constrained within $O(K)$.

The proof of the proposition is quite long; it is broken up into a sequence of lemmas which constitute the rest of the section. We begin with a pair of lemmas which effect a reduction: the existence of a certain type of element of $\mathscr{M}^{*}(p)$ will be enough to prove the proposition.

We shall preserve the notation of the preceding two sections. Thus, $P, Q, R, R^{*}$, and so forth, are as usual.

LEMMA 2. If $K$ is an element of $\mathscr{M}^{*}(p)$, then $K$ is p-constrained within $O(K)$ if and only if $T$ normalizes, but does not centralize, a Sylow p-subgroup of $O(K)$.

Proof. Since $K \in \mathscr{M}^{*}(p)$, we have that $O_{p^{\prime}}(H) \subseteq O(H)$. In particular, $O_{p^{\prime}, p}(H)$ $\subseteq O(H)$ and so $O_{p^{\prime}, p}(O(H))=O_{p^{\prime}, p}(H)$. Moreover, there is a $T$-invariant Sylow p-subgroup $X$ of $O(H)$, so $X_{0}=O_{p^{\prime}, p}(H) \cap X$ is a $T$-invariant Sylow $p$-subgroup of $O_{p^{\prime}, p}(H)$.

First, if $K$ is $p$-constrained within $O(K)$, then $C_{T}\left(X_{0}\right)=1$, by Lemma 2.6.3. Hence, $T$ normalizes, but does not centralize $X$. On the other hand, suppose that $K$ is not $p$-constrained within $O(K)$. Again, we apply Lemma 2.6 .3 and conclude that $T$ centralizes $X_{0}$. Hence $\left[X_{0}, T\right]=1$, so

$$
\left[X_{0}, X, T\right] \subseteq\left[X_{0}, T\right]=1, \quad\left[T, X_{0}, X\right] \subseteq[1, X]=1,
$$

and consequently, by the three subgroup lemma, $\left[X, T, X_{0}\right]=1$.

Thus, $[X, T]$ is a subgroup of $O(H)$ which centralizes $O_{p^{\prime}, p}(O(H)) / O_{p^{\prime}}(O(H))$ as $X_{0}$ covers this section. Since $O(H)$ is solvable, it follows that $O(H)$ is $p$-constrained and so $[X, T] \subseteq O_{p^{\prime}, p}(H)$. Hence, $[X, T] \subseteq X \cap O_{p^{\prime}, p}(H)=X_{0}$ and so

$$
[X, T, T] \subseteq\left[X_{0}, T\right]=1 .
$$

But $X$ is a $p$-group and $T$ is a 2-group, so $T$ centralizes $X$ and the lemma is proved.

Lemma 3. Suppose that there exists an element $K$ of $\mathscr{M}^{*}(p)$ with the following two properties:

(a) $K$ is p-constrained within $O(K)$;

(b) $K$ contains a $D$-invariant Sylow p-subgroup of $C$.

Under these conditions, every element $H$ of $\mathscr{M}^{*}(p)$ is p-constrained within $O(H)$.

Proof. Let $K$ be as described. Now any two $D$-invariant Sylow $p$-subgroups of $C$ are conjugate by an element of $C(D)$, so we may assume that $P$, our fixed $D$ invariant Sylow $p$-subgroup of $C$, is contained in $K$. Let $H$ be an arbitrary element of $\mathscr{M}^{*}(p)$; we shall assume that $H$ is not $p$-constrained within $O(H)$, and shall derive a contradiction. 
The subgroup $O_{p^{\prime}, p}(H)$ is of odd order and is normalized by $D$. Hence, there is a $D$-invariant Sylow $p$-subgroup $Z$ of $O_{p^{\prime}, p}(H)$. By Lemma 2, it follows that $T$ centralizes $Z$, so $Z$ is a $D$-invariant $p$-subgroup of $C$. Hence, there is $u \in C_{O(C)}(D)$ such that $Z^{u} \subseteq P$. However, $H^{u} \in \mathscr{M}^{*}(p)$, since $u$ centralizes $D$, so we may replace $H$ by $H^{u}$ and assume that $Z \subseteq P$.

To complete the normalization of the configuration in question, we let $U$ be a $D P$-invariant Sylow $p$-subgroup of $O(K)$. Such a subgroup exists since $O(K) D P$ is solvable and has $D$ as a Sylow 2-subgroup.

Since $K$ is $p$-constrained within $O(K)$, Lemma 2 yields that $T$ does not centralize $U$. Thus, no involution of $T$ centralizes $U$. Indeed, this follows immediately from the fact that any two $T$-invariant Sylow p-subgroups of $O(K)$ are conjugate by an element of $C \cap O(K)$ and the fact that the involutions of $T$ are conjugate in $K$, inasmuch as $K$ is a $D$-group with respect to $T$.

Since $P$ is a Sylow $p$-subgroup of $C$, it follows that $P=C_{P U}(T)$. Hence by Lemma 2.8.1, since no element of $T^{\#}$ centralizes $P U$, we have $F_{i}^{\prime} \neq 1,1 \leqq i \leqq 3$, where $F=C_{U}(P)$ and $F_{i}^{\prime}$ is the standard $T$-decomposition notation for $F$. Thus $F^{*}$ $=\left\langle F_{1}^{\prime}, F_{2}^{\prime}, F_{3}^{\prime}\right\rangle$ is a noncyclic $p$-group, normalized by $T,\left[F^{*}, T\right]=F^{*}$, and $F^{*}$ centralizes $P$.

However, $H$ is a $p$-local subgroup of $G$ since $H \in \mathscr{M}^{*}(p)$ by assumption. Thus, there is a nonidentity $p$-subgroup $Z_{0}$ of $H$ such that $H=N\left(Z_{0}\right)$. Hence $Z_{0} \subseteq Z$, so that, as $Z \subseteq P$, we have $C(P) \subseteq C\left(Z_{0}\right) \subseteq H$. In particular, $F^{*} \subseteq H$. But $H$ is a $D$-group, so that $F^{*} / F^{*} \cap O(H)$ is cyclic, by Lemma 2.5 .4 . Thus, there is an index $i, 1 \leqq i \leqq 3$, such that $x_{i}$ centralizes $F^{*} / F^{*} \cap O(H)$. Hence, $F_{j}^{\prime} \subseteq O(H)$ if $j \neq i, 1 \leqq j \leqq 3$. In particular, $F_{0}=\left\langle F_{j}^{\prime}\right\rangle$ for $j \neq i$ is a nonidentity $T$-invariant $p$-subgroup of $O(H)$ with $\left[F_{0}, T\right]=F_{0}$. It follows that $T$ normalizes but does not centralize some Sylow $p$-subgroup of $O(H)$; this contradicts Lemma 2 and completes the proof of this lemma.

LEMMA 4. If $p$ is not constrained, then $N(Q)$ is p-constrained within $O(N(Q))$.

This will establish the proposition in the case that $p$ is not constrained. Indeed, $N(Q) \in \mathscr{M}(p)$, by Definition 8.1 and Lemma 8.1. Hence, $N(Q) \in \mathscr{M}^{*}(p)$ by Lemma 1 , $N(Q)$ is $p$-constrained within $O(N(Q))$, and $N(Q)$ contains the $D$-invariant Sylow $p$-subgroup $P$ of $C$ since $Q$ is a characteristic subgroup of $P$. Thus Lemma 3 will imply the proposition in this case.

The proof of this lemma is fairly short.

Proof. By Lemma 2, it suffices to show that some element $t$ of $T^{\#}$ normalizes, but does not centralize, a $T$-invariant $p$-subgroup of $O(N(Q))$ as such a subgroup is contained in a $T$-invariant Sylow $p$-subgroup of $O(N(Q))$.

To prove this, observe first that $p$ is exceptional by assumption and so a $T$ invariant Sylow p-subgroup $U$ of $O(N(R))$ contains $R$ properly. Moreover, $N(R)$ covers $N / O(N)$ and contains both $D$ and $R$, so $N(R)$ is in $\mathscr{L}(p)$. The proof of Proposition 6.1 now shows that $N(R)$ is $p$-constrained within $O(N(R))$. As above, 
we may choose $U$ to be $D P$-invariant; indeed, $P$ normalizes $R$ by our definition of $R$ in $\S 7$ and $O(N(R)) P D$ is solvable. The constraint of $N(R)$ implies, by Lemma 2.6.2, that $x$ does not centralize $U$. Thus, in the $T$-decomposition notation, $U_{2}^{\prime} \neq 1$ or $U_{3}^{\prime} \neq 1$. But $d \in D$ interchanges $x_{2}$ and $x_{3}$, so $U_{2}^{\prime} \neq 1$ and $U_{3}^{\prime} \neq 1$ since $U$ is $D$ invariant. Since $P$ is a Sylow $p$-subgroup of $C$, we have $P=C_{P U}(T)$, so Lemma 2.8.1 forces $F_{i}^{\prime} \neq 1, i=2,3$, where $F=C_{U}(P)$. But $Q \subseteq P$, so $F_{0}=\left\langle F_{2}^{\prime}, F_{3}^{\prime}\right\rangle \subseteq N(Q)$. Since $N(Q)$ is in $\mathscr{M}(p)$, it is a $D$-group with respect to $T$ by Lemma 8.3, so $F_{0} / F_{0} \cap O(N(Q))$ is cyclic by Lemma 2.5.4. But $F_{0}$ is not cyclic as $F_{2}^{\prime} \neq 1$ and $F_{3}^{\prime} \neq 1$ and we conclude that $x$ does not centralize $F_{0} \cap O(N(Q))$. Since $F_{0} \cap O(N(Q))$ is $T$-invariant, the lemma is proved.

Thus we shall henceforth assume that $p$ is constrained. In particular, by Lemma 2.1, $C_{N}(R)$ lies in $S(N)$. Moreover, the argument following the statement of Lemma 4 shows that we may assume that $N(Q)$ is not $p$-constrained within $O(N(Q))$. We set $H=N(Q)$ and preserve that notation for the remainder of this section.

We begin by describing $H$ in some detail. We shall then return to the demonstration that there is an element of $\mathscr{M}^{*}(p)$ satisfying the hypotheses of Lemma 3.

LEMMA 5. Under the above hypotheses, we have

(i) $H$ is of linear type of characteristic power exceeding three. In particular, $G$ has quasi-dihedral Sylow 2-subgroups.

(ii) $P \cap O(H)$ is a Sylow p-subgroup of $O(H)$.

(iii) $C_{H}(P)$ is a $D$-group with respect to $T$.

Proof. (ii) Since $H$ is not $p$-constrained within $O(H)$, Lemma 2 implies that $T$ centralizes each Sylow $p$-subgroup of $O(H)$ that it normalizes. Moreover, $O(H) P T$ is a solvable subgroup of $H$, so there is a $P T$-invariant Sylow $p$-subgroup of $O(H)$. But $P$ is a Sylow $p$-subgroup of $C$ and $O(H)$ is normal in $O(H) P T$, so (ii) is proved.

(i) Observe that if $G$ has wreathed Sylow 2-subgroups, $H$ is necessarily of linear type of characteristic power three by Proposition 2.3.4 as $D \subseteq H$, so the first assertion of (i) will imply the second.

We shall assume that $H$ is not as described and derive a contradiction. Since $D \subseteq H$, it follows from Proposition 2.3.4 that $H / O(H)$ is isomorphic with $P G L_{n}(2,3)$ for some $n \geqq 1$, or with $A_{7}$.

First, we note that no element $u$ of $D-Z(D)$ centralizes $Q R \cap H=Q(R \cap H)$ $=Q N_{R}(Q)$. Indeed, if $u$ does not centralize $Q$, this is so. In any case $u$ does not centralize $R$ as $C_{N}(R) \subseteq S(N)$ and $D \cap S(N)=Z(D)$. Hence, if $u$ centralizes $Q$, then $u$ does not centralize $C_{R}(Q)$ by Theorem 5.3.4 of [19] and our assertion is valid.

Assume now that $\bar{H}=H / O(H)$ is isomorphic with $P G L_{n}(2,3)$ for some $n$. But $Q R \cap H$ is a $T$-invariant subgroup of $H$, so $\overline{Q R \cap H}$ is a $\bar{T}$-invariant subgroup of odd order in $\bar{H}$. Hence $\overline{Q R \cap H}=1$ by Lemma 2.5 .9 and so $Q R \cap H \subseteq O(H)$. However, it follows from the preceding paragraph that $T$ does not centralize $Q R \cap H$ inasmuch as $T \subset D$ and $T \nsubseteq Z(D)$. Thus $Q R \cap H$ is a $p$-subgroup of $O(H)$ normalized but not centralized by $T$. This contradicts our assumption that $H$ is not 
p-constrained within $O(H)$, in view of Lemma 2 . Hence, we may assume that $\bar{H}$ is isomorphic within $A_{7}$, in which case $D$ is dihedral of order eight and $G$ has quasidihedral Sylow 2-subgroups.

By an above paragraph, it is sufficient to prove that some four subgroup of $D$ centralizes $Q R \cap H$. However, $D$ is a Sylow 2-subgroup of $H$ since $|D|=8$ and $|\bar{H}|=2520$. Moreover, any subgroup of odd order in $A_{7}$ that is normalized by a Sylow 2-subgroup is centralized by a four subgroup of that Sylow subgroup by Lemma 2.5.9. Thus $\overline{Q R \cap H}$ is centralized by a four subgroup of $\bar{D}$. Hence, we need only prove that $D$ centralizes $Q R \cap O(H)$ to conclude that some four subgroup of $D$ centralizes $Q R \cap H$.

Thus, it suffices to show that $D$ centralizes any Sylow p-subgroup $U$ of $O(H)$ that it normalizes. However, $H=O(H) N_{H}(U)$ by the Frattini argument. Thus, $O(H) C_{H}(U)$ is a normal subgroup of $H$ containing $O(H)$. Moreover, it contains $T$, by Lemma 2, as $H$ is not $p$-constrained within $O(H)$. Hence, $O(H) C_{H}(U)=H$ as $H / O(H)$ is simple and so $A_{H}(U)$ is of odd order. Thus, $C_{H}(U) \supseteq D$ as $D \subseteq N_{H}(U)$ and this part of the lemma is proved.

(iii) We set $U=P \cap O(H)$ so $U$ is a Sylow $p$-subgroup of $O(H)$, by (ii). Hence, $H=O(H) N_{H}(U)$ by the Frattini argument. Thus, if we set $K=N_{H}(U)$ then $K$ is also a $D$-group with respect to $T$, contains $P$ and is of the same characteristic power $r$ as $H$. In particular, $K / O(K)$ contains a unique minimal normal subgroup, isomorphic to $P S L(2, r)$, which contains the image of $T$. Moreover, $O(K) \subseteq O(H)$ so that $U$ is a Sylow $p$-subgroup of $O(K)$. It will clearly suffice to show that $C_{K}(P)$ is a $D$-group with respect to $T$.

We now set $\bar{K}=K / O_{p^{\prime}}(O(K))$, so that we need only prove that $C_{R}(\bar{P})$ is a $D$-group with respect to $\bar{T}$ as $C_{K}(P)$ maps onto $\bar{K}$. We have $\bar{U}=O_{p}(\bar{K})$ is a Sylow $p$-subgroup of $O(\bar{K})=\overline{O(K)}$. Moreover, if $K_{1}=C_{K}(U)$, then $\bar{K}_{1}=C_{R}(\bar{U})$ and $\bar{T} \subseteq \bar{K}_{1}$ as $T$ centralizes $P \supseteq U$. Thus, $\bar{K}_{1}$ covers the unique minimal normal subgroup of $\bar{K} / O(\bar{K})$ isomorphic with $\operatorname{PSL}(2, r)$. Moreover, $O\left(\bar{K}_{1}\right) \subseteq O(\bar{K})$ now follows, so $\bar{K}_{1}$ is also a $D$-group with respect to $\bar{T}$ of characteristic power $r$. If $\bar{U}=\bar{P}$, then (iii) follows, so we may assume that $\bar{U} \subset \bar{P}$.

In particular, this implies that $r \neq 9$. Indeed, if $r=9$, then the image of $\bar{T}$ in $\bar{K} / O(\bar{K})$ is self-centralizing, by Lemma 2.5.7, part (iii), and Proposition 2.3.4. However, $\bar{U} \subset \bar{P}$ and $\bar{U}$ is a Sylow $p$-subgroup of $O(\bar{K})$, so the image $\bar{P}$ in $\bar{K} / O(\bar{K})$ is nontrivial. This contradiction forces $r \neq 9$.

The definition of $\bar{K}$ forces $O_{p^{\prime}}(O(\bar{K}))=1$. Thus, as $O(\bar{K})$ is $p$-constrained, being of odd order, and $\bar{U}=O_{p}(\bar{K})$, we have $C_{O(K)}(\bar{U})=Z(\bar{U})$. Moreover, $O\left(\bar{K}_{1}\right) \subseteq O(\bar{K})$, so $O\left(\bar{K}_{1}\right)=Z(\bar{U})$. Hence, $O\left(\bar{K}_{1}\right)$ is central in $\bar{K}_{1}$ as $K_{1}=C_{K}(U)$.

Hence, the structure of $\bar{K}_{1}$ enunciated above, the fact that $r \neq 9$, and Lemma 2.5.6 yield that $\bar{K}_{1}$ has a normal subgroup which is the direct product of $Z(\bar{U})$ and a group $\bar{L}_{0}$ containing $\bar{T}$ and isomorphic with $\operatorname{PSL}(2, r)$. Moreover, $\bar{L}_{0}$ is a characteristic subgroup of $\bar{L}_{0} Z(\bar{U})$, being the derived group of this product. Thus $\bar{P}$ normalizes $\bar{L}_{0}$. 
Thus, $X=\bar{P} / C_{\bar{P}}\left(\bar{L}_{0}\right)$ is a group of automorphisms of $\bar{L}_{0}$ centralizing $\bar{T}$. We form the semidirect product $\bar{L}_{0} X$; it suffices to show that $C_{L_{0}}(X)$ is a $D$-group with respect to $\bar{T}$. However, $C_{L_{0}}(\bar{T})=\bar{T}$, by Lemma 2.5.7, so $C_{L_{0} X}(\bar{T})=\bar{T} X$. But $O\left(\bar{L}_{0} X\right)$ centralizes $\bar{L}_{0}$ which contains $\bar{T}$, so $O\left(\bar{L}_{0} X\right)=1$. Hence, $\bar{L}_{0} X$ is a $D$-group with respect to $\bar{T}$ and $O\left(\bar{L}_{0} X\right)=1$.

Since $C_{L_{0} X}(\bar{T})=\bar{T} X$, Lemma 2.5.7 and Lemma 2.5.8 yield the desired conclusion: $C_{L_{0}}(X)$ is a $D$-group with respect to $\bar{T}$. Indeed, $X=E$, in the notation of those results. This proves the third assertion and establishes the lemma in all its parts.

The lemma shows, in particular, that the proposition is valid when $G$ has wreathed Sylow 2-subgroups. Thus it remains to establish the proposition in the case that $p$ is constrained and $G$ has quasi-dihedral Sylow 2-subgroups. This is the most difficult case.

We shall now choose and fix some more subgroups and notation. We note, first of all, that in the present case $D$ is dihedral of order eight, $Z(D)=\langle x\rangle$, and $y=d$. We set $K=N(R)$. The arguments of the proof of Lemma 4 show that $K$ is an element of $\mathscr{L}(p)$, so as usual, by Proposition $6.1, K$ is a $Q$-group of characteristic power $q$ and is $p$-constrained within $S(K)$. We also have the following obvious facts.

LEMma 6. The centralizer in $K$ of $R$ is contained in $S(K)$ and $S(K)=O(K) Z(D)$.

Proof. Indeed, $K$ is in $\mathscr{L}(p)$, so $K=O(K)(K \cap N)$, by Lemma 1.1. But we are assuming $C_{N}(R) \subseteq S(N)$, so $C_{K \cap N}(R) \subseteq S(K \cap N) \subseteq S(K)$ and $C_{K}(R) \subseteq O(K) S(K)$ $=S(K)$. Moreover, $S(K)=O(K) Z(D)$ by Lemma 2.4.14.

We now choose and fix two more subgroups. Let $U$ be a normal subgroup of $K$ contained in $R$ with $C_{K}(U) \subseteq S(K)$ and minimal subject to these conditions. Such a subgroup exists, by the previous lemma. If $U$ is abelian, we let $V$ be a normal subgroup of $K$ contained in $U$ such that $U / V$ is a chief factor of $K$. Such a subgroup exists since $U \neq 1$ inasmuch as $S(K) \subset K$. Finally, if $U$ is nonabelian, we let $V=Z(U)$.

We continue the proof by analyzing $U$ and $V$ in some detail in the next lemma.

LEMMA 7. If $U$ and $V$ are as described, then

(i) $U$ is of exponent $p$ and of class at most two;

(ii) $P \cap U$ and $P \cap V$ are Sylow p-subgroups of $C \cap U$ and $C \cap V$, respectively;

(iii) $P \cap U \neq 1$ and $P \cap V=1$.

Proof. (i) We let $U_{1}$ be a critical subgroup of $U$, in accordance with Theorem 5.3.11 of [19]. Thus, $U_{1}$ has class at most two. Moreover, $y$ does not centralize $U$ as $y \notin S(K)$, so $y$ does not centralize $U_{1}$. But $C_{K}\left(U_{1}\right) S(K)$ is a normal subgroup of $K$ and $K / S(K)$ has a unique minimal normal subgroup which contains the image of $y$. Hence, $C_{K}\left(U_{1}\right) \subseteq S(K)$ and $U_{1}=U$, by our minimal choice of $U$. Similarly, we can apply the same argument to $\Omega_{1}(U)$ as $y$ does not centralize $\Omega_{1}(U)$, by Theorem 5.3.10 of [19]. Thus, $U=\Omega_{1}(U)$. Since $p$ is odd, it follows that $U$ has exponent $p$ and so (i) is proved in full. 
(ii) The group $R$ is $P$-invariant by choice, so $P \subseteq K$. Thus $P$ normalizes $U$, as $U$ is normal in $K$. It follows that $P$ normalizes $C \cap U$ and $P$ is a $p$-subgroup of $(C \cap U) P$. However, $P$ is a Sylow $p$-subgroup of $C$ and $(C \cap U) P \subseteq C$, so $P$ is a Sylow $p$-subgroup of $(C \cap U) P$. However, $C \cap U$ is a normal subgroup of $(C \cap U) P$, so $P \cap U=C \cap U \cap P$ is a Sylow $p$-subgroup of $C \cap U$. Similarly, $P \cap V$ is a Sylow $p$-subgroup of $C \cap V$.

(iii) We assume that $P \cap U=1$ and derive a contradiction. Since $U$ is a $p$ subgroup, it follows that $C \cap U=1$. Hence, $x_{2}$ inverts $U$ as $x$ centralizes $R \subseteq N$ and $U \subseteq R$. If $S$ is a Sylow 2-subgroup of $K$ containing $D$, then $S$ is quasi-dihedral, since $K \in \mathscr{L}(p)$ forces $K$ to cover $L / O(N)$, by Lemma 1.1. Hence, $y=x_{2} x_{2}^{s}$ for some $s$ in $S$. But then $x_{2}^{s}$ also inverts $U$, so $y$ centralizes $U$, which is not the case. This contradiction forces us to accept that $P \cap U \neq 1$.

Finally, suppose that $P \cap V \neq 1$; again we shall derive a contradiction. Since $P \cap V \neq 1, N(P \cap V)$ is a $p$-local subgroup and

$$
N(P \cap V) \supseteq C(P \cap V) \supseteq C(P) \supseteq C_{H}(P) .
$$

But $C_{H}(P)$ is a $D$-group with respect to $T$, by Lemma 5 , so $N(P \cap V)$ is a $D$-group or $Q D$-group by Proposition 1.1. However, the minimal nature of $U$ yields that $C_{K}(V) \nsubseteq S(K)$, so $C_{K}(V)$ covers the unique minimal normal subgroup of $K / S(K)$. Hence, $C_{K}(V)$ is a $Q$-group of characteristic power $q$. But $C_{K}(V) \subseteq N(P \cap V)$, so $N(P \cap V)$ is a $Q D$-group of characteristic power $q$, contrary to Proposition 1.1.

LEMMA 8. If we set $H_{1}=N(P \cap U)$, then

(i) $H_{1}$ is an element of $\mathscr{M}^{*}(p)$.

(ii) $H_{1}$ contains the D-invariant Sylow p-subgroup $P$ of $C$.

Proof. Certainly the second assertion is valid. Indeed, $P \cap U$ is a normal subgroup of $P$ as $U$ is normal in $K$ and $P \subseteq K$ since $R$ is $P$-invariant by its very choice.

Thus, we must prove (i). But $H_{1}$ is a $p$-local subgroup since $P \cap U \neq 1$, by the previous result. Moreover $D$ normalizes $P$ and also normalizes $U$ since $D \subseteq K$. Hence, $D \subseteq H_{1}$. It remains to show that $H_{1}$ is a $D$-group with respect to $T$ and that $O_{p^{\prime}}(H)$ has odd order.

However, $C_{H}(P)$ is a $D$-group with respect to $T$, by Lemma 5 . Thus, the automizer in $C_{H}(P)$ of $T$ has order divisible by three. But $C_{H}(P) \subseteq C(P \cap U) \subseteq H_{1}$, so $A_{H_{1}}(T)$ has order divisible by three. Since $D \subseteq H_{1}$, it follows that $A_{H_{1}}(T)$ is Aut $(T)$. These conditions imply that $H_{1}$ is a $D$-group with respect to $T$ or is a $Q D$-group by Proposition 1.1. Hence, we shall assume that $H_{1}$ is a $Q D$-group and derive a contradiction.

However, $P$ is normal in $C$ and $U$ is normal in $K$, so $P \cap U$ is normal in $C \cap K$ and $C \cap K \subseteq H_{1}$. Since $K \in \mathscr{L}(p)$, Lemma 1.1 implies that $H_{1}$ covers $C \cap L / C \cap O(N)$. Since $T \subset H_{1}$ and $H_{1}$ is a local subgroup of $G$, it follows from Proposition 1.1 that $H_{1}$ is not a $Q D$-group. 
We now prove that $O_{p^{\prime}}\left(H_{1}\right) \subseteq O\left(H_{1}\right)$. We know that $T$ does not centralize $U$ as $C_{K}(U) \subseteq S(K)=\langle O(K), x\rangle$ and $T \subseteq K$. Hence, by Lemma 2.8.1, $T$ does not centralize $N_{U}\left(C_{U}(T)\right)$, which is $N_{U}(C \cap U)$. But $C \cap U=P \cap U$, as we pointed out above, so $T$ does not centralize $N_{U}(P \cap U)$. Let $S$ be a Sylow 2-subgroup of $H_{1}$ containing $D$, so that $S$ is either dihedral or quasi-dihedral and $Z(S)=\langle x\rangle$. If $O_{p^{\prime}}\left(H_{1}\right) \nsubseteq O\left(H_{1}\right)$, then $S \cap O_{p^{\prime}}\left(H_{1}\right) \neq 1$ and so $Z(S) \cap O_{p^{\prime}}(H) \neq 1$ as $S \cap O_{p^{\prime}}\left(H_{1}\right)$ is normal in $S$. Thus $x \in O_{p^{\prime}}\left(H_{1}\right)$. But as $H_{1}$ is a $D$-group with respect to $T$, all the involutions of $T$ are conjugate in $H_{1}$. Hence, if $O_{p^{\prime}}\left(H_{1}\right) \nsubseteq O\left(H_{1}\right)$, then $T \subseteq O_{p^{\prime}}\left(H_{1}\right)$. But then

$$
\left[N_{U}(P \cap U), T\right] \subseteq U \cap O_{p^{\prime}}\left(H_{1}\right)=1,
$$

which is a contradiction. The lemma is proved.

LEMMA 9. If $H_{1}$ is as above, then $H_{1}$ is p-constrained within $O\left(H_{1}\right)$.

Once this result is established, it will follow from Lemma 8 that if we set $K=H_{1}$, then $K$ satisfies all the hypotheses of Lemma 3. The conclusion of Lemma 3 will then show that Proposition 1 is valid. Hence, to prove Lemma 9, we shall assume for the remainder of the section that $H_{1}$ is not p-constrained within $O\left(H_{1}\right)$ and derive a contradiction. We shall, as usual, proceed by a sequence of lemmas. Our assumption implies, by Lemma 2, that $T$ centralizes every $p$-subgroup of $O\left(H_{1}\right)$ that it normalizes. We shall use this fact without reference.

LEMMA 10. One of the following holds:

(i) $U$ is elementary abelian, $V=1$, and $|U: U \cap P|=p$;

(ii) $U$ is extra-special, $V=Z(U)$ has order $p, U \cap P$ is abelian, and

$$
N_{U}(U \cap P)=(U \cap P) V .
$$

Proof. First, we claim that $V$ is cyclic. There were two possibilities in our definition of $V$, but in either case $V \subseteq Z(U)$, so $V \subseteq C(P \cap U) \subseteq H_{1}$. Moreover, $P \cap V=1$, by Lemma 7 , so $[V, T]=V$. But $V$ is abelian, so if $V_{0}=[V, T] \cap O\left(H_{1}\right)$, then $\left[V_{0}, T\right]=1$ and $V_{0}=1$, by our assumptions on $H_{1}$. Hence $V$ is isomorphic to $V O\left(H_{1}\right) / O\left(H_{1}\right)$, which is cyclic, by Lemma 2.5.4.

Suppose that $U$ is nonabelian; we shall prove that (ii) holds. In fact, $U$ is of exponent $p$ and class two exactly, by Lemma 7. Hence, $V=Z(U)$ is not the identity and is cyclic, so $V$ has order $p$. Thus $U$ is extra-special. Moreover, $C \cap V=1$, again by Lemma 7, so $[V, T]=V$ and $U \cap P$ intersects $V$ trivially; consequently, $U \cap P$ is abelian. Therefore, it remains to show that $N_{U}(U \cap P)=(U \cap P) V$. However, $U \subseteq R \subseteq N$, so $x$ centralizes $U$ and $U \cap P=C_{U}\left(x_{2}\right)$. But then $x_{2}$ inverts $Z(U)=V$, and $U /(U \cap P) V$. Thus, if the desired conclusion is false, then $(U \cap P) V$ is not a maximal abelian subgroup of $U$. However, in the latter case there are elements $u_{1}, u_{2}$ of $U$ inverted by $x_{2}$ which do not commute. But then $V=\left\langle\left[u_{1}, u_{2}\right]\right\rangle$, so $x_{2}$ centralizes $V$, a contradiction.

Finally, assume that $U$ is abelian, so $U \subseteq H_{1}$ as $H_{1}=N(P \cap V)$ and $U$ is elementary, by Lemma 7. Our choice of $U$ shows that $C_{K}(U) \subseteq S(K)$. Since $T \cap S(K)=\langle x\rangle$, 
it follows that $x_{2}$ does not centralize $U$ and hence $U \cap P \subset U$. Moreover, since $H_{1}$ is a $D$-group and $U$ is elementary, another application of Lemma 2.5.4 shows that $U / U \cap P$ is cyclic, so $|U: U \cap P|=p$. It remains to show that $V=1$; if this is not so, then $V$ has order $p$ and it follows that $U=(U \cap P) V$. In particular, $x_{2}$ centralizes $U / V$, so the normal closure of $x_{2}$ in $D$ does as well. Hence, $y$ centralizes $U / V$. But $C_{K}(V) \nsubseteq S(K)$, by our choice of $U$ and $V$, so $y$ centralizes $V$. Thus, $y$ centralizes $U$, which contradicts the containment $C_{K}(U) \subseteq S(K)$. This proves the lemma.

LEMMA 11. If $P_{0}$ is a subgroup of order $p$ in $P \cap U$ and we set $H_{0}=N\left(P_{0}\right)$, then the following conditions hold:

(i) $A_{H_{0}}(T)$ has order divisible by three.

(ii) $(P \cap U) V T \subseteq H_{0}$.

(iii) $T$ centralizes every p-subgroup of $O\left(H_{0}\right)$ that it normalizes.

The first assertion implies that $H_{0}$ is a $D$-group with respect to $T$ or a $Q D$-group by Proposition 1.1. We do not eliminate the latter possibility since we do not know that $H_{0}$ covers $C \cap L / C \cap O(N)$. Note that $P_{0}$ exists as $P \cap U \neq 1$ by Lemma 7 .

Proof. (ii) The previous lemma shows that $P \cap U$ is abelian and $V$ centralizes it, so $P \cap U$ and $V$ are contained in $H_{0}$. Furthermore, $T$ centralizes $P \cap U$, so $T \subseteq H_{0}$.

(i) By Lemma $5, C_{H}(P)$ is a $D$-group with respect to $T$. Thus, the automizer in $C_{H}(P)$ of $T$ has order divisible by three. However, $C_{H}(P) \subseteq H_{0}$ as $P_{0} \subseteq P$.

(iii) Suppose that there is a $p$-subgroup of $O\left(H_{0}\right)$ normalized but not centralized by $T$. Hence, condition (i) implies that no involution of $T$ centralizes any $T$ invariant Sylow $p$-subgroup of $O\left(H_{0}\right)$. Let $X$ be a $(P \cap U) T$-invariant Sylow $p$-subgroup of $O\left(H_{0}\right)$; it exists since $O\left(H_{0}\right)(P \cap U) T$ is solvable. In particular, if $Y=N_{X}(P \cap U)$, then $Y_{i}^{\prime} \neq 1,1 \leqq i \leqq 3$, by Lemma 2.8.1. As usual, $Y_{i}^{\prime} \subseteq O\left(H_{1}\right)$ for at least two values of $i$, by Lemma 2.5 .4 , and this is a contradiction.

LEMMA 12. The subgroup $U$ is abelian.

Proof. We shall assume $U$ is not abelian and derive a contradiction. In particular, $U$ has the structure given in part (ii) of Lemma 10 . We set $\bar{K}=K / C_{K}(U)$, so $\bar{K}$ is a $D$-group as $x$ centralizes $U \subseteq R$ and $C_{K}(U) \subseteq S(K)$. Thus, $\bar{K}$ is a $D$-group faithfully represented on $\bar{U}$, which is an elementary abelian group isomorphic to $U / V$. Hence, if $|\bar{U}|=p^{h}$, then $\bar{K}$ is isomorphic with a subgroup of $G L(h, p)$. Since $S L(2, p)$ is a $Q$-group, no subgroup of $S L(2, p)$ is a $D$-group by Lemma 2.4.17. Since $G L(2, p) / S L(2, p)$ is cyclic, the same is clearly true of $G L(2, p)$ and consequently $h>2$. Thus, $|U|=p^{2 m+1}$ with $m \geqq 2$.

This implies that $C_{U}\left(P_{0}\right)$ contains a subgroup $U_{0}$ with the following three properties:

(a) $U_{0}$ is extra-special;

(b) $V=Z\left(U_{0}\right)$;

(c) $U_{0}$ is $T$-invariant and $T$ does not centralize $U_{0} / V$. 
Indeed, $P \cap U$ is abelian and $C_{U}(P \cap U)=(P \cap U) V$. Since $m \geqq 2$ and $\left|P_{0}\right|=p$, $C_{U}\left(P_{0}\right)$ is nonabelian and so $C_{U}\left(P_{0}\right) \supset(P \cap U) V$. Thus $T$ does not centralize $C_{U}\left(P_{0}\right) / V$ and, as $x$ centralizes $C_{U}\left(P_{0}\right)$, we have that $C_{U}\left(P_{0}\right)$ contains a $T$-invariant subgroup $F$ of order $p$ inverted by $x_{2}$ with $F \neq V$. Then $F$ does not centralize $P \cap U$ and so there exists a subgroup $P_{1}$ of order $p$ in $P \cap U$ such that $\left[F, P_{1}\right] \neq 1$. Setting $U_{0}=\left\langle F, P_{1}\right\rangle$, it is immediate that $U_{0}$ has the required properties.

Since $T$ does not centralize $V$, we have $V \nsubseteq O\left(H_{0}\right)$ by the previous lemma. We shall derive a contradiction by showing that $V \subseteq O\left(H_{0}\right)$.

First, we note that $U_{0} \subseteq N \cap H_{0}$. Indeed, $U_{0} \subseteq U \subseteq R$ and $U_{0} \subseteq C_{U}\left(P_{0}\right) \subseteq H_{0}$. Moreover, the first assertion of the previous lemma yields that $H_{0}$ is a $D$-group with respect to $T$ or is a $Q D$-group. We shall show that $V \subseteq O\left(H_{0}\right)$ in either case.

Suppose first that $H_{0}$ is a $D$-group and set $\bar{H}_{0}=H_{0} / O\left(H_{0}\right)$. By Proposition 2.3.4, either $\bar{H}_{0}$ is isomorphic to $A_{7}$ or $\bar{H}_{0}=\bar{L} \bar{E}$, where $\bar{L}$ is isomorphic to $\operatorname{PSL}(2, t)$, $P G L(2, t)$, or $P G L^{*}(2, t)$ for some odd $t, \bar{L}$ is normal in $\bar{H}_{0}, \bar{E}$ is cyclic of odd order, $\bar{L} \cap \bar{E}=1$, and $\bar{E}$ centralizes $\bar{T}$. Moreover, in the latter case, if $\bar{L}_{0}$ is the normal subgroup of $\bar{L}$ isomorphic to $P S L(2, t)$, then as usual $\bar{L}_{0} \bar{T}$ is isomorphic to $\operatorname{PSL}(2, t)$ or $P G L(2, t)$. Now $U_{0}$ is a $T$-invariant extra-special $p$-subgroup of $H_{0} \cap N=C_{H_{v}}(x)$ and $V=U_{0}^{\prime}$. Hence if $\bar{H}$ is isomorphic to $A_{7}$, then $\bar{V}=\bar{U}_{0}^{\prime}=1$ as the Sylow subgroups of $A_{7}$ are abelian for all odd primes. On the other hand, if $\bar{H}$ is of the form $\bar{L} \bar{E}$, then $C_{L_{0}}(\bar{x})$ is dihedral by Lemma 2.5.7. Since $C_{H_{0}}(\bar{x})=C_{E}(\bar{x}) \bar{E}$ and $\bar{L}_{0}$ has index at most two in $\bar{L}$, it follows that the Sylow subgroups of $C_{B_{0}}(\bar{x})$ are either metacyclic or abelian on at most two generators for all odd primes. In either case, we see that $C_{H_{0}}(\bar{x})$ does not contain an extra-special $p$-group. This forces $\bar{V}=1$, since otherwise $\bar{U}_{0}$ would be an extra-special $p$-subgroup of $C_{H_{0}}(\bar{x})$. Thus $V \subseteq O\left(H_{0}\right)$ in this case as well.

Suppose, on the other hand, that $H_{0}$ is a $Q D$-group. We have $V \subseteq O(N)$ as $V \subseteq U \subseteq R \subseteq O(N)$. Therefore, using Proposition 1.1, we have

$$
\begin{aligned}
V=[V, T] & \subseteq\left[O(N) \cap H_{0}, T\right] \\
& \subseteq\left[O\left(N \cap H_{0}\right), T\right] \\
& \subseteq O\left(H_{0}\right) .
\end{aligned}
$$

This proves our assertion.

We now conclude the section by proving just the opposite, namely:

LEMMA 13. The subgroup $U$ is not abelian.

Proof. This time we assume that $U$ is abelian and derive a contradiction. Hence, $U$ is elementary abelian, $V=1$, and $|U: U \cap P|=p$, by Lemma 10 . We again set $\bar{K}=K / C_{K}(U)$. Now $K$ is a $Q$-group of characteristic power $q$ as $K$ covers $N / O(N)$. Since $x$ centralizes $U$ and $C_{K}(U) \subseteq S(K)=\langle O(K), x\rangle$, it follows from Lemma 2.4.15 that $\bar{K}$ is a $D$-group of linear type of the same characteristic power $q$. Moreover, $x_{2} \notin K^{\prime}$ by Lemma 2.4.1, applied to $K / O(K)$, and hence $\bar{x}_{2} \notin \bar{K}^{\prime}$. Since $q>5$ and since $C_{U}\left(\bar{x}_{2}\right)=U \cap P$, all the hypotheses of Lemma 2.5 .10 are satisfied and we 
conclude that there is an odd prime $r$ other than $p$ and a nonidentity $r$-element $\bar{v}$ in $\bar{K}$ inverted by $\bar{x}_{2}$ with $C_{P \cap U}(\bar{v}) \neq 1$. Since $\bar{x}=1$ and $\langle x\rangle$ is a Sylow 2-subgroup of $C_{K}(U)$, it follows that there is an $r$-element $v$ of $K$ with image $\bar{v}$ in $\bar{K}$ such that $x$ centralizes $v$ and $x_{2}$ inverts $v$.

We can therefore take $P_{0}$ to lie in $C_{P \cap U}(v)$ and do so. Hence $\langle U, v\rangle$ is a subgroup of $H_{0}$ of odd order centralized by $x$.

We now set $U_{1}=[U, v]$; we claim that $U_{1} \subseteq O\left(H_{0}\right)$. Since $v$ does not centralize $U$ by choice, $U_{1} \neq 1$ and $U_{1}=\left[U_{1}, v\right]$ as $U$ is a $p$-group, $v$ is an $r$-element, and $p, r$ are distinct primes. Moreover, $U_{1}\langle v\rangle$ is $T$-invariant. Again there are two possible structures for $H_{0}$. If $H_{0}$ is a $D$-group, we again set $\bar{H}_{0}=H_{0} / O\left(H_{0}\right)$, so that either $\bar{H}_{0}$ is isomorphic to $A_{7}$ or $\bar{H}_{0}=\bar{L} \bar{E}$, where $\bar{L}$ and $\bar{E}$ are as in the preceding lemma. Since $A_{7}$ possesses no nonabelian subgroups of odd order invariant under a four group, $\bar{U}_{1}=1$ in the first case and so $U_{1} \subseteq O\left(H_{0}\right)$. On the other hand, in the second case, $\bar{v} \in \bar{L}$ as $\bar{x}_{2}$ inverts $\bar{v}$ and $\bar{x}_{2} \in \bar{L}$. For the same reason, $\bar{v} \in \bar{L}_{0}$, where again $\bar{L}_{0}$ denotes the normal subgroup of $\bar{H}_{0}$ isomorphic to $\operatorname{PSL}(2, t)$ and of index at most two in $\bar{L}$. But then also $\bar{U}_{1} \subseteq \bar{L}_{0}$. Furthermore, $C_{L_{0}}(\bar{x})$ is again dihedral and so contains no nonabelian subgroups of odd order. Since $\left\langle\bar{U}_{1}, \bar{v}\right\rangle \subseteq C_{L_{0}}(\bar{x})$, we conclude that $\bar{U}_{1}=\left[\bar{U}_{1}, \bar{v}\right]=1$ and so $U_{1} \subseteq O\left(H_{0}\right)$.

On the other hand, if $H_{0}$ is a $Q D$-group, then we have, since $U \subseteq O(N)$, that

again by Proposition 1.1.

$$
\begin{aligned}
U_{1}=[U, v] & \subseteq\left[O(N) \cap H_{0}, N \cap H_{0}\right] \\
& \subseteq\left[O\left(N \cap H_{0}\right), N \cap H_{0}\right] \\
& \subseteq O\left(H_{0}\right),
\end{aligned}
$$

But $\left[U_{1}, T\right]$ is a $p$-subgroup not centralized by $T$. Indeed, if $T$ centralizes $U_{1}$, then $v$ does also since $v$ is inverted by $x_{2}$, contrary to the fact that $U_{1}=\left[U_{1}, v\right] \neq 1$. Thus $\left[U_{1}, T\right]$ is as described; but $\left[U_{1}, T\right] \subseteq O\left(H_{0}\right)$ and this contradicts Lemma 11. This completes the proof, the section, and the chapter.

\section{Chapter V. The Structure of $O(N)$. Proof of Theorem B*}

1. Outline of chapter. This chapter is devoted to a proof of Theorem B*; this section contains an outline of the chapter and explains the break-up of the proof into several stages. It is, of course, to be understood that our simple group $G$ with quasi-dihedral or wreathed Sylow 2-subgroups satisfies the working hypotheses listed in $\$ 1$ of Chapter IV. As a consequence, all results to Chapter IV hold for the group $G$.

In order to discuss the long proof to follow, we introduce yet another property of prime divisors of $|O(N)|$, namely:

Definition 1. If $p$ is a prime divisor of $|O(N)|$, then $p$ is totally inverted if $C \cap L$ is a $p^{\prime}$-group.

That is, if $P$ is a Sylow $p$-subgroup of $C$, then $P \cap L=1$. In particular, if $p$ is totally inverted, then it is certainly inverted. The relevance of this concept is as 
follows: the assumption on the prime $p$ in $\$ \S 7,8$ and 9 of the last chapter was that $p$ was exceptional and not totally inverted.

In order to establish Theorem B*, we must prove there are no exceptional primes. We prove this in three stages:

(1) If $p$ is not the characteristic of $G$ and $p$ is not totally inverted, then $p$ is ordinary.

(2) If $p$ is the characteristic and $p$ is not totally inverted, then $p$ is ordinary.

(3) If $p$ is totally inverted, then $p$ is ordinary.

The first assertion is shown in $\$ 2$; the next is proved in $\$ \$ 3,4,5$ and 6; the third statement is demonstrated in the next five sections.

The reader interested in the idea of the proof, but not wishing to go into all the details, might do well to read the chapter under the assumption that the characteristic, if it divides $|O(N)|$, is an ordinary prime. This considerably reduces the argument but leaves contact with all the essential ideas.

We preserve all the notation introduced in the preceding chapter.

2. Elimination of the first set of primes. As the title of the section suggests, we shall carry out the first step of the proof of Theorem $B^{*}$ as described above. We shall prove

Proposition 1. If $p$ is a prime divisor of $|O(N)|$ other than the characteristic of $G$ and $p$ is not totally inverted, then $p$ is ordinary.

This result has a consequence for the structure of $N$; namely, the following application of Proposition 4.4.1.

COROLlaRY 1. If $p$ is the characteristic of $G$ and $\bar{N}=N / O_{p}(N)$, then

(i) Any $\bar{T}$-invariant Sylow subgroup of $O(\bar{N})$ is centralized or inverted by $\bar{x}_{2}$.

(ii) $L_{0}$ has a normal subgroup $J$ containing $O_{p}(N)$ such that $L_{0}=J O(N)$ and either

(a) $\bar{J}$ is isomorphic to $\operatorname{SL}(2, q)$ and $\bar{J} \cap O(\bar{N})=1$, or

(b) $\bar{J}$ is isomorphic to $S L^{\wedge}(2,9), q=9$, and $|\bar{J} \cap O(\bar{N})|=3$.

(iii) $\bar{J}$ centralizes $O(\bar{N})$.

For the moment let us assume the proposition is valid.

Proof (of Corollary 1). We let $\pi$ be the set of prime divisors of $|O(N)|$ which are neither centralized nor inverted. Proposition 4.4.1 yields detailed information about the structure of $N / O_{\pi}(O(N))$. However, the above proposition implies that every prime divisor of $|O(N)|$, other than the characteristic of $G$, is ordinary or exceptional and totally inverted. But ordinary primes are centralized or inverted by Definition 4.1.3, while totally inverted primes are certainly inverted. Hence, $\pi$ is either empty or consists of the characteristic $p$ alone and so $O_{n}(O(N))=O_{p}(O(N))$ which, in turn, is $O_{p}(N)$ as $p$ is odd. The corollary is now a direct consequence of Proposition 4.4.1.

The proposition will follow from the next two lemmas. 
LEMMA 1. If $p$ is an exceptional prime and not totally inverted, then there is a Sylow p-subgroup $P$ of $G$ such that $N(Z(J(P)))$ is an element of $\mathscr{M}(p)$.

Proof. Choose $H \in \mathscr{M}(p)$ maximizing $|H|_{p}$. It is a consequence of Lemma 4.8.3 that $H$ is a $D$-group with respect to $T$ and that $O_{p^{\prime}}(H) \subseteq O(H)$. Moreover, $O_{p}(H) \neq 1$ as $H$ is a $p$-local subgroup, by Definition 4.8.1. Hence, $H$ is $p$-stable by Proposition 2.6.1. Moreover, $H$ is $p$-constrained by Corollary 4.9.1. Hence, if $P$ is a Sylow p-subgroup of $H$, then

$$
H=O_{p^{\prime}}(H) N_{H}(Z(J(P)))
$$

by Theorem 2.7.1. In particular, $O_{p^{\prime}}(H) Z(J(P))$ is a normal subgroup of $H$. Thus, by Corollary 4.8 .1 , replacing $P$ by a conjugate in $H$, if necessary, we may assume that $H_{1}=N(Z(J(P))) \in \mathscr{M}(p)$. To prove the lemma, we simply must now show that $P$ is a Sylow $p$-subgroup of $G$. However, if this is not the case, then $P$ is a proper normal subgroup of a $p$-subgroup $P_{1}$. Since $Z(J(P))$ is a characteristic subgroup of $P$, it is normal in $P_{1}$ and

$$
\left|H_{1}\right|_{p} \geqq\left|P_{1}\right|>|P|=|H|_{p},
$$

which contradicts the choice of $H$. This proves the lemma.

LEMMA 2. If $p$ is an exceptional prime, not totally inverted and not the characteristic of $G$, then there is a Sylow p-subgroup $P$ of $G$ such that $N(Z(J(P)))$ is an element of $\mathscr{L}(p)$.

Proof. This argument is very similar to the preceding one. Choose $H \in \mathscr{L}(p)$ maximizing $|H|_{p}$. Thus, $H$ is a $p$-local subgroup, by Definition 4.1.4, so $O_{p}(H) \neq 1$ and $H$ is a $Q$-group of characteristic power $q$, by Lemma 4.1.1. Moreover, $H$ is $p$-constrained within $S(H)$ by Proposition 4.6.1; in particular, $O_{p^{\prime}}(H) \subseteq S(H)$, by Definition 2.6.1. But $p$ is not the characteristic of $H$ by hypothesis and so $H$ is $p$-stable by Proposition 2.6.1 since $q>5$ is a constant assumption. Thus, if $P$ is a Sylow $p$-subgroup of $H$, then

$$
H=O_{p^{\prime}}(H) N_{H}(Z(J(P)))
$$

by Theorem 2.7.1. In particular, $O_{p^{\prime}}(H) Z(J(P))$ is a normal subgroup of $H$. Thus, we may assume, after replacing $P$ by a conjugate in $H$, if necessary, that $N(Z(J(P)))=H_{1} \in \mathscr{L}(p)$, by Corollary 4.6.1. Our choice of $H$ now implies that $P$ is a Sylow $p$-subgroup of $G$, just as in the proof of the previous lemma.

Proof (of Proposition 1). Let $p$ be a prime which violates the proposition, so $p$ is exceptional, not totally inverted, and $p$ is not the characteristic of $G$. Hence, by Lemma 1 , there is a Sylow $p$-subgroup $P_{1}$ of $G$ such that $N\left(Z\left(J\left(P_{1}\right)\right)\right)=H_{1} \in \mathscr{M}(p)$. Similarly, by Lemma 2 , there is a Sylow $p$-subgroup $P_{2}$ of $G$ such that $N\left(Z\left(J\left(P_{2}\right)\right)\right)$ $=H_{2} \in \mathscr{L}(p)$. But $H_{1}$ is then a $D$-group, by Lemma 4.8 .3 and $H_{2}$ is a $Q$-group, by Lemma 4.1.1. This is a contradiction as $H_{1}$ and $H_{2}$ are conjugate subgroups of $G$. 
3. A family of cyclic subgroups. We now fix $p$ as the characteristic of $G$ for the next four sections of this chapter. In particular, $N$ has the structure described in Corollary 1 of the previous section. In dealing with $\mathscr{M}(p)$ it was important to include the covering property. Since $N$ is now known to have a more restricted structure, we can simplify any work with the covering property.

We do this by introducing the following concept:

Definition 1. The set of all $D$-invariant subgroups of $J$ incident with

$$
(O(C) \cap J) O_{p}(N) / O_{p}(N)
$$

is denoted by $\mathscr{W}$.

Here $J$ is the subgroup which exists by virtue of Corollary 2.1, whence either $J \cap O(N)=O_{p}(N)$ and $J / O_{p}(N)$ is isomorphic to $S L(2, q)$ or $\left|J \cap O(N): O_{p}(N)\right|$ $=3, q=9$, and $J / O_{p}(N)$ is isomorphic to $S L^{\wedge}(2,9)$.

Lemma 1. The section $(C \cap J) O_{p}(N) /\langle x\rangle O_{p}(N)$ is cyclic of order $\frac{1}{2}(q+\delta \varepsilon)$. The collection $\mathscr{W}$ is nonempty and each element of $\mathscr{W}$ is cyclic of order $(q+\delta \varepsilon) / 2^{n}$, is centralized by $C_{D}(T)$, and is inverted by $d$.

Proof. We set $\bar{N}=N / O_{p}(N)$. Since $O_{p}(N)$ has odd order, the section in question is isomorphic to

$$
C_{\jmath}(\bar{T}) /\langle\bar{x}\rangle \text {. }
$$

There are two possible structures for $\bar{J}$. If $\bar{J}$ is isomorphic to $S L(2, q)$, then $C_{\mathcal{J}}(\bar{T})$ is cyclic of order $q+\delta \varepsilon$ by Lemma 2.4.2. Suppose, on the other hand, that $\bar{J}$ is isomorphic to $S L^{\wedge}(2,9)$, in which case $q=9$. Thus the characteristic of $G$ is three and so $p=3$. But $\bar{J} \cap O(\bar{N})=O(\bar{J})$ has order three and is normal in $O(\bar{N})$. Since $O_{3}(O(\bar{N})) \subseteq O_{3}(\bar{N})=O_{p}(\bar{N})$, it follows that $O_{p}(\bar{N}) \neq 1$, contrary to the fact that $\bar{N}=N / O_{p}(N)$ by definition. Thus this case cannot arise and so the first assertion is established.

Furthermore, by Lemma 2.4.2, $O\left(C_{J}(\bar{T})\right)=\bar{W}$ is cyclic of order $(q+\delta \varepsilon) / 2^{n}, \bar{W}$ centralizes $C_{\bar{D}}(\bar{T})$, and $\bar{W}$ is inverted by $\bar{d}$. Moreover, $\bar{D}=\left\langle C_{\bar{D}}(\bar{T}), \bar{d}\right\rangle$ as $C_{\bar{D}}(\bar{T})=\bar{T}$ and $\bar{d}=\bar{y}$ if $G$ has quasi-dihedral Sylow 2-subgroups, while $C_{\bar{D}}(\bar{T})$ is abelian of index two in $\bar{D}$ and $\bar{d} \in \bar{D}-C_{\bar{D}}(\bar{T})$ if $G$ has wreathed Sylow 2-subgroups. On the other hand, $(q+\delta \varepsilon) / 2^{n}$ is relatively prime to 2 and to $p$, since $p$ is the characteristic of $G$ and so divides $q$. Since $x$ is central in $(C \cap J) O_{p}(N)$, which is in turn $D$ invariant, there exist $D$-invariant Hall $\{2, p\}^{\prime}$-subgroups of $(C \cap J) O_{p}(N)$. If $W$ is such a subgroup, then $W$ is incident with $(O(C) \cap J) O_{p}(N) / O_{p}(N)$ and the remaining conditions of the second assertion hold for $W$. Thus $\mathscr{W}$ is nonempty and the lemma is proved.

We shall frequently make a choice of an element $W$ of $\mathscr{W}$. This leads to no ambiguity largely because of the following remark:

LEMMA 2. If $W$ and $W_{1}$ are elements of $\mathscr{W}$ and $P$ is a D-invariant Sylow p-subgroup of $C$, then $W_{1} \subseteq\langle W, P\rangle$. 
Proof. The definition of $\mathscr{W}$ and Lemma 1 yield immediately that any two elements $W$ and $W_{1}$ of $\mathscr{W}$ are certainly conjugate by an element of $C \cap O_{p}(N)$. Indeed, $W$ and $W_{1}$ are $D$-invariant Hall $p$-complements of $W O_{p}(N)$. However, $C \cap O_{p}(N)$ is a normal subgroup of $C$ as $O_{p}(N)$ is normal in $N$, so $C \cap O_{p}(N)=P \cap O_{p}(N)$ and hence $W_{1} \subseteq\langle W, P\rangle$.

We shall see in the next result that $\langle W, P\rangle$ is, in fact, just $W P$.

We now collect some properties of the elements of $\mathscr{W}$.

LEMMA 3. If $W$ is an element of $\mathscr{W}$, then the following conditions hold:

(i) $W \cap O(N)=1$;

(ii) $C O(N)=O_{p}(N) N_{C O(N)}(W)$;

(iii) $W$ is permutable with any Sylow p-subgroup of $C$;

(iv) $C=\left(C \cap O_{p}(N)\right) N_{C}(W)$;

(v) If $p$ is exceptional, but not totally inverted, then $W$ normalizes $Q$.

In statement (v), $Q$ is the subgroup which we proved existed in $\S 7$ of the previous chapter under the hypothesis that $p$ was exceptional, but not totally inverted.

Proof. (i) By definition, $W \subseteq J$ and the incidence condition (or the fact that $W$ is a $p^{\prime}$-group) forces $W \cap O_{p}(N)=1$. Hence, if $\bar{N}=N / O_{p}(N)$, it suffices to prove that $\bar{W} \cap O(\bar{N})=1$. But we have already noted in the proof of Lemma 1 that $\bar{J}$ is necessarily isomorphic to $S L(2, q)$, whence $\bar{J} \cap O(\bar{N})=1$. Since $\bar{W} \subseteq \bar{J}$, the desired conclusion $\bar{W} \cap O(\bar{N})=1$ holds.

(ii) First, $O(\bar{N})$ centralizes $\bar{J}$ by Corollary 2.1, so $O(\bar{N})$ centralizes $\bar{W}$. Moreover, $\bar{W}$ is normal in $\bar{C}$ as $\bar{W}=O(\bar{C}) \cap \bar{J}$ and $O(\bar{C}) \cap \bar{J}$ is normal in $\bar{C}$. Thus $\bar{W}$ is normal in $O(\bar{N}) \bar{C}$. However, $\overline{O(N) C}=O(\bar{N}) \bar{C}$ and so the product $W O_{p}(N)$ is normal in $O(N) C$. But $W$ is a Hall $p^{\prime}$-subgroup of $W O_{p}(N)$, so (ii) follows from the Frattini argument.

(iii) Let $P$ be a Sylow $p$-subgroup of $C$. Since $W O_{p}(N)$ is normal in $O(N) C$ by (ii), it follows that $W O_{p}(N) \cap C=W\left(O_{p}(N) \cap C\right)$. Since $O_{p}(N)$ is normal in $N$, we also have that $O_{p}(N) \cap C$ is normal in $C$ and so $O_{p}(N) \cap C=O_{p}(N) \cap P$ as $P$ is a Sylow $p$-subgroup of $C$. Hence $W\left(O_{p}(N) \cap C\right) P=W P$ is a group and (iii) is valid.

(iv) As we have just seen, $C \cap O_{p}(N)$ and $W\left(C \cap O_{p}(N)\right)$ are normal subgroups of $C$. Since $W$ is a Hall $p$-complement in $W\left(C \cap O_{p}(N)\right)$, (iv) follows by the Frattini argument.

(v) The subgroup $Q$ was a characteristic subgroup of a fixed $D$-invariant Sylow $p$-subgroup of $C$; let this Sylow $p$-subgroup be $P$. We claim that it suffices to show that there is an element $W_{1}$ of $\mathscr{W}$ such that $W_{1}$ normalizes $Q$. Indeed, if that is the case, then $W \subseteq W_{1} P$ by Lemma 2 and part (iii) of this lemma, so $W$ also normalizes $Q$ as $P$ certainly does.

We let $X / O(N)$ be the subgroup of $L_{0} / O(N)$ of order $(q+\delta \varepsilon) / 2^{n}$ in $O(C) O(N) \cap L_{0} / O(N)$. We now claim that we need only produce a $D$-invariant subgroup $W_{1}$ of $X$ incident with $X / O(N)$. Indeed, if $W_{1}$ is such a subgroup, then 
$W_{1} \in \mathscr{W}$ once we know that $W_{1} \subseteq J$, since $X / O(N)$ and $(O(C) \cap J) O_{p}(N) / O_{p}(N)$ are incident. However, the element $d$ of $D$ centralizes $O(N) / O_{p}(N)$ as it lies in $L_{0}$, so any subgroup of odd order in $L_{0} / O(N)$ inverted by $d$ lies in $J / O_{p}(N)$. Thus, if $W_{1}$ is as described, then $d$ inverts $W_{1}$ since $d$ inverts $X / O(N)$ and $W_{1}$ is $D$-invariant. Thus $W_{1} \subseteq J$ and our reduction is established.

Proposition 4.7.1 yields that $N(Q)$ covers $C \cap L / C \cap S(L)$, so certainly $N(Q)$ covers $X / O(N)$. Let $X_{0}=X \cap N(Q)$ and $Y_{0}=O(N) \cap N(Q)$. Thus $X_{0} / Y_{0}$ is incident with $X / O(N)$. In particular, $X_{0} / Y_{0}$ is cyclic of order $(q+\delta \varepsilon) / 2^{n}$, is centralized by $C_{D}(T)$ and is inverted by $d$. Moreover, by the reduction of the previous paragraph, it suffices to establish the existence of a $D$-invariant subgroup of $X$ incident with $X_{0} / Y_{0}$.

We set $Z_{0}=O_{p}(N) \cap N(Q)$, so $Z_{0}$ is a normal p-subgroup of $X_{0}$ contained in $Y_{0}$. Set $\bar{X}_{0}=X_{0} / Z_{0}$, so $\bar{Y}_{0}=Y_{0} / Z_{0}$. We now assert that we need only construct a $D$-invariant subgroup of $\bar{X}_{0}$ incident with $\bar{X}_{0} / \bar{Y}_{0}$. Indeed, if $K / Z_{0}$ is such a subgroup, then $K / Z_{0}$ is cyclic of order $(q+\delta \varepsilon) / 2^{n}$, is centralized by $C_{D}(T)$, and is inverted by $d$. In particular, $K / Z_{0}$ has order prime to $p$ and is $D$-invariant. Since $Z_{0}$ is a $p$-group, $K$ thus contains a $D$-invariant Hall $p$-complement and this subgroup clearly satisfies all the requirements.

Finally set $\bar{X}_{1}=C_{X_{0}}\left(C_{D}(T)\right)$ and $\bar{Y}_{1}=\bar{X}_{1} \cap \bar{Y}_{0}$. Since $C_{D}(T)$ centralizes $\bar{X}_{0} / \bar{Y}_{0}$ and $\bar{X}_{0}$ is of odd order, it follows that $\bar{X}_{1} / \bar{Y}_{1}$ is also cyclic of order $(q+\delta \varepsilon) / 2^{n}$. Moreover, as $d$ normalizes $C_{D}(T)$ and inverts $\bar{X}_{0} / \bar{Y}_{0}$, we also have that $d$ inverts $\bar{X}_{1} / \bar{Y}_{1}$. But $d$ centralizes $O(N) / O_{p}(N)$, as we have noted above, and so $d$ centralizes both $\bar{Y}_{1}$ and $\bar{Y}_{0}$. In particular, $\bar{Y}_{1}=C_{X_{1}}(d)$. Since $\bar{X}_{1}$ has odd order, the subset $\bar{W}_{1}$ of $\bar{X}_{1}$ inverted by $d$ covers $\bar{X}_{1} / \bar{Y}_{1}$. But $\bar{X}_{1} / \bar{Y}_{1}$ is cyclic and $\bar{W}_{1} \cap \bar{Y}_{1}=1$, which together imply that $\bar{W}_{1}$ is a subgroup. Thus $\bar{W}_{1}$ is cyclic of order $(q+\delta \varepsilon) / 2^{n}$ and $\bar{W}_{1}$ centralizes $C_{D}(T)$ as $\bar{W}_{1} \subseteq \bar{X}_{1}$, so $\bar{W}_{1}$ is invariant under $D=\left\langle C_{D}(T), d\right\rangle$. But $\bar{W}_{1} \cap \bar{Y}_{0}=1$ as $d$ inverts $\bar{W}_{1}$ and centralizes $\bar{Y}_{0}$, whence $\bar{X}_{0}=\bar{W}_{1} \bar{Y}_{0}$. Thus $\bar{W}_{1}$ is the required $D$-invariant subgroup of $\bar{X}_{0}$ incident with $\bar{X}_{0} / \bar{Y}_{0}$. This completes the proof of the lemma.

4. Properties of signalizers. We preserve our hypotheses on the prime $p$; we shall be studying the $D$-invariant $p$-subgroups of $G$, namely the elements of $U(D ; p)$. It is through use of these subgroups that we shall be able to prove later that $p$ is, in fact, an ordinary prime. We shall use the specialized signalizer notation which we defined in Chapter I.

Our main result in this section is as follows:

Proposition 1. Let $H$ be a subgroup of $G$ containing $D$ satisfying the following conditions:

(a) $O(H) \neq 1$;

(b) $P$ is a D-invariant Sylow p-subgroup of $C$ contained in $H$;

(c) $W$ is an element of $\mathscr{W}$ contained in $H$;

(d) $V$ is an element of $U_{H}(D, W ; p)$ containing $P$. 
Under these conditions we have

(i) There is an element $U$ of $\eta_{H}(D, W ; p)$ containing $V$ such that $U \in И_{H}^{*}(D ; p)$ and $U \cap O(H)$ is a D-invariant Sylow p-subgroup of $O(H)$.

(ii) If $U_{1}$ and $U_{2}$ are elements of $U_{H}(D, W ; p)$ containing $V$, both of which are elements of $U_{H}^{*}(D ; p)$, then $U_{1}$ and $U_{2}$ are conjugate by an element of $N_{C}(W)$ which centralizes $D$.

The subgroups $P$ and $W$ are permutable, by Lemma 3.3. Moreover, $W$ is $D$ invariant, by Definition 3.1, so $P W$ is also and $P W D$ is a group. The proof of the proposition depends on a few preliminary lemmas. Throughout these five lemmas we assume that the elements of $\mathscr{W}$ are nontrivial; equivalently, that $(q+\delta \varepsilon) / 2^{n}>1$.

First, we fix some notation. Let $\rho$ be the set of primes dividing $|W|=(q+\delta \varepsilon) / 2^{n}$ and let $\sigma=\rho \cup\{p\}$. As in the last chapter, let $N_{i}=C\left(x_{i}\right), 1 \leqq i \leqq 3$, so $N=N_{1}$. We begin with one more special fact about elements of $\mathscr{W}$.

LEMMA 1. If $W$ is an element of $\mathscr{W}$, then $W \subseteq O\left(N_{i}\right)$ for at most one value of $i$, $1 \leqq i \leqq 3$.

Proof. Indeed, $W \nsubseteq O(N)=O\left(N_{1}\right)$, by the definition of $\mathscr{W}$. Thus, if the lemma is false, then $W \subseteq O\left(N_{2}\right) \cap O\left(N_{3}\right)$. However, $N_{2}$ and $N_{3}$ have the same structure as does $N$, since all the involutions of $T$ are conjugate in $G$. In particular, if we set $\bar{N}_{i}=N_{i} / O_{p}\left(N_{i}\right), 2 \leqq i \leqq 3$, we have that $\bar{J}_{i}$ is normal in $\bar{N}_{i}$ and $\bar{J}_{i}$ centralizes $O\left(\bar{N}_{i}\right)$, by Corollary 2.1. Since $\bar{W} \subseteq O\left(\bar{N}_{i}\right)$, it follows that $\bar{W}$ centralizes $\bar{J}_{i}, 2 \leqq i \leqq 3$. However, $K_{i}=C_{J_{i}}(W)$ maps onto $\bar{J}_{i}$ as $W$ is a $p^{\prime}$-group; consequently, $K_{i}$ is a $Q$-group of characteristic power $q$ and $O\left(K_{i}\right) x_{i}$ is central in $K_{i} / O\left(K_{i}\right), 2 \leqq i \leqq 3$. Since $N(W)$ contains $K_{i}$ and $W \neq 1$, it follows from Proposition 4.1.1 that $N(W)$ is also a $Q$ group of characteristic power $q$ in which $O(N(W)) x_{i}$ is central in $N(W) / O(N(W))$, $2 \leqq i \leqq 3$. However, the center of that quotient group is cyclic by Lemma 2.4 .7 , so we have a contradiction. The lemma is proved.

The next result is to be used in proving the one that follows.

LEMMA 2. If $W$ is an element of $\mathscr{W}$, then $W \subseteq L_{i}$ for all $i, 1 \leqq i \leqq 3$.

Proof. Let $X$ be a Hall $p$-complement of $O(C)$ which contains $W$ and set $Y=C_{X}\left(X^{\prime}\right)$. It will suffice to show that $W \subseteq Y \subseteq L$. Indeed, suppose this is the case. Since $O(C)$ is a characteristic subgroup of $C$, it is normal in $M=N(C)$. Hence $M=O(C) N_{M}(X)$, by the Frattini argument, inasmuch as $X$ is a Hall subgroup of $O(C)$. Thus, it follows that $M=O(C) N_{M}(Y)$. Therefore, there is $a_{1} \in N_{M}(Y)$ so that $a_{1}$ cyclically permutes $x_{1}, x_{2}$ and $x_{3}$ by conjugation. We have now $W \subseteq L_{1}=L$ and $W^{a_{1}} \subseteq Y^{a_{1}}=Y \subseteq L$, so that $W \subseteq L_{1}^{a^{-1}}=L_{3}$. Similarly, $a_{1}^{-1} \in N_{M}(Y)$, so $W \subseteq L_{2}$.

We shall now show that $Y \subseteq L$ and we begin by setting $\bar{N}=N / O(N)$. Since $W \subseteq X \subseteq O(C)$, we have $\bar{W} \subseteq \bar{X} \subseteq O(\bar{C})$ and $\bar{W}=O(\bar{C}) \cap \bar{L}$; consequently, $\bar{X}=$ $O\left(C_{L X}(\bar{T})\right)$. It follows therefore from Lemma 2.4.2, applied to the $Q$-group $\bar{L} \bar{X}$, that $C_{\bar{X}}\left(\bar{X}^{\prime}\right)=\bar{W}$ and hence, that $C_{\bar{X}}\left(\bar{X}^{\prime}\right) \subseteq \bar{L}$. But clearly $\bar{Y} \subseteq C_{\bar{X}}\left(\bar{X}^{\prime}\right)$, so $\bar{Y} \subseteq \bar{L}$ and $Y \subseteq L$. 
It remains to prove that $W \subseteq Y$. To do this, we now set $\bar{N}=N / O_{p}(N)$. Since $X$ is a $p^{\prime}$-group, it is isomorphic with $\bar{X}$, so that it suffices to show that $\bar{W} \subseteq \bar{Y}$. However, $\bar{N} / \bar{L}$ is cyclic, by Proposition 2.3.3, and consequently $\bar{X}^{\prime} \subseteq \bar{L}$. Moreover, $\bar{Y}=C_{\bar{X}}\left(\bar{X}^{\prime}\right)$ since $X$ and $\bar{X}$ are isomorphic. Hence, it is enough to demonstrate that $\bar{W}$ is central in $O(\bar{C}) \cap \bar{L}$. But $\bar{W}=O(\bar{C}) \cap \bar{J}$, by Definition 3.1, and so $O(\bar{C}) \cap \bar{L}=$ $\bar{W}(O(\bar{C}) \cap O(\bar{N}))$. However, $\bar{W} \subseteq \bar{J}$ and $\bar{J}$ centralizes $O(\bar{N})$, by Corollary 2.1 ; in particular, $\bar{W}$ centralizes $O(\bar{C}) \cap O(\bar{N})$. Since $\bar{W}$ is cyclic, by Lemma 2.4 .2 , it follows that $\bar{W}$ is central in $O(\bar{C}) \cap \bar{L}$, as required.

We employ the usual $T$-decomposition notation in stating the next result:

LEMMA 3. If $X$ is a T-invariant $r$-subgroup of $G$ for some $r$ in $\rho, W$ is an element of $\mathscr{W}$ and $\langle X, W\rangle$ is of odd order, then the following conditions hold:

(i) If there is an integer $j, 1 \leqq j \leqq 3$, such that $X_{j}^{\prime} \neq 1$ and $W \nsubseteq O\left(N_{j}\right)$, then $X_{j}^{\prime} \subseteq O\left(N_{j}\right)$ and $r$ is ordinary and inverted.

(ii) There is an integer $i, 1 \leqq i \leqq 3$, such that $X \subseteq N_{i}$.

Proof. (i) First, suppose that $X_{j}^{\prime} \subseteq O\left(N_{j}\right)$; we shall show that $r$ is as required. Since $X_{j}^{\prime} \neq 1$ and $X_{j}^{\prime} \subseteq O\left(N_{j}\right)$, it follows that $r$ divides $|O(N)|$. Moreover, $r$ divides $|W|$ as $r \in \rho$. Hence, $r$ divides $|C \cap L|$ and $r$ is not totally inverted. Since $r \neq p$, as $p$ and $q+\delta \varepsilon$ are coprime, Proposition 2.1 yields that $p$ is ordinary. Furthermore, any $T$-invariant Sylow $r$-subgroup of $O(N)$ is centralized or inverted by $x_{2}$. However, $\left\langle X_{j}^{\prime}\right\rangle$ is a $T$-invariant $r$-subgroup of $O\left(N_{j}\right)$ not centralized by $T$, so $r$ is indeed inverted.

Thus it suffices to show that $Y=\left\langle X_{j}^{\prime}\right\rangle$ is contained in $O\left(N_{j}\right)$. Hence, we assume that $Y \nsubseteq O\left(N_{j}\right)$ and derive a contradiction. In any case, $Y$ is an $r$-subgroup of $N_{j}$ and $[Y, T]=Y$. Since $T \subset L_{j}$, we have, in fact, that $Y \subseteq L_{j}$.

We set $\bar{N}_{j}=N_{j} / O\left(N_{j}\right)$, so $\bar{Y}$ is a nonidentity $r$-subgroup of $\bar{L}_{j}$ and $[\bar{Y}, \bar{T}]=\bar{Y}$. Moreover, $W \subseteq C \subseteq N_{j}$ and $\bar{W} \neq 1$ as $W \nsubseteq O\left(\bar{N}_{j}\right)$. Thus, $\bar{W}$ is a nonidentity subgroup of $O(\bar{C})$. In addition, Lemma 2 implies that $\bar{W} \subseteq \bar{L}_{j}$. Hence, it will suffice to prove that $|\bar{Y}|$ divides $q+\delta \varepsilon$. Indeed, if this is the case, we have an immediate contradiction to Lemma 2.4 .6 with $\bar{W}, \bar{Y}, \bar{L}, \bar{T}$ in the roles of $W_{0}, X, L$, and $T$ respectively since $\langle\bar{W}, \bar{Y}\rangle$ has odd order.

But $\bar{Y}$ is an $r$-subgroup of $\bar{L}_{j}$ and $r$ is a divisor of $q+\delta \varepsilon$. Thus $|\bar{Y}|$ also divides $q+\delta \varepsilon$ by Lemma 3.1(v) of [22] applied to $\bar{L}_{j} /\left\langle\bar{x}_{j}\right\rangle$.

(ii) We assume the assertion is invalid, so that $X_{i}^{\prime} \neq 1$ for at least two values of $i$, $1 \leqq i \leqq 3$. By Lemma 1 , we may choose $j, 1 \leqq j \leqq 3$, such that $X_{j}^{\prime} \neq 1$ and $W \nsubseteq O\left(N_{j}\right)$. Hence, by (i), $X_{j}^{\prime} \subseteq O\left(N_{j}\right)$ and $r$ is ordinary and inverted. Therefore, by Lemma 4.2.3, if $u$ is a nonidentity element of $X_{j}^{\prime}$, then every $T$-invariant $r$-subgroup of $C(u)$ lies in $N_{j}$.

However, as $X_{i}^{\prime} \neq 1$ for at least two values of $i, 1 \leqq i \leqq 3$, we have $X_{j} \subset X$. Thus, by Lemma 2.8 .1 , there is a nonidentity $T$-invariant subgroup $Y$ of $X$ centralizing $X_{j}$ such that $\left[Y, x_{j}\right]=Y$. Therefore, $Y$ is a nonidentity $T$-invariant $r$-subgroup of 
$C(u)$ and $Y$ does not lie in $N_{j}$. This is the desired contradiction and the lemma is proved in full.

The next lemma is quite technical; however, it is only to be used in the argument of the last of the preliminary lemmas.

LEMMA 4. Let $R$ and $Y$ be subgroups of $G$ with the following properties:

(a) $R$ is a D-invariant p-subgroup;

(b) $Y$ is a D-invariant $r$-subgroup for some $r$ in $\rho$;

(c) $R$ and $Y$ are permutable;

(d) $\langle Y, W\rangle$ has odd order for some element $W$ of $\mathscr{W}$.

Under these conditions, we have that

$$
[R, T] \subseteq O_{p}(R Y) .
$$

Proof. We set $K=R Y$, so that $K$ is a subgroup of $G$. We let $\bar{K}=K / O_{p}(K)$; it must be shown that $[\overline{R, T}]=1$.

As a first step, in the usual $T$-decomposition notation, we prove that $R_{1}^{\prime}=1$. To do this, it suffices to demonstrate that $\bar{R}_{1}^{\prime}$ centralizes $O_{r}(\bar{K})$, since $K$ is a solvable $\{p, r\}$-group. However, set $Z=Y \cap O_{p, r}(K)$, so that $Z$ is a Sylow $r$-subgroup of $O_{p, r}(K)$ and $\bar{Z}=O_{r}(\bar{K})$. Moreover, let $F=C_{Z}\left(x_{2}\right)$ and let $I$ be the set of elements of $Z$ inverted by $x_{2}$, so that $Z=F I$.

We claim that in order to establish our assertion on $R_{1}^{\prime}$, it suffices to show that $\bar{R}_{1}^{\prime}$ centralizes $\bar{I}$. Indeed, suppose this is the case. Therefore, $x_{2}$ inverts each element of $\bar{R}_{1}^{\prime}$ and centralizes $\bar{Z} \mid\langle\bar{I}\rangle$, so that $\bar{R}_{1}^{\prime}$ centralizes $\bar{Z} \mid\langle\bar{I}\rangle=O_{p}(\bar{K}) /\langle\bar{I}\rangle$. However, $\bar{R}_{1}^{\prime}$ centralizes $\langle\bar{I}\rangle$, by assumption, so that $\bar{R}_{1}^{\prime}$ centralizes $\bar{Z}$, since each element of $\bar{R}_{1}^{\prime}$ is a $p$-element, while $\bar{Z}$ is an $r$-subgroup and $r \neq p$ as $r$ is in $\rho$.

We now shall show that $\bar{R}_{1}^{\prime}$ does centralize $\bar{I}$; we may, of course, assume that $\bar{I} \neq 1$, so that $Y_{1}^{\prime} \neq 1$. Hence, applying Lemma 3 , with $Y$ in place of $X$ and $j=1$, and using the fact that $W \nsubseteq O(N)$, we conclude that $Y_{1}^{\prime} \subseteq O(N)$ and that $r$ is ordinary and inverted. This yields that $Y_{1}^{\prime}$ is an abelian subgroup inverted by $x_{2}$.

Furthermore, $x$ centralizes $Y$. Indeed, some $x_{i}$ centralizes $Y$ by Lemma 3, $1 \leqq i \leqq 3$, so $C_{D}(Y)$ is a nontrivial normal subgroup of $D$. But then $C_{Z(D)}(Y) \neq 1$ and, as $x$ is the unique involution of $Z(D), x$ centralizes $Y$, as stated. It follows that $I=Z \cap Y_{1}^{\prime}$.

We now assert that in order to show that $\bar{R}_{1}^{\prime}$ and $\bar{I}$ do commute elementwise, it is enough to see that $R_{1}$ and $Y_{1}^{\prime}$ are permutable; indeed, assume this is the case. Thus $\bar{R}_{1}$ and $\bar{Y}_{1}^{\prime}$ are permutable, so $\bar{R}_{1}$ normalizes $\bar{I}=O_{r}(\bar{K}) \cap \bar{Y}_{1}^{\prime}$ by Lemma 2.8.3. Since $x_{2}$ inverts both $\bar{R}_{1}^{\prime}$ and $\bar{I}$, it follows that $x_{2}$ has no nontrivial fixed points on $\bar{R}_{1}^{\prime} \bar{I}$, which is a group as $\bar{R}_{1}^{\prime}$ normalizes $\bar{I}$. Thus $x_{2}$ inverts $\bar{R}_{1}^{\prime} \bar{I}$ and $\bar{R}_{1}^{\prime} \bar{I}$ is abelian, so $\bar{R}^{\prime}$ centralizes $\bar{I}$, as required.

Now $R$ is permutable with $Y$, by assumption, and $Y \subseteq N$, as we have shown above that $x$ centralizes $Y$. Moreover, we know that $Y_{1}^{\prime} \subseteq O(N)$ and that.r is an inverted prime, so that $Y_{1}^{\prime}=Y \cap O(N)$. Hence, $R_{1}$ and $Y_{1}^{\prime}$ are indeed permutable, by Lemma 2.8.3. Thus, at long last, we have established that $\bar{R}_{1}^{\prime}=1$. 
Moreover, $\bar{R}_{j}^{\prime}=1$ if $j=2$ or 3 . Indeed, since $x$ centralizes $Y$, it follows that $x$ centralizes $O_{r}(\bar{K})$. However, any element of $\bar{R}_{j}^{\prime}$ is inverted by $x$ and normalizes $O_{r}(\bar{K})$, so $\bar{R}_{j}^{\prime}$ centralizes $O_{r}(\bar{K})$ and therefore $\bar{R}_{j}^{\prime}=1,2 \leqq j \leqq 3$, as claimed.

Finally, $[R, T]=\left\langle R_{1}^{\prime}, R_{2}^{\prime}, R_{3}^{\prime}\right\rangle$. Indeed, if $R^{*}=\left\langle R_{1}^{\prime} \mid 1 \leqq i \leqq 3\right\rangle$, then $R^{*}=\left[R^{*}, T\right]$ $\subseteq[R, T]$. Moreover, $R=R_{0} R^{*}$, so $[R, T] \subseteq\left[R^{*}, T\right]$ and our assertion is valid. But each $R_{i}^{\prime} \subseteq O_{p}(R Y), 1 \leqq i \leqq 3$, since we have already shown that $\bar{R}_{i}^{\prime}=1,1 \leqq i \leqq 3$. Hence $R^{*} \subseteq O_{p}(R Y)$ and the lemma is proved.

The last result preliminary to the proof of the proposition is as follows:

Lemma 5. If $W$ is an element of $\mathscr{W}$ and $X$ is a $D W$-invariant $\sigma$-subgroup of $G$, then

(i) $X=O_{p}(X)(X \cap N)=O_{p}(X) N_{X}(W)$;

(ii) $O_{p}(X)(X \cap C)$ contains a Sylow p-subgroup of $X$.

Proof. First, we let $R$ be a $D$-invariant Sylow $p$-subgroup of $X$ and we claim that $[R, T] \subseteq O_{p}(X)$. Indeed, if $r$ is any prime divisor of $|X|$ other than $p$, let $Y$ be a $D$-invariant Sylow $r$-subgroup of $X$ permutable with $R$. Since $X$ is a $\sigma$-group and $r \neq p$, it follows that $r \in \rho$ and so $[R, T] \subseteq O_{p}(R Y)$ by the previous lemma. But now the desired conclusion $[R, T] \subseteq O_{p}(X)$ is a consequence of Lemma 2.8.7.

Moreover, preserving this notation for $r$ and $Y$, it is a consequence of Lemma 3 that $C_{T}(Y) \neq 1$ inasmuch as $\langle Y, W\rangle \subseteq\langle X, W\rangle=X W$ is of odd order. But $C_{D}(Y)$ is a normal subgroup of $D$ and so $x \in C_{D}(Y)$ for any $r$ and any $Y$. Thus

$$
X=R(X \cap N) \text {. }
$$

Therefore, since $R=[R, T](R \cap C)$, we have

$$
\begin{aligned}
X & =R(X \cap N)=[R, T](R \cap C)(X \cap N) \\
& =[R, T](X \cap N) \subseteq O_{p}(X)(X \cap N),
\end{aligned}
$$

so $X=O_{p}(X)(X \cap N)$ and half of (i) is established. Furthermore,

$$
R=[R, T](R \cap C) \subseteq O_{p}(X)(X \cap C)
$$

so the second assertion (ii) is also valid.

It remains to demonstrate the second factorization of $X$ in (i). However, $X \cap N$ is a $D W$-invariant subgroup of $N$, so $X \cap N \subseteq C O(N)$, by Lemma 2.4.9, applied to $N / O(N)$, inasmuch as $W(X \cap N)$ is of odd order, is normalized by $D$, and contains $W$. If we set $\bar{N}=N / O_{p}(N)$, then $\overline{X \cap N} \subseteq \bar{C} \overline{O(N)}=\bar{C} O(\bar{N})$. But $\bar{W} O(\bar{N})$ is the direct product of $\bar{W}$ and $O(\bar{N})$, by Corollary 2.1 , and $\bar{W}=O(\bar{C}) \cap \bar{L}$ is normal in $\bar{C}$, whence $\bar{W}$ is normal in $\overline{W(X \cap N) \text {. }}$

Hence, $W(X \cap N)$ normalizes $W O_{p}(N)$, so certainly $W(X \cap N)$ normalizes $W O_{p}(N) \cap W(X \cap N)$. However, $W \subseteq N$, so $W(X \cap N)=W X \cap N$ and

$$
\begin{aligned}
W O_{p}(N) \cap W(X \cap N) & =W O_{p}(N) \cap N \cap W X \\
& =W O_{p}(N) \cap W X
\end{aligned}
$$

and we have that $W(X \cap N)$ normalizes $W O_{p}(N) \cap W X$. 
We claim now that $W O_{p}(N) \cap W X=W\left(O_{p}(N) \cap X\right)$. Since $W\left(O_{p}(N) \cap X\right)$ $\subseteq W O_{p}(N) \cap W X$, it is enough to demonstrate the reverse inequality. Thus suppose that $u \in W O_{p}(N) \cap W X$, so that $u=w v$, where $w \in W$ and $v \in O_{p}(N)$. Since $W \subseteq W O_{p}(N) \cap W X$, it follows that $v \in W O_{p}(N) \cap W X$, so $v \in W X$. But $X$ is normal in $W X$, as $X$ is $W$-invariant. Thus $W X / X$ is a $p^{\prime}$-group, as $W$ is a $p^{\prime}$-group, so that $v \in X$ as $v$ is a $p$-element, being in $O_{p}(N)$. Hence, $v \in O_{p}(N) \cap X$, so $u \in W\left(O_{p}(N) \cap X\right)$ and $W O_{p}(N) \cap W X \subseteq W\left(O_{p}(N) \cap X\right)$, as claimed.

It now follows that $W(X \cap N)$ normalizes $W\left(O_{p}(N) \cap X\right)$. Since $W$ is a Hall subgroup of $W\left(O_{p}(N) \cap X\right)$, we conclude by the Frattini argument that

so

$$
X \cap N \subseteq\left(O_{p}(N) \cap X\right) N(W),
$$

$$
X \cap N=\left(O_{p}(N) \cap X\right) N_{X \cap N}(W) .
$$

But we already have proved the factorization

$$
X=O_{p}(X)(X \cap N) .
$$

In particular, this implies that $O_{p}(X \cap N) \subseteq O_{p}(X)$ and, as

$$
O_{p}(N) \cap X \subseteq O_{p}(N \cap X),
$$

it follows that $O_{p}(N) \cap X \subseteq O_{p}(X)$. Hence, $X \cap N \subseteq O_{p}(X) N_{X}(W)$ and we conclude that

$$
X=O_{p}(X) N_{X}(W) .
$$

This completes the proof of the lemma.

We are now ready to embark on the proof of the proposition.

Proof. We shall treat the case in which $H$ has a normal 2-complement at the end of the proof, so assume to begin with that $H$ is not of this type. Moreover, $H$ is not a $Q D$-group. Indeed, assume the contrary. Since $O(H) \neq 1$, by hypothesis, $O_{r}(H) \neq 1$ for some odd prime $r$ and so $H$ is contained in the local subgroup $K=N\left(O_{r}(H)\right)$. Since $H$ is a $Q D$-group, clearly so also is $K$. But this contradicts Proposition 4.1.1 as $W D \subseteq H \subseteq K$. Since $D \subseteq H$, we can therefore assume, in view of Proposition 4.1.1, that $H$ is a $Q$-group or a $D$-group.

We set $\bar{H}=H / O(H)$ and we claim that there is a unique element of $И_{H}^{*}(\bar{D} ; p)$ containing $\bar{V}$ and this subgroup lies in $U_{H}(\bar{D}, \bar{W} ; p)$; that is, it is permutable with $\bar{W}$. We shall prove this by examining separately each of the two possible types of structures for $H$.

First, suppose that $H$ is a $Q$-group. In this case $\bar{H}$ has characteristic power $q$ by Lemma 2.4.8, as $W \subseteq H$, and so $\bar{H}$ has characteristic $p$. Let $\bar{U}$ be any element of $\bigcup_{H}(\bar{D} ; p)$ which contains $\bar{V}$. Thus, $\bar{U} \subseteq C_{H}(\bar{T})$ by Lemma 2.4.9. However, $P$ is a Sylow $p$-subgroup of $C=C(T)$, so that $\bar{P}$ is a Sylow $p$-subgroup of $C_{H}(\bar{T})$. But $\bar{P} \subseteq \bar{V} \subseteq \bar{U}$, so $\bar{U}=\bar{P}$ and therefore $\bar{P}$ is the unique element of $\eta_{H}(\bar{D} ; p)$ containing $\bar{P}$. Moreover, $W$ and $P$ are permutable by Lemma 3.3, so certainly $\bar{W}$ and $\bar{P}$ are permutable. 
Next, suppose that $H$ is a $D$-group. There are three cases to consider. First, if $\bar{H}$ is isomorphic to $A_{7}$, then $\bar{D}$ is dihedral of order eight and $\bar{D}$ normalizes a unique nonidentity subgroup $\bar{R}$ of odd order in $\bar{H}$; namely $\bar{R}=O\left(C_{H}(\bar{x})\right)$ and $|\bar{R}|=3$, by Lemma 2.5.9. If $p=3$, then $\bar{W}=1$ and $\bar{R} \supseteq \bar{V}$; while if $p \neq 3$, then 1 is the unique element of $\eta_{H}^{*}(\bar{D} ; p)$ containing $\bar{V}$. Either way, the desired properties hold. Second, if $\bar{H}$ is isomorphic to $P G L_{n}(2,3)$ with $n \geqq 2$, which will be the case if $G$ has wreathed Sylow 2-subgroups, then $\bar{D}$ is a Sylow 2-subgroup of $\bar{H}$ and 1 is the unique element of $И_{H}^{*}(\bar{D} ; p)$ by Lemma 2.5.9. Again the desired properties hold.

Assume finally that $\bar{H}$ is not of one of these forms. Then by Proposition 2.3.4, $\bar{H}$ is isomorphic to a subgroup of $P \Gamma L(2, r)$ for some odd $r, \bar{D}$ is dihedral of order eight, and $\bar{H}=\bar{L} \bar{E}$, where $\bar{L}$ is isomorphic to $\operatorname{PSL}(2, r), P G L(2, r)$, or $P G L^{*}(2, r)$, $\bar{L}$ is normal in $\bar{H}, \bar{E}$ is cyclic of odd order, $\bar{L} \cap \bar{E}=1$, and $\bar{E}$ centralizes a Sylow 2-subgroup of $\bar{H}$, which without loss we may assume contains $\bar{D}$. Let $\bar{L}_{0}$ be the subgroup of $\bar{L}$ of index at most two isomorphic to $\operatorname{PSL}(2, r)$ and set $\bar{K}=\bar{L}_{0} \bar{D}$. Since a quasi-dihedral 2-group has only one conjugacy class of four groups and since $\bar{L}_{0}$ contains a four group, it follows, if $\bar{L}$ is isomorphic to $P G L^{*}(2, r)$, that $\bar{D} \subseteq \bar{L}_{0}$ inasmuch as $\bar{D}$ is generated by its four subgroups. Hence, in all cases, $\bar{K}$ is isomorphic to $\operatorname{PSL}(2, r)$ or $P G L(2, r)$. Furthermore, $\bar{E}$ normalizes $\bar{K}$ and $\bar{K} \bar{E}$ is isomorphic to a subgroup of $P \Gamma L(2, r)$. Moreover, since $\bar{K} \bar{E}$ is of index at most two in $\bar{H}$, it is normal in $\bar{H}$ and contains every subgroup of $\bar{H}$ of odd order. In particular, $\bar{K} \bar{E}$ contains $\bar{W}, \bar{V}$, and every element of $\eta_{H}(\bar{D} ; p)$.

Applying Lemma 3.1 (iii) and (vii) of [22] to $\bar{K} \bar{E}$ and using the fact that $\bar{D}$ centralizes $\bar{E}$, it follows now that every element of $И_{H}(\bar{D})$ lies in $C_{R E}(\bar{x})=C_{R}(\bar{x}) \bar{E}$ and that $C_{R}(\bar{x})$ is a dihedral group. Furthermore, $C_{R}(\bar{T})=\bar{T}$ by Lemma 3.1(iii) of [22] and so $C_{R E}(\bar{T})=\bar{T} \bar{E}$ as $\bar{T} \subset \bar{D}$ and $\bar{D}$ centralizes $\bar{E}$. Since $\bar{W}$ and $\bar{P}$ centralize $\bar{T}$, it also follows that $\bar{W} \bar{P} \subseteq \bar{E}$. Moreover, as $P$ is a Sylow $p$-subgroup of $C$ and $C_{H}(T)=C \cap H$ maps onto $C_{H}(\bar{T})$, we see that $\bar{P}$ is a Sylow $p$-subgroup of $\bar{E}$. Since $C_{\bar{R}}(\bar{x})$ is dihedral, we also have that $O_{p}\left(C_{R}(\bar{x})\right)$ is the unique Sylow $p$-subgroup of $C_{\bar{R}}(\bar{x})$ and consequently $\bar{U}=O_{p}\left(C_{R}(\bar{x})\right) \bar{P}$ is the unique Sylow $p$-subgroup of $C_{R E}(\bar{x})$ containing $\bar{P}$. In particular, $\bar{V} \subseteq \bar{U}$ as $\bar{V}$ contains $\bar{P}$. Since $\bar{U}$ is clearly $\bar{D}$-invariant, it allso follows that $\bar{U} \in{h_{H}^{*}}_{H}(\bar{D} ; p)$ and hence $\bar{U}$ is the unique element of $\eta_{H}^{*}(\bar{D} ; p)$ containing $\bar{V}$. Finally, $\bar{W}$ centralizes $\bar{P}$ as each is contained in $\bar{E}$, which is cyclic, and $\bar{W}$ normalizes $O_{p}\left(C_{R}(\bar{x})\right)$, so $\bar{W}$ normalizes $\bar{U}$. Thus $\bar{U} \in U_{H}(\bar{D}, \bar{W} ; p)$ and therefore the desired conclusions hold in this case as well.

Let $Y$ be the subgroup of $H$ containing $O(H)$ such that $\bar{Y}$ is the product of $\bar{W}$ and the unique element of $\eta_{H}^{*}(\bar{D} ; p)$ which we have just described. Thus, $D Y$ is a group and has $Y$ as a normal 2-complement. Moreover, $D Y$ contains $W$ and $V$. We claim that it suffices to verify the proposition with $H$ replaced by $H_{1}=D Y$ provided we show at the same time that $U, U_{1}, U_{2}$ are Sylow $p$-subgroups of $Y$.

Indeed, suppose that all these statements are true; we shall complete the proof. We begin by establishing (i). We have, by assumption, an element $U$ of $h_{H_{1}}(D, W ; p)$ containing $V$ such that $U \in И_{H_{1}}^{*}(D ; p)$ and $U$ is a Sylow $p$-subgroup of $H_{1}$. We 
shall demonstrate that $U$ fulfills all the conditions of (i). However, $O(H)$ is normal in $H_{1}$ as $O(H) \subseteq D Y \subseteq H$, so $U \cap O(H)$ is a Sylow p-subgroup of $O(H)$. Moreover, $U$ and $W$ are permutable as $U \in U_{H_{1}}(D, W ; p)$. Finally, let $U^{*}$ be an element of $\eta_{H}^{*}(D ; p)$ containing $U$. Since $U$ is a Sylow $p$-subgroup of $Y$ and because of the above description of $Y, \bar{U} \in \eta_{H}^{*}(\bar{D} ; p)$. Since $\bar{U}^{*} \in \eta_{H}(\bar{D} ; p)$ and $\bar{U}^{*} \supseteq \bar{U}$, it follows that $\bar{U}^{*}=\bar{U}$ and so $U^{*} \subseteq O(H) U$. But $U$ is a Sylow $p$-subgroup of $O(H) U$ as $U \subseteq O(H) U \subseteq Y$. Since $U \subseteq U^{*}$ and $U^{*}$ is a $p$-group, we conclude that $U^{*}=U$. Thus $U \in \eta_{H}^{*}(D ; p)$ and so all parts of (i) hold.

As for (ii), let $U_{1}$ and $U_{2}$ be as described in the statement of (ii). Thus, $\bar{U}_{1}$ and $\bar{U}_{2}$ are $\bar{D}$-invariant $p$-subgroups of $\bar{H}$ containing $\bar{V}$. Hence, by the uniqueness assertion proved above, we have $U_{1}, U_{2}$ contained in $Y$. Hence, $U_{1}, U_{2}$ are elements of $U_{H_{1}}^{*}(D ; p)$, so the conjugacy holds because it holds in $H_{1}$ by assumption.

Let us pause to state this reduction clearly. We need to establish the following two assertions:

(1) There is an element $U$ of ${U_{H_{1}}}_{(}(D, W ; p)$ containing $V$ such that $U$ is a Sylow p-subgroup of $H_{1}$;

(2) If $U_{1}$ and $U_{2}$ are elements of $\eta_{H_{1}}(D, W ; p)$ containing $V$, both of which are elements of $U_{H_{1}}^{*}(D ; p)$, then $U_{1}$ and $U_{2}$ are conjugate by an element of $N_{C}(W)$ which centralizes $D$.

Observe also that if $H$ itself has a normal 2-complement, the proposition will follow from (1) and (2) with $H_{1}=H$. Hence the proposition will be proved in its entirety once these two statements are established.

We make one further reduction. Let $X$ be a $D$-invariant Hall $\sigma$-subgroup of $Y$ containing $V W$. We claim that we need only show that $X$ has a $D$-invariant Sylow $p$-subgroup containing $V$ and permutable with $W$. Indeed, let $U$ be such a Sylow subgroup of $X$. Clearly $U$ fulfills (1), as $X$ is a Hall subgroup of $Y$ and $Y$ is the normal 2-complement of $H_{1}$. Next, suppose that $U_{1}$ and $U_{2}$ are as stated in the hypothesis of (2). Since $U_{i}$ is a $D$-invariant $p$-subgroup of $Y$ and $U$ is a $D$-invariant Sylow $p$-subgroup of $Y$, there exist elements $c_{i}$ in $C_{Y}(D)$ such that $U_{i}^{c_{i}} \subseteq U, 1 \leqq i \leqq 2$. Since $U_{i} \in \eta_{Y}^{*}(D ; p)$, this in turn implies that $U_{i}^{c_{i}}=U, 1 \leqq i \leqq 2$.

On the other hand, $C_{Y}(D) \subseteq C_{O(C)}(D)$ as $T \subset D$ and $C_{Y}(T) \subseteq O(C)$. But $O(C)$ $=P N_{O(C)}(W)$ by part (iv) of Lemma 3.3 and the fact that $O(C)$ is a normal 2complement in $C$. Since $P$ and $N_{O(C)}(W)$ are each $D$-invariant and $P$ is a Sylow p-subgroup of $O(C)$, Lemma 2.8 .8 now yields that

$$
C_{O(C)}(D)=C_{P}(D)\left(N_{O(C)}(W) \cap C(D)\right) .
$$

Hence $c_{i}=b_{i} u_{i}$, where $b_{i} \in N_{C}(W), b_{i}$ centralizes $D$, and $u_{i} \in P, 1 \leqq i \leqq 2$. Since $P \subseteq V \subseteq U$, this implies that $U_{i}^{b_{i}}=U, 1 \leqq i \leqq 2$, and so $U_{2}=U_{1}^{b}$, where $b=$ $b_{1} b_{2}^{-1} \in N_{C}(W)$ and $b$ centralizes $D$. Thus (2) also holds and our claim is valid.

Thus, in order to establish the proposition in full, it remains only to produce a $D$-invariant Sylow $p$-subgroup of $X$ which contains $V$ and is permutable with $W$. Since $V$ is $D$-invariant, there is a $D$-invariant Sylow $p$-subgroup $U$ of $X$ containing 
$V$. We assert that $U$ is automatically permutable with $W$. Indeed, if $W=1$, this is obvious. On the other hand, if $W \neq 1$, then $X=O_{p}(X) N_{X}(W)$ by Lemma 5 and again our assertion is obvious.

5. Construction of a certain signalizer. We shall demonstrate here the existence of a signalizer with certain special properties. We shall then prove a transitivity result which uses this constructed subgroup. These results will be proved under the following assumptions: the prime $p$ is, as in the preceding two sections, the characteristic of $G$; and, in addition, $p$ is exceptional, but not totally inverted.

Proposition 1. If $W \in \mathscr{W}$ and $P$ is a $D$-invariant Sylow p-subgroup of $C$, then there is $V \in U(D, W ; p)$ containing $P$ such that

(i) $O_{p^{\prime}}(V W)=1$;

(ii) $V W D$ is contained in an element of $\mathscr{L}(p)$ and in an element of $\mathscr{M}(p)$.

The results of $\$ \$ 7$ and 8 of Chapter IV apply to any $D$-invariant Sylow $p$-subgroup of $C$ and, in particular, to $P$. Let $Q$ be the characteristic subgroup of $P$ there constructed.

Proof. We begin by applying Proposition 4.1 with $N$ in place of $H$ and $V=P$. (Note that $O(N) \neq 1$ as our conditions imply that $p$ divides $|C \cap O(N)|$.) We deduce that there is $U \in \eta_{N}(D, W ; p)$ containing $P$ such that $U \in h_{N}^{*}(D ; p)$ and $R=U \cap O(N)$ is a $D$-invariant Sylow p-subgroup of $O(N)$. Moreover, $U$ and $W$ are of coprime order, so that $R=U \cap O(N)$ is normalized by $W$, by Lemma 2.8.3, as $W \cap O(N)=1$.

We shall construct $V$ by separate but similar consideration of two possibilities. First, suppose that $p$ is constrained ; that is, by Definition $4.2 .1, N$ is $p$-constrained within $S(N)$. In this case, we set $V=P N_{R}(Q)$.

Since $P \subseteq U$ and $R=U \cap O(N)$, it follows that $P$ normalizes $R$. Hence, as $Q$ is a characteristic subgroup of $P$, we have that $P$ normalizes $N_{R}(Q)$ and so $V$ is a nonidentity $p$-subgroup which certainly contains $P$.

The subgroup $R$ is $D$-invariant, as we saw above. Furthermore, $P$ is $D$-invariant by choice and $Q$ is a characteristic subgroup of $P$, so that $Q$ is also normalized by $D$. Hence $N_{R}(Q)$ is $D$-invariant, so $V=P N_{R}(Q)$ is also.

Moreover, $R$ is normalized by $W$, as we also saw, and $Q$ is $W$-invariant, by part (v) of Lemma 3.3, as $p$ is exceptional and not totally inverted by assumption, so that $N_{R}(Q)$ is normalized by $W$. Since $W$ and $P$ are permutable, by part (iii) of Lemma 3.3, it follows that $W$ and $V$ are permutable and so $V \in U(D, W ; p)$. In particular, as $W$ is $D$-invariant, being an element of $\mathscr{W}$, we have that $V W D$ is a subgroup of $G$.

We have seen that $P, W$ and $D$ normalize $R$; hence $V W D \subseteq N(R)$ as $N_{R}(Q) \subseteq R$ and $V=P N_{R}(Q)$. But $N(R)$ is an element of $\mathscr{L}(p)$, by the Frattini argument and Definition 4.1.4. Furthermore, $N(Q) \in \mathscr{M}(p)$, by Lemma 4.8.1 and Definition 4.8.1. 
Hence, in order to establish the proposition in the case under examination, it remains to show that $O_{p^{\prime}}(V W)=1$. However, $W$ normalizes $Q$ and $N_{R}(Q)$, so $V_{0}=Q N_{R}(Q)$ is a $p$-subgroup of $V$ normalized by $W$. Thus, if we set $W_{0}=C_{W}\left(V_{0}\right)$, then it suffices to show that $W_{0}=1$. However, $W_{0}$ is a $p^{\prime}$-group, $Q$ is a $p$-group, and $W_{0}$ centralizes $Q$ as $Q \subseteq V_{0}$. Moreover, $C_{N}(R) \subseteq S(N)$ by Lemma 4.2 .1 as $p$ is constrained, while $W \cap S(N)=1$ by part (i) of Lemma 3.3 and the fact that $O(N)$ is a normal 2-complement of $S(N)$. Thus $W$ acts faithfully on $R$, so certainly $W_{0}$ does also. Hence, $W_{0}$ acts faithfully on $C_{R}(Q)$ by Theorem 5.3.4 of [19]. But $C_{R}(Q) \subseteq V_{0}$, so $W_{0}=1$, as required.

Next, suppose that $p$ is not constrained and set $K=N(R)$. Thus, again $K \in \mathscr{L}(p)$ and now $K$ is $p$-constrained in $S(K)$ by Proposition 4.6.1. We claim that $O(K)$ possesses a $P W D$-invariant Sylow $p$-subgroup $F$. (Note that our above arguments show that $P W D$ is a group and a subgroup of $K$.) Indeed, there is $U_{1} \in \eta_{K}(D, W ; p)$ such that $U_{1}$ contains $P, U_{1} \in h_{K}^{*}(D ; p)$, and $U_{1} \cap O(K)$ is a $D$-invariant Sylow $p$-subgroup of $O(K)$, by Proposition 4.1. We set $F=U_{1} \cap O(K)$. Since $P \subseteq U_{1}$, we need only see that $F$ is normalized by $W$ to verify our assertion. But $W \cap O(K)$ $\subseteq N \cap O(K)$ and $N \cap O(K) \subseteq O(N)$, by Lemma 4.1.1, so that $W \cap O(K)=1$. Since $W$ and $U_{1}$ are permutable, it now follows from Lemma 2.8.3 that $W$ normalizes $F$.

The remainder of the proof in this case is very similar to the corresponding part of the previous argument. We set $V=P N_{F}(Q)$ and $V$ is a nonidentity $D$-invariant $p$-subgroup of $G$. Furthermore, $V$ certainly contains $P$ and again $V$ is permutable with $W$ since $W$ is permutable with $P$ and normalizes both $Q$ and $F$. Also $V W D$ $\subseteq K \in \mathscr{L}(p)$ and $V W D \subseteq N(Q) \in \mathscr{M}(p)$. Again $V_{0}=Q N_{F}(Q)$ is a $p$-subgroup of $V W$ normalized by $W$. Moreover, $C_{K}(F) \subseteq S(K)$ as $K$ is $p$-constrained within $S(K)$. Since $W \cap O(K)=1$ and $O(K)$ is a normal 2-complement in $S(K)$, it follows that $W \cap S(K)=1$ and so $W$ acts faithfully on $V_{0}$. Again, we derive that $O_{p^{\prime}}(V W)=1$ in the same way as before and the proposition is proved.

The next and last result of this section is a key transitivity theorem. The subgroups $V$ and $W$ are as in Proposition 1.

Proposition 2. Any two elements of $\mathrm{U}_{G}^{*}(D, W ; p)$ which contains $V$ are conjugate by an element of $N_{C}(W)$.

First, we require a consequence of a result of the previous section.

LEMMA 1. If $U$ is an element of $U(D, W ; p)$ and $U$ contains $P$, then $U=O_{p}(U W) P$.

Proof. We set $X=U W$, so $X$ is a $\sigma$-subgroup, since $U$ and $W$ are permutable, by hypothesis. Thus, $X$ is a $D W$-invariant $\sigma$-subgroup of $G$, so that $O_{p}(X)(X \cap C)$ contains a Sylow $p$-subgroup of $X$, by Lemma 4.5. However, $X=U W, W \subseteq C$, and $U$ contains the Sylow $p$-subgroup $P$ of $C$, so that $X \cap C=P W$. But $W$ is a $p^{\prime}$-group, so $O_{p}(X) P$ is a Sylow $p$-subgroup of $O_{p}(X)(X \cap C)$. However, $O_{p}(X) P$ 
$\subseteq U$ and $U$ is a Sylow $p$-subgroup of $X$, so that $O_{p}(X) P=U$ and the lemma is proved.

We now turn to the proposition.

Proof. We assume the result is false and argue to a contradiction. Choose elements $U_{1}, U_{2}$ of $U_{G}^{*}(D, W ; p)$, each of which contains $V$, but which are not conjugate by an element of $N_{C}(W)$. Furthermore, among all such pairs of subgroups, choose this one maximizing $\left|U_{1} \cap U_{2}\right|$.

We set $U=U_{1} \cap U_{2}$, so that $U$ is also $D$-invariant. Moreover, $U \subset U_{i}, i=1,2$, by the maximality of $U_{i}$ among elements of $\eta_{G}(D, W ; p)$ and since $U_{1} \neq U_{2}$. We now claim that even $U$ is permutable with $W$. Indeed, by the previous lemma, since $P \subseteq V \subset U_{i}, i=1,2$, we have that

$$
U_{i}=O_{p}\left(W U_{i}\right) P
$$

Hence, as $P \subseteq U$,

$$
U=U_{1} \cap U_{2}=\left(O_{p}\left(W U_{1}\right) \cap O_{p}\left(W U_{2}\right)\right) P .
$$

But $W$ is permutable with $P$, by Lemma 3.3, while $W$ normalizes $O_{p}\left(W U_{1}\right)$ and $O_{p}\left(W U_{2}\right)$. Thus, $W$ is certainly permutable with $U$, as asserted.

Hence, $U W$ is a subgroup of $G$. Moreover, $O_{p^{\prime}}(U W)=1$. Indeed, $V W \subseteq U W$, $W$ is a p-complement of both $V W$ and $U W$, while $O_{p^{\prime}}(V W)=1$, by Proposition 1 . Thus, since $U W$ has odd order and $U$ is a Sylow $p$-subgroup of it, we have by Theorem 2.7.1, that $Z(J(U))$ is a normal subgroup of $U W$.

We now set $H=N(Z(J(U)))$. It follows that $O(H) \neq 1$ as $1 \subset P \subseteq U$. Moreover, $W \subseteq H$ as we just saw that $U W \subseteq H$. Hence, since $W$ and $U_{i}, i=1,2$, are permutable, so are $W$ and $U_{i} \cap H$. Thus, as $D$ normalizes $U$, we have that $U_{i} \cap H \in \eta_{H}(D, W ; p)$. Furthermore, $U_{i} \cap H \supset U$ since $U_{i} \supset U$ and $Z(J(U))$ is a characteristic subgroup of $U$.

By Proposition 4.1, we may choose $P_{i}$ an element of $И_{H}(D, W ; p)$ which is in $U_{H}^{*}(D ; p)$ and contains $U_{i} \cap H, i=1,2$. Also, by the same result, there is $c \in N_{C}(W)$ with $c$ centralizing $D$ such that $P_{2}=P_{1}^{c}$. We now let $Q_{i}, i=1,2$, be an element of $\eta_{G}^{*}(D, W ; p)$ which contains $P_{i}$. Thus, $U_{1}, Q_{1}, Q_{1}^{c}, Q_{2}$ and $U_{2}$ are elements of $U_{G}^{*}(D, W ; p)$. Moreover,

$$
U_{1} \cap Q_{1} \supseteq U_{1} \cap P_{1} \supseteq U_{1} \cap H \supset U=U_{1} \cap U_{2},
$$

so that $U_{1}$ and $Q_{1}$ are conjugate by an element of $N_{C}(W)$, by our choice of $U_{1}$ and $U_{2}$. Also, $Q_{1}$ and $Q_{1}^{c}$ are clearly so conjugate. Furthermore,

$$
Q_{1}^{c} \cap Q_{2} \supseteq P_{1}^{c} \cap P_{2}=P_{2} \supseteq U_{2} \cap H \supset U,
$$

so $Q_{1}^{c}$ and $Q_{2}$ are conjugate by an element of $N_{C}(W)$. Finally,

$$
Q_{2} \cap U_{2} \supseteq P_{2} \cap U_{2} \supseteq U_{2} \cap H \supset U,
$$

and so $Q_{2}$ and $U_{2}$ are conjugate by an element of $N_{C}(W)$. Hence, so are $U_{1}$ and $U_{2}$, which is a contradiction. This proves the proposition. 
6. Elimination of the second set of primes. The end product of the argument developed in the previous three sections is as follows:

Proposition 1. If $p$ is a prime divisor of $|O(N)|$ and $p$ is not totally inverted, then, $p$ is ordinary.

Proof. If $p$ is other than the characteristic of $G$, then the assertion has already been dealt with in Proposition 2.1. Hence, we shall assume that $p$ is the characteristic of $G$, not totally inverted and exceptional; we need only derive a contradiction.

Since $p$ fulfills all the conditions of the last three sections, we choose $W \in \mathscr{W}$ and fix it and we choose $V \in \boldsymbol{U}_{G}(D, W ; p)$ in accordance with Proposition 5.1.

We shall, in fact, prove that there exist elements $U_{i}, i=1,2$ of $\eta_{G}^{*}(D, W ; p)$, each containing $V$ such that if $H_{i}=N\left(Z\left(J\left(U_{i}\right)\right)\right), i=1,2$, then $H_{1} \in \mathscr{L}(p)$ and $H_{2} \in \mathscr{M}^{*}(p)$. This will give the desired contradiction. Indeed, this yields that $H_{1}$ is a $Q$-group, by Lemma 4.1.1, $H_{2}$ is a $D$-group, by Definition 4.9.1, so certainly $H_{1}$ and $H_{2}$ are not conjugate. However, $H_{1}$ and $H_{2}$ are conjugate as $U_{1}$ and $U_{2}$ are conjugate by Proposition 5.2.

By Proposition 5.1 we know that $V W D$ is contained in an element $K_{1}$ of $\mathscr{L}(p)$ and in an element $K_{2}$ of $\mathscr{M}^{*}(p)$. Let $U_{i} \in U_{K_{i}}^{*}(D, W ; p), i=1,2$ with $V \subseteq U_{i}$. Among all such pairs of subgroups, we may assume that $\left|U_{1}\right|$ and $\left|U_{2}\right|$ are maximized.

We now assert that it suffices to show that $H_{1}=N\left(Z\left(J\left(U_{1}\right)\right)\right) \in \mathscr{L}(p), H_{2}=$ $N\left(Z\left(J\left(U_{2}\right)\right)\right) \in \mathscr{M}^{*}(p)$ and $W \subseteq H_{i}, i=1,2$. Indeed, assume that this is the case; we need only show that $U_{i} \in h_{G}^{*}(D, W ; p), i=1,2$. Thus, suppose that $U_{j} \notin$ $\eta_{G}^{*}(D, W ; p)$ for $j=1$ or 2 ; we shall derive a contradiction. We now have that there is $V_{j} \in U_{G}^{*}(D, W ; p)$ with $U_{j} \subset V_{j}$; consequently, $U_{j} \subset V_{j}^{*}=N_{V_{j}}\left(U_{j}\right)$. Thus, there are $D$-invariant $p$-subgroups of $H_{j}$ properly containing $U_{j}$. However, Proposition 4.1 implies that every element of ${И_{H}^{*}}_{H_{j}}(D, W ; p)$ containing $V$ is an element of $U_{H_{j}}^{*}(D ; p)$. In particular, there is $S_{j} \in U_{H_{j}}^{*}(D, W ; p)$ with $U_{j} \subset S_{j}$. Thus, $H_{j}$ contradicts the maximal nature of our choice of $K_{j}$. This contradiction establishes our assertion.

We begin by demonstrating that $W \subseteq H_{i}, i=1,2$. To do this, it suffices to show that $W \subseteq N_{W U_{i}}\left(Z\left(J\left(U_{i}\right)\right)\right)$. However, $O_{p^{\prime}}(V W)=1$, by Proposition 5.1, so that $O_{p},\left(U_{i} W\right)=1$ as $V \subseteq U_{i}$ and $W$ is a $p$-complement of $U_{i} W$. Since $U_{i} W$ is of odd order, Theorem 2.7.1 now yields that $Z\left(J\left(U_{i}\right)\right)$ is normal in $W U_{i}$, as required.

We now shall deal with $H_{1}$. In view of Proposition 4.6.2, in order to show that $H_{1} \in \mathscr{L}(p)$, it suffices to prove that $R_{1}=Z\left(J\left(U_{1}\right)\right)$ is a nonidentity $D$-invariant p-subgroup of $O\left(K_{1}\right)$ and that $N\left(R_{1}\right)=H_{1}$ covers $K_{1} / O\left(K_{1}\right)$ as well as contains a Sylow $p$-subgroup of $O\left(K_{1}\right)$.

However, $K_{1}$ is $p$-constrained in $S\left(K_{1}\right)$ by Proposition 4.6.1. Since $K_{1} \in \mathscr{L}(p)$, we have that $K_{1}$ is a $Q$-group of characteristic power $q$ and hence also of characteristic $p$. Moreover, $q>5$ by assumption and $U_{1} \in h_{K_{1}}^{*}(D ; p)$. Thus the hypotheses of Proposition 2.7.1 are satisfied and we conclude that $K_{1}=O_{p^{\prime}}\left(K_{1}\right) N_{K_{1}}\left(R_{1}\right)$. In particular, $H_{1}$ contains $U_{1}$ and so by Proposition $4.1 H_{1}$ contains a Sylow $p$ - 
subgroup of $O\left(K_{1}\right)$. Moreover, as $U_{1}$ is $D$-invariant, being an element of $\eta_{K_{1}}^{*}(D, W ; p)$ and as $U_{1} \neq 1$ since $U_{1} \supseteq P$, it follows that $R_{1}$ is a nonidentity $D$ invariant $p$-subgroup of $K_{1}$. Finally, $O_{p^{\prime}}\left(K_{1}\right) \subseteq S\left(K_{1}\right)$, by definition of $p$-constraint within $S\left(K_{1}\right)$ and $O_{p^{\prime}}\left(K_{1}\right) R_{1}$ is normal in $K_{1}$ by the above factorization of $K_{1}$. Hence $O_{p^{\prime}}\left(K_{1}\right) R_{1} \subseteq S\left(K_{1}\right)$. But $S\left(K_{1}\right)=O\left(K_{1}\right) Z(D)$ by Lemma 2.4.14 as $K_{1} \in \mathscr{L}(p)$ and consequently $R_{1} \subseteq O\left(K_{1}\right)$. Furthermore, as $Z(D)$ normalizes $R_{1}$, our factorization of $K_{1}$ implies that $N_{K_{1}}\left(R_{1}\right)$ covers $K_{1} / O\left(K_{1}\right)$.

It remains to show that $H_{2}$ is an element of $\mathscr{M}^{*}(p)$. Arguing now on $U_{2}$ as we did above on $U_{1}$, it follows that $R_{2}$ is a nonidentity $D$-invariant $p$-subgroup of $G$, so $H_{2}$ is a $p$-local subgroup of $G$ containing $D$. Hence, by Definition 4.9.1, we need only prove that $\mathrm{O}_{p^{\prime}}\left(\mathrm{H}_{2}\right) \subseteq O\left(\mathrm{H}_{2}\right)$ and that $\mathrm{H}_{2}$ is a $D$-group with respect to $T$.

However, $K_{2}$ is $p$-constrained within $O\left(K_{2}\right)$, by Proposition 4.9.1, as $K_{2} \in \mathscr{M}^{*}(p)$. Hence, by Proposition 2.7.2, we have $K_{2}=O_{p^{\prime}}\left(K_{2}\right)\left(K_{2} \cap H_{2}\right)$ inasmuch as $U_{2} \in$ $\eta_{K_{2}}^{*}(D ; p)$. But $O_{p^{\prime}}\left(K_{2}\right) \subseteq O\left(K_{2}\right)$ by the constraint, so $K_{2}=O\left(K_{2}\right)\left(K_{2} \cap H_{2}\right)$.

Since $K_{2} \in \mathscr{M}^{*}(p)$, it is a $D$-group with respect to $T$, so that $A_{K_{2}}(T)=$ Aut $(T)$. The last factorization stated for $K_{2}$ yields that $A_{K_{2} \cap H_{2}}(T)=$ Aut $(T)$ and so $A_{H_{2}}(T)=$ Aut $(T)$. Since $D \subseteq H_{2}$, it follows therefore from Proposition 4.1.1 that either $H_{2}$ is a $D$-group with respect to $T$ or a $Q D$-group. However, $W D \subseteq H_{2}$, so $\mathrm{H}_{2}$ is a local subgroup of $G$ which covers $C \cap L / C \cap O(N)$ and contains $T$. Proposition 4.1.1 therefore implies that $\mathrm{H}_{2}$ is not a $Q D$-group.

Finally, let $S$ be a Sylow 2-subgroup of $\mathrm{H}_{2}$ containing $D$, so that either $S$ is dihedral or quasi-dihedral or else $S=D$ and $S$ is wreathed. We conclude in each case that any nonidentity normal subgroup of $S$ intersects $T$ nontrivially. Hence if $S \cap O_{p^{\prime}}\left(H_{2}\right) \neq 1$, then $T \cap O_{p^{\prime}}\left(H_{2}\right) \neq 1$, so $T \subseteq O_{p^{\prime}}\left(H_{2}\right)$ as all elements of $T^{\#}$ are conjugate in $H_{2}$. Thus $T \subseteq K_{2} \cap O_{p^{\prime}}\left(H_{2}\right) \subseteq O_{p^{\prime}}\left(K_{2}\right)$, the latter inclusion holding because $H_{2}$ covers $K_{2} / O_{p^{\prime}}\left(K_{2}\right)$. This implies that $T \subseteq O\left(K_{2}\right)$ as $O_{p^{\prime}}\left(K_{2}\right) \subseteq O\left(K_{2}\right)$ and this is a contradiction. Thus $S \cap O_{p^{\prime}}\left(H_{2}\right)=1$ and we conclude that $O_{p^{\prime}}\left(H_{2}\right) \subseteq O\left(H_{2}\right)$. This completes the proof.

The proposition has a direct consequence for the structure of $N$; namely, the

COROLlaRY 1. The subgroup $N$ satisfies the following conditions:

(i) Any T-invariant subgroup of $N$ is centralized or inverted by $x_{2}$.

(ii) $L_{0}$ has a normal subgroup $J$ such that $L_{0}=J O(N)$ and either

(a) $J$ is isomorphic to $\operatorname{SL}(2, q)$ and $J \cap O(N)=1$, or

(b) $J$ is isomorphic to $S L^{\wedge}(2,9), q=9,|J \cap O(N)|=3$, and $G$ has quasidihedral Sylow 2-subgroups.

(iii) $J$ centralizes $O(N)$.

(iv) A Hall subgroup of $O(N)$, for the set of inverted primes, is normal in $N$.

The first three parts of the statement of this result (with the exception of the assertion concerning quasi-dihedral Sylow 2-subgroups in part (b) of (ii)) are obvious analogues of the three assertions of Corollary 2.1. Moreover, the proof is almost the same. 
Proof. The above proposition shows that every prime divisor of $|O(N)|$ is ordinary or exceptional and totally inverted. But ordinary primes are centralized or inverted by Definition 4.1.3, so every prime divisor of $|O(N)|$ is centralized or inverted. Thus, the set $\pi$ of primes which are neither centralized nor inverted is empty and $O_{\pi}(O(N))=1$. The statements (i), (ii), and (iii) are now direct consequence of Propositions 4.4.1 and 4.5.1.

We now prove the last statement of the corollary. In fact, let $A_{0}$ be a $T$-invariant Hall subgroup of $O(N)$ for the set of centralized primes, so that $x_{2}$ centralizes $A_{0}$. Let $B_{0}$ be a $T$-invariant Hall subgroup of $O(N)$ for the set of inverted primes. Hence $O(N)=A_{0} B_{0}$ and this implies that $B_{0}$ is precisely the set of elements of $O(N)$ inverted by $x_{2}$. Indeed, if an element $a b$ of $O(N)$ with $a \in A_{0}$ and $b \in B_{0}$ is inverted by $x_{2}$, then $(a b)^{-1}=a b^{-1}$ and so $b^{-1} a^{-1} b=a$, whence $a=1$ as $a$ and $b$ are of odd order. But the subset of $O(N)$ inverted by $x_{2}$ is always a normal subset, so $B_{0}$ is normal in $O(N)$, as required.

7. The structure of $N$. In this and the following four sections we again let $p$ be an exceptional prime. By the results of the previous section we know that $P$ is a totally inverted prime; that is, $x_{2}$ inverts a $T$-invariant Sylow p-subgroup of $O(N)(C \cap L)$. In particular, as $T$ certainly centralizes $C \cap L$, it follows that this $p$-subgroup is contained in $O(N)$. Our purpose in these final five sections is to show that no such prime $p$ exists, by deriving yet another contradiction. We shall do this much in the spirit of an earlier work (Lemma 6.12 of [22]).

The final result of the previous section is a detailed description of $N$ which is a consequence of our knowledge of the nature of the exceptional primes. In this section, we shall use this information to derive some useful facts about $N$. They will be used in the subsequent sections.

We begin by fixing a great deal of notation for the remainder of the chapter. Let $W$ denote the 2-complement of $C \cap J$, so that $W$ is cyclic of order $(q+\delta \varepsilon) / 2^{n}$, by Lemma 2.4.2. We note that $W$ is a $p^{\prime}$-group. Indeed, the Sylow $p$-subgroups of $O(N)(C \cap L)$ are contained in $O(N)$, by an above remark. In addition, $J \cap O(N)=1$ or $q=9, J \cap O(N)$ is of order three, and $S$ is quasi-dihedral by Corollary 1 of the preceding section. Furthermore, $J \cap O(N)$ is not contained in $C$ by Lemma 2.4.12.

Let $B^{*}$ be a $T$-invariant Hall subgroup of $O(N)$ for the set of inverted primes and let $A$ be the usual $T$-invariant Hall subgroup of $O(N)$ for the centralized ordinary primes. Proposition 1 and Corollary 1 of the previous section now imply that $O(N)=A B^{*}$. As a consequence, we have that $O(C \cap L)=W A$ as $J$ and $O(N)$ commute elementwise and $W=O(C) \cap J$. Moreover, $A$ is a $p^{\prime}$-group as $p$ is inverted. Hence, $O_{p}(C)=1$ as $W$ is also a $p^{\prime}$-group; this is a fact we shall constantly use.

Let $a$ be a fixed 3-element of $M, a \notin C$, so $a$ cyclically permutes the nonidentity elements $x=x_{1}, x_{2}, x_{3}$ of $T$; we may assume that $x_{1}^{a}=x_{2}, x_{2}^{a}=x_{3}$ and $x_{3}^{a}=x_{1}$. We set $N_{i}=C\left(x_{i}\right), 1 \leqq i \leqq 3$, so that $N=N_{1}$, while $N_{1}^{a}=N_{2}, N_{2}^{a}=N_{3}$ and $N_{3}^{a}=N_{1}$. Similarly, we set $J_{1}=J, J_{2}=J^{a}, J_{3}=J^{a^{2}}$ and define $D_{i}, W_{i}, A_{i}, B_{i}^{*}, 1 \leqq i \leqq 3$. Thus, 
each of the groups $N_{i}$ has the same structure as $N$ but with respect to $D_{i}, A_{i}, B_{i}^{*}$, and so on, instead of $D, A, B^{*}$ and so forth. Next, set $P_{i}=O_{p}\left(N_{i}\right), 1 \leqq i \leqq 3$, so $P_{i}$ is the unique Sylow $p$-subgroup of the abelian group $B_{i}^{*}$ and $P_{1}^{a}=P_{2}, P_{2}^{a}=P_{3}$, $P_{3}^{a}=P_{1}$.

If $\delta \varepsilon=-1$ and $p$ divides $q$ then, by Lemma 2.4 .10 , there are precisely two Sylow $p$-subgroups of $J_{i}, 1 \leqq i \leqq 3$, normalized by $C$ and these contain all the $C$-invariant $p$-subgroups of $J_{i}$. Let these be $Q_{i}$ and $Q_{i}^{*}$; so we may assume that $Q_{1}^{a}=Q_{2}, Q_{2}^{a}=Q_{3}$, $Q_{3}^{a}=Q_{1}$ and similar equations hold for $Q_{1}^{*}, Q_{2}^{*}, Q_{3}^{*}$, as $a^{3} \in C \subseteq N\left(Q_{1}\right) \cap N\left(Q_{1}^{*}\right)$. Moreover, by Lemma 2.4.10, $Q_{i}$ and $Q_{i}^{*}$ are conjugate by an element $z_{i}$ of $D_{i}$, while $J_{i}=\left\langle Q_{i}, Q_{i}^{*}\right\rangle, 1 \leqq i \leqq 3$. Note that $P_{i} Q_{i}, P_{i} Q_{i}^{*}$ are direct products. Indeed, $J$ and $O(N)$ form a direct product, for otherwise $q=9$ and $S$ is quasi-dihedral, in which case $\delta=1$ and $\varepsilon=1$, contrary to our present assumption that $\delta \varepsilon=-1$. Finally, recall by Lemma 2.4 .10 that $Z\left(D_{i}\right)$ acts trivially on $Q_{i}$ and $Q_{i}^{*}$ and that $C \cap L_{i} / Z\left(D_{i}\right)=W_{i} C_{D_{i}}(T) / Z\left(D_{i}\right)$ acts faithfully and irreducibly on $Q_{i}$ and $Q_{i}^{*}$, $1 \leqq i \leqq 3$.

We now examine the set of $p$-subgroups of $N_{i}, 1 \leqq i \leqq 3$, permutable with $C$.

LEMMA 1. If $1 \leqq i \leqq 3$, then the elements of ${И_{N}}_{i}^{*}(C ; p)$ are as follows:

(i) $P_{i} Q_{i}$ and $P_{i} Q_{i}^{*}$ if $\delta \varepsilon=-1$ and $p$ divides $q$;

(ii) $P_{i}$ in all other cases.

Proof. The subgroups given are elements of $И_{N_{i}}(C ; p)$; hence, we need only show that any element $U \in{И_{N}}_{N_{i}}(C ; p)$ is contained in one of them. First, we show,

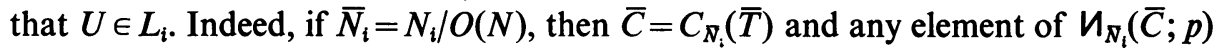
lies in $\bar{L}_{\boldsymbol{i}}$ by Lemma 2.4.9.

However, $U O\left(N_{i}\right) \cap J_{i}$ is a $C$-invariant $p$-subgroup of $J_{i}$, so $J_{i} \cap U O\left(N_{i}\right) \subseteq Q_{i}$ or $Q_{i}^{*}$ (where we set $Q_{i}=Q_{i}^{*}=1$ if $\delta \varepsilon=1$ or if $p$ does not divide $q$ ). Hence $U \subseteq Q_{i} O\left(N_{i}\right)$ or $Q_{i}^{*} O\left(N_{i}\right)$. Each of these products is a direct product (as $Q_{i}=Q_{i}^{*}=1$ if $q=9$ ) and $P_{i}$ is the unique Sylow $p$-subgroup of $O\left(N_{i}\right)$. The result now follows immediately.

Let $P$ be our usual $T$-invariant Sylow $p$-subgroup of $C$. Since $O(C) \cap L=W A$ is a $p^{\prime}$-group, we have that $P \cap L=1$ and $P \cap L_{i}=1,1 \leqq i \leqq 3$. Thus, $P$ "plays the same role" in all the groups $N_{i}, 1 \leqq i \leqq 3$. We reserve the notation $P_{i}, 1 \leqq i \leqq 3$, for the subgroups defined above. The final result of this section is as follows:

LEMMA 2. The elements of $И_{N_{i}}^{*}(T, C ; p)$ which contain $P, 1 \leqq i \leqq 3$, are as follows:

(i) $P Q_{i} P_{i}$ and $P Q_{i}^{*} P_{i}$ if $\delta \varepsilon=-1$ and $p$ divides $q$;

(ii) $P P_{i}$ otherwise.

Proof. Let $U \in \eta_{N_{i}}^{*}(T, C ; p)$ with $P \subseteq U$ and set $\bar{N}_{i}=N_{i} / O\left(N_{i}\right)$. Since $\bar{U}$ is permutable with $\bar{C}$, it follows from Lemma 2.4.11 that $\bar{U} \subseteq \bar{C} \bar{V}$, where $\left.\bar{V} \in{И_{N_{i}}}_{\bar{C}} ; p\right)$. Hence, $\bar{U} \subseteq \bar{C} \bar{Q}_{i}$ or $\bar{C} \bar{Q}_{i}^{*}$ by Lemma 2.4.10. But $\bar{U} \supseteq \bar{P}$ and $\bar{P} \bar{Q}_{i}, \bar{P} \bar{Q}_{i}^{*}$ are Sylow $p$-subgroups of $\bar{C} \bar{Q}_{i}$ and $\bar{C} \bar{Q}_{i}^{*}$, respectively. Thus $U \subseteq P Q_{i} O\left(N_{i}\right)$ or $P Q_{i}^{*} O\left(N_{i}\right)$. Again, the only Sylow $p$-subgroups of $P Q_{i} O\left(N_{i}\right)$ and $P Q_{i}^{*} O\left(N_{i}\right)$ which contain $P$ are $P Q_{i} P_{i}$ and $P Q_{i}^{*} P_{i}$ respectively, which proves the lemma. 
8. An omnibus lemma for signalizers. In eliminating the prime $p$ we shall be dealing extensively with the $p$-subgroups of $G$ normalized by the 2-groups $T$ and $D$. This section contains one result which collects all the necessary elementary facts about such signalizers in one long lemma. Only in the next section do we get deeper into the proof and prove a useful transitivity theorem.

Because we shall only be dealing with the one fixed prime $p$ we shall simplify the notation. We shall write $\eta_{X}(Y)$ instead of ${U_{X}}_{X}(Y, p)$ and systematically drop the symbol $p$ from all such notation for the remainder of the chapter. Of course, all the notation fixed in the previous section will be used fully with no further explanation.

The omnibus lemma is as follows:

Lemma 1. Let $H$ be any subgroup of $G$ containing $C$ and let $U \in \eta_{H}^{*}(T, C)$.

(i) In the T-decomposition of $U$ we have

(a) $U_{i} \in h_{H}(T, C), 1 \leqq i \leqq 3$;

(b) $U_{i}^{\prime}$ is a $C$-invariant subgroup of $P_{i}, P_{i} Q_{i}$ or $P_{i} Q_{i}^{*}$.

(ii) Moreover, $U$ contains the following subgroups:

(a) A T-invariant Sylow p-subgroup of $C$;

(b) A Sylow p-subgroup of $O(H)$.

(iii) If $O(H) \neq 1$ then

(a) $U=O_{p}(U C)(U \cap C)$;

(b) $U=(U \cap O(H))\left(U \cap N_{j}\right)$ for some $j, 1 \leqq j \leqq 3$.

(iv) If $O(H) \neq 1$ and $U^{*} \in h_{H}^{*}(T, C)$ then $U$ and $U^{*}$ are conjugate by an element of $M \cap H$.

(v) The subgroup UC has a normal 2-complement which is nontrivial if $W \neq 1$.

(vi) If $V=O_{p}(U C)$, then $V \in U_{H}^{*}(C)$ and $V$ contains every element of $U_{H}(C)$ contained in $U$.

We shall prove this lemma part by part but in an order different from the given one.

Proof of (i)(a). Since $U$ is $T$-invariant and $T$ is abelian, it follows that each $U_{i}, 1 \leqq i \leqq 3$, is a $T$-invariant subgroup of $H$. Hence, we need only see that $U_{i}$ and $C$ are permutable, that is, $C U_{i}$ is a subgroup. However, $C \subseteq N_{i}$, so that $N_{i} \cap C U$ $=C\left(N_{i} \cap U\right)=C U_{i}$.

Proof of (v). Set $F=C_{D}(T)$. Since $F$ is a Sylow 2-subgroup of $C$ and $U$ has odd order, $F$ is a Sylow 2-subgroup of $C U$. Moreover, $F$ is abelian of type $\left(2^{n}, 2^{n}\right)$ and $T=\Omega_{1}(F)$. We claim that $N_{C U}(F) \subseteq C_{C V}(F)$. Indeed, $N_{C U}(T)=C N_{U}(T) \subseteq C$ since $N_{U}(T)$ and $T$ commute elementwise as $U$ is $T$-invariant. Since $T$ is characteristic in $F$, it follows that $N_{C U}(F)$ centralizes $T$. Since $T=\Omega_{1}(F)$, every element of $N_{C U}(F)$ of odd order is thus forced to centralize $F$, whence $N_{C U}(F) \subseteq C_{C U}(F)$, as asserted. Hence, by a theorem of Burnside, $C U$ has a normal 2-complement. Since $W$ has odd order, $W \subseteq O(C U)$ and so this normal 2-complement is nontrivial if $W \neq 1$. 
Proof of (ii)(a). By (v), it follows that $U \subseteq U^{*}$, where $U^{*}$ is a $T$-invariant Sylow $p$-subgroup of $C U$. Thus $U^{*}=U\left(U^{*} \cap C\right)$, so $U^{*} C=C U^{*}$. But $U \in \eta_{U C}^{*}(T, C)$ as $U \in \eta_{H}^{*}(T, C)$, so $U=U^{*}$. Hence $U$ is a Sylow $p$-subgroup of $C U$, so $|C U: U|$ $=|C: C \cap U|$ is prime to $p$. Thus $C \cap U$ is a Sylow $p$-subgroup of $C$ and certainly is $T$-invariant.

Proof of (i)(b). By (i)(a), $C U_{i}$ is a subgroup, so it is a subgroup of $N_{i}$. By (ii)(a), there is a $T$-invariant Sylow $p$-subgroup $R$ of $C$ contained in $U$, so $R \subseteq U_{i}$. Applying Lemma 7.2, with $R$ in place of $P$, we have that $U_{i}$ is contained in $P_{i} R, P_{i} Q_{i} R$ or $P_{i} Q_{i}^{*} R$. But $T$ centralizes $R$, so $U_{i}^{\prime}$ is contained in $P_{i}, P_{i} Q_{i}$ or $P_{i} Q_{i}^{*}$ as these subgroups are the intersection of $L_{i}$ and $R P_{i}, R P_{i} Q_{i}$ and $R P_{i} Q_{i}^{*}$, respectively, and as $U_{i}^{\prime} \subseteq L_{i}$. However, each of the three subgroups of $L_{i}$ is inverted by an element of $T-\left\langle x_{i}\right\rangle$, so $U_{i}^{\prime}$ is the intersection of $U_{i}$ with the corresponding one of them and is therefore a subgroup and is $C$-invariant.

Proof of (iii)(a). With the usual notation for $T$-decompositions, we have $U=U_{0} U_{1}^{\prime} U_{2}^{\prime} U_{3}^{\prime}$. By (ii)(a), $U$ contains a $T$-invariant Sylow $p$-subgroup $R$ of $C$, so $U_{0}=R=U \cap C$. Moreover, $U_{i}^{\prime}, 1 \leqq i \leqq 3$, is a $C$-invariant subgroup, by (i)(b), so $\tilde{U}=\left\langle U_{1}^{\prime}, U_{2}^{\prime}, U_{3}^{\prime}\right\rangle \subseteq U$ is also $C$-invariant. Since $U=U_{0} \tilde{U}$, it remains only to show that $U_{i}^{\prime} \subseteq O_{p}(C U), 1 \leqq i \leqq 3$. However, $C U$ is solvable by (v), $C$ contains a $p$ complement of $C U$, which then automatically normalizes the $p$-group $U_{i}^{\prime}$, so that $U_{i}^{\prime} \subseteq O_{p}(C U)$, as required.

Most of the remaining parts of the lemma will be handled together. Before doing this we show that the last statement, namely (vi), is a consequence of the others. This will prove (vi) once we deal with these other parts.

Proof of (vi). By (v), $U C$ is solvable, so that $V=O_{p}(C U)$ is the unique element of $\eta_{U C}^{*}(C)$, inasmuch as $C$ contains a $p$-complement of $C U$. If the result is false, then

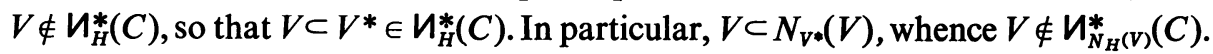
Hence, there is $U^{*} \in{U_{N}(V)}_{N^{\prime}}^{*}(T, C)$ with $O_{p}\left(C U^{*}\right) \supset V=O_{p}(C U)$. But $U \in И_{N_{H}(V)}^{*}(T, C)$ as $V$ is normal in $U C$ and $U \in \eta_{H}^{*}(T, C)$. This contradicts (iv) applied to $N_{H}(V)$ and the subgroups $U, U^{*}$ as $C$ is normal in $M \cap H$.

This leaves (ii)(b), (iii)(b), and (iv) to be proved. Since $O(H) \neq 1$ is assumed in these assertions except (ii)(b) and since (ii)(b) is clear if $O(H)=1$, we shall assume for the remainder of the section that $O(H) \neq 1$. We shall now proceed by a sequence of lemmas to verify the remaining three parts of the omnibus lemma. We set $\bar{H}=H / O(H)$.

LeMMA 2. The group $\bar{H}$ is a 2-group, a $Q$-group or a D-group, and $O(\bar{H})=1$.

Proof. We need only see that $H$ is not a $Q D$-group inasmuch as $H$ is a subgroup of $G$. However, $C \subseteq H$, whence $H$ covers $C \cap L / C \cap O(N)$ and $H$ contains $T$, so that $H$ is not a $Q D$-group by Proposition 4.1.1.

LEMMA 3. For some $i, 1 \leqq i \leqq 3, \bar{U} \subseteq C_{H}\left(\bar{x}_{i}\right)$.

Proof. If $\bar{H}$ is a 2-group, then $\bar{U}=1$ and the result is trivial. If $\bar{H}$ is a $Q$-group, then $\bar{H}=C_{H}\left(\bar{x}_{i}\right)$ for some $i, 1 \leqq i \leqq 3$, by Proposition 2.3.1 and the fact that the 
center of a Sylow 2-subgroup of $\bar{H}$ intersects $\bar{T}$ nontrivially. Again the result is clear. Finally, suppose that $\bar{H}$ is a $D$-group. If $\bar{H}$ is isomorphic to $A_{7}$, then $|\bar{U}| \leqq 3$ and the result is clear by inspection. In any other type of $D$-group, $\bar{U}$ is a subgroup of odd order normalized by the four subgroup $\bar{T}$ of $\bar{H}$ and the result follows directly from Lemma 2.5.7.

Proof of (iii)(b). Indeed, by Lemma $3, \bar{x}_{i}$ centralizes $\bar{U}$ for some $i, 1 \leqq i \leqq 3$, so $x_{i}$ centralizes $U / U \cap O(H)$. Hence, $U=C_{U}\left(x_{i}\right)(U \cap O(H))=\left(U \cap N_{i}\right)(U \cap O(H))$.

We now let $X$ be a subgroup of $H$ containing $O(H)$ and $U$ and such that $\bar{X} \in И_{H}^{*}(\bar{T}, \bar{C})$. We may do this as certainly $\bar{U} \in И_{H}(\bar{T}, \bar{C})$. Since $\bar{X}$ is permutable with $\bar{C}, X C$ is clearly a subgroup of $H$.

LEMMA 4. Under the above conditions we have the following:

(i) The group XC has a normal 2-complement.

(ii) There is a T-invariant Sylow p-subgroup $V$ of $X C$ containing $U$.

(iii) The subgroups $V$ and $C$ are permutable.

Proof. Since $X$ has odd order, $F=C_{D}(T)$ is a Sylow 2-subgroup of $X C$, being one of $C$. As in the proof of (v), $N_{X C}(F) \subseteq C_{X C}(F)$ and so (i) follows from Burnside's theorem.

In particular, $X C$ is solvable and $U \subseteq O(X C)$. Thus, part (ii) of the lemma is immediate.

In order to establish (iii), it suffices to show, in the usual $T$-decomposition notation, that $C$ and $\left\langle V_{i}^{\prime}\right\rangle, 1 \leqq i \leqq 3$, are permutable. Indeed, $V=V_{0} V_{1}^{\prime} V_{2}^{\prime} V_{3}^{\prime}$, so $V=V_{0}\left\langle V_{1}^{\prime}\right\rangle\left\langle V_{2}^{\prime}\right\rangle\left\langle V_{3}^{\prime}\right\rangle$ and $V_{0}=C_{V}(T) \subseteq C$ is certainly permutable with $C$.

We shall prove that $V_{i}^{\prime}=\left\langle V_{i}^{\prime}\right\rangle$ and is normalized by $C$. First, $V_{i}^{\prime} \subseteq N_{i}$, so certainly $V_{i}^{\prime} \subseteq L_{i}$ as $T$ centralizes $N_{i} / L_{i}$. We set $\bar{N}_{i}=N_{i} / O\left(N_{i}\right)$. Then $\left\langle\overline{O(C)}, \bar{V}_{i}^{\prime}\right\rangle$ is of odd order as $O(C)$ and $V$ are contained in the normal 2-complement $O(X C)$ of $X C$. Hence, by Lemmas 2.4.10 and 2.4.11, $\bar{V}_{i}^{\prime} \subseteq \bar{Q}_{i}$ or $\bar{Q}_{i}^{*}$, so $V_{i}^{\prime} \subseteq Q_{i} O\left(N_{i}\right)$ or $V_{i}^{\prime} \subseteq$ $Q_{i}^{*} O\left(N_{i}\right)$. However, $Q_{i} O\left(N_{i}\right)$ and $Q_{i}^{*} O\left(N_{i}\right)$ have normal Sylow $p$-subgroups $Q_{i} P_{i}$ and $Q_{i}^{*} P_{i}$ respectively, so $V_{i}^{\prime} \subseteq Q_{i} P_{i}$ or $V_{i}^{\prime} \subseteq Q_{i}^{*} P_{i}$. Since each of these Sylow subgroups is abelian, certainly $\left\langle V_{i}^{\prime}\right\rangle=V_{i}^{\prime}$.

Set $R_{i}=\left[V_{i}^{\prime}, C\right]$, so $R_{i}$ is a $p$-group of elements inverted by $x_{j}, j \neq i$. In particular, $R_{i} \supseteq V_{i}^{\prime}$ and $R_{i}$ is $C$-invariant. Hence to prove (iii), we need only show that $R_{i}=V_{i}^{\prime}$, and so it suffices to demonstrate that $\left|R_{i}\right| \leqq\left|V_{i}^{\prime}\right|$. However, $R_{i} \subseteq X C$, so $R_{i}$ is contained in a $T$-invariant Sylow $p$-subgroup $V^{*}$ of $X C$ and certainly $R_{i} \subseteq\left(V^{*}\right)_{i}^{\prime}$. But $V$ and $V^{*}$ are conjugate by an element of $C_{X C}(T)=C$, so $\left|\left(V^{*}\right)_{i}^{\prime}\right|=\left|V_{i}^{\prime}\right|$ and $\left|R_{i}\right| \leqq\left|V_{i}^{\prime}\right|$, as required.

As an immediate consequence we have

LEMMA 5. With the above notation, we have $U=V$ and $\bar{U}=\bar{X}$.

Proof. The previous lemma shows that $V \in И_{H}(T, C)$ and $V \supseteq U$. But

$$
U \in И_{H}^{*}(T, C),
$$


by our choice of $U$, so $U=V$. Next, $U \subseteq X$ by our choice of $X$, so $U$ is a Sylow $p$-subgroup of $X$. However, $\bar{X}$, the image of $X$ in $\bar{H}$, is a $p$-group, again by our choice of $X$, so $\bar{U}=\bar{X}$ and the lemma is proved.

Proof of (ii)(b). We have that $O(H) \subseteq X C \subseteq H$, so $O(H)$ is a normal subgroup of $X C$. Since $U=V$ is a Sylow $p$-subgroup of $X C$, it follows that $U \cap O(H)$ is a Sylow $p$-subgroup of $O(H)$.

This now leaves only part (iv) of the omnibus lemma to be proved. We choose $U^{*} \in \eta_{H}^{*}(T, C)$ as there stated.

LEMMA 6. If $U^{*} \subseteq X$, then $U$ and $U^{*}$ are conjugate by an element of $M \cap H$.

Proof. We have seen that $U$ is a $T$-invariant Sylow $p$-subgroup of $X C$. The same, of course, is true of $U^{*}$, as it was chosen in the same way $U$ was. But $X C$ has a normal 2-complement which is $T$-invariant, so $U$ and $U^{*}$ are conjugate by an element of $C \cap O(X C) \subseteq C \cap H \subseteq M \cap H$, as required.

LEMmA 7. The subgroups $\bar{U}$ and $\bar{U}^{*}$ are conjugate by an element of $\overline{M \cap H}$.

Before proving this let us stop to note that this completes the proof of the omnibus lemma.

Proof of (iv). By Lemma 7, there is an element $m$ of $M \cap H$ such that $\overline{\left(U^{*}\right)^{m}}=\bar{U}$. In particular, $\left(U^{*}\right)^{m} \subseteq X$ as $\bar{U}=\bar{X}$. But, by Lemma 6 , the subgroups $\left(U^{*}\right)^{m}$ and $U$ are conjugate by an element of $M \cap H$, inasmuch as $\left(U^{*}\right)^{m} \in U_{H}^{*}(T, C)$ since $M$ normalizes $C$ as well as $T$.

Proof of Lemma 7. Let $\bar{U}^{*}=\bar{X}^{*}$, so that $\bar{X}$ and $\bar{X}^{*}$ are elements of $\eta_{H}^{*}(\bar{T}, \bar{C})$. Indeed, $\bar{U}=\bar{X}$ by Lemma 5 , so $\bar{U} \in \eta_{H}^{*}(\bar{T}, \bar{C})$ by our choice of $\bar{X}$, and the same is, of course, true for $U^{*}$. Moreover, $O(H)$ has odd order, so $\overline{M \cap H}=N_{H}(\bar{T})$. Hence, we need to show that the elements $\bar{X}$ and $\bar{X}^{*}$ of $И_{H}^{*}(\bar{T}, \bar{C})$ are conjugate by an element of $N_{H}(\bar{T})$. Since we shall be dealing with $\bar{H}$ in the remainder of the proof, we shall omit all the "bars" throughout.

By Lemma 2 there are three possible structures for $H$. However, if $H$ is a 2-group, then $X=X^{*}=1$ and the result is immediate. Hence, $H$ is a $Q$-group or a $D$-group such that $O(H)=1$.

First, suppose that $H$ is a $Q$-group. Then $H=C_{H}\left(x_{i}\right)$ for some $i, 1 \leqq i \leqq 3$, as $T \subseteq H$. Moreover, $H$ has characteristic power $q$, since $C \subseteq H$. Hence, by Lemmas 2.4.10 and 2.4.11, $X \subseteq C Q_{i}$ or $C Q_{i}^{*}$ if $\delta \varepsilon=-1$ and $p$ divides $q$, while $X \subseteq C$ in all other cases. But $Q_{i}$ and $Q_{i}^{*}$ are conjugate by an element of $N_{H}(T)$, by Lemma 2.4.10, so we may assume that $X$ and $X^{*}$ are both contained in $C$ or $C Q_{i}$. But $X, X^{*} \in$ $\eta_{H}^{*}(T, C)$, so $X$ and $X^{*}$ are Sylow $p$-subgroups of $C$ or $C Q_{i}$ and each contains $Q_{i}$ in the latter case inasmuch as $Q_{i}$ is $C$-invariant. It follows that $X$ and $X^{*}$ are conjugate as desired.

Next, assume that $H$ is a $D$-group. First, suppose that $H$ is isomorphic to $A_{7}$. Thus, the only nontrivial subgroup of odd order normalized by $T$ has order three, so either $|X|=1$ or $p=3,|X|=3$ and $X=O\left(C_{H}\left(x_{i}\right)\right)$ for some $i, 1 \leqq i \leqq 3$. A similar 
statement holds for $X^{*}$, so $X$ and $X^{*}$ are conjugate by an element of $N_{H}(T)$ since all the elements of $T^{\#}$ are conjugate in $H$.

If $H$ is isomorphic to $P G L_{n}(2,3)$, then $\eta_{H}(T)$ is trivial by Lemma 2.5.9, in which case the desired conclusion is obvious. Hence we can assume that $S$ is quasi-dihedral and that $H$ is of linear type.

In this case, $H=K C_{H}(T)$, where $K$ is isomorphic to $\operatorname{PSL}(2, r), \operatorname{PGL}(2, r)$, or $P G L^{*}(2, r), r$ odd, by Lemma 2.5.7. Moreover, $C_{H}(T)=C$ is the direct product of $T$ and the cyclic group $O(C)$, while $K \cap O(C)=1$, by the same lemma. Now $X$ and $X^{*}$ each contain a $T$-invariant Sylow $p$-subgroup of $C$, by (ii)(a), and we may assume, without loss of generality, that it is the same Sylow $p$-subgroup $P$ of $C$. Furthermore, by Lemma 2.5.7 any element of $U_{H}(T)$ is contained in $C_{H}\left(x_{i}\right)$, for some $i, 1 \leqq i \leqq 3$. However, $C_{H}\left(x_{i}\right)=C_{K}\left(x_{i}\right) C$ as $H=K C$. Moreover, $C_{K}\left(x_{i}\right)$ is a dihedral group, by the same lemma. Thus, $X=R_{i} P$, where $R_{i}$ is the Sylow $p$-subgroup of $C_{K}\left(x_{i}\right)$ for some $i, 1 \leqq i \leqq 3$. If $R_{i}=1$, then $X=P$, so $X^{*}=P$ by the maximality of $X$ and since $X^{*} \supseteq P$. Thus we may assume $X=R_{i} P, R_{i} \neq 1$ and similarly $X^{*}=R_{j} P, R_{j} \neq 1$, for some $j, 1 \leqq j \leqq 3$. If $i=j$, then again $X=X^{*}$ and we are done, so we may also assume that $i \neq j$.

If $H$ is a $D$-group with respect to $T$, then $\left|N_{H}(T): C\right|$ is divisible by three. Since $C$ is normal in $N_{H}(T)$, there is a 3-element of $N_{H}(T)$ which normalizes $P$ and cyclically permutes the elements of $T^{\#}$. In this case we again have that $X$ and $X^{*}$ are conjugate as desired.

This leaves the possibility that $H$ is not a $D$-group with respect to $T$, whence $T \nsubseteq K_{0}$, where $K_{0}$ is the subgroup of $K$ isomorphic to $P S L(2, r)$. Hence, $\left|T \cap K_{0}\right|=2$. Thus if $x_{i}$ and $x_{j}$ both lie in $K_{0}$, then $x_{i}=x_{j}$ and consequently $X=X^{*}$. Therefore we can assume without loss that $x_{i} \notin K_{0}$. But then $x_{j} \notin K_{0}$ or else $C_{K}\left(x_{j}\right)$ would be a $p^{\prime}$-group by Lemma 2.5.7. Furthermore, in this case, $T$ is not a Sylow 2-subgroup of $K$, so $N_{K}(T)$ contains a 2-element $v$ not in $T$. Since $x_{i}$ and $x_{j}$ are the two elements of $T^{\#}$ not in $K_{0}$ and $v$ does not centralize $T, v$ necessarily interchanges $x_{i}$ and $x_{j}$. We can clearly choose $v$ to normalize $P$. Again $X$ and $X^{*}$ are conjugate as desired.

9. A subgroup which is not a $p$-group. This section begins the elimination of the prime $p$. We shall prove two results, the first of which is a transitivity theorem for $G$ and the second shows that the subgroups $P_{i}, 1 \leqq i \leqq 3$, generate a subgroup of $G$ that is not a $p$-group.

The first of these is as follows:

Proposition 1. If $U_{1}$ and $U_{2}$ are elements of $U_{G}^{*}(T, C)$, then $U_{1}$ and $U_{2}$ are conjugate by an element of $M$.

This will be used to show

Proposition 2. The subgroup of $G$ generated by $P_{1}, P_{2}$ and $P_{3}$ is not a p-group.

We shall prove these in turn; however, the second proof will be broken up into a sequence of lemmas. 
The heart of the proof of Proposition 1 is as follows:

LEMMA 1. If $V_{1}$ and $V_{2}$ are elements of $U_{G}^{*}(C)$, then $V_{1}$ and $V_{2}$ are conjugate by an element of $M$.

Proof. Assume the assertion is false and among all pairs of subgroups $V_{1}$ and $V_{2}$ which satisfy the hypothesis and not the conclusion of the lemma, choose $V_{1}$ and $V_{2}$ maximizing the order of $\left|V_{1} \cap V_{2}\right|$. Set $Y=V_{1} \cap V_{2}$, so that $Y \subset V_{1}$, $i=1,2$.

We claim that $Y=1$. Indeed, suppose $Y \neq 1$ and let $H=N(Y)$. Thus, $Y \subset V_{i} \cap H$ $\in U_{H}(C), i=1,2$, so we may choose $X_{i} \in U_{H}^{*}(T, C)$ containing $V_{i} \cap H$. By (iv) of Lemma 8.1, there is $m \in M \cap H$ such that $X_{2}^{m}=X_{1}$. Now $V_{\imath} \cap H \in U_{H}(C), i=1,2$, so if we set $Y_{i}=O_{p}\left(C V_{i}\right)$, then $V_{i} \cap H \subseteq Y_{i}$ and $Y_{i} \in U_{H}^{*}(C)$, by Lemma 8.1, part (vi). Hence, $Y_{2}^{m}=Y_{1}$. Choose $R_{i} \in{U_{G}^{*}}_{G}(C)$ with $R_{i} \supseteq Y_{\mathfrak{i}}, i=1,2$, so $Y_{2}^{m}$ is also an element of $h_{G}^{*}(C)$. Now $V_{1} \cap R_{1} \supseteq V_{1} \cap H \supset Y, \quad R_{1} \cap R_{2}^{m} \supseteq Y_{1} \supseteq V_{1} \cap H \supset Y$, $R_{2} \cap V \supseteq V_{2} \cap H \supset Y$, so by our choice of $V_{1}$ and $V_{2}$ it follows that $V_{1}$ and $R_{1}$, $R_{1}$ and $R_{2}^{m}, R_{2}$ and $V_{2}$ are respectively conjugate by an element of $M$. Since $m \in M$, it follows immediately that $V_{1}$ and $V_{2}$ are conjugate by an element of $M$, which is a contradiction. Hence $Y=V_{1} \cap V_{2}=1$, as asserted.

Each of the groups $V_{1}, V_{2}$ is certainly $T$-invariant and so is generated by its intersections with $N_{1}, N_{2}$ and $N_{3}$. Since the elements of $T^{\#}$ are all conjugate in $M$, we may assume, without any loss of generality that $V_{1} \cap N \neq 1, V_{2} \cap N \neq 1$. Thus, $V_{i} \cap N, i=1,2$, is an element of $\eta_{N}(C)$. By Lemma 7.1, since $D \subseteq M$ and $Q_{1}$ and $Q_{1}^{*}$ are conjugate by an element of $D$, we may also assume that $V_{1} \cap N$ and $V_{2} \cap N$ generate a $p$-subgroup of $N$. This subgroup is therefore $C$-invariant, so there is $V \in h_{G}^{*}(C)$ which contains $V_{1} \cap N$ and $V_{2} \cap N$. In particular, $V_{1} \cap V \neq 1$ and $V_{2} \cap V \neq 1$, so $V_{i}$ and $V$ are conjugate by an element of $M$, by our choice of $V_{1}$ and $V_{2}$ and as $V_{1} \cap V_{2}=1$. Hence, $V_{1}$ and $V_{2}$ are also conjugate by an element of $M$, which is yet another contradiction. This proves the lemma.

Proof of Proposition 1. The subgroup $C U_{i}, i=1,2$ is solvable, by (v) of Lemma 8.1. Moreover, $U_{i}$ is certainly an element of $\eta_{C U_{i}}^{*}(T, C), i=1,2$, so that $U_{i}=$ $O_{p}\left(U_{i} C\right)\left(U_{i} \cap C\right)$, by part (iii) of that lemma. Furthermore, $V_{i}=O_{p}\left(U_{i} C\right) \in \eta_{G}^{*}(C)$ by part (vi) and $U_{i} \cap C$ is a Sylow $p$-subgroup of $C$ by part (ii).

Hence, by Lemma 1 , there is $m \in M$ such that $V_{1}^{m}=V_{2}$ and by Sylow's theorem in $C$, there is $c \in C$ such that $\left(\left(U_{1} \cap C\right)^{m}\right)^{c}=U_{2} \cap C$. Thus,

as required.

$$
\begin{aligned}
U_{1}^{m c} & =\left(V_{1}\left(U_{1} \cap C\right)\right)^{m c} \\
& =\left(V_{2}\left(U_{1} \cap C\right)^{m}\right)^{c} \\
& =V_{2}\left(U_{2} \cap C\right) \\
& =U_{2},
\end{aligned}
$$

Turning to Proposition 2, we set $F=\left\langle P_{1}, P_{2}, P_{3}\right\rangle, K=N(F)$ and preserve this notation for the remainder of the section. We shall assume that $F$ is a $p$-subgroup and derive a contradiction. 
LEMMA 2. The subgroup $K$ is a D-group containing $M$.

Proof. Indeed, $C$ normalizes $P_{i}$ as $C \subseteq N_{i}$ and $P_{i}=O_{p}\left(N_{i}\right), 1 \leqq i \leqq 3$, so that $C \subseteq K$. Also the element $d$ of $D$ centralizes $x_{1}=x$ and interchanges $x_{2}$ and $x_{3}$, so $P_{1}^{d}=P_{1}$, $P_{2}^{d}=P_{3}, P_{3}^{d}=P_{2}$ and so $d \in K$. Finally, the element $a$ of $M$ cyclically permutes $x_{1}, x_{2}$, and $x_{3}$, so $a \in K$. But $M=\langle C, d, a\rangle$ and therefore $M \subseteq K$, as claimed.

In particular, $C \subseteq M$, so $K$ is not a $Q D$-group by Proposition 4.1.1. But $M \subseteq K$, so the elements of $T^{\#}$ are all conjugate in $K$ and $K$ is not a $Q$-group and does not have a normal 2-complement. Hence, the lemma is proved.

Lemma 3. If $U \in U_{K}^{*}(T, C)$ then $[U, T] \subseteq O(K)$.

Proof. In the $T$-decomposition notation, $[U, T]=\left\langle U_{1}, U_{2}^{\prime}, U_{3}^{\prime}\right\rangle$, so it suffices to show that $U_{i}^{\prime} \subseteq O(K), 1 \leqq i \leqq 3$; by symmetry it suffices to prove this for $i=1$.

By Lemma 8.1, $U_{1}^{\prime}$ is a $C$-invariant subgroup of $P_{1}, Q_{1} P_{1}$ or $Q_{1}^{*} P_{1}$. But $P_{1} \subseteq K$ and $x_{2}$ inverts $P_{1}$, so that $P_{1} \subseteq U_{1}^{\prime}$. Hence, $U_{1}^{\prime}$ equals $P_{1}, Q_{1} P_{1}$ or $Q_{1}^{*} P_{1}$ as $C$ acts irreducibly on $Q_{1}$ and $Q_{1}^{*}$.

If $S$ is wreathed, then $D=S$ and $U_{1}^{\prime} \subseteq O(K)$ by Lemma 2.5.9, applied to $\bar{K}=K / O(K)$. Hence we may assume that $S$ is quasi-dihedral. In this case, $W \neq 1$, $W$ is inverted by the element $d=y$ of $D$, and $W$ acts irreducibly and nontrivially on $U_{1}^{\prime} / P_{1}$. Since $P_{1} \subseteq O(K)$, we need only show that $\left[U_{1}^{\prime}, W\right] \subseteq O(K)$. If $K$ is of linear type, the second condition on $W$ implies that $\bar{W}$ lies in the normal subgroup $\bar{K}_{0}$ of $\bar{K}$ isomorphic to $\operatorname{PSL}(2, r)$ for some odd $r$. But $C_{E_{0}}(\bar{T}) \subseteq \bar{T}$ by Lemma 2.5.7 and consequently $\bar{W}=1$, whence $W \subseteq O(K)$ and hence also $\left[U_{1}^{\prime}, W\right] \subseteq O(K)$, as required.

On the other hand, suppose $\bar{K}$ is isomorphic to $A_{7}$. If $\bar{W}=1$, then as above $\left[U_{1}^{\prime}, W\right] \subseteq O(K)$. In the contrary case, $|\bar{W}|=3$ and $W O(K)$ is the unique maximal $T$-invariant subgroup of $K$ of odd order containing $W$. Hence also $U_{1}^{\prime} \subseteq W O(K)$. But $\overline{W O(K)}=\bar{W}$ is abelian, so again we conclude that $\left[U_{1}^{\prime}, W\right] \subseteq O(K)$ and the lemma follows in this case as well.

Lemma 4. If $U \in И_{K}^{*}(T, C)$, then $U \in И_{G}^{*}(T, C)$.

Proof. Choose $X \in \eta_{G}^{*}(T, C)$ with $U \subseteq X$; we shall prove that $U=X$. By Lemma 8.1, part (ii)(a), we know that $U_{0}$ is a Sylow $p$-subgroup of $C$, so that $U_{0}=X_{0}$. It remains to show that $U_{i}^{\prime}=X_{i}^{\prime}, 1 \leqq i \leqq 3$.

Now $U_{i}^{\prime} \supseteq P_{i}, 1 \leqq i \leqq 3$, as we saw above. First, suppose $U_{i}^{\prime} \supset P_{i}$ for some $i$, $1 \leqq i \leqq 3$, say $i=1$. By choice of notation, we may assume that $U_{1}^{\prime}=Q_{1} P_{1}$. But $a \in K$ and $Q_{1} \subseteq O(K)$, by Lemma 3, so $Q_{2}=Q_{1}^{a}$ and $Q_{3}=Q_{2}^{a}$ are contained in $O(K)$. This forces $U_{2}^{\prime}=Q_{2} P_{2}$ since otherwise $U_{2}^{\prime}=Q_{2}^{*} P_{2}$ and $Q_{2}^{*} \subseteq O(K)$, whence $\left\langle Q_{2}, Q_{2}^{*}\right\rangle$ has odd order, contrary to Lemma 2.4.10. Similarly, $U_{3}^{\prime}=Q_{3} P_{3}$. In any event, $U_{i}^{\prime} \in И_{N_{i}}^{*}(C), 1 \leqq i \leqq 3$, by Lemma 7.1. However, $U_{i}^{\prime} \subseteq X_{i}^{\prime} \subseteq N_{i}$ and $X_{i}^{\prime}$ is also an element of $\eta_{N_{i}}(C)$, by Lemma 8.1, part (i)(b). This yields that $U_{i}^{\prime}=X_{i}^{\prime}$ as required. 
Hence, we may assume that $U_{i}^{\prime}=P_{i}, 1 \leqq i \leqq 3$, and again we must demonstrate that $U_{i}^{\prime}=X_{i}^{\prime}$. We set $U^{*}=\left\langle U_{1}^{\prime}, U_{2}^{\prime}, U_{3}^{\prime}\right\rangle$, so $U^{*}=F$ and we let $X^{*}=\left\langle X_{1}^{\prime}, X_{2}^{\prime}, X_{3}^{\prime}\right\rangle$. It suffices to prove that $U^{*}=X^{*}$. In any case, $U^{*} \subseteq X^{*}$.

We observe that $U^{*} \in \eta_{K}^{*}(C)$. Indeed, $U^{*}$ is $C$-invariant as each $U_{i}^{\prime}$ is, $1 \leqq i \leqq 3$, and so $U^{*} \subseteq O_{p}(C U)$. But $O_{p}(C)=1$ and $C U=(C \cap U) O_{p}(C U)$, so that $C \cap U \cap O_{p}(C U)=1$. Hence, $U^{*}=O_{p}(C U)$ and our assertion is immediate from Lemma 8.1(vi).

However, $X^{*} \cap K$ is $C$-invariant as $X^{*}$ and $K$ are, so $X^{*} \cap K=U^{*}$. Thus, as $K=N(F)$ and $F=U^{*}$ in this case, we have that $N_{X^{*}}\left(U^{*}\right)=X^{*} \cap K=U^{*}$. But $X^{*}$ is a $p$-group, so this implies that $U^{*}=X^{*}$ and we are done.

LemMA 5. There is a $D$-invariant element of $U_{K}^{*}(T, C)$.

Proof. Choose $U \in \mathrm{U}_{R}^{*}(T, C)$ and let $V$ be a $D$-invariant Sylow p-subgroup of $O(K)$ with $T$-decomposition $V=V_{0} V_{1}^{\prime} V_{2}^{\prime} V_{3}^{\prime}$. But $O(K) \cap U$ is a $T$-invariant Sylow $p$-subgroup of $O(K)$, by Lemma 8.1(ii)(b), so $\left|V_{i}^{\prime}\right|=\left|U_{i}^{\prime}\right|$ for all $i$. Moreover, as we saw above, $U_{i}^{\prime}=P_{i}$ or $P_{i} Q_{i}$, with suitable choice of notation. Also, $V$ and $U \cap O(H)$ are conjugate by an element of $C \cap O(K)$, so that $V_{i}^{\prime}$ is a subgroup since $U_{i}^{\prime}$ is a subgroup by Lemma 8.1(i)(b). Furthermore, $\left\langle C, V_{i}^{\prime}\right\rangle \subseteq C\left(O(K) \cap N_{i}\right)$. Applying Lemmas 2.4.10 and 2.4.11 to $N_{i} / O\left(N_{i}\right)$, we conclude at once that $V_{i}^{\prime}$ equals $P_{i}$, $P_{i} Q_{i}$ or $P_{i} Q_{i}^{*}, 1 \leqq i \leqq 3$. But $\left\langle U_{i}^{\prime}, V_{i}^{\prime}\right\rangle$ is also of odd order, so $V_{i}^{\prime} \neq P_{i} Q_{i}^{*}$ since $\left\langle Q_{i}, Q_{i}^{*}\right\rangle$ is of even order, by Lemma 2.4.10. Hence $V_{i}^{\prime}=U_{i}^{\prime}, 1 \leqq i \leqq 3$.

In particular, $D$ normalizes $U^{*}=\left\langle U_{1}^{\prime}, U_{2}^{\prime}, U_{3}^{\prime}\right\rangle$. Moreover, $U^{*}$ is also $C$-invariant by Lemma 8.1(i)(b). Hence, if $P$ is a $D$-invariant Sylow $p$-subgroup of $C$, then $P$ normalizes $U^{*}$ and $D$ normalizes the subgroup $P U^{*}$. Moreover, $P U^{*}$ is clearly permutable with $C$ and is a Sylow $p$-subgroup of $C U$. Thus, $P U^{*} \in \eta_{K}^{*}(T, C)$ and is $D$-invariant. This proves the lemma.

We now let $U$ be a fixed $D$-invariant element of $h_{K}^{*}(T, C)$ and let $P=U_{0}=U \cap C$, a $D$-invariant Sylow $p$-subgroup of $C$, also be fixed.

Lemma 6. The group $U^{*}$ is $M$-invariant.

Proof. The subgroups $U \cap O(K)$ and $(U \cap O(K))^{a}$ are $T$-invariant Sylow $p$ subgroups of $O(K)$, where $a$ is the element of $M-C$ chosen in $\$ 7$. Hence, there is $c \in C$ such that $a c$ normalizes $U \cap O(K)$. But $a c$ normalizes $T$, so $a c$ normalizes $U^{*}$ as $U^{*} \subseteq U \cap O(K)$ by Lemma 3. Thus, $a c, D$ and $C$ normalize $U^{*}$ and the lemma is proved as $M=\langle C, D, a\rangle$.

Lemma 7. The subgroup $U$ is the unique element of $U_{G}^{*}(T, C)$ containing $P$.

Proof. We already have that $P \subseteq U$ and that $U \in \eta_{G}^{*}(T, C)$, the latter by Lemma 4 . Choose $V \in \eta_{G}^{*}(T, C)$ with $V \supseteq P$. By Proposition 1 , there is $m \in M$ such that $V=U^{m}$. Thus, in our above notation, $U=P U^{*}$, so $V=P^{m}\left(U^{*}\right)^{m}=P^{m} U^{*}$, as $U^{*}$ is $M$-invariant, by Lemma 6 . In particular, $[V, T]=U^{*}$ as $P^{m} \subseteq C$. On the other hand, if $V^{*}$ is defined for $V$ as $U^{*}$ was for $U$ then $V^{*}=O_{p}(C V)=[V, T]$ and 
$V=P V^{*}$. But then $V^{*}=U^{*}$ as each is equal to $[V, T]$ and so $V=P U^{*}=U$, as required.

LEMMA 8. For each $i, 1 \leqq i \leqq 3, Q_{i}=Q_{i}^{*}=1$.

Proof. The subgroups $Q_{i}$ and $Q_{i}^{*}$ are $C$-invariant, by Lemma 7.1, so $P Q_{i}$, $P Q_{i}^{*} \in U_{G}(T, C)$. Thus, by Lemma 7 ,

$$
\left\langle Q_{i}, Q_{i}^{*}\right\rangle \subseteq\left\langle P Q_{i}, P Q_{i}^{*}\right\rangle \subseteq U .
$$

But $\left\langle Q_{i}, Q_{i}^{*}\right\rangle$ has even order if $Q_{i} \neq 1$ and $U$ is a $p$-subgroup. Thus, $Q_{i}=Q_{i}^{*}=1$, as required.

LEMMA 9. The subgroup $K$ is an element of $\mathscr{L}(p)$.

This result contradicts Lemma 2, inasmuch as elements of $\mathscr{L}(p)$ are $Q$-groups; hence, once we prove this lemma, then Proposition 2 will be established and the section will be complete.

Proof. We first note that $N\left(P_{1}\right)$ is an element of $\mathscr{L}(p)$. Indeed, $N\left(P_{1}\right)$ covers $L_{0} / O(N)$, contains $D$ and $P_{1}$ itself is a Sylow $p$-subgroup of $O(N)$, so $N\left(P_{1}\right)$ fulfills all the conditions of Definition 2.1.4. Hence, $N\left(P_{1}\right)$ is an element of $\mathscr{L}(p)$ and also contains $C$.

We also assert that $U^{*}$ has $T$-decomposition $U^{*}=P_{1} P_{2} P_{3}$. Indeed, $P P_{1} \in$ $\eta_{G}(T, C)$, so $P_{1} \subseteq U$ by Lemma 7 . Thus, $P_{1} \subseteq U_{1}^{\prime}$; however, $Q_{1}=Q_{1}^{*}=1$, by Lemma 8 , so $P_{1}=U_{1}^{\prime}$. But $U^{*}$ is $M$-invariant, by Lemma 6 , so $U_{2}^{\prime}=P_{2}$ and $U_{3}^{\prime}=P_{3}$ and our assertion is valid. In particular, $F=U^{*}$. Moreover, $U$ has $T$-decomposition $U=P P_{1} P_{2} P_{3}$ and $P \cap U^{*}=1$ as $U^{*}=O_{p}(C U)$, by Lemma 8.1(iii)(a), and since $O_{p}(C)=1$.

We choose a $C$-invariant subgroup $X$ of $U^{*}$ maximal such that $P_{1} \subseteq X$ and $N(X) \in \mathscr{L}(p)$. We may do this as $N\left(P_{1}\right) \in \mathscr{L}(p)$, as we saw above. It suffices to show that $X=U^{*}$, as $K=N(F)$ by definition and $F=U^{*}$. Hence, we shall assume that $X \subset U^{*}$ and derive a contradiction.

We set $H=N(X)$ and choose $V \in U_{H}^{*}(T, C)$ with $V \supseteq P X$; we claim that $V=U \cap H$. Indeed, $P \subseteq V$ and $V \in U_{G}(T, C)$, so $V \subseteq U$ by Lemma 7 . On the other hand, $U \cap H$ is $T$-invariant as $U$ is $T$-invariant and $T \subseteq D \subseteq H$ as $H \in \mathscr{L}(p)$. Moreover, $C \subseteq H$ as $X$ is $C$-invariant, so $C(U \cap H)=C U \cap H$ and $U \cap H$ is permutable with $C$. Thus, $U \cap H \in U_{H}(T, C)$, so $V=U \cap H$, as desired, since $V \subseteq U \cap H$ and $V \in И_{H}^{*}(T, C)$. In particular, as $P \subseteq V$, we have $V^{*}=U^{*} \cap H$, where $V^{*}=[V, T]$, as usual. But $V^{*}=U^{*} \cap N(X)$ and $X \subset U^{*}$, so that $X \subset V^{*}$.

We observe now that $P \cap O(H)=1$. Indeed,

$$
P \cap O(H) \subseteq P \cap O(H) \cap N \subseteq P \cap O(N),
$$

by Lemma 4.1.1(iv). But $P \cap O(N)=1$ as $p$ is inverted, even totally inverted.

On the other hand, we have $V^{*} \subseteq O(H)$. Indeed, $V_{2}^{\prime}$ and $V_{3}^{\prime}$ are inverted by $x=x_{1}$ and $O(H) x$ is central in $H / O(H)$, so that $V_{2}^{\prime} \subseteq O(H)$ and $V_{3}^{\prime} \subseteq O(H)$. Moreover, 
$V_{1}^{\prime} \subseteq U_{1}^{\prime}=P_{1}$, as we saw above, so that

$$
V_{1}^{\prime} \subseteq P_{1} \subseteq X \subseteq O(H) .
$$

Hence, $V^{*}=\left\langle V_{1}^{\prime}, V_{2}^{\prime}, V_{3}^{\prime}\right\rangle \subseteq O(H)$, as claimed.

However, $V \cap O(H)$ is a Sylow p-subgroup of $O(H)$, by Lemma 8.1(ii)(b). But $V^{*} \subseteq O(H)$ and $P \cap O(H)=1$, so it follows that $V^{*}=V \cap O(H)$ and $V^{*}$ is a Sylow $p$-subgroup of $O(H)$.

We claim that $N\left(V^{*}\right) \in \mathscr{L}(p)$. Indeed, $U^{*}$ is $D$-invariant so that $V^{*}=U^{*} \cap H$ is as well and so $D \subseteq N\left(V^{*}\right)$. Moreover, $V^{*}$ is a Sylow $p$-subgroup of $O(H)$, so $N\left(V^{*}\right)$ covers $H / O(H)$. Hence, $N\left(V^{*}\right) \in \mathscr{L}(p)$, by Proposition 4.6.2, as all the requirements of that result are satisfied.

But $V^{*}$ is also $C$-invariant, as $U^{*}$ is $C$-invariant and $V^{*}=U^{*} \cap H$, and $X \subset V^{*}$; thus, we have contradicted the maximality of $X$ and the proof is complete.

10. A transitivity theorem. The last two sections of this chapter are devoted to developing a contradiction to Proposition 2 of the preceding section and so remove the possibility that $p$ exists.

Here we prove the following important preliminary result:

Proposition 1. If $R_{1} \in \mathrm{U}_{N}^{*}(C)$ and $U$ and $V$ are elements of $И_{G}^{*}(T, C)$ which contain $R_{1}$, then one of the following holds:

(i) $U$ and $V$ are conjugate by an element of $C$.

(ii) $U \cap V$ contains $P_{2}$ or $P_{3}$.

First, however, we establish the

LEMMA 1. If $R$ is a nonidentity element of $\eta_{N}(C), H=N(R)$, and $U \in \eta_{H}(T)$, then $[U, x] \subseteq O(H)$. Moreover, $H$ has a normal 2-complement or is a $Q$-group.

Proof. Since $H$ contains $C$, it follows that $H$ is not a $Q D$-group. Moreover, if $H$ has a normal 2-complement then the assertion is immediate. Hence, we may assume that $H$ is a $Q$-group or $D$-group.

First, suppose that $H$ is a $Q$-group. By the results and descriptions of $\S 7$ we see that $R$ is inverted by $x_{2}$ and $x_{3}$, while $x$ centralizes $R$. However, $R$ is certainly normal in $H$, so $x$ must be in the center of a Sylow 2-subgroup of $H$ and thus $H=O(H)(N \cap H)$. Hence, $[U, x] \subseteq O(H)$.

Second, assume that $H$ is a $D$-group. If $S$ is wreathed, the lemma follows at once from Lemma 2.5.9, so we may suppose that $S$ is quasi-dihedral, in which case $\delta=1$. We claim that $R \nsubseteq P_{1}$. Indeed, if $R \subseteq P_{1}$ then $J \subseteq C\left(P_{1}\right)$, so $J \subseteq H$, contradicting the fact that $H$ is a $D$-group. Thus, as a consequence, we conclude from Lemma 7.1 that $\varepsilon=\delta \varepsilon=-1$ and $p$ divides $q$.

By the remarks at the beginning of $\S 4$ of Chapter II, $N=L E$, where $E=O(C(S))$. Since $E \subseteq C$, it follows that $C=(C \cap L) E$. But $C \cap L$ is a $p^{\prime}$-group, inasmuch as $p$ is totally inverted, and therefore $E$ contains a Sylow $p$-subgroup $P$ of $C$. In particular, $P$ centralizes some Sylow 2-subgroup of $N$. Thus, $P$ centralizes a Sylow 2-subgroup of $G$. 
On the other hand, since $H$ is a $D$-group and $x_{2}$ inverts $R$, we have from Lemma 2.5.3 that $C_{H}(R)$ contains a four subgroup $T^{*}$. But $T$ and $T^{*}$ are conjugate in $G$ as $S$ is quasi-dihedral, so $R$ is conjugate to a subgroup of $P$. In particular, $R$ also centralizes a Sylow 2-subgroup of $G$. Thus $|N(R): C(R)|$ must be odd. But $x_{2}$ inverts $R$, so $|N(R): C(R)|$ is even, a contradiction.

We now begin the proof of the proposition. We assume it is false and derive a contradiction. Hence, choose $U, V \in \eta_{G}^{*}(T, C)$ which violate the result and maximize $|U \cap V \cap C|$ first and $|U \cap V|$ second subject to these conditions. As usual, by Lemma 8.1, we have $U=U_{0} U^{*}, V=V_{0} V^{*}$, where $U_{0}=C \cap U$ and $V_{0}=C \cap V$ are Sylow $p$-subgroups of $C$ and where $U^{*}=[U, T]$ and $V^{*}=[V, T]$ are $C$ invariant. We also set $R=U \cap V$ and keep all this notation fixed for the remainder of the section.

We now proceed as usual to prove the proposition by a lengthy sequence of lemmas.

LEMMA 2. We have that

(i) $|U|=|V|$.

(ii) $U_{0}=V_{0}$.

Proof. Indeed, $U$ and $V$ are conjugate by an element of $M$, by Proposition 9.1, so (i) is valid. Choose $c \in C$ such that $V_{0}^{c}=U_{0}$. We may do this as $U_{0}$ and $V_{0}$ are Sylow $p$-subgroups of $C$. Hence, $V^{c}=\left(V_{0} V^{*}\right)^{c}=U_{0} V^{*}$. Now, $R_{1}$ is $C$-invariant by hypothesis, so $R_{1} \subseteq U \cap V^{c}$. Also, $U, V^{c} \in \eta_{G}^{*}(T, C)$ as $c \in C$. Furthermore, $U \cap V^{c} \cap C=U_{0}$, a Sylow $p$-subgroup of $C$. Hence, by our choice of $U$ and $V$, we have that $U \cap V \cap C$ is a Sylow p-subgroup of $C$; for otherwise either $U$ and $V^{c}$ are conjugate by an element of $C$ or $U \cap V^{c}$ contains $P_{2}$ or $P_{3}$. Since $P_{2}$ and $P_{3}$ are $C$-invariant, we see that in either case the proposition holds for $U$ and $V$, which proves the assertion. However, $U \cap V \cap C=(U \cap C) \cap(V \cap C)=U_{0} \cap V_{0}$, so $U_{0}=V_{0}$, as desired.

We shall denote $P=U_{0}=V_{0}$ for the remainder of the section. Hence, $U=P U^{*}$, $V=P V^{*}$, and $R=U \cap V$.

LEMMA 3. $R \in U_{G}(T, C)$.

Proof. Since $U$ and $V$ are $T$-invariant, so is their intersection $R$. Moreover,

$$
\begin{aligned}
C(U \cap V) & =C\left(P U^{*} \cap P V^{*}\right) \\
& =C\left(P\left(U^{*} \cap V^{*}\right)\right) \\
& =C\left(U^{*} \cap V^{*}\right) .
\end{aligned}
$$

But $U^{*}$ and $V^{*}$ are $C$-invariant, so that $U^{*} \cap V^{*}$ is as well. Hence $C(U \cap V)$ is a subgroup and $C$ and $U \cap V=R$ are permutable.

We now fix yet some more notation. We let $R^{*}=[R, T]$ and $H=N\left(R^{*}\right)$. 
LEMMA 4. Under the above conditions,

(i) $R_{1} \subseteq R^{*}$,

(ii) $R^{*}=O_{p}(C R)=U^{*} \cap V^{*}$,

(iii) $U \cap H \supset R, V \cap H \supset R$,

(iv) $P \cap U^{*}=P \cap V^{*}=P \cap R^{*}=1$.

Proof. First, $R_{1} \in U_{N}^{*}(C)$ and $R_{1} \subseteq U \cap V$ by the hypothesis of the proposition. In particular, $R_{1} \subseteq R$. Moreover, since $R_{1}$ is a $C$-invariant $p$-subgroup of $N=N_{1}$, we have that $x_{2}$ inverts $R_{1}$, by Lemma 7.1. Hence, $R_{1}=\left[R_{1}, T\right] \subseteq[R, T]=R^{*}$ and (i) is valid.

By Lemma 8.1, $U^{*}=O_{p}(C U)$. In particular, $U^{*}$ is normal in $C U^{*}$, so that $C \cap U^{*}=P \cap U^{*}$ is normal in $C$. But $O_{p}(C)=1$, as we noted above, so $P \cap U^{*}=1$. Similarly $P \cap V^{*}=1$. Now $R^{*} \subseteq U^{*} \cap V^{*}$, as $U^{*}=[U, T], V^{*}=[V, T]$ and $R=U \cap V$, so $P \cap R^{*}=1$ as well and (iv) holds.

Since $R$ is $T$-invariant, certainly $R=(C \cap R)[R, T]$; that is, $R=P R^{*}$. Moreover, $R^{*} \subseteq U^{*} \cap V^{*} \subseteq R$, so $R=P\left(U^{*} \cap V^{*}\right)$. However, $P \cap R^{*}=P \cap\left(U^{*} \cap V^{*}\right)=1$, so we conclude that $R^{*}=U^{*} \cap V^{*}$. Since $U^{*}$ and $V^{*}$ are $C$-invariant, it follows that $R^{*}$ is as well. Hence $R^{*} \subseteq O_{p}(C R)$. But $C \cap O_{p}(C R)=1$ as $O_{p}(C)=1$ and $P R^{*}$ is a Sylow p-subgroup of $C R$ as $R$ and $C$ are permutable, by Lemma 3. Hence, $O_{p}(C R)=R^{*}$ and (ii) is verified.

We have that $U \neq V$, so $U \supset R$. Since $U=P U^{*}$ and $R=P R^{*}$, this implies that $U^{*} \supset R^{*}$. Hence, $N_{U^{*}}\left(R^{*}\right) \supset R^{*}$. However, $U^{*} \cap R=R^{*}$ as $R^{*} \subseteq U^{*}$ and $P \cap U^{*}=1$. Thus, $N_{U^{*}}\left(R^{*}\right) \nsubseteq R$, so $N_{U}\left(R^{*}\right) \supset R$, which is $H \cap U \supset R$. Similarly, $H \cap V \supset R$ and the lemma is proved in full.

LEMma 5. There is no element of $\eta_{H}(T, C)$ which contains both $U \cap H$ and $V \cap H$.

Proof. If this is false, then there is $X \in \eta_{G}^{*}(T, C)$ which contains $U \cap H$ as well as $V \cap H$. Thus, $U \cap X \supseteq U \cap H \supset R$, by Lemma 4(iii), so the proposition is valid for $U$ and $X$ by our maximal choice of $U$ and $V$. Similarly, it holds for $X$ and $V$.

Suppose first that $U$ and $X$ are conjugate by an element of $C$, that is, condition (i) of Proposition 1 holds. Hence, $X$ and $V$ are not conjugate by an element of $C$, since $U$ and $V$ violate the proposition and so are not conjugate by an element of $C$. Thus, $X \cap V$ contains $P_{2}$ or $P_{3}$. Choose $c \in C$ such that $X^{c}=U$. Since $P_{2}$ and $P_{3}$ are $C$-invariant, we have that if one of them is contained in $X \cap V$ then it is also in $U$ and again we have contradicted our choice of $U$ and $V$. Thus, $U$ and $X$ are not conjugate by an element of $C$. Similarly, $X$ and $V$ are not conjugate by an element of $C$.

Hence, $P_{i} \subseteq U \cap X, i=2$ or 3 and $P_{j} \subseteq X \cap V, j=2$ or 3. Moreover, $i \neq j$ as $P_{2} \nsubseteq U \cap V$ and $P_{3} \nsubseteq U \cap V$. Thus, $\left\langle P_{2}, P_{3}\right\rangle \subseteq X$. But $R_{1} \subseteq R=U \cap V$ by Lemma 4(i). Since $P_{1} \subseteq R_{1}$, it follows that $P_{1} \subseteq U \cap H \subseteq X$, so $\left\langle P_{1}, P_{2}, P_{3}\right\rangle \subseteq X$. This contradicts Proposition 9.2 and the lemma is proved. 
Lemma 6. If $X, Y \in \eta_{H}^{*}(T, C)$ with $U \cap H \subseteq X$ and $V \cap H \subseteq Y$, then $X$ and $Y$ are not conjugate by an element of $C$.

Proof. In fact, suppose that $c \in C$ such that $Y^{c}=X$. Since $P \subseteq U \cap H \subseteq X$ and $P \subseteq V \cap H \subseteq Y$, we have that $C \cap X=C \cap Y=P$, so that $P^{c}=P$. Hence $U$ and $V^{c}$ are elements of $\eta_{G}^{*}(T, C)$, which both contain $P$. Moreover, $R^{*} \subseteq U \cap V$ and $R^{*}$ is $C$-invariant by Lemma 4(ii). Since $R=P R^{*}$, we have $R \subseteq U$ and $R \subseteq V^{c}$. The proposition cannot hold for $U$ and $V^{c}$ or it will hold for $U$ and $V$ as $P_{2}$ and $P_{3}$ - are $C$-invariant. Hence, by our maximal choice of $U$ and $V$ we have $\left|U \cap V^{c}\right|$ $=|U \cap V|$, since $R \subseteq U \cap V^{c}$, and consequently $R=U \cap V^{c}$. Thus, all the above results apply equally well to $U$ and $V^{c}$ as to $U$ and $V$. In particular, we have $\left\langle U \cap H,(V \cap H)^{c}\right\rangle \subseteq X$, inasmuch as $H=N\left(R^{*}\right)$ contains $C$, which contradicts the previous lemma and so this lemma is now proved.

We now fix elements $X$ and $Y$ of $U_{H}^{*}(T, C)$ with $U \cap H \subseteq X$ and $V \cap H \subseteq Y$. We keep this notation for the remainder of the section. As usual, $X^{*}=[X, T]$ and $Y^{*}=[Y, T]$, so $P X^{*}=X, P Y^{*}=Y$ and $P \cap X^{*}=P \cap Y^{*}=1$.

LEMMA 7. With the usual T-decomposition notation, we have that $\left\langle X_{2}^{\prime}, X_{3}^{\prime}\right\rangle \nsubseteq O(H)$.

Proof. We assume the contrary and derive a contradiction. By Lemma 8.1(i)(b), $X_{1}^{\prime}$ is a $C$-invariant subgroup of $N$. Since $R_{1} \subseteq X$, we must have $X_{1}^{\prime}=R_{1}$, since $R_{1} \in V_{N}^{*}(C)$. But $R_{1} \subseteq R^{*}$, by Lemma 4(i), so $X_{1}^{\prime} \subseteq O(H)$, as certainly $R^{*} \subseteq O(H)$. Hence, $X^{*} \subseteq O(H)$.

By part (iv) of Lemma 8.1 (which applies as $R_{1} \neq 1$, so $O(H) \neq 1$ ), we have that $X$ and $Y$ are conjugate by an element of $M \cap H$. Hence, $Y^{*}=[Y, T] \subseteq O(H)$ as well. Moreover, $X \cap O(H)$ and $Y \cap O(H)$ are Sylow p-subgroups of $O(H)$, by Lemma 8.1(ii)(b). Thus, $X$ and $Y$ are $T$-invariant Sylow p-subgroups of the $T$ invariant subgroup $P O(H)$. Since $P O(H)$ has odd order, it follows that $X$ and $Y$ are conjugate by an element of $C$, which contradicts Lemma 6. Hence, this result is proved.

We shall now choose our notation so that $X_{3}^{\prime} \nsubseteq O(H)$. We may clearly do this without any loss of generality.

We shall study the structure of $H$. Since $O(H) \neq 1$, there are four possibilities for $H$. We shall show that none of these occur; this contradiction will complete the proof of Proposition 1.

LEMMA 8. The subgroup $H$ is not a $Q D$-group and it does not have a normal 2-complement.

Proof. Indeed, $C \subseteq H$ as $R^{*}$ is $C$-invariant, by Lemma 4(ii). Hence $H$ is not a $Q D$-group by Proposition 4.1.1. Finally, if $H$ has a normal 2-complement, then $X$ and $Y$ are $T$-invariant Sylow $p$-subgroups of $H$, by Lemma 8.1(ii)(b). In particular, $X$ and $Y$ are then conjugate by an element of $C \cap O(H)$, which contradicts Lemma 6. 
LEMMA 9. The subgroup $H$ is not a $Q$-group.

Proof. We have seen that $X_{3}^{\prime}$ is a subgroup not contained in $O(H)$. But $x_{1}$ and $x_{2}$ invert $X_{3}^{\prime}$, so if $H$ is a $Q$-group, then $O(H) x_{3}$ is central in $H / O(H)$ and $H=O(H)\left(H \cap N_{3}\right)$. Thus, $T$ is a four subgroup of a Sylow 2-subgroup $T^{*}$ of $H$, so that $\Omega_{1}\left(Z\left(T^{*}\right)\right)=\left\langle x_{3}\right\rangle$ and $T^{*}$ is either quasi-dihedral or a subgroup of a wreathed 2-group. In either case, $x_{1}$ and $x_{2}$ are conjugate in $T^{*}$, so certainly there is $v \in H$ with $x_{1}^{v}=x_{2}$. But $P_{1} \subseteq R_{1}$, so we have $P_{2}=O_{p}\left(N_{2}\right)=P_{1}^{v}$ and $P_{2}=P_{1}^{v} \subseteq R_{1}^{v}$ $\subseteq\left(R^{*}\right)^{v}=R^{*} \subseteq U \cap V$, contradicting our choice of $U$ and $V$. Hence, $H$ is not a $Q$-group.

LEMMA 10. The following conditions hold:

(i) $S$ is quasi-dihedral.

(ii) $H$ is a D-group.

Proof. Suppose, by way of contradiction, that $S$ is wreathed, in which case $C_{S}(T)=C_{D}(T)$ is abelian of type $\left(2^{n}, 2^{n}\right)$. Since $C \subseteq H$, we have $C_{D}(T) \subseteq H$. Hence if $T^{*}$ is a Sylow 2-subgroup of $H$ containing $C_{D}(T)$, then either $T^{*}$ is wreathed or $T^{*}=C_{D}(T)$. If $T^{*}$ is wreathed, it follows from the preceding two lemmas that $H$ must be a $D$-group, whence $H / O(H)$ is isomorphic to $P G L_{n}(2,3)$ and, by Lemma 2.5.9, every element of $U_{H}(T)$ is contained in $O(H)$. But then $X$ and $Y$ are $T$ invariant Sylow $p$-subgroups of $O(H)$ and so are conjugate by an element of $C \cap O(H)$, contrary to Lemma 6.

On the other hand, suppose $T^{*}=C_{D}(T)$. Since $H$ does not have a normal 2-complement, Brauer's theorem ([6,II, Theorem 1, §VI]) implies that $O(H) T^{*}$ has index 3 in $H$. It follows at once from this that every element of $\eta_{H}(T)$ is contained in $O(H)$ and we reach the same contradiction as in the preceding case.

Since $S$ is quasi-dihedral and $H$ is neither a $Q D$-group nor a $Q$-group nor does $H$ have a normal 2-complement, $H$ must be a $D$-group. Thus both parts of the lemma hold.

LEMMA 11. The element $x \notin H^{\prime}$.

Proof. Suppose $x \in H^{\prime}$. Since $H$ is a $D$-group, a Sylow 2-subgroup of $H^{\prime}$ is dihedral, so $x \in T^{*}$ for some four subgroup $T^{*}$ of $H^{\prime}$. But then by Lemma 2.5.1 $A_{H}\left(T^{*}\right)$ has order divisible by three. Since $T^{*} \subseteq N$ and $S$ is quasi-dihedral, there is $v \in N$ such that $\left(T^{*}\right)^{v}=T$, inasmuch as $S$ has one conjugacy class of four subgroups.

We now set $K=H^{v}$. By our remark on $A_{H}\left(T^{*}\right)$, it follows that $A_{K}(T)$ also has order divisible by three, so there is $k \in K$ such that $x_{1}^{k}=x_{2}, x_{2}^{k}=x_{3}$. However, $P_{1} \subseteq R_{1} \subseteq R^{*} \subseteq H$ and $P_{1}^{v}=P_{1}$ as $P_{1}=O_{p}(N)$, so $P_{1} \subseteq O_{p}(K)$ as $R^{*} \subseteq O_{p}(H)$. Hence $P_{2}=O_{p}\left(N_{2}\right)=O_{p}\left(N_{1}\right)^{k}=P_{1}^{k}$ and $P_{2} \subseteq O_{p}(K)$. Similarly, $P_{3} \subseteq O_{p}(K)$ and $\left\langle P_{1}, P_{2}, P_{3}\right\rangle$ is a $p$-group, contradicting Proposition 9.2. This proves the lemma.

This next result clearly completes the proof of the proposition. 
LEMMA 12. The subgroup $H$ is not a D-group.

Proof. To prove the lemma, we shall contradict the fact that $H$ is a $D$-group, which we have established in Lemma 10. By the preceding lemma, we have that $x \notin H^{\prime}$. In particular, $\bar{H}=H / O(H)$ is not isomorphic to $A_{7}$ and so $H$ is of linear type. Hence $C_{H}(\bar{x})$ has a normal 2-complement, by Lemma 2.5.7. Thus, $H \cap N$ $=C_{H}(x)$ also has a normal 2-complement since $O(H)$ is of odd order.

We claim that $P R_{1}$ is a Sylow $p$-subgroup of $H \cap N$. In fact, assume the contrary and let $\tilde{R}$ be a Sylow $p$-subgroup of $H \cap N$ with $\widetilde{R} \supset P R_{1}$. However, $L_{0} P / L_{0}$ is a Sylow $p$-subgroup of $N / L_{0}$, by Lemma 2.4.2(i) applied to $N / O(N)$. Thus, $\tilde{R} \cap L_{0}$ $\supset R_{1}$, as $R_{1} \subseteq L_{0}$ by Lemma 7.1. In particular, $R_{1}$ is not a Sylow $p$-subgroup of $L_{0}$, so $R_{1}=P_{1}$ and either $\varepsilon \delta=\varepsilon=1$ or $\varepsilon=-1$ with $p$ not dividing $q$, by Lemmas 2.4.1(i) and 2.4.10 applied to $N / O(N)$. (Here $\delta=1$ as $S$ is quasi-dihedral.) Hence, as $\widetilde{R} \supseteq P_{1}$ it follows that $\tilde{R} \cap J$ is a nonidentity p-subgroup of $J$.

But $\tilde{R} \cap J \subseteq H$ and $C \subseteq H$ as $R^{*}$ is $C$-invariant. We claim that $\tilde{R} \cap J$ and $W T$ generate $J T$. Once this is shown, then $J \subseteq H$, so $H$ is a $Q D$-group, contradicting Lemma 8, and so $P R_{1}$ will be a Sylow $p$-subgroup of $H \cap N$.

However, $W$ is a $p^{\prime}$-group, so that $p$ divides $q$ or $q-\varepsilon$. If $p$ divides $q$, then $\varepsilon=1$, by our remarks above. Hence in either case, Lemma 2.4.6(ii), applied to $J T / O(J)$, yields that $\langle W T, \tilde{R} \cap J\rangle=J T$ inasmuch as $W T \cap J T=C \cap J T$.

We next observe that $\bar{X}$ and $\bar{Y}$ are elements of $\eta_{H}^{*}(\bar{T}, \bar{C})$. Indeed, the arguments of Lemma 8.4 and Lemma 8.5 with $X$ in place of $U$ clearly show that $\bar{X} \in U_{H}^{*}(\bar{T}, \bar{C})$. Of course, the same holds equally well for $\bar{Y}$.

Suppose that $\bar{X}=\bar{Y}$. Thus, $X$ and $Y$ are $T$-invariant Sylow $p$-subgroups of the $T$-invariant group $X O(H)$, since $X \cap O(H)$ and $Y \cap O(H)$ are Sylow $p$-subgroups of $O(H)$, by Lemma 8.1(ii)(b). Since $X O(H)$ is of odd order, $X$ and $Y$ are conjugate by an element of $C$, which contradicts Lemma 6 . Hence, the proof of this lemma and of the proposition will be complete once we establish the final result of this section, namely:

LEMMA 13. Under the above conditions, we have that $\bar{X}=\bar{Y}$.

Proof. Since we shall be working in $\bar{H}$ throughout the argument, we shall drop all the "bars". First, $x \notin H^{\prime}$, so $H$ has a normal subgroup $K$ of odd index such that $K$ is isomorphic to $\operatorname{PGL}(2, r)$ for some odd $r$, by Proposition 2.3.4 and Lemma 2.5.7(vi).

We now assert that $X^{*} \subseteq C_{K}\left(x_{3}\right)$. Indeed, $X \in И_{H}(T, C)$, so $X \subseteq C_{H}\left(x_{i}\right)$ for some $i$, $1 \leqq i \leqq 3$, by Lemma 2.5.7. Furthermore, $C_{H}\left(x_{i}\right)=C_{K}\left(x_{i}\right) C_{H}(T)$, by the same lemma, Hence, $X^{*}=[X, T] \subseteq C_{K}\left(x_{i}\right)$ as $C_{K}\left(x_{i}\right)$ is normal in $C_{H}\left(x_{i}\right)$. However, $X_{3}^{\prime} \neq 1$, by Lemma 7 and the choice of notation following. Since $x_{1}$ and $x_{2}$ invert $X_{3}^{\prime}$, it now follows that $i=3$ and our assertion is valid. Thus also $X \subseteq C_{H}\left(x_{3}\right)$.

The next step is to show that $C_{K}\left(x_{j}\right)$ is a $p^{\prime}$-group if $j=1,2$. In fact, as we saw in the beginning of the proof of Lemma 12, $X$ contains a Sylow $p$-subgroup of 
$H \cap N$. Hence, as $X \subseteq C_{H}\left(x_{3}\right)$, it follows that $C_{H}\left(x_{3}\right)$ contains a Sylow $p$-subgroup of $C_{H}(x)$. Thus a Sylow $p$-subgroup of $C_{K}(x)$ is contained in $C_{K}(T)$. But $C_{K}(T)$ is a 2-group by Lemma 2.5.7, so that $C_{K}(x)$ is a $p^{\prime}$-group. It remains therefore to see that $C_{K}\left(x_{2}\right)$ is a $p^{\prime}$-group.

However, this will be immediate once we prove that $x=x_{1}$ and $x_{2}$ are conjugate in $H$. But $x \notin H^{\prime}$ and so $T \cap H^{\prime}$ has order two. Thus there are exactly two involutions of $T$ not in $H^{\prime}$, one of which is $x_{1}$. But these two involutions are conjugate in $H$ since a Sylow 2-subgroup of $H$ has order at least eight as $K$ is isomorphic to $P G L(2, r)$. Hence, we need only see that these two involutions are just $x_{1}$ and $x_{2}$. They cannot be $x_{1}$ and $x_{3}$ as $C_{K}\left(x_{1}\right)$ is a $p^{\prime}$-group and $C_{K}\left(x_{3}\right)$ has order divisible by $p$, so the involutions are as described and thus $C_{K}\left(x_{j}\right)$ is a $p^{\prime}$-group for $j=1,2$.

It follows that $Y^{*} \subseteq C_{K}\left(x_{3}\right)$. Indeed, if $Y^{*}=1$, this is clear, while if $Y^{*} \neq 1$, then $Y^{*}$ is a nonidentity $p$-subgroup of $C_{K}\left(x_{i}\right)$, for some $i, 1 \leqq i \leqq 3$, since $Y \in U_{H}(T, C)$, by the same reasoning as was applied to $X$ above. But $C_{K}\left(x_{j}\right)$ is of order prime to $p$ if $j=1,2$. Thus, $\left\langle X^{*}, Y^{*}\right\rangle \subseteq C_{K}\left(x_{3}\right)$.

However, $C_{K}\left(x_{3}\right)$ is a dihedral group, by Lemma 2.5.7(i) and (ii), so it has a unique Sylow $p$-subgroup $Q$. Moreover, $C_{K}\left(x_{3}\right)$ is $C$-invariant, so $Q$ is as well. Hence $P Q \in \mathrm{U}_{H}(T, C)$. But $X^{*} \subseteq Q, Y^{*} \subseteq Q$ and $X=P X^{*}, Y=P Y^{*}$, so $X \subseteq P Q$, $Y \subseteq P Q$. But $X, Y \in \mathrm{U}_{H}^{*}(T, C)$ by their definition. Hence $X=P Q=Y$; the lemma is proved and the section is complete.

11. Elimination of the last set of primes. We come at last to the final section of the chapter and the contradiction that shows that no exceptional totally inverted primes do exist.

In order to describe the main result of the section we fix some more notation. We let $R_{i}=R_{i}^{*}=P_{i}, 1 \leqq i \leqq 3$, unless $\delta \varepsilon=-1$ and $p$ divides $q$, in which case we set $R_{i}=Q_{i} P_{i}$ and $R_{i}^{*}=Q_{i}^{*} P_{i}$.

The chief end of our analysis is the

Proposition 1. The subgroup generated by $R_{1}$ and $R_{3}$ is a p-group.

We begin by noting the important fact that this result gives the final contradiction. Indeed, suppose the proposition is true. Since $C$ normalizes $R_{1}$ and $R_{3}$, we certainly have $\left\langle R_{1}, R_{3}\right\rangle \in \eta_{G}(T, C)$, so there is $U \in \eta_{G}^{*}(T, C)$ with $\left\langle R_{1}, R_{3}\right\rangle \subseteq U$. If we set $V=U^{a}$, where the element $a$ is the element fixed in $\S 7$, then

$$
\left\langle P_{1}, P_{3}\right\rangle \subseteq\left\langle R_{1}, R_{3}\right\rangle \subseteq U
$$

yields

$$
\left\langle P_{2}, P_{1}\right\rangle=\left\langle P_{1}, P_{3}\right\rangle^{a} \subseteq U^{a}=V .
$$

If $U$ and $V$ are conjugate by an element of $C$, then $\left\langle P_{1}, P_{2}, P_{3}\right\rangle \subseteq U \cap V$ since $P_{1}, P_{2}, P_{3}$ are certainly $C$-invariant. But $\left\langle P_{1}, P_{2}, P_{3}\right\rangle$ is not a $p$-group, by Proposition 9.2. Hence $U$ and $V$ are not conjugate by an element of $C$. Thus, by Proposition 
10.1, $U \cap V$ contains $P_{2}$ or $P_{3}$. If $P_{2} \subseteq U \cap V$, then $P_{2} \subseteq U$, so $\left\langle P_{1}, P_{2}, P_{3}\right\rangle \subseteq U$ as $\left\langle P_{1}, P_{3}\right\rangle \subseteq U$ from above; while if $P_{3} \subseteq U \cap V$, then $P_{3} \subseteq V$ and $\left\langle P_{1}, P_{2}, P_{3}\right\rangle \subseteq V$ as $\left\langle P_{2}, P_{1}\right\rangle \subseteq V$. Hence, in either case we have contradicted Proposition 9.2.

Before embarking on the proof of the proposition, we remark that it suffices to show that $\left\langle R_{i}, R_{j}\right\rangle$ or $\left\langle R_{i}^{*}, R_{j}^{*}\right\rangle$ is a $p$-group for some $i, j, 1 \leqq i, j \leqq 3$ with $i \neq j$. To see this, we first note that the element $z_{1} \in D_{1}$ which interchanges $Q_{1}$ and $Q_{1}^{*}$ also interchanges $Q_{2}$ and $Q_{3}^{*}, Q_{2}^{*}$ and $Q_{3}$, while similar statements hold for $z_{2}$ and $z_{3}$. In fact, $z_{1} \notin C$ as $Q_{1}, Q_{1}^{*}$ are $C$-invariant and so $z_{1}$ inverts $a$ modulo $C$, inasmuch as $D_{1}=D \subseteq M$ and $M / C$ is isomorphic to the symmetric group $S_{3}$. That is, $a z_{1}=z_{1} a^{-1} c$ for some $c \in C$. Thus,

$$
\begin{aligned}
Q_{2}^{z} & =Q_{1}^{a z_{1}}=Q_{1^{1}}^{z^{-1} a^{-1} c}=\left(Q_{1}^{*}\right)^{a^{-1} c} \\
& =\left(Q_{3}^{*}\right)^{c}=Q_{3}^{*},
\end{aligned}
$$

and $\left(Q_{2}^{*}\right)^{z_{1}}=Q_{3}$ similarly may be shown. Now, if $\left\langle R_{i}, R_{j}\right\rangle$ is a $p$-group, $1 \leqq i, j \leqq 3$, $i \neq j$, then conjugation of this $p$-group by $a$ or $a^{-1}$ shows that $\left\langle R_{1}, R_{3}\right\rangle$ is a $p$-group. Similarly if $\left\langle R_{i}^{*}, R_{j}^{*}\right\rangle$ is a $p$-group, $1 \leqq i, j \leqq 3, i \neq j$, then conjugation by $z_{i}$ shows $\left\langle R_{i}, R_{k}\right\rangle$ is a $p$-group, where $1 \leqq k \leqq 3, k \neq i, j$. Thus, again $\left\langle R_{1}, R_{3}\right\rangle$ is a $p$-group. We shall use this comment often without further mention.

We now proceed with the usual sequence of lemmas.

LEMMA 1. If $H$ is a subgroup of $G$ containing $C$, with $R_{1} \subseteq H$ and $R_{3} \subseteq O(H)$, then $\left\langle R_{1}, R_{3}\right\rangle$ is a p-group.

Proof. Choose $U \in U_{H}^{*}(T, C)$ with $U \supseteq R_{1} \cdot$ By Lemma 8.1(ii)(b), $U \cap O(H)$ is a Sylow $p$-subgroup of $O(H)$. Hence, as $R_{3} \subseteq O(H), R_{3}$ is $T$-invariant and $U \cap O(H)$ is a $T$-invariant Sylow $p$-subgroup of $O(H)$, there is $c \in C \cap O(H)$ such that $R_{3}^{c} \subseteq U \cap O(H)$. But $R_{3}$ is $C$-invariant, so $R_{3} \subseteq U$ and $\left\langle R_{1}, R_{3}\right\rangle \subseteq U$. This proves the lemma.

LEMMA 2. If there are integers $i, j, 1 \leqq i, j \leqq 3, i \neq j$, such that $C_{P_{j}}\left(R_{i}\right) \neq 1$ or $C_{P_{j}}\left(R_{i}^{*}\right) \neq 1$, then $\left\langle R_{1}, R_{3}\right\rangle$ is a p-group.

Proof. We may as well assume that $j=1, i=3$ and $R=C_{P_{1}}\left(R_{3}\right) \neq 1$. We set $H=N(R)$. Thus, $H$ is a subgroup which contains $C$, since $P_{1}$ and $R_{3}$ are $C$-invariant. Moreover, $R_{3} \subseteq H$ as $R_{3}$ centralizes $R$, by definition of $R$. Furthermore, $P_{1} \subseteq H$ as $P_{1}$ is abelian, so $R_{1} \subseteq H$ since $J$ and $P_{1}$ commute elementwise. Thus, in view of Lemma 1, it suffices to show that $R_{3} \subseteq O(H)$.

However, the element $d$ lies in $J$ and hence centralizes $P_{1}$, so $D \subseteq H$. Thus, $H \in \mathscr{L}(p)$ by Definition 4.1.4 as $D \subseteq H, P_{1} \subseteq H$, and $H$ covers $L_{0} / O(N)$. Hence, $H=O(H)\left(H \cap N_{1}\right)$, so $\left[R_{3}, x\right] \subseteq O(H)$. But, $x=x_{1}$ inverts $R_{3}$, so $R_{3}=\left[R_{3}, x\right]$ and the lemma is proved.

We now fix some further notation. We set $K=N\left(P_{1}\right)$, choose $V \in U_{R}^{*}(T, C)$ with $R_{1} \subseteq V$, as we may do since $R_{1}$, being abelian, lies in $K$. Thus, with the usual $T$-decomposition notation, $V_{0}$ is a Sylow p-subgroup of $C$, by Lemma 8.1(ii)(a), 
and we set $P=V_{0}$. Moreover, by part (i)(b) of that lemma, $V_{i}^{\prime}$ is a $C$-invariant subgroup of $R_{i}$ or $R_{i}^{*}$ and, in particular, is abelian. We set $X_{i}=C_{V_{i}}\left(R_{1}\right), i=2,3$. Moreover, we shall be using Lemma 8.1 throughout the rest of the section and shall now do so without any further reference.

LEMMA 3. With the above notation, we have

(i) $K \in \mathscr{L}(p)$ and $K$ is p-constrained within $O(K)$.

(ii) $P \cap O(K)=1, \quad V \cap O(K)=P_{1} V_{2}^{\prime} V_{3}^{\prime}, \quad V_{1} \cap O(K)=P_{1}$, and $V \cap O(K)$ is

\section{$C$-invariant.}

(iii) $V_{1}^{\prime}=R_{1}, V_{i}^{\prime} \subseteq O(K), i=2,3$.

(iv) $X_{i} \neq 1, i=2$ or 3 , and $X_{i}$ is $C$-invariant.

Proof. We have $N \subseteq K$ as $P_{1}=O_{p}(N)$, so $K$ contains $D$, covers $L_{0} / O(N)$, and contains a Sylow $p$-subgroup of $O(N)$. Hence, $K \in \mathscr{L}(p)$.

Moreover, $p$ is exceptional, so $p$ is either not stationary or it is stationary and neither centralized nor inverted, by Definition 4.1.3. But $p$ is inverted, even totally inverted. Hence, $p$ is not stationary, so $P_{1}$ is not a Sylow $p$-subgroup of $O(K)$, by Definition 4.1.1. Thus, there is a $D$-invariant Sylow $p$-subgroup of $O(K)$ properly containing $P_{1}$. From Lemma 4.3.3 it follows that $K$ is $p$-constrained in $O(K)$ and (i) is established.

Now $V \supseteq R_{1}$ by choice. Moreover, $V_{i}^{\prime} \in h_{N_{i}}(C), 1 \leqq i \leqq 3, R_{1} \subseteq V_{1}^{\prime}$ as $x_{2}$ inverts $R_{1}$, and $R_{1} \in V_{N_{1}}^{*}(C)$. Thus $V_{1}^{\prime}=R_{1}$. Furthermore, $x$ inverts $V_{2}^{\prime}$ and $V_{3}^{\prime}$, while $O(K) x \in Z(K / O(K))$ as $K \in \mathscr{L}(p)$. Thus, $V_{2}^{\prime} \subseteq O(K)$ and $V_{3}^{\prime} \subseteq O(K)$. This shows that (iii) is valid.

We have $P \cap O(K) \subseteq P \cap O(K) \cap N$, as $P \subseteq N$. But $O(K) \cap N \subseteq O(N)$, by Lemma 4.1.1(v), so $P \cap O(K) \subseteq P \cap O(N)$. However, $p$ is inverted and thus $P \cap O(N)=1$ and we have that $P \cap O(K)=1$.

We also have $V_{1}=V_{0} V_{1}^{\prime}=P R_{1}$, by part (iii), so $V_{1} \cap O(K)=V_{1} \cap N \cap O(K)$ and $V_{1} \cap O(K) \subseteq V_{1} \cap O(N)$, again by Lemma 4.1.1. However, $P R_{1} \cap O(N)=P_{1}$ since $P \cap L_{0}=1$ and $R_{1}=Q_{1} P_{1}$ or $P_{1}$. Hence, $V_{1} \cap O(K) \subseteq P_{1}$. Since $P_{1}$ is normal in $K$, we deduce that $V_{1} \cap O(K)=P_{1}$.

Consequently, $V \cap O(K)=V_{1} V_{2}^{\prime} V_{3}^{\prime} \cap O(K)$, so $V \cap O(K)=\left(V_{1} \cap O(K)\right) V_{2}^{\prime} V_{3}^{\prime}$ since $\left\langle V_{2}^{\prime}, V_{3}^{\prime}\right\rangle \subseteq O(K)$ by (iii). Since $V_{1} \cap O(K)=P_{1}$ it follows that $V \cap O(K)$ $=P_{1} V_{2}^{\prime} V_{3}^{\prime}$. Since $C$ normalizes $P_{1}$ and each $V_{i}^{\prime}$, we also have that $V \cap O(K)$ is $C$-invariant. Thus all the assertions of (ii) have been proved and it remains to establish (iv).

The intersection $V \cap O(K)$ is a Sylow p-subgroup of $O(K)$. However, it contains $P_{1}$, so the containment is proper, as we saw above. Hence, $N_{V \cap O(K)}\left(R_{1}\right) \supset R_{1} \cap O(K)$ as $R_{1} \cap O(K)=P_{1}$. Since $V \cap O(K)$ and $R_{1}$ are $T$-invariant, so is $N_{V \cap O(K)}\left(R_{1}\right)$. The $T$-decomposition of this group shows that $N_{V_{2}^{\prime}}\left(R_{1}\right) \neq 1$ or $N_{V_{3}^{\prime}}\left(R_{1}\right) \neq 1$. However, $x$ inverts $V_{2}^{\prime}$ and $V_{3}^{\prime}$ and centralizes $R_{1}$, so $N_{V_{i}^{\prime}}\left(R_{1}\right) \subseteq X_{i}, i=2,3$. Thus, $X_{2} \neq 1$ or $X_{3} \neq 1$, as required. Furthermore, $X_{i}$ is $C$-invariant as $R_{1}$ and $V_{i}^{\prime}$ are both $C$ invariant. 
LEMMA 4. If $X_{2} \neq 1$, then one of the following holds:

(i) $\left\langle R_{1}, R_{3}\right\rangle$ is a p-group;

(ii) The characteristic of $G$ is $p, \delta \varepsilon=-1, X_{2}=Q_{2}^{*}$ and $\left\langle R_{1}, R_{2}^{*}\right\rangle$ is a p-group.

Note that $\left\langle R_{1}, R_{2}^{*}\right\rangle$, being a $p$-group, does not trivially imply the desired assertion that $\left\langle R_{1}, R_{2}\right\rangle$ is a $p$-group. One must have that $\left\langle R_{i}, R_{j}\right\rangle$ or $\left\langle R_{i}^{*}, R_{j}^{*}\right\rangle$ is a $p$-group, $i \neq j, 1 \leqq i, j \leqq 3$, in order to deduce that.

Also observe that the proposition is now proved unless $p$ divides $q$ and $\delta \varepsilon=-1$ since a similar argument applies if $X_{3} \neq 1$, while we already know that $X_{2} \neq 1$ or $X_{3} \neq 1$.

Proof. If $X_{2} \cap P_{2} \neq 1$, then $C_{P_{2}}\left(R_{1}\right) \neq 1$, so $\left\langle R_{1}, R_{3}\right\rangle$ is a $p$-group, by Lemma 2 , and the proof is complete.

On the other hand, if $X_{2} \cap P_{2}=1$, then $X_{2}$ is either $Q_{2}$ or $Q_{2}^{*}$. Indeed, $X_{2}$ is $C$-invariant by Lemma 3(iv), so $X_{2}$ is a $C$-invariant subgroup of $R_{2}$ or $R_{2}^{*}$. However, $C \cap J$ acts trivially on $P_{2}$, while by Lemma 2.4 .10(iii), it acts irreducibly and nontrivially on $Q_{2}$ and $Q_{2}^{*}$, so $X_{2}=Q_{2}$ or $Q_{2}^{*}$, as asserted. In particular, $Q_{2} \neq 1$ and consequently the characteristic of $G$ is $p$ and $\delta \varepsilon=-1$.

Suppose first that $X_{2}=Q_{2}$. By definition of $X_{2}$, we have $R_{1} \subseteq C\left(X_{2}\right)$. Moreover, since $X_{2}=Q_{2}$, also $R_{2} \subseteq C\left(X_{2}\right)$. Thus, $\left\langle R_{1}, R_{2}\right\rangle \subseteq C\left(Q_{2}\right)$.

We now assert that $C\left(Q_{2}\right)$ has a normal 2-complement. Indeed, this will follow from Burnside's theorem if we show that a Sylow 2-subgroup of $C\left(Q_{2}\right)$ is cyclic. We know that $x_{2} \in C\left(Q_{2}\right)$. Hence if $S^{*}$ is a Sylow 2-subgroup of $C\left(Q_{2}\right)$ containing $x_{2}$ and if $S^{*}$ is not cyclic, then $C_{S^{*}}\left(x_{2}\right)$ is also not cyclic, whence $S^{*} \cap N_{2}$ is not cyclic. However, $C_{N_{2}}\left(Q_{2}\right)$ has a cyclic Sylow 2-subgroup by Lemma 2.4.4, applied to $N_{2} / O\left(N_{2}\right)$. Thus $S^{*}$ is cyclic and $C\left(Q_{2}\right)$ has a normal 2-complement, so our assertion is valid.

In particular, $R_{2} \subseteq O\left(C\left(Q_{2}\right)\right)$. But $Q_{2}$ is $C$-invariant, so $C\left(Q_{2}\right)$ is a normal subgroup of the product $H$ of $C\left(Q_{2}\right)$ and $C$. Hence $R_{2} \subseteq O(H)$ and $R_{1} \subseteq H$. Thus, by Lemma 1 , with $R_{2}$ in place of $R_{3}$, we have that $\left\langle R_{1}, R_{2}\right\rangle$ is a $p$-group. Conjugation by $a^{-1}$ now yields (i).

Finally, if $X_{2}=Q_{2}^{*}$, we argue in the same way and conclude that $\left\langle R_{1}, R_{2}^{*}\right\rangle$ is a $p$-group. The lemma is proved.

The last bit of the argument will be useful later on. We have proved

LEMMA 5. If $1 \leqq i \leqq 3$, then $R_{i} \subseteq O\left(N\left(Q_{i}\right)\right)$ and $R_{i}^{*} \subseteq O\left(N\left(Q_{i}^{*}\right)\right)$.

We shall now assume that $\left\langle R_{1}, R_{3}\right\rangle$ is not a $p$-group and derive a contradiction with the final lemmas. Moreover, as $X_{2} \neq 1$ or $X_{3} \neq 1$, we may as well assume that $X_{2} \neq 1$. Thus, we deduce from Lemma 4 that $p$ divides $q, \delta \varepsilon=-1, X_{2}=Q_{2}^{*}$, and $\left\langle R_{1}, R_{2}^{*}\right\rangle$ is a $p$-group.

We now choose and fix an element $U$ of $U_{G}^{*}(T, C)$ which contains $V$. We use the usual $T$-decomposition notation for $U$ and we set $U^{*}=\left\langle U_{1}^{\prime}, U_{2}^{\prime}, U_{3}^{\prime}\right\rangle, V^{*}=$ $\left\langle V_{1}^{\prime}, V_{2}^{\prime}, V_{3}^{\prime}\right\rangle$. 
LEMMA 6. With the above notation, we have

(i) $U_{1}^{\prime}=R_{1}$;

(ii) $U_{0}=P, P \cap U^{*}=P \cap V^{*}=1$;

(iii) $U^{*}=U_{1}^{\prime} U_{2}^{\prime} U_{3}^{\prime}, V^{*}=V_{1}^{\prime} V_{2}^{\prime} V_{3}^{\prime}$.

Proof. The set $U_{1}^{\prime}$ is an element of $\eta_{N}(C)$ and contains $R_{1}$ as $R_{1}=V_{1}^{\prime} \subseteq U_{1}^{\prime}$, by Lemma 3. Hence, $U_{1}^{\prime}=R_{1}$ and (i) holds.

Similarly, $P \subseteq V \subseteq U$, so $U_{0}=P$. We know that $U^{*}=O_{p}(C U)$ and $V^{*}=O_{p}(C V)$. But $O_{p}(C)=1$, so $P \cap U^{*}=P \cap V^{*}=1$ and (ii) is also valid.

Finally, $U^{*} \supseteq U_{1}^{\prime} U_{2}^{\prime} U_{3}^{\prime}$ and $U=P U_{1}^{\prime} U_{2}^{\prime} U_{3}^{\prime}$, so that if $U^{*} \supset U_{1}^{\prime} U_{2}^{\prime} U_{3}^{\prime}$ then $P \cap U^{*}$ $\neq 1$. This does not happen, by (ii), so $U^{*}$ factors as required. Similarly, the factorization for $V^{*}$ holds.

We now have considerable information on the $T$-decompositions of $U$ and $V$, having determined $U_{0}, V_{0}, U_{1}^{\prime}, V_{1}^{\prime}$ and some information about $V_{2}^{\prime}$, namely that $X_{2}=Q_{2}^{*} \subseteq V_{2}^{\prime}$. We shall now determine the other components of the $T$-decompositions of $U$ and $V$ as well as study $Z\left(U^{*}\right)$. This latter group will lead us to the final contradiction.

LEMMA 7. We have

(i) $V_{3}^{\prime} \supseteq Q_{3}$;

(ii) $U_{2}^{\prime}=R_{2}^{*}$.

Proof. We already have that $V_{2}^{\prime} \supseteq Q_{2}^{*}$. Moreover, $z_{1} \in D_{1}=D \subseteq K$ as $D \subseteq N$ and $P_{1}=O_{p}(N)$. Hence, $Q_{3}=\left(Q_{2}^{*}\right)^{z_{1}} \subseteq V^{z_{1}}$. But $Q_{2}^{*} \subseteq O(K)$, by Lemma 3(ii), so $Q_{3}$ is contained in the $T$-invariant Sylow p-subgroup $V^{z_{1}} \cap O(K)$ of $O(K)$. However, $V \cap O(K)$ is also such a subgroup of $O(K)$, so $V \cap O(K)$ and $V^{z_{1}} \cap O(K)$ are conjugate by an element of $C$. Since $V^{*}$ is $C$-invariant, and $V \cap O(K) \subseteq V^{*}$ by Lemma 3(ii), it follows that $V^{*} \supseteq Q_{3}$, so $V_{3}^{\prime} \supseteq Q_{3}$ and (i) is proved.

As for (ii), it suffices to show that $U \supseteq R_{2}^{*}$. However, $\left\langle R_{1}, R_{2}^{*}\right\rangle$ is a $p$-group and is certainly an element of $\eta_{G}(T, C)$. However, any two elements of $\eta_{G}^{*}(T, C)$ are conjugate by an element of $M$, by Proposition 9.1 , so that $U$ contains the conjugates of $R_{1}$ and $R_{2}^{*}$ by an element of $M$. Thus, $U$ contains an element of $\eta_{N_{2}}^{*}(C)$ or $\eta_{N_{3}}^{*}(C)$. But $Q_{2}^{*} \subseteq U$ and $Q_{3} \subseteq U$ and we know that $\left\langle Q_{2}, Q_{2}^{*}\right\rangle$ and $\left\langle Q_{3}, Q_{3}^{*}\right\rangle$ are not $p$-groups. Hence $R_{2}^{*} \subseteq U$ or $R_{3} \subseteq U$. But $R_{3} \nsubseteq U$ as $R_{1} \subseteq U$ and $\left\langle R_{1}, R_{3}\right\rangle$ is not a $p$-group, by assumption. This proves the lemma.

Before continuing with the analysis of the factors of the $T$-decompositions of $U$ and $V$ we require two results, the second of which is a determination of the important subgroup $Z\left(U^{*}\right)$.

LEMMA 8. We have $J \subseteq N(V \cap O(K))$.

Proof. We begin by observing that any subgroup of $N$ which covers $L_{0} / O(N)$ certainly contains $J=J_{1}$. Indeed, since $q>3$, we have that $J$ is the ultimate term of 
the derived series of $J O(N)$. However, the ultimate term of the derived series of a subgroup of $L_{0}$ which covers $L_{0} / O(N)$ is not only contained in $J$, but also covers $L_{0} / O(N)$ as $L_{0} / O(N)$ is perfect and so is actually equal to $J$. Thus, our assertion is true.

We now set $K_{0}=O(K)\left(L_{0} \cap K\right)$, so that $L_{0} \cap K_{0}$ covers $L_{0} / O(N)$ since $K \in \mathscr{L}(p)$. Now $V \cap O(K)$ is a Sylow $p$-subgroup of $O(K)$, so $K_{0}=O(K) N_{K_{0}}(V \cap O(K))$. Moreover, applying the Frattini argument to the Sylow 2-subgroup $\langle x\rangle$ of the normal subgroup $N_{S\left(K_{0}\right)}(V \cap O(K))$ of $N_{K_{0}}(V \cap O(K))$, we conclude that

$$
K_{0}=O(K)\left(N \cap N_{K_{0}}(V \cap O(K))\right) .
$$

Hence,

$$
\begin{aligned}
K_{0} \cap L_{0} & \subseteq(O(K) \cap N)\left(N \cap N_{K_{0}}(V \cap O(K))\right) \\
& \subseteq O(N)\left(N \cap N_{K_{0}}(V \cap O(K))\right. \\
& \subseteq O(N) N_{K_{0}}(V \cap O(K))
\end{aligned}
$$

Thus, $N_{K_{0}}(V \cap O(K))$ covers $L_{0} / O(N)$ since $K_{0} \cap L_{0}$ covers that section. The lemma now follows from the preceding paragraph.

This last result is now used to prove the

LEMMA 9. The center of $U^{*}$ is $Q_{2}^{*}$.

Proof. Indeed, $Z\left(U^{*}\right)$ is a nonidentity $C$-invariant group. Moreover, $P \cap U^{*}=1$, by Lemma $6\left(\right.$ ii), so $Z\left(U^{*}\right) \cap C=1$. Thus, $Z\left(U^{*}\right)$ has $T$-decomposition

$$
Z\left(U^{*}\right)=\left(R_{1} \cap Z\left(U^{*}\right)\right)\left(R_{2}^{*} \cap Z\left(U^{*}\right)\right)\left(U_{3}^{\prime} \cap Z\left(U^{*}\right)\right)
$$

since $U_{1}^{\prime}=R_{1}$, by Lemma 6(i) and $U_{2}^{\prime}=R_{2}^{*}$, by Lemma 7(ii). Moreover, $Z\left(U^{*}\right) \cap P_{i}$ $=1,1 \leqq i \leqq 3$, by Lemma 2 . Furthermore, each factor is $C$-invariant, so that $R_{1} \cap Z\left(U^{*}\right)$ is $Q_{1}$ or 1 and $R_{2}^{*} \cap Z\left(U^{*}\right)$ is $Q_{2}^{*}$ or 1 . Finally, $V_{3}^{\prime} \supseteq Q_{3}$, by Lemma 7(i), so $U_{3}^{\prime} \subseteq R_{3}$ and thus, $U_{3}^{\prime} \cap Z\left(U^{*}\right)$ is $Q_{3}$ or 1 . Hence, we need only show that $Q_{1} \nsubseteq Z\left(U^{*}\right)$ and that $Q_{3} \nsubseteq Z\left(U^{*}\right)$.

However, suppose that $Q_{3} \subseteq Z\left(U^{*}\right)$. Thus, $R_{1} \subseteq N\left(Q_{3}\right)$ as $R_{1}=U_{1}^{\prime} \subseteq U^{*}$ by Lemma 6(i). Moreover, by Lemma $5, R_{3} \subseteq O\left(N\left(Q_{3}\right)\right)$. Hence, as $Q_{3}$ is $C$-invariant means $C \subseteq N\left(Q_{3}\right)$, we have from Lemma 1 that $\left\langle R_{1}, R_{3}\right\rangle$ is a $p$-group, which is a contradiction. Thus, $Q_{3} \nsubseteq Z\left(U^{*}\right)$.

Finally, $x=x_{1}$ inverts $V \cap O(K) / P_{1}$ as $P_{1}$ is certainly normal in $K=N\left(P_{1}\right)$ and $V \cap O(K)=P_{1} V_{2}^{\prime} V_{3}^{\prime}$, by Lemma 3. Moreover, $V \cap O(K) / P_{1}$ is nontrivial as $V_{3}^{\prime} \supseteq Q_{3}$, by Lemma 7(i). However, $J$ normalizes $V \cap O(K)$, by Lemma 8 , so $J$ acts on $V \cap O(K) / P_{1}$. Furthermore, as we have noted at the beginning of $\S 7$, $J \cap O(N)=1$ when $\delta \varepsilon=-1$. But $\delta \varepsilon=-1$ by Lemma 4(ii). Since $O(J) \subseteq J \cap O(N)$, it follows that $O(J)=1$ and hence that $\langle x\rangle$ is the unique minimal normal subgroup of $J$. We conclude that $J$ acts faithfully on $V \cap O(K) / P_{1}$. Thus, $Q_{1}$ does not centralize $V \cap O(K)$, so $Q_{1} \nsubseteq Z\left(U^{*}\right)$ as desired. This proves the lemma. 
We can now complete our determination of the $T$-decompositions of $U$ and $V$.

LEMMA 10. We have

(i) $U_{3}^{\prime}=V_{3}^{\prime}=Q_{3}$;

(ii) $V_{2}^{\prime}=Q_{2}^{*}$.

Once we prove this result, all the $T$-decompositions we have are as follows:

$$
\begin{array}{cc}
U=P R_{1} R_{2}^{*} Q_{3}, & U^{*}=R_{1} R_{2}^{*} Q_{3}, \\
V=P R_{1} Q_{2}^{*} Q_{3}, & V^{*}=R_{1} Q_{2}^{*} Q_{3}, \\
V \cap O(K)=P_{1} Q_{2}^{*} Q_{3} .
\end{array}
$$

Proof. We have $U_{3}^{\prime} \supseteq V_{3}^{\prime} \supseteq Q_{3}$, by Lemma 7(i), so $U_{3}^{\prime} \subseteq R_{3}$. Assume that $U_{3}^{\prime} \nsubseteq Q_{3}$, so that $U_{3}^{\prime} \cap P_{3} \neq 1$. Since $Q_{2}^{*}=Z\left(U^{*}\right)$, by Lemma 9 , this implies that $C_{P_{3}}\left(Q_{2}^{*}\right) \neq 1$. Conjugation by the element $a$ yields that $\tilde{R}=C_{P_{1}}\left(Q_{3}^{*}\right) \neq 1$. Thus, $\tilde{R}$ is a nonidentity subgroup of $P_{1}$, so $C(\tilde{R}) \supseteq J_{1}=J$ and $C(\tilde{R}) \supseteq Q_{3}^{*}$ as we just have seen.

Moreover, $Q_{3}$ centralizes $P_{1}$. Indeed, $V_{3}^{\prime} \supseteq Q_{3}$, so $Q_{3} \supseteq K=N\left(P_{1}\right)$. But $x=x_{1}$ inverts $Q_{3}$ and centralizes $P_{1}$, so $\left[Q_{3}, P_{1}\right]=1$ as claimed. In particular, $H=C(\tilde{R})$ $\supseteq Q_{3}$, so $H \supseteq\left\langle Q_{3}, Q_{3}^{*}\right\rangle$ and therefore $H \supseteq J_{3}$, by Lemma 2.4.10. Hence $H \supseteq\left\langle J_{1}, J_{3}\right\rangle$ and so $H$ is a $Q$-group of characteristic power $q$ with $H=O(H)(H \cap N)$ by Proposition 4.1.1(iii). Thus $\bar{x}$ is in the center of $\bar{H}=H / O(H)$, contrary to the fact that $\bar{x}$ is not in the center of $\bar{J}_{3}$. We conclude that $U_{3}^{\prime} \subseteq Q_{3}$, so $Q_{3} \subseteq V_{3}^{\prime} \subseteq U_{3}^{\prime} \subseteq Q_{3}$ and (i) is established.

We know that $J$ normalizes $V \cap O(K)$ by Lemma 8 , so $y \in J$ normalizes $V \cap O(K)$. Furthermore, $C$ normalizes $V \cap O(K)$ by Lemma 3(ii) and hence $D_{1}=D=$ $\langle C \cap D, y\rangle$ normalizes $V \cap O(K)$. In particular, $(V \cap O(K))^{z_{1}}=V \cap O(K)$. Hence, $C_{V \cap O(K)}\left(x_{2}\right)^{z_{1}}=C_{V \cap O(K)}\left(x_{3}\right)$ and $\left|C_{V \cap O(K)}\left(x_{2}\right)\right|=\left|C_{V \cap O(K)}\left(x_{3}\right)\right|$. However, $C_{V \cap O(K)}\left(x_{2}\right)$ $=V_{2}^{\prime}$ and $C_{V \cap O(K)}\left(x_{3}\right)=V_{3}^{\prime}=Q_{3}$, by (i). Hence, $\left|V_{2}^{\prime}\right|=\left|Q_{3}\right|=q$. Since $V_{2}^{\prime} \supseteq Q_{2}^{*}$, by Lemma 4(ii), this implies that $V_{2}^{\prime}=Q_{2}^{*}$ as $\left|Q_{2}^{*}\right|=q$.

We reach the penultimate lemma:

Lemma 11. The center of $V^{*}$ is $P_{1} Q_{2}^{*}$.

Proof. First, $Z\left(V^{*}\right) \supseteq P_{1} Q_{2}^{*}$. Indeed, $Q_{2}^{*}=Z\left(U^{*}\right)$, by Lemma 9 , and $Q_{2}^{*} \subseteq V^{*}$, so $Q_{2}^{*} \subseteq Z\left(V^{*}\right)$. Moreover, $P_{1}$ is normal in $V^{*}$, while $x$ centralizes $P_{1}$ and inverts $Q_{2}^{*}$ and $Q_{3}$, so $Q_{2}^{*}$ and $Q_{3}$ centralize $P_{1}$. Since $R_{1}$ is abelian and $V^{*}=R_{1} Q_{2}^{*} Q_{3}$, it follows that $P_{1} \subseteq Z\left(V^{*}\right)$.

To conclude the proof we need only show that $Z\left(V^{*} / P_{1}\right)$ has order $q$ as then $Z\left(V^{*}\right) \subseteq P_{1} Q_{2}^{*}$. However, $V \cap O(K) / P_{1}$ is elementary of order $q^{2}$ as $V \cap O(K)$ $=P_{1} Q_{2}^{*} Q_{3}$ and as $x=x_{1}$ inverts $Q_{2}^{*}$ and $Q_{3}$. Moreover, as in the previous lemma, $J$ acts faithfully on $V \cap O(K) / P_{1}$. Since $Q_{1}$ is a Sylow $p$-subgroup of $J$, it follows therefore from Lemma 2.4.18 that the centralizer of $Q_{1}$ on $V \cap O(K) / P_{1}$ has order $q$. Since $V^{*}=Q_{1}(V \cap O(K))$, we conclude that $Z\left(V^{*} / P_{1}\right)$ has order $q$, as required, and the lemma is proved. 
The next and final result of the section-and of the chapter-contradicts Lemma 9 and so completes all the proofs.

LEMMA 12. The center of $U^{*}$ contains $P_{1} Q_{2}^{*}$.

Proof. Choose a $T$-invariant subgroup $F$ of $U^{*}$ containing $V^{*}$ maximal subject to the condition that $Z(F) \supseteq P_{1} Q_{2}^{*}$. We may do this as $Z\left(V^{*}\right) \supseteq P_{1} Q_{2}^{*}$ by the previous lemma. It suffices to show that $F=U^{*}$, so we assume $F \subset U^{*}$ and derive a contradiction. We set $Y=N_{U^{*}}(F)$, so $Y \supset F$.

However, we have the containments

$$
U^{*}=R_{1} R_{2}^{*} Q_{3} \supset F \supseteq V^{*}=R_{1} Q_{2}^{*} Q_{3},
$$

so $Y=R_{1} N_{R_{2}^{*}}(F) Q_{3}$. But $F \supseteq Q_{2}^{*}$, so $Y=F N_{P_{2}}(F)$. Thus we will have that $Z(Y)$ $\supseteq P_{1} Q_{2}^{*}$, and a contradiction, once we show that $N_{P_{2}}(F)$ centralizes both $P_{1}$ and $Q_{2}^{*}$.

However, $Y \cap P_{2}=N_{P_{2}}(F) \subseteq R_{2}^{*}=P_{2} Q_{2}^{*}$, which is abelian. Thus, $Y \cap P_{2}$ certainly centralizes $Q_{2}^{*}$. It remains to show that $Y \cap P_{2}$ centralizes $P_{1}$.

The group $Y \cap P_{2}$ normalizes $Z(F)$ and centralizes $x_{2}$ and thus normalizes $\left[Z(F), x_{2}\right]$. However, $Z(F) \supseteq P_{1} Q_{2}^{*}$, by assumption. Suppose first that $Z(F) \subseteq P_{1} R_{2}^{*}$. Since $x_{2}$ centralizes $R_{2}^{*}$ and inverts $P_{1}$, we have $\left[Z(F), x_{2}\right]=\left[P_{1}, x_{2}\right]=P_{1}$, whence $Y \cap P_{2}$ normalizes $P_{1}$. But $x$ inverts $Y \cap P_{2}$ and centralizes $P_{1}$, so $Y \cap P_{2}$ centralizes $\boldsymbol{P}_{1}$.

Hence, in order to complete the proof, we need only see that $Z(F) \subseteq P_{1} R_{2}^{*}$. However, $F$ is $T$-invariant, $V^{*} \subseteq F \subseteq U^{*}$, so $F$ has $T$-decomposition

$$
F=R_{1} Q_{2}^{*}\left(F \cap P_{2}\right) Q_{3}
$$

Hence, $Z(F)$ has $T$-decomposition

$$
Z(F)=\left(R_{1} \cap Z(F)\right)\left(Q_{2}^{*}\left(F \cap P_{2}\right) \cap Z(F)\right)\left(Q_{3} \cap Z(F)\right) .
$$

But $R_{1} \cap Z(F) \subseteq Z\left(V^{*}\right)$ as $R_{1} \subseteq V^{*}$ and $Q_{3} \cap Z(F) \subseteq Z\left(V^{*}\right)$ as $Q_{3} \subseteq V^{*}$. Hence, $R_{1} \cap Z(F) \subseteq P_{1}$ and $Q_{3} \cap Z(F)=1$. But $Q_{2}^{*}\left(F \cap P_{2}\right) \subseteq R_{2}^{*}$, so $Z(F) \subseteq P_{1} R_{2}^{*}$, as required.

\section{Chapter VI. Structure of $N$. Proof of Theorem C}

1. Outline of chapter and some consequences of Theorem B. In this, the final chapter of the paper, we consider a simple group $G$ with quasi-dihedral Sylow 2-subgroups which satisfies the conclusions of the expanded form of Theorem B, stated in $\$ 1$ of Chapter IV; and we shall prove that such a group $G$ necessarily satisfies the conclusions of Theorem $\mathrm{C}$. This will complete the proof of all of our Main Theorems, for as we have shown in Chapter I, Theorems A, B, and C together with certain results of Chapter III imply the three Main Theorems. In particular, the characteristic power $q$ of $G$ can be assumed to be greater than 5 , 
the Main Theorems having been established in $\S 8$ of Chapter III in the cases $q=3$ and 5.

We use the same notation as before. In particular, we know from Theorem B that $O(N)=A B$, where $A$ is cyclic of order dividing $\frac{1}{2}(q+\varepsilon), B$ is abelian, and if $A$ and $B$ are both nontrivial, then $O(N)$ is a Frobenius group with kernel $B$ and complement $A$. Furthermore, $T$ centralizes $A$, the involution $x_{2}=z$ of $T$ inverts $B$, and $C^{*}(u) \subseteq N$ for any element $u \neq 1$ of $O(N)$. Moreover, $L_{0}=J O(N)$, where $J$ is normal in $N, J$ centralizes $O(N)$, and either $J$ is isomorphic to $S L(2, q)$ and $J O(N)$ $=J \times O(N)$ or $q=9, J$ is isomorphic to $S L^{\wedge}(2,9)$, and $|J \cap O(N)|=3$. We note also that since $S=(S \cap J) T, A$ centralizes $S$. Since the element $y$ of $S$ inverts $O(C) \cap J$, it follows that $A=O(C(S)) \cap L=E \cap L$.

In this section, we shall give a brief outline of the chapter and shall then derive a few direct consequences of Theorem B. The last of these (Lemma 3 below), when combined with Corollary 3.9.2, will immediately yield part (i) of Theorem $\mathrm{C}$.

As in Chapters I and III, we have $a=|A|=|C \cap O(N)|, b=|O(N): C \cap O(N)|$ $=|B|, e=|E / E \cap L|=|E / A|$, and $|N|=2 a b e q(q-1)(q+1)$ (cf. equation 3.3.2). We also set $C_{0}=O(C)$ and let $t$ be a 3-element of $N(T)$ which cyclically permutes the involutions of $T$.

$\$ 2$ consists of several, somewhat independent results, which it is convenient to establish at the outset. First, we prove that for any prime $p$ dividing $b$, a Sylow $p$-subgroup of $N$ is a Sylow $p$-subgroup of $G$. This result, in conjunction with the order formula for a regular $Q D$-group given in Proposition 3.2.6 is then used to prove that part (ii) of Theorem $\mathrm{C}$ holds whenever $G$ is regular. A similar conclusion is then obtained in the cases $q=7$ and $q=9$, even if $G$ is not regular, using the order formulas for $Q D$-groups of characteristic power 7 and 9 developed in $\$ 8$ of Chapter III. For $q=7$, it then follows that $G$ is regular. After that, we argue that part (iii) of Theorem $\mathrm{C}$ holds if $A \neq 1$. Thus if $A \neq 1, G$ is regular and therefore part (ii) of Theorem $\mathrm{C}$ holds in this case by one of the preceding results of the section. Finally we establish an omnibus lemma concerning the structure of $N\left(C_{0}\right)$ in the case that $A=1$.

In $\$ \S 3,4$, and 5 , we establish parts (ii) and (iii) of Theorem $C$. In view of the results of $\S 2$, we can assume that $q>7$. $\S 3$ is devoted to a proof that the case $A=1, B=1$ is impossible, the argument being primarily character-theoretic. Then in $\$ \$ 4$ and 5 , we argue that the case $A=1, B \neq 1$ is also impossible. This argument is largely local group-theoretic, but does require one particular fact whose proof is charactertheoretic: Namely, every prime divisor of $b$ is necessarily a divisor of $q(q-1)(q+1)$. Together, these results force $A \neq 1$ and therefore parts (ii) and (iii) of Theorem C will follow from the results of $\S 2$.

In the final two sections we investigate the case $\varepsilon=1$ in more detail. In $\$ 6$ we show that $G$ is a doubly transitive permutation group of the type described in part (iv) of Theorem C, while in $\S 7$ we prove that the order of $A$ is $(q+1) / 2 d$, where $d=(q+1,3)$. Together these results will establish part (iv) of Theorem $\mathrm{C}$. 
In $\$ \$ 2$ and 7, we shall point out in two remarks what is required to establish the corresponding analogue of Theorem $\mathrm{C}$ in the case of simple $Q D$-groups with wreathed Sylow 2-subgroups.

We turn now to some consequences of Theorem B.

LEMMA 1. If $B=1$, then $L=(J T) \times O(N)=(J T) \times A$.

Proof. If $J$ is isomorphic to $S L^{\wedge}(2,9)$, then $J T$ is isomorphic to $S U_{1}^{\wedge}(2,9)$ by Lemma 2.2.2. But then $z$ inverts $O(J)$ by Lemma 2.4.12. However, $O(J)$ has order 3 and lies in $O(N)$, so $O(J) \subseteq B$, contrary to our assumption that $B=1$. We conclude that $J$ is not isomorphic to $S L^{\wedge}(2,9)$ and hence that $L_{0}=J \times O(N)$. Since $B=1, O(N)=A$ and therefore $T$ centralizes $O(N)$. Since $L=L_{0} T=J T O(N)$, the lemma follows.

As a corollary, we have

Lemma 2. If $B=1$ and $E=A$ (equivalently, $b=1$ and $e=1$ ), then $N=(J T) \times A$ and $N$ is isomorphic to a quotient of $G L(2, q)$ if $\varepsilon=-1$ and of $G U(2, q)$ if $\varepsilon=1$ by a central subgroup of odd order $d=(q+\varepsilon) / 2 a$.

Proof. We know that $N=L E$. Since $E=A$ by assumption and since $A \subseteq L$, it follows that $N=L$. Hence by the preceding lemma, $L=(J T) \times A$. Moreover, $J T$ is isomorphic to $L / O(N)$ and hence to $S L^{ \pm}(2, q)$ if $\varepsilon=-1$ and to $S U^{ \pm}(2, q)$ if $\varepsilon=1$. Finally, $A$ is cyclic of order $a$ dividing $(q+\varepsilon) / 2$. But now the lemma follows at once from the structure of $G L(2, q)$ and $G U(2, q)$ as described in Chapter II.

We next prove

LEMMA 3. The following conditions hold:

(i) $C_{0}=\left(C_{0} \cap L\right) E$;

(ii) $C_{0} \cap L$ is normal in $N(T)$.

Proof. Since $N=L E$ and $E \subseteq C_{0}$, we have $C_{0}=\left(C_{0} \cap L\right) E$, proving (i). To prove (ii), set $W=C_{0} \cap J$, so that $C_{0} \cap L=W A$. In $\S 5$ of Chapter IV we have argued that assertions (i) and (ii) of Theorem $B$ are a consequence of assertion (iii). In carrying out this argument, we have shown in Lemma 4.5.1 that the conclusion that $W A$ is normal in $N(T)$ follows from part (iii) of Theorem B. Since $W A$ $=C_{0} \cap L$, and since $G$ satisfies all the conclusions of Theorem $\mathrm{B}$, we see that (ii) holds.

This enables us to prove

Proposition 1. We have $N=L$ and $E=A$.

Proof. Since $C_{0} \cap L$ is normal in $N(T)$ by the lemma, it is invariant under the element $t$ of $N(T)$. But now as $G$ is simple, Corollary 3.9.2 implies that $C_{0}=C_{0} \cap L$. Since $C_{0}=\left(C_{0} \cap L\right) E$ by the preceding lemma, it follows that $E \subseteq L$. On the other hand, $N=L E$ and $E \cap L=A$; and we conclude at once that $N=L$ and $E=A$. 
2. Preliminary results. We begin this section with the following key result:

Proposition 1. If $P$ is a Sylow p-subgroup of $B$ for some prime divisor $p$ of $b$ and $Q$ is a p-subgroup of $N$ containing $P$, then $N(Q) \subseteq N$. In particular, a Sylow p-subgroup of $N$ is a Sylow p-subgroup of $G$.

Proof. Set $H=N(Q)$ and $K=C(Q)$. We shall argue that $H \subseteq N$. Indeed, by part (iii) of Theorem $\mathrm{B}$, which holds for $G, C(P) \subseteq N$. Since $C(Q) \subseteq C(P)$, it follows that $K \subseteq N$. Furthermore, $L=L_{0}\langle z\rangle$ and $z$ inverts $P$, so a Sylow 2-subgroup $S_{0}$ of $K$ is contained in $L_{0}$ and therefore is either cyclic or generalized quaternion. Since $x \in K$, we can choose $S_{0}$ to contain $x$. But then in either case $x$ is the unique involution of $S_{0}$, so $\langle x\rangle$ is characteristic in $S_{0}$. Since $K$ is normal in $H$, it follows therefore by the Frattini argument that

$$
H=K N_{H}\left(S_{0}\right)=K N_{H}(\langle x\rangle)=K C_{H}(x) .
$$

Since $N=C(x)$ and $K \subseteq N$, we conclude that $H \subseteq N$, as asserted.

As for the final assertion of the proposition, take $Q$ now to be a Sylow $p$-subgroup of $N$ and let $R$ be a Sylow. $p$-subgroup of $G$ containing $Q$. By the preceding argument, $N_{R}(Q) \subseteq N$, whence $N_{R}(Q)=Q$ as $Q$ is a Sylow $p$-subgroup of $N$. This forces $Q=R$ and so $Q$ is a Sylow $p$-subgroup of $G$.

As a corollary, we have

Proposition 2. If $G$ is regular, then $B=1$.

Proof Since $G$ is regular by assumption and since $e=1$ by Proposition 1.1, Proposition $3.3 .2^{*}$ yields

$$
|G|=2 a b^{3} q^{3}(q+1)(q-1)\left(q^{2}-\varepsilon q+1\right) .
$$

On the other hand,

$$
|N|=2 a b q(q+1)(q-1) .
$$

Equations (1) and (2) together imply that $b^{2}$ divides $|G: N|$. Hence a Sylow $p$-subgroup of $N$ for $p$ dividing $b$ is certainly not a Sylow $p$-subgroup of $G$, contrary to Proposition 1.

REMARK. If $G$ is a simple $Q D$-group with wreathed Sylow 2-subgroups, which satisfies the conclusions of Theorem B, then it is very easy to establish the corresponding Proposition 1 above. Moreover, the general character theory of $Q D$ groups with wreathed Sylow 2-subgroups, based on an analysis of the principal 2-block of $G$, will show that $G$ is always "regular", in the sense that $|G|$ is necessarily given by a formula analogous to that of Proposition 3.3.2*. Hence the same argument as above will show that $B=1$. Thus for simple $Q D$-groups with wreathed Sylow 2-subgroups, parts (ii) and (iii) of the corresponding Theorem $C$ will follow very much more easily than in the quasi-dihedral case, once the general character theory of such groups is developed. 
A similar argument yields

Proposition 3. If $G$ has characteristic power $q=7$ or $q=9$, then $B=1$.

Proof. We apply Propositions 3.8.3 and 3.8.4. In view of the preceding proposition, we need only consider the nonregular cases, of which there are three. However, in each of these cases, it is still true that $b^{3}$ divides $|G|$ and that $b^{2}$ divides $|G: N|$, so we reach the same contradiction from Proposition 1.

As a consequence, we also have

Proposition 4. If $G$ has characteristic power $q=7$, then $G$ is regular.

Proof. This is immediate from Proposition 3.8.7 since we now know that $b=1$ and that $a$ divides $(q+\varepsilon) / 2=3$.

Since parts (ii) and (iii) of Theorem $C$ have been verified in the case $q=7$, we shall assume for the balance of the proof of these parts of the theorem that $q>7$.

We wish next to show that $G$ is regular whenever $A \neq 1$. We require the following preliminary lemma:

LEMMA 1. The following conditions hold:

(i) $C_{0} / A$ is cyclic of order $\frac{1}{2}(q+\varepsilon)$ and is inverted by the element $y$ of $D$;

(ii) If $A \neq 1$, the element $t$ does not normalize $A$.

Proof. Since $E=A$ by Proposition 1.1, we have $C_{0}=C_{0} \cap L$ by Lemma 1.3. Since $A=C \cap O(N)$, it follows therefore from Lemma 2.4.2, applied to $L / O(N)$, that $C_{0} / A$ is cyclic of order $\frac{1}{2}(q+\varepsilon)$ and is inverted by $y$. Thus (i) holds.

Suppose next that $A \neq 1$ and that $t$ normalizes $A$. Since $S$ centralizes $A$, so therefore does $S^{t}$. However, $C(A) \subseteq N$ since $G$ satisfies the conclusions of Theorem B. Thus $S^{t} \subseteq N$ and so $S^{t u}=S$ for some element $u$ of $N$. It follows that $t u \in N(S)$ $\subseteq N(Z(S))=C(x)=N$. We conclude that $t \in N$, contrary to the fact that $x^{t}=x_{2}$ by the definition of $t$. (Alternately, one can argue, under the given conditions, that $N(A)$ is a $Q D$-group of characteristic power $q$ and then invoke Theorem $\mathrm{A}$ to obtain a contradiction.)

We now prove

Proposition 5. If $A \neq 1$, then $G$ is regular.

Proof. We shall show that the following two conditions are satisfied:

(\#) For each block $b$ of $N$ with the defect group $T$, the (unique) degree of the characters in $b$ is coprime to $q$ (cf. Chapter III, §9).

(\#\#) There exist irreducible characters $\omega$ of $C$ which are trivial on $T$ and which have the stabilizer $C$ in $N(T)$.

If these conditions are established, we can apply Proposition 3.9.4 and obtain Proposition 5.

Suppose that (\#) is false for the block $b$. Then the degree of a character $\xi \in b$ is divisible by the prime $p$ dividing $q$. Since $b$ has defect $2, \xi(1)$ is divisible by $2^{n-1}$ 
and hence by 4 . By Proposition 1.1, $N=L$ and hence $\left|N: L_{0}\right|=2$. If $\xi_{0}$ is an irreducible constituent of $\xi \mid L_{0}$, we have either $\xi(1)=\xi_{0}(1)$ or $\xi(1)=2 \xi_{0}(1)$. In any case, $\xi_{0}(1)$ is divisible by $2 p$.

Assume first that $q \neq 9$. Then $L_{0}=J \times O(N)$. Hence $\xi_{0}$ can be written as product of an irreducible character $\eta_{1}$ of $J$ and an irreducible character $\eta_{2}$ of $O(N)=A B$. We note that $\eta_{2}(1)$ is not divisible by $p$. This is clear if $a=1$ or $b=1$, since then $O(N)$ is abelian. If $a \neq 1, b \neq 1$, then as we have seen from Theorem $\mathrm{B}, O(N)$ is a Frobenius group with Frobenius kernel $B$ and complement $A$. Since $B$ is abelian, it follows that $\eta_{2}(1)$ divides $|A|=a$, cf. [19, Theorem 4.5.3]. Since we also know that $a$ divides $\frac{1}{2}(q+\varepsilon), \eta_{2}(1)$ is indeed coprime to $p$.

It now follows that $\eta_{1}(1)$ is divisible by $2 p$. However, this yields a contradiction since $J \simeq S L(2, q)$ and the only degree of an irreducible character of $S L(2, q)$ divisible by $p$ is $q$ itself, [26, p. 128] or [27]. This establishes the condition (\#) for $q \neq 9$.

If $q=9$, then by Proposition $2.3, b=1$ and by Lemma $1.1, N=L=(J T) \times A$. Again, $L_{0}=J \times A$ does not have an irreducible character of a degree divisible by $2 p$. Again, condition (\#) holds.

By Proposition 1.1 and Lemma $1, C_{0} / A$ is cyclic of order $\frac{1}{2}(q+\varepsilon)$. Let $\omega_{0}$ denote an irreducible character of $C_{0}$ with the kernel $A$. Let $\omega$ be the extension of $\omega_{0}$ to $C=T \times C_{0}$ which is trivial on $T$. Then $\omega$ has the kernel $T \times A$. This implies that $\omega^{t}$ has the kernel $T \times A^{t}$ and $\omega^{t^{-1}}$ has the kernel $T \times A^{t^{-1}}$. Moreover, the three kernels are distinct by Lemma 1 and hence the irreducible characters $\omega, \omega^{t}, \omega^{t-1}$ of $C$ are distinct. Since $y$ inverts $C_{0} / A$, again by Lemma 1 , the character $\omega^{y}$ is the conjugate complex $\bar{\omega}$ of $\omega$. Since $a \neq 1, \bar{\omega}$ is different from $\omega, \omega^{t}, \omega^{t^{-1}}$. (note that $t$ is a 3-element). Hence $\omega$ has at least four distinct conjugates in $N(T)$. We then must have six distinct conjugates of $\omega$ in $N(T)$. The stabilizer of $\omega$ in $N(T)$ is necessarily $C_{0}$ and $\omega$ satisfies condition (\#\#).

As remarked in the beginning of the proof, this completes the proof of the proposition.

Corollary 1. We have $A=1$ or $B=1$.

Indeed, if $A \neq 1$, by Proposition 5, $G$ is regular and then $B=1$ by Proposition 2 .

We conclude this section with an omnibus lemma concerning the structure of $N\left(C_{0}\right)$ in the case that $A=1$.

Lemma 2. Assume that $A=1$ and set $H=N\left(C_{0}\right), K=C\left(C_{0}\right)$. Then the following conditions hold:

(i) $C_{0}$ is a nontrivial cyclic group of order $\frac{1}{2}(q+\varepsilon)$ which is inverted by $y$ and centralized by $t$.

(ii) $|H: K|=2, D$ is a Sylow 2-subgroup of $H$ and $T$ is a Sylow 2-subgroup of $K$.

(iii) $H / O(H)$ is isomorphic to $P G L\left(2, q^{\prime}\right)$ with $q^{\prime} \equiv 3,5(\bmod 8)$ and $K / O(H)$ is isomorphic to $\operatorname{PSL}\left(2, q^{\prime}\right)$. Moreover, if $B=1$, then $q^{\prime}=3$ or 5 . 
(iv) $H \cap N=C_{0} B D$ and $B$ is cyclic of order $\frac{1}{4}\left(q^{\prime}-\varepsilon^{\prime}\right)$, where $q^{\prime} \equiv \varepsilon^{\prime}(\bmod 4)$.

(v) $C_{H}(z y)$ is a dihedral group and $O\left(C_{H}(z y)\right)$ is cyclic of order $\frac{1}{2}\left(q^{\prime}+\varepsilon^{\prime}\right)$ and is inverted by $x$.

(vi) $O\left(C_{H}(z y)\right)$ and $B$ are of relatively prime orders.

(vii) For any element $w \neq 1$ of $C_{0}, C(w) \subseteq K, T$ is a Sylow 2-subgroup of $C(w)$, $O(C(w))=C_{0}, C_{0}$ is normal in $C(w)$, and $N(\langle w\rangle)=H$.

(viii) If $w_{1}$ and $w_{2}$ are elements of $C_{0}$ that are conjugate in $G$, then $w_{2}=w_{1}^{ \pm 1}$.

Proof. Since $A=1, C_{0}$ is cyclic of order $\frac{1}{2}(q+\varepsilon)$ and is inverted by $y$ by Lemma 1 . Moreover, $C_{0} \neq 1$ since $q>7$ by our present assumption. Furthermore, $M=N(T)$ $=\left\langle C_{0}, T, y, t\right\rangle$ normalizes $C_{0}=O(C)$ and $y$ inverts $t$ (modulo $C$ ). In particular, $t \in M^{\prime}$. But $M^{\prime} \subseteq K=C\left(C_{0}\right)$ as $C_{0}$ is cyclic. Thus $t$ centralizes $C_{0}$ and so all parts of (i) hold.

Let $W_{0}$ be any nontrivial subgroup of $C_{0}$ and set $F=N\left(W_{0}\right)$. We shall analyze the structure of $F$. Since $W_{0}$ is characteristic in $C_{0}$ and $C_{0}$ is normal in $M$, we have $M \subseteq F$. In particular, $D=\langle T, y\rangle \subseteq F$ and so a Sylow 2-subgroup $S_{0}$ of $F$ containing $D$ is either dihedral or quasi-dihedral. In either case, $Z(F)=Z(D)=\langle x\rangle$ and so if $\bar{N}=N / O(N)$, then $N_{L_{0}}\left(\bar{W}_{0}\right)=\left\langle\bar{C}_{0}, \bar{y}\right\rangle$ by Lemma 2.4 .2 (i). However, $O(N)=B$ as $A=1$ and $B$ centralizes $C_{0}$ as $C_{0} \subseteq J$. It follows therefore that $N_{L_{0}}\left(W_{0}\right)=\left\langle C_{0} B, y\right\rangle$ and hence that

$$
N_{N}\left(W_{0}\right)=N \cap F=C_{0} B D
$$

In particular, $D$ is a Sylow 2-subgroup of $N \cap F$ and therefore $S_{0}=D$. Thus $D$ is a Sylow 2-subgroup of $F$. Since $T$ centralizes $W_{0}$ and the involution $z y$ inverts $W_{0}$, it also follows that $F$ has a normal subgroup of index 2. On the other hand, $t \in F$ by (i) and $t$ normalizes, but does not centralize $T$. Hence $F$ does not have a normal 2-complement and so $F$ is a $D$-group with dihedral Sylow 2-subgroup of order eight.

Next let $P$ be a $T$-invariant Sylow $p$-subgroup of $O(F)$ with $T$-decomposition $P=P_{1} P_{2} P_{3}=P_{0} P_{1}^{\prime} P_{2}^{\prime} P_{3}^{\prime}$. Since $t$ cyclically permutes the involutions $x_{i}$ of $T, 1 \leqq i \leqq 3$, either each $P_{i}^{\prime}=1$ or each $P_{i}^{\prime} \neq 1$. Suppose $P_{1}^{\prime} \neq 1$, in which case $P_{1} \subset P$. Since $P_{1}^{\prime}$ is inverted by $x_{2}=z$ and $P_{1}^{\prime}$ normalizes $W_{0}, P_{1}^{\prime}$ centralizes $W_{0}$ and so $P_{1}^{\prime} \subseteq C_{L_{0}}\left(W_{0}\right)$. Since $N_{N}\left(W_{0}\right)=C_{0} B D$, we obtain that $P_{1}^{\prime} \subseteq C_{0} B=C_{0} \times B$. Since $z$ inverts $B$ and centralizes $C_{0}$, it follows that $P_{1}^{\prime} \subseteq B$. Since $G$ satisfies the conclusions of Theorem B, we conclude that $C\left(P_{1}^{\prime}\right) \subseteq N$. On the other hand, $N_{P}\left(P_{1}\right) \supset P_{1}$ and so $x$ inverts some element $u \neq 1$ of $N_{P}\left(P_{1}\right)$. Since $x$ centralizes $P_{1}, u$ is forced to centralize $P_{1}$ and, in particular, $P_{1}^{\prime}$. Hence $u \in C\left(P_{1}^{\prime}\right) \subseteq N$, so $x$ centralizes $u$ which is not the case. This contradiction shows that $P_{i}^{\prime}=1$ for all $i, 1 \leqq i \leqq 3$, and hence that $P=P_{0}$ $\subseteq C_{0}$. Since the prime $p$ was arbitrary, we conclude that $O(F) \subseteq C_{0}$.

We argue next that $F=H$. Since $C_{0}$ is abelian, we have $C_{0} \subseteq F$. It will suffice to show that $C_{0} \subseteq O(F)$. Indeed, if this is the case, then $C_{0}=O(F)$ by the preceding paragraph. But then $C_{0}$ is normal in $F$ and so $F \subseteq H=N\left(C_{0}\right)$. However, the reverse 
inclusion also holds as $W_{0}$ is characteristic in $C_{0}$. Thus the desired conclusion $F=H$ will follow.

Set $\bar{F}=F / O(F)$. Since $\bar{F}$ is a $D$-group having a normal subgroup of index 2 and dihedral Sylow 2-subgroup $\bar{D}$ of order eight, Proposition 2.3.4 implies that $\bar{F}=\bar{L}^{*} \bar{E}^{*}$, where $\bar{L}^{*}$ is isomorphic to $\operatorname{PGL}\left(2, q^{\prime}\right), q^{\prime}$ odd, $\bar{L}^{*}$ is normal in $\bar{F}, \bar{E}^{*}$ is cyclic of odd order, and $\bar{E}^{*}$ centralizes $\bar{D}$. In particular, $\bar{E}^{*} \subseteq \bar{C}_{0}$. However, the element $\bar{y}$ of $\bar{D}$ inverts $\bar{C}_{0}$ and consequently $\bar{E}^{*}=1$. Thus $\bar{F}=\bar{L}^{*}$ and $\bar{C}_{0} \subseteq \bar{L}^{*}$. But $\bar{T}$ centralizes $\bar{C}_{0}$ and so $\bar{C}_{0}=1$ by Lemma 2.5 .7 (iii). Thus $C_{0} \subseteq O(F)$ and the desired assertion $\boldsymbol{F}=\boldsymbol{H}$ follows.

We can now easily verify the various parts of the lemma. We have shown that $\bar{H}=H / O(H)=\bar{F}$ is isomorphic to $P G L\left(2, q^{\prime}\right)$. Since $|\bar{D}|=8$ and $\bar{D}$ is a Sylow 2-subgroup of $\bar{F}$, we must have $q^{\prime} \equiv 3,5(\bmod 8)$. Furthermore, $\bar{H}^{\prime}$ is of index 2 in $\bar{H}$ and acts trivially on $C_{0}$ as $C_{0}$ is cyclic. Since $y$ does not centralize $C_{0}$, it follows at once that $\bar{K}=\bar{H}^{\prime}$ and $|H: K|=2$. Since $\bar{H}^{\prime}$ is isomorphic to $\operatorname{PSL}\left(2, q^{\prime}\right)$, we see that (ii) and the first assertion of (iii) hold. Furthermore, taking $W_{0}=\langle w\rangle$ in (vii), the various parts of (vii) follow directly from the fact that $F=H$ and $C(w)$ is normal in $F$.

Since $O(H)=C_{0}, B \cap O(H)=1$ and hence $B$ and $\bar{B}$ are isomorphic. Furthermore, we have shown above that $C_{H}(x)=H \cap N=C_{0} B D$, whence $C_{H}(\bar{x})=\bar{B} \bar{D}$. However, since $\bar{x} \in \bar{D}^{\prime}$, Lemma 2.5 .7 (i) implies that $C_{H}(\bar{x})$ is a dihedral group of order $2\left(q^{\prime}-\varepsilon^{\prime}\right)$, where $q^{\prime} \equiv \varepsilon^{\prime}(\bmod 4)$. Thus $\bar{B}$ is cyclic of order $\frac{1}{4}\left(q^{\prime}-\varepsilon^{\prime}\right)$ and consequently $B$ is cyclic of the same order. Thus all parts of (iv) hold. Furthermore, if $B=1$, then $\frac{1}{4}\left(q^{\prime}-\varepsilon^{\prime}\right)=1$, whence $q^{\prime}=3$ or 5 and the final assertion of (iii) also holds.

Since the involution $z y$ inverts $C_{0}$, it lies in $H-K=H-H^{\prime}$. But now (v) follows at once from Lemma 2.5.7 (ii). Clearly (iv) and (v) imply (vi).

Finally we verify (viii). Suppose then that $w_{1}, w_{2}$ are elements of $C_{0}$ with $w_{2}=w_{1}^{u}$ for some $u$ in $G$. Clearly we may assume that $w_{1} \neq 1$. By (vii), $N\left(\left\langle w_{i}\right\rangle\right)=H$ for $i=1$ and 2 and consequently $N\left(\left\langle w_{2}\right\rangle\right)$ is equal to both $H$ and $H^{u}$. Thus $u \in N(H)$. Since $C_{0}=O(H), u$ normalizes $C_{0}$ and therefore $u \in H=N\left(C_{0}\right)$. But $H=K\langle y\rangle$ with $K$ centralizing and $y$ inverting $C_{0}$. We conclude at once that $u$ either centralizes or inverts $C_{0}$. Hence $w_{2}=w_{1}^{ \pm 1}$ and (viii) holds. This completes the proof of the lemma.

3. Elimination of the case $O(N)=1$. The object of this section is the proof of the following proposition.

Proposition 1. If $G$ is a simple $Q D$-group of characteristic power $q>7$, then $O(N) \neq 1$.

Proof. Assume that this is false for some $G$. Then clearly the conclusions of Theorem B hold trivially for $G$ with $A=B=1$. In particular, Proposition 1.1 holds for $G$ and hence $N=L$ and $E=A=1$.

Our proof is based on a method used already in $[8, \S 10]$ in the case $\varepsilon=-1$. We shall refer to this here indicating the changes which have to be made when $\varepsilon=1$. 
There is a simplification at the end where it was necessary in [8] to deal directly with some special values of $q$. It may be remarked that we use the results of Chapter III only as far as they refer to the principal 2-block $B_{0}(2, G)$ and are stated in Chapter III, §2. The notation $\chi_{0}=1, \chi_{1}, \ldots, \chi_{4}, \chi^{(j)}$, etc. will always refer to $B_{0}(2, G)$. If the condition $e=1$ is added to the hypotheses of Proposition 1 , the proof does not require Theorems $\mathrm{A}$ and $\mathrm{B}$ any further and, in particular, it is independent of the induction approach used in Chapters IV and V.

It should be observed that the notation in [8] differs from our notation. The group elements denoted by

$$
x, z, y, y z, t
$$

in the present paper are denoted in [8] by

$$
J, J_{1}, F, T, Y^{-1}
$$

respectively. The subgroup $T$ is denoted by $D$ in [8] and $C_{0}$ is denoted by $\mathfrak{W}$. The exponent $n+1$ of the order of the Sylow 2-subgroup of $G$ is taken in [8] as $n$. The 2-regular core $O(M)$ of a group $M$ is designated by $K_{2}(M)$. Finally, the degree $f_{i}=\chi_{i}(1)$ are called $x_{i}$ in [8].

Since we have $a=b=e=1$, we have

$$
|N|=|C(x)|=2 q(q-1)(q+1) ; \quad|C|=4\left|C_{0}\right|=2(q+\varepsilon) ;
$$

cf. Lemmas 2.4.1 (i) and 2.4.2 (ii). If this is substituted in Proposition 3.2.6, we have

(2) $\quad g=|G|=2 q^{2}(q+\varepsilon)^{2}(q-\varepsilon)^{3} \frac{f_{1}\left(f_{1}+\delta_{1}\right)}{\left(f_{1}-\delta_{1} \varepsilon q\right)^{2}}=2 q^{2}(q+\varepsilon)(q-\varepsilon)^{4} \frac{f_{2}\left(f_{2}+\delta_{2}\right)}{\left(f_{2}+\delta_{2} q \varepsilon\right)^{2}}$.

Since $q>7$, we have $C_{0} \neq 1$. By Lemma 2.2 (i), the group $C_{0}$ is cyclic.

No change is necessary in the proof of the Propositions (10A), (10B), (10C) and (10G) of [8]. We may also obtain them alternatively from Lemma 2.2. In particular, all elements $w \neq 1$ of $C_{0}$ have the same centralizer $K$ and we have

$$
K / C_{0} \simeq \operatorname{PSL}(2,3) \simeq A_{4} \text { or } K / C_{0} \simeq \operatorname{PSL}(2,5) \simeq A_{5}
$$

We refer to the first possibility as Case $A$ and to the second possibility as Case B.

In order to obtain the analogue of $[8,(10 \mathrm{D})]$ for both cases $\varepsilon=-1$ and $\varepsilon=1$, we apply Proposition 3.2.3.

First of all, since $\psi(1)=q$, we have

$$
\chi_{1}(x)=\delta_{1} \varepsilon q, \quad \chi_{2}(x)=-\delta_{2} \varepsilon q, \quad \chi_{3}(x)=-\delta_{3}, \quad \chi_{4}(x)=\delta_{2}(\varepsilon q-1) .
$$

If we use the same notation as in Proposition 3.2.2 for the $Q$-group $N$ with asterisks added, then $\psi$ can be taken as the character $\chi_{1}^{*}$, which has degree $q$. Moreover, for 2-regular elements $r$ of $N$, we have

$$
\delta_{1}^{*}+\psi(r)=\delta_{1}^{*}+\chi_{1}^{*}(r)=\chi^{(1) *}(r),
$$


where $\delta_{1}^{*}$ is a fixed sign. Then $\chi^{(1) *}(1)=\delta_{1}^{*}+q$ and, as $\chi^{(1) *}(1)$ is not divisible by 4 , necessarily $\delta_{1}^{*}=\varepsilon$. It is now clear from (1) that for any odd prime $p$ dividing $q+\varepsilon$, the character $\chi^{(1) *}$ of $N$ is of defect 0 and then vanishes for all $p$-singular elements of $N$, cf. [9]. If $r \in C_{0}$ and $r \neq 1$, we can choose a prime factor $p$ of $\frac{1}{2}(q+\varepsilon)$ which divides the order of $r$. Then $\chi^{(1) *}(r)=0$ and as (4) holds for $r$, we have

$$
\psi(r)=-\delta_{1}^{*}=-\varepsilon .
$$

(This can also be seen in other ways.) It now follows from Proposition 3.2.3 that if $r \in C_{0}$ and $r \neq 1$, then

$$
\chi_{1}(x r)=-\delta_{1}, \quad \chi_{2}(x r)=\delta_{2}, \quad \chi_{3}(x r)=-\delta_{3}, \quad \chi_{4}(x r)=-2 \delta_{2} .
$$

This is actually the same as the equation (10.5) in [8, (10D)].

We now come to the proof of the crucial Lemma (10E) of [8], which states that if $P$ is the Sylow $p$-group of $G$ for some prime $p$ dividing $\frac{1}{2}(q+\varepsilon)$ and if $P$ is abelian, it is impossible that $N(P)=C(P)\left\langle x_{0}\right\rangle$, where $x_{0}$ is an involution which inverts $P$.

Suppose by contradiction that $N(P)$ is of this form. Then the results of [6, III, $\S \mathrm{IX]}$ apply with $m=2$. It follows that the principal $p$-block $B_{0}(p, G)$ consists of $(|P|+3) / 2$ characters which as in [8] will be denoted by

$$
\chi_{0}=1, \psi_{1}, \psi^{(i)} \quad(i=1,2, \ldots,(|P|-1) / 2)
$$

(which are not to be confused with the character $\psi$ of $N$ in Proposition 3.2.3). The characters (6) are real-valued. Moreover, there exists a sign $\delta^{*}$ such that for all $p$-singular elements $w$ of $G$, we have

$$
\psi_{1}(w)=\delta^{*},
$$

while for all $p$-regular elements $r$ of $G$ and all $i$,

$$
\delta^{*}+\psi_{1}(r)=\psi^{(i)}(r)
$$

In particular, all $\psi^{(i)}(r)$ with the same $r$ are equal. Finally, if we set

$$
\alpha=\psi_{1}(1)\left(\psi_{1}(1)+\delta^{*}\right) /\left(\psi_{1}(1)-\psi_{1}\left(x_{0}\right)\right)^{2}
$$

and if $w \neq 1$ is an element of $P$, we have

$$
|G|=g=\alpha \frac{\left|C\left(x_{0}\right)\right|^{2}}{|C(w)|} \mu^{2}
$$

where $\mu$ denotes the number of conjugates of $x_{0}$ which invert $w$.

Since $p$ divides the order $(q+\varepsilon) / 2$ of $C_{0}$, we may assume without restriction that $P \cap C_{0} \neq 1$ and we can choose $w \neq 1$ in $P \cap C_{0}$. By the results of $[8, \S 10]$ discussed or by Lemma 2.2 , the centralizer $C(w)$ is $K$ and the extended centralizer $C^{*}(w)$ of $w$ is $H$. Hence $\mu$ is the number of involutions in $H-K$. Since $H$ has the dihedral Sylow 2-subgroup $D=\langle y, z\rangle$ and since $x, z, x z$ lie in $K$ while the other two involutions $y z$ and $y x z$ are conjugate in $H$, the number $\mu$ is the number of conjugates of $y z$ in $H$. Thus,

$$
\mu=\left|H: C_{H}(y z)\right|
$$


Since $|H: K|=2$ and $y z \in H-K$, it is then clear that $\mu=\left|K: C_{K}(y z)\right|$. If we use $[8,(10 \mathrm{~A})]$ and $[8,(10 \mathrm{C})]$ it is now clear that

$$
\frac{\mu^{2}}{|C(w)|}=\frac{|K|^{2}}{|K| \cdot\left|C_{K}(y z)\right|^{2}}=\frac{|K|}{\left|C_{K}(y z)\right|^{2}}=\gamma(q+\varepsilon) / 2,
$$

where $\gamma=3$ in the Case A and $\gamma=5 / 3$ in the Case B.

Since $\left|C\left(x_{0}\right)\right|=|N|$, it follows from (10) and (1) that

$$
|G|=g=2 \alpha \gamma q^{2}(q-\varepsilon)^{2}(q+\varepsilon)^{3} .
$$

If $\nu$ denotes the 2-adic exponential valuation of the rationals, then as $\nu(g)=n+1$, $\nu(q+\varepsilon)=1$, and $\nu(q-\varepsilon)=n-1$, we find

$$
v(\alpha)=-n-1 \text {. }
$$

It follows from (8) that either $\psi_{1}(1)$ or $\psi^{(i)}(1)$ is odd. Now all real-valued irreducible characters of odd degree lie in $B_{0}(2, G)$. If $|P| \neq 3$, the degrees $\psi^{(i)}(1)$ cannot be odd since by Corollary 3.2.1, $B_{0}(2, G)$ does not contain two different characters of the same odd degree. If $|P|=3$, we can interchange $\psi_{1}$ and $\psi^{(1)}$, if necessary, and we may assume that $\psi_{1}(1)$ is odd. It follows that $\psi_{1} \in B_{0}(2, G)$. Then $\psi_{1}$ must be one of the characters $\chi_{1}, \chi_{2}, \chi_{3}$ in $B_{0}(2, G)$.

If $\psi_{1}=\chi_{2}$, comparison of (5) and (7) for $w=x r$ with $r \neq 1$ in $P \cap C_{0}$ yields

$$
\delta_{2}=\chi_{2}(x r)=\chi_{2}(w)=\psi_{1}(w)=\delta^{*}
$$

By (3),

Now, (9) becomes

$$
\psi_{1}\left(x_{0}\right)=\chi_{2}\left(x_{0}\right)=-\delta_{2} \varepsilon q
$$

$$
\alpha=f_{2}\left(f_{2}+\delta_{2}\right) /\left(f_{2}+\delta_{2} \varepsilon q\right)^{2} .
$$

Since $\nu(\varepsilon q-1)=n-1$, the congruence for $f_{2}$ in Proposition 3.2.6 shows that $\nu\left(f_{2}+\delta_{2}\right)=n-1, v\left(f_{2}+\delta_{2} \varepsilon q\right) \geqq n+1$ and then $\nu(\alpha) \leqq n-1-2(n+1)=-n-3$, which is not consistent with (12).

Similarly, if $\psi_{1}=\chi_{3}$, we have

$$
-\delta_{3}=\chi_{3}(x r)=\psi_{1}(x r)=\delta^{*}, \quad \psi_{1}\left(x_{0}\right)=\chi_{3}\left(x_{0}\right)=\chi_{3}(x)=-\delta_{3},
$$

and then

$$
\alpha=f_{3}\left(f_{3}-\delta_{3}\right) /\left(f_{3}+\delta_{3}\right)^{2} .
$$

Here, by Proposition 3.2.6, $v\left(f_{3}+\delta_{3}\right)=n$ and we find $v(\alpha)=1-2 n$, which again is inconcistent with (12).

Thus, only the possibility $\psi_{1}=\chi_{1}$ remains. Here by (5) and (7)

$$
-\delta_{1}=\chi_{1}(x r)=\psi_{1}(x r)=\delta^{*},
$$

while by (3) $\psi_{1}\left(x_{0}\right)=\chi_{1}(x)=\delta_{1} \varepsilon q$. Thus,

$$
\alpha=f_{1}\left(f_{1}-\delta_{1}\right) /\left(f_{1}-\delta_{1} \varepsilon q\right)^{2} .
$$


On substituting this in (11) and comparing the result with (2), we obtain

$$
(q-\varepsilon)\left(f_{1}+\delta_{1}\right)=\gamma(q+\varepsilon)\left(f_{1}-\delta_{1}\right)
$$

In the Case $\mathrm{A}, \gamma=3$ and (13) shows that $\frac{1}{2}(q+\varepsilon)$ divides $f_{1}+\delta_{1}$. Since $f_{1}+\delta_{1}$ is even, $q+\varepsilon$ also divides $f_{1}+\delta_{1}$. If we set

$$
f_{1}+\delta_{1}=k(q+\varepsilon)
$$

with integral $k$, then (13) yields

$$
3\left(f_{1}-\delta_{1}\right)=k(q-\varepsilon) .
$$

It follows from the last two equations that

$$
6 \delta_{1}=3\left(f_{1}+\delta_{1}\right)-3\left(f_{1}-\delta_{1}\right)=2 k(q+2 \varepsilon) .
$$

Since $q>7$, this is clearly impossible.

In the Case $\mathrm{B}$, (13) with $\gamma=5 / 3$ implies that $\frac{1}{2}(q+\varepsilon)$ divides $3\left(f_{1}+\delta_{1}\right)$. Again, $q+\varepsilon$ also divides $3\left(f_{1}+\delta_{1}\right)$ and if we set

with integral $k$, we find

$$
3\left(f_{1}+\delta_{1}\right)=k(q+\varepsilon)
$$

$$
5\left(f_{1}-\delta_{1}\right)=k(q-\varepsilon)
$$

The last two equations imply

$$
15 \delta_{1}=k(q+4 \varepsilon) .
$$

Since $q+4 \varepsilon$ then divides 15 , and since $q>7$, we can only have $q=19$. Then $k=1$, $\delta_{1}=1, f_{1}=5$. By Propositions 3.2.4 and 3.2.6, then $6=f_{2}+f_{3}, 5 f_{2}=361 f_{3}$. This is absurd and we see that $[8,(10 \mathrm{E})]$ holds in our case.

If we take here $l$ as $\frac{1}{2}(q+\varepsilon)$, then the statement $[8,(10 \mathrm{~F})]$ is obtained as a corollary as in [8]. We now know that $l=\frac{1}{2}(q+\varepsilon)$ is divisible only by the primes $p=3$ or 5 . If 5 divides $l$, we must have Case $\mathrm{B}$.

It was already mentioned that $[8,(10 \mathrm{G})]$ holds. Again, the proof of $[8,(10 \mathrm{H})]$ applies. Actually, we need it only for $p=3$ where it can be simplified slightly. As in [8, p. 148, lines 12 and 13], it follows that 9 does not divide $l$. (An analogous proof is carried out in detail below in Propositions 7.1 and 7.2.)

We now see that we must have

$$
l=\frac{1}{2}(q+\varepsilon)=3^{h} \cdot 5^{m}
$$

with $h=0$ or 1 and with $m \geqq 0$. Since $q>7$, necessarily $m>0$. Then $5 \mid q+\varepsilon$ and, as remarked above, we then have Case B. Since $\left|K: C_{0}\right|=60$ and since $\left|C_{0}\right|=\frac{1}{2}(q+\varepsilon)$ is divisible by $5^{m}$, it follows that $5^{m+1}$ divides $g=|G|$.

Let $v$ now denote the 5-adic exponential valuation of the rational numbers $\left({ }^{12}\right)$.

${ }^{(12)}$ The following discussion also appears in [8]. We repeat it here to make it clear that it does not depend on the hypothesis $25 \mid l$. 
Then $\nu(q+\varepsilon)=m, \nu(q)=0, \nu(q-\varepsilon)=0$. On account of (2), we have

$$
m+1 \leqq \nu(g)=m+\nu\left(f_{2}\right)+\nu\left(f_{2}+\delta_{2}\right)-2 \nu\left(f_{2}+\delta_{2} \varepsilon q\right) \text {. }
$$

Hence, we have one of the following two cases: $\nu\left(f_{2}\right)>0$ or $\nu\left(f_{2}+\delta_{2}\right)>0$. We refer to the former case as Case I and to the latter case as Case II.

Case I. $v\left(f_{2}\right)>0$.

Then

$$
\nu\left(f_{2}+\delta_{2}\right)=0, \quad \nu\left(f_{2}+\delta_{2} \varepsilon q\right)=0 .
$$

Let $P_{0}$ denote the Sylow 5-subgroup of $C_{0}$ and set $P_{0}=\langle r\rangle$. Since (5) shows that $\chi_{2}(x r) \neq 0$, it follows that $r$ belongs to a defect group $D$ of the 5-block $B(5)$ to which $\chi_{2}$ belongs, cf. $[5, \mathrm{II}]$. On the other hand,

$$
\nu\left(\frac{g}{|C(x r)|} \frac{\chi_{2}(x r)}{f_{2}}\right)=\nu(g)-\nu\left(f_{2}\right)-v(|C(x r)|) .
$$

Since $C(x r) \subseteq C(x)$, we have

$$
\nu(|C(x r)|) \leqq \nu(|N|)=\nu(q+\varepsilon)=m .
$$

On the other hand, $C_{0} \subseteq C(x r)$ and we conclude that we have equality. Now (15) and (16) show that the right side of (17) vanishes. It follows from the definition of defect groups in [5, I] that $C(x r)$ contains a conjugate of the defect group $D$ of $B(5)$. It is now clear that we can choose $D=\langle r\rangle=P_{0}$.

Case II. $\nu\left(f_{2}+\delta_{2}\right)>0$.

Here, $\nu\left(f_{2}\right)=0$. As we have $\left(f_{2}+\delta_{2}\right)-\left(f_{2}+\delta_{2} \varepsilon q\right)=-\delta_{2} \varepsilon(q-\varepsilon), \nu\left(f_{2}+\delta_{2} q\right)=0$. Thus,

$$
\nu\left(f_{2}\right)=\nu\left(f_{2}+\delta_{2} \varepsilon q\right)=0 .
$$

Note that by Proposition 3.2.4, $f_{2}+\delta_{2}=f_{4}$. If $P_{0}=\langle r\rangle$ is the Sylow 5-subgroup of $C_{0}$ as before, by (5) $\chi_{4}(x r)=-2 \delta_{2}$. Let $B(5)$ here denote the 5-block of $G$ to which $\chi_{4}$ belongs. The same arguments as in the Case I show that $B(5)$ has the defect group $P_{0}$.

We follow [8] in studying 5-blocks of $G$ with defect group $P_{0}$. On account of [8, (10A), (10B), (10G)] or of Lemma 2.2 (vii), we have $C\left(P_{0}\right)=C\left(C_{0}\right)=K, N\left(P_{0}\right)$ $=K\langle y z\rangle$, and as we have Case B, $K / C_{0} \simeq P S L(2,5)$. We can then set $K=K_{0} \times C_{0}$ cf. [25], where $K_{0} \simeq P S L(2,5)$. It follows from (14) that we may set $C_{0}=P_{0} \times V$, where $V$ is a group of order $3^{h}, h=0$ or 1 . (In [8], the group $V$ is denoted by $\mathfrak{W}_{0}$.)

The group $K_{0} \simeq P S L(2,5)$ has a unique irreducible character $\theta$ of degree 5 and we interpret $\theta$ as a character of $K$ which is trivial on $C_{0}$. Let $\omega$ be a faithful linear character of the cyclic group $P_{0}$ and interpret $\omega$ as a character of $K$ which is trivial on $K_{0} \times V$. Then the $5^{m}$ irreducible characters $\theta \omega^{i}$ with $0 \leqq i<5^{m}$ form a 5-block $b$ of $K$ with the defect group $P_{0}$. The arguments in [8, p. 149] show that in both Cases I and II, $B(5)$ is the block $b^{G}$ of $G$. If $w \in P_{0}, w \neq 1$, and if $r$ is a 5-regular element of $C\left(P_{0}\right)=K$, we can set

$$
\chi_{\mu}(w r)=d_{\mu}(w) \theta(r)
$$


for $\chi_{\mu} \in B(5)$, where the $d_{\mu}(w)$ are the generalized decomposition numbers for $w$, cf. $[8,(10.12)]$. Since $\theta(x)=1$, then $d_{\mu}(w)=\chi_{\mu}(w x)$ for $\chi_{\mu} \in B(5)$. If $u \in G$, we consider the values $\chi_{u}(u)$ with $\chi_{\mu} \in B(5)$ as entries of a column $X(u)$ for $B(5)$, cf. [6, I, p. 165]. Then $X(w x)$ is the column $X(w)$ with the entries $d_{\mu}(w)$. It follows that the column $R$ denoted by $\mathfrak{a}(\omega-1)$ in [8] can be written as follows:

$$
R=5^{-m} \sum_{w} X(w x)(\bar{\omega}(w)-1)
$$

with $w$ ranging over $P_{0}$. As in [8], we see that $R$ is an integral column, that $(R, R)$ $=3$, and that $(R, X(u))=0$ for 5-regular $u \in G$. Then $R$ has three nonzero entries and these are \pm 1 . This implies that the Case II is impossible, cf. the first paragraph of $[8$, p. 150].

If we have Case I, the arguments in [8] show that $\chi_{2}, \chi_{3} \in B(5)$ and that we have a further character $\chi_{\beta}$ such that, with a suitable sign $\eta$, we have the entries

\begin{tabular}{c|ccc|}
\multicolumn{1}{c}{} & \multicolumn{1}{c}{$R$} & $X(1)$ & $X(x)$ \\
\cline { 2 - 4 }$\chi_{2}$ & $-\delta_{2}$ & $f_{2}$ & $-\delta_{2} \varepsilon q$ \\
$\chi_{3}$ & $\delta_{3}$ & $f_{3}$ & $-\delta_{3}$ \\
$\chi_{\beta}$ & $\eta$ & $\eta\left(\delta_{2} f_{2}-\delta_{3} f_{3}\right)$ & $\eta(1-\varepsilon q)$ \\
\cline { 2 - 4 } & &
\end{tabular}

Here, the entries of $R$ for $\chi_{2}$ and $\chi_{3}$ are obtained from (18) and (5), those of $X(1)$ are obvious, and those of $X(x)$ are obtained from Proposition 3.2.3. The character $\chi_{\beta}$ is chosen as the character in $B(5)$ different from $\chi_{2}$ and $\chi_{3}$ for which the third nonzero entry appears in $R$. The entries of $X(1)$ and $X(x)$ for $\chi_{\beta}$ are obtained from the orthogonality with $R$.

We wish to apply [6, III $(2 \mathrm{~A})]\left({ }^{13}\right)$ using the block $B(5)$ and the column $R$ which is a linear combination of columns $X(w)$ with $w \in P_{0}, w \neq 1$. Both involutions are to be chosen as $x$. Finally, we use the subgroup $H=K\langle y z\rangle$ which is the extended centralizer $C^{*}(w)$ for each $w \neq 1$ in $P_{0}$.

Let $B^{*}(5)$ denote the 5-block $b^{H}$ of $H$. Then $\left(B^{*}(5)\right)^{G}=B(5)$. Then $B^{*}(5)$ covers the block $b$ of the normal subgroup $K$ of $H$, e.g. by [7, I, (4D)] and $B^{*}(5)$ consists of the irreducible constituents of the characters of $H$ induced by the characters in $b$. Since $\theta$ is the unique irreducible character of degree 5 of $K$ which is trivial on $C_{0}$, $\theta$ is stable in $H$ and hence there exist two extensions $\theta_{1}$ and $\theta_{2}$ of $\theta$ to characters of $H$. These belong to $B^{*}(5)$. Let $R^{*}$ and $X^{*}(u)$ have analogous significance for $B^{*}(5)$ and $H$ as $R$ and $X(u)$ have for $B(5)$ in $G$. Then

$$
R^{*}=5^{-m} \sum_{w} X^{*}(w x)(\bar{\omega}(w)-1)
$$

${ }^{(13)}$ Since this part of the proof was given in [8] without full details, we shall present it here completely. 
with $w$ ranging over $P_{0}$. Moreover, $R^{*}$ is an integral column for $B^{*}(5),\left(R^{*}, R^{*}\right)=3$ and $\left(R^{*}, X^{*}(u)\right)=0$ for 5-regular $u$ in $H$. Again, $R^{*}$ has three nonzero entries and these are all \pm 1 . Since $\theta$ is trivial on $P_{0}$, we have $\theta(x w)=\theta(x)=1$ for $w \in P_{0}$ and (19) shows that $R^{*}$ has entries -1 for $\theta_{1}$ and $\theta_{2}$. Let $\chi_{u}^{*}$ denote the third character of $H$ for which $R^{*}$ has a nonzero entry. Since $\theta_{1}$ and $\theta_{2}$ can be identified with the two irreducible characters of degree 5 of $H / C_{0} \simeq P G L(2,5) \simeq S_{5}$, we obtain the entries in the row belonging to $\theta_{1}$ and $\theta_{2}$ in the following table.

\begin{tabular}{c|rrrr|}
\multicolumn{1}{c}{} & \multicolumn{1}{c}{$R^{*}$} & $X^{*}(1)$ & $X^{*}(x)$ & $X^{*}(y z)$ \\
\cline { 2 - 5 }$\theta_{1}$ & -1 & 5 & 1 & 1 \\
$\theta_{2}$ & -1 & 5 & 1 & -1 \\
$\chi_{\mu}^{*}$ & 1 & 10 & 2 & 0 \\
\cline { 2 - 5 } & &
\end{tabular}

The orthogonality of $R^{*}$ with $X^{*}(1)$ shows that the entry of $R^{*}$ for $\chi_{\mu}^{*}$ cannot be -1 . Then this entry is 1 and the other entries for $\chi_{u}^{*}$ are obtained from the orthogonality of $R^{*}$ with $X^{*}(1), X^{*}(x)$, and $X^{*}(y z)$.

Note that the intersection of the conjugate class of $x$ in $G$ with $H$ consists of the conjugate classes of $x$ and of $y z$ in $H$. We are now ready to apply [6, III (2A)] and find

$$
\frac{g}{|N|^{2}}\left(\frac{-\delta_{2} q^{2}}{f_{2}}+\frac{\delta_{3}}{f_{3}}+\frac{(1-\varepsilon q)^{2}}{\delta_{2} f_{2}-\delta_{3} f_{3}}\right)=|H|\left(-\frac{1}{5} h\left(\theta_{1}\right)^{2}-\frac{1}{5} h\left(\theta_{2}\right)^{2}+\frac{1}{10} h\left(\chi_{u}^{*}\right)^{2}\right),
$$

where

$$
\begin{aligned}
& h\left(\theta_{1}\right)=\frac{1}{\left|C_{H}(x)\right|^{2}} \theta_{1}(x)+\frac{1}{\left|C_{H}(y z)\right|} \theta_{1}(y z)=\frac{1}{\left|C_{H}(x)\right|}+\frac{1}{\left|C_{H}(y z)\right|}, \\
& h\left(\theta_{2}\right)=\frac{1}{\left|C_{H}(x)\right|^{2}} \theta_{2}(x)+\frac{1}{\left|C_{H}(y z)\right|^{2}} \theta_{2}(y z)=\frac{1}{\left|C_{H}(x)\right|}-\frac{1}{\left|C_{H}(y z)\right|}, \\
& h\left(\chi_{\mu}^{*}\right)=\frac{1}{\left|C_{H}(x)\right|} \chi_{\mu}^{*}(x)+\frac{1}{\left|C_{H}(y z)\right|^{2}} \chi_{\mu}^{*}(y z)=\frac{2}{\left|C_{H}(x)\right|} .
\end{aligned}
$$

Our result can then be written in the form

$$
\frac{g}{|N|^{2}} \frac{-\delta_{2} q^{2} f_{3}\left(\delta_{2} f_{2}-\delta_{3} f_{3}\right)+\delta_{3} f_{2}\left(\delta_{2} f_{2}-\delta_{3} f_{3}\right)+f_{2} f_{3}(1-\varepsilon q)^{2}}{f_{2} f_{3}\left(\delta_{2} f_{2}-\delta_{3} f_{3}\right)}=-\frac{2}{5}|H| \frac{1}{\left|C_{H}(y z)\right|^{2}} .
$$

Since we have Case $\mathrm{B}$, we have $\left|C_{K}(y z)\right|=6$ by $[8,(10 \mathrm{C})]$ and since $y z \in H,|H: K|$ $=2$, this implies $\left|C_{H}(y z)\right|=12$. Also, $|H|=120\left|C_{0}\right|=60(q+\varepsilon)$. Thus, the last term in our equation is $-(q+\varepsilon) / 6$.

The numerator of the factor by $g /|N|^{2}$ on the left can be written in the form

$$
\delta_{2} \delta_{3} f_{2}^{2}+\delta_{2} \delta_{3} q^{2} f_{3}^{2}+f_{2} f_{3}(-2 \varepsilon q) \text {. }
$$

Since $f_{1} f_{2}=q^{2} f_{3}$ and $\delta_{1} \delta_{2}=\delta_{3}$ (cf. Chapter III, §2) this can be written in the form

$$
\delta_{1} f_{2} f_{3} f_{1}^{-1}\left(q^{2}+f_{1}^{2}-2 \delta_{1} \varepsilon q f_{1}\right)=\delta_{1} f_{2} f_{3} f_{1}^{-1}\left(f_{1}-\delta_{1} \varepsilon q\right)^{2} .
$$


If we use this, and substitute $|N|$ and $g$ from (1) and (2), we obtain

$$
\frac{2 q^{2}\left(q+\varepsilon^{2}\right)(q-\varepsilon)^{3}}{4 q^{2}(q-\varepsilon)^{2}(q+\varepsilon)^{2}} \frac{f_{1}\left(f_{1}+\delta_{1}\right)}{\left(f_{1}-\delta_{1} \varepsilon q\right)^{2}} \delta_{1} \frac{\left(f_{1}-\delta_{1} \varepsilon q\right)^{2}}{f_{1}\left(\delta_{2} f_{2}-\delta_{3} f_{3}\right)}=-(q+\varepsilon) / 6
$$

Since $\delta_{1}\left(f_{1}+\delta_{1}\right)=-\delta_{2} f_{2}-\delta_{3} f_{3}$ by Proposition 3.2.4, our equation becomes

$$
3\left(\delta_{2} f_{2}+\delta_{3} f_{3}\right)(q-\varepsilon)=(q+\varepsilon)\left(\delta_{2} f_{2}-\delta_{3} f_{3}\right)
$$

Hence

$$
2 \delta_{2} f_{2}(q-2 \varepsilon)=-2 \delta_{3} f_{3}(2 q-\varepsilon) .
$$

On multiplying with $f_{1}$ and using $f_{1} f_{2}=q^{2} f_{3}$, we have

$$
q^{2}(q-2 \varepsilon)=f_{1}(2 q-\varepsilon)
$$

This implies that $2 q-\varepsilon$ divides $q-2 \varepsilon$ and hence that $2 q-\varepsilon$ divides 3. This is impossible and the proof of Proposition 1 is complete.

4. Elimination of the case $A=1, B \neq 1$. We continue with our assumption that the characteristic power $q$ of $G$ exceeds 7 together, of course, with the assumption that $G$ is a simple $Q D$-group satisfying the conclusions of Theorem B. We know from Proposition 3.1 that $O(N) \neq 1$. Hence either $A \neq 1$ or $B \neq 1$. In the next two sections we shall demonstrate that the case $A=1, B \neq 1$ does not occur. This will imply that $A \neq 1$, in which case parts (ii) and (iii) of Theorem $\mathrm{C}$ will then follow from Propositions 2.2 and 2.5. Since part (i) of Theorem $C$ has been proved in $\S 1$, all that will then remain is to establish part (iv) of Theorem $\mathrm{C}$.

We assume by way of contradiction that $A=1$ and $B \neq 1$. In view of Proposition 2.3 , it follows that $q \neq 9$. Hence, under the given conditions, our assumptions imply that $q>9$. In particular, this implies that $L_{0}$ is a direct product. Since $O(N)$ $=B$ in the present case, we have

$$
L_{0}=J \times B .
$$

Our argument will be largely group-theoretic. However, at one point in the next section we require a character-theoretic result which will restrict the possible prime divisors of $b=|B|$. We shall make considerable use of the omnibus Lemma 2.2. We again set $H=N\left(C_{0}\right)$ and $K=C\left(C_{0}\right)$. In particular, by the lemma, $O(H)=C_{0}$, $H / C_{0}$ is isomorphic to $P G L\left(2, q^{\prime}\right)$, where $q^{\prime} \equiv \varepsilon^{\prime}(\bmod 4), \varepsilon^{\prime}= \pm 1, K / C_{0}$ is isomorphic to $\operatorname{PSL}\left(2, q^{\prime}\right)$, and $D$ is a Sylow 2-subgroup of $H$. Moreover, $B$ is cyclic of order

$$
b=\frac{1}{4}\left(q^{\prime}-\varepsilon^{\prime}\right) .
$$

In this section, we shall treat the special case

$$
q^{\prime}=q \text { and } \varepsilon^{\prime}=\varepsilon .
$$

However, we begin with an easy consequence of Proposition 2.1 and Lemma 2.2 (vii), which is valid in all cases. 
LEMMA 1. The following conditions hold for any subgroup $R$ of $G$ of odd order:

(i) If $C_{0} \subseteq R$ and $C_{0}$ is a Hall subgroup of $R \cap H$, then $C_{0}$ is a Hall subgroup of $R$ and $R$ possesses a normal complement to $C_{0}$;

(ii) If $B \subseteq R$, then $R$ possesses a normal complement to $B$.

Proof. Let the assumptions be as in (i). Let $P$ be a nontrivial Sylow $p$-subgroup of $C_{0}$ for some prime $p$, and let $Q$ be a Sylow $p$-subgroup of $R$ containing $P$. By Lemma 2.2 (vii), $N(P) \subseteq H$. In particular, $N_{Q}(P) \subseteq H \cap R$. Hence $N_{Q}(P)=P$ and consequently $Q=P$ and $P$ is a Sylow $p$-subgroup of $R$. Moreover, $N_{R}(P) \subseteq K$ as $R$ is of odd order and $K$ is of index 2 in $H$. But $K$ centralizes $P$ since it centralizes $C_{0}$. Thus $P \subseteq Z\left(N_{R}(P)\right)$ and so $R$ has a normal $p$-complement by Burnside's transfer theorem. Since $p$ is arbitrary, both parts of (i) follow at once.

The proof of (ii) is similar. Let $P$ be a nontrivial Sylow $p$-subgroup of $B$ and let $U$ denote a Sylow $p$-subgroup of $N$ containing $P$. Since $L_{0}=J \times B$ is of index 2 in $N=L$, it follows that $U=(U \cap J) \times P$. Since $U \cap J$ is abelian by Lemma 2.4.1 and since $B$ is abelian, we see that $U$ is abelian. Moreover, by Proposition 2.1, $U$ is a Sylow $p$-subgroup of $G$.

Next let $Q$ be a Sylow $p$-subgroup of $R$ containing $P$. Since the Sylow $p$-subgroups of $G$ are abelian, $Q \subseteq N(P)$. But $N(P) \subseteq N$ by Proposition 2.1 and so $Q \subseteq N$. Hence by the same proposition, $N(Q) \subseteq N$. Furthermore, since $L_{0}$ is of index 2 in $N$ and $R$ is of odd order, $N \cap R=L_{0} \cap R$ and consequently $N_{R}(Q) \subseteq L_{0} \cap R$. But $L_{0}=J \times B$ and $B$ is abelian, so $P \subseteq Z\left(N_{R}(Q)\right)$. Since $Q$ is abelian, Theorem 7.4.4 of [19] now yields that $R$ possesses a normal complement to $P$. Since $p$ is again arbitrary, (ii) also follows.

For the balance of the section we assume that $q^{\prime}=q$ and $\varepsilon^{\prime}=\varepsilon$. We let $p$ be the prime divisor of $q$ and fix this notation. As the first step in the argument, we prove

Proposition 1. $\varepsilon=-1$.

We carry out the proof by contradiction in a sequence of lemmas. Thus throughout we assume that $\varepsilon=+1$.

LEMMA 2. The following conditions hold:

(i) A Sylow 2-subgroup of $G$ has order 16;

(ii) $\boldsymbol{U}_{N}\left(C_{0} ; p\right)$ and $\boldsymbol{U}_{N}\left(C_{0} B\right)$ are trivial;

(iii) $B\langle y\rangle$ is a cyclic subgroup of $H$ of order $q-1$ and acts regularly on some Sylow p-subgroup of $H$ which is elementary abelian of order $q$;

(iv) If $P$ is a nontrivial p-subgroup of $G$, then $N(P)$ does not contain a four subgroup.

Proof. In the present case, $|N|=2 b q(q+1)(q-1)$. Furthermore, by Lemma 2.2 (iii), $q^{\prime}=q \equiv 3,5(\bmod 8)$. Since $\varepsilon=1$, we have, in fact, $q \equiv 5(\bmod 8)$, whence 16 is the exact power of 2 dividing $N$. Since $N$ contains a Sylow 2-subgroup of $G$, (i) holds. 
By Lemma 2.2 (iv), $b=\frac{1}{4}(q-\varepsilon)$ and so $B$ has order prime to $p$. Furthermore, since $\varepsilon=1$, Lemma 2.4.6 (i) implies that $\eta_{J}\left(C_{0}\right)$ is trivial. Since $N=L_{0} T=(J \times B) T$, both parts of (ii) follow at once.

Since $y \in J, y$ centralizes $B$. Hence by Lemma 2.2 (iv), $B\langle y\rangle$ is a cyclic subgroup of $H$ of order $q^{\prime}-\varepsilon^{\prime}=q-1$. Since $\bar{H}=H / C_{0}$ is isomorphic to $\operatorname{PGL}(2, q)$, it follows from Lemma 3.1 (vi) of [22] that $\bar{B}\langle\bar{y}\rangle$ acts regularly on a Sylow $p$-subgroup $\bar{P}$ of $\bar{H}$ and $\bar{P}$ is elementary abelian of order $q$. But $\left|C_{0}\right|=\frac{1}{2}(q+\varepsilon)$ is prime to $p$. Since $B\langle y\rangle \cap C_{0}=1$, (iii) follows at once.

To prove (iv), it will suffice to show that $T$ normalizes no nontrivial $p$-subgroup of $J$. Indeed, if this is the case, then $И_{N}(T ; p)$ is trivial as $B$ is a $p^{\prime}$-group. Since the involutions $x=x_{1}, x_{2}, x_{3}$ are conjugate in $N(T)$, it follows that $\eta_{C\left(x_{i}\right)}(T ; p)$ is trivial for all $i, 1 \leqq i \leqq 3$. But then if $T$ normalizes the $p$-subgroup $Q$ of $G$, our conditions force $C_{Q}\left(x_{i}\right)=1$ for all $i$. Therefore $Q=1$ and so $T$ normalizes no nontrivial $p$-subgroup of $G$. However, $G$ has only one conjugacy class of four subgroups by Lemma 2.1.1 and hence no four subgroup of $G$ will normalize a nontrivial $p$-subgroup of $G$, as asserted in (iv).

Suppose then that $T$ normalizes a nontrivial $p$-subgroup of $J$. Since the Sylow $p$-subgroups of $S L(2, q)$ with $q=p^{m}$ are known to be disjoint from their conjugates, it follows that $T$ normalizes a Sylow $p$-subgroup $Q_{1}$ of $J$. Since $\bar{J} \bar{T}=J T /\langle x\rangle$ is isomorphic to $\operatorname{PGL}(2, q)$, Lemma 3.1 (vi) of [22] implies that $N_{J T}\left(\bar{Q}_{1}\right)=\bar{Q}_{1} \bar{Y}$, where $\bar{Y}$ is cyclic of order $q-1$ and acts regularly on $\bar{Q}_{1}$. If $Y$ is the inverse image of $\bar{Y}$ in $J T$, then $T \subseteq Y,|Y|=2(q-1)$, and $Y$ has a normal 2-complement. Furthermore, since $\varepsilon=1$ and $q \equiv 3,5(\bmod 8)$, it follows that $|O(Y)|=\frac{1}{4}(q-1)=b$. In particular, $O(Y) \neq 1$ and $O(Y)$ has order relatively prime to $\frac{1}{2}(q+1)=\left|C_{0}\right|$. Hence $x_{2}$ must invert $O(Y)$. But $O(Y)$ acts regularly on $Q_{1}$ and consequently if we consider the action of $O(Y)\left\langle x_{2}\right\rangle$ on $Q_{1}$, we must have $C_{Q_{1}}\left(x_{2}\right) \neq 1$. Since $C_{Q_{1}}\left(x_{2}\right)$ centralizes $T$, we conclude that $p$ divides $\left|C_{0}\right|$, which is not the case. This contradiction completes the proof of (iv).

Let $P$ be a Sylow $p$-subgroup of $H$ normalized by $B\langle y\rangle$. By Lemma 2.2 (ii), $P$ centralizes $C_{0}$ and so $C_{0} B\langle y\rangle$ normalizes $P$. We next prove

LEMMA 3. If $Q$ is a $C_{0} B\langle y\rangle$-invariant p-subgroup of $G$ of maximal order containing $P$ and $F=N(Q)$, then the following conditions hold:

(i) $Q$ is abelian and inverted by $x$;

(ii) $F$ has a normal 2-complement;

(iii) $B$ is a Hall subgroup of $O(F)$;

(iv) $Q$ is a Sylow p-subgroup of $G$.

Proof. First, $C_{Q}(x)$ is a $C_{0}$-invariant $p$-subgroup of $N=C(x)$, so $C_{Q}(x)=1$ by Lemma 2 (ii). Hence $x$ inverts $Q$ and so $Q$ is abelian, proving (i).

By Lemma 2 (iv), $F$ does not contain a four group, so a Sylow 2-subgroup of $F$ is either cyclic or generalized quaternion. Thus $F$ is either a $Q$-group or has a 
normal 2-complement. Consider the first case. Then $F=O(F)(F \cap N)$ as $x=y^{2}$ is in the center of some Sylow 2-subgroup of $K$. Likewise we have that $F \cap J$ is a $Q$-group. Since $C_{0} \subseteq F \cap J$ and $C_{0}$ is cyclic of order $\frac{1}{2}(q+1)$, an argument entirely analogous to that of Lemma 2.4.2 (ii) yields that $F \cap J=J$. Since also $B \subseteq F \cap N$ and $F$ does not contain a four group, it follows that $L_{0}=J \times B=F \cap N$. Thus $B \subseteq O(F)$ and $F=O(F) J$.

In either case, set $R=O(F) C_{0}$. Then $P C_{0} B \subseteq R \cap H$. Since $\bar{H}=H / C_{0}$ is isomorphic to $P G L(2, q)$, Lemma 3.1 (viii) of [22] implies that $\bar{P} \bar{B}$ is a maximal subgroup of $\bar{H}$ of odd order, whence $P C_{0} B$ is a maximal such subgroup of $H$. We conclude therefore that $P C_{0} B=R \cap H$. Since $|P|=q,\left|C_{0}\right|=\frac{1}{2}(q+1)$ and $|B|=\frac{1}{4}(q-1)$, it also follows that $C_{0}$ is a Hall subgroup of $R \cap H$.

We can therefore apply Lemma 1 to $C_{0}$ as well as to $B$ to conclude that $C_{0}$ is a Hall subgroup of $R$ and that $C_{0} B$ has a normal complement $U$ in $R$. Since $x$ centralizes $C_{0} B$, we have $C_{R}(x)=C_{U}(x) C_{0} B$. Since $C_{R}(x)$ is $C_{0} B$-invariant, it follows that $C_{U}(x)$ is as well. Thus $C_{U}(x) \in \mathrm{U}_{N}\left(C_{0} B\right)$ and hence $C_{U}(x)=1$ by Lemma 2 (ii). (We do not yet know that $x$ leaves $U$ invariant.)

We claim that $B$ is a Hall subgroup of $R$. Indeed, if $r$ is a prime divisor of $b$ and $V$ is a Sylow $r$-subgroup of $R$ such that $V \cap B$ is a Sylow $r$-subgroup of $B$, Proposition 2.1 implies that $V \subseteq N$. In particular, $V \cap U \subseteq N$, whence $V \cap U$ $\subseteq C_{U}(x)=1$. But $V \cap U$ is a Sylow $r$-subgroup of $U$ as $U$ is normal in $R$ and $V$ is a Sylow $r$-subgroup of $R$. We conclude that $U$ is an $r^{\prime}$-group. Thus $U$ has order prime to $b$. Since $R=U B C_{0}$ and $C_{0}$ also has order prime to $b$, we see that $B$ is a Hall subgroup of $R$, as asserted. In particular, since $B \subseteq O(F) \subseteq R, B$ is a Hall subgroup of $O(F)$ and so (iii) holds.

Furthermore, since $U$ has order prime to $\left|C_{0} B\right|$, it is characteristic in $R$ and so is $y$-invariant. Since $C_{U}(x)=1, x$ inverts $U$ and $U$ is abelian. If $Q_{0}$ denotes the Sylow $p$-subgroup of $U$, then $Q_{0}$ is characteristic in $U$ and hence is invariant under $C_{0} B\langle y\rangle$. But $Q \subseteq U$ as $Q$ is normal in $F$ and so $Q \subseteq Q_{0}$. It follows therefore from our maximal choice of $Q$ that $Q=Q_{0}$ and we conclude that $Q$ is a Sylow $p$-subgroup of $R$ and hence of $O(F)$. If $F$ has a normal 2-complement, $Q$ is a Sylow $p$-subgroup of $F=N(Q)$ and hence $Q$ is a Sylow $p$-subgroup of $G$, so (iv) holds in this case.

Thus the lemma will be completely proved once we establish (ii). However, if $F$ does not have a normal 2-complement, then the above argument shows that $F=O(F) J$. Since $x$ inverts $Q, J$ is faithfully represented on $Q_{1}=\Omega_{1}(Q)$ regarded as a vector space over $G F(p)$. Lemma 2.4 .18 now yields that $\left|Q_{1}\right| \geqq q^{2}$. Since $J \cap Q_{1}=1$ and $q$ divides $|J|$, it follows that $q^{3}$ divides $|G|$.

On the other hand, $|C|=2(q+\varepsilon)$ and $|N|=2 b q(q-1)(q+1)$ with $b=\frac{1}{4}(q-\varepsilon)$. Hence $C$ has order prime to $p$ and a Sylow $p$-subgroup of $N$ has order $q$. Since $q^{3}$ divides $|G|$, we conclude at once from the definition that $G$ is regular. But then Proposition 2.2 implies that $B=1$, contrary to assumption.

We shall now construct a Sylow $p$-subgroup of $G$ by an alternative procedure. We need the following preliminary result: 
LEMMA 4. If $Q_{1}$ is a Sylow p-subgroup of $J$, then $N_{N}\left(Q_{1}\right)=Y B$, where $Y$ is cyclic of order $2(q-1)$ and $O(Y) B$ is abelian of type $(b, b)$. In particular, the Sylow 2-subgroup of $Y$ is cyclic of order 8 .

Proof. Arguing as in the final paragraph of the proof of Lemma 2, we have that $N_{J T}\left(Q_{1}\right)=Q_{1} Y$, where $|Y|=2(q-1), Y \mid\langle x\rangle$ is cyclic, and $Y$ has a cyclic normal 2-complement of order $\frac{1}{4}(q-1)=b$. Furthermore, by Lemma 2 (iv) itself, $Y$ does not contain a four subgroup. It follows at once that the Sylow 2-subgroups of $Y$ are cyclic of order 8 . Since $Y \mid\langle x\rangle$ is cyclic, this implies that $Y$ is cyclic. Since $O(Y) \subseteq J, O(Y)$ centralizes $B$ and $O(Y) \cap B=1$. Since $B$ is also cyclic of order $b$, $O(Y) B$ is therefore abelian of type $(b, b)$ and all parts of the lemma are proved.

We now prove

LEMMA 5. If $R$ is a $Y B$-invariant p-subgroup of $G$ of maximal order containing $Q_{1}$, then $R$ is a Sylow p-subgroup of $G$.

Proof. Set $V=N(R)$. Since $R \neq 1, V$ does not contain a four group by Lemma 2 (iv) and hence $V$ does not contain a Sylow 2-subgroup of $G$. Lemma 2 (i) now implies that a Sylow 2-subgroup of $V$ has order at most 8. But $Y \subseteq V$ and the Sylow 2-subgroup of $Y$ is cyclic of order 8. We conclude that $V$ has cyclic Sylow 2-subgroups and hence that $V$ possesses a normal 2-complement.

We shall argue next that $X=O(Y) B$ is a Hall subgroup of $O(V)$ and that $X$ has a normal complement in $O(V)$. Indeed, let $r$ be a prime divisor of $b$ and let $U$ be a Sylow $r$-subgroup of $O(V)$ containing one of $X$. Since $U \cap B$ is then a Sylow $r$-subgroup of $B$, Proposition 2.1 implies that $U \subseteq N$. But $O(Y)$ is a Hall subgroup of $J$ inasmuch as $|O(Y)|=b=\frac{1}{4}(q-1)$ and $|J|=q(q-1)(q+1)$. Hence $X$ is a Hall subgroup of $N$ and so $X$ contains a Sylow $r$-subgroup of $N$. It follows that $U \subseteq X$, whence $X$ contains a Sylow $r$-subgroup of $O(V)$. Since $r$ was arbitrary, we conclude that $X$ is a Hall subgroup of $O(V)$.

Furthermore, $C_{V}(U) \subseteq C_{V}(U \cap B) \subseteq N$ since $U \cap B \neq 1$ and $G$ satisfies the conclusions of Theorem B. But $x \in C_{V}(U)$ as $U \subseteq N$. Since $C_{V}(U)$ is normal in $N_{V}(U)$ and since a Sylow 2-subgroup of $C_{V}(U)$ is cyclic, it follows at once by the Frattini argument that

$$
N_{V}(U)=C_{V}(U)\left(N_{V}(U) \cap N\right),
$$

whence $N_{V}(U) \subseteq N$. In particular, $N_{O(V)}(U) \subseteq N$. On the other hand, $U \cap O(Y) \neq 1$ as $r$ divides $b=|O(Y)|$. Applying Lemma 3.1 (v) of [22] to $J T /\langle x\rangle$, it follows at once that $N_{J T}(U \cap O(Y))$ has $O(Y)$ as a normal complement. Since $U=(U \cap O(Y))$ $\times(U \cap B)$, it follows that $N_{N}(U)$ has $O(Y) B=X$ as a normal complement. Since $N_{O(V)}(U) \subseteq N_{N}(U)$, we conclude that $N_{O(V)}(U) \subseteq X$. But $X$ is abelian and hence $U$ is in the center of its normalizer in $O(V)$. Burnside's transfer theorem now yields that $O(V)$ possesses a normal $r$-complement. Since this holds for each prime divisor of $b$ and since $X$ is a Hall subgroup of $O(V)$, we conclude at once that $X$ has a normal complement $V_{0}$ in $O(V)$. 
Finally, our conditions imply that $V_{0}$ has order prime to $|X|$. In particular, $V_{0}$ is characteristic in $O(V)$ and $V_{0}$ contains a Sylow $p$-subgroup of $V$. Since $V_{0}$ has order prime to $Y X=Y B$, it follows that $Y B$ normalizes some Sylow $p$-subgroup $R_{0}$ of $V_{0}$ and hence of $V$. But $R \subseteq R_{0}$ as $R$ is normal in $V$, whence $R=R_{0}$ by our maximal choice of $R$. Since $R$ is a Sylow p-subgroup of its normalizer, we conclude that $R$ is a Sylow p-subgroup of $G$.

We can now easily complete the proof of the proposition. Let $Q, F, Y$, and $R$ be as above. Since $Q$ and $R$ are Sylow $p$-subgroups of $G$ by Lemmas 3 and 5 and since $Y B$ normalizes $R$, we see that $(Y B)^{u}$ normalizes $Q$ for some $u$ in $G$, whence $(Y B)^{u} \subseteq F=N(Q)$. Since $F$ has a normal 2-complement by Lemma 3 (ii), it follows that $(O(Y) B)^{u} \subseteq O(F)$. But $O(Y) B$ is abelian of type $(b, b)$ by Lemma 4 and therefore $b^{2}$ divides $|O(F)|$. Since $|B|=b$, this implies that $B$ is not a Hall subgroup of $O(F)$, contrary to Lemma 3 (iii). This completes the proof of the proposition.

We next prove

Proposition 2. A Sylow p-subgroup of $G$ has order $q$.

Proof. Since $B \neq 1, G$ is not regular by Proposition 2.2. As in Lemma 3 above, this implies that $q^{3}$ does not divide the order of $G$. Thus a Sylow $p$-subgroup of $G$ has order less than $q^{3}$. We use this fact to establish the proposition.

Since $\varepsilon=-1$, Lemma 2.4.10 implies that $C=C_{0} T$ normalizes a Sylow $p$-subgroup $Q_{1}$ of $N$. Moreover, $Q_{1}$ is elementary abelian of order $q$ and $C$ acts irreducibly on $Q_{1}$. Assume by way of contradiction that $Q_{1}$ is not a Sylow $p$-subgroup of $G$. We shall argue that a Sylow $p$-subgroup of $G$ has order at least $q^{3}$ and this will contradict the conclusion of the preceding paragraph.

Set $F=N\left(Q_{1}\right)$, so that $C_{0} B T \subseteq F$. We shall argue that $F$ has a normal 2-complement. Since $C_{0}=O(C)$ has order prime to $p$ and since $G$ has only one conjugacy class of four groups, $Q_{1}$ does not centralize any four subgroup of $G$. Hence a Sylow 2-subgroup $S_{1}$ of $C\left(Q_{1}\right)$ is either cyclic or quaternion (since a Sylow 2-subgroup of $G$ is quasi-dihedral of order 16). Since $x \in C\left(Q_{1}\right)$, we can choose $S_{1}$ to contain $x$, in which case $x \in Z\left(S_{1}\right)$ and hence $S_{1} \subseteq N$. Since $N=(J \times B) T$ with $J T$ isomorphic to $S L^{ \pm}(2, q)$, it is immediate that $\langle x\rangle$ is a Sylow 2-subgroup of $C_{N}\left(Q_{1}\right)$. Hence $\langle x\rangle=S_{1}$ and $C\left(Q_{1}\right)$ has a normal 2-complement. Furthermore, since $C\left(Q_{1}\right)$ is normal in $F$, it follows by the Frattini argument that $F=C\left(Q_{1}\right)(F \cap N)$. But $F \cap N \supseteq C B Q_{1}$ by Lemma 2.4.10. Furthermore, if we set $\bar{N}=N / B\langle x\rangle$, Lemma 3.1 (vi) of [22] shows that $N_{\bar{N}}\left(\bar{Q}_{1}\right)=\bar{C} \bar{Q}_{1}$. Hence $F \cap N=C B Q_{1}$ and so $F \cap N$ also has a normal complement. Since $\langle x\rangle$ is a Sylow 2-subgroup of $C\left(Q_{1}\right)$, we conclude at once that $F$ itself has a normal 2-complement, as asserted.

We know that $\bar{H}=H / C_{0}$ is isomorphic to $\operatorname{PGL}(2, q)$ and that $\bar{B}$ is cyclic of order $\frac{1}{4}(q-\varepsilon)=\frac{1}{4}(q+1)$. Lemma 3.1 of [22] implies therefore that $\bar{B}$ is a maximal subgroup of $\bar{H}$ of odd order. Hence $C_{0} B$ is a maximal such subgroup of $H$. Since $C_{0} B \subseteq O(F)$, it follows that $C_{0}$ is a Hall subgroup of $H \cap O(F)$. We conclude 
therefore from Lemma 1 that $C_{0} B$ has a normal complement $U$ in $O(F)$. Clearly $Q_{1} \subseteq U$. Since $O(F) \cap N=C_{0} B Q_{1}$, we also have that $U \cap N=Q_{1}$.

We argue next that also $B$ is a Hall subgroup of $O(F)$. As in Lemma 3, if $V$ is a Sylow $r$-subgroup of $O(F)$ with $V \cap B$ a Sylow $r$-subgroup of $B, r$ a prime divisor of $b$, we have by Proposition 2.1 and the normality of $U$ in $O(F)$ that $V \cap U \subseteq N$ and $V=(V \cap U)(V \cap B)$. Since $U \cap N=Q_{1}$ and $r$ is prime to $p$, it follows that $V \cap U=1$ and $V \subseteq B$. Thus $B$ is a Hall subgroup of $O(F)$, as asserted. Since $O(F)=U C_{0} B$ and $C_{0} B$ is a Hall subgroup of $O(F)$, this implies that $U$ is characteristic in $O(F)$. In particular, $U$ is $T$-invariant. Since $C_{U}(x)=U \cap N=Q_{1}$, $x$ inverts $U / Q_{1}$ and so we also have that $U / Q_{1}$ is abelian.

Set $Q=O_{p}(U)$. Since $Q_{1}$ is a $p$-group and $U / Q_{1}$ is abelian, it follows that $Q$ is a Sylow $p$-subgroup of $U$. Since $F$ has a normal 2-complement and $O(F)=U C_{0} B$, $Q$ is, in fact, a Sylow $p$-subgroup of $F$. Moreover, $Q$ is invariant under $C_{0} B T$ inasmuch as $U$ is normal in $O(F)$ and is $T$-invariant.

By assumption, $Q_{1}$ is not a Sylow $p$-subgroup of $G$. Since $Q$ is a Sylow $p$-subgroup of $F=N\left(Q_{1}\right)$, it follows that $Q \supset Q_{1}$. We shall determine the order of $Q$. The involutions of $T$ are $x=x_{1}, x_{2}, x_{3}$ and $Q$ has the corresponding $T$-decomposition $Q=Q_{1} Q_{2} Q_{3}$. We claim that $Q_{i} \neq 1, i=2$ and 3. Indeed, suppose, say, that $Q_{2}=1$. Then $x_{2}$ inverts $\bar{Q}=Q / Q_{1}$. But $x_{2}$ also inverts $B$ and consequently $B$ is forced to centralize $\bar{Q}$. Since $B$ centralizes $Q_{1}$, this implies that $B$ centralizes $Q$. However, $C(B) \subseteq N$ as $G$ satisfies the conclusions of Theorem B. Since $Q_{1}$ is a Sylow $p$-subgroup of $N$, it follows that $Q=Q_{1}$, which is not the case. Thus $Q_{i} \neq 1$, $i=2$ and 3 , as asserted.

Finally, $Q_{i} \subseteq N_{i}=C\left(x_{i}\right)$ and $Q_{i}$ is $C$-invariant, $i=2$ or 3. But $N_{i}=\left(J_{i} T\right) \times B_{i}$, where $J_{i}$ is isomorphic to $J$ and $B_{i}$ to $B$. Hence $Q_{i} \subseteq J_{i}$ and we conclude now from Lemma 2.4 .10 that $Q_{i}$ is necessarily a Sylow $p$-subgroup of $J_{i}$. Thus $\left|Q_{i}\right|=q$ for $i=2$ and 3. Furthermore, $C_{Q}(T)=1$ as $C_{0}$ is a $p^{\prime}$-group. Hence $Q=Q_{1} Q_{2} Q_{3}$ has order $q^{3}$. However, this is impossible since a Sylow $p$-subgroup of $G$ has been shown to have order less than $q^{3}$.

We can now easily establish the main result of the section.

Proposition 3. If $A=1$ and $B \neq 1$, then either $q^{\prime} \neq q$ or $\varepsilon^{\prime} \neq \varepsilon$.

Proof. Assume by way of contradiction that $q^{\prime}=q$ and $\varepsilon^{\prime}=\varepsilon$. Let $P$ be a Sylow p-subgroup of $H=N\left(C_{0}\right)$. Since $H / C_{0}$ is isomorphic to $P G L(2, q), P$ is elementary abelian of order $q$. Hence by Proposition 2, $P$ is a Sylow $p$-subgroup of $G$. Likewise a Sylow $p$-subgroup $Q_{1}$ of $N$ has order $q$ and is a Sylow $p$-subgroup of $G$. By Sylow's theorem, $P$ and $Q_{1}$ are conjugate. Since $Q_{1}$ centralizes $x$, we conclude that $P$ centralizes some involution $u$ of $G$.

We have already argued in Proposition 2 that $\langle x\rangle$ is a Sylow 2-subgroup of $C\left(Q_{1}\right)$. Hence $\langle u\rangle$ is a Sylow 2-subgroup of $F=C(P)$ and consequently $F$ has a normal 2-complement. On the other hand, $K=C\left(C_{0}\right)$ has index 2 in $H$, whence $P \subseteq K$ and so $P$ centralizes $C_{0}$. Thus $C_{0} \subseteq F$ and hence $C_{0} \subseteq O(F)$. If $\bar{H}=H / C_{0}$, 
then $C_{H}(\bar{P})=\bar{P}$ by Lemma 3.1 (vi) of [22] and so $F \cap H=C_{H}(P)=P C_{0}$. Hence $C_{0}$ is a Hall subgroup of $F \cap H$ and now Lemma 1 (i) yields that $C_{0}$ is a Hall subgroup of $O(F)$. But then by the Frattini argument, some involution $v$ of $F$ normalizes $C_{0}$. Thus $v \in F \cap H=P C_{0}$, which is impossible as $P C_{0}$ is of odd order. This completes the proof of the proposition.

5. The case $A=1, B \neq 1$ (continued). We continue the notation and initial assumptions of the preceding section. In particular, $A=1, B \neq 1$, and $q>7$. By Proposition 4.3 , either $q^{\prime} \neq q$ or $\varepsilon^{\prime} \neq \varepsilon$. The elimination of this remaining case is based in part on the following proposition which imposes a restriction on the prime divisors of $b$. Its proof is primarily character-theoretic.

Proposition 1. If $p$ is a prime divisor of $b$, then $p$ divides $q(q-1)(q+1)$.

Proof. As noted at the beginning of the preceding section, our conditions imply that $q \neq 9$, that $L_{0}=J \times B$, that $N=L$, and that $E=1$. In particular, $B$ is centralized by the Sylow 2-subgroup $\left\langle s^{2}, s z\right\rangle$ of $J$. On the other hand, $B$ is inverted by $z$. This implies that $B$ is abelian. If $u_{1}$ and $u_{2}$ are two elements of $B$ which are conjugate in $G$, then as $S$ is a Sylow 2-group of the extended centralizers $C^{*}\left(u_{1}\right)$ and $C^{*}\left(u_{2}\right)$ of $u_{1}$ and $u_{2}, u_{1}$ and $u_{2}^{ \pm 1}$ are conjugate in $N(S)=S \times E$. Since $E=A=1$ under our present conditions, the remark above shows that $u_{2}=u_{1}^{ \pm 1}$.

Suppose that $p$ is a prime which divides $b$, but not $q(q-1)(q+1)$, and let $P$ be a Sylow $p$-subgroup of $B$. Since

$$
|N|=2 b q(q-1)(q+1),
$$

$P$ then is a Sylow $p$-subgroup of $N$ and, by Proposition 2.1, $P$ is a Sylow $p$-subgroup of $G$. As a subgroup of $B, P$ is abelian. If $r \in N(P)$ and if $u \in P$, the elements $u$ and $u^{r}$ are conjugate. As we have seen, then $u^{r}=u^{ \pm 1}$. If $u_{1}, u_{2} \in P-\{1\}$ and $u_{1}^{r}=u_{1}$, we cannot have $u_{2}^{r}=u_{2}^{-1}$ since then $\left(u_{1} u_{2}\right)^{r}=u_{1} u_{2}^{-1} \neq\left(u_{1} u_{2}\right)^{ \pm 1}$. It follows that $r$ either centralizes $P$ or inverts $P$. Since for $r=z$ we have the latter case, we conclude that

$$
N(P)=\langle C(P), z\rangle .
$$

The following argument is very similar to one used in $\$ 3$. It follows from (1) that we can apply the results of [6, III, §IX], in particular the Theorems (9A) (9B), (9C) of that paper with $m=2$. It follows that the principal $p$-block $B_{0}(p, G)$ consists of $(|P|+3) / 2$ characters $\chi_{0}=1, \psi_{1}, \psi^{(i)}$ with $i=1,2, \ldots,(|P|-1) / 2$ (which are not to be confused with the character $\psi$ of $N$ in Proposition 3.2.3). They are real-valued. Moreover, there exists a sign $\delta^{*}$ such that, for every $p$-singular element $w$ of $G$.

$$
\psi_{1}(w)=\delta^{*},
$$

while for all $p$-regular elements $r$ of $G$ and all $i$

$$
\delta^{*}+\psi_{1}(r)=\psi^{(i)}(r)
$$


In particular, all $\psi^{(i)}(r)$ with the same $r$ are equal. Finally, if we set

$$
\alpha=\psi_{1}(1)\left(\psi_{1}(1)+\delta^{*}\right) /\left(\psi_{1}(1)-\psi_{1}(z)\right)^{2}
$$

and if $u \neq 1$ is an element of $P$, we have

$$
|G|=\alpha \frac{|C(z)|^{2}}{|C(u)|} \mu^{2}
$$

where $\mu$ denotes the number of conjugates of $z$ which invert $u$.

It follows from Theorem B that $C(u) \subseteq N$. Since $J$ and $B$ centralize $u$, while $z$ inverts $u$, the group $C(u)$ is the normal subgroup $L_{0}$ of $N$ of index 2 and $\mu$ is the number of involutions which lie in $N-L_{0}$. Since $x \in L_{0}$ and $z \notin L_{0}, \mu$ then is the number of elements in the conjugate class of $z$ in $N$. Thus

$$
\mu=\left|N: C_{N}(z)\right|=|N: C| .
$$

Since $|C(z)|=|N|$ and $|C(u)|=\frac{1}{2}|N|$, it follows that

$$
|G|=2 \alpha \frac{|N|^{3}}{|C|^{2}}
$$

The equation (3) shows that either $\psi_{1}(1)$ or all $\psi^{(i)}(1)$ are odd. Since the characters are real-valued, accordingly either $\psi_{1} \in B_{0}(2, G)$ or $\psi^{(i)} \in B_{0}(2, G)$ for all $i$, cf. [6, IV]. If $|P| \neq 3$, the latter case is impossible, since Corollary 3.2.1 shows that $B_{0}(2, G)$ does not contain two different irreducible characters of the same odd degree. Thus, $\psi_{1} \in B_{0}(2, G)$ for $|P| \neq 3$. We may assume that this is still true for $|P|=3$, since we can here interchange $\psi_{1}$ and $\psi^{(1)}$, if necessary.

It follows from $\psi_{1} \in B_{0}(2, G)$ that $\psi_{1}$ is one of the characters $\chi_{1}, \chi_{2}, \chi_{3}$ of the principal 2-block $B_{0}(2, G)$. Now (2) implies that

$$
\psi_{1}(x u)= \pm 1 \text {. }
$$

On the other hand, as $u \in O(N)$, Proposition 3.2.3 shows that

$$
\chi_{i}(x u)= \pm q \text { for } i=1,2 \text {. }
$$

Hence necessarily $\psi_{1}=\chi_{3}$. Now Proposition 3.2.3 yields

$$
\psi_{1}(z)=\chi_{3}(z)=\chi_{3}(x)=-\delta_{3}, \quad \psi_{1}(x u)=\chi_{3}(x u)=-\delta_{3} .
$$

On comparing the second equation with (2), we find $\delta^{*}=-\delta_{3}$ and (4) becomes

Thus

$$
\alpha=f_{3}\left(f_{3}-\delta_{3}\right) /\left(f_{3}+\delta_{3}\right)^{2} \text {. }
$$

$$
|G|=\frac{|N|^{3}}{|C|^{2}} 2 \frac{f_{3}\left(f_{3}-\delta_{3}\right)}{\left(f_{3}+\delta_{3}\right)^{2}}
$$

On comparing this formula with the expressions for $|G|$ in Proposition 3.2.6, we have

$$
\frac{f_{1}\left(f_{1}+\delta_{1}\right)}{\left(f_{1}-\delta_{1} \varepsilon q\right)^{2}} \frac{q+\varepsilon}{q}=\frac{f_{2}\left(f_{2}-\delta_{2}\right)}{\left(f_{2}+\delta_{2} \varepsilon q\right)^{2}} \frac{q-\varepsilon}{q}=2 \frac{f_{3}\left(f_{3}-\delta_{3}\right)}{\left(f_{3}+\delta_{3}\right)^{2}} .
$$


Suppose first that $\delta_{3}=-1$. Then the last expression in (6) is more than 2. Since $(q+\varepsilon) / q<2$, it follows that $\delta_{1} \varepsilon=1$. Moreover

Hence

$$
f_{1}\left(f_{1}^{2}+\delta_{1}\right)(q+\varepsilon)>2 q\left(f_{1}-q\right)^{2} .
$$

whence

$$
\left(f_{1}^{2}+f_{1}\right)(q+1)>2 q\left(f_{1}^{2}-2 q f_{1}+q^{2}\right)
$$

$$
f_{1}^{2}(q-1)<f_{1}\left(4 q^{2}+q+1\right)
$$

Since for $q \geqq 7$, we have

we obtain

$$
4 q^{2}+q+1 \leqq 4 q^{2}+2 q-6=(q-1)(4 q+6)
$$

$$
f_{1}<4 q+6 \text {. }
$$

Since $\delta_{1} \delta_{2} \delta_{3}=1$, it follows from $\delta_{3}=-1, \delta_{1}=\varepsilon$ that $\delta_{2}=-\varepsilon$. Then (6) yields

$$
(q-\varepsilon) f_{2}\left(f_{2}-\delta_{2}\right)>2 q\left(f_{2}-q\right)^{2} \text {. }
$$

As in the case of $f_{1}$, we see that

$$
f_{2}<4 q+6
$$

Since $f_{3} q^{2}=f_{1} f_{2}$, it follows that

$$
f_{3} q^{2}<(4 q+6)^{2}<(5 q)^{2} .
$$

Hence $f_{3}<25$. However, in the proof of Lemma 3.7.3, we have seen that $f_{3}>100$ for $q \geqq 7$. Hence we have a contradiction.

Assume next that $\delta_{3}=1$. Then $\delta_{1}=\delta_{2}=-1$. It follows from Proposition 3.2.8 that $f_{1}>q^{2}, f_{2}>q^{2}$.

If $\varepsilon=1$, the expression on the left in (6) is less than $(q+1) / q<8 / 7$. If $\varepsilon=-1$, then as $f_{1}\left(f_{1}-1\right) /\left(f_{1}-q\right)^{2}$ is increased if $f_{1}$ is replaced by a smaller value lying above $q$, we find

$$
\frac{f_{1}\left(f_{1}-1\right)}{\left(f_{1}-q\right)^{2}} \frac{q-1}{q} \leqq \frac{q^{2}\left(q^{2}-1\right)}{\left(q^{2}-q\right)^{2}} \frac{q-1}{q}=\frac{q+1}{q}<\frac{8}{7} .
$$

In either case, (6) implies

$$
2 \frac{f_{3}\left(f_{3}-1\right)}{\left(f_{3}+1\right)^{2}}<\frac{8}{7}
$$

This is false for $f_{3}=6$ and hence for all $f_{3} \geqq 6$. Thus, $f_{3}<6$. However, this is impossible and the proposition is established.

We use this to prove

Proposition 2. $q^{\prime}+\varepsilon^{\prime} \neq q+\varepsilon$.

Proof. Assume, by way of contradiction, that $q^{\prime}+\varepsilon^{\prime}=q+\varepsilon$. If $\varepsilon^{\prime}=\varepsilon$, this would force $q^{\prime}=q$, contrary to Proposition 4.3. Thus

$$
\varepsilon^{\prime}=-\varepsilon \text { and } q^{\prime}=q+2 \varepsilon
$$


By Lemma 2.2 (iv), $b=\frac{1}{4}\left(q^{\prime}-\varepsilon^{\prime}\right)$ and hence by (7),

$$
b=\frac{1}{4}(q+3 \varepsilon) .
$$

But by Proposition 1, any prime divisor $r$ of $b$ is a divisor of $q(q-1)(q+1)$ $=q(q-\varepsilon)(q+\varepsilon)$. Since $r$ divides $q+3 \varepsilon$ by (8) and $r$ is odd, the only possibility is that $r=3$ and $r$ divides $q$. Thus $q=3^{m}$ and $b=3^{h}$ for suitable positive integers $m$ and $h$.

Substitution in (8) yields

$$
4 \cdot 3^{h-1}=3^{m-1}+\varepsilon
$$

the only solution of which is $h=1, m=2$, and $\varepsilon=1$. Thus $b=3$ and $q=9$. However, by Proposition 2.3, $b=1$ when $q=9$. This contradiction establishes the proposition.

By Lemma $2.2(\mathrm{v}), C_{H}(z y)$ has a cyclic normal 2-complement $U$ of order $\frac{1}{2}\left(q^{\prime}+\varepsilon^{\prime}\right)$ which is inverted by $x$. Note that since $b=\frac{1}{4}\left(q^{\prime}-\varepsilon^{\prime}\right)$ and $b \neq 1, q^{\prime}>3$ and consequently $U \neq 1$. We shall now use Proposition 2 to establish the following property of $U$ :

LEMMA 1. U does not centralize a four subgroup of $G$.

Proof. Suppose, by way of contradiction, that $U$ centralizes a four subgroup $T_{0}$ of $G$. We set $C_{0}^{*}=O\left(C\left(T_{0}\right)\right)$, so that $C_{0}^{*}$ is a normal 2-complement in $C\left(T_{0}\right)$. In particular, $U \subseteq C_{0}^{*}$ as $U$ has odd order and $U$ centralizes $T_{0}$. Since $G$ has only one conjugacy class of four subgroups, $T_{0}$ and $T$ are conjugate in $G$ and therefore so are $C_{0}^{*}$ and $C_{0}$. Hence if we set $H^{*}=N\left(C_{0}^{*}\right)$, it follows that $H^{*}$ and $H$ are conjugate in $G$. As a consequence, Lemma 2.2 applies as well for $H^{*}$ and $C_{0}^{*}$.

Since $U \subseteq C_{0}^{*}$, Lemma 2.2 (vii) implies that $H^{*}=N(U)$. But $U$ centralizes $C_{0}$ by Lemma 2.2 (ii), applied to $H$, whence $C_{0} \subseteq H^{*}$. It follows now from Lemma 2.2 (ii), applied to $H^{*}$, that $C_{0}$ centralizes $C_{0}^{*}$. Hence $C_{0}^{*} \subseteq H$. Observe next that since $z y$ centralizes $U$, Lemma 2.2 (vii), applied to $H^{*}$, yields that $z y$ centralizes $C_{0}^{*}$. Thus $C_{0}^{*} \subseteq C_{H}(z y)$. But $U$ is a normal 2-complement in $C_{H}(z y)$, so $C_{0}^{*} \subseteq U$. Since $U \subseteq C_{0}^{*}$, we conclude that $U=C_{0}^{*}$.

On the other hand, $|U|=\frac{1}{2}\left(q^{\prime}+\varepsilon^{\prime}\right)$, while $\left|C_{0}^{*}\right|=\left|C_{0}\right|=\frac{1}{2}(q+\varepsilon)$. Since $U=C_{0}^{*}$, it follows that $q^{\prime}+\varepsilon^{\prime}=q+\varepsilon$. However, this contradicts Proposition 2 .

Now set $T^{*}=\langle x, z y\rangle$, so that $T^{*}$ is a four group. Since $T^{*}$ is conjugate to $T$, there exists a 3-element $t^{*}$ in $N\left(T^{*}\right)$ such that $x^{* *}=z y$. Setting $N^{*}=C(z y)$, it follows that $N^{*}=N^{t^{*}}$. But $N=J T B=J T^{*} B$ and hence $N^{*}=J^{* *} T^{*} B^{t^{*}}$. We set $J^{*}=J^{t^{*}}$ and $B^{*}=B^{t^{*}}$ and fix all this notation. In particular, $B^{*}$ is cyclic of order $b$, $J^{*}$ is isomorphic to $\operatorname{SL}(2, q), N^{*}=\left(J^{*} \times B^{*}\right) T^{*}$, and $x$ inverts $B^{*}$.

We next prove

LEMMA 2. The following conditions hold:

(i) $U \subseteq J^{*}$;

(ii) $|U|$ divides $q(q-\varepsilon)$. 
Proof. We know that $U=O\left(C_{H}(z y)\right) \subseteq N^{*}$, so $U \subseteq J^{*} \times B^{*}$. Since $|U|$ is prime to $b=\left|B^{*}\right|$ by Lemma 2.2 (vi), it follows that $U \subseteq J^{*}$, proving (i).

A cyclic subgroup of $S L(2, q)$ of odd order necessarily has order dividing $q$, $q-\varepsilon$, or $q+\varepsilon$. Since $U$ is cyclic and $J^{*}$ is isomorphic to $\operatorname{SL}(2, q)$, either (ii) holds or $|U|$ divides $q+\varepsilon$. However, $O\left(C\left(T^{*}\right)\right)$ is a cyclic subgroup of $J^{*}$ of order $\frac{1}{2}(q+\varepsilon)$. By the structure of $S L(2, q)$, if $|U|$ divides $q+\varepsilon$, a conjugate of $U$ must lie in $O\left(C\left(T^{*}\right)\right)$ and hence $U$ centralizes a conjugate of $T^{*}$, contrary to Lemma 1 .

We next prove

LEMMA 3. If $H^{*}=C^{*}(U)$, then we have

(i) $H^{*}$ has a normal 2-complement containing $C_{0} B^{*}$;

(ii) $C_{0}$ is a Hall subgroup of $O\left(H^{*}\right)$ and $O\left(H^{*}\right)$ has a normal $C_{0}$-complement;

(iii) If $V^{*}$ is a Hall subgroup of $O\left(H^{*}\right)$ containing $B^{*}$ for the set of primes dividing $b$, then $V^{*} \subseteq N^{*}$;

(iv) $H \cap O\left(H^{*}\right)=U C_{0}$.

Proof. By Lemma 1, $C(U)$ does not contain a four group. Hence if $S_{0}$ is a Sylow 2-subgroup of $C(U)$ containing $z y$, then $S_{0}$ is either cyclic or generalized quaternion. In either case, $z y \in Z\left(S_{0}\right)$, so $S_{0} \subseteq N^{*}$. If $S_{0}$ is generalized quaternion, then $S_{0}$ necessarily lies in $J^{*}$. But no nontrivial subgroup of $J^{*}$ of odd order centralizes a quaternion subgroup of $J^{*}$ as $J^{*}$ is isomorphic to $S L(2, q)$, as is easily verified. Since $U \subseteq J^{*}$ by Lemma 2 (i) and $U \neq 1$, we conclude that $S_{0}$ cannot be generalized quaternion. Hence $S_{0}$ is cyclic and so $C(U)$ has a normal 2-complement. Since $\left|H^{*}: C(U)\right|=2$, so also does $H^{*}$. Furthermore, $U$ centralizes $C_{0}$ by Lemma 2.2 (ii) and centralizes $B^{*}$ by Lemma 2 (i), so $C_{0} B^{*} \subseteq O\left(H^{*}\right)$. This proves (i).

Since $\bar{H}=H / O(H)$ is isomorphic to $\operatorname{PGL}\left(2, q^{\prime}\right), \bar{U} \neq 1$, and $\bar{U}=O\left(C_{B}(\bar{z} \bar{y})\right)$, $\bar{U}=O\left(C_{H}(\bar{U})\right)$ by Lemma $3.1(\mathrm{v})$ of [22]. Hence $U C_{0}=O\left(C_{H}(U)\right)$. But $O\left(C_{H}(U)\right)$ $=H \cap O\left(H^{*}\right)$ as $O\left(H^{*}\right)$ is a normal 2-complement in $H^{*}$. Therefore $U C_{0}=$ $H \cap O\left(H^{*}\right)$, which proves (iv).

We use (iv) to establish (ii). Indeed, $|U|$ divides $q(q-\varepsilon)$ by Lemma 2 (ii). Since $\left|C_{0}\right|=\frac{1}{2}(q+\varepsilon)$, it follows that $C_{0}$ and $U$ have coprime orders. But then $C_{0}$ is a Hall subgroup of $U C_{0}=H \cap O\left(H^{*}\right)$. Now (ii) is a direct consequence of Lemma 4.1 (i).

Finally let $V^{*}$ be as in (iii). Since $B^{*}$ is conjugate to $B$, Proposition 2.1 holds for $B^{*}$ and we conclude at once that $V^{*} \subseteq N^{*}$. Thus (iii) also holds and the lemma is proved.

We can now readily establish the main objective of $\$ \$ 4$ and 5:

Proposition 3. The case $A=1, B \neq 1$ does not occur.

Proof. In view of the results of this and the preceding section, the proposition will be established provided we show that the situation described in Lemmas 2 and 3 above leads to a contradiction. This we shall now do. We preserve the notation of those lemmas. 
By Lemma 3, $C_{0}$ and $V^{*}$ are each Hall subgroups of $O\left(H^{*}\right)$ and $V^{*}$ is centralized by $z y$. Since $z y$ inverts $C_{0}$, it follows that $C_{0}$ and $V^{*}$ have coprime orders. Indeed, if not, then $z y$ would normalize some nontrivial Sylow $r$-subgroup $R_{0}$ of $C_{0}$ and $R^{*}$ of $V^{*}$ for a suitable prime $r$. But then $R_{0}$ and $R^{*}$ would be $z y$-invariant Sylow $r$-subgroups of $O\left(H^{*}\right)$ and so would be conjugate by an element of $C_{O\left(H^{*}\right)}(z y)$. However, this is impossible as $z y$ centralizes $R^{*}$ and inverts $R_{0}$ and each is nontrivial.

By Lemma 3 (ii), $C_{0}$ has a normal complement $X^{*}$ in $O\left(H^{*}\right)$. Furthermore, $V^{*} \subseteq X^{*}$ as $V^{*}$ and $C_{0}$ have coprime orders. Since $V^{*}$ and $C_{0}$ are $z y$-invariant Hall subgroups of $O\left(H^{*}\right)$, it follows that $C_{0}$ leaves invariant some conjugate $V_{0}^{*}$ of $V^{*}$ by an element of $C_{X^{*}}(z y)$. In particular, $z y$ also centralizes $V_{0}^{*}$. Since $C_{0}=\left[C_{0}, z y\right]$, we see that $C_{0}$ also centralizes $V_{0}^{*}$. Hence $V_{0}^{*} \subseteq N\left(C_{0}\right)=H$. Thus $V_{0}^{*} \subseteq H \cap O\left(H^{*}\right)$ and consequently $V_{0}^{*} \subseteq U C_{0}$ by Lemma 3 (iv). However, $V_{0}^{*}$ and $C_{0}$ have relatively prime order and therefore $\left|V_{0}^{*}\right|$ divides $|U|$.

On the other hand, $\left|V_{0}^{*}\right|=\left|V^{*}\right|$ and $b$ divides $\left|V^{*}\right|$ as $B^{*} \subseteq V^{*}$. Hence $b$ divides $|U|$. But $U=O\left(C_{H}(z y)\right)$ by definition and so $|U|$ is relatively prime to $b$ by Lemma 2.2 (vi). This contradiction completes the proof of the proposition.

6. Double transitivity of $G$ in the case $\varepsilon=1$. As we have already noted at the beginning of $\$ 4$, the results of $\$ \$ 1-5$ show that parts (i), (ii), and (iii) of Theorem $C$ hold for $G$ and so it remains only to establish part (iv) of Theorem $C$. This we shall do in the next two sections.

We thus have that $G$ is a simple $Q D$-group of characteristic power $q$ which satisfies the conclusions of Theorem $B$ and we now assume that $\varepsilon=1$, where, by definition, $q \equiv \varepsilon(\bmod 4)$. Our results imply that $E=A$, that $G$ is regular, that $B=1$, and that $A$ is cyclic of order $a$ dividing $\frac{1}{2}(q+1)($ as $\varepsilon=1)$. In particular, $N=(J T) \times A$.

In this section we shall prove the following basic result:

Proposition 1. If $\varepsilon=1, G$ has a doubly transitive permutation representation of degree $q^{3}+1$ such that if $G_{1}$ denotes the subgroup leaving one letter fixed, then $G_{1}$ has a normal subgroup $Q$ of order $q^{3}$ which acts regularly on the set of remaining letters and $G_{1} / Q$ is cyclic of order $\left(q^{2}-1\right) / d$ with $d=(q+1) / 2 a$.

We shall carry out the proof in a sequence of lemmas. However, we first collect some known facts concerning $N$ and $G$, which we shall need. First of all, in view of the results of Chapter III, we may, as usual, assume that $q>5$. We have

$$
J T /\langle x\rangle \simeq P G L(2, q)
$$

and also

$$
|N|=2 a q(q-1)(q+1) \text {. }
$$

Since $G$ is regular, the degrees of the characters $\chi_{1}, \chi_{2}, \ldots$ in $B_{0}(2, G)$ have the following values:

$$
\begin{gathered}
f_{1}=q^{3}, \quad f_{2}=q^{2}-q+1, \quad f_{3}=q\left(q^{2}-q+1\right), \quad f_{4}=q^{2}-q, \\
f=q^{3}+1 .
\end{gathered}
$$


In addition, $\delta_{1}=1, \delta_{2}=\delta_{3}=-1$ (Proposition 3.3.1). Since $b=e=1$, Proposition 3.3.2* reads

$$
|G|=2 a q^{3}(q-1)(q+1)\left(q^{2}-q+1\right) .
$$

Note that

$$
q-1=2^{n-1} k
$$

where $k$ is an odd integer.

Suppose that $q=p^{m}$, where $p$ is a prime. Let $Q_{0}$ denote a Sylow $p$-subgroup of $J T$. This is then a Sylow $p$-subgroup of $N$. If $\bar{J} \bar{T}=J T /\langle x\rangle$, then by (1), $N_{J T}\left(\bar{Q}_{0}\right)=\bar{Q}_{0} \bar{W}$, where $\bar{W}$ is cyclic of order $q-1$. It follows therefore from (1) that

$$
N_{J T}\left(Q_{0}\right)=Q_{0} W
$$

where the complement $W$ of $Q_{0}$ is an abelian group which is either cyclic of order $2(q-1)$ or the direct product of $\langle x\rangle$ with a cyclic group of order $q-1$. In the latter case, as shown by (5), $J T$ would contain an abelian subgroup of type $\left(2^{n-1}, 2\right)$. Since $n \geqq 3$, no such subgroup occurs in the Sylow 2-subgroup $S$ of $G$. Hence we have the former case. In particular, $W$ contains a cyclic subgroup of order $2^{n}$. After replacing $Q_{0}$ by a suitable conjugate, we may assume that $S_{0}=\langle s\rangle$ occurs in $W$. Then $W=S_{0} \times W_{0}$, where $W_{0}$ is cyclic of order $k$ and

$$
N_{J T}\left(Q_{0}\right)=Q_{0} W=Q_{0}\left(S_{0} \times W_{0}\right) .
$$

Since $N=(J T) \times A$, it follows at once that

$$
N_{N}\left(Q_{0}\right)=Q_{0}\left(S_{0} \times W_{0} \times A\right) .
$$

Since $q-1$ and $a=|A|$ are coprime, the group

$$
H=S_{0} \times W_{0} \times A
$$

is cyclic of order $2(q-1) a$.

It follows at once from the structure of $\operatorname{PGL}(2, q)$ that $Q_{0}$ is elementary abelian of order $q$ and that all elements of $Q_{0}^{\#}$ are conjugate under the action of $H$. Since $\langle x\rangle \times A$ centralizes $Q_{0}$, it is now clear that $W$ acts transitively on $Q_{0}^{\#}$ with $x$ the only element of $W^{\#}$ centralizing any element of $Q_{0}^{\#}$. In particular,

$$
C_{N}\left(Q_{0}\right)=Q_{0} \times\langle x\rangle \times A .
$$

This is an abelian group of order $2 a q$.

Since $E=A$, we also have

$$
N(S)=S \times A \text {. }
$$

We also note that

$$
C_{N}\left(S_{0}\right)=C_{N}(y)=S_{0} \times W_{0} \times A
$$

and that

$$
N\left(S_{0}\right)=\left\langle C\left(S_{0}\right), z\right\rangle .
$$


We now begin the proof of the proposition.

LEMMA 1. If $p_{0}$ is a prime dividing $\mathscr{K}=(q-1) / 2^{n-1}$, then $G$ does not contain elements of order $p p_{0}$.

Proof. Let $P$ be a Sylow $p_{0}$-subgroup of $W_{0}$. Since $k$ is coprime to $2, a, q, q+1$ and $q^{2}-q+1$, it follows that $P$ is a Sylow $p_{0}$-subgroup of $G$.

If $u \in P$ and $u \neq 1$, we have $S_{0} \subseteq C(u)$. Since $\left(p_{0}, a\right)=1$, the equation (9) shows that $u$ cannot centralize a Sylow 2-subgroup of $G$. Hence $C(u)$ has the Sylow 2subgroup $S_{0}$. If $u$ is conjugate in $G$ to $u^{\prime} \in P$, a Sylow group argument shows that $u$ and $u^{\prime}$ are conjugate in $N\left(S_{0}\right)$. Then (10) and (11) show that $u^{\prime}=u$ or $u^{\prime}=u^{z}$. Also, since $z$ normalizes $S_{0}$, it normalizes $W_{0}$ and $P$. A remark above show that $z$ cannot centralize any $u \neq 1$ in $P$. Hence $z$ inverts $P$ and we have $u^{\prime}=u^{ \pm 1}$.

If $v \in N(P)$, then for $u \in P, u$ and $u^{v}$ are conjugate and hence $u^{v}=u^{ \pm 1}$. If $u_{1}$ and $u_{2}$ are nonidentity elements of $P$, then $\left(u_{1} u_{2}\right)^{v}=\left(u_{1} u_{2}\right)^{ \pm 1}$. It is then impossible that $u_{1}^{v}=u_{1}, u_{2}^{v}=u_{2}^{-1}$. Hence $v$ either centralizes all elements of $P$ or $v$ inverts all elements of $P$. Since we have the latter case for $v=z$, we conclude that

$$
N(P)=\langle C(P), z\rangle \text {. }
$$

We can now apply the results of [6, III, §IX with $m=2$, in particular (9A) and (9B)]. It follows that the principal $p_{0}$-block $B_{0}\left(p_{0}, G\right)$ consists of $(|P|+3) / 2$ characters

$$
\chi_{0}=1, \psi^{(1)}, \psi^{(i)} \quad(i=1,2, \ldots,(|P|-1) / 2) .
$$

These are real-valued. There exists a sign $\delta^{*}$ such that

$$
\psi_{1}(v)=\delta^{*}
$$

for all $p_{0}$-singular $v \in G$. We have

$$
\delta^{*}+\psi_{1}(1)=\psi^{(i)}(1)
$$

for all $i$. Either $\psi_{1}(1)$ or $\psi^{(i)}(1)$ is odd. Now any real-valued irreducible character of odd degree lies in $B_{0}(2, G)$, [6, IV]. Hence either $\psi_{1}$ or all $\psi^{(i)}$ lie in $B_{0}(2, G)$. Again the latter case is impossible for $|P| \neq 3$, since Corollary 3.2.1 shows that $B_{0}(2, G)$ does not contain two different irreducible characters of the same odd degree. If $|P|=3$, we may interchange $\psi_{1}$ and $\psi^{(1)}$, if necessary. We can then assume that then, too, $\psi_{1} \in B_{0}(2, G)$.

It is now clear that $\psi_{1}$ is one of the characters $\chi_{1}, \chi_{2}, \chi_{3}$ in $B_{0}(2, G)$. If $u \neq 1$ is an element of $P$, then, by (12)

$$
\psi_{1}(s u)=\psi_{1}\left(s^{2} u\right)=\delta^{*} .
$$

Now, Proposition 3.2.2 implies that this is not true for $\psi_{1}=\chi_{2}$ or $\psi_{1}=\chi_{3}$. Hence $\psi_{1}=\chi_{1}$. However, $\chi_{1}$ of degree $q^{3}$ has $p$-defect 0 and vanishes for all $p$ singular elements of $G$. Now (12) shows that we cannot have elements of $G$ which are both $p$-singular and $p_{0}$-singular. In particular, there do not exist elements of order $p p_{0}$ in $G$. 
LeMma 2. If $u \neq 1$ is a p-element of $G$ then $|C(u)|$ divides $2 a q^{3}$.

Proof. As we have already used, $\chi_{1}$ of degree $q^{3}$ has $p$-defect 0 and vanishes for all $p$-singular elements of $G$. Since $1+\chi_{1}(u)=\chi^{(1)}(u)$ by Proposition 3.2.4, we have $\chi^{(1)}(u)=1$. We now find

$$
\frac{|G|}{|C(u)|} \frac{\chi^{(1)}(u)}{f}=\frac{|G|}{|C(u)|} \frac{1}{q^{3}+1}=\frac{2 a q^{3}(q-1)}{|C(u)|} ;
$$

(cf. (4)). As is well known, this expression is an algebraic integer. Thus, $|C(u)|$ divides $2 a q^{3}(q-1)$. It follows from Lemma 1 that $|C(u)|$ does not contain an odd prime factor of $q-1=2^{n-1} k$. If 4 divides $|C(u)|$, then $C(u)$ contains a conjugate of $T$ or of $\langle y\rangle$. However, $|C(T)|=2(q+1) a$ is not divisible by $p$. Neither is $|C(y)|$, cf. (10). Hence, 4 does not divide $|C(u)|$ and $|C(u)|$ must divide $2 a q^{3}$ as had been stated.

LEMMA 3. If $Q$ is a Sylow p-subgroup of $C\left(Q_{0}\right)$, then $Q$ is characteristic in $C\left(Q_{0}\right)$ and

$$
C\left(Q_{0}\right)=Q(A \times\langle x\rangle) .
$$

Proof. Since $Q_{0}$ is abelian, we have $Q_{0} \triangleleft C\left(Q_{0}\right)$ and hence $Q_{0} \subseteq Q$. By (8), $\langle x\rangle \times A$ belongs to $C\left(Q_{0}\right)$ and Lemma 2 shows that $\langle x\rangle \times A$ is a Hall subgroup of $C\left(Q_{0}\right)$. If $r \in\langle x\rangle \times A$ and if $r$ has even order, then $C(r) \subseteq C(x)=N$ and hence $C(r)=N$. If $r$ has odd order, then $r \in A$ and if $r \neq 1$, by Theorem $\mathrm{B}, C(r) \subseteq N$ and again $C(r)=N$. A Sylow group argument shows that if two elements $r_{1}$ and $r_{2}$ of $\langle x\rangle \times A$ are conjugate in $G$, they are conjugate in $N(S)$. It follows from (9) that then $r_{1}=r_{2}$. We conclude therefore from Burnside's transfer theorem that $\langle x\rangle \times A$ has a normal complement in $C\left(Q_{0}\right)$. Since this normal complement is a Sylow $p$-subgroup of $C\left(Q_{0}\right)$ by Lemma 2, $C\left(Q_{0}\right)$ has a unique Sylow $p$-subgroup $Q$ and $Q$ is characteristic in $C\left(Q_{0}\right)$. Also, (14) holds.

On account of (6) and (7), $H$ normalizes $Q_{0}$. Then $H$ normalizes $C\left(Q_{0}\right)$. On account of Lemma 3, $H$ normalizes $Q$. Thus, we have

LEMma 4. The group $Q$ is normalized by the cyclic group $H$ of order $2(q-1) a$.

We show next

LemMA 5. The group $Q$ is a Sylow p-subgroup of $G$.

Proof. Suppose first that we had $Q=Q_{0}$. By (14)

$$
C\left(Q_{0}\right)=Q_{0} \times A \times\langle x\rangle .
$$

Since the elements of $N\left(Q_{0}\right)$ normalize $C\left(Q_{0}\right)$, they normalize $\langle x\rangle$, i.e. they lie in $N$. Then $Q_{0}$ is a Sylow $p$-subgroup of $N\left(Q_{0}\right)$. As $Q_{0}$ is certainly not a Sylow $p$ subgroup of $G$, this is impossible by the elementary properties of $p$-groups.

Hence $Q_{0} \subset Q$. Since $Q_{0}$ and $Q$ are normalized by $H$, the set $Q-Q_{0}$ consists of full classes of elements conjugate with regard to $H$. Suppose that $u \in Q-Q_{0}$ is 
centralized by an element $r \neq 1$ of $H$. Lemma 1 implies that $r$ has order prime to $k$ and then (7) shows that $r \in S_{0} \times A$. If $r$ has even order, then $u$ centralizes $x$ and then $u \in Q \cap N=Q_{0}$, a contradiction. Thus $r \in A^{\#}$. By Theorem B, $C(r)=N$ and again $u \in Q \cap N=Q_{0}$, which is impossible.

Hence $u \in Q-Q_{0}$ is not centralized by any element $r$ of $H^{\#}$. It follows that the class of $H$-conjugates of $u$ consists of $|H|=2(q-1) a$ elements. Hence $|Q|-\left|Q_{0}\right|$ is divisible by $2(q-1) a$.

Set $\left|Q: Q_{0}\right|=p^{h} ; h \geqq 1$. It follows that $p^{h} q-q=2(q-1) a c$ with integral $c>0$. Then $q$ divides $2 a c$ and as $(q, 2 a)=1, q$ divides $c$, say $c=q c_{0}$ with integral $c_{0}>0$. We now have

$$
p^{h}-1=2(q-1) a c_{0} .
$$

Since $p^{h}-1$ is divisible by $q-1=p^{m}-1$, we see that $h$ is divisible by $m$. Since $a \neq 1$, we have, $p^{h}-1>p^{m}-1$ and consequently $h \geqq 2 m$. Thus $\left|Q: Q_{0}\right| \geqq p^{2 m}=q^{2}$ and therefore $|Q| \geqq q^{3}$. But now it follows from (4) that $|Q|=q^{3}$ and that $Q$ is a Sylow $p$-subgroup of $G$, as we wished to show.

Now Lemma 4 yields that

$$
N(Q) \supseteq Q H
$$

and that $|N(Q)| \geqq q^{3} 2(q-1) a$.

We are now in a position to establish Proposition 1 . Since $G$ has an irreducible character $\chi_{1}$ of $p$-defect 0, a theorem of Green (cf. [23] or [29]) implies that there exists a Sylow $p$-subgroup $Q_{1}$ of $G$ with $Q \cap Q_{1}=1$.

This implies that the $q^{3}$ groups $Q_{1}^{u}$ with $u \in Q$ are distinct. Since these groups are different from $Q$, the set $\Omega$ of all Sylow $p$-subgroups consists of at least $q^{3}+1$ members. On the other hand, $|\Omega|=|G: N(Q)|$, whence, by (4),

$$
q^{3}+1 \leqq 2 a q^{3}(q-1)(q+1)\left(q^{2}-q+1\right) /|N(Q)| .
$$

Thus $|N(Q)| \leqq 2 a q^{3}(q-1)$. Since we have proved the reverse inequality, we have equality and (15) becomes

$$
N(Q)=Q H \text {. }
$$

It also follows that $|\Omega|=q^{3}+1$ and that $\Omega=\{Q\} \cup\left\{Q_{1}^{u} \mid u \in Q\right\}$.

Now Proposition 1 is evident. Indeed, if we let $G$ act by conjugation on $\Omega$, we obtain a transitive permutation representation $R$ of $G$ of degree $q^{3}+1$. As $G$ is simple, this representation is faithful. The subgroup $G_{1}$ of $G$ fixing the element $Q \in \Omega$ is $N(Q)$. The normal subgroup $Q$ of $N(Q)$ is transitive on the set $\Omega-\{Q\}$. Thus, $R$ is doubly transitive. It is also clear that no element of

$$
\Omega-\{Q\}=\left\{Q_{1}^{u} \mid u \in Q\right\}
$$

is fixed by a nonidentity element of $Q$. This means that $Q$ acts regularly on $\Omega-\{Q\}$. Finally, (16) shows that $N(Q) / Q$, which is isomorphic to $H$, is a cyclic group of order $|H|=2 a(q-1)$, i.e. of order $\left(q^{2}-1\right) / d$ with $d=(q+1) / 2 a$.

This concludes the proof of Proposition 1. 
REMARK. It should be pointed out that work on a characterization of $P S U(3, q)$, $q$ odd, by means of centralizers of involutions was begun by Suzuki a number of years ago. The earlier parts of the work had some overlap with Proposition 1 above and also paralleled that of an earlier, unpublished version of [8]. In particular, he was able to construct a projective space on which the group $G$ acted. However, it was not possible to prove that the space was Desarguesian. It was his later investigation [28] which enabled him to complete this portion of the work in the case that the subgroup $H$ above has order $q^{2}-1($ that is, $q \not \equiv-1(\bmod 3)$ and $d=1)$ using a nongeometric approach involving generators and relations for $G$. Using the method of block designs, O'Nan [24*] has recently been able to treat the case that $|H|=\left(q^{2}-1\right) / d$, where $d=(q+1,3)$. Combined with the results of the following section, his work completes the characterization of $P S U(3, q), q$ odd.

7. The value of $d$ in the case $\varepsilon=1$. In this section $G$ will be a group which satisfies the same assumptions as in $\S 6$. We shall use the same notation. To complete the proof of part (iv) of Theorem $\mathrm{C}$, it remains to establish that the integer $d=(q+1,3)$. Here

$$
d=(q+1) / 2 a
$$

with $a=|A|$ dividing $(q+1) / 2$.

Proposition 1. Let $p_{0}$ be an odd prime which divides $(q+1) / 2$. If $p_{0} \neq 3$, then $p_{0} \mid a$.

Proof. Let $P_{0}$ denote a Sylow $p_{0}$-subgroup of $J$. Then $\left|P_{0}\right|$ is the highest power of $p_{0}$ dividing $q+1$. Suppose that $p_{0}$ does not divide $a$. Then the formula $\S 6$, (4) shows that $P_{0}$ is a Sylow $p_{0}$-subgroup of $G$.

It follows from Lemma 2.4.2 that $P_{0}$ is cyclic and that for a suitable choice of $P_{0}$

$$
N\left(P_{0}\right)=\left\langle C\left(P_{0}\right), y z\right\rangle,
$$

where the involution $y z$ inverts $P_{0}$. Again, the results of [6, III, §IX] apply with $m=2$. The principal $p_{0}$-block $B_{0}\left(p_{0}, G\right)$ then consists of $\left(\left|P_{0}\right|+3\right) / 2$ characters

$$
1, \psi_{1}, \psi^{(i)} \quad\left(i=1,2, \ldots,\left(\left|P_{0}\right|-1\right) / 2\right)
$$

These are real-valued. As $\left|P_{0}\right| \neq 3, \psi_{1}(1)$ is odd.

Again, it follows that $\psi_{1}$ is one of the characters $\chi_{1}, \chi_{2}, \chi_{3}$ of the principal 2-block $B_{0}(2, G)$. There exists a sign $\delta^{*}$ such that

$$
\delta^{*}+\psi_{1}(1)=\psi^{(i)}(1)
$$

For all $p_{0}$-singular elements $u$ of $G$, we have

$$
\psi_{1}(u)=\delta^{*} .
$$

The orthogonality relations applied to $\psi_{1} \mid P_{0}$ then show that

$$
\psi_{1}(1) \equiv \delta^{*}\left(\bmod \left|P_{0}\right|\right)
$$


Since $q \equiv-1\left(\bmod \left|P_{0}\right|\right)$, we have

$$
f_{2}=q^{2}-q+1 \equiv 3, \quad f_{3}=q\left(q^{2}-q+1\right) \equiv-3 \quad\left(\bmod \left|P_{0}\right|\right)
$$

and on comparing this with (3), we see that $\psi_{1} \neq \chi_{2}, \chi_{3}$. Hence $\psi_{1}=\chi_{1}, \psi_{1}(1)=f_{1}=q^{3}$ and (3) shows that $\delta^{*}=-1$. Now, by (1)

$$
\psi^{(i)}(1)=q^{3}-1
$$

As a degree of an irreducible character, $q^{3}-1$ divides $|G|$. Then $q^{2}+q+1$ divides $|G|$. However, $q^{2}+q+1$ is coprime to $q, q+1$, and $q^{2}-q+1$ while the greatest common divisor of $q^{2}+q+1$ and $q-1$ is 1 or 3 . Since $q^{2}+q+1>3$, the formula $\S 6$, (4) for $|G|$ yields a contradiction.

In the case $p_{0}=3$, we have

Proposition 2. If $q+1 \equiv 0(\bmod 9)$, then $3 \mid a$.

Proof. Suppose that $a \not \equiv 0(\bmod 3)$. Let $P_{0}$ be a Sylow 3-subgroup of $C_{0}$. Then the order $\left|P_{0}\right|=3^{h}$ is the highest power of 3 dividing $q+1$ and, by assumption, $h \geqq 2$. Since $P_{0} \subseteq J$, by Lemma $2.4 .2, P_{0}$ is cyclic, say, $P_{0}=\langle u\rangle$.

Since $t$ normalizes the abelian group $C_{0}$, it normalizes $P_{0}$. As $y$ inverts $P_{0} \subseteq C_{0} \cap J$ and inverts $t(\bmod C)$, it follows directly that $t$ centralizes $P_{0}$.

Since $t \notin P_{0}$, the group $P=\left\langle P_{0}, t\right\rangle$ is abelian of order at least $3^{h+1}$. On the other hand, formula (4) of $\S 6$ shows that $3^{h+1}$ is the highest power of 3 dividing $|G|$. Hence $|P|=3^{h+1}$ and $P$ is a Sylow 3-subgroup of $G$. Either $P$ is cyclic of order $3^{h+1}$ or it is of type $\left(3^{h}, 3\right)$.

All fusion in the abelian Sylow group $P$ takes place in $N(P)$. Since $u$ and $u^{y}=u^{-1}$ are conjugate in $G$, they are conjugate in $N(P)$ and $N(P)$ contains a 2-element $x_{0}$ which inverts $u$. Hence

$$
N(P) \supseteq\left\langle C(P), x_{0}\right\rangle \supset C(P) .
$$

If $P$ is cyclic, $N(P) / C(P)$ is isomorphic to a subgroup of the multiplicative group of coprime residue classes of integers $\bmod 3^{h+1}$. Since $|N(P) / C(P)|$ is prime to 3 , this order must be 2 and $x_{0}$ inverts $P$. Furthermore, $|C(P)|$ must be odd; otherwise $P$ would centralize an involution and hence a conjugate of $P$ would be contained in $N$. However, under our present assumptions, $P_{0}$ is a Sylow 3-subgroup of $N$ and, as $\left|P_{0}\right|<|P|$, we would reach a contradiction. Thus $|C(P)|$ is odd. Since $x_{0}^{2}$ centralizes $P$, it follows that $x_{0}^{2}$ is odd and hence that $x_{0}$ is an involution.

We shall show that the same conclusions hold if $P$ is of type $\left(3^{h}, 3\right)$. We let $N(P)$ act by conjugation on $P$ and obtain a faithful representation of $N(P) / C(P)$ by automorphisms of $P$. Since $N(P) / C(P)$ has order prime to 3 and since $h>1$ by assumption, it follows that this representation is decomposable (cf. [19, Theorem 5.2.2]). Hence there exists a basis $\left\{w_{1}, w_{2}\right\}$ of $P$, say with $w_{1}$ of order $3^{h}$ and $w_{2}$ of order 3 such that every element of $N(P)$ maps $w_{i}$ on a power of $w_{i}(i=1,2)$. In particular, this implies that $w_{2}$ is not conjugate in $G$ to a power of $w_{1}$. 
Furthermore, we see in this case that $N(P) / C(P)$ is an elementary abelian 2-group of order 2 or 4 . However, if this order were 4 , it would follow that $\left|C\left(w_{2}\right)\right|$ is even. But then $w_{2}$ would be conjugate to an element of $N$. Since $P_{0}$ is a Sylow 3-subgroup of $N$ and $\Omega_{1}\left(P_{0}\right)=\left\langle w_{1}^{3^{n-1}}\right\rangle, w_{2}$ would thus be conjugate to a power of $w_{1}$, which we have just shown not to be the case. Therefore $|N(P) / C(P)|=2$ and we conclude that

$$
N(P)=\left\langle C(P), x_{0}\right\rangle,
$$

where $x_{0}$ is a 2-element which inverts $P$. We have just noted that $\left|C\left(w_{2}\right)\right|$ is odd. Since $x_{0}^{2}$ centralizes $w_{2}, x_{0}^{2}$ has odd order and so $x_{0}$ is an involution.

Now the results of [6, III, $\S I X]$ can be applied and, as in the proof of Proposition 1 , we obtain a contradiction. This establishes the lemma.

As before, let $p_{0}$ denote an odd prime dividing $q+1$ and let $P_{0}$ denote a Sylow $p_{0}$-subgroup of $W$. Then $P_{0}$ is a cyclic group of order $\left|P_{0}\right|=p_{0}^{h}$, where $p_{0}^{h}$ is the highest power of $p_{0}$ dividing $q+1$. Let $P_{1}$ denote a Sylow $p_{0}$-subgroup of $A$. Then $\left|P_{1}\right|=p_{0}^{k}$ is the highest power of $p_{0}$ dividing $a$. Then $P_{1}$, too, is a cyclic group. We shall assume that $P_{1} \neq 1$. As we have shown, this is so if $p_{0} \neq 3$ and if $p_{0}=3$ and $h \geqq 2$. Since $a \mid q+1$, we have $h \geqq k$. We set $P_{0}=\left\langle u_{0}\right\rangle, P_{1}=\left\langle u_{1}\right\rangle$ and $P=P_{1} \times P_{2}$.

If $\eta$ is an automorphism of $P$, we have formulas

$$
\eta: u_{0} \rightarrow u_{0}^{\alpha} u_{1}^{\beta}, \quad u_{1} \rightarrow u_{0}^{\gamma} u_{1}^{\delta},
$$

where $\alpha, \beta, \gamma, \delta$ are integers with $\alpha$ and $\gamma$ to be taken modulo $p_{0}^{h}$ and with $\beta$ and $\delta$ to be taken modulo $p_{0}^{k}$. We then have a representation of the automorphism group of $P$ by matrices

$$
\left(\begin{array}{ll}
\alpha & \beta \\
\gamma & \delta
\end{array}\right) ; \quad\left(\alpha, \gamma \bmod p_{0}^{h} ; \beta, \delta \bmod p_{0}^{k}\right)
$$

of this type. Since automorphisms of $N(P)$ map $u_{1}$ on elements of order $p_{0}^{k}$, we see that $\gamma$ is divisible by $p_{0}^{h-k}$, say,

$$
\gamma=p_{0}^{h-k} \gamma_{0}
$$

In particular, if we let $N(P)$ act on $P$ by conjugation, we obtain a representation $R$ of $N(P)$ with the kernel $C(P)$. We prove

LEMMA 1. The elements $y$ and $t$ belong to $N(P)$. We have

$$
R(y)=\left(\begin{array}{rr}
-1 & 0 \\
0 & 1
\end{array}\right)
$$

Set

$$
R(t)=\left(\begin{array}{ll}
\alpha & \beta \\
\gamma & \delta
\end{array}\right) .
$$

If $p_{0} \neq 3$, we must have $h=k$ and

$$
2 \alpha+1 \equiv 0, \quad \alpha \equiv \delta, \quad 4 \beta \gamma \equiv-3 \quad\left(\bmod p_{0}^{h}\right) .
$$


If $p_{0}=3$ and if $h \neq k+1$, then $k=1, h \geqq 3$, and

$$
\alpha=1+3^{h-1} \alpha_{0}, \quad \gamma=3^{h-1} \gamma_{0}, \quad \alpha_{0}+\beta \gamma_{0} \equiv 0, \quad \alpha_{0} \not \equiv 0 \quad(\bmod 3),
$$

where $\alpha_{0}$ is an integer.

Proof. Since $y$ and $t$ normalize $C_{0}=W_{0} \times A$ (cf. $\S 1$ ), they normalize the unique Sylow $p_{0}$-subgroup $P=P_{0} \times P_{1}$ of $C_{0}$. Hence $y, t \in N(P)$. The formula (6) is immediate as $y$ inverts $W$ and centralizes $A$. Define $\alpha, \beta, \gamma, \delta$ by (4). Since $t^{2} \equiv t^{-1}$ $(\bmod C)$ then

$$
R\left(t^{-1}\right)=R\left(t^{2}\right)=\left(\begin{array}{ll}
\alpha^{2}+\beta \gamma, & (\alpha+\delta) \beta \\
(\alpha+\delta) \gamma, & \delta^{2}+\beta \gamma
\end{array}\right) .
$$

Also, $t y \equiv y t^{-1}(\bmod C)$ and hence

$$
R(t y)=\left(\begin{array}{ll}
-\alpha & \beta \\
-\gamma & \delta
\end{array}\right)=R\left(y t^{-1}\right)=\left(\begin{array}{cc}
-\left(\alpha^{2}+\beta \gamma\right), & -(\alpha+\delta) \beta \\
(\alpha+\delta) \gamma, & \delta^{2}+\beta \gamma
\end{array}\right) .
$$

Thus,

$$
\begin{aligned}
& \alpha^{2}+\beta \gamma \equiv \alpha, \quad(\alpha+\delta) \gamma \equiv-\gamma \quad\left(\bmod p_{0}^{h}\right), \\
& (\alpha+\delta) \beta \equiv-\beta, \quad \delta^{2}+\beta \gamma \equiv \delta \quad\left(\bmod p_{0}^{k}\right) \text {. }
\end{aligned}
$$

Now $R\left(t^{-1}\right)$ can be written in the form

$$
R\left(t^{-1}\right)=\left(\begin{array}{rr}
\alpha & -\beta \\
-\gamma & \delta
\end{array}\right)
$$

On multiplying with $R(t)$, we find

$$
\begin{aligned}
& \alpha^{2}-\beta \gamma \equiv 1, \quad(\alpha-\delta) \gamma \equiv 0 \quad\left(\bmod p_{0}^{h}\right), \\
& (\alpha-\delta) \beta \equiv 0, \quad \delta^{2}-\beta \gamma \equiv 1 \quad\left(\bmod p_{0}^{k}\right) \text {. }
\end{aligned}
$$

Before discussing these congruences, we observe that in (5), $\gamma_{0} \not \equiv 0\left(\bmod p_{0}\right)$. Indeed, suppose that $\gamma_{0} \equiv 0\left(\bmod p_{0}\right)$. The definition of $R(t)$ shows that

$$
u_{1}^{t}=u_{0}^{\gamma} u_{1}^{\delta}
$$

Raising this to the exponent $p_{0}^{k-1}$ and using (5) and $\gamma_{0} \equiv 0\left(\bmod p_{0}\right)$, we see that if $u^{*}$ is the $p_{0}^{k-1}$ st power of $u_{1}$, we have

$$
\left(u^{*}\right)^{t}=\left(u^{*}\right)^{\delta} .
$$

Now $u^{*}$ is an element different from 1 of $P_{1} \subseteq A$. Then $C\left(u^{*}\right)$ has the Sylow 2-group $S$ and since $u^{*}$ and $\left(u^{*}\right)^{\delta}$ are conjugate in $G$, they are conjugate in $N(S)=S C(S)$ $=S \times E=S \times A$. (Here we have used Lemma 2.1.1 and Proposition 1.1 to determine the structure of $N(S)$.) Hence $\left(u^{*}\right)^{\delta}=u^{*}$ i.e. $t$ centralizes $u^{*}$. But then by Theorem $\mathrm{B}, t \in N$. This is not true as $x^{t}=x z$. Thus, $\gamma_{0} \not \equiv 0\left(\bmod p_{0}\right)$.

As a second preliminary remark, we shall show that if $\delta \equiv 1\left(\bmod p_{0}\right)$, we have $\beta \not \equiv 0\left(\bmod p_{0}\right)$. In order to do this, we form a linear character $\theta$ of $E=A$ whose 
value for a generator of $A$ is a primitive $p_{0}$ th root of unity. Then $\theta$ defines a 2-block $B_{\theta}$ of $G$ of full defect, cf. Chapter III, $\S 5$. Since $N=T J \times A$ by Lemma 1.1 as $B=1$, $\theta$ can be extended to a character $\theta_{N}$ of $N$ which is trivial on $T J$ and which then is trivial on $S \subseteq T J$. Thus $B_{\theta}$ satisfies the condition $\left(^{*}\right)$ of Chapter 3 , $\S$. Since $\theta \neq 1$, Proposition 3.7.6 shows that $B_{\theta}$ has a minor subsection $(x, b)$. Now Proposition 3.6.2 shows that $t$ does not stabilize $\theta_{C}$. Here, $\theta_{C}$ is trivial on $P_{0}$ and if we set $A=P_{1} \times A^{*}, \theta_{C}$ is trivial on the group $A^{*}$ of order prime to $p_{0}$. On the other hand, $\theta_{C}\left(u_{1}\right)$ is a primitive $p_{0}$ th root of unity. Now

$$
\theta_{C}\left(t^{-1} u_{0} t\right)=\theta_{C}\left(u_{0}^{\alpha} u_{1}^{\beta}\right)=\theta_{C}\left(u_{1}\right)^{\beta}, \quad \theta_{C}\left(t^{-1} u_{1} t\right)=\theta_{C}\left(u_{0}^{\gamma} u_{1}^{\delta}\right)=\theta_{C}\left(u_{1}\right)^{\delta} .
$$

If we had $\beta \equiv 0\left(\bmod p_{0}\right), \delta \equiv 1\left(\bmod p_{0}\right)$, we would have $\left(\theta_{C} \mid P\right)^{t-1}=\theta_{C} \mid P$ and as $\theta_{C}^{t}$ and $\theta_{C}$ are trivial on the set of elements of $C$ of order prime to $p_{0}$, then $\theta_{C}^{t-1}=\theta_{C}$, a contradiction. Hence if $\delta \equiv 1\left(\bmod p_{0}\right)$, we must have $\beta \equiv 0\left(\bmod p_{0}\right)$.

We now discuss the congruences (9) and (10). They imply that

$$
\begin{aligned}
& (2 \alpha+1)(\alpha-1)=2 \alpha^{2}-\alpha-1 \equiv 0 \quad\left(\bmod p_{0}^{h}\right), \\
& (2 \delta+1)(\delta-1)=2 \delta^{2}-\delta-1 \equiv 0 \quad\left(\bmod p_{0}^{k}\right) .
\end{aligned}
$$

Moreover, we have $\alpha^{2} \equiv 1+\beta \gamma \equiv \delta^{2}\left(\bmod p_{0}^{k}\right)$, i.e. $\alpha \equiv \pm \delta\left(\bmod p_{0}^{k}\right)$.

Suppose first that $p_{0} \neq 3$. Since the greatest common divisor of $2 \alpha+1$ and $\alpha-1$ divides 3 , we have either $\alpha \equiv 1\left(\bmod p_{0}^{h}\right)$ or $2 \alpha \equiv-1\left(\bmod p_{0}^{h}\right)$.

If $\alpha \equiv 1\left(\bmod p_{0}^{h}\right)$, (9) implies $\beta \gamma \equiv 0\left(\bmod p_{0}^{h}\right)$. On account of $(5)$, then $\beta \gamma_{0} \equiv 0$ $\left(\bmod p_{0}^{k}\right)$. This is impossible since $k>0$ by Proposition 1 and since we have shown that $\beta \not \equiv 0\left(\bmod p_{0}\right), \gamma_{0} \not \equiv 0\left(\bmod p_{0}\right) .\left(\right.$ We here have $\delta \equiv 1\left(\bmod p_{0}\right)$ )

Thus, $2 \alpha+1 \equiv 0\left(\bmod p_{0}^{h}\right)$ or, if we use fractions $\left(\bmod p_{0}^{h}\right), \alpha \equiv-\frac{1}{2}\left(\bmod p_{0}^{h}\right)$. Then $\delta \equiv \pm \alpha \equiv \mp \frac{1}{2}\left(\bmod p_{0}^{k}\right)$. Now (11) shows that we have $\delta \equiv-\frac{1}{2}\left(\bmod p_{0}^{k}\right)$. By (9),

$$
\beta \gamma \equiv \alpha-\alpha^{2} \equiv-\frac{3}{4} \quad\left(\bmod p_{0}^{h}\right) .
$$

It follows from (5) that $h=k$. This shows that the lemma holds for $p_{0} \neq 3$.

Assume now that $p_{0}=3$. If $h \geqq 2$, Proposition 2 shows that $k \geqq 1$. For $h=1$, we still have $k>0$ since we assume $h \neq k+1$. It now follows from (11) that $\alpha \equiv 1$ $(\bmod 3), \delta \equiv 1(\bmod 3)$.

If $h=1$, we must have $k=1$. It follows from (9) and (5) that

$$
\beta \gamma_{0}=\beta \gamma \equiv 0 \quad(\bmod 3) .
$$

This is impossible since, as was shown, $\beta \not \equiv 0, \gamma_{0} \not \equiv 0(\bmod 3)$.

Suppose then $h \geqq 2$. Since $\alpha \equiv 1(\bmod 3), \alpha-1$ and $2 \alpha+1$ have the greatest common divisor 3 . Now $(11)$ shows that $\alpha \equiv 1\left(\bmod 3^{h-1}\right)$ or that $\alpha \equiv-\frac{1}{2}\left(\bmod 3^{h-1}\right)$.

Suppose first that $\alpha \equiv-\frac{1}{2}\left(\bmod 3^{h-1}\right)$. By (9) and (5)

$$
3^{h-k} \beta \gamma_{0}=\beta \gamma \equiv-\frac{1}{2}-\frac{1}{4}=-\frac{3}{4}\left(\bmod 3^{h-1}\right) .
$$


If $h>2$, then $h-k=1$, which was excluded. If $h=2$, we see that $h \neq k$ and then $k=1$, i.e. we would still have $h-k=1$.

Thus, our assumption $h-k \neq 1$ implies that $\alpha \equiv 1\left(\bmod 3^{h-1}\right)$. Set $\alpha=1+\alpha_{0} 3^{h-1}$. Then by (9) and (5)

$$
3^{h-k} \beta \gamma_{0} \equiv \alpha-\alpha^{2} \equiv-\alpha_{0} 3^{h-1} \quad\left(\bmod 3^{h}\right)
$$

This implies $k=1$ and $\beta \gamma_{0} \equiv-\alpha_{0}(\bmod 3)$. Since $\beta \not \equiv 0, \gamma_{0} \not \equiv 0(\bmod 3)$, we have the situation described in Lemma 1 and the proof is complete.

As an immediate consequence of the lemma, we prove

Proposition 3. If $q \not \equiv-1(\bmod 3)$, we have $d=1$. If $q+1$ contains 3 with the exact exponent $h>0$, we have either $d=3$ or $d=3^{h-1}$.

Proof. We know that $d$ has the value $(q+1) / 2 a$. It follows from Lemma 1 that for any odd prime $p_{0}$ dividing $q+1$, the exponent $h$ of $p_{0}$ in $q+1$ and the exponent $k$ of $p_{0}$ in $a$ are equal. Hence $(q+1) / 2 a$ can only be divisible by the prime 3 . For $p_{0}=3$, we have either $h=k+1$ and then $d=3$ or else $k=1$, i.e. $d=3^{h-1}$.

In view of Proposition 3, either part (iv) of Theorem $\mathrm{C}$ holds or $q+1$ is divisible by 3 to the exact exponent $h>2$ and $d=3^{h-1}$. In the balance of the section, we shall show that this case cannot occur by proving

Proposition 4. If $q+1$ is divisible by 3 , then $d=3$.

We shall carry out the proof by contradiction in a sequence of lemmas. Thus we assume $h>2$ and $d=3^{h-1}$. Since $d=(q+1) / 2 a$, this implies that $a$ is divisible by 3 to the first power only. We preserve the notation $P_{0}, P_{1}, P=P_{0} \times P_{1}, u_{0}, u_{1}$. Under the present assumptions, $P_{0}$ is cyclic of order $3^{h}$ and $P_{1}$ has order $3=3^{k}$. By Lemma 1, $Q=\langle P, t\rangle$ has order $3^{h+2}$. Formula (6.4) for $|G|$ shows that $Q=$ $\left\langle P_{0} \times P_{1}, t\right\rangle$ is a Sylow 3-subgroup of $G$.

Furthermore, the proof of Lemma 1 shows that we have formulas

$$
t^{-1} u_{0} t=u_{0}^{\alpha} u_{1}^{\beta}, \quad t^{-1} u_{1} t=u_{0}^{\gamma} u_{1}^{\delta}
$$

where

$\gamma=3^{h-1} \gamma_{0}, \quad \alpha=1+3^{h-1} \alpha_{0}, \quad \delta \equiv 1 \quad(\bmod 3), \quad \alpha_{0}+\beta \gamma_{0} \equiv 0 \quad(\bmod 3)$, and

$$
\gamma_{0} \not \equiv 0 \quad(\bmod 3), \quad \alpha_{0} \not \equiv 0 \quad(\bmod 3), \quad \beta \not \equiv 0 \quad(\bmod 3) .
$$

Since $(\beta, 3)=1$, we can replace $u_{1}$ by $u_{1}^{\beta}$. Then $\beta$ is replaced by 1 . We can also choose $\delta=1$. For simplicity, we set

$$
l=3^{h-1} .
$$

Thus we have

$$
t^{-1} u_{0} t=u_{0}^{1+l \alpha_{0}} u_{1}, \quad t^{-1} u_{1} t=u_{0}^{-l \alpha_{0}} u_{1}
$$


The proof of Lemma 1 also shows that

$$
y^{-1} u_{0} y=u_{0}^{-1}, \quad y^{-1} u_{1} y=u_{1},
$$

and

$$
t u_{0} t^{-1}=u_{0}^{1+l \alpha} 0 u_{1}^{-1}, \quad t u_{1} t^{-1}=u_{0}^{l \alpha} 0 u_{1} .
$$

Note that formulas (13) and (15) remain the same when $t$ is replaced by an arbitrary element of $t P=t\left(P_{0} \times P_{1}\right)$.

LeMmA 2. We have $Q^{\prime}=\left\langle u_{0}^{l}, u_{1}\right\rangle=\Omega_{1}(P)$ and $Z(Q)=\left\langle u_{0}^{3}\right\rangle=\mho^{1}(P)$.

Proof. These assertions follow immediately from (12) and (13).

The next lemma is very close to Lemma 2.2.

LEMMA 3. If $w$ is a nonidentity element of $P$, then we have

(i) If $w \in P_{1}^{t^{v}}$ with $\nu=0,1$, or -1 , then $C(w)=N^{t^{v}}$.

(ii) If $w \in \mho^{1}(P)$, then $C(w)=\langle C, t\rangle$.

(iii) In all other cases, $C(w)=C$.

Proof. First of all, if $w \in P_{1}, C(w)=N$ as $G$ satisfies the conclusion of Theorem B and (i) follows at once.

We can therefore assume that $w \notin P_{1}^{t^{\nu}}$ for any $\nu$. We set $K=C(w)$, so that $C=$ $C_{0} T=(W \times A) T \subseteq K$. Let $R$ be a Sylow 2-subgroup of $K$ containing $T$ and suppose $R \supset T$. Since $R$ is dihedral or quasi-dihedral, $x_{i} \in Z(R)$ for some $i, 1 \leqq i \leqq 3$. Since $t$ cyclically permutes $x_{1}, x_{2}, x_{3}$, it follows that $R_{1}=R^{t} \subseteq N$ for some $j=0,1$, or -1 and that $R_{1}$ centralizes $w_{1}=w^{t \prime}$. But $R_{1} \supset T$ and $w_{1} \in P$. However, by the structure of $N=(J T) \times A$, we clearly have $C_{P}\left(R_{1}\right)=P_{1}$, whence $w_{1} \in P_{1}$ and so $w \in P_{1}^{t^{v}}, \nu=0,1,-1$, contrary to assumption. We conclude that $T$ is a Sylow 2-subgroup of $K$.

Since $w \notin P_{1}$, Lemma 2.4.2(i), applied to $N / O(N)=N / A$, implies that $N \cap K$ $=C_{N}(w)=C$. Since $w \notin P_{1}^{t}$ or $P_{1}^{t^{-1}}$, it follows similarly that $C\left(x_{i}\right) \cap K=C, 1 \leqq i \leqq 3$. In particular, since $O(K)$ is $T$-invariant, it follows from the $T$-decomposition of $O(K)$ that $O(K) \subseteq C_{0}$.

Suppose first that $K$ has a normal 2-complement, whence $K=C_{0} T=C$ and $i \notin K$. Moreover, $w \notin\left\langle u_{0}^{3}\right\rangle=\mho^{1}(P)$. Indeed, $y$ inverts $t(\bmod C), y$ inverts $P_{0}$, and $t$ normalizes $\mho^{1}(P)=\mho^{1}\left(P_{0}\right)$, which forces $t$ to centralize $\mho^{1}\left(P_{0}\right)$. Since $t \notin K$, the assertion follows and so (iii) holds in this case.

Assume then that $K$ does not have a normal 2-complement, in which case $N_{K}(T) \supset C_{K}(T)=C$ and so $t \in K$. Equation (13) now implies that $w \in\left\langle u_{0}^{3}\right\rangle=\mho^{1}(P)$. Thus it remains only to show that (ii) holds in this case. We set $\bar{K}=K / O(K)$ and apply the main theorem of [22] to obtain that $\bar{K}$ contains a normal subgroup $\bar{L}^{*}$ of odd index isomorphic to $\operatorname{PSL}\left(2, q^{\prime}\right), q^{\prime}$ odd. Since $C_{K}(x)=K \cap N=C$, as we have shown above, $C_{L^{*}}(\bar{x}) \subseteq \bar{C}$. However, $C_{L^{*}}(\bar{x})$ is dihedral of order $q^{\prime}-\varepsilon^{\prime}$, where $q^{\prime} \equiv \varepsilon^{\prime}(\bmod 4)$ and $\varepsilon^{\prime}= \pm 1$ by Lemma $2.5 .7(\mathrm{i})$ and consequently $C_{L^{*}}(\bar{x})=\bar{T}$, 
whence $q^{\prime}-\varepsilon^{\prime}=4$. Thus $q^{\prime}=3$ or 5 and it follows in either case that $\bar{K}=\bar{L}^{*}$ and that $\bar{C}_{0}=1$. In particular, $C_{0}=O(K)$.

If $q^{\prime}=3$, then $K=\left\langle C_{0}, T, t\right\rangle=\langle C, t\rangle$ and (ii) holds. To complete the proof, we show that the case $q^{\prime}=5$ cannot occur. Indeed, since $C_{K}\left(C_{0}\right)=C_{K}(O(K))$ is normal in $K$ and contains $T$, the assumption $q^{\prime}=5$ would force $C_{K}\left(C_{0}\right)=K$ inasmuch as $K / O(K)$ is simple. But then $t$ would centralize $C_{0}$ and so would also centralize $P$, contrary to equation (13). Thus all parts of the lemma hold.

LEMMA 4. We have $C_{C}(t) \subseteq Q$.

Proof. If false, $t$ centralizes an element $u^{*}$ of $C_{0}$ of prime order $p^{*} \neq 3$. The Sylow $p^{*}$-subgroup $P^{*}$ of $C_{0}$ is the direct product of the Sylow $p^{*}$-subgroups $P_{0}^{*}$ of $W$ and $P_{1}^{*}$ of $A$, which by Proposition 3 are cyclic of the same order. We claim that $t$ centralizes $R^{*}=\Omega_{1}\left(P^{*}\right)$. Indeed, $t$ centralizes $u^{*} \in R^{*}$; so either this is true or $C_{R^{*}}(t)=\left\langle u^{*}\right\rangle$. However, as $y$ inverts $t(\bmod C), C_{R^{*}}(t)$ is $y$-invariant. Since $R^{*} \mid\left\langle u^{*}\right\rangle$ has order $p^{*}$ and since $y$ inverts $t(\bmod C)$, it would follow in the latter case that $t$ centralizes $R^{*} \mid\left\langle u^{*}\right\rangle$. But then as $t$ is a 3-element and $p^{*} \neq 3$, this would imply that $t$ centralizes $R^{*}$. We thus conclude that $t$ centralizes $R^{*}$ and hence centralizes $R_{1}^{*}=\Omega_{1}\left(P_{1}^{*}\right)$. Since $R_{1}^{*}$ is normal in $N$ and $G=\langle N, t\rangle$ by Proposition 3.7.8, $R_{1}^{*}$ is normal in $G$, contrary to the simplicity of $G$.

LEMMA 5. We have

$$
\begin{array}{ll}
C(P)=C, & N(P)=N(T)=\langle C, y, t\rangle, \\
C(Q)=Z(Q), & N(Q)=\left\langle Q, z^{*}\right\rangle,
\end{array}
$$

where $z^{*}$ is an involution of $C y$ which inverts $P_{0}$ and centralizes $P_{1}$.

Proof. Since $C=C_{0} T$ and $C_{0}$ is abelian, $C$ centralizes $P$. Since by Lemma 2 (iii), $C\left(u_{0}\right)=C$, we find $C(P)=C$. An element $w$ of $N(P)$ normalizes $C(P)$ and hence the unique Sylow 2-subgroup $T$ of $C(P)=C$. Thus,

$$
w \in N(T)=\langle C, y, t\rangle .
$$

On the other hand, every element of $N(T)$ normalizes $C=C(T)$ and hence the unique Sylow 3-subgroup $P$ of $C$. Thus, $N(P)=N(T)$.

We have $Q=\langle P, t\rangle$ and so

$$
C(Q)=C(P) \cap C(t)=C \cap C(t)=C_{C}(t) .
$$

However, by the preceding lemma, $C_{C}(t) \subseteq Q$. Thus, $C(Q)=Z(Q)$.

The subgroup $C Q$ is normal of index 2 in $N(T)=\langle C, y, t\rangle$ and so by the Frattini argument $N_{N(T)}(Q)$ contains an involution $z^{*}$. Since $t$ cyclically permutes the involutions of $T$, clearly no involution of $C$ normalizes $Q$, so $z^{*} \notin C$. Replacing $Q$ (equivalently $t$ ) by a suitable conjugate, we can assume without loss that $z^{*} \in C D-C$ $=C y$. Thus, $z^{*}$ inverts $P_{0}$ and centralizes $P_{1}$. 
We have $\left\langle Q, z^{*}\right\rangle \subseteq N_{N(T)}(Q)$, and we argue next that equality holds. Indeed, either this is the case or there exists an element $u^{*}$ of $C$ of prime order $p^{*} \neq 3$ which normalizes $Q$. But $t$ normalizes the unique Sylow $p^{*}$-subgroup $P^{*}$ of $C$ and consequently $\left[t, u^{*}\right] \in Q \cap P^{*}=1$. Thus $t$ centralizes $u^{*}$ and so $u^{*} \in C_{C}(t)$, contrary to Lemma 4 . Hence

$$
N_{N(T)}(Q)=\left\langle Q, z^{*}\right\rangle \text {. }
$$

Thus to complete the proof of the lemma, we need only show that $N(Q) \subseteq N(T)$, for then $N(Q)=\left\langle Q, z^{*}\right\rangle$ will follow from (16). Let $w$ be a $3^{\prime}$-element of $N(Q)$. Then $w$ normalizes $Z(Q)$ and, by Lemma $2, Z(Q)=\left\langle u_{0}^{3}\right\rangle=\mho^{1}(P)$. But $Z(Q)$ is a cyclic 3-group inverted by $z^{*}$. Hence $w$ either centralizes or inverts $\mho^{1}(P)$; and correspondingly $w$ or $w z^{*}$ centralizes $\mho^{1}(P)$. However, by Lemma 3 (ii), $C\left(\mho^{1}(P)\right)$ $=\langle C, t\rangle \subseteq N(T)$. Since $z^{*} \in N(T)$, it follows in either case that $w \in N(T)$. Thus, $N(T)$ contains every $3^{\prime}$-element of $N(Q)$. Since $Q$ is a Sylow 3-subgroup of $G$, this yields the desired conclusion $N(Q) \subseteq N(T)$.

LeMma 6. If $v \in Q-P$, then $v$ is not conjugate in $G$ to an element of $P$ and, in addition, $C(v)$ has odd order.

Proof. Assume to the contrary that there exists $r \in G$ for which $v^{r}=u$ with $u \in P$. Then $r$ can be chosen so that it transforms a given Sylow 3-subgroup $R^{(1)}$ of $C(v)$ into a given Sylow 3-subgroup $R^{(2)}$ of $C(u)$. Lemma 3 shows that we may take $R^{(2)}$ either as $P$ or as $Q$. Choose $R^{(1)}$ so that it contains the 3-subgroup $\langle v, Z(Q)\rangle$ of $C(v)$. Then $Z(Q)^{r} \subseteq Q$ and, in particular, $\left(u_{0}^{3}\right)^{r} \in Q$. This implies that $\left(u_{0}^{9}\right)^{r} \in P$ and, as $l=3^{h-1} \geqq 9,\left(u_{0}^{l}\right)^{r}$ is an element of order 3 in $P$. Thus,

$$
\left(u_{0}^{l}\right)^{r}=u_{0}^{i l} u_{1}^{j} \neq 1 \quad(i, j=0,1, \text { or }-1) .
$$

By Lemma 3, $u_{0}^{l}$ is not conjugate to $u_{1}^{ \pm 1}$ in $G$, hence $i \neq 0$. Furthermore, by (13), the element $t$ or $t^{-1}$ conjugates $u_{0}^{i l} u_{1}^{j}$ with $j= \pm 1$ into $u_{0}^{ \pm l}$. Since $t \in Q$, we can replace $r$ by $r t$ or $r t^{-1}$, if necessary, and so can assume without loss that

$$
\left(u_{0}^{l}\right)^{r}=u_{0}^{ \pm l} \text {. }
$$

Hence either $r$ or $r y$ belongs to $C\left(u_{0}^{l}\right)=\langle C, t\rangle$. In both cases, $r$ normalizes $P$. We conclude that $v^{r}=u \in P$ implies $v \in P$, a contradiction. This proves the first statement of the lemma.

Finally, if $|C(v)|$ were even, $v$ would commute with an involution. Then a conjugate of $v$ lies in $N$ and hence some conjugate of $v$ lies in $P$, as $P$ is a Sylow 3-subgroup of $N$, which has been excluded.

LEMMA 7. The element $t$ can be chosen of order 3. If this is done and if we set $\alpha^{*}=1+2 \cdot 3^{n-2} \alpha_{0}$, then

$$
\left(t u_{0}^{i} u_{1}^{j}\right)^{3}=u_{0}^{3 i \alpha^{*}}
$$

for all integral $i, j$. 
Proof. In any case, $t^{3} \in P \cap C(t)=Z(Q)=\left\langle u_{0}^{3}\right\rangle$. We have

$$
\left(t u_{0}^{i} u_{1}^{j}\right)^{3}=t^{2} \cdot t^{-1} u_{0}^{i} u_{1}^{j} t u_{0}^{i} u_{1}^{j} t u_{0}^{i} u_{1}^{j} t^{-1} \cdot t .
$$

Now (13) and (15) yield

$$
\begin{aligned}
\left(t u_{0}^{i} u_{1}^{j}\right)^{3} & =t^{2} u_{0}^{i\left(1+l \alpha \alpha_{0}\right)} u_{1}^{i} u_{0}^{i} u_{1}^{j} u_{0}^{i\left(1+l \alpha_{0}\right)} u_{1}^{-j} \\
& =t^{2} u_{0}^{3 i+2 l l \alpha_{0}} t=t^{2} u_{0}^{3 i \alpha^{*}} t
\end{aligned}
$$

Since $u_{0}^{3} \in Z(Q)$, we obtain

$$
\left(t u_{0}^{i} u_{1}^{j}\right)^{3}=t^{3} u_{0}^{3 i \alpha^{*}}
$$

As $\left(3, \alpha^{*}\right)=1$ and $t^{3} \in\left\langle u_{0}^{3}\right\rangle$, we may choose $i$ such that $u_{0}^{3 i \alpha^{*}}$ is the inverse of $t^{3}$. Since we can replace $t$ by any element of the coset $t P$, we may assume $t^{3}=1$. Then, for arbitrary $i$ and $j$, (17) follows from (18).

LEMMA 8. If $v \in Q-P$, then $C(v)$ has the Sylow 3-subgroup $\langle v, Z(Q)\rangle$ of order $3^{h}$.

Proof. There exists a Sylow 3-subgroup $P^{*}$ of $C(v)$ which contains $\langle v, Z(Q)\rangle$. Since $v^{3} \in P \cap C(v)=Z(Q)$, we have $|\langle v, Z(Q)\rangle|=3 \cdot 3^{h-1}=3^{h}$. Suppose we have $\left|P^{*}\right|>3^{h}$. There exists $r \in G$ for which $\left(P^{*}\right)^{r} \subseteq Q$. Because of our assumption on $\left|P^{*}\right|$, we have $\left|\left(P^{*}\right)^{r} \cap P\right|>3^{h-1}$, and consequently there exists an element $w \in\left(P^{*}\right)^{r}$ with $w \in P-Z(Q)$. Since $v^{r} \in C(w)$, Lemma 3 implies that $v^{r}$ is conjugate to an element of $P$. However, this is inconsistent with Lemma 6. Hence $P^{*}=$ $\langle v, Z(Q)\rangle$ is a Sylow 3-subgroup of $C(v)$, as we wished to show.

LEMMA 9. Any two elements of $Q-P$ that are conjugate in $G$ are conjugate in $N(Q)=\left\langle Q, z^{*}\right\rangle$.

Proof. Let $v_{1}, v_{2}$ be two elements of $Q-P$ that are conjugate in $G$. By Lemma 8, $C\left(v_{i}\right)$ has the Sylow 3-subgroup $\left\langle v_{i}, Z(Q)\right\rangle,(i=1,2)$. Hence there exists $r \in G$ such that $v_{1}^{r}=v_{2}$ and $\left\langle v_{1}, Z(Q)\right\rangle^{r}=\left\langle v_{2}, Z(Q)\right\rangle$. In particular, $\left(u_{0}^{l}\right)^{r} \in\left\langle v_{2}, Z(Q)\right\rangle$. Now Lemma 6 implies that $\left(u_{0}^{l}\right)^{r} \in Z(Q)$, whence $\left(u_{0}^{l}\right)^{r}=u_{0}^{ \pm l}$. Since $C\left(u_{0}^{l}\right)=\langle C, t\rangle$ by Lemma 3 and $y$ inverts $u_{0}^{l}$, it follows that $r \in\langle C, t, y\rangle=N(T)$.

Now let $H$ denote the normal 3-complement of the abelian group $C$. Then $C=P \times H$ and $H$ is normal in $N(T)$. Since $N(T)=\langle y\rangle Q H=\left\langle z^{*}, Q\right\rangle H$, we can set $r=r_{1} r_{2}$ with $r_{1} \in\left\langle Q, z^{*}\right\rangle$ and $r_{2} \in H$. Then $r^{-1} v_{1} r=v_{2}$ implies $r_{1}^{-1} v_{1} r_{1} \equiv v_{2}$ $(\bmod H)$. But $r_{1}^{-1} v_{1} r_{1} \in Q, v_{2} \in Q$, and $Q \cap H=1$. Hence $r_{1}^{-1} v_{1} r_{1}=v_{2}$. Since $r_{1} \in\left\langle Q, z^{*}\right\rangle$ and $\left\langle Q, z^{*}\right\rangle=N(Q)$ by Lemma 5 , the lemma is proved.

As our final lemma, we have

LEMMA 10. If $v$ is an element of $Q-P$, then $C(v)$ has a normal 3-complement.

Proof. By the focal subgroup theorem, it suffices to show that if $v_{1}, v_{2}$ are two distinct elements of the Sylow 3-subgroup $P^{*}=\langle v, Z(Q)\rangle$ of $C(v)$, then $v_{1}$ and $v_{2}$ are not conjugate in $C(v)$. Suppose to the contrary that $r^{-1} v_{1} r=v_{2}$ with $r \in C(v)$. 
Since $P^{*}$ is abelian, we may asume that $r$ normalizes $P^{*}$. As in the proof of the preceding lemma, it follows that $r \in N(T)$. If $H$ has the same significance as in that lemma and if we set $r=r_{1} r_{2}$ with $r_{1} \in\left\langle Q, z^{*}\right\rangle, r_{2} \in H$, we again have $r_{1}^{-1} v_{1} r_{1}$ $\equiv v_{2}(\bmod H)$ and also $r_{1}^{-1} v r_{1} \equiv v(\bmod Q)$.

Since $Q \cap H=1$, we conclude as before that $r_{1} v_{1} r_{1}=v_{2}$ and $r_{1}^{-1} v r_{1}=v$. By Lemma 6, $C(v)$, and hence $r_{1}$, has odd order. Since $r_{1} \in\left\langle Q, z^{*}\right\rangle$, this implies that $r_{1} \in Q$. But $Q=\langle P, v\rangle$, and so the intersection of $C(v)$ with $Q$ is $\langle Z(Q), v\rangle=P^{*}$. Thus, $r \in P^{*}$. Since $v_{1}$ and $v_{2}$ also lie in $P^{*}$ and $P^{*}$ is abelian, we have $v_{1}=v_{2}$, a contradiction.

We are now ready to prove Proposition 4. If $v \in Q-P$, it follows from Lemma 8 that the conjugacy class of $v$ in $Q$ consists of $3^{h+2} / 3^{h}=9$ elements. Since $\left|Q^{\prime}\right|=9$ by Lemma 2 , the conjugacy class of $v$ in $Q$ is $v Q^{\prime}$. Now Lemma 9 shows that the intersection of the conjugacy class $\Gamma(v)$ of $v$ in $G$ with $Q$ is equal to

$$
\Gamma(v) \cap Q=v Q^{\prime} \cup v^{z^{*}} Q^{\prime} .
$$

We next note that $z^{*-1} t z^{*}$ is an element of order 3 in $Q-P$. It follows from formula (17) of Lemma 7 that

$$
z^{*-1} t z^{*} \in t Q^{\prime} \cup t^{-1} Q^{\prime}
$$

If we had $z^{*-1} t z^{*} \in t Q^{\prime}$, then $z^{*-1} t Q^{\prime} z^{*}=t Q^{\prime}$ and the involution $z^{*}$ would centralize one of the nine elements of $t Q^{\prime}$. This is contrary to Lemma 6 . Hence $z^{*-1} t z^{*} \in t^{-1} Q^{\prime}$ and then $z^{*-1} t Q^{\prime} z^{*}=t^{-1} Q^{\prime}$. It is now clear that one of the conjugate classes of $Q$ in (19) belongs to $t P$ and the other one to $t^{-1} P$.

We now work with columns belonging to the principal 3-block $B_{0}(3, G)$. In particular, let $X(r)$ denote the column whose entry for $\chi \in B_{0}(3, G)$ is the value $\chi(r), r \in G$. In particular for $r=v \in Q-P$, it follows from [6, I, §III] and Lemma 10 that $X(v)$ is also the column of generalized decomposition numbers for the block $B_{0}(3, G)$ and the 3-section of $v$. With the product of two columns defined in the usual manner, we have

$$
(X(v), X(v))=3^{h},
$$

as the Sylow 3-subgroup of $C(v)$ has order $3^{h}$, (Lemma 8). If $v^{\prime} \in Q-P$ and if $v$ and $v^{\prime}$ are not conjugate in $G$,

$$
\left(X(v), X\left(v^{\prime}\right)\right)=0
$$

Finally,

$$
(X(1), X(v))=0 \text {. }
$$

Let $\lambda$ denote the linear character of $Q$ with the kernel $P$ for which $\lambda(t)$ is a primitive third root of unity $\rho$ and form the column

$$
R=3^{-(h+2)} \sum_{u \in Q} X(u)(1-\lambda(u)) .
$$


Since the entry of $R$ for the character $\chi \in B_{0}(3, G)$ is the inner product $(\chi \mid Q, 1-\lambda)$, the column $R$ is integral. Since $1-\lambda$ vanishes on $P$, it suffices to let $u$ range over $Q-P$. Clearly, $Q-P$ is the union of the sets (19) for $v=t u_{0}^{j}$ with $j=0,1,2, \ldots$, $3^{h-1}-1$ and this union is disjoint. If $u \in t u_{0}^{j} Q^{\prime}$, we have $X(u)=X\left(t u_{0}^{j}\right)$, and $1-\lambda(u)$ $=1-\rho$, while for $u \in\left(t u_{0}^{j}\right)^{z^{*}} Q^{\prime}$, we have $X(u)=X\left(t u_{0}^{j}\right), 1-\lambda(u)=1-\rho^{-1}$. As $\left|Q^{\prime}\right|=9$, we find

$$
R=3^{-(h+2)} \sum_{j=0}^{3^{h-1}-1} 9 X\left(t u_{0}^{j}\right)\left(1-\rho+1-\rho^{-1}\right)=3^{-h+1} \sum_{j=0}^{3^{n-1}-1} X\left(t u_{0}^{j}\right) .
$$

Since (19) shows that the $3^{h-1}$ elements $t u_{0}^{j}$ lie in $3^{h-1}$ distinct conjugate classes of $G$, it follows from (20) and (21) that

$$
(R, R)=3^{-2 h+2} \cdot 3^{h} \cdot 3^{h-1}=3 .
$$

Thus, $R$ has exactly three nonzero entries and each of them is \pm 1 . It is clear from (23) that the entry $R_{x_{0}}$ of $R$ for the principal character $\chi_{0}$ of $G$ is 1 . Moreover, if $v \in Q-P$ and if $r$ is a 3-regular element of $C(v)$, then by Lemma $6, v r$ has odd order and then

$$
1+\chi_{1}(v r)=\chi^{(1)}(v r)
$$

by Proposition 3.2.4; (the notation $\chi_{1}, \chi^{(1)}$ refers to $B_{0}(2, G)$ ). Here $\chi^{(1)}$ has degree $q^{3}+1=(q+1)\left(q^{2}-q+1\right)$, which is divisible by $3^{h+1}$, but not by $3^{h+2}$. Hence $\chi^{(1)}$ belongs to a 3-block of defect 1 . Now the main result of $\left[5\right.$, II] implies that $\chi^{(1)}(v r)$ $=0, \chi_{1}(v r)=-1$ and that $\chi_{1} \in B_{0}(3, G)$. In particular, $\chi_{1}(v)=-1$ for all $v \in Q-P$. Now (23) shows that the entry $R_{\chi_{1}}$ of $R$ for $\chi_{1}$ is -1 .

Suppose that the third character of $B_{0}(3, G)$ which gives a nonzero entry for $R$ is $\chi_{j}$. Since $\chi_{1}(1)=q^{3}$, then (22) reads

It follows that $\chi_{j}(1)=q^{3}-1$.

$$
1-q^{3} \pm \chi_{j}(1)=0
$$

Then $q^{3}-1=(q-1)\left(q^{2}+q+1\right)$ divides $|G|=2 a(q+1)(q-1) q^{3}\left(q^{2}-q+1\right)$.

However, $q^{2}+q+1$ is coprime to $q+1$, to the divisor $a$ of $q+1$, to $q$ and to $q^{2}-q+1$. The greatest common divisor of $q^{2}+q+1$ and $q-1$ divides 3. Since $q+1 \equiv 0(\bmod 3)$, we have $\left(q^{2}+q+1, q-1\right)=1$ and therefore $q^{2}+q+1$ is coprime to $|G|$. This is a contradiction and the proof of Proposition 4 is complete.

This completes the proof of part (iv) of Theorem $C$.

REMARK. In conclusion, we should like to mention what remains to be done to obtain the analogue of Theorem $\mathrm{C}$ for a simple $Q D$-group $G$ with wreathed Sylow 2-subgroup $S$. We have already pointed out in $\$ 2$ that parts (ii) and (iii) of the corresponding Theorem $\mathrm{C}$ will follow from the group order formulas for such a group $G$ provided $G$ satisfies the conclusions of Theorem B.

As for part (i) (i.e. $N=L$ and $E=A$ ), there are two reasonable approaches to this problem. The first method would be to proceed as in the quasi-dihedral case, which would require an analysis of certain 2-blocks of full defect, other than 
the principal 2-block, comparable to that carried out in $\$ 3.5,3.6$, and 3.7 above. A feasible alternative approach also exists. Since one will know at the outset that $B=1$ (part (ii) of the corresponding Theorem C), which was not true in the quasidihedral case, one can attempt to carry through the analogue of part (iv) of Theorem $\mathrm{C}$ in the case $q \equiv-1(\bmod 4)$ without assuming that $E=A$. If this can be done, the desired conclusion $E=A$ will then follow from the simplicity of $G$.

It should be pointed out that to complete the proof of the Third Main Theorem, the case $q \equiv 1(\bmod 4)$ must be shown to lead to the group $L_{3}(q)$. In the quasidihedral case, we used a result of Brauer [8] to achieve this. The analogous result for the wreathed case has at present been established only when $N$ is isomorphic to $G L(2, q)$ and remains to be completed when $N$ is isomorphic only to a homomorphic image of $G L(2, q)$. Furthermore, if the second approach to the proof that $E=A$ is to be taken, it will also be necessary to extend this analysis to the case $E \supset A$.

Added in proof. The necessary work in the wreathed case has now been completed. Thus the analogues of our three Main Theorems hold for groups with wreathed Sylow 2-subgroups. The proofs will appear in subsequent papers.

\section{BIBLIOGRAPHY}

1. J. L. Alperin, Sylow intersections and fusion, J. Algebra 6 (1967), 222-241. MR 35 \#6748.

2. H. Bender, Doubly transitive groups with no involution fixing two points (to appear).

3. - Finite groups having a strongly embedded subgroup (to appear).

4. R. Brauer, On groups whose order contains a prime number to the first power. I, II, Amer. J. Math. 64 (1942), 401-440. MR 4, 1; MR 4, 2.

5. —_ Zur Darstellungstheorie der Gruppen endlicher Ordnung. I, II, Math. Z. 63 (1956), 406-444; ibid., 72 (1959/60), 25-46. MR 17, 824; MR 21 \#7258.

6. - Some applications of the theory of blocks of characters of finite groups. I, II, III, IV, J. Algebra 1 (1964), 152-167, 307-334; ibid., 3 (1966), 225-255 (IV to appear). MR 29 \#5920; MR 30 \#4836; MR 34 \#2716.

7. - On blocks and sections in finite groups. I, II, Amer. J. Math. 89 (1967), 11151136; ibid., 90 (1968), 895-925. MR 36 \#2716.

8. — On finite Désarguesian planes. I, II, Math. Z. 90 (1965), 117-151; ibid., 91 (1966), 124-151. MR 33 \#1373; MR 33 \#1374.

9. R. Brauer and C. Nesbitt, On the modular characters of groups, Ann. of Math. (2) 42 (1941), 556-590. MR 2, 309.

10. R. Brauer and M. Suzuki, On finite groups of even order whose 2-Sylow group is a quaternion group, Proc. Nat. Acad. Sci. U.S.A. 45 (1959), 1757-1759. MR 22 \#731.

11. R. Brauer and H. F. Tuan, On simple groups of finite order. I, Bull. Amer. Math. Soc. 51 (1945), 756-766. MR 7, 371.

12. H. Cartan and S. Eilenberg, Homological algebra, Princeton Univ. Press, Princeton, N. J., 1956. MR 17, 1040.

13. R. Carter and P. Fong, The Sylow 2-subgroups of the finite classical groups, J. Algebra 1 (1964), 139-151. MR 29 \#3548.

14. J. Dieudonné, La géométrie des groupes classiques, Ergebnisse der Mathematik und ihrer Grenzgebiete, Heft 5, Springer-Verlag, Berlin and New York, 1955. MR 17, 236. 
15. W. Feit and J. G. Thompson, Solvability of groups of odd order, Pacific J. Math. 13 (1963), 775-1029. MR 29 \#3538.

16. P. Fong and W. Wong, A characterization of the finite simple groups $P S p(4, q), G_{2}(q)$, $D_{4}^{2}(q)$. I, Nagoya Math. J. 36 (1969), 143-184.

17. G. Glauberman, A characteristic subgroup of a p-stable group, Canad. J. Math. 20 (1968), 1101-1135. MR 37 \#6365.

18. ——, Central elements in core-free groups, J. Algebra 4 (1966), 403-420. MR 34 \#2681.

19. D. Gorenstein, Finite groups, Harper \& Row, New York, 1968. MR 38 \#229.

20. - Finite groups the centralizers of whose involutions have normal 2-complements, Canad. J. Math. 21 (1969), 335-357.

21. D. Gorenstein and J. Walter, On finite groups with dihedral Sylow 2-subgroups, Illinois J. Math. 6 (1962), 553-593. MR 26 \#188.

22. - The characterization of finite groups with dihedral Sylow 2-subgroups. I, II, III, J. Algebra 2 (1965), 85-151, 218-270, 354-393. MR 31 \#1297a, b; MR 32 \#7634.

23. J. A. Green, Blocks of modular representations, Math. Z. 79 (1962), 100-115. MR 25 \#5114.

24. K. Harada, Finite simple groups with short chains of subgroups, J. Math. Soc. Japan 20 (1968), 655-672. MR 37 \#6369.

$24^{\prime}$. - A characterization of $U_{3}(5)$, Nagoya J. Math. 38 (1970), $27-40$.

24*. M. O'Nan, $A$ characterization of $U_{3}(q), q$ odd, Doctoral Thesis, Princeton University, Princeton, N. J., 1969.

25. I. Schur, Uber eine Klasse von endlichen Gruppen linearer Substitutionen, S.-B. Preuss. Akad. Wiss. Berlin 1905, 77-91.

26. ——, Untersuchungen über die Darstellung der endlichen Gruppen durch gebrochene lineare Substitutione, Crelle J. 132 (1907), 85-137.

27. R. Steinberg, The representation of $G L(3, q), G L(4, q), P G L(3, q)$, and $P G L(4, q)$, Canad. J. Math. 3 (1951), 225-235. MR 13, 10.

28. M. Suzuki, A characterization of the 3-dimensional projective unitary group over a finite of odd characteristic, J. Algebra 2 (1965), 1-14. MR 31 \#2336.

29. J. G. Thompson, Defect groups are Sylow intersections, Math. Z. 100 (1967), 146. MR 35 \#4296.

30. W. Wong, On finite groups whose 2-Sylow subgroups have cyclic subgroups of index 2, J. Austral. Math. Soc. 4 (1964), 90-112. MR 28 \#4026.

31. H. Zassenhaus, The theory of groups, 2nd ed., Chelsea, New York, 1958. MR 19, 939.

INSTITUTe for AdVANCEd Study,

Princeton, New Jersey 08540

UNIVERSITY OF CHICAGO,

ChicAGo, IllinoIs 60637

HARVARD UNIVERSITY,

CAmbridge, Massachusetts 02138

NORTHEASTERN UNIVERSITY, Boston, MassachuSETTS 02115

Rutgers, THe STATE UNIVERSITY,

New Brunswick, NeW Jersey 08903 